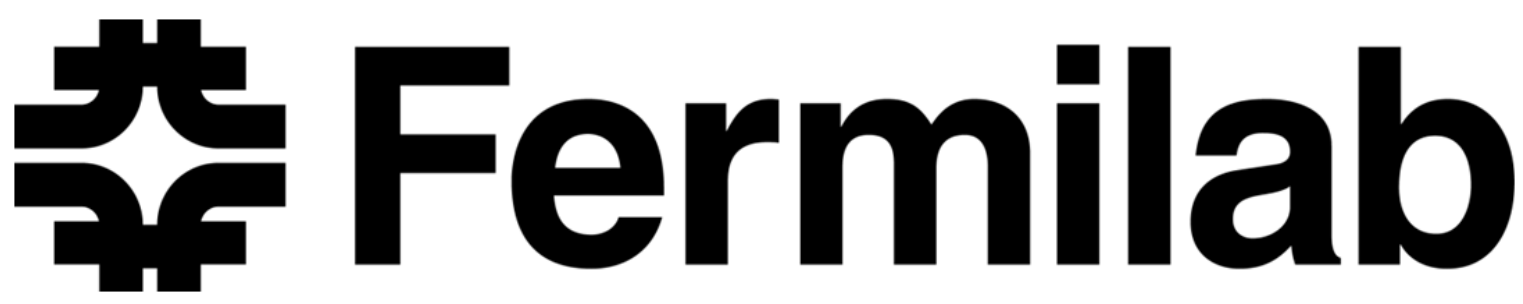

\title{
RADIATION PHYSICS FOR PERSONNEL AND ENVIRONMENTAL PROTECTION
}

FERMILAB REPORT TM-1834

Revision 13

March 2014

J. Donald Cossairt

Fermi National Accelerator Laboratory

\author{
Presented at Sessions of the \\ U. S. PARTICLE ACCELERATOR SCHOOL
}




\section{ACKNOWLEDGMENTS}

This text is dedicated to my wife Claudia and our children, Joe and Sally, who provided me with love, cheerfulness, and support during the initial preparation of these materials. I acknowledge the encouragement of John Peoples, Jr. and A. Lincoln Read to initially participate in the U. S. Particle Accelerator School. The support of my teaching in the USPAS by Mel Month, Shyh-Yuan Lee, Helmut Wiedemann, Bill Barletta, Bill Griffing, Nancy Grossman, and Martha Michels has been sincerely appreciated. Members of the Fermilab Environment, Safety, Health and Quality Section have greatly assisted me during the preparation and revision of these materials. Alex Elwyn deserves special recognition for his helpful advice during his entire distinguished career at Fermilab. In so many ways, he has been my mentor both in science and in our mutual hobby of photography. Nancy Grossman, Kamran Vaziri, Vernon Cupps, Diane Reitzner, Matthew Quinn (all of Fermilab), Reginald Ronningen (National Superconducting Cyclotron Laboratory at Michigan State University), Scott Schwahn (Oak Ridge National Laboratory), Sayed Rokni (SLAC National Accelerator Laboratory), and Vaclav Vylet (Thomas Jefferson National Accelerator Facility) have provided me with very constructive criticism in connection with their assistance in presenting these materials to students in the USPAS. Many others at Fermilab have given me good helpful comments along the way. Sam Childress of Fermilab was of special assistance with the section on beam optics. Vaclav Vylet of TJNAF and James Liu of SLAC have enriched the material on radiation fields due to electrons. This work hopefully continues the great legacy of the late Miguel Awschalom who both anticipated and attacked nearly all conceivable problems in accelerator radiation physics in addition to his considerable contribution to medical physics.

The original version of this text was presented as part of a course taught at the session of the USPAS held at Florida State University in January 1993. Subsequently, the material was further refined and presented as a course at Fermilab in the spring of 1993 and autumn of 1994. The course was offered at USPAS sessions held at Duke University (January 1995), the University of California Berkeley (January 1997), Vanderbilt University (January 1999), Rice University (January 2001), Indiana University (January 2003), the University of Wisconsin Madison (June 2004), Arizona State University (January 2006), Michigan State University (June 2007), the University of California Santa Cruz (January 2010), the University of Texas Austin (January 2012), and the University of Tennessee Knoxville (January 2014). It has also been presented as a lecture series on several occasions over the years at Fermilab and at Brookhaven National Laboratory (2005). This publication is based on the work of numerous people whose works populate the reference list. Notable among these in no particular order of prominence are Bill Swanson, Wade Patterson, Ralph Thomas, Geoffrey Stapleton, Graham Stevenson, Nikolai Mokhov, Ralph Nelson, James Liu, Vaclav Vylet, Theodore Jenkins, Keran O'Brien, Manfred Höfert, Anthony Sullivan, and Klaus Tesch.

\section{J. Donald Cossairt March 2014}




\section{PREFACE}

The advancement of particle accelerators is now well into its second century if Röntgen's $\mathrm{x}$-ray tube is correctly considered to be a particle accelerator. This field of human endeavor has achieved maturity, but not stagnation. Accelerators now pervade nearly every facet of both modern scientific research and everyday life. They are utilized in virtually all branches of science ranging from the frontiers of physics (particle, nuclear physics, atomic, and condensed matter) to engineering, chemistry, biology, geology, and the environmental sciences. Very important practical applications of accelerators are now found in many industrial applications and in agriculture. The prominent and longstanding contribution to medicine is well-known as community hospitals of moderate size now utilize accelerators extensively. This makes particle accelerators by far the type of "radiological" installation most commonly encountered by members of the public. The historical development of accelerator radiation physics has accompanied that of the machines themselves, as is well-described by Patterson and Thomas (Pa94). A stated goal of the USPAS is to provide quality education in beam physics and associated accelerator technology. It is therefore quite proper that the USPAS has included a course on accelerator radiation physics in its curriculum. Those who develop, operate, and utilize the accelerators of the future will be able to do this far more effectively if the associated radiological hazards are better understood and mitigated. To that end, the content of this textbook has been selected to address the major elements of radiation physics issues that are encountered at accelerators of all particle types and energies. Some topics not commonly thought to be within the domain of "health physics" such as charged particle optics, synchrotron radiation, hydrogeology, and meteorology are included along with the more familiar subjects that might be anticipated by the readers. The problem sets were developed to promote better understanding of the content. 
Chapter 1 Basic Radiation Physics Concepts and Units of Measurement

1.1 Introduction

1.2 Units of Measure for Physical Quantities

1.3 Units of Measure for Radiological Quantities

1.3.1 Synopsis of the 1973 Radiation Protection System

1.3.2 Synopsis of the 1990 Radiation Protection System

1.3.3 Values of Radiation Protection Quantities

1.4 Physical Constants and Atomic and Nuclear Properties 11

1.5 Summary of Relativistic Relationships 11

1.6 Energy Loss by Ionization and Multiple Coulomb Scattering 14

$\begin{array}{lll}\text { 1.6.1 Energy Loss by Ionization } & 14\end{array}$

1.6.2 Multiple Coulomb Scattering 20

$\begin{array}{lll}1.7 & \text { Radiological Standards } & 21\end{array}$

$\begin{array}{ll}\text { Problems } & 22\end{array}$

\section{Chapter 2 General Considerations of Radiation Fields at Accelerators}

2.1 Introduction 23

2.2 Primary Radiation Fields At Accelerators-General Considerations 23

$\begin{array}{lll}2.3 & \text { Theory of Radiation Transport } & 25\end{array}$

2.3.1 General Considerations of Radiation Transport 25

$\begin{array}{ll}\text { 2.3.2 The Boltzmann Equation } & 26\end{array}$

2.4 The Monte Carlo Method 28

2.4.1 General Principles of the Monte Carlo Technique 28

2.4.2 Monte Carlo Example; A Sinusoidal Angular Distribution of Beam Particles $\quad 30$

2.5 Review of Magnetic Deflection and Focusing of Charged Particles 32

2.5.1 Magnetic Deflection of Charged Particles $\quad 32$

2.5.2 Magnetic Focusing of Charged Particles 34

$\begin{array}{ll}\text { Problems } & 40\end{array}$

\section{Chapter 3 Prompt Radiation Fields Due to Electrons}

3.1 Introduction 41

3.2 Unshielded Radiation Produced by Electron Beams 41

3.2.1 Dose Rate in a Direct Beam of Electrons 41

$\begin{array}{lll}3.2 .2 & \text { Bremsstrahlung } & 42\end{array}$

$\begin{array}{lll}3.2 .3 & \text { Neutrons } & 47\end{array}$

3.2.3.1 Giant Photonuclear Resonance Neutrons 47

3.2.3.2 Quasi-Deuteron Neutrons 49

3.2.3.3 High Energy Particles $\quad 50$

$\begin{array}{lll}3.2 .4 & & 50\end{array}$

3.2.5 Summary of Unshielded Radiation Produced by Electron
Beams 


\section{TABLE OF CONTENTS}

3.3 The Electromagnetic Cascade-Introduction 52

3.4 The Electromagnetic Cascade Process 56

3.4.1 Longitudinal Shower Development 57

3.4.2 Lateral Shower Development 60

3.5 Shielding of Hadrons Produced by the Electromagnetic Cascade 62

3.5.1 Neutrons 62

3.5.2 High Energy Particles 63

3.6 Synchrotron Radiation 64

3.6.1 General Discussion of the Phenomenon 64

3.6.2 Insertion Devices 68

3.6.3 Radiation Protection Issues Specific to Synchrotron

Radiation Facilities $\quad 72$

3.6.3.1 Operating Modes $\quad 72$

3.6.3.2 Gas Bremsstrahlung - Straight Ahead 74

3.6.3.3 Gas Bremsstrahlung - Secondary Photons 75

3.6.3.4 Gas Bremsstrahlung - Neutron Production Rates 77

3.6.3.5 Importance of Ray Tracing $\quad 79$

$\begin{array}{ll}\text { Problems } & 80\end{array}$

\section{Chapter 4 Prompt Radiation Fields Due to Protons and Ions}

$\begin{array}{lll}4.1 & \text { Introduction } & 82\end{array}$

4.2 Radiation Production by Proton Accelerators 82

4.2.1 The Direct Beam; Radiation Hazards and Nuclear Interactions $\quad 82$

4.2.2 Neutrons and Other Hadrons at High Energies $\quad 82$

4.2.2.1 $E_{o}<10 \mathrm{MeV} \quad 82$

4.2.2.2 $10<E_{o}<200 \mathrm{MeV} \quad 84$

4.2.2.3 $200 \mathrm{MeV}<E_{o}<1.0 \mathrm{GeV}$; ("Intermediate" Energy) 84

4.2.2.4 $\quad E_{O}>1.0 \mathrm{GeV}$ ("High" Energy Region) 84

$\begin{array}{lll}4.2 .3 & \text { Sullivan's Formula } & 89\end{array}$

$\begin{array}{lll}\text { 4.2.4 Muons } & 90\end{array}$

4.3 Primary Radiation Fields at Ion Accelerators 92

4.3.1 Light Ions (Ion Mass Number $A<5$ ) 92

4.3.2 Heavy Ions (Ions with $A>4$ ) 92

4.4 Hadron (Neutron) Shielding for Low Energy Incident Protons $\begin{array}{ll}\left(E_{o}<15 \mathrm{MeV}\right) & 97\end{array}$

4.5 Limiting Attenuation at High Energy 98

4.6 Intermediate and High Energy Shielding-The Hadronic Cascade 100

4.6.1 The Hadronic Cascade from a Conceptual Standpoint 100

4.6.2 A Simple One-Dimensional Model 102

4.6.3 A Semiempirical Method; the Moyer Model for a Point Source 105

4.6.4 The Moyer Model for a Line Source 110

4.7 The Use of Monte Carlo Shielding Codes for Hadronic Cascades 111

4.7.1 Examples of Results of Monte Carlo Calculations 111

4.7.2 General Comments on Monte Carlo Star-to-Dose Conversions 113

4.7.3 Shielding Against Muons at Proton Accelerators 118 
Problems

\section{Chapter 5 Low Energy Prompt Neutron Radiation Phenomena}

$\begin{array}{lll}5.1 & \text { Introduction } & 127\end{array}$

5.2 Transmission of Photons and Neutrons Through Penetrations 127

$\begin{array}{ll}\text { 5.2.1 Albedo Coefficients } & 127\end{array}$

5.2.1.1 Usage of Photon Albedo Coefficients 130

5.2.2 Neutron Attenuation in Labyrinths-General Considerations 131

5.2.3 Attenuation in the First Legs of Straight Penetrations 132

5.2.4 Attenuation in Second and Successive Legs of Straight Penetrations 135

$\begin{array}{llr}\text { 5.2.5 Attenuation in Curved Tunnels } & 139\end{array}$

$\begin{array}{ll}\text { 5.2.6 Attenuation Beyond the Exit } & 140\end{array}$

$\begin{array}{ll}\text { 5.2.7 Determination of the Source Factor } & 143\end{array}$

$\begin{array}{lll}5.3 & \text { Skyshine } & 144\end{array}$

$\begin{array}{lll}\text { 5.3.1 Simple Parameterizations } & 144\end{array}$

$\begin{array}{ll}\text { 5.3.2 A More Rigorous Treatment } & 146\end{array}$

5.3.3 Examples of Experimental Verifications 149

$\begin{array}{ll}\text { Problems } & 154\end{array}$

\section{Chapter 6 Shielding Materials and Neutron Energy Spectra}

$\begin{array}{lll}6.1 & \text { Introduction } & 155\end{array}$

6.2 Discussion of Shielding Materials Commonly Used at Accelerators 155

$\begin{array}{lll}6.2 .1 & \text { Earth } & 155\end{array}$

$\begin{array}{ll}\text { 6.2.2 Concrete } & 156\end{array}$

$\begin{array}{ll}\text { 6.2.3 Other Hydrogenous Materials } & 157\end{array}$

6.2.3.1 Polyethylene and Other Materials That Can Be Borated 157

$\begin{array}{ll}\text { 6.2.3.2 Water, Wood, and Paraffin } & 158\end{array}$

$\begin{array}{lll}6.2 .4 & \text { Iron } & 158\end{array}$

6.2.5 High Atomic Number Materials (Lead, Tungsten, and Uranium) 158

6.2.6 Miscellaneous Materials (Beryllium, Aluminum, and Zirconium) 159

$\begin{array}{lll}6.3 & \text { Neutron Energy Spectra Outside of Shields } & 160\end{array}$

$\begin{array}{lll}\text { 6.3.1 General Considerations } & 160\end{array}$

6.3.2 Example of Neutron Spectra Due to Incident Electrons 160

6.3.3 Examples of Neutron Spectra Due to Low and Intermediate $\begin{array}{ll}\text { Energy Protons } & 161\end{array}$

6.3.4 Examples of Neutron Spectra Due to High Energy Protons 161

6.3.5 Leakage of Low Energy Neutrons Through Iron Shielding 169

$\begin{array}{lll}\text { 6.3.6 Neutron Spectra Due to Ions } & 172\end{array}$

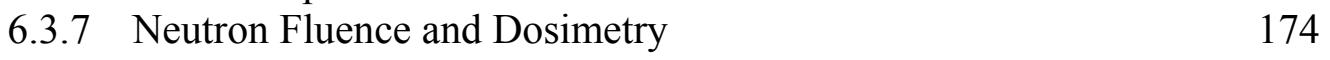

\section{Chapter 7 Induced Radioactivity in Accelerator Components}

$\begin{array}{lll}7.1 & \text { Introduction } & 176\end{array}$ 
$\begin{array}{lll}7.2 & \text { Fundamental Principles of Induced Radioactivity } & 176\end{array}$

$\begin{array}{ll}7.3 & \text { Activation of Components at Electron Accelerators } \\ & 178\end{array}$

7.3.1 General Phenomena 178

7.3.2 Results for Electrons at Low Energies 181

7.3.3 Results for Electrons at High Energies 181

7.4 Activation of Components at Proton and Ion Accelerators 184

7.4.1 General Phenomena 184

7.4.2 Methods of Systematizing Activation Due to High Energy 194

7.4.2.1 Gollon's Rules of Thumb 198

7.4.2.2 The Barbier Danger Parameter 200

7.4.3 The Utilization of Monte Carlo Star Densities in Activation
Calculations

7.4.4 Uniform Irradiation of the Walls of an Accelerator Enclosures 207 Problems

\section{Chapter 8 Induced Radioactivity in Environmental Media}

8.1 Introduction 211

8.2 Airborne Radioactivity 211

8.2.1 Production 211

8.2.2 Accounting for Ventilation 212

8.2.3 Propagation of Airborne Radionuclides in the Environment 214

8.2.3.1 Propagation of Airborne Radioactivity-Tall Stacks 215

8.2.3.2 Propagation of Airborne Radioactivity-Short Stacks 217

8.2.4 Radiation Protection Standards for Airborne Radioactivity 218

8.2.5 Production of Airborne Radionuclides at Electron Accelerators $\quad 220$

8.2.6 Production of Airborne Radionuclides at Proton Accelerators 223

$\begin{array}{lll}8.3 & \text { Water and Geological Media Activation } 224\end{array}$

8.3.1 Water Activation at Electron Accelerators 224

8.3.2 Water and Geological Media Activation at Proton Accelerators 225

8.3.2.1 Water Activation at Proton Accelerators 225

8.3.2.2 Geological Media Activation 225

8.3.3 Regulatory Standards 228

8.3.4 The Propagation of Radionuclides Through Geological Media 230

Problems

\section{Chapter 9 Radiation Protection Instrumentation at Accelerators}

$\begin{array}{lll}9.1 & \text { Introduction } & 239\end{array}$

$\begin{array}{lll}9.2 & \text { Counting Statistics } & 239\end{array}$

9.3 Special Considerations for Accelerator Environments 241

9.3.1 Large Range of Flux Densities, Absorbed Dose Rates, etc. 241

9.3.2 Possible Large Instantaneous Values of Flux Densities, Absorbed Dose Rates, etc. 241

9.3.3 Large Energy Domain of Neutron Radiation Fields 242 
9.3.4 Presence of Mixed Radiation Fields 242

9.3.5 Directional Sensitivity 242

9.3.6 Sensitivity to Features of the Accelerator Environment Other Than Ionizing Radiation $\quad 242$

9.4 Standard Instruments and Dosimeters 243

9.4.1 Ionization Chambers 243

9.4.2 Geiger-Müller Detectors 247

9.4.3 Thermoluminescent Dosimeters (TLDs) 247

9.4.4 Nuclear Track Emulsions 248

9.4.5 Track Etch Dosimeters 249

9.4.6 CR-39 Dosimeters 249

9.4.7 Bubble Detectors 249

9.5 Specialized Detectors 250

9.5.1 Thermal Neutron Detectors 250

9.5.1.1 Boron-10 251

9.5.1.2 Lithium-6 252

9.5.1.3 Helium-3 253

9.5.1.4 Cadmium 254

9.5.1.5 Silver 254

9.5.2 Moderated Neutron Detectors 254

9.5.2.1 Spherical Moderators, Bonner Spheres, and Related Detectors 255

9.5.2.2 Long Counters 263

9.5.3 Activation Detectors 264

9.5.4 Fission Counters 267

9.5.5 Proton Recoil Counters 268

9.5.6 TEPCs and LET Spectrometry 269

9.5.7 The Recombination Chamber Technique 270

9.5.8 Counter Telescopes 274

Problems 276

Appendix A Summary Descriptions of Commonly Used Monte Carlo Codes 278

Appendix B Examples of Results of Star Density Calculations 282

B.1 Results for Solid CONCRETE Cylinders 283

B.2 Results for Solid IRON Cylinders 285

B.3 Results for 1.0 TeV Protons Incident on Magnets and Pipes 287

B.4 Results for $120 \mathrm{GeV}$ Protons Incident on Magnets and Pipes 289

References $\quad 292$

$\begin{array}{ll}\text { Index } & 310\end{array}$ 


\section{Chapter 1 Basic Radiation Physics Concepts and Units of Measurement}

\subsection{Introduction}

Our study begins with a review of the standard terminology of radiation physics. The most important physical and radiological quantities and the system of units used to measure them are introduced. Due to its importance at most accelerators, the results of the special theory of relativity are reviewed. The energy loss by ionization along with the multiple Coulomb scattering of charged particles is also summarized.

\subsection{Units of Measure for Physical Quantities}

In order to develop an understanding of accelerator radiation physics, it is necessary to introduce the quantities of importance and the units by which they are measured. Over the years various systems of units have been employed. Presently in the United States there is a slow migration toward the use of the Système Internationale (SI) commonly employed elsewhere. This requires the practitioner to understand the interconnections of the units, both "customary" and SI, due to the diversity found in both the scientific literature and government regulation. As always in technical work the wise practitioner conducts a careful unit analysis of all calculations to assure meaningful results.

A number of quantities commonly used in physics are relevant to the subdiscipline of radiation physics. The unit of measure of the energy of particles is nearly always the electron volt $(\mathrm{eV})$. This choice is usually much more convenient than the use of the Joule, the SI unit of energy. $1.0 \mathrm{eV}$ is the kinetic energy of a particle carrying one electron's worth of electric charge (positive or negative) after acceleration through an electric potential of one volt. It is equal to $1.602 \times 10^{-12} \mathrm{ergs}$ or $1.602 \times 10^{-19}$ Joules. Multiples in common use at accelerators are the $\mathrm{keV}\left(10^{3} \mathrm{eV}\right), \mathrm{MeV}\left(10^{6} \mathrm{eV}\right), \mathrm{GeV}\left(10^{9} \mathrm{eV}\right)$, and TeV $\left(10^{12} \mathrm{eV}\right)$. Nearly always in discussions of particles at accelerators, the "energy" of an accelerated particle refers to its kinetic energy (see Section 1.5).

One needs to be able to describe the number of particles that transverse a unit area per unit time. This is called the flux density and is generally denoted by the symbol $\phi$;

$$
\phi=\frac{d^{2} n}{d A d t},
$$

where $d^{2} n$ is the differential number of particles crossing surface area element $d A$ during time $d t$. For radiation fields where the particles move in a multitude of directions rather than in a parallel or nearly parallel beam, $\phi$ is similarly related to the number crossing a sphere of cross-sectional area $d A$ per unit time. The units of flux density are $\mathrm{cm}^{-2} \mathrm{~s}^{-1}$ or $\mathrm{m}^{-}$ ${ }^{2} \mathrm{~S}^{-1}$ (SI) with other units of time (e.g., hours, minutes, days, years) often encountered. The fluence $\Phi$, the number of particles that cross such a surface area element during some time interval, $t_{i}<t<t_{f}$, is simply the time integral of the flux density,

$$
\Phi=\int_{t_{i}}^{t_{f}} d t \phi(t)
$$




\section{Chapter 1 Basic Radiation Physics Concepts and Units of Measurement}

The units of fluence are, of course, inverse area (e.g., $\left.\mathrm{cm}^{-2}, \mathrm{~m}^{-2}\right)$.

When particles interact with matter, the cross section $\sigma$ is an extremely important quantity. It represents the effective "size" of the atom or nucleus for some particular interaction. Consider a beam of particles of fluence $\Phi$ (particles $\mathrm{cm}^{-2}$ ) incident on a thin slab of absorber of thickness $d x$. The absorbing medium has $N$ atoms $\mathrm{cm}^{-3}$. The number of incident particles that interact and are "lost" from the original fluence $-d \Phi$ is given by

$$
-d \Phi=\sigma N \Phi d x
$$

$\sigma$ is most commonly measured in units of $\mathrm{cm}^{2} . N=\rho N_{A} / A$, where $\rho$ is the material density $\left(\mathrm{g} \mathrm{cm}^{-3}\right), N_{A}$ is Avogadro's number (6.02x1023 atoms g-mole-1, see Table 1.1), and $A$ is the atomic weight (i.e., the mass number). Cross sections are often tabulated in units of barns. 1.0 barn is $10^{-24} \mathrm{~cm}^{2}$. Submultiples such as the $\mathrm{mb}\left(10^{-3} \mathrm{barn}, 10^{-27} \mathrm{~cm}^{2}\right)$ are in common use. If only one physical process is present and if one starts with an initial fluence $\Phi_{o}$, after some distance $x(\mathrm{~cm})$ of material a simple integration reveals the fluence $\Phi(x)$ to be

$$
\Phi(x)=\Phi_{o} e^{-N \sigma x}
$$

Exponential attenuation, ubiquitous in radiation physics, can be characterized by the linear absorption coefficient $\mu$. It and its reciprocal the attenuation length $\lambda$ are given by

$$
\mu=N \sigma\left(\mathrm{cm}^{-1}\right) \text { and } \lambda=1 / N \sigma(\mathrm{cm}) \text {. }
$$

Alternatively, the mass attenuation length $\lambda_{m}=\rho / N \sigma\left(\mathrm{g} \mathrm{cm}^{-2}\right)$ is used where $\rho$ is the density $\left(\mathrm{g} \mathrm{cm}^{-3}\right)$. Some authors use $\lambda$ for $\lambda_{m}$; thus care with units is always needed. The term interaction length for this quantity is commonly used for high energy hadrons.

\subsection{Units of Measure for Radiological Quantities}

Absorbed dose, usually denoted $D$, is the energy absorbed per unit mass of material. It is a purely physical quantity, one that is directly measurably at least in principle. The customary unit of absorbed dose is the rad while the SI unit is the Gray (Gy). $1.0 \mathrm{rad}$ is defined to be 100 ergs $\operatorname{gram}^{-1}\left(6.24 \times 10^{13} \mathrm{eV} \mathrm{g}^{-1}\right)$. One Gray (Gy) is defined as $1.0 \mathrm{~J} \mathrm{~kg}^{-1}$ (100 rads). Thus $1.0 \mathrm{~Gy}=6.24 \times 10^{15} \mathrm{eV} \mathrm{g}^{-1}$. The concept of absorbed dose is applicable to any material and is also used for some forms of nonionizing radiation. It is commonly used to quantify both radiation exposures to human beings and to quantify potential radiation damage in the delivery of energy to materials and equipment components.

Radiation protection would be a very simple indeed if the deleterious effects of ionizing radiation were correlated in a very simple way, ideally linearly, with absorbed dose. Unfortunately, the results of a large body of science support the conclusion that these effects are also correlated with the types of particles and their energies. This correlation, a 
topic of ongoing scientific research, is known to be complex and remains incompletely resolved. In order to meet the pressing needs of assuring workplace and environmental radiation safety, approximations have been developed and special dosimetric quantities and units invented. The definition and usage of these quantities has evolved with time along with the subfield of accelerator radiation protection as the experts in the field represented principally by the International Commission on Radiation Protection (ICRP), the International Commission on Radiation Units and Measurements (ICRU), and in the United States the National Council on Radiation Protection and Measurements (NCRP) have worked to improve the system of radiological protection. Thus, in reading archival scientific literature specifically on accelerator radiation protection, one finds that older papers sometimes utilize slightly different concepts and units than do more modern ones.

The ongoing development of this topic is rather complicated and mostly beyond the scope of this text. Using the publications of ICRP as time markers, the older system was established by about 1973 (IC73) and will here be called the 1973 Radiation Protection System (1973 System). The more current system was initially announced in 1990 in ICRP Publication 60 (IC91) and is here called the 1990 Radiation Protection System (1990 System). The refinement of the 1990 System has continued in ICRP Publications 74 (IC96) and 103 (IC07). In addition to external radiation fields of major interest here, these systems also address internal radiation exposures due to the uptakes of radionuclides, here germane only to a small part of the content of Chapters 7 and 8. While the 1990 System is fully adopted outside the United States, its use within the U. S. can be said to be "piecemeal". As of 2011 the 1990 System now applies to radiation protection at U. S. Department of Energy facilities [(CFR07), (DOE11)] while the 1973 System remains in use by the U. S. Nuclear Regulatory Commission and by the Occupational Health and Safety Administration. The U. S. Environmental Protection Agency uses a mixture of the two systems as of 2013.

\subsubsection{Synopsis of the 1973 Radiation Protection System}

In the 1973 System dose equivalent $H_{\text {equiv }}$ is used to take into account the fact that different particle types have biological effects that are enhanced, per given absorbed dose, over those due to the standard reference radiation taken to be $200 \mathrm{keV}$ photons. This quantity has the same physical dimensions as absorbed dose. The customary unit of measure is the rem while the SI unit is the Sievert (Sv) (1.0 Sv=100 rem). The concept of dose equivalent is applied only to radiation exposures received by human beings. The dimensionless quality factor, usually denoted by $Q$, is used to reflect this enhancement by connecting $H_{\text {equiv }}$ with $D$ through

$$
H_{\text {equiv }}=Q D \text {. }
$$

Thus, $H_{\text {equiv }}(\mathrm{rem})=Q D$ (rads) or $H_{\text {equiv }}(\mathrm{Sv})=Q D(\mathrm{~Gy}) . Q$ is dependent on both particle type and energy and for any radiation field its value is an average over all components. It is formally defined to have a value of unity for $200 \mathrm{keV}$ photons (Ce69). In the 1973 System, $Q$ ranges from unity for photons, electrons of most energies, and high energy muons to a value as large as 20 for $\alpha$-particles (i.e., ${ }^{4} \mathrm{He}$ nuclei) of a few $\mathrm{MeV}$ in kinetic energy. For 
neutrons, $Q$ ranges from 2 to greater than 10 in the 1973 system. $Q$ is defined to be a function of linear energy transfer (LET) $L$. LET is approximately equivalent to the stopping power, or rate of energy loss for charged particles and is conventionally expressed in units of $\mathrm{keV} \mathrm{\mu m}^{-1}$ (see Section 1.6). All ionizing radiation ultimately manifests itself through charged particles so LET is a good measure of localized radiation damage to materials not limited to biological structures.

For the common situation where a spectrum of energies and a mixture of particle types is present, the value of $Q$ for the complete radiation field is an average over the spectrum of LET present weighted by the absorbed dose as a function of LET, $D(L)$;

$$
\langle Q\rangle=\frac{\int_{0}^{\infty} d L Q(L) D(L)}{\int_{0}^{\infty} d L D(L)} .
$$

The dose equivalent per fluence conversion factor for various particles $P_{\text {equiv. }}$, as a function of energy, is a very important quantity quite useful in practical work. It is the connection between the fluence and the dose equivalent;

$$
H_{\text {equiv }}=P_{\text {equiv }} \Phi
$$

Values of $P_{\text {equiv }}$ as well as the relationship between $Q$ and LET consistent with the 1973 System for neutron radiation fields were published by the National Council on Radiation Protection and Measurements in NCRP Report 38 (NC71).

\subsubsection{Synopsis of the 1990 Radiation Protection System}

The 1990 System revises the details, but not the character, of this system in an attempt to more accurately incorporate results from radiobiology. For example, a more complicated model is used to better reflect the role of exposures to individual organs in human radiation risk. Thus several quantities in addition to $H_{\text {equiv }}$ have been defined as the metric of radiation detriment. These quantities are also applicable only to exposures to humans.

Since these quantities are encountered in the scientific literature, it is relevant to define them here. Ambient dose equivalent $H_{a m b}(d)\left(\mathrm{J} \mathrm{kg}^{-1}\right.$, i.e., Sv) is the dose equivalent, measured at each point in a radiation field that would be produced in the corresponding expanded and aligned field in the ICRU sphere ${ }^{1}$ at depth $d$ on the radius opposing the direction of the aligned field. Personal dose equivalent $H_{\text {pers }}(d)\left(\mathrm{J} \mathrm{kg}^{-1}\right.$, i.e., Sv) is the equivalent dose in soft tissue defined at depth, $d$, below a specified point in the body. For the primary concern of whole body exposures, $d$ is taken here to be $10 \mathrm{~mm}$ for both of these

\footnotetext{
${ }^{1}$ The ICRU sphere, a mathematical construct developed as a surrogate for the human body by the ICRU, has a diameter of $0.3 \mathrm{~m}$, a density of $1.0 \mathrm{~g} \mathrm{~cm}^{-3}$ and a "tissue equivalent" elemental composition of $76.2 \%$ oxygen, $11.1 \%$ carbon, $10.1 \%$ hydrogen, and $2.6 \%$ nitrogen. "Expanded" means the radiation field encompasses the sphere and "aligned" means that the measurement is independent of the angular distribution of the radiation field $[(\mathrm{Sa} 95),(\mathrm{Ka} 08)]$.
} 
quantities. Equivalent dose $E_{\text {equiv }}\left(\mathrm{J} \mathrm{kg}^{-1}\right.$, i.e., $\left.\mathrm{Sv}\right)$ is the absorbed dose in an organ or tissue multiplied by the relevant radiation weighting factor $w_{R}$ analogous to $Q$ above and discussed in more detail later. Effective dose $H_{\text {eff }}\left(\mathrm{J} \mathrm{kg}^{-1}\right.$, i.e., Sv) is the summation of equivalent doses in tissues or organs each multiplied by the appropriate organ weighting factors specified in the ICRP Publications cited. $H_{\text {eff }}$ is also the sum of all absorbed doses weighted by radiation weighting factors and by the correct organ weighting factors of the entire body. Where relevant it can include internal dose from uptakes of radioactive materials. These quantities are of two types. Operational quantities such as $H_{a m b}(d)$ and $H_{\text {pers }}(d)$ are measurable, at least in principle, and can be used to determine the properties of radiation fields to estimate and demonstrate compliance with specified standards. Protection quantities such as $H_{\text {eff }}$ and $E_{\text {equiv }}$ are used to determine conformance with numerical limits and action levels published in radiation protection standards. They are theoretical, not directly measurable.

Given our primary interest here in external radiation fields generated by particle accelerators, $H_{\text {eff }}$ is selected as the metric of radiation detriment in this book under the 1990 System. Thus, one is interested in the effective dose per fluence $P_{\text {eff }}$ as a function of particle energy as used in

$$
H_{e f f}=P_{e f f} \Phi
$$

This choice is confounded by an additional complexity. The use of the dosimetry quantities requires a selection of exposure geometry from standardized models. These models specify the orientation of the exposed person relative to the radiation source, a condition likely undefined in a typical workplace or environmental setting. Two of the models, called ROT and ISO, appear to best match workplace conditions at accelerators. ROT (rotational) geometry is defined to be that where the body is irradiated by a parallel beam of ionizing radiation from a direction orthogonal to the long axis of the body rotating at a uniform rate about its long axis (IC96). While this "shish kebab" picture is preferable to the alternatives that involve a static orientation, it is clearly imperfect. In ISO (isotropic) geometry the fluence per unit solid angle is independent of direction (IC96). Using results available from the literature based on ROT (preferred) and ISO (when values for ROT were unavailable) Cossairt and Vaziri (Co09a) have provided values of $P_{\text {eff }}$ for neutrons from "thermal" energies to the energies of the accelerated beams. The importance of neutron radiation fields at accelerators will be justified in subsequent chapters.

The 1990 System includes radiation weighting factors $w_{R}$ used to connect absorbed dose to the protection quantity $H_{\text {eff }}$ by replacing $Q$ with $w_{R}$ and $H_{\text {equiv }}$ with $H_{\text {eff }}$ in Eq. (1.6). As with $Q$, a value of unity for $w_{R}$ is tied to the radiobiological effects of low energy photons $(\approx 200 \mathrm{keV})$ (IC07). In general, the values of $w_{R}$ in the 1990 System are larger than those of $Q$ for the same radiation field. However, the most recent ICRP guidance (IC07) has served to reduce some of this increase in $w_{R}$ for some energy domains of neutrons. In contrast, for most neutron energies, the values of $P_{\text {eff }}$ are less than those for $P_{\text {equiv }}$. Readers should note that people continue to refer to "quality factors" and "effective quality factors" and use the symbol $Q$. In this system the effective quality factor of a given radiation field is still be 


\section{Chapter 1 Basic Radiation Physics Concepts and Units of Measurement}

determined with Eq. (1.7). Furthermore, the use of terminology is not always precise as practitioners sometimes even refer to $H_{\text {eff }}$ as "dose equivalent". It is also common to see the symbol $H$ without subscripts, forcing the reader to precisely identify the system or quantity being used. Clearly, the system of radiation protection dosimetry begs for simplicity and stability, a problem understood by the ICRP and the NCRP! Fortunately the differences are small in many practical circumstances where averages over a large domain of particle energies are involved. Thus, it is often not important to distinguish between the 1973 and 1990 Systems $^{2}$.

\subsubsection{Values of Radiation Protection Quantities}

Fig. 1.1 gives the relationship between $Q$ and LET in the 1973 and 1990 Systems. Fig. 1.2 provides values of $Q$ in the 1973 System as a function of particle energy for a variety of particles and energies. The results shown in Fig. 1.2 are based upon ionization due to the primary particles only. For particles subject to the strong (or nuclear) interaction, the inclusion of secondary particles produced in numbers that increase as a function of energy results in larger values of $Q$ at higher energy. (The distinction between primary and secondary particles will be clarified in subsequent chapters.) For example, under the 1973 System, with secondary particles included, the value of $Q$ for protons rises with energy to a value of 1.6 at $400 \mathrm{MeV}$ and 2.2 at $2000 \mathrm{MeV}$ (Pa73). Fig. 1.3 gives the values of quality $Q$ and radiation weighting factors $w_{R}$ for neutrons as a function of energy in two systems.

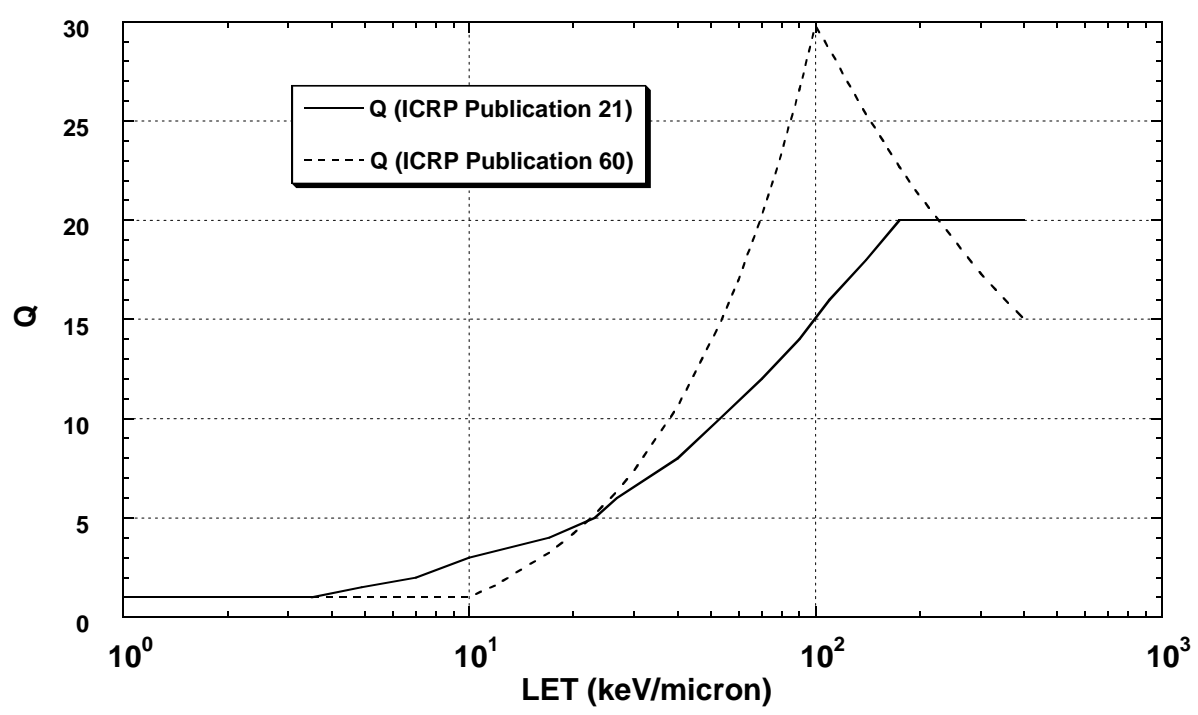

Fig. 1.1 Quality Factor $Q$ of charged particles as a function of collision stopping power (LET) in water as recommended by the ICRP in Publication 21 [1973 System (IC73)] and later as revised in Publication 60 [1990 System (IC91)].

\footnotetext{
${ }^{2}$ Other references including the ICRP reports cited commonly use more esoteric, less intuitive symbols for the dosimetric quantities. These are: $H$ for dose equivalent, $H^{*}(d)$ for ambient dose equivalent, $H_{p}(d)$ for personal dose evivalent, $E$ for effective dose, and $H_{T, R}$ for equivalent dose. In particular, this author has avoided the use of $E$ for effective dose because of the obvious confusion with its common association with energy in the physical sciences!
} 


\section{Chapter 1 Basic Radiation Physics Concepts and Units of Measurement}

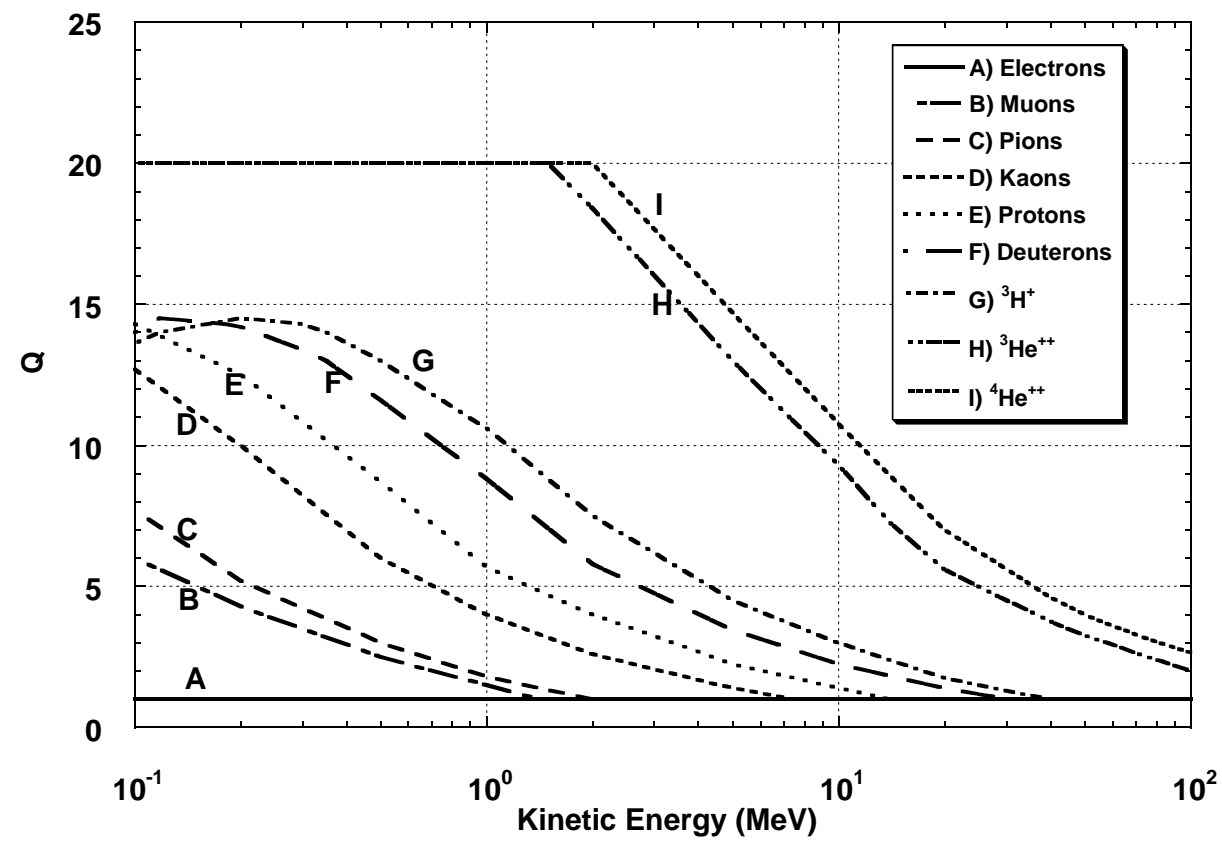

Fig. 1.2 Quality factors $Q$ of several types of charged particles as a function of energy, as recommended by the ICRP according to the 1973 System. [Adapted from (IC73)].

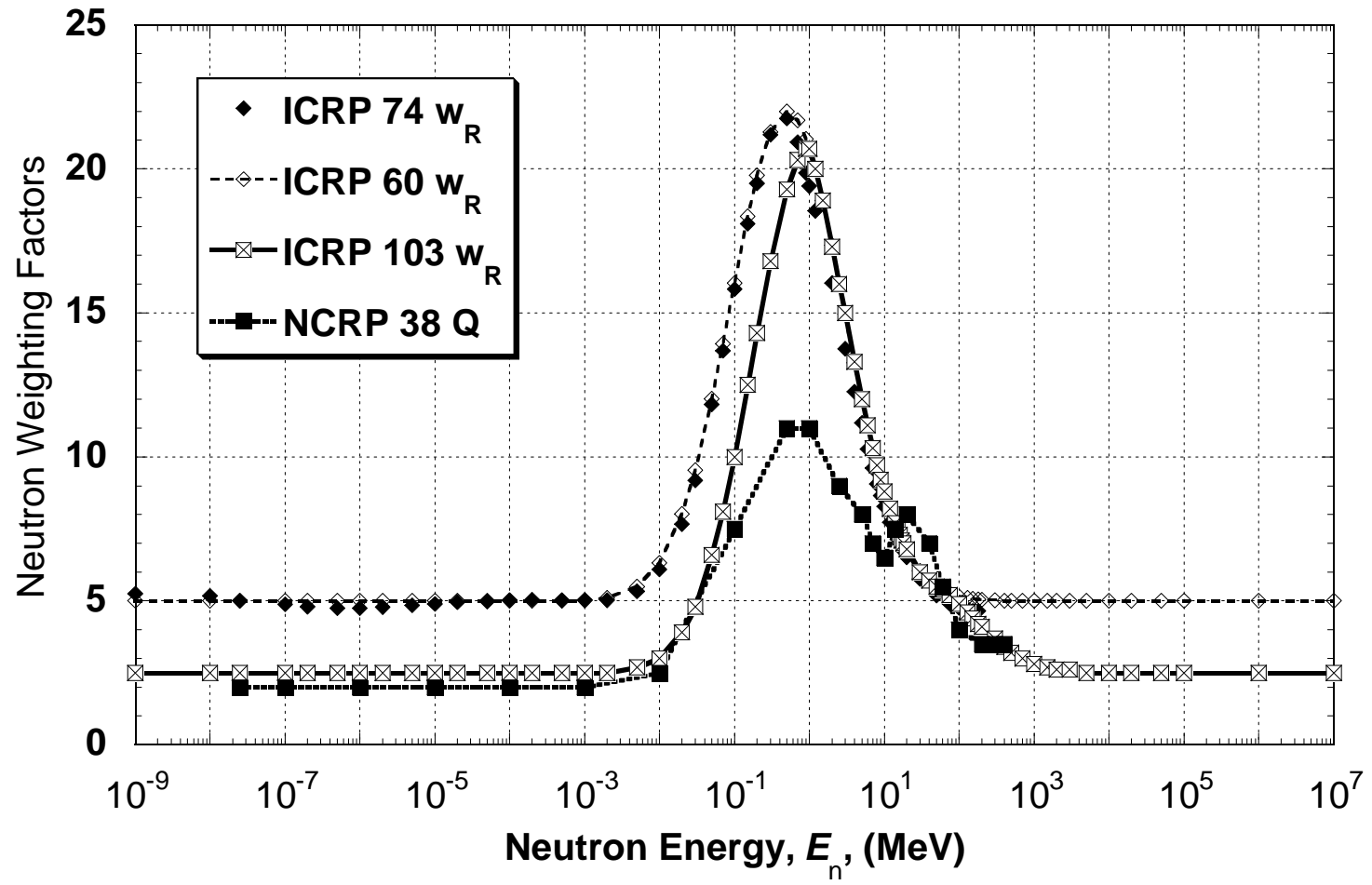

Fig. 1.3 Radiation weighting factors for neutrons as a function of neutron kinetic energy. "NCRP $38 Q$ " values are those of the 1973 System and were obtained from (NC71). Those labeled "ICRP 60 $w_{R}$ ", "ICRP $74 w_{R}$ ", and "ICRP $103 w_{R}$ " are found in (IC91), (IC96), and (IC07), respectively. [Adapted from (Co09a).] 
Dose per fluence factors $P$ are often more useful than are radiation weighting or quality factors in practical work. Fig. 1.4 provides dose equivalent per fluence values for a representative sample of charged particles over a wide range of energies according to the 1990 System. Muons, as will be seen later, are of particular importance at high energy accelerators. For these particles, the dose equivalent per fluence $P$ has been found by Stevenson (St83) to be about $40 \mathrm{fSv} \mathrm{m}^{2}\left(4.0 \times 10^{-4} \mu \mathrm{Sv} \mathrm{cm}{ }^{2}\right)$ for $100 \mathrm{MeV}<E_{\mu}<200 \mathrm{GeV}$. This is equivalent to $2.5 \times 10^{4}$ muons $\mathrm{cm}^{-2} \mathrm{mrem}^{-1}$. At lower energies range-out of muons in the human body with consequential higher energy deposition (see Section 1.6) gives a dose per fluence factor of $260 \mathrm{fSv} \mathrm{m}{ }^{2}$ (equivalent to 3850 muons $\mathrm{cm}^{-2} \mathrm{mrem}^{-1}$ ). Fig. 1.5 provides dose per fluence factors for neutrons and photons. In principle, they can be calculated for any particle. The behavior for other particles is similar to that of neutrons. Representative results have been documented by the Pelliccioni Group [(Fe96), (Fe97a), (Fe97b), (Fe97c), and (Pe00)] As an example of this for more "exotic" particles possibly of importance at future accelerators, Fig. 1.6 gives values of $P$ for muon neutrinos, $v_{\mu}$ 's, [(Co97), (Mo99)].

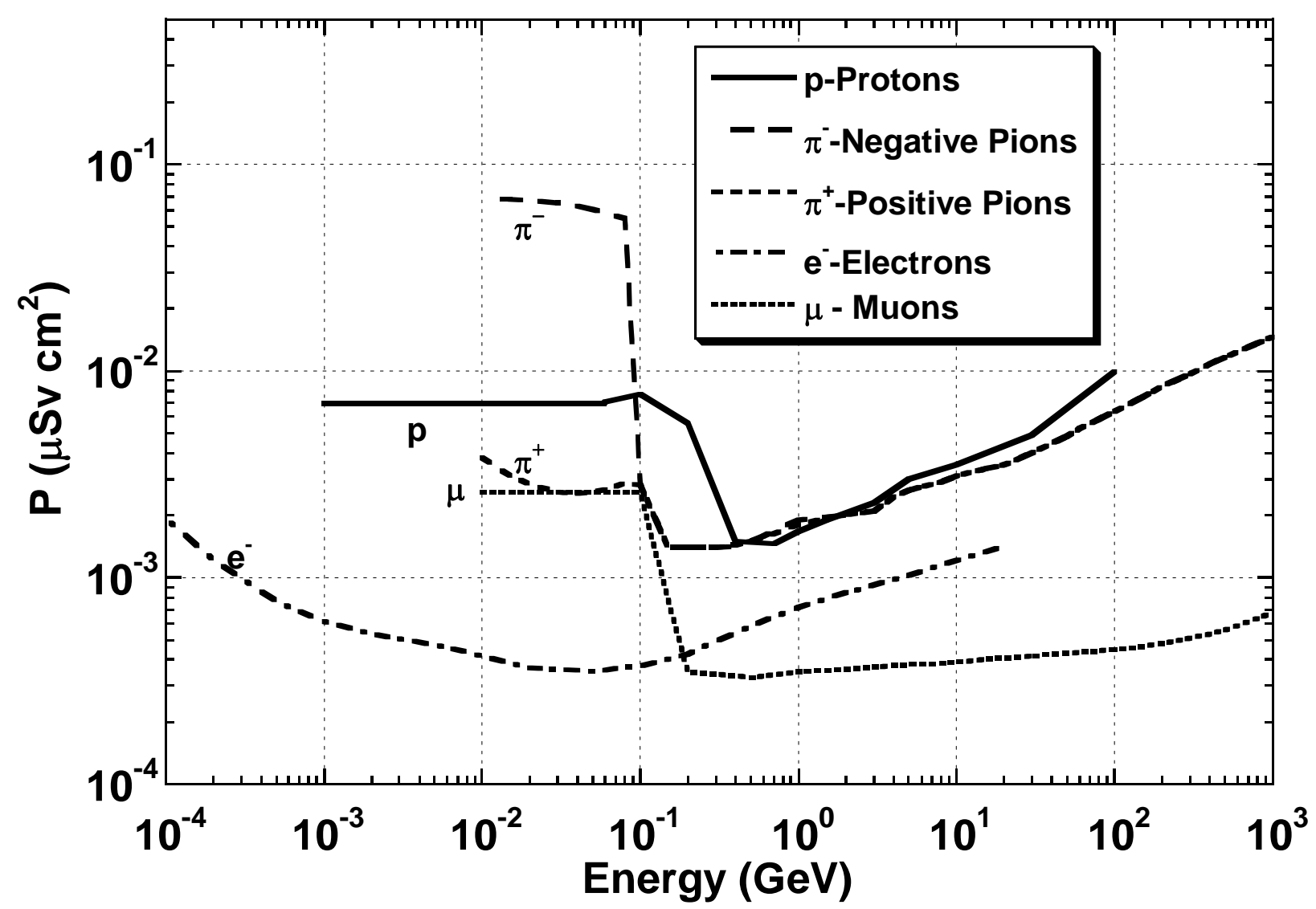

Fig. 1.4 Dose equivalent per fluence for various charged particles $P$ as a function of energy. The curve for muons is valid for both negative and positively-charged muons. [Adapted from (Fa90).] 


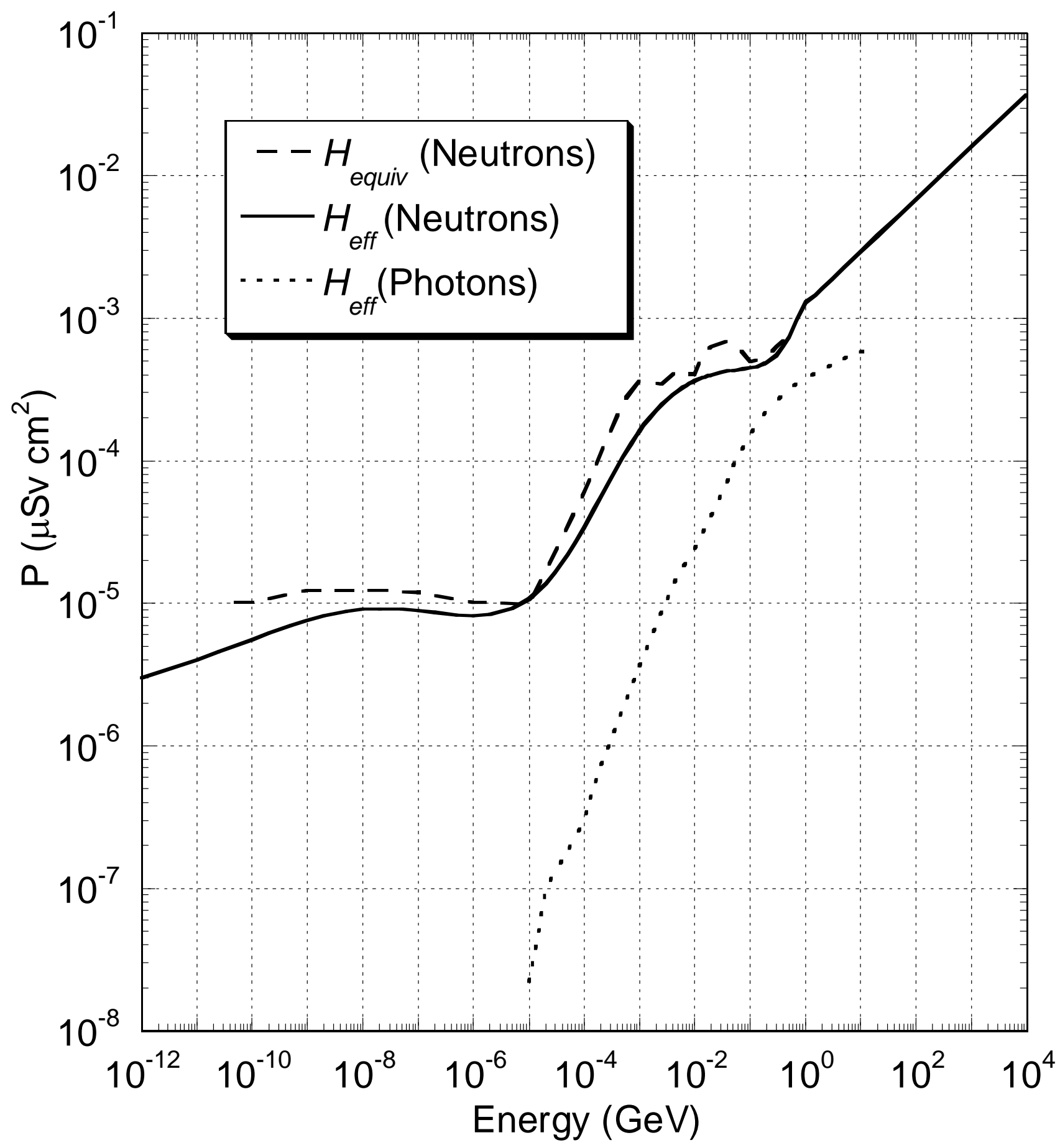

Fig. 1.5 Dose per fluence factors for photons and neutrons $P$ as a function of energy. [Adapted from $(\mathrm{Co} 09 \mathrm{a})$ and $(\mathrm{Fa} 90)$.] 


\section{Chapter 1 Basic Radiation Physics Concepts and Units of Measurement}

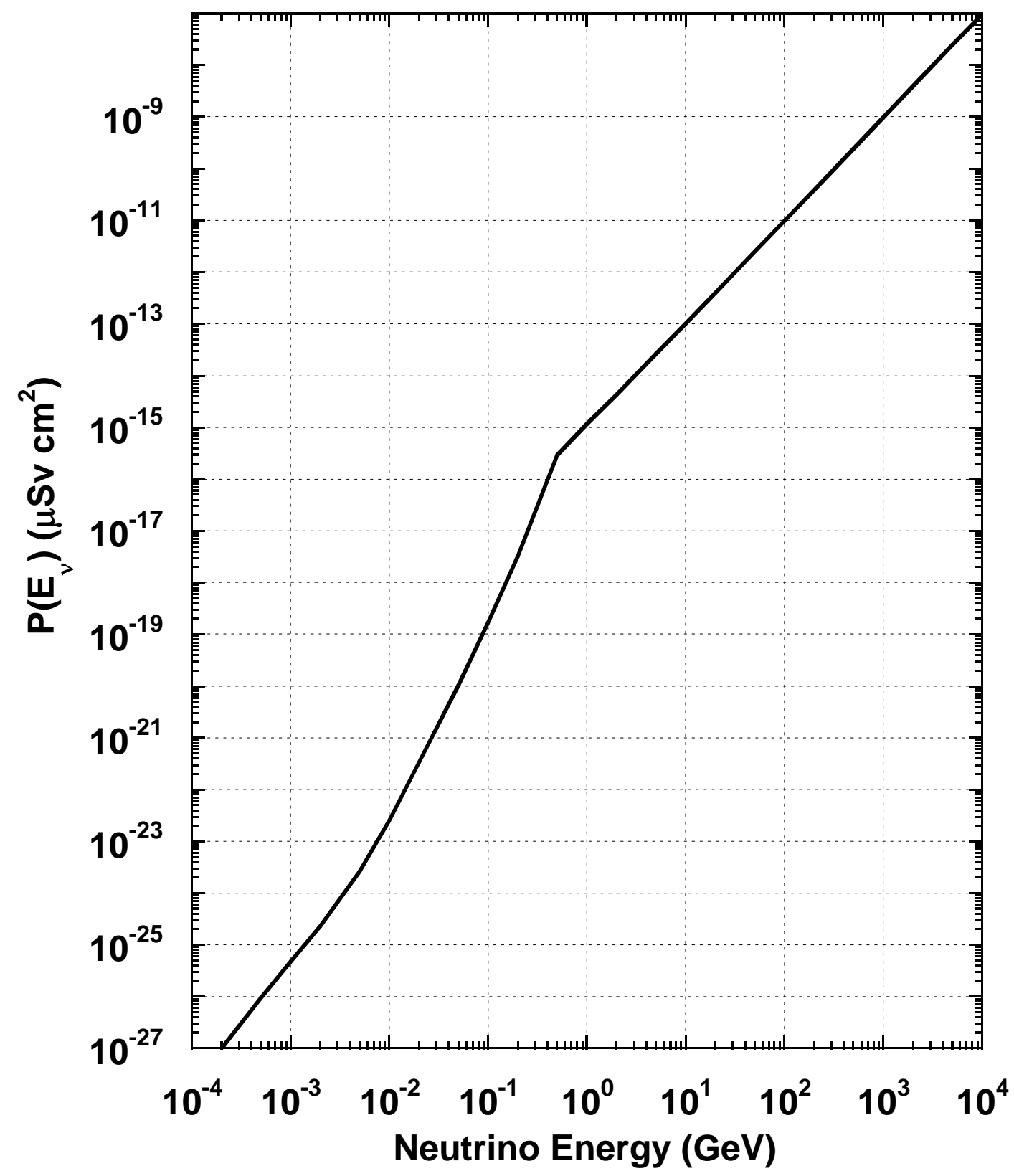

Fig. 1.6 Dose equivalent per fluence factors for muon neutrinos $\left(v_{\mu}\right) P$ as a function of energy. [Adapted from (Co97).]

For a radiation field containing a mixture of $n$ different components (e.g., different particle types), one determines the dose equivalent $H$ (or $H_{\text {eff }}$ in the 1990 System) by

$$
H=\sum_{i=1}^{n} \int_{E_{\min }}^{E_{\max }} d E P_{i}(E) \Phi_{i}(E),
$$

where $\Phi_{i}(E)$ is the fluence of particles of type $i$ with energy between $E$ and $d E$ and $P_{i}(E)$ is the dose per fluence. In the remainder of this text, unless otherwise specified, the term dose equivalent will be used for the radiation-weighted quantity. 


\section{Chapter 1 Basic Radiation Physics Concepts and Units of Measurement}

\subsection{Physical Constants and Atomic and Nuclear Properties}

Tables 1.1 and 1.2 give physical constants and atomic and nuclear properties as tabulated by the Particle Data Group (PDG04) ${ }^{3}$. The content of these tables is used throughout the rest of this text and in the problem sets. Most of these quantities will be discussed subsequently in more detail.

\subsection{Summary of Relativistic Relationships}

The results of the special theory of relativity are evident at nearly all accelerators. The rest energy $W_{O}$ of a particle of rest mass $m_{o}$ is given by

$$
W_{o}=m_{o} c^{2}
$$

where $c$ is the velocity of light. The total energy in free space $W$ is given by

$$
W=m c^{2}=\frac{m_{o} c^{2}}{\sqrt{1-\beta^{2}}}=\gamma m_{o} c^{2}, \text { with } \gamma=\frac{W}{W_{o}}=\frac{1}{\sqrt{1-\beta^{2}}}
$$

where $\beta=v / c$ and $v$ is the velocity of the particle in a given frame of reference. The relationship between the quantities $\beta$ and $\gamma$ is obvious. Similarly, the relativistic mass $m$ of a particle moving at velocity $\beta$ is given by

$$
m=\frac{m_{o}}{\sqrt{1-\beta^{2}}}=\gamma m_{o}
$$

The kinetic energy $E$ is $\quad E=W-W_{o}=\left(m-m_{o}\right) c^{2}$ and

$$
\beta=\sqrt{1-\left(\frac{W_{0}}{W}\right)^{2}}
$$

the momentum $p$ of a particle in terms of its relativistic mass $m$ and velocity $v$ is;

$$
p=m v=\gamma m_{o} \beta c=\frac{W m_{o}}{m_{o} c^{2}}\left[\sqrt{1-\left(\frac{W_{o}}{W}\right)^{2}}\right] c=\frac{1}{c}\left[\sqrt{W^{2}-W_{o}^{2}}\right]=\frac{1}{c} \sqrt{E\left(E+2 W_{o}\right)}
$$

so that at high energies $\left(E>>W_{o}\right), p \approx E / c \approx W / c$ while at low energies $\left(E<<W_{o}\right)$ one has the familiar nonrelativistic $p^{2} \approx 2\left(W_{o} / c^{2}\right) E=2 m_{o} E$.

\footnotetext{
${ }^{3}$ The Particle Data Group based at the Lawrence Berkeley National Laboratory maintains many tabulations on its website which are regularly updated. The reference list entry for (PDG04) provides the web link to this important source of information.
} 


\section{Chapter 1 Basic Radiation Physics Concepts and Units of Measurement}

Table 1.1 Physical constants. [Adapted from (PDG04).]

\begin{tabular}{|c|c|c|}
\hline Quantity & Symbol, Equation & Value $^{\text {a,b }}$ \\
\hline speed of light & $c$ & $2.99792458 \times 10^{8} \mathrm{~m} \mathrm{~s}^{-1}$ \\
\hline Planck constant & $h$ & $6.6260693(11) \times 10^{-34} \mathrm{~J} \mathrm{~s}$ \\
\hline Planck constant, reduced & $\hbar=h / 2 \pi$ & $\begin{array}{l}1.05457168(18) \times 10^{-34} \mathrm{~J} \mathrm{~s} \\
=6.58211915(56) \times 10^{-22} \mathrm{MeV} \mathrm{s}\end{array}$ \\
\hline electron charge & $\bar{e}$ & $\begin{array}{l}1.60217653(14) \times 10^{-19} \mathrm{C} \\
=4.80320441(41) \times 10^{-10} \mathrm{esu}\end{array}$ \\
\hline useful constant & $\hbar c$ & $197.326968(17) \mathrm{MeV} \mathrm{fm}$ \\
\hline useful constant & $(\hbar c)^{2}$ & $0.389379323(67) \mathrm{GeV}^{2}$ mbarn \\
\hline electron mass & $m_{e}$ & $\begin{array}{l}0.510998918(44) \mathrm{MeV} / \mathrm{c}^{2} \\
=9.1093826(16) \times 10^{-31} \mathrm{~kg}\end{array}$ \\
\hline proton mass & $m_{p}$ & $\begin{array}{l}938.272029(80) \mathrm{MeV} / \mathrm{c}^{2} \\
=1.67262171(29) \times 10^{-27} \mathrm{~kg} \\
=1.00727646688(13) \mathrm{u} \\
=1836.15267261(85) \mathrm{m}\end{array}$ \\
\hline neutron mass & $m_{n}$ & $\begin{array}{l}939.565360(81) \mathrm{MeV} / \mathrm{c}^{2} \\
=1.00866491560(55) \mathrm{u}\end{array}$ \\
\hline neutron mean -life & $\tau_{n}$ & $885.70(80) \mathrm{s}$ \\
\hline deuteron mass & $m_{d}$ & $1875.61282(16) \mathrm{MeV} / \mathrm{c}^{2}$ \\
\hline unified atomic mass unit (u) & $\begin{array}{l}\left(\mathrm{mass}{ }^{12} \mathrm{C} \text { atom }\right) / 12 \\
=(1 \mathrm{~g}) / N_{A}\end{array}$ & $\begin{array}{l}931.494043(80) \mathrm{MeV} / \mathrm{c}^{2} \\
=1.66053886(28) \times 10^{-27} \mathrm{~kg}\end{array}$ \\
\hline permittivity of free space & $\varepsilon_{0}$ & 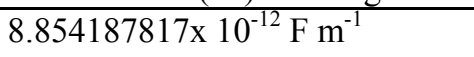 \\
\hline permeability of free space & $\mu_{\mathrm{o}},\left[\varepsilon_{\mathrm{o}} \mu_{\mathrm{o}}=1 / \mathrm{c}^{2}\right]$ & $4 \pi \times 10^{-7} \mathrm{~N} \mathrm{~A}^{-2}$ \\
\hline fine structure constant & $\alpha=e^{2} / 4 \pi \varepsilon_{0} \hbar c$ & $1 / 137.03599911(46)$ \\
\hline classical electron radius & $r_{e}=e^{2} / 4 \pi \varepsilon_{o} m_{e} c^{2}$ & $2.817940325(28) \times 10^{-15} \mathrm{~m}$ \\
\hline electron Compton wavelength & $\lambda=\hbar / m_{e} c=r_{e} / \alpha$ & $3.86159678(26) \times 10^{-13} \mathrm{~m}$ \\
\hline wavelength of $1 \mathrm{eV} / \mathrm{c}$ particle & $h c / e$ & $1.23984191(11) \times 10^{-6} \mathrm{~m}$ \\
\hline Thomson cross section & $\sigma_{T}=8 \pi r_{e}^{2} / 3$ & $0.665245873(13)$ barn \\
\hline Avogadro number & $\overline{N_{A}}$ & $6.0221415(10) \times 10^{23} \mathrm{~mol}^{-1}$ \\
\hline Boltzmann constant & $k$ & $\begin{array}{l}1.3806505(24) \times 10^{-23} \mathrm{~J} \mathrm{~K}^{-1} \\
=8.617343(15) \times 10^{-5} \mathrm{eV} \mathrm{K}^{-1}\end{array}$ \\
\hline & 1 barn & $10^{-28} \mathrm{~m}^{2}=10^{-24} \mathrm{~cm}^{2}$ \\
\hline & $1 \mathrm{eV}$ & $1.60217653(14) \times 10^{-19} \mathrm{~J}$ \\
\hline & 1 Gauss & $10^{-4}$ Tesla \\
\hline & $1 \mathrm{erg}$ & $10^{-7} \mathrm{~J}$ \\
\hline & $1 \mathrm{fm}$ & $10^{-15} \mathrm{~m}$ \\
\hline & 1 atmosphere & 760 torr $=1.01325 \times 10^{5} \mathrm{~N} \mathrm{~m}^{-2}(\mathrm{~Pa})$ \\
\hline & $0^{\circ} \mathrm{C}$ & $273.15^{\circ} \mathrm{K}$ \\
\hline
\end{tabular}

${ }^{\mathrm{a}}$ The one-standard deviation uncertainties in the last digits are given in parentheses.

${ }^{\mathrm{b}} \mathrm{N}=$ Newton, $\mathrm{F}=$ Farad, $\mathrm{A}=$ Ampere, $\mathrm{C}=$ Coulomb, $\mathrm{J}=\mathrm{J}$ oule, esu=electrostatic unit, $\mathrm{u}=$ atomic mass unit 


\section{Chapter 1 Basic Radiation Physics Concepts and Units of Measurement}

Table 1.2 Atomic and nuclear properties of materials. [Adapted from (PDG04).]

\begin{tabular}{|c|c|c|c|c|c|c|c|c|c|}
\hline Material & $Z$ & $A^{\mathrm{b}}$ & 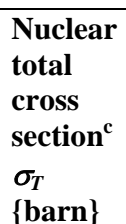 & $\begin{array}{l}\text { Nuclear } \\
\text { inelastic } \\
\text { cross } \\
\text { section }^{c} \\
\sigma_{\text {in }} \\
\{\text { barn\} }\end{array}$ & 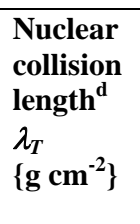 & $\begin{array}{l}\text { Nuclear } \\
\text { inter- } \\
\text { action } \\
\text { length } \\
\lambda_{\text {in }} \\
\left\{\mathrm{g} \mathrm{cm}^{-2}\right\}\end{array}$ & $\begin{array}{l}\text { Minimum } \\
\text { stopping } \\
\text { power }^{\mathrm{e}} \\
d E / d x \\
\{\mathrm{MeV} \\
\left.\mathrm{cm}^{2} \mathrm{~g}^{-1}\right\}\end{array}$ & $\begin{array}{l}\text { Radiation } \\
\text { Length } \\
X_{\mathbf{o}} \\
\left\{\mathrm{g} \mathrm{cm}^{-2}\right\}\end{array}$ & $\begin{array}{l}\text { Density } \\
\rho\left\{\text { cm }^{-3}\right\} \\
\left(\mathrm{g} \mathrm{I}^{-1}\right) \text { or }\left[\mathrm{g} \mathrm{I}^{-1}\right] \text { for } \\
\text { gas }\end{array}$ \\
\hline $\mathrm{H}_{2}{ }^{\mathrm{a}}$ & 1 & 1.00794 & 0.0387 & 0.033 & 43.3 & 50.8 & $(4.103)$ & 63.047 & $(0.0838)[0.0899]$ \\
\hline $\mathrm{D}_{2}{ }^{\mathrm{a}}$ & 1 & 2.014 & 0.073 & 0.061 & 45.7 & 54.7 & $(2.052)$ & 122.4 & $0.169[0.179]$ \\
\hline $\mathrm{He}^{\mathrm{a}}$ & 2 & 4.00260 & 0.133 & 0.102 & 49.9 & 65.1 & $(1.937)$ & 94.32 & $0.1249[0.1786]$ \\
\hline $\mathrm{Li}$ & 3 & 6.941 & 0.211 & 0.157 & 54.6 & 73.4 & 1.693 & 82.76 & 0.534 \\
\hline $\mathrm{Be}$ & 4 & 9.01218 & 0.268 & 0.199 & 55.8 & 75.2 & 1.594 & 65.19 & 1.848 \\
\hline $\mathrm{C}$ & 6 & 12.011 & 0.331 & 0.231 & 60.2 & 86.3 & 1.745 & 42.70 & $2.265^{\mathrm{f}}$ \\
\hline $\mathrm{N}_{2}^{\mathrm{a}}$ & 7 & 14.0067 & 0.379 & 0.265 & 61.4 & 87.8 & $(1.825)$ & 37.99 & $0.8073[1.250]$ \\
\hline $\mathrm{O}_{2}{ }^{\mathrm{a}}$ & 8 & 15.9994 & 0.420 & 0.292 & 63.2 & 91.0 & $(1.675)$ & 34.24 & $1.141[1.428]$ \\
\hline $\mathrm{Al}$ & 13 & 26.9815 & 0.634 & 0.421 & 70.6 & 106.4 & 1.615 & 24.01 & 2.70 \\
\hline $\mathrm{Si}$ & 14 & 28.0855 & 0.660 & 0.440 & 70.6 & 106.0 & 1.664 & 21.82 & 2.33 \\
\hline $\mathrm{Ar}^{\mathrm{a}}$ & 18 & 39.948 & 0.868 & 0.566 & 76.4 & 117.2 & $\begin{array}{l}(1.519) \\
\end{array}$ & 19.55 & $1.396[1.782]$ \\
\hline $\mathrm{Fe}$ & 26 & 55.845 & 1.120 & 0.703 & 82.8 & 131.9 & 1.451 & 13.84 & 7.87 \\
\hline$\overline{\mathrm{Cu}}$ & 29 & 63.546 & $1.232^{\prime}$ & 0.782 & 85.6 & 134.9 & 1.403 & 12.86 & 8.96 \\
\hline $\mathrm{Ge}$ & 32 & 72.61 & 1.365 & 0.858 & 88.3 & 140.5 & 1.371 & 12.25 & 5.323 \\
\hline $\mathrm{W}$ & 74 & 183.84 & 2.767 & 1.65 & 110.3 & 185 & 1.145 & 6.76 & 19.3 \\
\hline $\mathrm{Pb}$ & 82 & 207.2 & 2.960 & 1.77 & 116.2 & 194 & 1.123 & 6.37 & 11.35 \\
\hline $\bar{U}$ & 92 & 238.029 & 3.378 & 1.98 & 117.0 & 199 & 1.082 & 6.00 & 18.95 \\
\hline \multicolumn{5}{|l|}{$\operatorname{Air}^{a}$} & 62.0 & 90.0 & $(1.815)$ & 36.66 & $(1.205)[1.2931]$ \\
\hline \multicolumn{5}{|l|}{$\mathrm{H}_{2} \mathrm{O}$} & 60.1 & 83.6 & 1.991 & 36.08 & 1.00 \\
\hline \multicolumn{5}{|c|}{ Shielding concrete $^{\mathrm{g}}$} & 67.4 & 99.9 & 1.711 & 26.7 & 2.5 \\
\hline \multicolumn{5}{|c|}{$\mathrm{SiO}_{2}$ (quartz) } & 66.5 & 97.4 & 1.699 & 27.05 & 2.64 \\
\hline \multicolumn{5}{|l|}{$\mathrm{NaI}$} & 94.6 & 151 & 1.305 & 9.49 & 3.67 \\
\hline \multicolumn{5}{|c|}{ Polystyrene, scintillator $(\mathrm{CH})$} & 58.5 & 81.9 & 1.936 & 43.72 & 1.032 \\
\hline \multicolumn{5}{|c|}{ Polyethylene $\left(\mathrm{CH}_{2}\right)$} & 57.0 & 78.4 & 2.076 & 44.6 & $0.92-0.95$ \\
\hline \multicolumn{5}{|c|}{ Mylar $\left(\mathrm{C}_{5} \mathrm{H}_{4} \mathrm{O}_{2}\right)$} & 60.2 & 85.7 & 1.848 & 39.95 & 1.39 \\
\hline \multicolumn{5}{|l|}{$\mathrm{CO}_{2}{ }^{\mathrm{a}}$} & 62.4 & 89.7 & $(1.819)$ & 36.2 & [1.977] \\
\hline \multicolumn{5}{|c|}{ Methane $^{\mathrm{a}}\left(\mathrm{CH}_{4}\right)$} & 54.8 & 73.4 & $(2.417)$ & 46.22 & $0.4224[0.717]$ \\
\hline \multicolumn{5}{|c|}{ Ethane $^{\mathrm{a}}\left(\mathrm{C}_{2} \mathrm{H}_{6}\right)$} & 55.8 & 75.7 & $(2.304)$ & 45.45 & $0.509(1.356)$ \\
\hline \multicolumn{5}{|c|}{$\mathrm{NaF}$} & 66.9 & 98.3 & 1.69 & 29.87 & 2.558 \\
\hline \multicolumn{5}{|l|}{$\mathrm{LiF}$} & 62.2 & 88.2 & 1.614 & 39.25 & 2.632 \\
\hline
\end{tabular}

${ }^{\mathrm{a}}$ Parameters for materials that are gases at NTP are evaluated at $20{ }^{\circ} \mathrm{C}$ and $1 \mathrm{~atm}$ (value) or at STP [value] or as cryogenic liquids at their 1.0 atmosphere boiling point if the value is given without parentheses.

${ }^{\mathrm{b}}$ Averaged over naturally occurring isotopes.

${ }^{\mathrm{c}}$ These are energy dependent. The values tabulated are for the high energy limit. The inelastic cross section is obtained by subtracting the elastic and quasi-elastic cross sections from the total cross section.

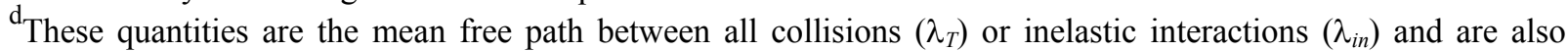
energy dependent The values quoted are for the high energy limit.

${ }^{\mathrm{e}}$ This is the minimum value of the ionization stopping power for heavy particles. It is calculated specifically for pions and the results are slightly different for other particles.

${ }^{\mathrm{f}}$ The tabulated values are for pure graphite; industrial graphite may vary between $2.1-2.3 \mathrm{~g} \mathrm{~cm}^{-3}$.

${ }^{\mathrm{g}}$ This is for so-called "standard" shielding blocks, typical composition of $\mathrm{O}_{2}(52 \%), \mathrm{Si}(32.5 \%), \mathrm{Ca}(6 \%), \mathrm{Na}$ $(1.5 \%), \mathrm{Fe}(2 \%), \mathrm{Al}(4 \%)$, plus reinforcing iron bars. 


\section{Chapter 1 Basic Radiation Physics Concepts and Units of Measurement}

It is usually most convenient to work in a system of units where energy is in units of eV, $\mathrm{MeV}$, etc. Velocities are then expressed in units of the speed of light $(\beta)$, momenta are expressed as energy divided by $c$ (e.g., $\mathrm{MeV} / \mathrm{c}$, etc.), and masses are expressed as energy divided by $\mathrm{c}^{2}$ (e.g., $\mathrm{MeV} / \mathrm{c}^{2}$, etc.). In these so-called energy units, the $W$ is equal to $m$. One thus avoids the explicit inclusion of numerical values for $c$ or $c^{2}$.

The decay length at a given velocity of a particle with a finite mean-life (at rest) $\tau$ is given by $\gamma \beta c \tau$, where relativistic time dilation is taken into account by inclusion of the factor $\gamma$. The product of the speed of light and the mean-life $c \tau$ is often tabulated. The decay length is the mean distance traveled by a particle in vacuum prior to its decay. This length must be distinguished from the decay path. The decay path represents a distance in space in which a given particle is allowed to decay with no or minimal competition from competing mechanisms such as scattering or absorption. Thus, the decay length is determined by the fundamental physics of the decay process while the decay path is defined by the physical configuration of the accelerator components present.

\subsection{Energy Loss by Ionization and Multiple Coulomb Scattering}

\section{$\underline{1.6 .1 ~ E n e r g y ~ L o s s ~ b y ~ I o n i z a t i o n ~}$}

For moderately relativistic heavy $\left(m_{o}>>m_{e}\right)$ particles, the mean rate of energy loss, the ionization stopping power or rate of energy deposition, is given approximately by

$$
-\frac{d E}{d x}=4 \pi N_{A} r_{e}^{2} m_{e} c^{2} z^{2} \frac{Z}{A} \frac{1}{\beta^{2}}\left[\ln \left\{\frac{2 m_{e} c^{2} \gamma^{2} \beta^{2}}{I}\right\}-\beta^{2}-\frac{\delta}{2}\right]\left(\mathrm{MeV} \mathrm{cm}^{2} \mathrm{~g}^{-1}\right),
$$

where $N_{A}$ is Avogadro's number (atoms g-mole ${ }^{-1}$ ), $Z$ and $A$ are the atomic number and weight (mass number) of the material traversed, $z$ is the charge state of the projectile in units of electron charge, $m_{e}$ and $r_{\mathrm{e}}$ are the rest mass and classical radius of the electron (see Table 1.1), and $I$ is the ionization constant. For $Z>1, I \approx 16 Z^{0.9} \mathrm{eV}$. For diatomic hydrogen $\left(\mathrm{H}_{2}\right), I=19 \mathrm{eV} . \beta$ and $\gamma$ are as defined in Section 1.5. $\delta$ is a small correction factor that can be approximated by $2 \ln \gamma$. Substituting constants, for $I$ in $\mathrm{eV}$;

$$
-\frac{d E}{d x}=0.3071 z^{2} \frac{Z}{A} \frac{1}{\beta^{2}}\left[\ln \left\{\frac{1.022 \times 10^{6} \gamma^{2} \beta^{2}}{I}\right\}-\beta^{2}-\frac{\delta}{2}\right]\left(\mathrm{MeV} \mathrm{cm}^{2} \mathrm{~g}^{-1}\right)
$$

This is the stopping power ${ }^{4}$ due to ionization, the process in which a charged particle transfers its energy to atomic electrons in the absorbing medium. In these units, the dependence upon the absorbing material is rather weak given the fact that $I$ appears only in

\footnotetext{
${ }^{4}$ The argument of the logarithmic term of Eqs (1.17) and (1.18) must be dimensionless. Hence, the rest energy of the electron $m_{e} c^{2}$ and $I$ must be in the same units (e.g., both in $\mathrm{eV}$ ). These equations are found in Phys Rev. D45 (1992) S1, the 1992 edition of reference (PDG04). This version of these equations is somewhat simpler than, but equivalent to, that found in (PDG04) and thus is adopted for use here.
} 


\section{Chapter 1 Basic Radiation Physics Concepts and Units of Measurement}

the logarithmic term and the ratio $Z / A$ ranges between 0.4 and 0.5 over most of the periodic table for stable nuclides. Thus, for a given projectile charge $z$ the value of the stopping power $d E / d x$ is most strongly dependent on $\beta \gamma$. A broad minimum is found at an approximate value of $\gamma=3.2$. At this value of $\gamma$, the particles are said to be minimum ionizing and the corresponding minimum stopping powers are provided in Table 1.2.

The absorption of the energy of charged particles by ionization is characterized by a parameter called the range $R$ in material. The range is the length of the path followed by the particle while it is coming to rest while losing all of its kinetic energy. Simplistically one might think that one could calculate the value of $R$ by a numerical integration of the reciprocal of the stopping power. However, as the particles lose energy by ionization and thus slow down, other mechanisms prominent at very low energies become important that are not included in Eq. (1.17). It is therefore prudent to consult explicit tabulations to determine the particle ranges. For charged particles much more massive than electrons, the trajectory through the material to first approximation is a straight line modified only by multiple Coulomb scattering (see Section 1.6.2) since the mass of the moving particle is so much larger than the mass of the atomic electrons. For a moving electron, the range is the sum of many divergent line segments through the material since its mass is identical to that of the atomic electrons encountered so that the individual angular deflections are generally much larger. As will be seen in Section 3.2.2, for electrons the loss of energy in matter due to the radiation of photons increases rapidly with electron kinetic energy and becomes much more important than the ionization stopping power or range at relatively low energies. The situation is also different for particles such as protons that participate in the nuclear interaction. For these particles, as the kinetic energy of the particle increases, the absorption of the particles through strong interaction processes has a high probability of occurring prior to their depositing all of their energy by ionization. This will be discussed further in Section 4.2.1. Figs 1.7 and 1.8 give stopping power and range values as a function of momentum or energy for common high energy particles and for some light ions, respectively. Detailed tables of the values of stopping power and ranges for many heavy ions have been given by Northcliffe and Schilling (No70). Also, the Monte Carlo computer code SRIM is currently easily obtained and may be used to generate similar tables as well as do simulations of protons or heavy charged ions interacting with elemental or compound materials (Zi96).

For muons ( $\mu$ 's) the situation is rather unique. The muon rest energy is $105.66 \mathrm{MeV}$, its mean-life $\tau=2.1970 \times 10^{-6} \mathrm{~s}$, and the mean-life times the speed of light $c \tau=658.65 \mathrm{~m}$ (PDG04). Due to their large rest mass compared to that of the electron and the fact that these particles, to first order, do not participate in the strong (nuclear) interaction, muons tend to penetrate long distances in matter without being absorbed by other mechanisms. Muons, due to their heavier masses, are also far less susceptible to radiative effects. Thus, over a very large energy domain, the principal energy loss mechanism is that of ionization. This, as shall be seen later, makes the shielding of muons matter of considerable importance at high energy accelerators. The range-energy relation of muons is given in Fig. 1.9 . 

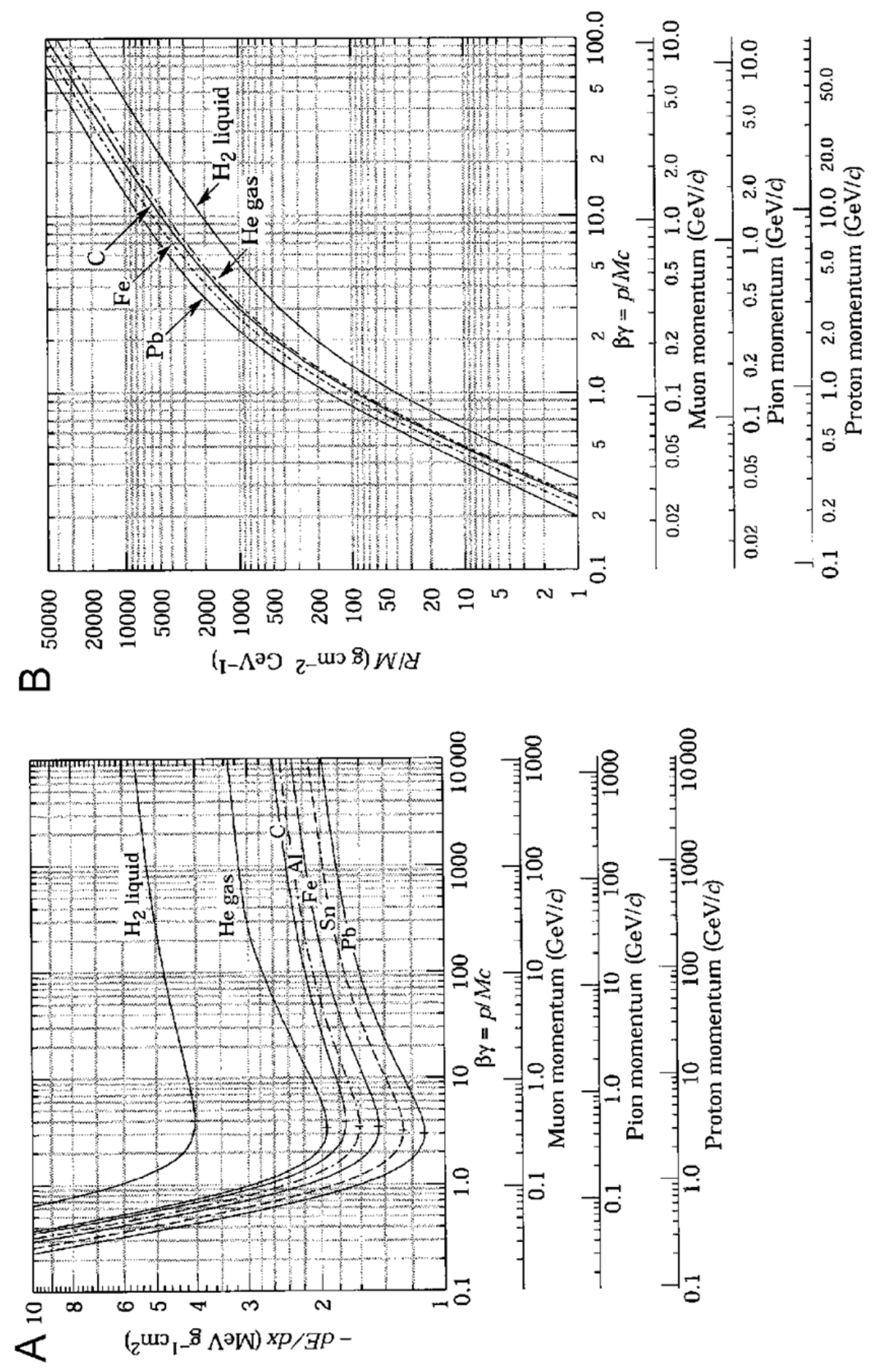

Fig. 1.7 Frame A: Mean ionization stopping power in various media as a function of particle momenta. Radiative effects are not included. Frame B: Ionization range of heavy charged particles in various media. The abscissa values of these plots are scaled to the ratio of particle momenta $p$ to particle rest mass $M$. [Reproduced from (PDG04).] 

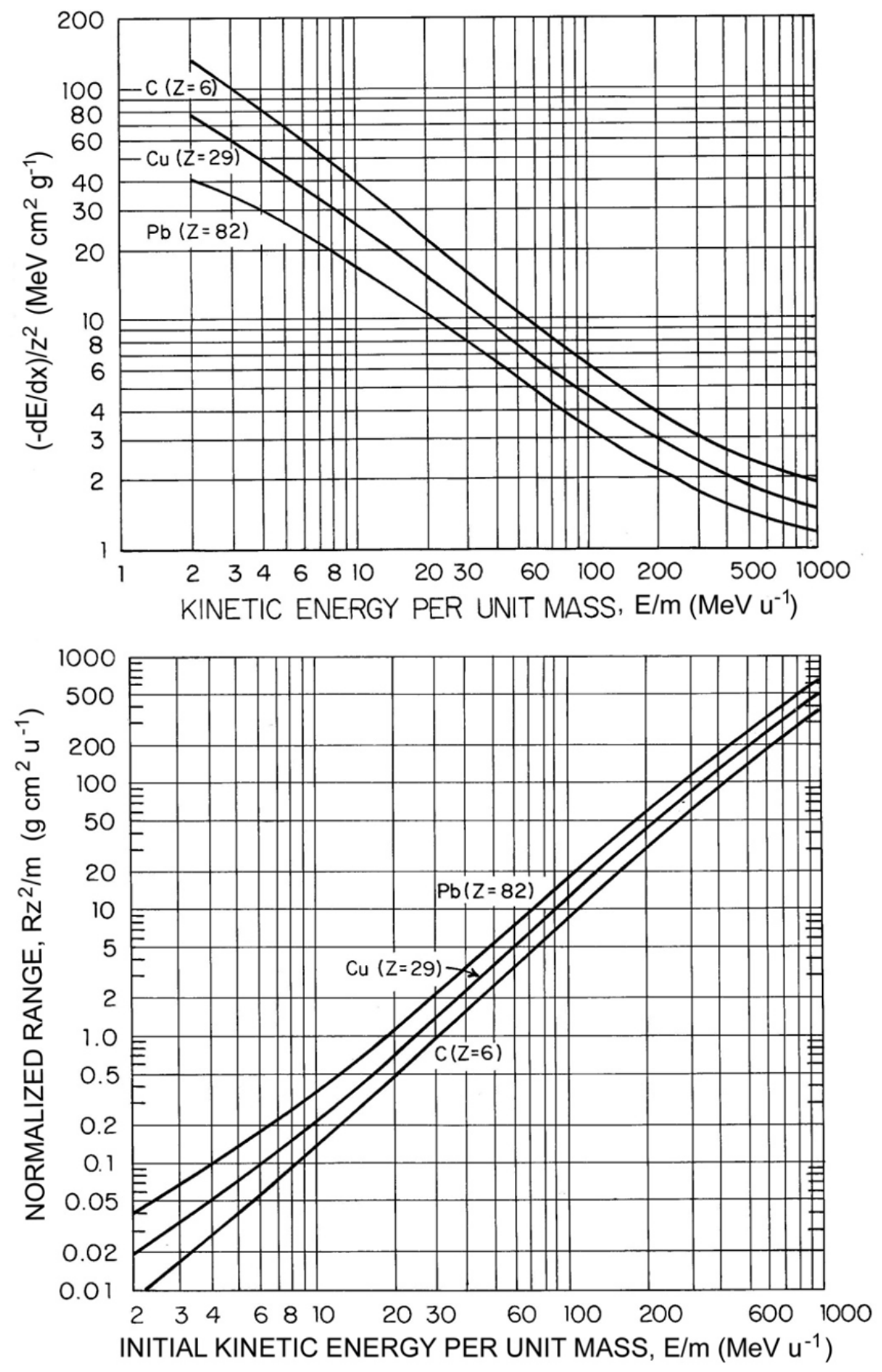

Fig. $1.8 \quad$ Stopping power (top) and ranges $R$ (bottom) for protons in three different materials. These curves can be used for other incident particles by taking their atomic number $z$ and mass $m$ (in atomic mass units), into account. The incident energy is thus expressed as the kinetic energy per unit nucleon mass $E / m$. The curves are approximately correct except at the very lowest energies where charge exchange effects can be important. The results are most valid for projectile mass, $m \leq 4$ [Reproduced from (En66).] 


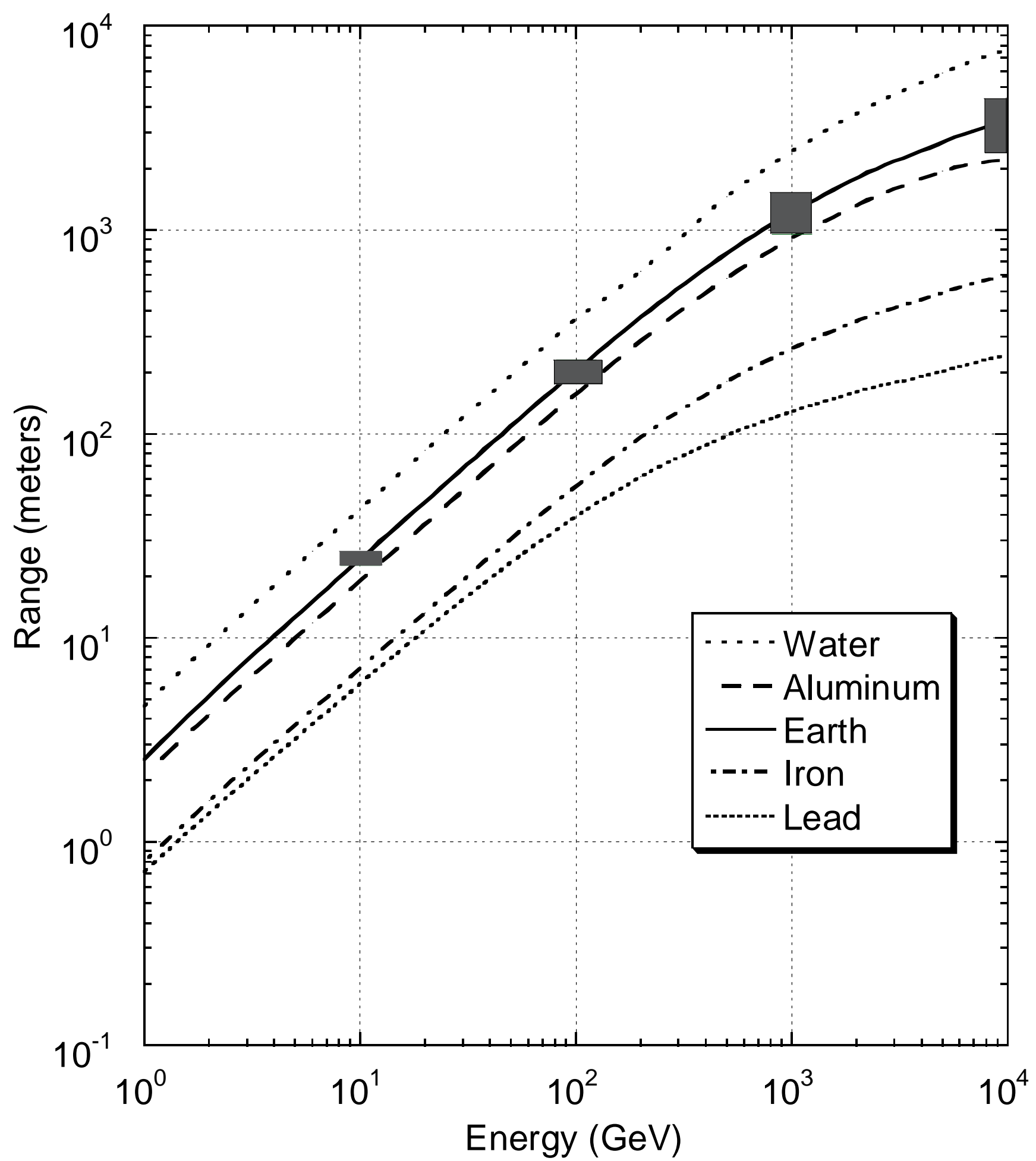

Fig. 1.9 Range-energy curves for muons in various materials. On the curve labeled "Earth", the gray boxes are indicative the approximate spread in the range due to range straggling at one standard deviation at the indicated muon energy. The density of "earth" was taken to be $2.0 \mathrm{~g} \mathrm{~cm}^{-2}$. [Adapted from ( $\mathrm{Fa} 90)$.] 


\section{Chapter 1 Basic Radiation Physics Concepts and Units of Measurement}

At high energies $\left(E_{\mu}>100 \mathrm{GeV}\right)$, the distribution of the ranges of individual muons about the mean range, called the range straggling, becomes severe [(Va87), (Fa90)]. Also, above muon energies of several hundred $\mathrm{GeV}$, radiative losses begin to dominate such that the stopping power $d E / d x$ is given by (PDG 04);

$$
-\frac{d E}{d x}=a(E)+b(E) E
$$

where $a(E)$ is the collisional ionization energy loss [from Eq. (1.18), in iron approximately $\left.0.002 \mathrm{GeV} \mathrm{cm}^{2} \mathrm{~g}^{-1}\right]$, and $b(E)$ is the radiative coefficient for $E$ in $\mathrm{GeV}$. The latter parameter separated into contributions from the important physical mechanisms is plotted in Fig. 1.10.

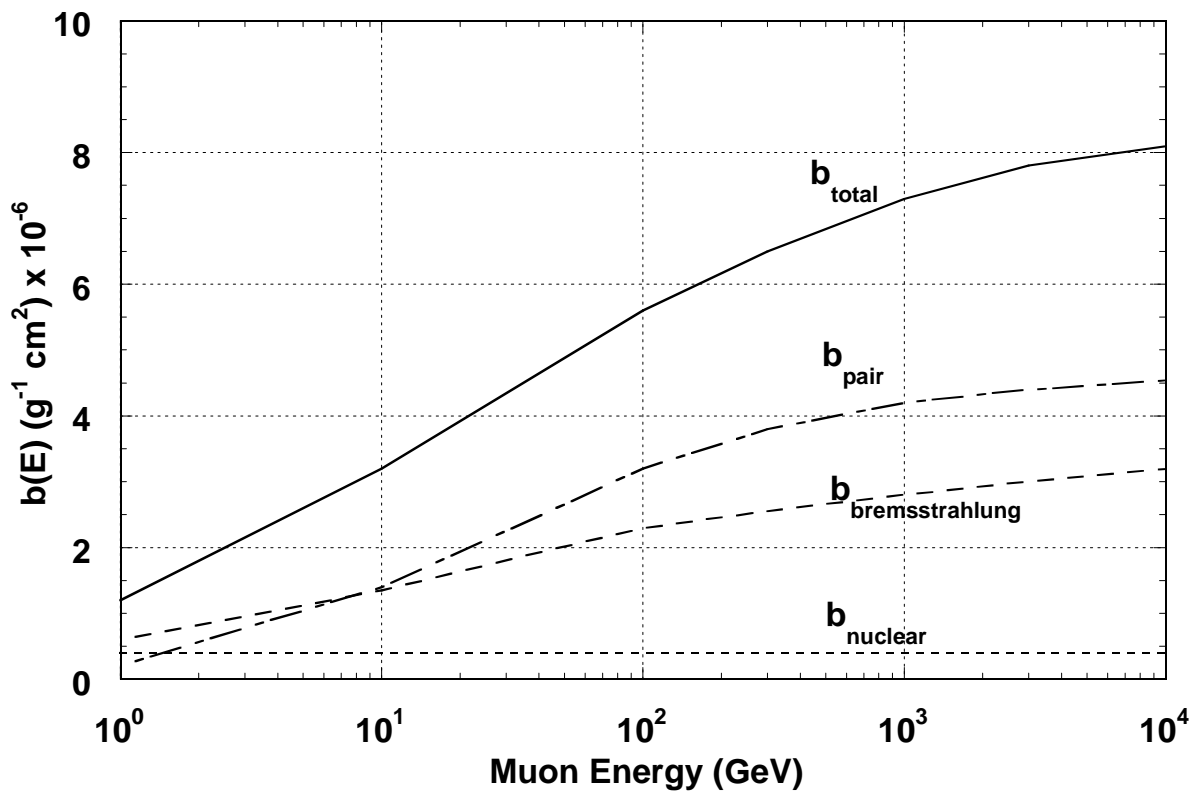

Fig. 1.10 Contributions to the fractional energy loss by muons in iron due to $\mathrm{e}^{+} \mathrm{e}^{-}$pair production, bremsstrahlung, and photonuclear interactions. See Eq. (1.19). [Adapted from (PDG04).]

The mean range $R_{\mu}$ of a muon of kinetic energy $E(\mathrm{GeV})$, is approximated by

$$
R_{\mu}(E)=\frac{1}{b(E)} \ln \left[1+\frac{b(E)}{a(E)} E\right]\left(\mathrm{g} \mathrm{cm}^{-2}\right) .
$$

Muon range straggling [(Va87), (Fa90)] is chiefly due to the fact that for muon kinetic energies greater than about $100 \mathrm{GeV}$, electron-positron pair production, bremsstrahlung, and deep inelastic nuclear reactions become the dominant energy loss mechanisms. Although these processes have low probabilities, when they do occur they involve large energy losses. Tables 1.3 and 1.4 give fractional energy loss and comparisons of muon ranges at high energies for different physical mechanisms. Range straggling can be very important since shielding calculations based upon using the mean range values can significantly underestimate the muon fluence that can penetrate a shield. 
Table 1.3 Fractional energy loss of muons in soil $\left(\rho=2.0 \mathrm{~g} \mathrm{~cm}^{-3}\right)$. The fractions of the total energy loss due to the four dominant energy loss mechanisms are given at selected energies. [Adapted from (Va87) and (Fa90).]

\begin{tabular}{|c|c|c|c|c|}
\hline Energy & Ionization & Bremsstrahlung & $\begin{array}{c}\text { Pair } \\
\text { production }\end{array}$ & $\begin{array}{c}\text { Deep inelastic } \\
\text { nuclear } \\
\text { scattering }\end{array}$ \\
\hline $\mathbf{G e V})$ & & 0.037 & $8.8 \times 10^{-4}$ & $9.7 \times 10^{-4}$ \\
\hline 10 & 0.972 & 0.086 & 0.020 & 0.0093 \\
\hline 100 & 0.888 & 0.193 & 0.168 & 0.055 \\
\hline 1000000 & 0.580 & 0.335 & 0.388 & 0.110 \\
\hline
\end{tabular}

Table 1.4 Comparison of muon ranges (meters) in heavy soil $\left(\rho=2.24 \mathrm{~g} \mathrm{~cm}^{-3}\right)$ at selected energies. [Adapted from (Va87) and (Fa90).]

\begin{tabular}{|c|c|c|c|c|c|}
\hline Energy & \multicolumn{2}{|c|}{} & \multicolumn{3}{c|}{ Mean Ranges from dE/dx in Heavy } \\
\hline$(G e V)$ & $\begin{array}{c}\text { Mean Range } \\
\text { (meters) }\end{array}$ & $\begin{array}{c}\text { Standard } \\
\text { Deviation } \\
\text { (meters) }\end{array}$ & All Processes & $\begin{array}{c}\text { Coulomb } \\
\text { Losses Only }\end{array}$ & $\begin{array}{c}\text { Coulomb \& } \\
\text { Pair } \\
\text { Production } \\
\text { Losses }\end{array}$ \\
\hline 10 & 22.8 & 1.6 & 21.4 & 21.5 & 21.5 \\
\hline 30 & 63.0 & 5.6 & 60.3 & 61.1 & 60.8 \\
\hline 100 & 188 & 23 & 183 & 193 & 188 \\
\hline 300 & 481 & 78 & 474 & 558 & 574 \\
\hline 1000 & 1140 & 250 & 1140 & 1790 & 1390 \\
\hline 3000 & 1970 & 550 & 2060 & 5170 & 2930 \\
\hline 10,000 & 3080 & 890 & 3240 & 16,700 & 5340 \\
\hline 20,000 & 3730 & 1070 & & & \\
\hline
\end{tabular}

\subsubsection{Multiple Coulomb Scattering}

Multiple Coulomb scattering from nuclei is an important effect in the transport of charged particles through matter. A charged particle traversing a medium is deflected by many small-angle scattering events and only occasionally by ones involving large-angle scattering. These small-angle scattering events are largely due to Coulomb scattering from nuclei, hence the name of this phenomenon. This simplification is not quite completely correct for hadrons since it ignores the contribution of strong interactions to multiple scattering. For present purposes, a Gaussian approximation adequately describes the distribution of deflection angles of the final trajectory compared with the incident trajectory for all charged particles. The distribution as a function of deflection angle $\theta$ is as follows:

$$
f(\theta) d \theta=\left(\frac{d \theta}{\theta_{0} \sqrt{2 \pi}}\right) \exp \left(-\frac{\theta^{2}}{2 \theta_{0}^{2}}\right) .
$$

The mean width of the projected angular distribution $\theta_{O}$, on a particular plane is 


\section{Chapter 1 Basic Radiation Physics Concepts and Units of Measurement}

approximated by

$$
\theta_{o}=\frac{13.6 z}{p c \beta} \sqrt{\frac{x}{X_{o}}}\left\{1+0.038 \ln \left(\frac{x}{X_{o}}\right)\right\} \text { (radians) }
$$

where $z$ is the charge of the projectile in units of the charge of the electron, $p$ is the particle momentum in $\mathrm{MeV} / \mathrm{c}$ and $x$ is the absorber thickness in the same units as the quantity $X_{O}$ (PDG04). $X_{O}$ is a material-dependent parameter, to be discussed further in Section 3.2.2 called the radiation length. This description of multiple Coulomb scattering has been validated experimentally for particles having momenta up to $200 \mathrm{GeV} / \mathrm{c}$ by Shen et al. (Sh79). The best values of the radiation length are probably those of Tsai (Ts74), the values tabulated in Table 1.2. A compact, approximate formula for calculating the value of $X_{o}$ as a function of atomic number $\mathrm{Z}$ and atomic weight $A$ of the material medium (PDG04) is

$$
X_{o}=\frac{716.4 A}{Z(Z+1) \ln (287 / \sqrt{Z})}\left(\mathrm{g} \mathrm{cm}^{-2}\right) \text {. }
$$

Results obtained using this formula agree to those of Tsai within about $2.5 \%$ for all elements except helium, where the result is about 5\% low. An alternative method of calculating $X_{o}$ using a somewhat different approximation to the atomic wave functions is given by Seltzer and Berger (Se85). It provides results similar to those given by Eq. (1.23).

\subsection{Radiological Standards}

While the discussion of radiological standards is not a topic of great emphasis in this text, some mention of it is appropriate. Standards or limits on occupational and environmental exposure to ionizing radiation are now instituted worldwide. In general, individual nations, or sub-national entities, incorporate guidance provided by international or national bodies into their laws and regulations. The main international body that develops radiological standards is the International Commission on Radiation Protection (ICRP). The International Commission on Radiation Units and Measurements (ICRU) also has an important role. In the U. S, the corresponding body chartered by the U. S. Congress is the National Council on Radiation Protection and Measurements (NCRP). In the U.S. the Environmental Protection Agency (EPA) is the primary federal agency for establishing radiation protection requirements. These are further implemented by other U. S. federal agencies, notably the U. S. Department of Energy for its facilities, and by individual states. Currently the U. S. Nuclear Regulatory Commission (NRC) does not regulate the operation of particle accelerators. However, certain aspects of state regulations pertaining to accelerators are reflective of general NRC requirements. The regulation of accelerator facilities varies considerably between individual states and some local jurisdictions, the authority having jurisdiction should be consulted to obtain an accurate understanding of applicable regulatory requirements. 


\section{Problems}

1. a) Express 1.0 kilowatt $(1.0 \mathrm{~kW})$ of beam power in $\mathrm{GeV} \mathrm{s}^{-1}$.

b) To how many singly charged particles per second does 1.0 ampere of beam current correspond?

c) Express an absorbed dose of $1.0 \mathrm{~Gy}$ in $\mathrm{GeV} \mathrm{kg}^{-1}$ of energy deposition.

2. In the 1973 System, which has the higher quality factor, a $10 \mathrm{MeV}$ (kinetic energy) $\alpha$-particle or a $1.0 \mathrm{MeV}$ neutron? Write down the quality factors for each particle. What is the quality factor of a 1.0 MeV neutron in the 1990 System?

3. Calculate the number of ${ }^{12} \mathrm{C}$ and ${ }^{238} \mathrm{U}$ atoms in a cubic centimeter of solid material.

4. Calculate the velocity and momenta of a $200 \mathrm{MeV}$ electron, proton, iron ion, $\pi^{+}$, and $\mu^{+}$. The $200 \mathrm{MeV}$ is kinetic energy and the answers should be expressed in units of the speed of light (velocity) and $\mathrm{MeV} / \mathrm{c}$ (momenta). Iron ions have an isotope-averaged mass (rest energy) of $52,021 \mathrm{MeV}(A=55.847 \times 931.5 \mathrm{MeV} / \mathrm{amu})$. The $\pi^{+}$mass is $140 \mathrm{MeV}$ and the $\mu^{+}$mass $=106 \mathrm{MeV}$. Do the same calculation for $20 \mathrm{GeV}$ protons, iron ions, and muons. It is suggested that these results be presented in tabular form. Make general comments on the velocity and momenta of the particles at the two energies. (The table may help you notice any algebraic errors that you may have made.)

5. Calculate the mass stopping power of a $20 \mathrm{MeV}$ electron (ionization only) and a $200 \mathrm{MeV}$ proton in ${ }^{28} \mathrm{Si}$.

6. Calculate the fluence of minimum ionizing muons necessary to produce an effective dose of 1.0 mrem assuming a radiation weighting factor of unity and that tissue is equivalent to water for minimum ionizing muons. (Hint: Use Table 1.2.) Compare with the results given in Fig. 1.4 for high energies. 


\section{ChAPTER 2 General Considerations of RADiATION FieldS AT ACCELERATORS}

\section{$2.1 \quad$ Introduction}

In this chapter general properties of the radiation fields at accelerators will be discussed. To do this, the concept of particle yield into a given solid angle is introduced. Following that, a theoretical approach to particle transport will be introduced. The Monte Carlo technique will be described and illustrated by simple examples. The manipulation of charged particles using electromagnetic fields will be reviewed due to its importance in understanding the handling of the charged particle beams.

\subsection{Primary Radiation Fields at Accelerators-General Considerations}

Accelerated charged particles, except in the singular phenomenon of synchrotron radiation (see Section 3.6), do not produce radiation unless there is some interaction with matter. The charged particles directly accelerated, and otherwise manipulated by the electromagnetic fields within the accelerator, are referred to as the primary particles or primary beam. All other particles that are produced from this beam either result from interactions of these primary particles in matter or are due to synchrotron radiation and are referred to as secondary particles or secondary beam. Sometimes one finds references to tertiary particles or tertiary beam that result from interactions in matter of the secondary particles or their radioactive decay. Confusion can also arise when secondary or tertiary particles are collected into beams of their own and sometimes even accelerated. When this is done and the secondary or tertiary particles are employed at some location separated from the place where they were initially produced, they can obviously play the role of primary particles.

If one considers primary particles incident upon material such as a target, the yield $Y$ of secondary particles is a crucial parameter. For a given type of secondary particle, the yield is typically a function of primary particle type and energy, the angle of emission, and the secondary particle energy. It is defined according to Fig. 2.1. Scattered reaction products are found at a "point of interest" located at radius $r$ and polar angle $\theta$ relative to the direction of the incident particle along the positive $z$-axis. In general, particle differential yields are expressed in terms of particles per unit solid angle at the point of interest and are commonly normalized to the number of incident particles or to the beam current or total delivered charge. Such particle yields, dependent upon both target material and thickness, are reported in terms of particle type, energy, and angular distribution. The rate of production of the desired reaction products and their energy spectra is, in general, a strong function of both $\theta$ and the incident particle energy $E_{O}$. Almost always, the particle energy is the kinetic energy (see Section 1.5). There is usually no dependence on the azimuthal angle in a spherical coordinate system. ${ }^{5}$

\footnotetext{
${ }^{5}$ There are exceptions. One is the situation in which the spins of the target nuclei and/or the incident particles are oriented along some chosen direction in a so-called polarization experiment. Interactions of colliding beams involving spin-polarized particles have azimuthal dependencies. Secondary particles resulting from multipole emission/deexcitation processes from excited atomic or nuclear states will also have a dependence on azimuthal angle. Processes involving parity violation constitute other examples.
} 


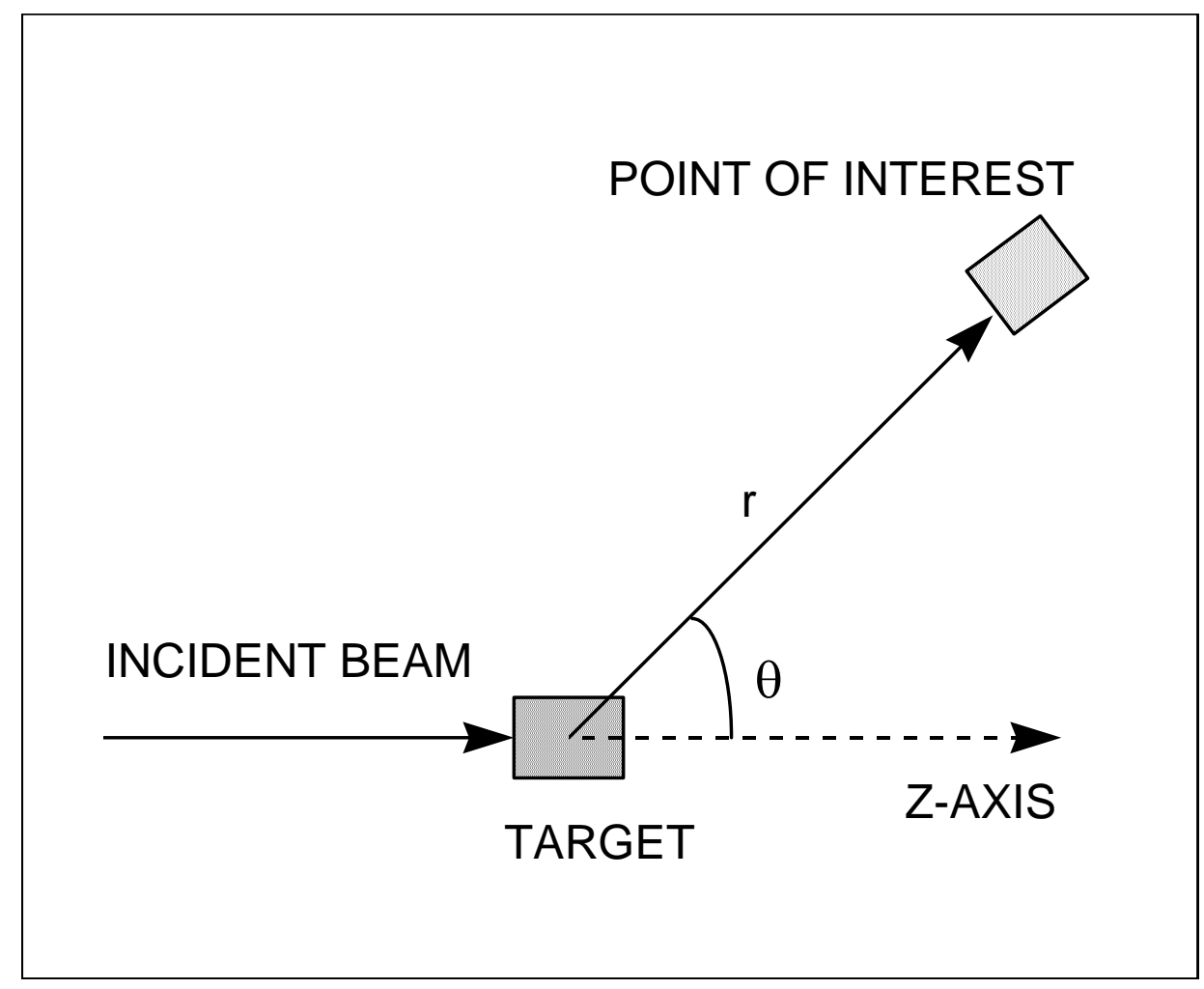

Fig. 2.1 Conceptual interaction of incident beam with material (target) which produces radiation at the point of interest located at polar coordinates $(r, \theta)$.

In principle, the particle yield could be obtained directly from differential cross sections for given incident particle kinetic energy $E$;

$$
\frac{d \sigma(E, \theta)}{d \Omega}
$$

where $\sigma(E, \theta)$ is the cross section as a function of energy and angle and $\Omega$ is the solid angle into which the secondary particles are directed. For example, $Y$ could in principle be obtained from an integration of this cross section as it varies with energy while the incident particle loses energy in passing through the target material.

Calculations of the radiation field that directly use the cross sections may not always be practical because targets hit by beam are not really "thin". One cannot always ignore energy loss or secondary interactions in the target. Furthermore, the knowledge of cross sections at all energies is often incomplete with the unfortunate result that one cannot always perform an integration over $\theta$ and $E$ to get the total yield.

For many applications, the details of the angular distributions of total secondary particle yield $d Y(\theta) / d \Omega$ and the angular dependence of the emitted particle energy spectrum $d^{2} Y(E, \theta) / d E d \Omega$ are very important. 


\section{ChAPTER 2 GENERAL Considerations of RADiATION FieldS AT ACCELERATORS}

Often, the particle fluence is needed at a particular location at coordinates $(r, \theta)$ from a known point source ${ }^{6}$ of beam loss while the angular distributions of $d Y / d \Omega$ are generally expressed in units of particles steradian ${ }^{-1}$ incident particle ${ }^{-1}$. To obtain the total fluence $\Phi(\theta)$ [e.g., particles $\mathrm{cm}^{-2}$.(incident particle) $)^{-1}$ ] or differential fluence $d \Phi(E, \theta) / d E$ [e.g., particles $\mathrm{cm}^{-2} \cdot \mathrm{MeV}^{-1}$.(incident particle) $\left.{ }^{-1}\right]$ at a given distance $r(\mathrm{~cm})$ at a specified angle $\theta$ from such a point source, one must simply multiply the yield values by $1 / r^{2}$ since an area $d A$ subtends a solid angle $d \Omega$ of $d A / r^{2}$ at distance $r$ from an arbitrary point in space;

$$
\Phi(\theta)=\frac{1}{r^{2}} \frac{d Y(\theta)}{d \Omega} \text { and } \frac{d \Phi(E, \theta)}{d E}=\frac{1}{r^{2}} \frac{d^{2} Y(E, \theta)}{d E d \Omega} .
$$

Given the fact that secondary, as well as primary, particles can create radiation fields, it is clear that the transport of particles through space and matter can be a complex matter. In the following section, the advanced techniques for handling these issues are described.

\subsection{Theory of Radiation Transport}

The theoretical material in this section largely follows O'Brien (OB80). It is included here to show clearly the mathematical basis of the contents of shielding codes, especially those that use the Monte Carlo method. Vector notation is used in this section.

\subsubsection{General Considerations of Radiation Transport}

Stray and direct radiations at any location are distributed in particle type, direction, and energy. To determine the amount of radiation present for radiation protection purposes one must assign a magnitude to this multidimensional quantity. This is done by forming a double integral over energy and direction of the product of the flux density and an approximate dose per unit fluence conversion factor, summed over particle type;

$$
\frac{d H(\vec{x}, t)}{d t}=\sum_{i} \int_{4 \pi} d \vec{\Omega} \int_{0}^{\infty} d E f_{i}(\vec{x}, E, \vec{\Omega}, t) P_{i}(E),
$$

where the summation index $i$ is over the various particle types, $\vec{\Omega}$ is the direction vector of particle travel, $\vec{x}$ is the coordinate vector of the point in space where the dose rate $d H / d t$ is to be calculated, $E$ is the particle energy, $t$ is time, and $i$ is the particle type. $P_{i}(E)$ is the dose per fluence conversion factor for the radiation dosimetry quantity desired (e.g., dose equivalent, effective dose, etc., here generically denoted by $H$ ) expressed as a function of energy and particle type for the $i^{t h}$ particle. The inner integral is over all energies while the outer integral is over all spatial directions from which contributions to the radiation field at the location specified by $\vec{x}$ originate. The result of the integration is $d H(\vec{x}, t) / d t$, the dose rate at location $\vec{x}$ and time $t$. Values of $P_{i}(E)$ are given in Figs. $1.4,1.5$, and 1.6.

\footnotetext{
${ }^{6}$ A point source is one in which the dimensions of the source are small compared with the distance to some other location of interest.
} 


\section{CHAPTER 2 GENERAL CONSIDERATIONS OF RADIATION FiELDS AT ACCELERATORS}

The angular flux density $f_{i}(\vec{x}, E, \vec{\Omega}, t)$ is the number of particles of type $i$ per unit area, per unit energy, per unit solid angle, per unit time at location $\vec{x}$, with a energy $E$, at a time $t$, traveling in a direction $\vec{\Omega}$. It is related to the total flux density $\phi(\vec{x}, t)$ by integrating over direction and particle energy;

$$
\phi(\vec{x}, t)=\sum_{i} \int_{4 \pi} d \vec{\Omega} \int_{0}^{\infty} d E f_{i}(\vec{x}, E, \vec{\Omega}, t) .
$$

$f_{i}(\vec{x}, E, \vec{\Omega}, t)$ is connected to the total fluence $\Phi(\vec{x})$ by integrating over a relevant interval of time (from $t_{i}$ to $t_{f}$ ), as well as direction and energy by

$$
\Phi(\vec{x})=\sum_{i} \int_{4 \pi} d \vec{\Omega} \int_{0}^{\infty} d E \int_{t_{i}}^{t_{f}} d t f_{i}(\vec{x}, E, \vec{\Omega}, t) .
$$

$f_{i}(\vec{x}, E, \vec{\Omega}, t)$ is connected to the energy spectrum expressed as a flux density for particle type $i$ at point $\vec{x}$ at time $t, \phi_{i}(\vec{x}, t, E)$ by

$$
\phi_{i}(\vec{x}, t, E)=\int_{4 \pi} d \vec{\Omega} f_{i}(\vec{x}, E, \vec{\Omega}, t)
$$

To determine the proper dimensions and composition of a shield, the amount of radiation, expressed in terms of the dose (i.e., dose equivalent, effective dose, etc.) that penetrates the shield and reaches locations of interest must be calculated. This quantity must be compared with the maximum allowed by the design objectives or by regulation. If the calculated result is too large, either the conditions associated with the source of the radiation or the physical properties of the shield must be changed. The latter could be a change in shield materials, dimensions, or both. If the shield cannot be adjusted, then the amount of beam loss allowed by the beam control instrumentation, the amount of residual gas in the vacuum system, or the amount of beam accelerated must be reduced. It is difficult and expensive, especially in the case of the larger accelerators, to alter permanent shielding or operating conditions if the determination of shielding dimensions and composition has not been done correctly. The methods for determining these quantities have been described by a number of workers with only a summary of this important work given in the next section.

\subsubsection{The Boltzmann Equation}

The primary tool for determining the amount of radiation reaching a given location is the stationary form of the Boltzmann equation (for present purposes simply the Boltzmann equation). The solution to this equation yields the angular flux density $f_{i}$. The angular flux density is then converted to dose rate by means of Eq. (2.2). This section reviews the theory that yields the distribution of radiation in matter, and discusses some of the methods for extracting detailed numerical values for elements of this distribution such as 


\section{ChAPTER 2 General Considerations of RADiATION FieldS AT ACCELERATORS}

the particle flux, or related quantities, such as dose, radioactivation or instrument response. The Boltzmann equation includes all the processes that the particles of various types that comprise the radiation field can undergo. More details are given by (OB80) and a summary can be found in (NC03).

The Boltzmann equation was originally derived by Ludwig Boltzmann in 1872 to describe the properties of gases but is well-adapted to address radiation fields. The equation is an integral-differential equation describing the behavior of a dilute assemblage of moving particles. It is a continuity equation of the angular flux density $f_{i}$ in a phase space made up of the three space coordinates of Euclidean geometry, the three corresponding direction cosines (i.e. the cosines of the angles between the trajectory vector and each of the three standard axes of Cartesian coordinates), the kinetic energy, and the time. The density of radiation in a volume of phase space may change in the following five ways:

- uniform translation, where the spatial coordinates change, but the energy-angle coordinates remain unchanged;

- collisions, as a result of which the energy-angle coordinates change, but the spatial coordinates remain unchanged, or the particle may be absorbed and disappear altogether;

- continuous slowing down, in which uniform translation is combined with continuous energy loss;

- decay, where particles are changed through radioactive transmutation into particles of another kind; and

- introduction, involving the direct emission of a particle from a source into the volume of phase space of interest: electrons or photons from radioactive materials, neutrons from an $\alpha-n$ emitter, the "appearance" of beam particles, or particles emitted from a collision at another (usually higher) energy.

Combining these five elements yields

$$
\tilde{\mathrm{B}}_{i} f_{i}(\vec{x}, E, \vec{\Omega}, t)=Q_{i j}+Y_{i}
$$

where the mixed differential-integral Boltzmann operator for particles of type $i, \tilde{\mathrm{B}}_{i}$, is

$$
\begin{gathered}
\tilde{\mathrm{B}}_{i}=\vec{\Omega} \cdot \nabla+\sigma_{i}+d_{i}-\frac{\partial S_{i}}{\partial E}, \\
Q_{i j}=\sum_{j} \int_{4 \pi} d \overrightarrow{\Omega^{\prime}} \int_{o}^{E_{\max }} d E_{B} \sigma_{i j}\left(E_{B} \rightarrow E, \vec{\Omega}^{\prime} \rightarrow \vec{\Omega}\right) f_{j}\left(\vec{x}, E, \vec{\Omega}^{\prime}, t\right), \\
\text { and } \quad d_{i}=\frac{\sqrt{1-\beta_{i}^{2}}}{\tau_{i} \beta_{i} c} .
\end{gathered}
$$

The gradient operator $\nabla$ inherently results in a vector, not scalar, quantity. Not surprisingly, except for a few special cases the Boltzmann equation is difficult to solve! 


\section{CHAPTER 2 GenERAL CONSIDERATIONS OF RADIATION FiELDS AT ACCELERATORS}

In Eqs. (2.6) and (2.7):

- $Y_{i}$ is the number of particles of type $i$ introduced by a source per unit area, time, energy, and per unit solid angle;

- $\sigma_{i}$ is the absorption cross section for particles of type $i$. To be dimensionally correct, this is really the macroscopic cross section or linear absorption coefficient $\mu=N \sigma$ as defined in Eq. (1.5);

- $d_{i}$ is the decay probability per unit flight path of radioactive particles (such as muons, pions, or radionuclide ions) of type $i$;

- $S_{i}$ is the stopping power for charged particles of type $i$ (assumed to be zero for uncharged particles);

- $Q_{i j}$ is the "scattering-down" integral; the production rate of particles of type $i$ with a direction $\vec{\Omega}$, an energy $E$ at a location $\vec{x}$, by collisions with nuclei or decay of $j$-type particles having a direction $\vec{\Omega}^{\prime}$ at a higher energy $E_{B}$;

- Rarely, there are analogous "scattering-up" integrals of similar form for exothermic processes such as thermal neutron capture and some others;

- $\sigma_{i j}$ is the doubly-differential inclusive cross section for the production of $i$-type particles with energy $E$ and a direction $\vec{\Omega}$ from nuclear collisions or decay of $j$ type particles with an energy $E_{B}$ and a direction $\vec{\Omega}^{\prime}$; and

- $\quad \beta_{i}$ is the velocity of a particle of type $i$ divided by the speed of light $c$; and $\tau_{i}$ is the mean-life of a radioactive particle of type $i$ in the rest frame.

\subsection{The Monte Carlo Method}

\subsubsection{General Principles of the Monte Carlo Technique}

The Monte Carlo method is the most common approach in radiation physics to solving the Boltzmann equation for realistic geometries that are difficult to characterize using analytic techniques (i.e., with equations in closed form). The method proceeds by constructing a series of trajectories or histories, choosing each step at random from a distribution of applicable processes. In its most widely used form, the inverse transform method, one starts with calculating a set of travel distances between collisions using known cross sections. Then, the cross sections for changing energy, particle type, or direction are used to create a set of possible outcomes of each collision. The result of the interaction may be a number of particles of varying types, energies, and directions each of which will be followed in turn. Along the way, decay processes are included. The results of many histories are tabulated, leading typically to mean values and standard deviations of particle types, locations, directions, energies, etc.

If $p(x) d x$ is the differential probability of an occurrence at $x_{-1 / 2} d x$ within the interval $[a, b]$ then the integration

$$
P(x)=\int_{a}^{x} d x^{\prime} p\left(x^{\prime}\right)
$$




\section{ChAPTER 2 General Considerations of RADiATION FieldS AT ACCELERATORS}

gives $P(x)$, the cumulative probability of the event occurring in the interval $[a, x]$. The cumulative probability increases monotonically with $x$ and must satisfy the conditions $P(a)=0, P(b)=1.0$. If a random number $R$ uniform on the interval $[0,1]$ is chosen,

$$
R=P(x)
$$

corresponds to a random choice of the value of $x$, since the distribution function for the cumulative $P(x)$ can, in principle, be inverted as a unique one-to-one mapping;

$$
x=P^{-1}(R) .
$$

To illustrate, in determining when an uncharged particle undergoes a reaction in a onedimensional system with no decays $(d=0)$, no competing processes $(S=0)$, and no "inscattering" $(Q=0)$, one recognizes from Eqs. (1.3), (2.6), and (2.7) that a simple application of the Boltzmann equation is evident;

$$
\tilde{\mathrm{B}} \Phi=\left\{\vec{\Omega} \cdot \nabla+\sigma_{i}\right\} \Phi
$$

This simple situation reduces to the following, when one understands $\sigma_{i}$ to be the macroscopic cross section otherwise denoted by $N \sigma$,

$$
\tilde{\mathrm{B}} \Phi=\frac{d \Phi}{d x}+N \sigma \Phi=0
$$

The solution to this equation is the familiar

$$
\Phi=\Phi_{0} \exp (-x / \lambda)
$$

where $\lambda=1 / N \sigma$ as in Eq. (1.5). Replacing $x / \lambda$ with $r$, the number of mean-free-paths the particle travels in the medium, the differential probability per unit mean-free-path for an interaction is given by

$$
\begin{gathered}
p(r)=\exp (-r), \text { then; } \\
\left.P(r)=\int_{0}^{r} d r^{\prime} \exp \left(-r^{\prime}\right)=-\exp \left(-r^{\prime}\right)\right]_{0}^{r}=1-\exp (-r)=R \Rightarrow r=-\ln (1-R)
\end{gathered}
$$

Selecting a random number $R$ then determines a depth $r$ that has the proper distribution. Obviously, identical results apply to other processes described by an exponential function such as radioactive decay. In this simple situation, it is clear that one can solve the above for $r$ as a function of $R$ and thus obtain individual values of $r$ from a corresponding set of random numbers. For many processes, an inversion this simple is not possible analytically. In those situations, other techniques exemplified by successive approximations and table look-up procedures can be employed. 


\section{ChAPTER 2 General Considerations of RADiATION FieldS AT ACCELERATORS}

In a real Monte Carlo calculation, further sampling processes might select the scattering of the particles being followed, particle production, decays, etc. Deflections by magnetic fields can be readily included for each segment of the trajectory.

The Monte Carlo result is the number of times the event of interest occurred in a given number of so-called trajectories or histories. As a counting process it has an uncertainty. The reciprocal of the relative error will tend to be roughly proportional to the square root of the number of trajectories calculated. Thus high probability processes are more accurately simulated than are low probability ones. This becomes a problem for calculations of radiation field properties external to a thick shield in which the particle fluences are attenuated over many orders of magnitude. Sophisticated techniques are often used that temporarily give enhanced probabilities, called weights, to the lowprobability events during the calculation in order to study them. The correct probabilities are then restored at the end of the calculation by removing the weights. It is common practice to evaluate the accuracy of a Monte Carlo result by repeating calculations using different initial values of the random number generator, the so-called "seed".

\subsubsection{Monte Carlo Example; A Sinusoidal Angular Distribution of Beam Particles}

Suppose one has a distribution of beam particles such as exhibited in Fig 2.2.

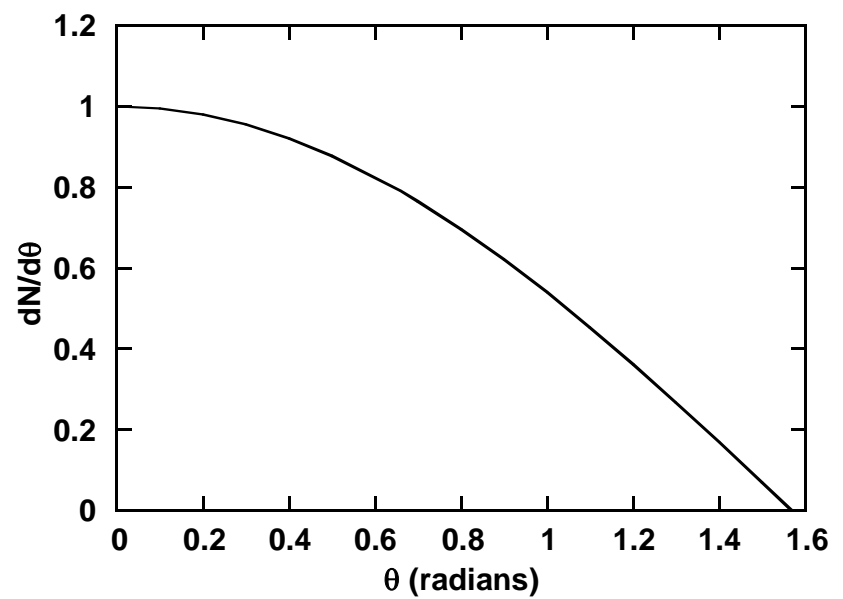

Fig. 2.2 Hypothetical angular distribution of particles obeying a distribution proportional to $\cos \theta$.

For this distribution, $p(\theta)=A \cos \theta$ for $0<\theta<\pi / 2$. The fact that the integral of $p(\theta)$ over the relevant interval $0 \leq \theta \leq \pi / 2$ to get the cumulative probability $P(\theta=\pi / 2)$ must be unity implies $A=1$ since

$$
P(\pi / 2)=\int_{0}^{\pi / 2} d \theta A \cos \theta=\left.A \sin \theta\right|_{0} ^{\pi / 2} \stackrel{\text { def }}{=} 1
$$

Thus, $p(\theta)=\cos \theta$. The cumulative probability, $P(\theta)$, is then given by

$$
P(\theta)=\int_{O}^{\theta} d \theta^{\prime} p\left(\theta^{\prime}\right)=\int_{O}^{\theta} d \theta^{\prime} \cos \theta^{\prime}=\left.\sin \theta^{\prime}\right|_{O} ^{\theta}=\sin \theta
$$




\section{ChAPTER 2 GENERAL Considerations of RADiATION FieldS AT ACCELERATORS}

If $R$ is a random number, then $R=P(\theta)$ determines a unique value of $\theta$; hence

$$
\theta=\sin ^{-1}(R)
$$

It is instructive to do a Monte Carlo calculation with, say, 50 random numbers. One can proceed by setting up a table such as Table 2.1 populated by a particular set of 50 numbers where the results have been tallied ( $2^{\text {nd }}$ column) into "bins" of ranges of $\theta$ values $\left(1^{\text {st }}\right.$ column $)$. The tallies in the second column collect those "events" for which an individual random number $R$ results in a value of $\theta$ within the associated $1^{\text {st }}$ column bin. $\theta_{\text {mid }}$ is the midpoint of the bin $(0.1,0.3, \ldots)$. The $4^{\text {th }}$ column is the normalized result determined from the following:

$$
\begin{aligned}
& N=\frac{\text { Number found in Monte Carlo bin }}{\text { (Total number of events })(\text { bin width })} \\
& =\frac{\text { Number found in bin in Monte Carlo }}{(50)(0.2 \text { radians })} .
\end{aligned}
$$

Table 2.1 Tally sheet for Monte Carlo example.

\begin{tabular}{|c|c|c|c|c|}
\hline$\theta$ (radians) & $R$ (random \#) & Total $R$ 's in Bin & $N$ (norm. \#) & $\theta_{\text {mid }}$ \\
\hline $0.0-0.199$ & $1114+1111$ & 11 & 1.1 & 0.1 \\
\hline $0.2-0.399$ & $1114+111-111$ & 13 & 1.3 & 0.3 \\
\hline $0.4-0.599$ & $1114+1141$ & 11 & 1.1 & 0.5 \\
\hline $0.6-0.799$ & 1111 & 4 & 0.4 & 0.7 \\
\hline $0.8-0.999$ & 111111 & 7 & 0.7 & 0.9 \\
\hline $1.0-1.199$ & 1111 & 4 & 0.4 & 1.1 \\
\hline $1.2-1.399$ & & & & 1.3 \\
\hline $1.4-1.57$ & & & & 1.485 \\
\hline
\end{tabular}

One can calculate exactly the mean value of $\theta$ for the specified distribution:

$$
\begin{gathered}
\langle\theta\rangle=\frac{\int_{0}^{\pi / 2} \theta p(\theta) d \theta}{\int_{0}^{\pi / 2} p(\theta) d \theta}=\frac{\int_{0}^{\pi / 2} \theta \cos (\theta) d \theta}{1}=[\cos \theta+\theta \sin \theta]_{0}^{\pi / 2} \\
\langle\theta\rangle=\left[(0-1)+\left(\frac{\pi}{2}-0\right)\right]=0.57
\end{gathered}
$$

To calculate the same quantity from the Monte Carlo result, one proceeds first by multiplying the frequency of Monte Carlo events for each eight angular bins from the table by the midpoint value of the bins. Then one sums over the eight bins and divides by the number of incident particles ( 50 in this example). Thus one can determine the average 


\section{ChAPTER 2 General Considerations of RADiATION FieldS AT ACCELERATORS}

value of $\theta,<\theta>_{\mathrm{MC}}$, calculated by the Monte Carlo technique:

$<\theta>_{\mathrm{MC}}=[(11)(0.1)+(13)(0.3)+(11)(0.5)+(4)(0.7)+(7)(0.9)+(4)(1.1)] / 50=0.48$.

In spite of the very coarse bins and a very small number of histories used in this example, the agreement is perhaps surprisingly good. This example also illustrates that the statistical errors are generally larger for the more rare events here represented by large values of $\theta$ (i.e., $\theta>1$ radian).

Practical Monte Carlo calculations generally involve the need to follow a huge number of histories. This requires the use of computers perform useful calculations in nearly all circumstances. $^{7}$ As the speed of computer processors has increased, the ability to model the physical effects in more detail and with ever improving statistical accuracy has resulted. In subsequent chapters, results obtained using some specific codes will be presented. Descriptions of these codes, accurate as of this writing, are given in Appendix A. It should be noted that most of these codes are being constantly improved with the result that the wisest practice in using them is to consult with their authors directly.

\subsection{Review of Magnetic Deflection and Focusing of Charged Particles}

\subsubsection{Magnetic Deflection of Charged Particles}

Particle accelerators of all types utilize electromagnetic forces to accelerate, deflect, and focus charged particles. The physics has been well-described in textbooks such as those by Carey (Ca87), Chao and Tigner (Ch99), Edwards and Syphers (Ed93), and Lee (Le04). A review of this topic tailored to the needs of radiation physics at accelerators has been given by Cossairt (Co08). In accelerator radiation protection, an understanding of these forces is motivated by the need to be able to determine the deflection of particles by electric or magnetic fields to be able to assure that particles in a deflected particle beam either interact with material where such interactions are desired or avoid such beam loss. Doing this is interconnected with the design of the accelerator and for those purposes advanced texts such as those cited above should be consulted. This is especially important when radiofrequency (RF) electromagnetic fields are applied to the particle beams where a full treatment using electrodynamics is needed ${ }^{8}$. However, some of the simpler issues are discussed in this section for static, or slowly varying electric and magnetic fields.

The Lorentz force $\vec{F}$ (Newtons) on a given charge $q$ (Coulombs) in SI units at any point in space is

\footnotetext{
${ }^{7}$ Historically some Monte Carlo calculations predating the advent of computers were performed successfully using wheels of chance and hand-tallying techniques exemplified by that of Wilson (Wi52).

${ }^{8}$ As the reader should recall, Maxwell's Equations interconnect the electric and magnetic fields when they vary with time.
} 


$$
\vec{F}=q(\vec{v} \times \vec{B}+\vec{E})=\frac{d \vec{p}}{d t}
$$

where the electric field $\vec{E}$ is in Volts meter ${ }^{-1}$, the magnetic field $\vec{B}$ is in Tesla (1.0 Tesla $=10^{4}$ Gauss), and $\vec{v}$ is the velocity of the charged particle in $\mathrm{m} \mathrm{s}^{-1}, \vec{p}$ is the momentum of the particle in SI units, and $t$ is the time $(\mathrm{sec})$. The direction of the force due to the cross product in Eq. (2.24) is, of course, determined by the usual right-hand rule. Static electric fields (i.e., $d \vec{E} / d t=0$ ), if present, serve to accelerate or decelerate the charged particles. Electrostatic deflection according to Eq. (2.24) is used and is of considerable importance even at large accelerators. This has been discussed in the references cited above including (Co08). In a uniform magnetic field without the presence of an electric field, due to the cross product in this equation, any component of $\vec{p}$ which is parallel to $\vec{B}$ will not be altered by the magnetic field. Typically, charged particles are deflected by dipole magnets in which the magnetic field is, to high order, spatially uniform and constant with time, or slowly-varying compared with the time during which the particle is present. For this situation, if there is no component of $\vec{p}$ which is parallel to $\vec{B}$, the motion is circular and the magnetic force serves to supply the requisite centripetal acceleration. The presence of a component of $\vec{p}$ which is parallel to $\vec{B}$ results in a trajectory that is a spiral rather than a circle. Fig. 2.3 illustrates the condition of circular motion.

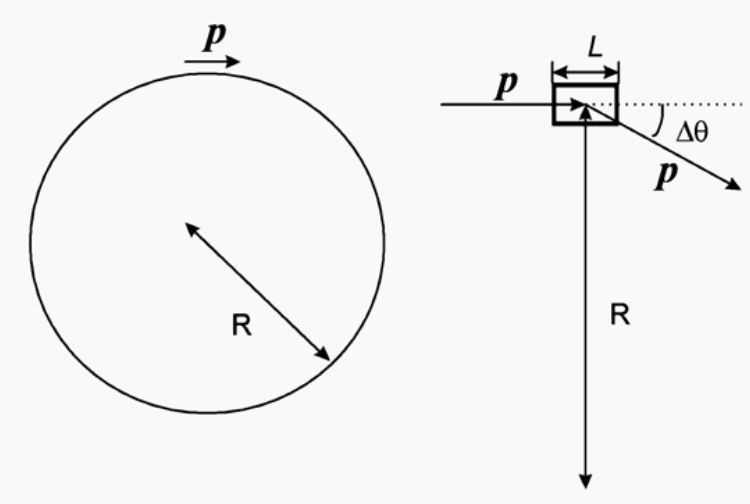

$\boldsymbol{B}$ is perpendicular to the paper and directed toward the reader

Fig. 2.3 A particle of positive charge $q$ having momentum $\vec{p}$ follows a circular path when directed perpendicular to a static, uniform magnetic field $\vec{B}$. The panel on the left illustrates this for a complete circle. On the right, a particle of momentum $\vec{p}$ enters a magnet of length $L$ that has field integral value of $B L$. For this example, $L<<R$ and the particle experiences a small angular deflection $\Delta \theta$. The angular deflection is exaggerated in this figure for clarity. [Reproduced from (Co08).]

Equating the centripetal force to the magnetic force and recognizing that $\vec{p}$ is perpendicular to $\vec{B}$ leads to

$$
\frac{m v^{2}}{R}=q v B
$$




\section{ChAPTER 2 General Considerations of RADiATION FieldS AT ACCELERATORS}

where $m$ is the relativistic mass (see Eq. 1.13). Solving for the radius of the circle $R$ (meters), recognizing that $p=m v$, and changing the units of measure for momentum,

$$
R(\text { meters })=\frac{p}{q B}(\text { SI units })=\frac{p(\mathrm{GeV} / \mathrm{c})}{0.29979 q B}
$$

where $q$ in the denominator of the right hand side is now the number of electronic charges carried by the particle and $B$ remains expressed in Tesla. The numerical factor in the denominator is just the value of the speed of light in SI units divided by $10^{9}$.

At large accelerators, one is often interested in the angular deflection of a magnet of length $L$ that provides such a uniform field orthogonal to the particle trajectory. Such a situation is also shown in Fig. 2.3. If $L$ is only a small piece of the complete circle (i.e., $L<<R$ ), one can consider the circular path over such a length to be two straight line segments "bent" by the deflection. Doing this, the change in direction $\Delta \theta$ is given by

$$
\Delta \theta=\frac{L}{R}=\frac{0.29979 q B L}{p} \text { (radians), }
$$

where the product $B L$ (Tesla-meters) is commonly referred to the field integral of the magnet system and $p$ remains in GeV/c. $B L$ could just as well be obtained by integrating a non-uniform field over the length of a particular magnet system. This angle of deflection can be used to determine if a particle beam will interact with some solid object near its path, a matter of practical importance for radiation protection.

\subsubsection{Magnetic Focusing of Charged Particles}

Charged particle beams can be focused by a variety of devices including the edge fields of dipole magnets and electrostatic quadrupoles (Co08). However, the use of quadrupole magnets is the most common method. The references cited in Section 2.5.1 describe in much more detail these systems and those of higher order that focus particle beams. Mathematical methods analogous to those found in the study of geometrical optics are used to describe the optics of charged particles. Where time-varying electric and magnetic fields are involved, the full complement of Maxwell's equations must, of course, be used to describe the motion of charged particles. The application of higher order multipole fields and the employment of radiofrequency (RF) electromagnetic fields to accelerate, decelerate, and otherwise manipulate charged particle beams is left to the specialized texts.

An idealized quadrupole magnet has the transverse cross section shown in Fig. 2.4, which also defines the Cartesian coordinate system to be used in the remainder of this section. As one can see, the polarities of the pole pieces alternate. Following the usual convention, the longitudinal coordinate, $z$, is taken to be directed along the beam and, in this case, "into the paper" along the optic axis of the quadrupole. Positive values of the $y$ coordinate measure upward deviations from the optic axis while positive values of the $x$ coordinate measure deviations from the optic axis to "beam left", to maintain consistency 
with the right-hand rule.

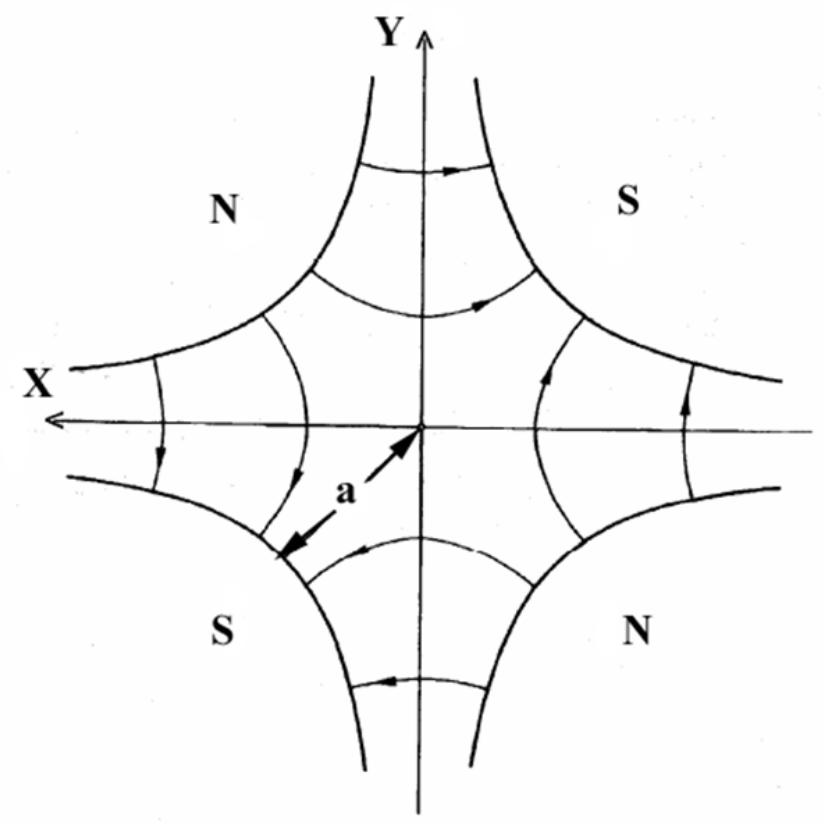

Fig. 2.4 Cross section of a typical quadrupole magnet. The pole pieces are of opposite magnetic polarities, denoted $\mathrm{N}$ and $\mathrm{S}$, and are of hyperbolic shapes. A Cartesian coordinate system is used in which $x$ and $y$ denote transverse coordinates while $z$ is along the desired beam trajectory, the optic axis of the beam optical system. In this figure, the beam enters the quadrupole into the paper along the positive $z$ axis. The curves with arrows denote magnetic field lines. [Reproduced from (Co08).]

Often in the accelerator magnets themselves and nearly always in beam lines transmitting extracted particles, the electromagnetic fields vary only slowly with time or are static compared with the particle transit times. Under these conditions, it is shown in the references that if the shape of the pole pieces are hyperbolae described by equations of form $x y= \pm k$, where $k$ is a constant, and if the pole pieces are uniformly magnetized, then the components of the magnetic field within the gap containing the beam are;

$$
\begin{aligned}
& B_{x}=-\frac{B_{o}}{a} y=-g y, \text { and } \\
& B_{y}=-\frac{B_{o}}{a} x=-g x .
\end{aligned}
$$

Here, $a$ is the gap dimension defined in Fig. 2.4 and $B_{o}$ is the magnitude of the magnetic field strength at the pole pieces. The parameter $g$ is, quite naturally, called the gradient of the quadrupole and in this scheme has units of Tesla meter ${ }^{-1}$. This ideal quadrupole has length $L$ along the z-coordinate, the optic axis of the system.

Now examine qualitatively what happens to a particle having positive charge that enters this magnet parallel to the $z$-axis. If the particle trajectory is on the optic axis, then it will 


\section{CHAPTER 2 GENERAL CONSIDERATIONS OF RADIATION FiELDS AT ACCELERATORS}

not be deflected at all since $B_{x}=B_{y}=0$. If, however, a particle enters the magnet parallel to the optic axis but with some finite positive value of $y$, it will receive a deflection toward smaller values of $y$ in accordance with the right hand rule and Eq. (2.24). Likewise, if it enters with a finite negative value of $y$, it will receive a deflection toward less negative values of $y$. Thus, a beam of such particles is said to be focused in the $y z$ plane. However, if the particle enters with a finite positive value of $x$, it will be deflected toward a larger value of $x$, away from the optic axis. Finally, a particle incident with a finite negative value of $x$ will similarly be deflected away from the optic axis. Thus, a beam of such particles is said to be defocused in the $x z$ plane. Even qualitatively, it is clear that more than one quadrupole is needed to achieve a net focusing effect.

Considering just the situation in the $y z$ plane, it is easy to see that the analogy with geometrical optics is instructive even in mathematical detail. For a particle entering with coordinate $y$, one can substitute into Eq. (2.27) and find the angular deflection to be

$$
\Delta \theta=\frac{0.29979 q L g y}{p} \text { (radians), }
$$

where the same units as Eq. (2.27) are used, with $g$ (Tesla meter ${ }^{-1}$ ) and $y$ (meters) inserted and the negative sign of Eq. (2.28) now implicit. If the incident particle trajectory is parallel with the $z$-axis, the situation is schematically shown in Fig. $2.5 \mathrm{a}^{9}$. Applying trigonometry, one finds that after deflection in this situation, the particle trajectory will intercept the $z$-axis at a distance $f$ is

$$
f=\frac{y}{\tan \Delta \theta} \approx \frac{y}{\Delta \theta}=\frac{p}{0.29979 q L g}
$$

since the deflection $\Delta \theta$ is small. This approximation is called the thin lens approximation. Recognizing that $f$ is independent of the $y$ coordinate, $f$ is called the focal length of the quadrupole. By analogy with optical thin lenses, the thin lens equation connecting the image distance $z_{i}$, with the object distance $z_{o}$ for other rays is as follows:

$$
\frac{1}{z_{o}}+\frac{1}{z_{i}}=\frac{1}{f}
$$

In this equation, $z_{o}$ and $z_{i}$ are $>0$ if the object is to the left of the lens and the image is to the right of the lens, forming a real image, for a focusing lens with $f>0$. The situation for the defocusing plane, here the $x z$ plane, is shown in Fig. $2.5 \mathrm{~b}$ as a concave lens. For that plane, the equations are still workable if one applies a negative sign to the value of $f$ and understands that a value of $z_{i}<0$ describes a virtual image.

\footnotetext{
${ }^{9}$ In schematic drawings of beam optics, it is customary to show convex lenses to denote focusing elements and concave lenses to represent defocusing elements pertinent to a given plane. Bending magnets are similarly represented by prisms in such drawings.
} 
a)

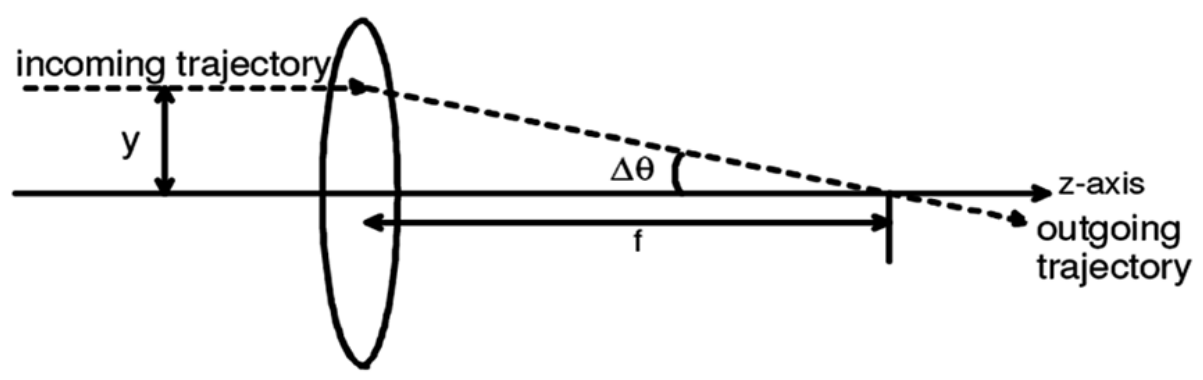

b)

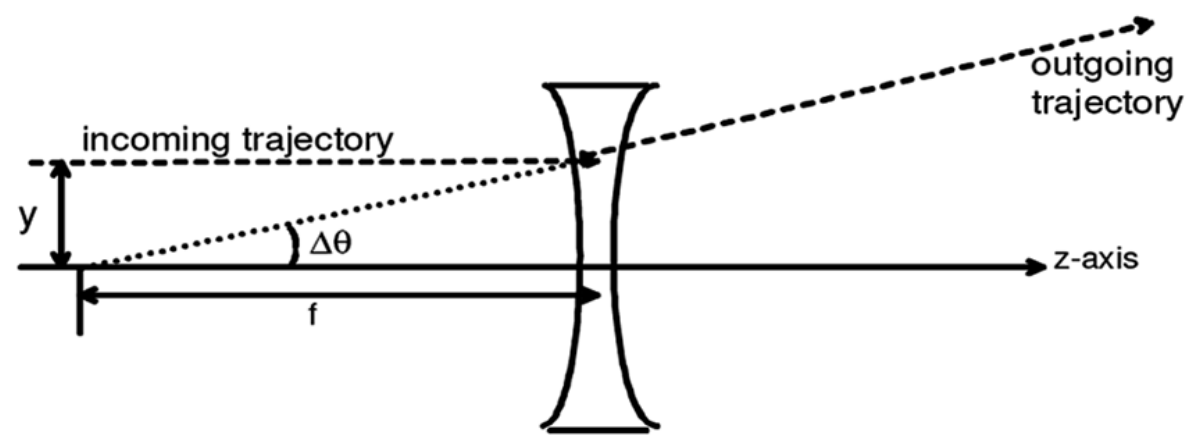

c)

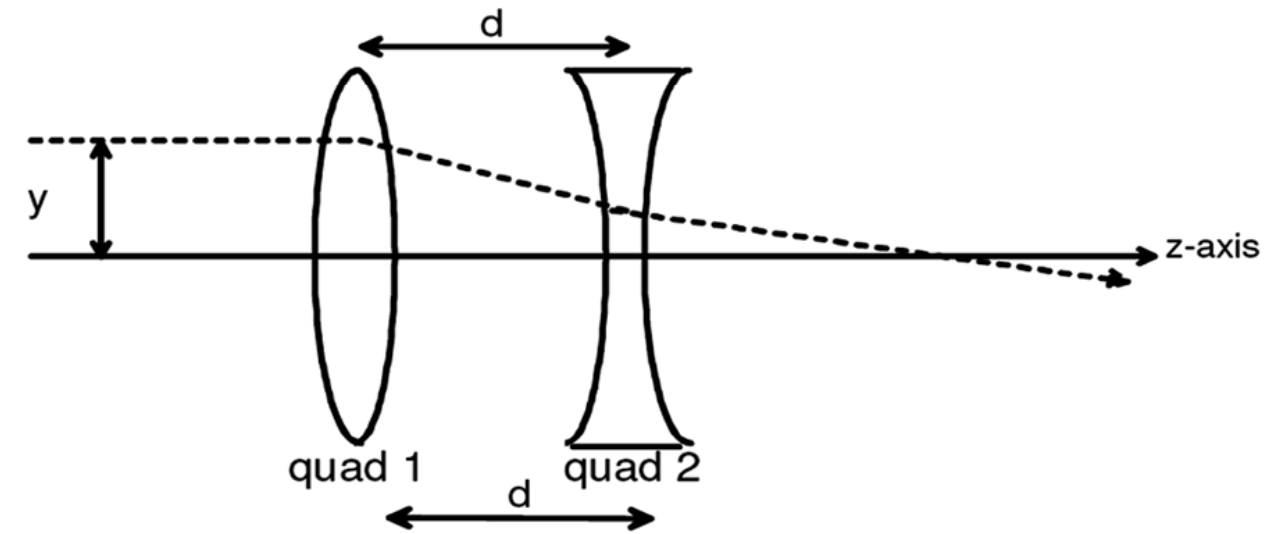

d)

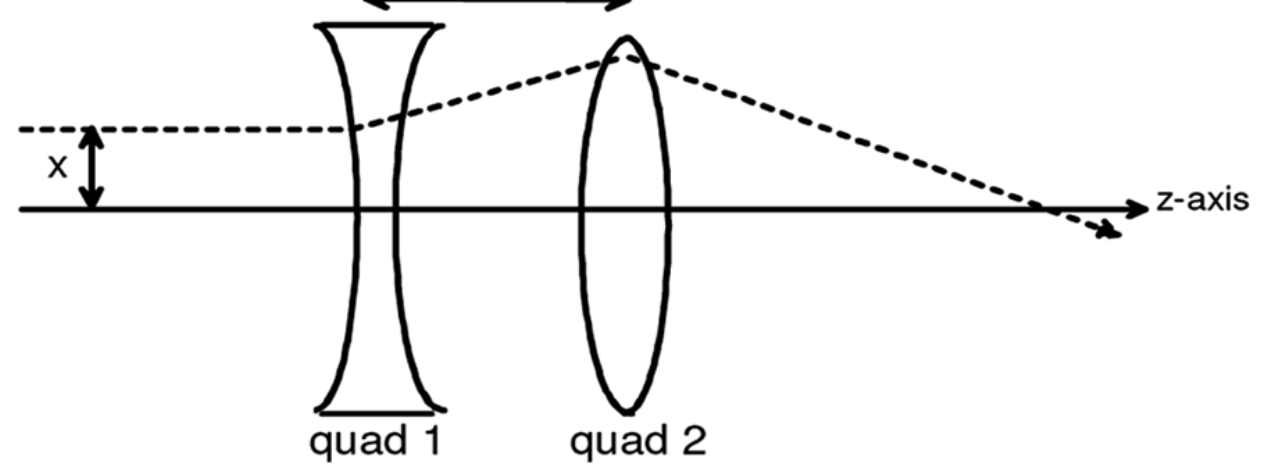

Fig. 2.5 Configurations of quadrupole lenses are shown with the symbolism explained in the text: a) Representation of focusing in the $y z$ plane of a beam trajectory incident from the left parallel to the $z$-axis. A real image is formed at the focal length $f$ from the lens. b) Representation of defocusing in the $y z$ plane. The parallel beam is deflected so that it appears to emerge from a point a distance $f$ before the lens, thus, forming a virtual image. c) Representation of a particle trajectory in the $y z$ plane of a quadrupole doublet. The particle enters a quadrupole doublet parallel to the $z$-axis from the left. First a focusing quadrupole (quad 1) is encountered and then a defocusing quadrupole (quad 2) follows. d) Representation of a particle trajectory in the $x z$ plane of the same doublet. The particle enters the doublet parallel to the $z$-axis. In this plane, the defocusing quadrupole is encountered first. [Reproduced from (Co08).] 


\section{ChAPTER 2 General Considerations of RADiATION FieldS AT ACCELERATORS}

The simplest configuration of quadrupole magnets is a pair of two. In a given plane, say the $y z$, the first might be focusing while the second is defocusing. In the orthogonal plane, here the $x z$, the defocusing quadrupole would thus be encountered first. Commonly, these magnets are of identical dimensions and have the same or similar gradients. Such a quadrupole doublet is shown in Figs $2.5 \mathrm{c}$ and $2.5 \mathrm{~d}$ for the $y z$ and $x z$ planes, respectively.

Eq. (2.32) can now be used to see how a quadrupole doublet can focus a parallel beam in both the $x z$ and $y z$ planes in a simple example. For this discussion, the two quadrupoles, quad 1 and quad 2, have different focal lengths $f_{1}$ and $f_{2}$, respectively, and are separated by distance $d$. Quad 1 is focusing in the $y z$ plane. As in geometrical optics, for an incoming parallel beam, the object distance relative to quad 1 is $z_{y o 1} \rightarrow \infty$. Thus, the image distance from quad 1 is at $z_{y i l}=f_{1}$. The object distance of this image from quad 2 is then $z_{y o}=d-f_{1}$. From Eq. (2.32), relative to quad 2, the final image will be at $z_{y i 2}$;

$$
\frac{1}{z_{y i 2}}=\frac{1}{-f_{2}}-\frac{1}{d-f_{1}},
$$

where the negative coefficient of $f_{2}$ explicitly incorporates the fact that lens 2 is defocusing in the $y z$ plane. Solving,

$$
z_{y i 2}=\frac{f_{2}\left(f_{1}-d\right)}{f_{2}-f_{1}+d} .
$$

If the quadrupoles are identical $\left(f=f_{1}=f_{2}\right)$, then

$$
z_{y i 2}=\frac{f(f-d)}{d}
$$

Following the same procedure for the $x z$ plane to obtain the corresponding image distance $z_{x i 2}$;

$$
z_{x i 2}=\frac{f_{2}\left(f_{1}+d\right)}{f_{1}-f_{2}+d}
$$

With identical quadrupoles, this becomes

$$
z_{x i 2}=\frac{f(f+d)}{d}
$$

One notices that with identical quadrupoles,

$$
z_{x i 2}-z_{y i 2}=2 f
$$

a result that is expected since that particles in $x z$ plane are first subject to defocusing, and thus become more divergent, prior to their being focused. The average focal length of 


\section{ChAPTER 2 General Considerations of RADiATION FieldS AT ACCELERATORS}

the system for both the $x z$ and $y z$ planes is thus $f^{2} / d$. More sophisticated schemes like quadrupole triplets and non-identical magnets can be used to obtain a specialized beam envelope. These advanced methods are discussed in great detail in the references.

In quadrupoles, the beam axis should coincide with the optic axis. If a beam enters a quadrupole far off-axis, the entire beam will be deflected nearly as if a quadrupole were a dipole magnet of equivalent field strength and length (see Fig. 2.4). Beams that are deflected in this manner by a quadrupole are said to have suffered steering. The steering of beams can constitute significant loss points in the beam transport system.

In this simple exposition, some significant effects have been ignored. First, a typical particle beam will contain some spread in particle momenta. The derivation given above ignores the fact that dispersion will occur in the magnetic fields in the same way that prism disperses a visible beam of "white" light into the various colors. There also may be aberrations or distortions of an image. An example is chromatic aberration, analogous to its namesake encountered in geometrical optics. For particle beams chromatic aberration is due to the dependence of focal length on particle momentum in Eq. (2.31). Also, no particle beam is ever completely parallel or emergent from a geometrical point.

All particle beams possess a property called transverse emittance. This quantity is expressed as the product of angular divergence and physical size, typically in units of $\pi$ mm-milliradian. The explicit display of the factor $\pi$ is a matter of custom. Emittance is used to describe both longitudinal and transverse phenomena but the discussion here is limited to transverse emittance. During the acceleration process, the beam emittance becomes smaller because the normalized transverse emittance [the emittance when multiplied by the relativistic factors $\gamma \beta$ from Eqs. (1.12)] is an invariant. Thus, as velocity and $\gamma \beta$ increases, the unnormalized emittance must decrease. There are exceptions to this generalization not discussed here. Once a beam is no longer undergoing acceleration (e.g., in an extracted beamline), the emittance remains constant or increases due to processes such as multiple Coulomb scattering, space charge effects, etc. ${ }^{10}$ When the emittance remains constant, the product of the angular divergence of the beam envelope and the transverse size of the beam envelope is conserved. ${ }^{11}$ Thus efforts made to focus the beam into a smaller cross-sectional size will unavoidably result in a beam with a correspondingly larger angular spread. Likewise, attempts to create a parallel beam (one with essentially no angular spread) will result in a correspondingly larger beam size.

\footnotetext{
${ }^{10}$ Under some conditions not discussed further in this text, synchrotron radiation can, in fact, reduce the transverse emittance.

${ }^{11}$ This is a consequence of Liouville's theorem of classical dynamics as applied to the coordinate system defined here which requires that the volume of the phase space defined by the transverse spatial coordinates; $x$ and $y$, and their corresponding "conjugate" momentum components, $p_{x}$ and $p_{y}$; is conserved. Since in this Cartesian coordinate system, the momentum components $p_{x}$ and $p_{y}$ are, in small angle approximation, directly proportional to the angles (in radians) between the momentum vector and the $x$ and $y$ coordinate axes, respectively, the assertion made above directly follows. Textbooks on accelerator physics discuss this in more detail [e.g., (Ca87), (Ch99)].
} 


\section{Problems}

1. This problem gives two elementary examples of Monte Carlo techniques that are almost "trivial". In this problem, obtaining random numbers from a standard table or from a hand calculator should be helpful.

a) First, use a random number table or random number function on a calculator along with the facts given about the cumulative probability distribution for exponential attenuation to demonstrate that, even for a sample size as small as, say, 15, the mean value of paths traveled is "within expectations" if random numbers are used to select those path lengths from the cumulative distribution. Do this, for example, by calculating the mean and standard deviation of your distribution.

b) An incident beam is subjected to a position measurement in the coordinate $x$. It is desirable to "recreate" incident beam particles for a shielding study using Monte Carlo. The $x$ distribution as measured is as follows:

\begin{tabular}{|l|l|}
\hline $\boldsymbol{x}$ & $\#$ \\
\hline 0 & 0 \\
\hline 1 & 1 \\
\hline 2 & 2 \\
\hline 3 & 3 \\
\hline 4 & 4 \\
\hline 5 & 5 \\
\hline 6 & 4 \\
\hline 7 & 3 \\
\hline 8 & 2 \\
\hline 9 & 1 \\
\hline 10 & 0 \\
\hline
\end{tabular}

Determine, crudely, $p(x)$ and $P(x)$ and then use 50 random numbers to "create" particles intended to represent this distribution. Then compare with the original one which was measured in terms of the average value of $x$ and its standard deviation. Do not take the time to use interpolated values of $x$, simply round off to integer values of $x$ for this demonstration.

2. A beam of protons having a kinetic energy of $100 \mathrm{GeV}$ is traveling down a beam line. The beam is entirely contained within a circle of diameter $1.0 \mathrm{~cm}$. All of the beam particles have the same kinetic energy. An enclosure further downstream must be protected from the beam or secondary particles produced by the beam by shielding it with a large diameter iron block that is $20 \mathrm{~cm}$ in radius centered on the beam line. The beam passes by this block by being deflected by a uniform field magnet that is 3.0 meters long, the longitudinal center of which is located 30 meters upstream of the iron block. Calculate the magnetic field $B$ that is needed to accomplish this objective. 


\section{ChAPTER 3 PRompt RAdiation FieldS Due to ElECTRONS}

\subsection{Introduction}

In this chapter the major features of the prompt radiation fields produced by electrons are described. The development of the electromagnetic cascade and the shielding of photoneutrons and high energy particles that result are discussed. The utilization of Monte Carlo calculations in electron shielding problems is also addressed. The material presented in this chapter is useful for understanding electron, photon, and photoneutron radiation from electron accelerators used in medicine and in research. Some of this information is also useful in understanding the radiation produced by certain accelerator components, such as RF cavities operated apart from the main accelerator, as pointed out by Silari, et al. (Si99). A section on synchrotron radiation completes this chapter.

\subsection{Unshielded Radiation Produced by Electron Beams}

At all energies photons produced by bremsstrahlung dominate the unshielded radiation field aside from the hazard of the direct beam. As the energy increases, neutrons become a significant problem. For electrons having kinetic energy $E_{O}$ approaching $100 \mathrm{MeV}$ or higher the electromagnetic cascade is of great importance. A useful rule of thumb is that electrons have a finite ionization range $R$ in any material that monotonically increases with the initial kinetic energy $E_{o}(\mathrm{MeV})$. For $2<E_{o}<10 \mathrm{MeV}$,

$$
R \approx 0.6 E_{O}\left(\mathrm{~g} \mathrm{~cm}^{-2}\right) \text {. }
$$

In air at standard temperature and pressure (STP) over this energy domain, $R($ meters $) \approx 5 E_{O}(\mathrm{MeV})$. At energies above $10 \mathrm{MeV}$ or so, a threshold that we will see is dependent upon the absorbing medium, the loss of energy begins to be dominated by radiative processes, whereby the emission of photons begins to dominate over those losses of energy due to collisions, a phenomenon discussed further in Section 3.2.2.

\section{$\underline{\text { 3.2.1 }}$ Dose Rate in a Direct Beam of Electrons}

At all accelerators, the dose rate in the direct particle beam is generally larger than in any purely secondary radiation field. This is certainly true at electron accelerators. Swanson (Sw79a) has given a rule of thumb, said to be "conservative", for electrons in the energy domain of $1.0<E_{O}<100 \mathrm{MeV}$;

$$
\frac{d H_{\text {equiv }}}{d t}=1.6 \times 10^{-4} \phi
$$

where $d H_{\text {equiv }} / d t$ is the dose equivalent rate $\left(\mathrm{rem} \mathrm{h}^{-1}\right)$ and $\phi$ is the flux density $\left(\mathrm{cm}^{-2} \mathrm{~s}^{-1}\right)$ in the electron beam. One of the problems at the end of this chapter examines the domain of validity of this approximation. The coefficient is $1.6 \times 10^{-6}$ if $d H_{\text {equiv }} / d t$ is to be in $\mathrm{Sv} \mathrm{h}^{-1}$ with $\phi$ still expressed as $\mathrm{cm}^{-2} \mathrm{~s}^{-1}$. For electrons, there is no significant difference between dose equivalent and effective dose. 


\section{ChAPTER 3 PROMPt RADiATION Fields DUe To ELECTRONS}

\subsubsection{Bremsstrahlung}

Bremsstrahlung is the radiative energy loss of charged particles, especially electrons, as they interact with materials. It appears in the form of photons. An important parameter when considering the radiative energy loss of electrons in matter is the critical energy $E_{c}$ above which the radiative loss of energy exceeds that due to ionization. There are several formulae used to calculate $E_{c}$ with representative ones given here. For electrons, the value of $E_{c}$ is a smooth function of atomic number, approximated by

$$
E_{c}=\frac{800(\mathrm{MeV})}{Z+1.2},
$$

where $Z$ is the atomic number of the material. For muons (see Sections 1.6.1 and 3.2.4) the corresponding critical energy $E_{c, \text { тио }}$ is much larger and differs for solid and gaseous media (PDG04);

$$
E_{c, \text { mио }}=\frac{5700 \mathrm{GeV}}{(\mathrm{Z}+1.47)^{0.838}}(\text { solids }), \text { and } E_{c, \text { mио }}=\frac{7980 \mathrm{GeV}}{(\mathrm{Z}+2.03)^{0.879}} \text { (gases). }
$$

The transition from dominance by ionization to dominance by radiation is a smooth one. The total stopping power for electrons or muons may be written as the sum of collisional and radiative components, respectively;

$$
\left(\frac{d E}{d x}\right)_{\text {tot }}=\left(\frac{d E}{d x}\right)_{\text {coll }}+\left(\frac{d E}{d x}\right)_{\mathrm{rad}} .
$$

Another parameter of significant importance is the radiation length $X_{O}$, the mean thickness of material in which a high energy electron loses all but $1 / e$ of its energy by bremsstrahlung. This parameter is the approximate scale length for describing high energy electromagnetic cascades, supplanting the ionization range for even moderate electron energies. It also plays a role in the "scaling" of multiple Coulomb scattering for all charged particles and was discussed in that context in Section 1.6.2. The radiation length is approximated by Eq. (1.23). For electrons with energies well above $E_{c}$, the fractional energy loss is equal to the fraction of a radiation length it penetrates;

$$
\frac{d E_{r a d}}{E}=-\frac{d x}{X_{0}}, \text { thus }\left(\frac{d E}{d x}\right)_{r a d}=-\frac{E}{X_{0}}
$$

so that under these conditions (i.e., where loss by ionization can be neglected), the energy of the electron $E$ as a function of thickness of shield penetrated $x$ is given by

$$
E(x)=E_{o} \exp \left(-x / X_{o}\right)
$$

where the energy of the incident particle is $E_{o}$ and $x$ and $X_{o}$ are in the same units. 


\section{ChAPTER 3 PRompt RAdiATION Fields Due to Electrons}

Fig. 3.1 gives the percentage of energy $E_{o}$ that appears as radiation for various materials as a function of energy for electrons stopped in the material. Bremsstrahlung develops as a function of target thickness and is described by a transition curve. As the thickness increases, the intensity of the radiation increases until re-absorption begins to take effect. Then, self-shielding begins to take over. One talks about the maximal conditions as being a "thick-target" bremsstrahlung spectrum. This phenomenon becomes dominant above energies of about $100 \mathrm{MeV}$ for low atomic number ("low-Z") materials and above 10 $\mathrm{MeV}$ for high atomic number ("high-Z”) materials.

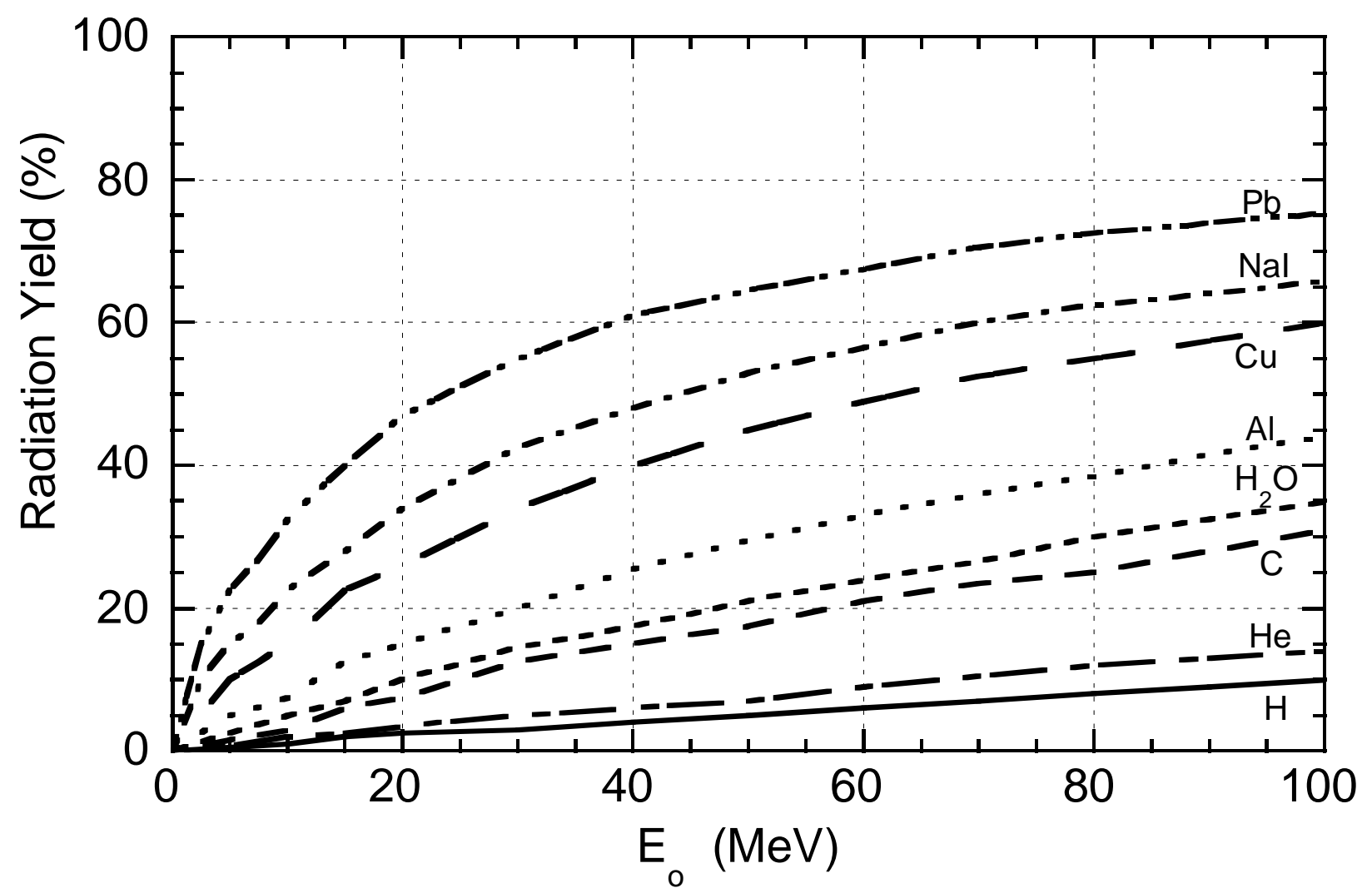

Fig. 3.1 Bremsstrahlung efficiency for electrons stopped in various materials. This is the percentage of the kinetic energy of incident electrons converted to radiation as a function of incident energy $E_{o}$. The remainder of the kinetic energy is transferred to the medium by ionization. [Adapted from (Sw79a).]

The energy spectrum of the radiated photons ranges from zero to the energy of the incident electron and the number of photons in a given energy interval is approximately inversely proportional to the photon energy. The amount of energy radiated per energy interval is practically constant according to Fassò et al. (Fa90). Detailed spectral information for bremsstrahlung photons has been provided by various workers. Figs. 3.2 and 3.3 are provided as examples of such spectra at moderate electron beam energies. Bremsstrahlung spectra are noticeably more energetic (i.e., "harder") at forward angles. 


\section{ChAPTER 3 PROMPt RAdiATION Fields DUe to ElECTRONS}

For thin targets of thickness $x\left(x<<X_{o}\right)$, the spectrum of photons of energy $k$ per energy interval $d k, d N / d k$, can be approximated by

$$
\frac{d N}{d k} \propto \frac{x}{X_{o} k} .
$$

Thick targets may require consideration of the electromagnetic cascade. In general, the spectra fall as $1 / k^{2}$ at $\theta=0$ and even faster at larger angles (Sw79a).

A more detailed parameterization of the normalized total photon differential yield per incident electron $d N / d \Omega$ for photons of all energies has been reported by Swanson and Thomas (Sw90), with improvements suggested by Nelson (Ne97);

$\frac{1}{E_{o}} \frac{d N}{d \Omega}=4.76 E_{o} \exp \left(-\theta^{0.6}\right)+1.08 \exp (-\theta / 72)\left({\text { photons } \mathrm{sr}^{-1} \mathrm{GeV}^{-1} \text { electron }}^{-1}\right)$

This expression is normalized to results involving iron and copper targets of thicknesses of about $17 X_{o}$ at $E_{o}=15 \mathrm{GeV}$. In Eq. (3.8), $E_{\mathrm{o}}$ is in $\mathrm{GeV}$ and $\theta$ is in degrees. As will be shown below, this semi-empirical parameterization is especially useful as a source term in thick shields and is particularly valid for production angles around 90 degrees.

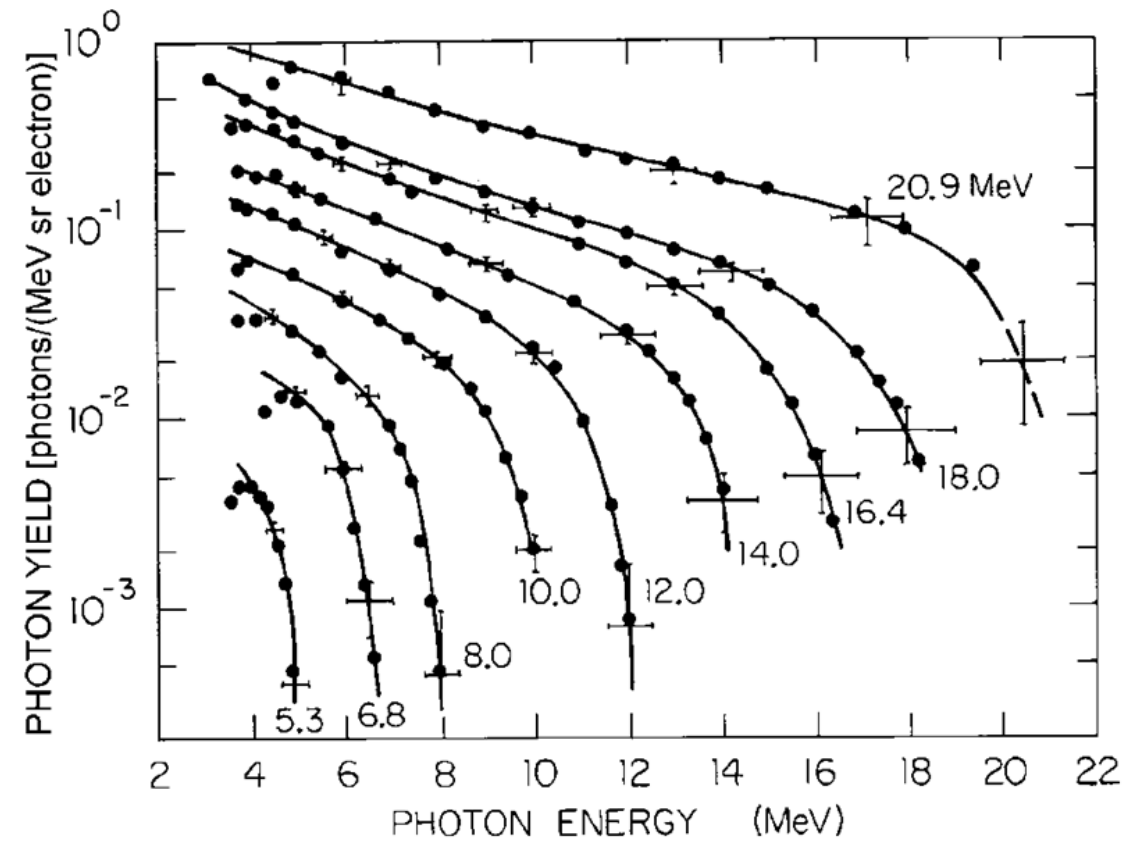

Fig. 3.2 Bremsstrahlung spectra measured at $\theta=0$ degrees from intermediate thickness $\left(0.2 X_{o}\right)$ targets of high atomic number $(Z)$ material. The data points are measurements of O'Dell et al. (OD68) [adapted by Swanson (Sw79a)]. 


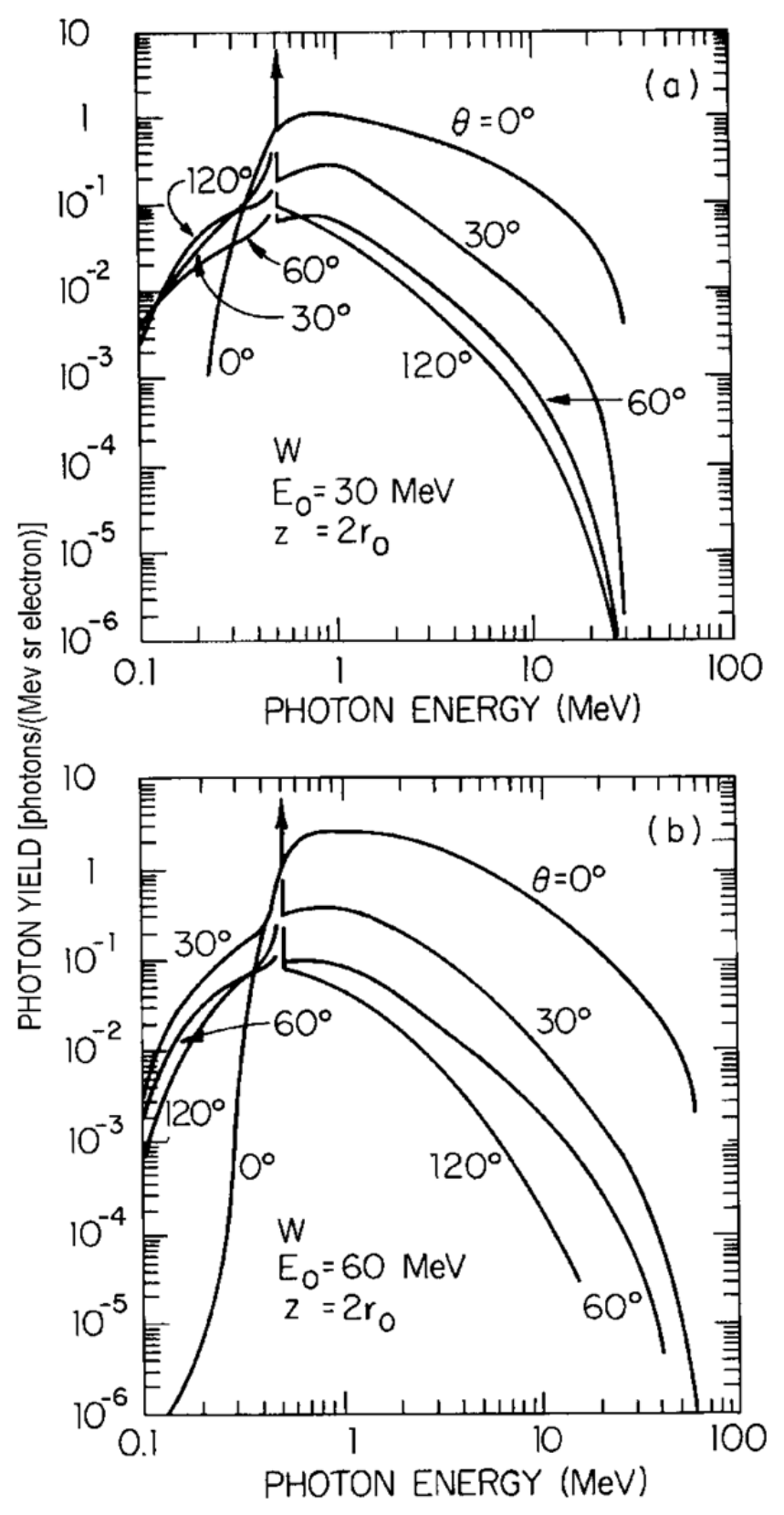

Fig. 3.3 Spectra of bremsstrahlung photons emerging in various directions from thick tungsten targets irradiated by normally incident monoenergetic electron beams at two different energies. The target thickness $z$ at both energies is twice the mean electron ionization range $r_{o}$ given by the continuous slowing down approximation. The arrows indicate the abundant positron annihilation radiation at $0.511 \mathrm{MeV}$. a) Kinetic energy $30 \mathrm{MeV}$, thickness $=24 \mathrm{~g} \mathrm{~cm}^{-2}\left(3.6 X_{o}\right)$; b) $60 \mathrm{MeV}$, thickness $=33 \mathrm{~g} \mathrm{~cm}^{-2}\left(4.9 X_{o}\right)$. [Adapted from Berger and Seltzer (Be70) by Swanson (Sw79a).] 


\section{ChAPTER 3 Prompt RAdiATION Fields Due to Electrons}

The three Swanson's Rules of Thumb parameterize this behavior for the absorbed dose rates $d D / d t$ normalized to one $\mathrm{kW}$ of incident beam power for $E_{o}$ in $\mathrm{MeV}$, expected at one meter from a point "target" of high atomic number $Z$ (Sw79a):

Swanson's Rule of Thumb 1;

$$
\frac{d D}{d t} \approx 20 E_{o}^{2}\left(\mathrm{~Gy} \mathrm{~m}^{2}\right)\left(\mathrm{kW}^{-1} \mathrm{~h}^{-1}\right) \text { at } \theta=0^{\circ}, E_{o}<15 \mathrm{MeV} \text {. }
$$

Swanson's Rule of Thumb 2;

$$
\frac{d D}{d t} \approx 300 E_{o}\left(\mathrm{~Gy} \mathrm{~m}^{2}\right)\left(\mathrm{kW}^{-1} \mathrm{~h}^{-1}\right) \text { at } \theta=0^{\circ}, E_{o}>15 \mathrm{MeV} \text {. }
$$

Swanson's Rule of Thumb 3;

$$
\frac{d D}{d t} \approx 50 \quad\left(\mathrm{~Gy} \mathrm{~m}^{2}\right)\left(\mathrm{kW}^{-1} \mathrm{~h}^{-1}\right) \text { at } \theta=90^{\circ}, E_{O}>100 \mathrm{MeV} .
$$

Higher absorbed dose rates at $\theta=90^{\circ}$ can arise in certain circumstances due to the presence of softer radiation components. In Eq. (3.11), the constant value of 50 is sometimes increased to 100 to better describe measurements (Fa84). For point-like sources, one can scale these results to other distances (in meters) using the inverse square law. Fig. 3.4 shows the behavior for a high- $Z$ target. The forward intensity is a slowly varying function of target material except at very low values of $Z$.

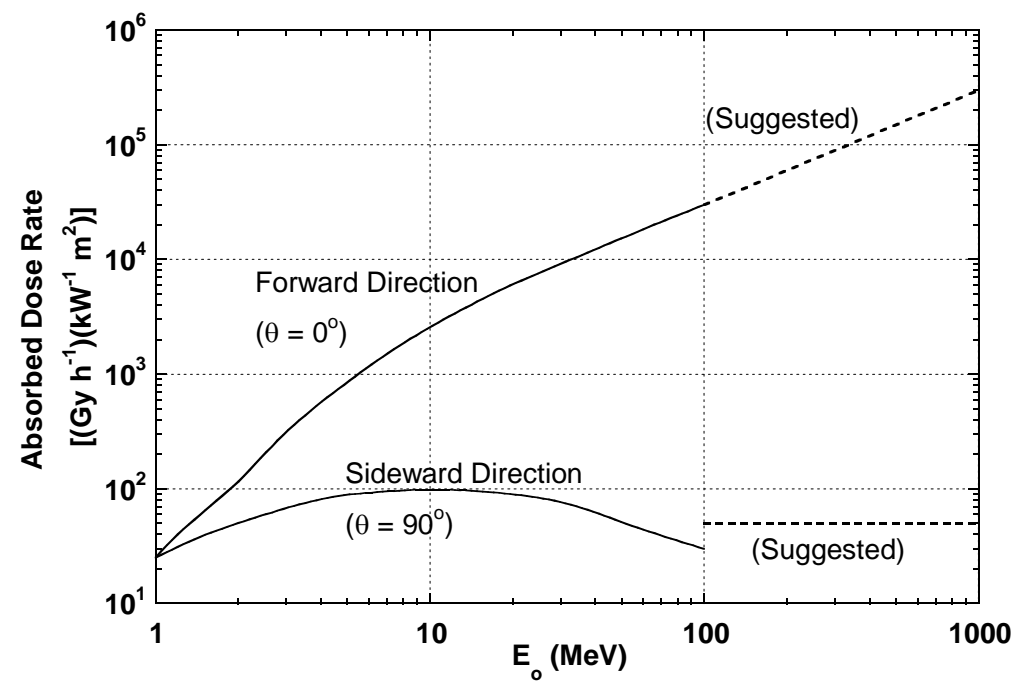

Fig. 3.4 Thick target bremsstrahlung from a high atomic number target. Absorbed dose rates at 1.0 meter per unit incident electron beam power $(\mathrm{kW})$ are given as a function of incident electron kinetic energy $E_{O}$. The dashed lines represent a reasonable extrapolation. The dose rates measured in the sideward direction (smoothed for this figure) depend strongly on target and detector geometry and can vary by more than a factor of two. [Adapted from (Sw79a).]

The value of $\theta$ where the intensity in the forward lobe has half of its maximum intensity $\theta_{1 / 2}$ is approximately given by a relationship with $E_{o}(\mathrm{MeV})$ due to Swanson (Sw79a);

$$
\theta_{1 / 2}=\frac{100}{E_{0}} \text { (degrees) }
$$




\section{ChAPTER 3 Prompt RAdiATION Fields Due to Electrons}

Alternatively, according to Fassò et al. (Fa90) the average angle of emission is of the order of $m_{e} / E_{o}$ (radians) where $m_{e}$ is the rest mass (in energy units, e.g., MeV) of the electron. At higher energies ( $E_{O}$ greater than approximately $100 \mathrm{MeV}$ ), the electromagnetic cascade development in accelerator components is very important and can result in a forward "spike" of photons of characteristic angle of $\theta_{C}=29.28 / E_{O}$ (degrees, for $E_{O}$ in $\mathrm{MeV}$ ). At $\theta=\theta_{c}$ the intensity of the spike has fallen to $1 / e$ of its value at $\theta=0$.

A formula for calculating the unshielded bremsstrahlung dose equivalent at one meter $H_{\text {brem }}$ from a point source is needed. An approximation found to be in good agreement with Eqs. (3.10), (3.11), and (3.12) for all angles for $E_{0} \geq 100 \mathrm{MeV}$ is

$$
\begin{aligned}
& H_{b r e m}=E_{0}\left[1.33 E_{0} \exp \left(-E_{0} \theta / 2.51\right)\right. \\
& +133 \exp (-\theta / 0.159)+3 \exp (-\theta / 0.834)] \\
& {\left[\left(\mathrm{Sv} \mathrm{m}^{2} \text { electron }^{-1}\right) \times 10^{-17}\right]}
\end{aligned}
$$

where $E_{o}$ is in $\mathrm{MeV}$ and $\theta$ is here in radians (NC03). Eq. (3.13) includes the "doubling" suggested by (Fa84) for Eq. (3.11).

\section{$\underline{3.2 .3 \quad \text { Neutrons }}$}

\subsubsection{Giant Photonuclear Resonance Neutrons}

Neutron production can be expected to occur in any material irradiated by electrons in which bremsstrahlung photons above the material-dependent threshold are produced. This threshold varies from 10 to $19 \mathrm{MeV}$ for light nuclei and 4 to $6 \mathrm{MeV}$ for heavy nuclei. Thresholds of $2.23 \mathrm{MeV}$ for deuterium and $1.67 \mathrm{MeV}$ for beryllium are exceptions. Between this threshold and approximately $30 \mathrm{MeV}$, a production mechanism known as the giant photonuclear resonance is the most important source of neutron emission from material. Swanson (Sw79a) has given a detailed description of this process that is summarized here. A simple picture of this phenomenon is that the electric field of the photon produced by bremsstrahlung transfers its energy to the nucleus by inducing an oscillation in which the protons as a group move oppositely to the neutrons as a group. This process has a broad maximum cross section at photon energies $k_{o}$ between about 20$23 \mathrm{MeV}$ for light nuclei for materials having mass numbers $A$ less than about 40. For heavier targets, the peak is at an energy of approximately $k_{o}=80 A^{-1 / 3} \mathrm{MeV}$. Fassò et al. (Fa90) have provided a great deal of data on the relevant cross sections. It turns out that the yield $Y$ of giant resonance neutrons at energies above approximately $2 k_{o}$ is nearly independent of energy and nearly proportional to the beam power.

This process may be thought of as one in which the target nucleus is excited by the electron and then decays somewhat later by means of neutron emission. It is a $(\gamma, n)$ nuclear reaction, written in the scheme of notation in which the first symbol in the parentheses represents the incoming particle in a reaction while the second represents the outgoing particle. The directionality of the incident electron or photon becomes "lost" so that these emissions are isotropic, with no dependence on $\theta$. Because of this isotropicity, 


\section{ChAPTER 3 PRompt RAdiATION Fields Due to ElECtrons}

the inverse square law may be used to estimate the flux density at any given distance $r$. The spectrum of neutrons of energy $E_{n}$ is similar to that seen in a fission neutron spectrum and can be described as a Maxwellian distribution;

$$
\frac{d N}{d E_{n}}=\frac{E_{n}}{T^{2}} \exp \left(-E_{n} / T\right),
$$

where $T$ is a nuclear "temperature" characteristic of the target nucleus. Its excitation energy $T$ in energy units is generally in the range $0.5<T<1.5 \mathrm{MeV}$. For this distribution, the most probable value of $E_{n}=T$ and the average value of $E_{n}=2 T$. This process generally is the dominant one for incident photon kinetic energies $E_{O}<150 \mathrm{MeV}$. The energy dependences, called excitation functions, of total neutron yields in various materials are plotted in Fig. 3.5. Table 3.1 gives the high energy limits for total yield rate $d Y_{n} / d t$ of giant resonance neutrons per watt of beam power $\left(\mathrm{s}^{-1} \mathrm{~W}^{-1}\right)$, the isotropic differential neutron yield $d Y_{n} / d \Omega\left(\mathrm{GeV}^{-1} \mathrm{sr}^{-1}\right)$ per unit of beam energy per electron, and a recommended dose equivalent source term $S_{n}\left(\mathrm{~Sv} \mathrm{~cm}^{2} \mathrm{GeV}^{-1}\right)$ per unit beam energy per electron to be used as follows:

$$
H=\frac{S_{n}}{r^{2}} E_{0} I
$$

where $H$ is the dose equivalent in Sieverts, $r$ is the radial distance from the target in $\mathrm{cm}$, $E_{O}$ is in $\mathrm{GeV}$, and $I$ is the total number of beam particles incident (e.g., during some time interval). For electron energies below $500 \mathrm{MeV}$, appropriate values can be obtained by scaling the Table 3.1 entries according to the Fig. 3.5 curves. The agreement with various experiments is quite good according to Fassò et al. (Fa90). The use of these "saturation" values can support reasonable, but conservative, estimates.

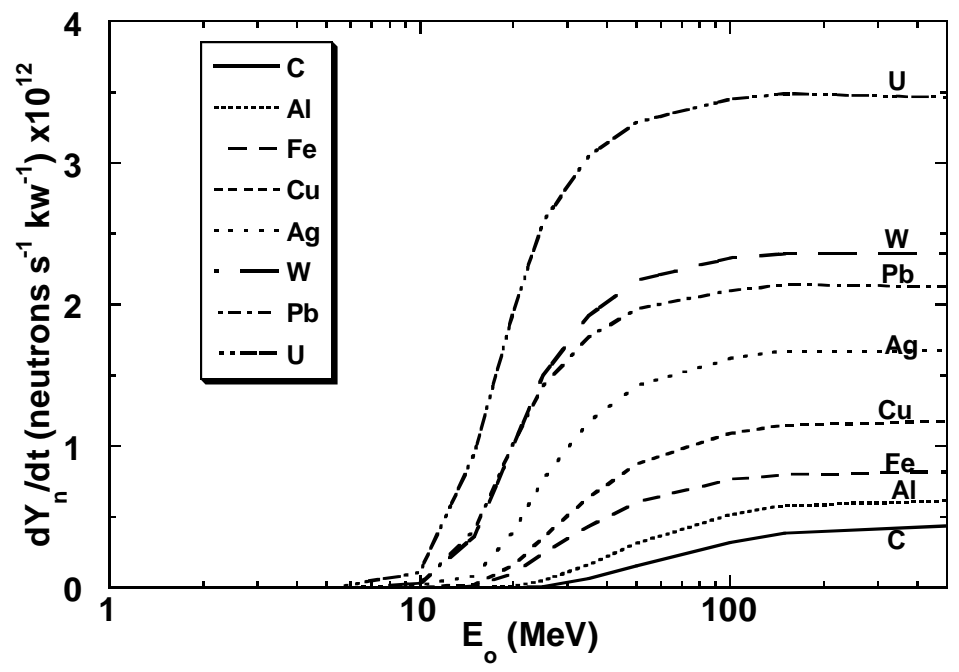

Fig. 3.5 Neutron yield rate $d Y_{n} / d t$ from infinitely thick targets per $\mathrm{kW}$ of electron beam power as a function of electron beam energy $E_{O}$, ignoring target self-shielding. [Adapted from (Sw79b).] 


\section{ChAPTER 3 PRompt RAdiAtion Fields Due to Electrons}

Table 3.1 Total neutron yield rate per unit beam power $d Y_{n} / d t\left(\mathrm{~s}^{-1}\right.$ watt $\left.^{-1}\right)$, differential yield per unit electron energy $d Y_{n} / d \Omega\left(\mathrm{GeV}^{-1} \mathrm{sr}^{-1}\right)$ per electron, and source term $S_{n}$ per unit electron energy $\left(\mathrm{Sv} \mathrm{cm}^{2} \mathrm{GeV}^{-1}\right)$ per electron for giant resonance neutrons in an optimum target. No energy dependence "near threshold" is assumed. The neutrons are distributed isotropically over all directions. These results are best used for $E_{o} \geq 0.5 \mathrm{GeV}$. [Adapted from (Sw79a),(Fa90).]

\begin{tabular}{|c|c|c|c|}
\hline Material & $\begin{array}{l}\text { Total Neutron } \\
\text { Production } \\
d Y_{n} / d t\left(s^{-1} W^{-1}\right)\end{array}$ & $\begin{array}{c}\text { Differential Neutron Yield } \\
d Y_{n} / d \Omega \\
\left(\mathrm{GeV}^{-1} \mathrm{sr}^{-1}\right) \text { per electron }\end{array}$ & $\begin{array}{c}\text { Recommended Source } \\
\text { Terms a }, S_{n} \\
\left(\mathrm{~Sv} \mathrm{~cm}^{2} \mathrm{GeV}^{-1}\right) \text { per electron }\end{array}$ \\
\hline $\mathrm{C}$ & $4.4 \times 10^{8}$ & $5.61 \times 10^{-3}$ & $4.3 \times 10^{-12}$ \\
\hline $\mathrm{Al}^{\mathrm{b}}$ & $6.2 \times 10^{8}$ & $7.90 \times 10^{-3}$ & $6.0 \times 10^{-12}$ \\
\hline $\mathrm{Fe}$ & $8.18 \times 10^{8}$ & $1.04 \times 10^{-2}$ & $7.7 \times 10^{-12}$ \\
\hline $\mathrm{Ni}$ & $7.36 \times 10^{8}$ & $9.38 \times 10^{-3}$ & $6.9 \times 10^{-12}$ \\
\hline$\overline{\mathrm{Cu}}$ & $1.18 \times 10^{9}$ & $1.50 \times 10^{-2}$ & $1.1 \times 10^{-11}$ \\
\hline $\mathrm{Ag}$ & $1.68 \times 10^{9}$ & $2.14 \times 10^{-2}$ & $1.5 \times 10^{-11}$ \\
\hline $\mathrm{Ba}$ & $1.94 \times 10^{9}$ & $2.47 \times 10^{-2}$ & $1.8 \times 10^{-11}$ \\
\hline $\mathrm{Ta}$ & $2.08 \times 10^{9}$ & $2.65 \times 10^{-2}$ & $1.8 \times 10^{-11}$ \\
\hline $\mathrm{W}$ & $2.36 \times 10^{9}$ & $3.01 \times 10^{-2}$ & $2.0 \times 10^{-11}$ \\
\hline $\mathrm{Au}$ & $2.02 \times 10^{9}$ & $2.58 \times 10^{-2}$ & $1.8 \times 10^{-11}$ \\
\hline $\mathrm{Pb}$ & $2.14 \times 10^{9}$ & $2.73 \times 10^{-2}$ & $1.9 \times 10^{-11}$ \\
\hline $\mathrm{U}$ & $3.48 \times 10^{9}$ & $4.44 \times 10^{-2}$ & $3.0 \times 10^{-11}$ \\
\hline
\end{tabular}

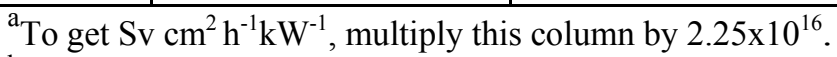

${ }^{\mathrm{b}}$ The value for aluminum is also recommended for concrete.

\subsubsection{Quasi-Deuteron Neutrons}

At energies above the giant resonance, the dominant neutron production mechanism is one in which the photon interacts with a neutron-proton pair within the nucleus rather than with the whole nucleus. The quasi-deuteron effect is so-named because for $E_{O} \approx 30$ $\mathrm{MeV}$ the photon wavelength nearly matches the average inter-nucleon distance so that the photon interactions tend to occur with "pairs" of nucleons. Only neutron-proton pairs have a nonzero electric dipole moment, a fact that favors interactions of photons with such pairs (pseudo-deuterons). This mechanism is important for $30<E_{o}<300 \mathrm{MeV}$ as described by Swanson (Sw79a). An important effect due to this mechanism is to add a tail of higher-energy neutrons to the giant resonance spectrum. For $5<E_{n}<E_{o} / 2(\mathrm{MeV})$, the nearly isotropic spectrum of quasi-deuteron neutrons is given by

$$
\frac{d N}{d E_{n}} \approx E_{n}^{-\alpha} \text { where, approximately, } 1.7<\alpha<3.6 \text {. }
$$

The slope becomes steeper as $E_{O}$, the kinetic energy of the incident electron, is approached. Eq. (3.16) is for thin targets. For thick target situations, the fall-off with $E_{n}$ is generally steeper. Since the reaction is $(\gamma, \mathrm{np})$ and the neutron and the proton are nearly identical in mass, they share the available energy equally so that the yield of neutrons due this mechanism is essentially zero for neutrons having kinetic energy $E_{n}>E_{o} / 2$. In general 


\section{ChAPTER 3 PRompt RAdiATION FieldS Due to ELECTRONS}

the quasi-deuteron neutrons are fewer in number and generally less important than are the giant resonance neutrons. Shielding against the latter will usually provide adequate protection against the former for shielding purposes, but should not be neglected when the fluence of particularly energetic neutrons may be important.

\subsubsection{High Energy Particles}

There are interactions in which the production of other elementary particles, perhaps best typified by pions, becomes energetically possible at still higher energies (i.e., $E_{O}>300$ $\mathrm{MeV}$ ). These particles can then produce neutrons, and other particles, through secondary interactions as discussed in Chapter 4. The neutrons from this source tend to dominate the lateral shielding requirements in the GeV region. Fassò et al. (Fa90), based on the earlier results of DeStaebler (De65), give a parameterization of the measured yield per incident electron on iron for high energy particles of

$$
\frac{d^{2} Y_{n}}{d E d \Omega}=\frac{7.5 \times 10^{-4}}{(1-0.75 \cos \theta)^{2} A^{0.4}}\left(\mathrm{GeV}^{-1} \mathrm{sr}^{-1}\right)
$$

where $A$ is the atomic mass $\left(\mathrm{g} \mathrm{mole}^{-1}\right.$ ) of the target material and $\theta$ is the production angle. It is conservative to use a dose per fluence factor of approximately $10^{-13} \mathrm{~Sv} \mathrm{~m}^{2}\left(10^{-3} \mu \mathrm{Sv}\right.$ $\mathrm{cm}^{2}$ ) for these particles that are mostly high energy neutrons (see Fig. 1.5). Obviously, these neutrons are forward-peaked, not isotropic.

In general photons are produced more copiously with the neutrons more difficult to shield.

\section{$\underline{3.2 .4 \quad \text { Muons }}$}

With electron beams, muons become of significance above an electron energy of approximately $211 \mathrm{MeV}$, the threshold of the process in which a $\mu^{+}$and a $\mu^{-}$are produced in a pair production process quite analogous to the more familiar one in which an electron-positron pair results from photon interactions. Muons can also be produced with much smaller yields at electron accelerators by means of the decay of $\pi_{-}^{+}$and $\mathrm{K}_{-}^{+}$. The latter are due to secondary particle production processes exemplified by photo-pion creation. Such decay muons, more prominent at hadron accelerators, will be discussed in Section 4.2.4. A detailed theoretical treatment of muon production by incident electrons is given by Nelson et al. [(Ne68a), (Ne74)]. Fig. 3.6 gives the muon flux density as a function of electron energy at $\theta=0^{\circ}$ while Fig. 3.7 shows an example of the angular dependence of these yields at $E_{O}=20 \mathrm{GeV}$. The reasonableness of scaling with energy to larger values of $E_{O}$ is well demonstrated.

Obviously, the range-energy relation of muons and considerations related to their energy loss mechanisms discussed in Section 1.6.1 is relevant to shielding against them. 


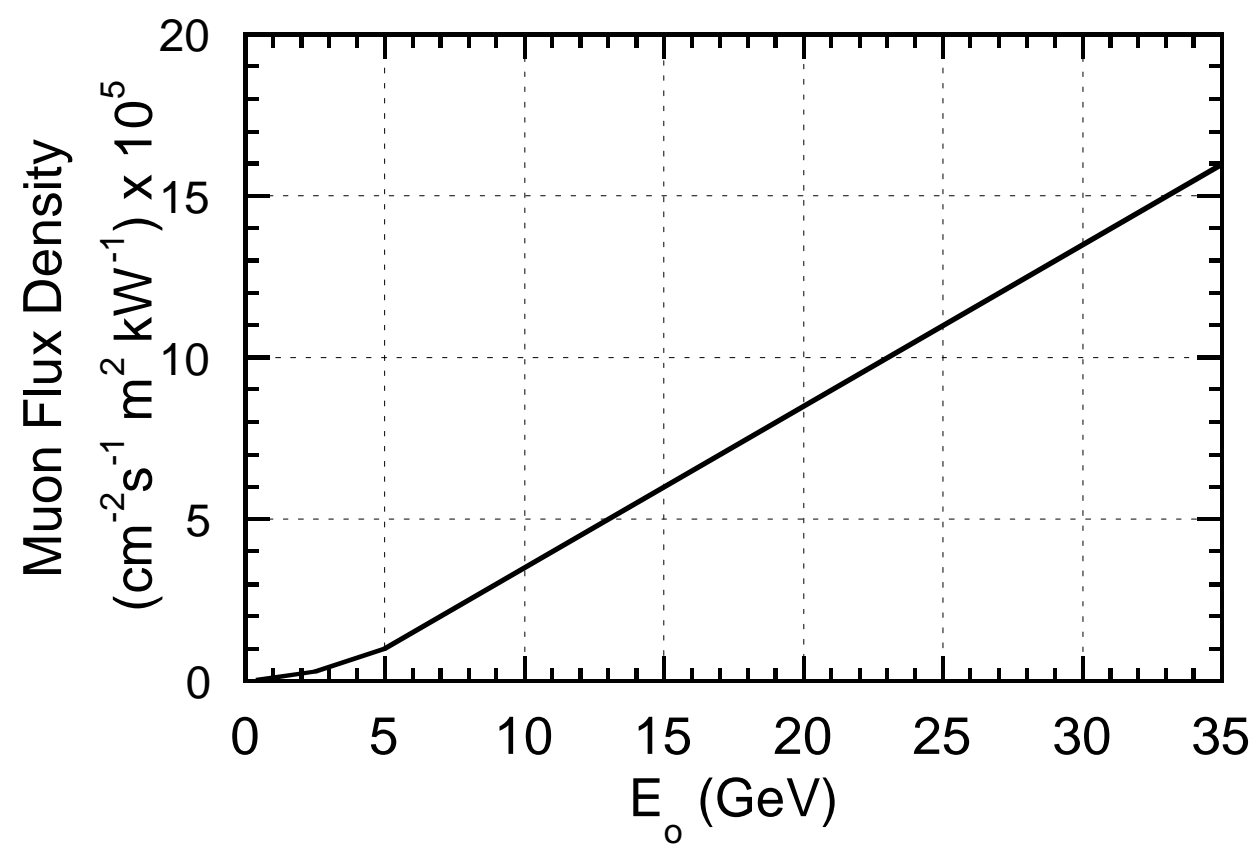

Fig. 3.6 Muon production at $\theta=0^{\circ}$ from an unshielded thick iron target at one meter, as a function of electron energy $E_{O}$ in units of integrated flux density per $\mathrm{kW}$ at 1.0 meter. [Adapted from (Ne68a) and (Ne74).]

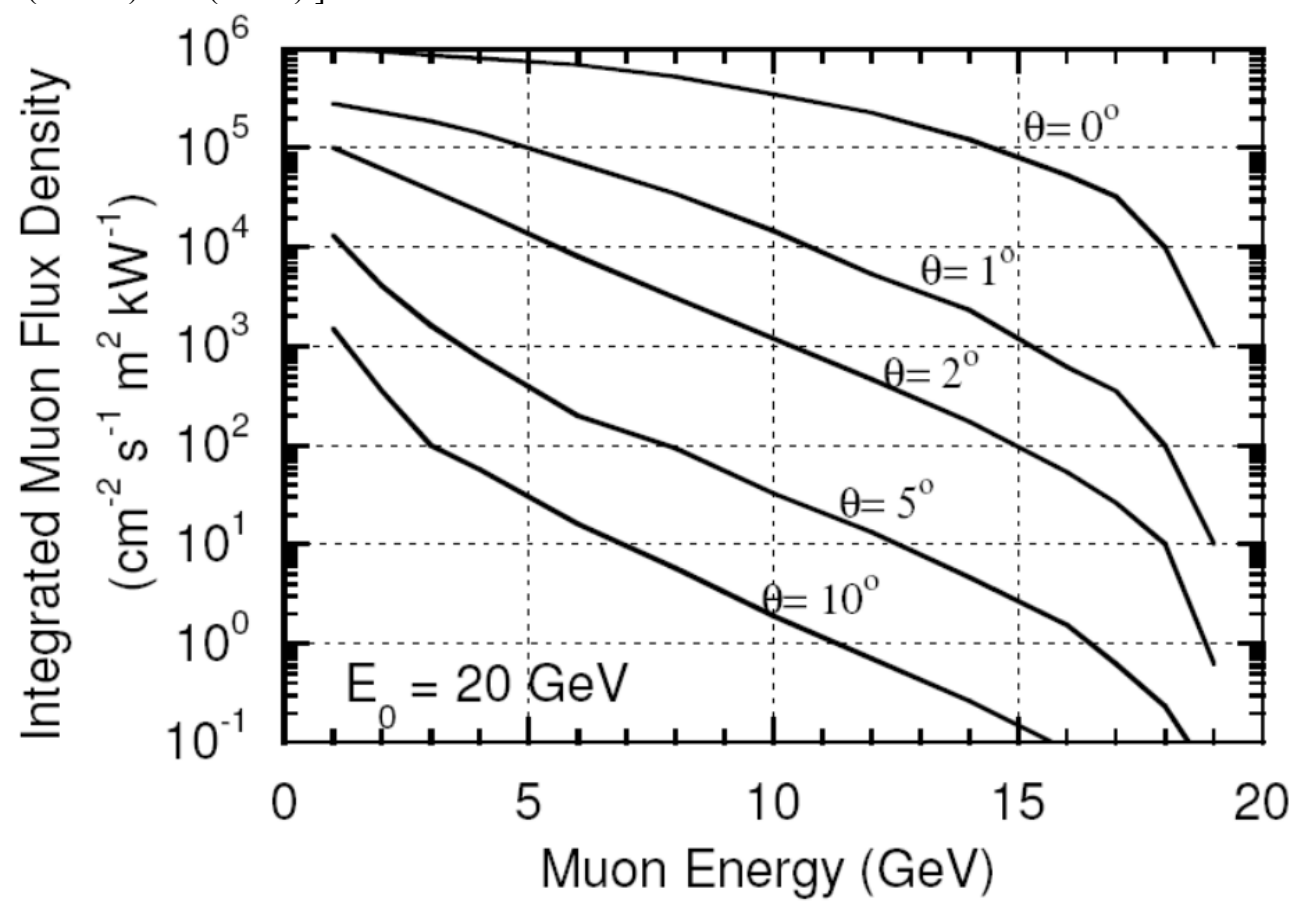

Fig. 3.7 Integrated muon flux density at 1.0 meter per $\mathrm{kW}$ of electron beam power as a function of muon energy for $20 \mathrm{GeV}$ electrons incident on a thick iron target at several values of $\theta$. The integral of the flux density over energy includes all muons that have energies that exceed the value of the abscissa at the specified value of $\theta$. [Adapted from (Ne68a) and (Ne74).] 


\section{ChAPTER 3 PRompt RAdiATION FiELdS DUE TO ELECTRONS}

\subsubsection{Summary of Unshielded Radiation Produced by Electron Beams}

Swanson (Sw79a) has illustrated the broad features of the radiation field due to the unshielded initial interactions of electrons that is given in Fig. 3.8, a diagram intended to only be qualitative. As one can see, at large angles, from the standpoint of dose equivalent, the unshielded field is always dominated by photons. At small angles, the field is dominated by photons at the lower energies with muons increasing in importance as the energy increases to large values. The production of induced radioactivity is discussed in Chapters 7 and 8.

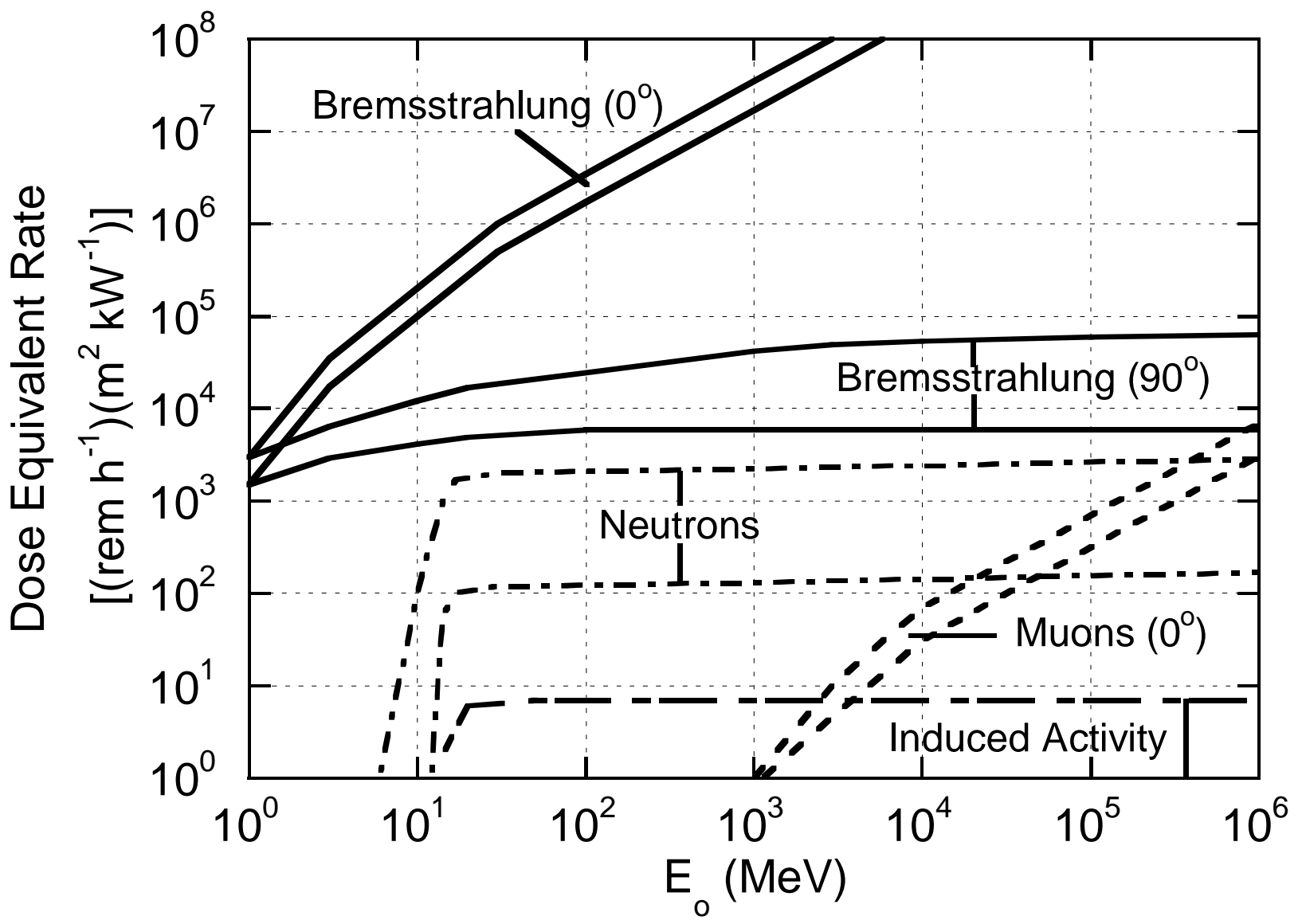

Fig. 3.8 Dose equivalent rates per unit primary electron beam power at one meter produced by various types of "secondary" radiations from a high-Z target as a function of primary beam energy, if no shielding were present (qualitative). The width of the bands suggests the degree of variation found, depending on such factors as target material and thickness. The angles at which the various processes are most important are indicated. Dose due to neutrons and induced activity have essentially no angular dependence. [Adapted from (Sw79a).]

\subsection{The Electromagnetic Cascade-Introduction}

As a prelude to discussing the electromagnetic cascade process, one must look a bit more at the dose equivalent due to thick target bremsstrahlung at large values of $\theta$ for targets surrounded by cylindrical shields. The situation is given in Fig. 3.9. 


\section{ChAPTER 3 Prompt RAdiation Fields Due to Electrons}

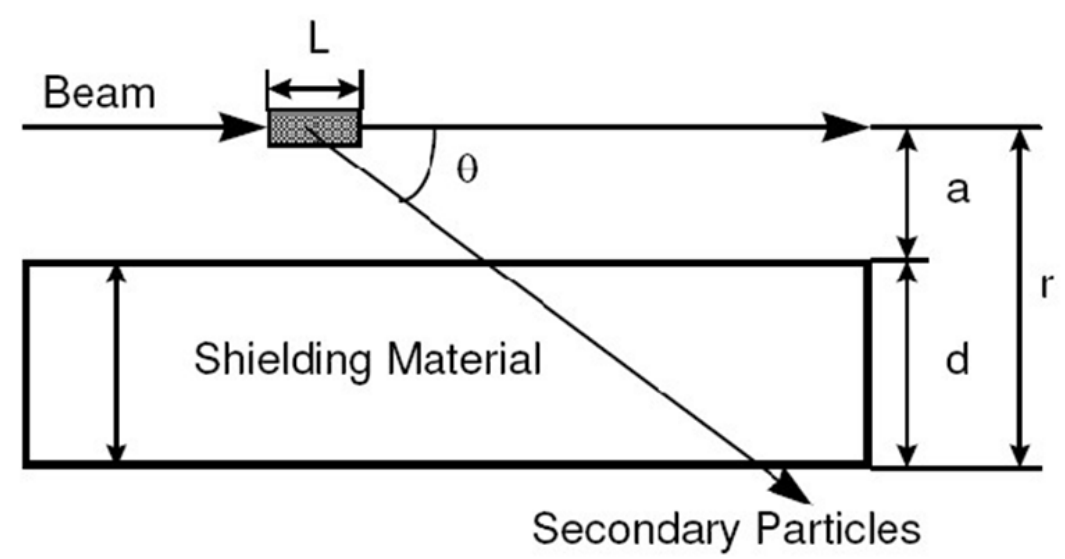

Fig. 3.9 Target and shielding geometry for the estimation of dose equivalent due to electron beam interactions with a target surrounded by a cylindrical shielding. $L$ is the length of the target and the other parameters specify the geometry.

Following Eq. (3.8), the results of Swanson and Thomas (Sw90) as improved by Nelson (Ne97) give the photon absorbed dose per incident electron $D$ external to such a shield;

$$
\begin{gathered}
D(\theta)=\left(1 \times 10^{-11}\right)\left\{10.2 E_{o} \exp \left(-\theta^{0.6}\right)+2.3 \exp (-\theta / 72)\right\} E_{o}\left(\frac{\sin \theta}{a+d}\right)^{2} \exp \left(-\frac{\mu}{\rho} \frac{\rho d}{\sin \theta}\right) \\
\left(\text { Gy electron }^{-1}\right) .
\end{gathered}
$$

As was the case for Eq. (3.8), this expression is normalized to results involving thick iron and copper targets at $E_{0}=15 \mathrm{GeV}$. Here, $E_{\mathrm{o}}$ is the electron energy in $\mathrm{GeV}, \theta$ is in degrees, $a$ is the target-to-shield distance $(\mathrm{cm}), d$ is the shield thickness $(\mathrm{cm}), \rho$ is the shielding material density $\left(\mathrm{g} \mathrm{cm}^{-3}\right)$, and $\mu / \rho$ is the value of the attenuation coefficient at the socalled Compton minimum, the energy where the total photon cross section is at a minimum and the photon mean free path $\lambda_{\gamma}$ is thus a maximum. The use of this term is somewhat inaccurate since the cross section for the Compton scattering process monotonically decreases with energy. However, the contribution of the pair production mechanism to the total photon cross section increases with energy, thus creating the "minimum" in the attenuation coefficient. For concrete $\mu / \rho=2.4 \times 10^{-2} \mathrm{~cm}^{2} \mathrm{~g}^{-1}$ at the Compton minimum. Figs. 3.10 and 3.11 give values of the photon mean free path for a variety of materials as a function of energy. Values for more materials, energies, and mixtures, are available from the National Institute of Standards and Technology. ${ }^{12}$

The major feature that needs to be considered in the shielding design at electron accelerators is the electromagnetic cascade. One should recall the definitions of critical energy $E_{c}$ and radiation length $X_{o}$ that were given in Eqs. (3.3a), (3.3b), and (1.23).

\footnotetext{
${ }^{12}$ Specifically, these data are found at: http://physics.nist.gov/PhysRefData. (Accessed October 29, 2013.)
} 


\section{ChAPTER 3 Prompt RAdiATION Fields Due to Electrons}

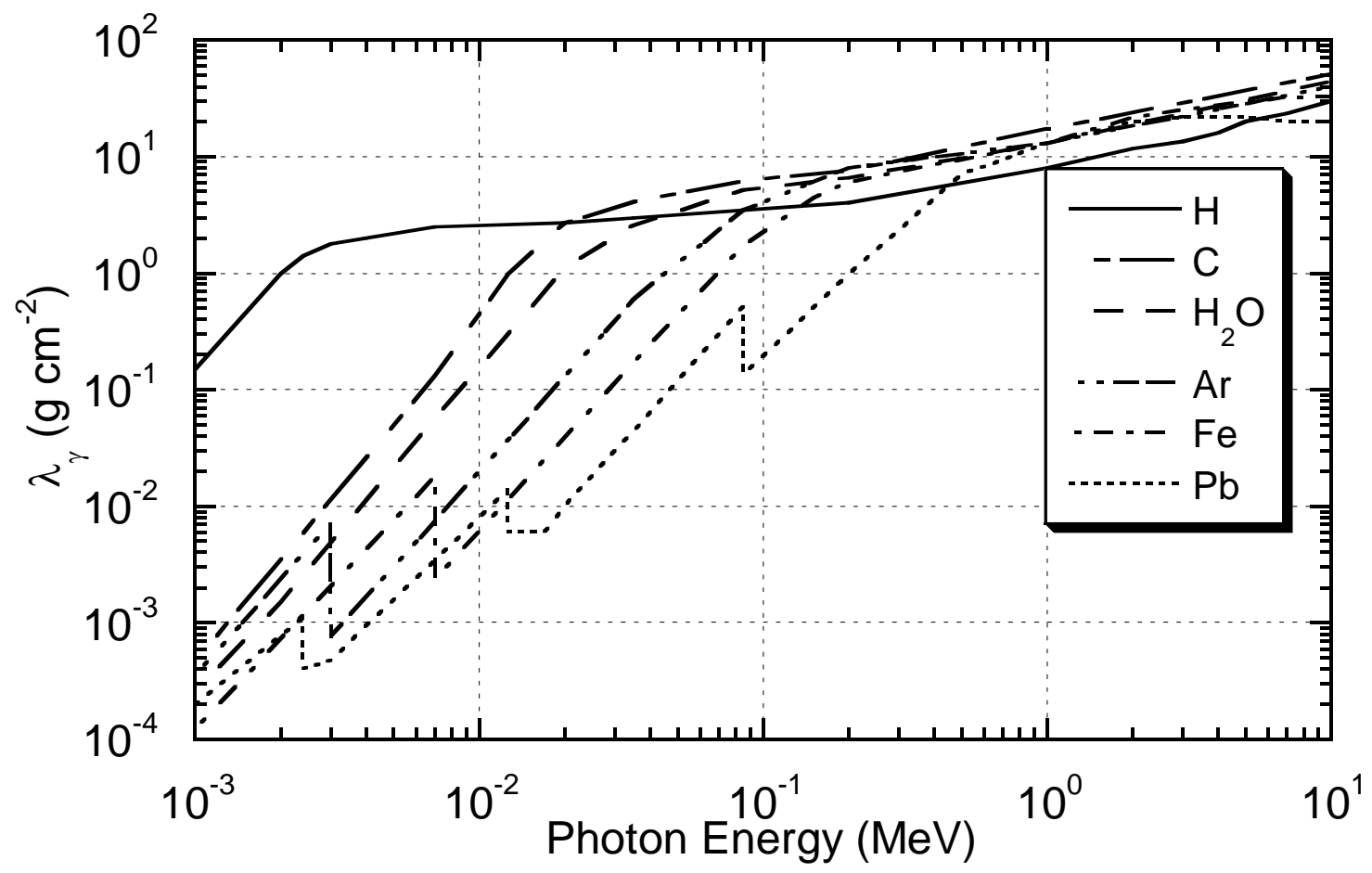

Fig. 3.10 Photon mean free path as a function of photon energy in various materials for low energies. [Adapted from (PDG04).]

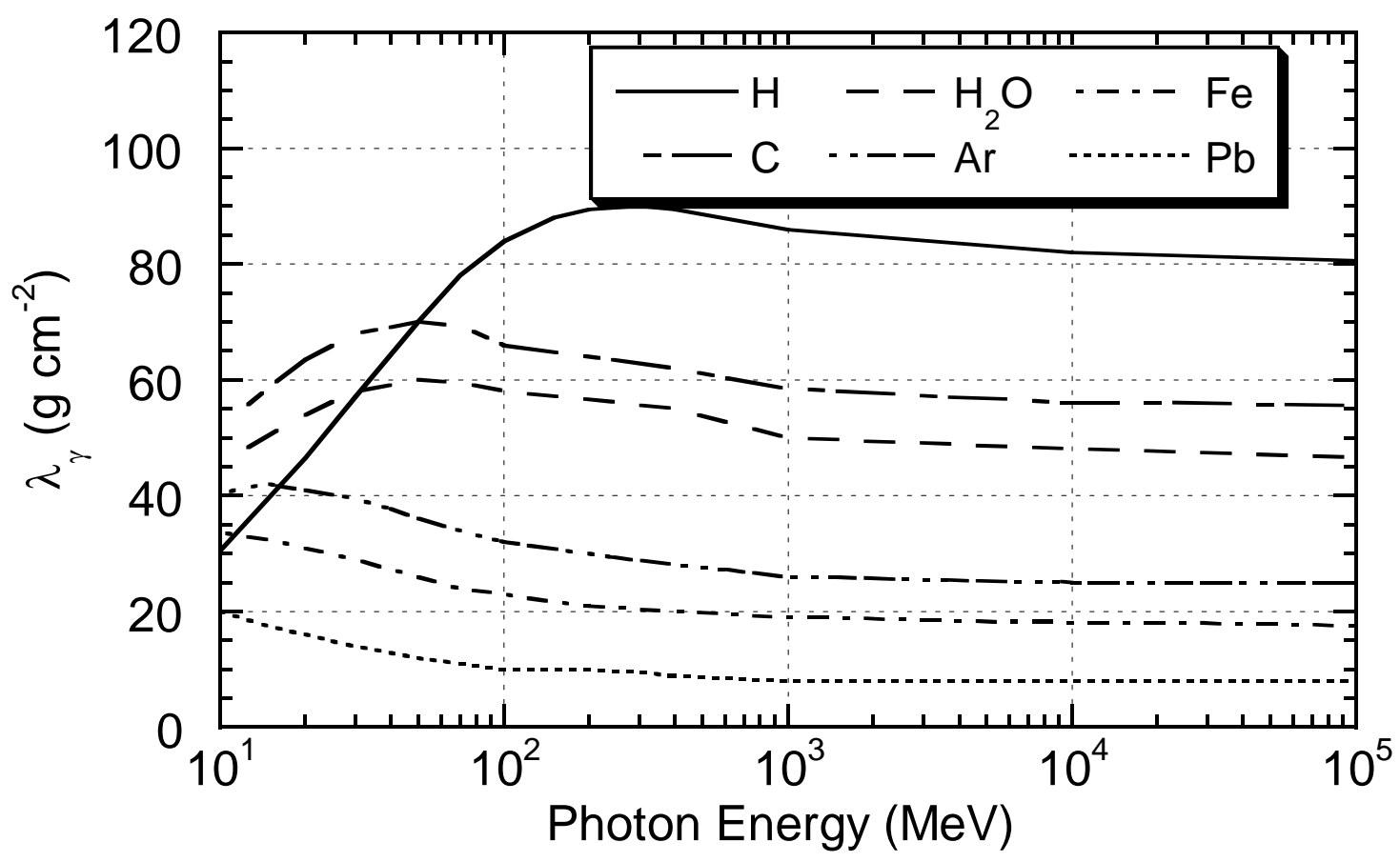

Fig. 3.11 Photon mean free path as a function of photon energy in various materials for high energies. [Adapted from (PDG04).] 


\section{ChAPTER 3 PRompt RAdiAtion Fields Due to Electrons}

A related parameter of importance for describing the electromagnetic cascade is the Molière radius $X_{m}$;

$$
\begin{gathered}
X_{m}=X_{o} E_{S} / E_{c} \\
\text { where } E_{s}=\left(\sqrt{\frac{4 \pi}{\alpha}}\right) m_{e} c^{2}=21.2 \mathrm{MeV} .
\end{gathered}
$$

$\alpha$ is the fine structure constant of atomic physics (see Table 1.1) and $m_{e}$ is the mass of the electron. $X_{m}$ is a good characteristic length for describing radial distributions in electromagnetic showers. Two additional dimensionless scaling variables are commonly introduced to describe electromagnetic shower behavior;

$$
\begin{array}{ll}
t=x / X_{O} & \text { (for longitiudinal distance scaling) } \\
\text { and } y=E / E_{c} \quad \text { (for energy scaling). }
\end{array}
$$

For mixtures of $n$ elements these quantities and also the stopping power $d E / d x$ scale according to the elemental fractions by mass $f_{i}$ as follows:

$$
\frac{d E}{d x}=\sum_{i=1}^{n} f_{i}\left(\frac{d E}{d x}\right)_{i}
$$

where all stopping powers are expressed as energy loss per unit areal density (e.g., $\mathrm{MeV}$ $\mathrm{cm}^{2} \mathrm{~g}^{-1}$ ) (PDG04).

For photons of energies $E_{o}$ greater than about one $\mathrm{GeV}$, the total $\mathrm{e}^{+} \mathrm{e}^{-}$pair production cross section $\sigma_{\text {pair }}$ is approximately given, for a single constituent element, by

$$
\sigma_{\text {pair }}=\frac{7}{9}\left(\frac{A}{X_{0} N_{A}}\right) \quad\left(\mathrm{cm}^{2}\right)
$$

where $A$ is the atomic weight, $N_{A}$ is Avogadro's number, and $X_{O}$ is the radiation length expressed in units of $\mathrm{g} \mathrm{cm}^{-2}$. For energies larger than a few $\mathrm{MeV}$, the pair production process dominates the total photon attenuation. The mean free path length for pair production $\lambda_{\text {pair }}$ is thus given by

$$
\lambda_{\text {pair }}=\frac{\rho}{N \sigma}\left(\mathrm{g} \mathrm{cm}^{2}\right)=\frac{\rho}{\frac{\rho N_{A}}{A} \frac{7}{9}\left(\frac{A}{X_{0} N_{A}}\right)}=\frac{9}{7} X_{0} .
$$




\section{ChAPTER 3 PROMPt RAdiATION Fields DUe to ElECTRONS}

The energy-independence and near-equality of $\lambda_{\text {pair }}$ and $X_{o}$ leads to the most important fact about the electromagnetic cascade:

\section{The electrons radiatively produce photons with almost the same characteristic length for which the photons produce more $\mathrm{e}^{+} \mathbf{e}^{-}$pairs.}

This first order approximation is important it means that the "size" in physical space is independent of energy. For hadronic cascades (see Chapter 4) the results are considerably different and perhaps more complicated.

\subsection{The Electromagnetic Cascade Process}

Fig. 3.12 conceptually illustrates the electromagnetic cascade process.

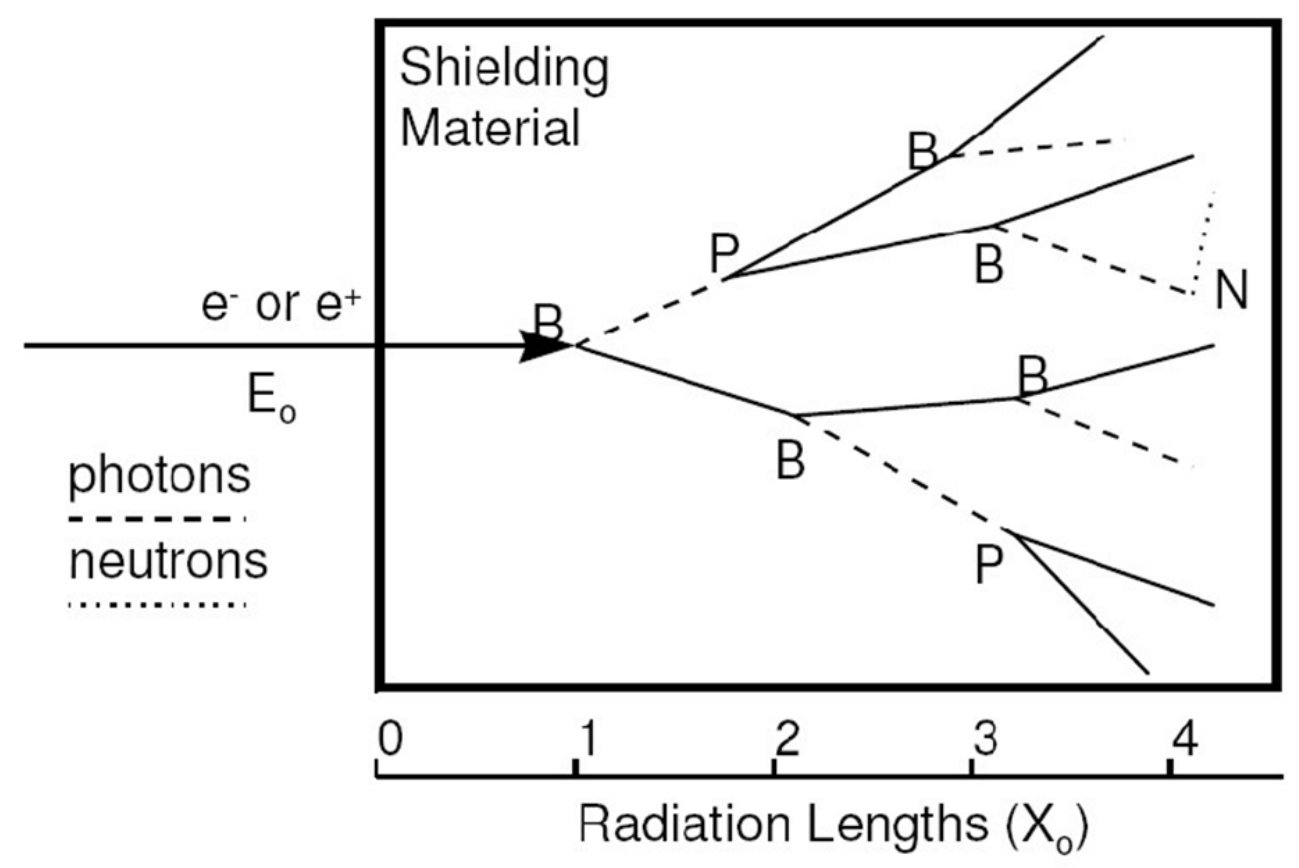

Fig. 3.12 Conceptual view of the development of an electromagnetic cascade in a semi-infinite medium with no magnetic or electric fields present. The solid lines represent electrons or positrons, the dashed lines represent photons, and the dotted lines represent neutrons. The shower is initiated by an electron or positron of energy $E_{o}$ incident on the medium from the left. The spreading in the transverse direction is greatly exaggerated for clarity. Bremsstrahlung and pair production events are denoted by $\mathbf{B}$ and $\mathbf{P}$, respectively. Compton scattering, ionization, and the production of other hadrons in addition to neutrons are not shown but also play a roles in the dispersal of energy. Photonuclear reactions, as illustrated by the $(\gamma, n)$ reaction at point $\mathbf{N}$ also play a role, albeit much more infrequently than inferred from this illustration. The process could just as well be initiated by a photon. [Adapted from (Sw79a).]

In the simplest terms, the electromagnetic cascade at an electron accelerator proceeds qualitatively according to the following steps: 


\section{ChAPTER 3 PRompt RAdiATION FieldS Due to ELECTRONS}

1. A high energy electron $\left(E_{o}>>m_{e} c^{2}\right)$ produces a high energy photon by means of bremsstrahlung after traveling an average distance of $X_{o}$.

2. This photon produces an $\mathrm{e}^{+} \mathrm{e}^{-}$pair after traveling an average distance of $9 / 7 X_{o}$. Each member of the pair will have, on average, half the energy of the photon.

3. After traveling an average distance of $X_{o}$, each member of the $\mathrm{e}^{+} \mathrm{e}^{-}$pair will produce yet another bremsstrahlung photon.

4. Each electron or positron may continue on to interact again and release yet more photons before its energy is totally absorbed.

This chain of events can equally well be initiated by a high energy photon, even one produced in secondary interactions at a hadron accelerator. Eventually, after a number of generations, the individual energies of the electrons and positrons will be degraded to values below $E_{c}$ so that ionization processes then begin to dominate and terminate the shower. Likewise, the photon energies eventually are degraded so that Compton scattering and the photoelectric effect compete with the further $\mathrm{e}^{+} \mathrm{e}^{-}$pair production.

Of course, there are subtleties representing many different physical processes, such as the production of other particles, which must be taken into account and are best handled by Monte Carlo calculations. A general discussion of the use of Monte Carlo techniques for such problems has been given by Rogers and Bielajew (Ro90). A widely-used code incorporating the Monte Carlo method applied to electromagnetic cascades is EGS (electron gamma shower), written by W. R. Nelson and co-workers (Ne85, Ne90) (see Appendix A). Van Ginneken developed the Monte Carlo program called AEGIS (Va78), which was very effective for calculating the propagation of such cascades through thick shields. Analytical approximations have been developed and are summarized elsewhere [e.g., (Sw79a), (Fa90)]. The results of published calculations are used in the following discussion to aid in improving the reader's understanding of electromagnetic cascades.

\subsubsection{Longitudinal Shower Development}

The dosimetric properties of the calculations of an electromagnetic cascade may be summarized in curves that give fluence, dose, or other quantities of interest as functions of shower depth or distance from the axis. Fig. 3.13 shows the fraction of total energy deposited (integrated over all radii about the shower axis) versus longitudinal depth as calculated by Van Ginneken and Awschalom (Va75). They introduced a longitudinal scaling parameter $\zeta$ given by

$$
\zeta=325(\ln Z)^{-1.73} \ln E_{0} \quad\left(\mathrm{~g} \mathrm{~cm}^{-2}\right)
$$

where $E_{o}$ is in $\mathrm{MeV}$ and $Z$ is the atomic number of the absorber. When the longitudinal coordinate is expressed in units of $\zeta$, all curves approximately merge into this universal 


\section{Chapter 3 Prompt RAdiation Fields Due to Electrons}

one and are rather independent of target material.

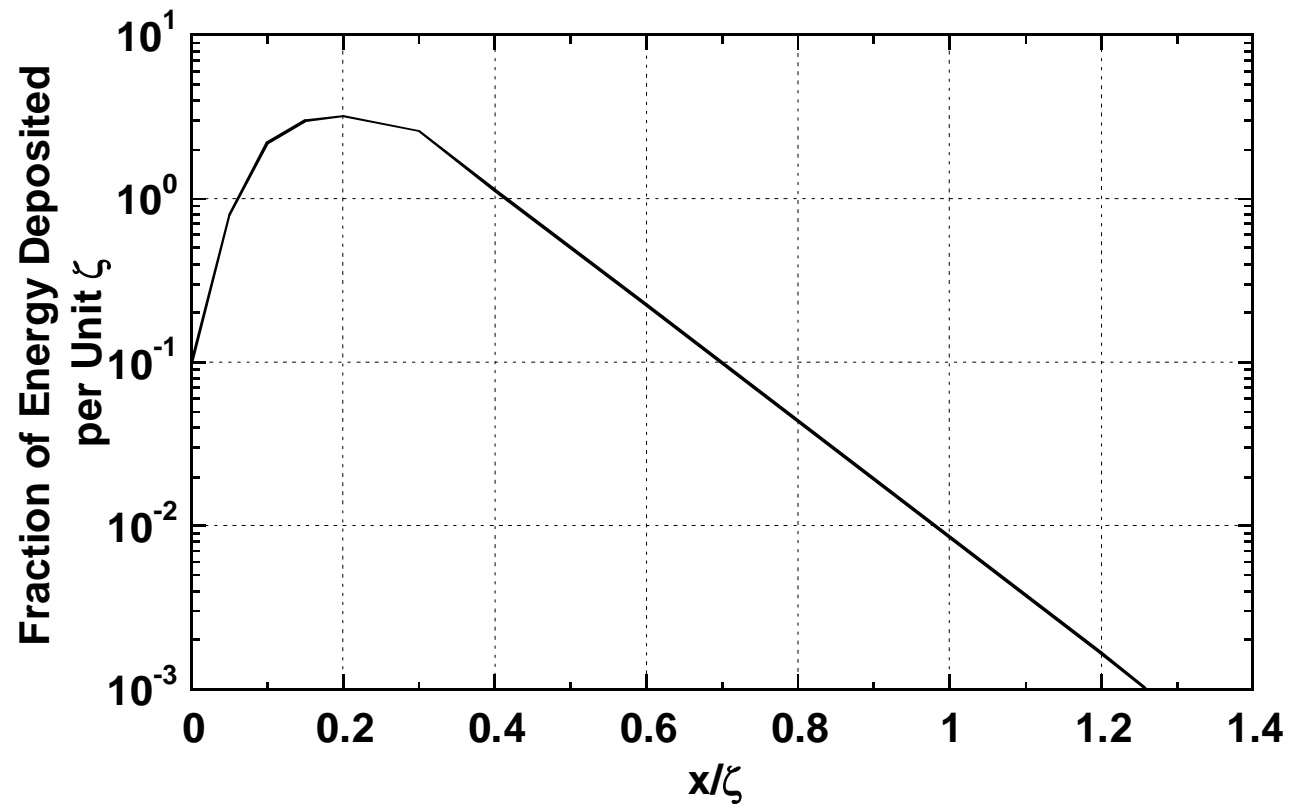

Fig. 3.13 Fraction of total energy deposited by an electromagnetic cascade versus longitudinal depth $x$ integrated over all radii about the shower axis. See Eq. (3.26). [Adapted from (Va75).]

In their epic development of analytical shower theory, Rossi and Griesen (Ro41) using their so-called Approximation B, a more advanced formalism than their parallel Approximation A, predicted for an electron-initiated shower that the total number of electrons and positrons at the shower maximum $N_{\text {show }}$ is proportional to the primary energy as follows:

$$
N_{\text {show }}=\frac{0.31 E_{o} / E_{c}}{\sqrt{\ln \left(E_{o} / E_{c}\right)-0.37}} .
$$

For a photon-initiated shower, a value of 0.18 should replace that of 0.37 in the denominator of Eq. (3.27). This distinction related to the initiator of the shower (electron/positron or photon) and others reflect the deeper penetration of an initiating photon implied by the $9 / 7$ factor in Eq. (3.25). The result embodied in the mathematical language of this equation is intuitively sensible since the final outcome of the shower is to divide the energy at the shower maximum among a number of particles with energies near $E_{c}$. One can obtain the maximum energy deposited per radiation length from Eq. (3.27) as the product $E_{c} N_{\text {show }}(\mathrm{Fa} 90)$.

Also from the Rossi-Griesen work, the location of the shower maximum $t_{\max }$ along the longitudinal coordinate in units of radiation length [see Eq. (3.21)] should be given by

$$
t_{\max }=1.01 \ln \left(\frac{E_{o}}{E_{c}}\right)-C_{\text {show }}, \text { with } C_{\text {show }}=1
$$




\section{ChAPTER 3 PRompt RAdiation Fields Due to EleCtrons}

Experimentally Bathow et al. (Ba67) found that values of $C_{\text {show }}=0.77$ for copper and $C_{\text {show }}=0.47$ for lead fit data better. Not surprisingly, photon-initiated showers penetrate about 0.8 radiation lengths deeper than do the electron-initiated showers. Fassò et al. (Fa90) simply give values of $C_{\text {show }}=1.0$ and $C_{\text {show }}=0.5$ for electron- and photon-initiated showers, respectively.

The longitudinal "center of gravity" (i.e. the mean depth in the shield) $\bar{t}$ of all the shower electrons is given by

$$
\begin{array}{ll}
\bar{t}=1.009 \ln \left(\frac{E_{o}}{E_{c}}\right)+0.4 & \text { (electron-induced shower), and } \\
\bar{t}=1.012 \ln \left(\frac{E_{o}}{E_{c}}\right)+1.2 \quad \text { (photon-induced shower) } .
\end{array}
$$

Fassò et al. (Fa90) gives the mean-squared longitudinal spread $\tau^{2}$ (squared standard deviation in units of $X_{o}^{2}$ ) about $\bar{t}$ to be

$$
\begin{aligned}
\tau^{2} & =1.61 \ln \left(\frac{E_{o}}{E_{c}}\right)-0.2 \quad \text { (electron-induced shower), and } \\
\tau^{2} & =1.61 \ln \left(\frac{E_{o}}{E_{c}}\right)+0.9 \quad \text { (photon-induced shower) } .
\end{aligned}
$$

There are other, perhaps less important, differences between photon and electron-induced showers. EGS4 results tabulated by Fassò et al. (Fa90) have been parameterized to determine source terms $S_{i}$ for longitudinal distributions of absorbed dose in various materials and for the associated dose equivalent within shields comprised of these materials over the energy region of $1.0 \mathrm{GeV}<E_{o}<1.0 \mathrm{TeV}$. This has been done for the dose on the $z$-axis (subscripts " $a$ ") and for the dose averaged over a $15 \mathrm{~cm}$ radius about the $z$-axis (subscripts "15"). Table 3.2 gives parameters for calculating dose equivalent $H_{\text {long }}(\mathrm{Sv}$ per electron), at the end of a beam absorber of length $L(\mathrm{~cm})$ of density $\rho(\mathrm{g}$ $\left.\mathrm{cm}^{-3}\right)$, and gives fitted values of the various "attenuation lengths" $\lambda_{i}\left(\mathrm{~g} \mathrm{~cm}^{-2}\right)$ to be used with the corresponding tabulated values of $S_{i}$. For absorbed dose calculations, the factor $C$, which is the ratio of dose equivalent in tissue (Sv) to absorbed dose in the material (not tissue) (Gy), should be set to unity. The formula in which these parameters from Table 3.2 are to be used is as follows:

$$
H_{\text {long }}=C S_{i} \exp \left(-\rho L / \lambda_{i}\right)
$$

This equation is valid in the longitudinal region beyond the shower maximum. 


\section{ChAPTER 3 PRompt RAdiATION Fields Due to ElECtrons}

Table 3.2 Source terms $S_{a}$ and $S_{15}$ and corresponding recommended longitudinal attenuation lengths, $\lambda_{a}$ and $\lambda_{15}$ for doses on the axis and averaged over a radius of $15 \mathrm{~cm}$ in the forward direction for beam absorbers and end-stops, respectively. These results are most valid in the region of incident electron energy $E_{o}$ from 1.0 $\mathrm{GeV}$ to 1.0 TeV. Conversion factors $C$ from absorbed dose in the shielding material to dose equivalent within the shield are given. $E_{o}$ is the beam kinetic energy in $\mathrm{GeV}$. These parameters are to be used with Eq. (3.33). [Adapted from (Fa90).]

\begin{tabular}{|c|c|c|c|c|c|}
\hline Material & $\begin{array}{c}C \\
\left(\operatorname{Sv} G y^{-1}\right)\end{array}$ & $\begin{array}{c}S_{a} \\
\left(\text { Gy electron }^{-1}\right)\end{array}$ & $\begin{array}{c}\lambda_{a} \\
\left(\mathrm{~g} \mathrm{~cm}^{-2}\right)\end{array}$ & $\begin{array}{c}S_{15} \\
\left(\text { (Gy electron }^{-1}\right)\end{array}$ & $\begin{array}{c}\lambda 15 \\
\left(\mathrm{~g} \mathrm{~cm}^{-2}\right)\end{array}$ \\
\hline Water & 0.95 & $1.9 \times 10^{-10} \mathrm{E}_{\mathrm{o}} 2.0$ & 58 & $1.5 \times 10^{-11} \mathrm{E}_{\mathrm{o}} 2.0$ & 59.9 \\
\hline Concrete & 1.2 & $1.9 \times 10^{-9} \mathrm{E}_{\mathrm{o}}^{1.8}$ & 44 & $2.2 \times 10^{-11} \mathrm{E}_{\mathrm{o}} 1.8$ & 45.6 \\
\hline Aluminum & 1.2 & $2.3 \times 10^{-9} \mathrm{E}_{\mathrm{o}} 1.7$ & 46 & $3.4 \times 10^{-11} \mathrm{E}_{\mathrm{o}} 1.7$ & 46.3 \\
\hline Iron & 1.3 & $2.9 \times 10^{-8} \mathrm{E}_{\mathrm{o}} 1.7$ & 30 & $1.8 \times 10^{-10} \mathrm{E}_{\mathrm{o}} 1.7$ & 33.6 \\
\hline Lead & 1.8 & $1.9 \times 10^{-7} \mathrm{E}_{\mathrm{o}} 1.4$ & 18 & $4.6 \times 10^{-10} \mathrm{E}_{\mathrm{o}} 1.4$ & 24.2 \\
\hline
\end{tabular}

\subsubsection{Lateral Shower Development}

Fig. 3.14 shows the fraction $U / E_{O}$ of the incident electron energy that escapes laterally from infinitely long cylinders made of various materials as a function of cylinder radius, $R$ for showers caused by electrons of various energies that bombard the front face of the cylinder. On this graph $R$ is in units of the Molière radius $X_{m}$. According to Neal et al. (Ne68b), a function that fits data between $100 \mathrm{MeV}$ and $20 \mathrm{GeV}$ for electrons incident on targets ranging from aluminum to lead is

$$
\frac{U\left(R / X_{m}\right)}{E_{o}}=0.8 \exp \left[-3.45\left(R / X_{m}\right)\right]+0.2 \exp \left[0.889\left(R / X_{m}\right)\right]
$$

Results similar to this universal curve have been obtained using EGS4 (Fa90). For values of $R / X_{m}$ greater than about four, a material-dependent phenomenon emerges in which the photons having the largest mean free paths determined by the photon cross section at the Compton minimum for the absorber material will dominate the slopes of these curves. These extrapolations, normalized to $X_{m}$, are also included in Fig. 3.14. As was done for the longitudinal situation, EGS4 (Fa90) has been similarly used to give the maximum energy deposition (and by extension, the maximum absorbed dose and dose equivalent) as a function of radius $r$. Over the energy range $1.0 \mathrm{GeV}<E_{o}<1.0 \mathrm{TeV}$, there is direct scaling with energy in the formula for maximum dose equivalent at $\theta \approx 90^{\circ}$;

$$
H_{\text {lat }}=C E_{0} S_{\text {lat }} \frac{\exp \left(-\rho d / \lambda_{\text {lat }}\right)}{r^{2}}
$$

where $H_{\text {lat }}$ is the maximum dose equivalent laterally ( $\mathrm{Sv}$ per electron), $C$ is the same as in Eq. (3.33), $E_{o}$ is the electron kinetic energy in $\mathrm{GeV}, S_{\text {lat }}$ is the source term from the 
EGS4 calculations, $d$ is the lateral dimension of the shield (shield thickness) in $\mathrm{cm}, \rho$ is the density $\left(\mathrm{g} \mathrm{cm}^{-3}\right), \lambda_{\text {lat }}$ is the attenuation length $\left(\mathrm{g} \mathrm{cm}^{-2}\right)$, and $r$ is the distance from the axis, in $\mathrm{cm}$, where the dose equivalent is desired (see Fig. 3.9). Table 3.3 gives the parameters needed for Eq. (3.35).

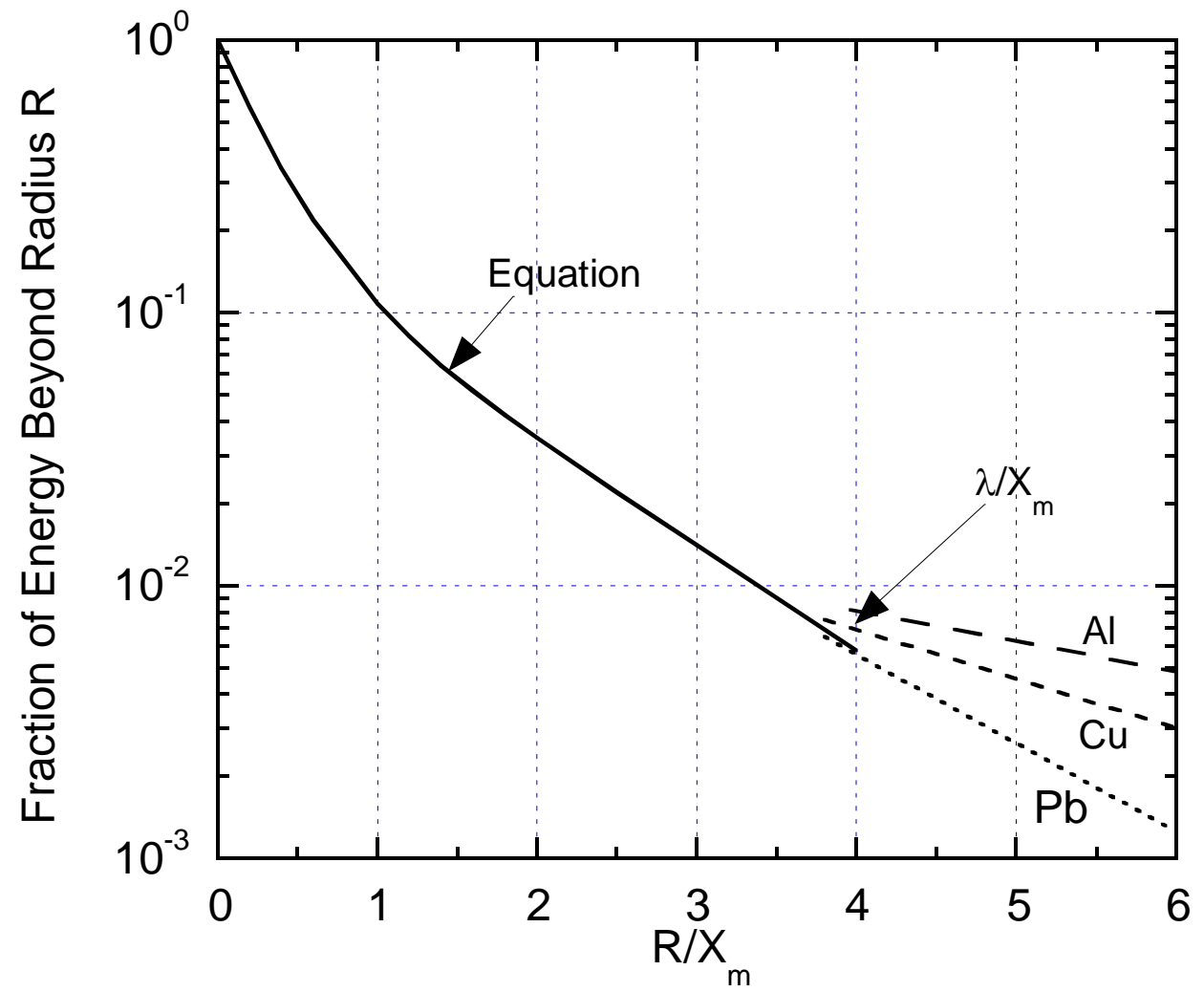

Fig. 3.14 Fraction of total energy deposited beyond a cylindrical radius $R / X_{m}$, as a function of radius $R$ for showers caused by 0.1 to $20 \mathrm{GeV}$ electrons incident on various materials. The curve labeled "Equation" refers to Eq. (3.34). [Adapted from (Ne68b).]

Table 3.3 Conversion factors $C$ from absorbed dose in shielding material to dose equivalent, source terms $S_{\text {lat }}$ for the maximum of the electromagnetic component, and recommended lateral attenuation lengths $\lambda_{\text {lat }}$ for the electron energy range $E_{o}$ from 1.0 GeV to 1.0 TeV laterally for beam absorbers or end-stops. These parameters are to be used with Eq. (3.35). [Adapted from (Fa90).]

\begin{tabular}{|c|c|c|c|}
\hline Material & $\begin{array}{c}C \\
\left(\mathrm{~Sv} G y^{-1}\right)\end{array}$ & $\begin{array}{c}S_{\text {lat }} \\
\left(\mathrm{Gy} \mathrm{cm}^{2} \mathrm{GeV}^{-1} \text { electron }^{-1}\right)\end{array}$ & $\begin{array}{c}\lambda_{\text {lat }} \\
\left(\mathrm{g} \mathrm{cm}^{-2}\right)\end{array}$ \\
\hline Water & 0.95 & $2.5 \times 10^{-12}$ & 26 \\
\hline Concrete & 1.2 & $3.6 \times 10^{-12}$ & 27 \\
\hline Aluminum & 1.2 & $3.4 \times 10^{-12}$ & 29 \\
\hline Iron & 1.3 & $4.7 \times 10^{-11}$ & 33 \\
\hline Lead & 1.8 & $1.3 \times 10^{-10}$ & 26 \\
\hline
\end{tabular}




\section{ChAPTER 3 Prompt RAdiATION Fields Due to Electrons}

\subsection{Shielding of Hadrons Produced by the Electromagnetic Cascade}

\section{$\underline{3.5 .1 \quad \text { Neutrons }}$}

As discussed before, neutrons are produced by high energy electrons and photons. These neutrons must be taken into account to properly shield electron accelerators. Tesch summarized shielding against these neutrons by developing simple analytical relations for cases where thick targets are struck by the electron beam (Te88). Fig. 3.9 defines the shielding geometry. For lateral concrete shielding, the maximum dose equivalent outside of shield thickness $d(\mathrm{~cm})$ which begins at radius $a(\mathrm{~cm})$ from a thick iron or copper target struck by electrons having primary energy $E_{o}(\mathrm{GeV})$ per incident electron is

$$
H(d, a)=\frac{4 \times 10^{-13}}{[(a+d)]^{2}} E_{o} \exp (-\rho d / 100) \quad\left(\mathrm{Sv} \text { electron }{ }^{-1}\right)
$$

This equation is valid for $E_{o}$ greater than about $0.4 \mathrm{GeV}$ and $\rho d$ greater than about $200 \mathrm{~g}$ $\mathrm{cm}^{-2}$. The maximum dose equivalent will be found at $\theta \approx 90^{\circ}$ as defined in Fig. 3.9. For other target materials one can scale this equation in the following way: The neutron production is proportional to the photoproduction cross section, the track length in $\mathrm{cm}$, and the atom number density (atoms $\mathrm{cm}^{-3}$ ). The interaction cross section is generally proportional to the atomic weight $A$. Since the track length is proportional to $X_{O}$, the production becomes proportional to the radiation length in units of $\mathrm{g} \mathrm{cm}^{-2}$. Thus, for rough estimates of dose equivalent in the environs of targets of materials other than iron, obtain results by scaling this value for iron by the factor $f$;

$$
f=\frac{X_{o}(\text { material })}{X_{o}(\text { iron })} \text {. }
$$

For shields comprised of other materials, one can simply adjust the implicit attenuation length (i.e., the value of $100 \mathrm{~g} \mathrm{~cm}^{-2}$ in the exponential function) to that appropriate to the material.

Fassò et al. (Fa90) give a more detailed treatment separately handling the giant resonance neutrons and high energy particle components of dose while deriving "source terms" and appropriate formulae. The formula for the dose equivalent $H_{n}$ due to the giant resonance neutrons given below is held to be valid for $1 \mathrm{GeV}<E_{o}<1.0 \mathrm{TeV}$ and for $30<\theta<120$ degrees. For the giant resonance neutrons, per incident electron;

$$
H_{n}=\eta_{n} S_{n} E_{o}\left(\frac{\sin \theta}{a+d}\right)^{2} \exp \left(-\frac{\rho d}{\lambda_{n} \sin \theta}\right), \quad\left(\text { Sv electron }^{-1}\right)
$$

where $E_{o}$ is the beam energy $(\mathrm{GeV}), \rho\left(\mathrm{g} \mathrm{cm}^{-3}\right)$ is the density, and the quantities $a(\mathrm{~cm})$ 


\section{ChAPTER 3 PROMPt RADIATION Fields DUe To ELECTRONS}

and $d(\mathrm{~cm})$ are defined in Fig. 3.9. $S_{n}\left(\mathrm{~Sv} \mathrm{~cm}^{2} \mathrm{GeV}^{-1}\right)$ is the source term from Table 3.1 and $\lambda_{n}\left(\mathrm{~g} \mathrm{~cm}^{-2}\right)$ is the attenuation length recommended for giant resonance neutrons listed in Table 3.4. Values of $\lambda_{n}$ are given as follows for representative materials. This formula is regarded as being valid for $30<\theta<120$ degrees.

\section{Table 3.4 Recommended attenuation lengths for use in Eq. (3.38) for various materials. [Adapted from (Fa90).]}

\begin{tabular}{|c|c|}
\hline Material & $\lambda_{n}\left(\mathrm{~g} \mathrm{~cm}^{-2}\right)$ \\
\hline Water & 9 \\
\hline concrete & 42 \\
\hline Iron & 130 \\
\hline Lead & 235 \\
\hline
\end{tabular}

The factor $\eta_{n}$ where $\eta_{n} \leq 1$ is dimensionless and gives an estimate of the "efficiency" for the production of neutrons by the target. It is generally connected with details of a given configuration. For "conservative" calculations, it can be taken to have a value of unity. It smoothly increases from very small values to unity as the target thickness approaches $X_{0}$.

\subsubsection{High Energy Particles}

In this situation no correction for target thickness is generally employed. These particles tend to drive the shielding requirements of large electron accelerators. The following formula for the dose equivalent per incident electron external to such a shield is based upon Eq. (3.17) augmented with exponential attenuation;

$$
H_{h}=\frac{7.5 \times 10^{-13} E_{O}}{(1-0.75 \cos \theta)^{2} A^{0.4}}\left[\frac{\sin \theta}{a+d}\right]^{2} \exp \left[-\frac{\rho d}{\lambda_{h} \sin \theta}\right]\left(\text { Sv electron }^{-1}\right) .
$$

The cylindrical geometry is as defined in Fig. 3.9. $H_{h}$ is the dose equivalent due to these particles ( $\mathrm{Sv}), E_{\mathrm{o}}$ is the beam energy $(\mathrm{GeV}), A$ is the atomic weight of the target, and $\lambda_{h}$ $\left(\mathrm{g} \mathrm{cm}^{-2}\right)$ is the attenuation length typical of these particles. Table 3.5 gives values of $\lambda_{h}$ for representative materials. Fassò et al. (Fa90) go further and describes a variety of special cases. The neutrons from this source tend to dominate the lateral shielding requirements in the $\mathrm{GeV}$ region. 


\section{ChAPTER 3 PRompt RAdiation Fields Due to Electrons}

Table 3.5 Attenuation lengths $\lambda_{h}$ in $\mathrm{g} \mathrm{cm}^{-2}$ for the high energy particle component. [Adapted from (Fa90).]

\begin{tabular}{|c|c|c|c|c|}
\hline Material & $\begin{array}{c}\text { Energy Limit } \\
>15 \mathrm{MeV} \text { or } \\
>25 \mathrm{MeV} \\
\left(\mathrm{g} \mathrm{cm}^{-2}\right)\end{array}$ & $\begin{array}{c}\text { Energy Limit } \\
>100 \mathrm{MeV}\end{array}$ & $\begin{array}{c}\text { Nuclear } \\
\text { Interaction } \\
\text { Length } \\
\left(\mathrm{g} \mathrm{cm}^{-2}\right)\end{array}$ & $\begin{array}{c}\text { Recommended } \\
\lambda_{h}[\mathrm{Eq} \cdot(3.39)] \\
\left(\mathrm{g} \mathrm{cm}^{-2}\right)\end{array}$ \\
\hline Water & & & 84.9 & 86 \\
\hline Aluminum & & & 106.4 & 128 \\
\hline Soil (sand) & $\begin{array}{l}101,104^{\mathrm{a}} \\
102,105^{\mathrm{b}}\end{array}$ & $\begin{array}{c}117 \\
96 \\
\end{array}$ & 99.2 & 117 \\
\hline Concrete & $\begin{array}{c}101,105^{\mathrm{a}} \\
91 \\
82,100^{\mathrm{b}}\end{array}$ & $\begin{array}{l}120 \\
105 \\
100\end{array}$ & 99.9 & 117 \\
\hline Iron & $139^{b}$ & & 131.9 & 164 \\
\hline Lead & $244^{\mathrm{b}}$ & & 194 & 253 \\
\hline
\end{tabular}

${ }^{\mathrm{a}}$ Attenuation lengths for the indicated values are slightly dependent on angle with the higher value at $\theta=0^{\circ}$ and the smaller value in the backward direction for $E>15 \mathrm{MeV}$.

${ }^{\mathrm{b}}$ Same remark as in footnote a, but for $E>25 \mathrm{MeV}$.

\subsection{Synchrotron Radiation}

While the physics of synchrotron radiation has long been understood, its importance in modern research, both basic and applied has grown dramatically with the advent of electron accelerators designed for use as photon sources. Wiedemann (Wi03) and Margaritondo (Ma88) provide much detailed information on both the synchrotron radiation itself and the modern facilities that have been built to utilize it. An important review of radiation protection considerations at synchrotron radiation facilities has been provided by Swanson (Sw90) and Liu and Vylet (Li01).

\subsubsection{General Discussion of the Phenomenon}

The movement of electrons in a curved orbit results in their centripetal acceleration. This gives rise to emission of photons. At nonrelativistic energies this radiation is largely isotropic. However, for relativistic energies, a condition readily achieved by accelerated electrons, the photons emerge in a tight bundle along a tangent to any point on a circular orbit. For a single electron, or a small bunch of electrons orbiting together, the photon beam will sweep around like a searchlight. Fig. 3.15 shows this bundle. The exact shape of this bundle is a somewhat complicated function of the energy of the radiation emitted and the electron energy.

The characteristic angle $\theta_{c}$ (i.e., the angle of $1 / \mathrm{e}$ of the zero degree intensity) of this "lobe" is:

$$
\theta_{c}=\frac{1}{\gamma}=\sqrt{1-\beta^{2}} \text { radians, }
$$




\section{ChAPTER 3 PRompt RAdiAtion Fields Due to Electrons}

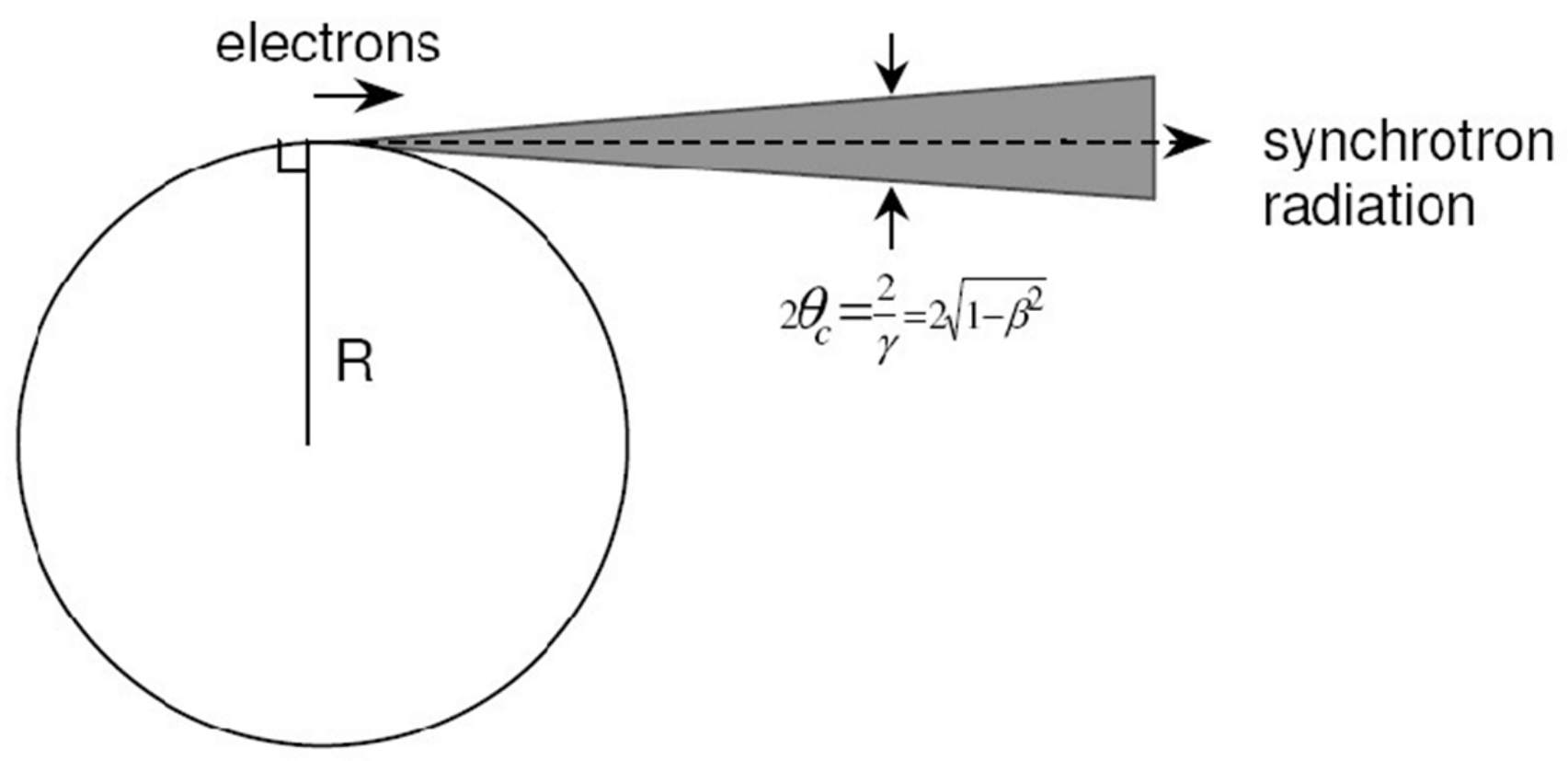

Fig. 3.15 Synchrotron radiation pattern for relativistic particles at the instantaneous orbit location denoted by "electrons". Twice the characteristic opening angle $\theta_{c}$ is shown as the conical shaded region.

with $\gamma$ being the relativistic parameter defined in Eq. (1.12). The energy spectrum of the photons emitted by electrons captured in such a circular orbit turns out to be a standard mathematical function the shape of which is independent of the electron beam energy. It is an integral of a modified Bessel function of the third kind with numerical tabulations of it available. For the present discussion the mathematical details are not needed. Fig. 3.16 shows this function. This photon spectrum is called the bending magnet spectrum.

The median energy of the power spectrum, sometimes called the characteristic energy or critical energy $\varepsilon_{C}$ is given in terms of the total energy $W(\mathrm{GeV})$ and bending radius $R$ (meters), by

$$
\varepsilon_{c}=\frac{2.218 W^{3}}{R}(\mathrm{keV})
$$

For singly-charged particles other than electrons of rest mass $m_{x}$, the characteristic energy is obtained by multiplying this result by a factor of $\left(m_{e} / m_{x}\right)^{3}$ where $m_{e}$ is the rest mass of the electron. The characteristic energy for synchrotron radiation for protons having the same total energy as electrons is thus far lower. As will become obvious, it is often convenient to specify these and other quantities as functions of the magnetic field 


\section{Chapter 3 Prompt RAdiation Fields Due to Electrons}

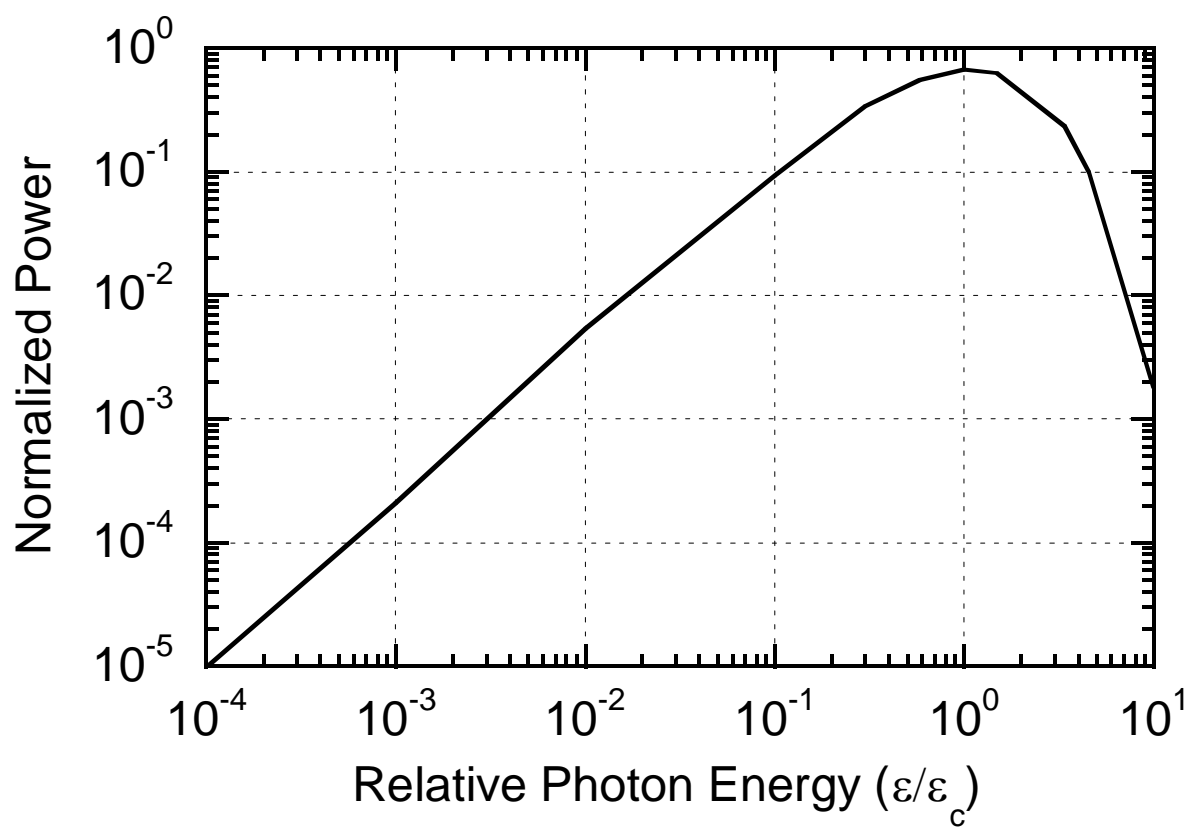

Fig. 3.16 Universal synchrotron radiation spectrum. The graph gives the relative power as a function of photon energy in units of characteristic energy, $\varepsilon_{\mathcal{C}}$. This spectrum yields unity if integrated over all energies. [Adapted from (Sw90).]

$B$ (Tesla) that creates the circular orbit of radius $R$ for particles of momentum $p(\mathrm{GeV} / \mathrm{c})$ by recalling Eq. (2.26):

$$
R=\frac{p}{0.29979 q B} \text { (meters). }
$$

If one substitutes for $R$; recalls that $q=1$ for electrons (ignoring the negative sign of the charge); disregards the small distinctions between kinetic energy, momentum, and total energy for relativistic electrons; and combines constants;

$$
\varepsilon_{c}=0.6649 W^{2} B(\mathrm{keV})
$$

For relativistic conditions (i.e., $\gamma>>1$ ) the mean number of photons emitted per complete revolution is

$$
N_{\gamma}=\frac{5 \pi}{\sqrt{3}} \alpha \gamma
$$

where $\alpha$ is the fine structure constant of atomic physics $(\approx 1 / 137$, see Table 1.1$)$. Since 


\section{ChAPTER 3 PRompt RAdiATION FieldS DUE To ELECTRONS}

the bending magnet spectrum has considerable "skewness", again for $\gamma>>1$, the mean energy per photon $\langle\varepsilon\rangle$ is

$$
\langle\varepsilon\rangle=\frac{8}{15 \sqrt{3}} \varepsilon_{c}
$$

As an electron circulates in this circular orbit, the energy loss per revolution is given by

$$
\delta E=\frac{0.08846}{R} W^{4}(\mathrm{MeV})
$$

with $W$ in $\mathrm{GeV}$ and $R$ in meters. An alternative form commonly used arises from substituting for $R$ employing Eq. (3.42);

$$
\delta E=0.02652 W^{3} B(\mathrm{MeV})
$$

with $B$ in Tesla.

If the orbit is a circle with continuous, uniform bending around the circumference and with straight sections of "negligible" length, it should be clear that a circulating current $I$ (milliamperes) can be connected with the radiated power $P$ (watts). First, one needs to determine the number of electrons $\mathrm{s}^{-1}$ per milliampere current;

$$
I(\mathrm{~mA})=I\left(\frac{10^{-3} \text { Coulombs }}{\mathrm{sec}}\right) \times\left(\frac{1 \text { electron }}{1.602 \times 10^{-19} \text { Coulombs }}\right)=I\left(\frac{\text { electrons }}{\sec \times\left\{1.602 \times 10^{-16}\right\}}\right)
$$

Then one can derive the radiated power from Eq. (3.46);

$$
\begin{gathered}
P=\frac{0.08846 W^{4}}{R} \frac{\mathrm{MeV}}{\text { electron }} I\left(\frac{\text { electrons }}{\sec \times\left\{1.602 \times 10^{-16}\right\}}\right) \times \frac{1.602 \times 10^{-13} \text { Joule }}{\mathrm{MeV}} \\
=\frac{88.46 W^{4} I}{R} \frac{\text { Joules }}{\mathrm{sec}}=\frac{88.46 W^{4} I}{R} \text { watts. }
\end{gathered}
$$

Again using Eq. (3.42), this can be expressed in terms of the magnetic field;

$$
P=26.52 W^{3} B I \text { watts. }
$$

For singly-charged particles other than electrons of rest mass $m_{x}$, the radiated power is obtained by multiplying this result by a factor of $\left(m_{e} / m_{x}\right)^{4}$ where $m_{e}$ is the rest mass of 


\section{ChAPTER 3 PRompt RAdiation Fields Due to ElECTRONS}

the electron. Again, one can see why all synchrotron radiation facilities (i.e., "light" sources) are based upon circulating electrons, not protons or heavier particles. However for ultra high energy proton accelerators (e.g., the Large Hadron Collider at CERN), the need to replenish the energy lost through synchrotron radiation can be a significant electrical power demand.

Synchrotron radiation possesses an additional property not further discussed in detail here; the fact that the photons are polarized to rather high degree, greater than $80 \%$ is typical, in the plane of the ring in which they orbit. These large polarizations can be further manipulated and are extremely beneficial to the users of light sources. They also can result in asymmetries in the radiation production by these accelerators.

\subsubsection{Insertion Devices}

The researchers who use the modern light sources are not limited to the broad band of photons obtained from the general bending of the electron beam around its circular orbit. It was realized at an early stage in the development of this technology that if one were to insert a set of bending magnets of alternating polarities into a straight section of a ring, smaller bending radii over short distances could be produced that would result in radiation of higher energy photons according to Eqs. (3.41) or (3.43). Fig. 3.17 shows such a wavelength shifter schematically:

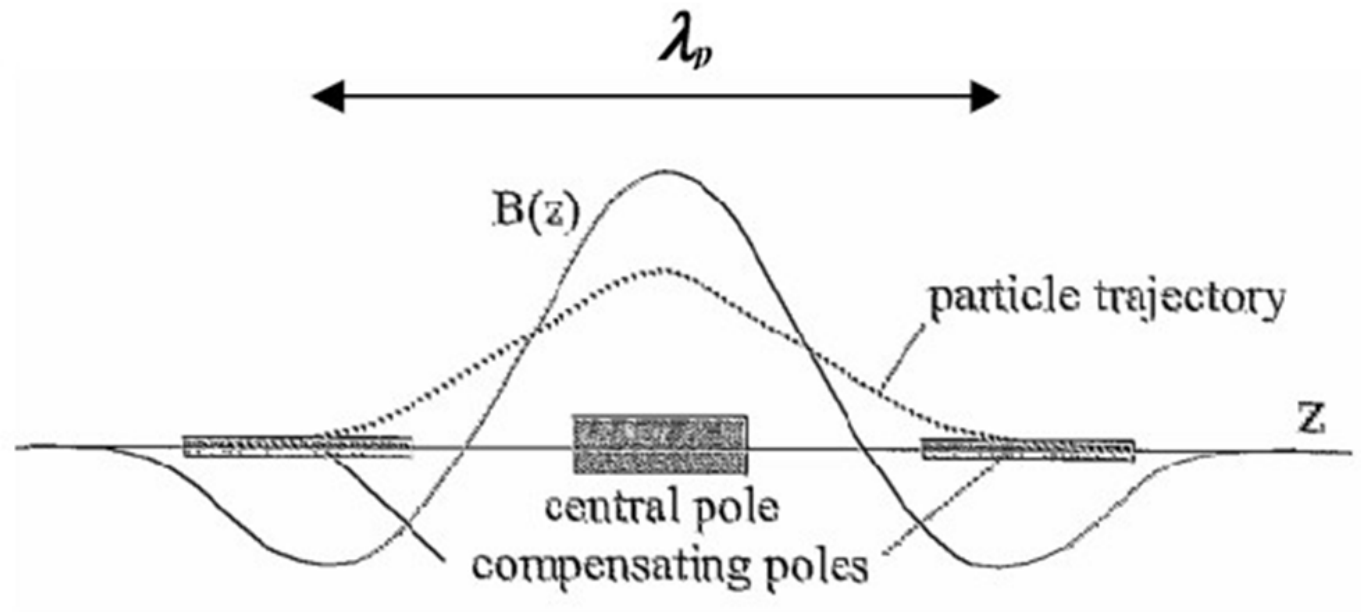

Fig. 3.17 Schematic of a wavelength shifter showing the three magnets involved; the magnetic field strength $B(z)$ as a function of longitudinal coordinate $z$; and the electron trajectory. $\lambda_{p}$ is the length of a period of group of such magnets, here consisting of a pair. Since the pole pieces are typically short, the magnetic field strength may have an approximately sinusoidal dependence on $z$ as depicted here. [Adapted from (Wi03).]

It is clear while more energetic photons would be emitted, their intensity would be limited by the short fraction of the time of each orbit the individual beam electrons are deflected by this higher magnetic field. It was discovered that if one were to line up a series of such magnets of alternating polarities in a row, the intensity could be increased. 


\section{Chapter 3 Prompt RAdiation Fields Due to Electrons}

If there are $N_{m}$ such pairs of magnet poles (i.e., "periods") in the system, then one will get $2 N_{m}$ times the photons provided by one of them, neglecting end effects. These magnets could be dipoles of alternating polarities lined up in any plane. In practice they are generally set to bend charged particles in the bending plane of the storage ring. This avoids some complications for accelerator operations since it limits the coupling between horizontal and vertical betatron oscillations in the storage ring.

The magnetic field strength in these three magnets can be of any strength if there is no net deviation of the overall orbit of the electrons, aside from corrections that might be needed to compensate for additional dispersion and aberrations introduced by this "device" (see Section 2.5.2). Components of this type are placed in storage rings to create specialized photon energy spectra are called insertion devices. Typical modern light sources contain a number of such devices designed to create particular photon beam properties. Some employ permanent magnets while others may utilize superconducting magnets to achieve high magnetic fields. Sophisticated, improved versions not discussed here have been developed. Fig. 3.18 is a schematic picture of an insertion device.

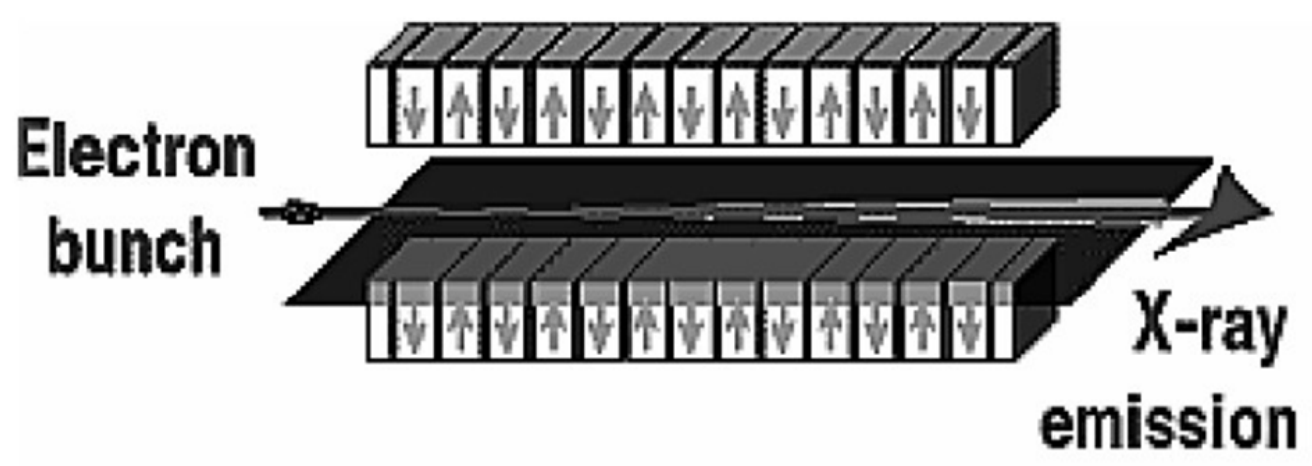

Fig. 3.18 Sketch of a typical insertion device. The vertical arrows show the orientation of the magnetic field in the individual gaps. [J. Liu and V. Vylet, private communication.]

A special parameter is useful in this discussion. Consider a device consisting of a large number of alternating magnet poles. The spacing of each pair of poles $\lambda_{p}$ as defined in Fig. 3.17 constitutes the length of the period. Because the individual pole pieces are often short compared with the dimensions of the field gaps, truly "uniform" dipole field conditions are generally not achieved. Instead, the magnetic field component perpendicular to the bending plane, here denoted $B_{y}$, is often approximated by a sinusoidal dependence on the longitudinal coordinate $z$ as shown in Fig. 3.17;

$$
B_{y}=B_{o} \sin \frac{2 \pi z}{\lambda_{p}} .
$$

Now one can calculate the angle $\alpha_{m}$, the maximum deflection of the electrons away from the central axis, as they proceed along the insertion device using Eq. (3.42) by performing an integration over the longitudinal coordinate $z$. Given the size of practical 


\section{ChAPTER 3 PROMPt RAdiATION Fields DUe to ElECTRONS}

insertion device pole pieces, it is useful to work with $z$ and $\lambda_{p}$ in centimeters. Performing the integration after making the unit conversion;

$$
\begin{aligned}
& \alpha_{m}=\frac{2.9979 \times 10^{-3} B_{o}}{p(\mathrm{GeV} / \mathrm{c})} \int_{o}^{\lambda_{p} / 4} d z \sin \frac{2 \pi z}{\lambda_{p}}=\frac{\lambda_{p}}{2 \pi} \frac{2.9979 \times 10^{-3} B_{o}}{p(\mathrm{GeV} / \mathrm{c})}\left[-\cos \frac{2 \pi z}{\lambda_{p}}\right]_{0}^{\lambda_{p} / 4} \\
& =\left(4.771 \times 10^{-4}\right) \frac{B_{o} \lambda_{p}}{p} \text { (radians). }
\end{aligned}
$$

One multiplies this by the relativistic parameter of the electron beam $\gamma$ to define a new dimensionless parameter $K$. Since the electrons that produce useful synchrotron radiation are highly relativistic, working in energy units as was done in Eq. (3.51);

$$
\gamma=\frac{W}{m_{o}} \approx \frac{p}{m_{o}} \text {, and } K=\gamma \alpha_{m}=\left(4.771 \times 10^{-4}\right) \frac{B_{o} \lambda_{p}}{m_{o}}=0.934 B_{o} \lambda_{p} \text {, }
$$

with $B_{o}$ in Tesla, $\lambda_{p}$ in $\mathrm{cm}$, and the rest energy of the electron $\left(\mathrm{m}_{o} \mathrm{c}^{2}\right)$ in GeV. $K$ is called the wiggler strength parameter or deflection parameter. Its role can be better understood with the help of Fig. 3.19. If one recalls that the cone of emission of the synchrotron radiation has an approximate half-width of $1 / \gamma$, for $K>1$, the maximum deflection $\alpha_{m}$ is thus larger than the cone of emission (as illustrated in the bottom frame of Fig. 3.19. In such circumstances the insertion device is called a wiggler and the synchrotron radiation produced has a bending magnet energy spectrum shape.
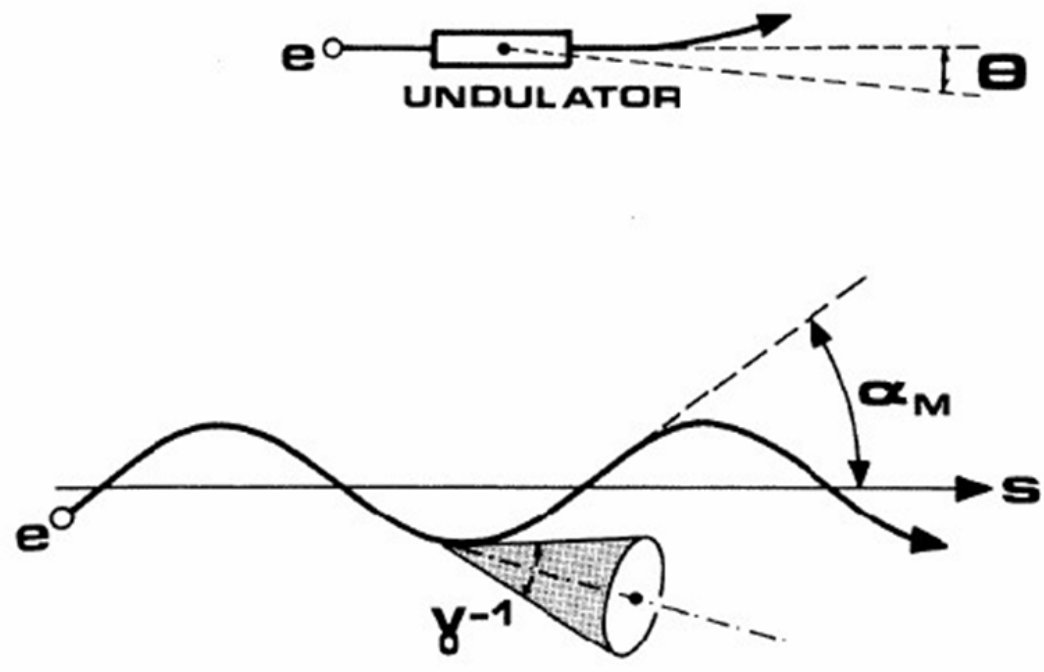

Fig. 3.19 Top: Definition of the angle $\theta$ at which radiation is emitted by an undulator inserted in a straight section of a storage ring. Bottom: Angular amplitude of the half-angle of the cone of emission of radiation $\approx \gamma^{-1}$ and maximum deflection angle of the electron trajectory caused by an insertion device $\alpha_{m}$. [Adapted from (Ma88).] 


\section{ChAPTER 3 PRompt RAdiation Fields Due to EleCtrons}

For $K<1$, the divergence due to the magnetic deflections is smaller than the intrinsic cone of emission and the device is then called an undulator. In an undulator, since the deflections occur within the cone of emission, interference effects can occur. In fact, these are exploited to provide approximately monochromatic photons or spectra with other desired properties. While wigglers are useful for making the energy spectrum of the photons more energetic (i.e., "hardening" the spectrum), undulators can be used to create very "bright" beams of nearly monoenergetic photons or a spectrum of photons delivered in a few narrow bands. The increased brightness is due to the smaller dispersion due to the bending magnet deflections. Avoiding the details of a somewhat complicated derivation, the undulator frequency $v_{1}$ of the photons produced is given by (Wi03)

$$
v_{1} \approx \frac{2 c \gamma^{2}}{\lambda_{p}} \frac{1}{\left(1+K^{2} / 2+\gamma^{2} \theta^{2}\right)}(\mathrm{Hz})
$$

for small, but not negligible, values of $K$, angles of emission $\theta$ in radians and $\lambda_{p}$ in $\mathrm{cm}$. Since $K$ is a function of magnetic field strength and magnet pole spacing, this frequency can be adjusted to some degree by altering those parameters. At "intermediate" values of $K$, other spectral peaks at harmonics of the above frequencies become possible. It is of course easy to obtain the corresponding photon energy $E_{1}$ by applying Planck's constant $h$;

$$
E_{1}=h v_{1} \approx \frac{\left(2.480 \times 10^{-7}\right) \gamma^{2}}{\lambda_{p}} \frac{1}{\left(1+K^{2} / 2+\gamma^{2} \theta^{2}\right)} \quad(\mathrm{keV})
$$

One should consider the power that can be emitted in the tightly focused undulator beam. For an undulator of $N_{m}$ periods, the power emitted (Ma88) is given by:

$$
P_{t o t}=\frac{\left(1.9 \times 10^{-12}\right) N_{m} \gamma^{2} K^{2} I(\mathrm{~mA})}{\lambda_{\mathrm{p}}(\mathrm{cm})} \quad(\mathrm{kW}) .
$$

Unlike for the bending magnet situation, this power would be emitted into a very small $1 / \gamma$ cone, not in the "pancake-shaped" distribution around the entire circumference representative of the bending magnet situation.

Fig. 3.20 shows some examples of spectra emitted by different types of insertion devices. Obviously, these devices continue to evolve and more complicated ones are being developed to address specific applications. Collimation is often used to select desired portions of these spectra, optimized for their intended use. 


\section{Chapter 3 Prompt Radiation Fields Due to Electrons}

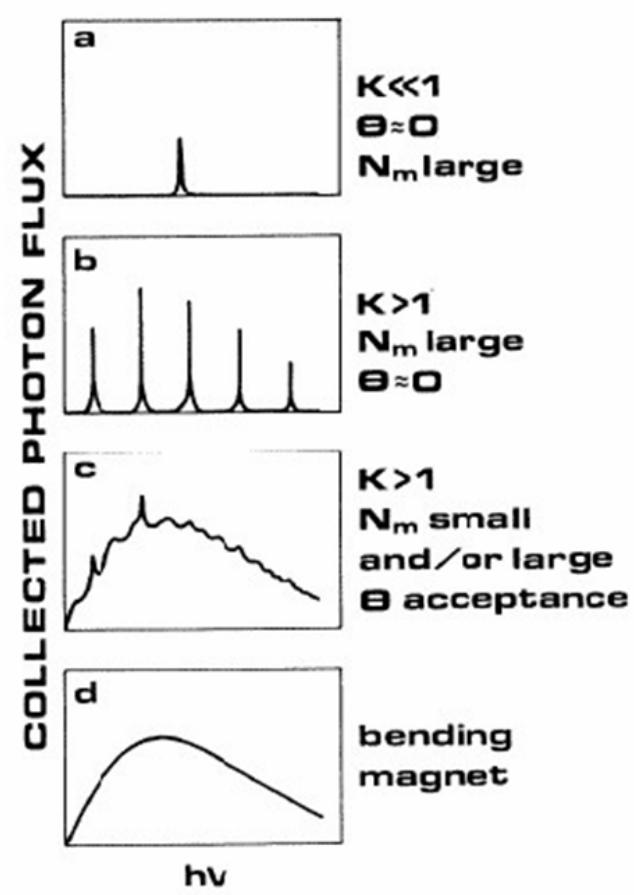

Fig. 3.20 Different examples of insertion device emission, compared to bending magnet radiation. Case $\mathbf{a}$ is the line emission from an undulator, seen through a pinhole which limits the angular acceptance. Case $\mathbf{b}$ is a strong field device (not further described here), again seen through a pinhole so as to limit the angular acceptance. In Case c a limited number of periods are used to create a spectrum of small peaks atop the bending magnet spectrum. Case $\mathbf{d}$ is the pure bending magnet spectrum. Unlike Fig. 3.16, the scale of the abscissa is linear, not logarithmic [Adapted from (Ma88).]

\subsubsection{Radiation Protection Issues Specific to Synchrotron Radiation Facilities}

Obviously, all the radiation protection concerns discussed elsewhere in this text pertinent to electron accelerators of the same energies and intensities apply to synchrotron radiation facilities. These include the production of bremsstrahlung photons, the production of neutrons and high energy particles, the development of electromagnetic cascades, and the production of induced radioactivity. However, there are unique phenomena prominent at these facilities. Though reviewed here, these and related topics are discussed in more detail elsewhere [e.g. in (Ri82), (Ba89), (Tr90), (Ip94), (Li95), and (Li05)].

\subsubsection{Operating Modes}

Synchrotron radiation sources largely operate as storage rings. To accommodate insertion devices and experimental apparatus, these storage rings often have relatively long straight sections. Operating modes need to be considered. Typically, the electron beam is produced by an injector accelerator of some type and injected into the main storage ring in an injection event. Following injection the beam is typically smoothed out for several thousand turns before being added to the stored beam. Electrons are typically lost during the injection process on limiting apertures designed to "clean-up" the beam for storage or are otherwise lost around the ring. Then, the beam is used for the intended research 


\section{ChAPTER 3 Prompt RAdiation FieldS Due to Electrons}

purposes for long periods of time. At the end of a defined period of storage ring operations called a store, the beam is generally disposed of in a beam absorber, and the cycle is repeated. A relatively recent advancement made to improve the efficiency of operations is the capability to replenish the beam without ending a store by delivering additional electrons from the injector to the storage ring in a so-called top-off mode.

Often the personnel protection requirements and beamline access restrictions imposed on the researchers are considerably different during injection events and storage ring operations due to the differences in the levels and types of radiological hazards presented. Typically, radiological problems are most prominent during the injection events because at that time, errors of beam tuning may result in point losses and the rate of beam delivery can be large, perhaps up to the output of the injector. This also applies to the top-off mode of operation. During pure storage ring operations, since accelerator orbits have been established to achieve a useful beam lifetime, inductive time constants render sudden, large losses due to mistuning or collapse of the magnetic field (e.g., during a power failure) much less probable. Point losses can, however, occur due to other types of events such as the sudden closure of vacuum valves or some other unintended movement of material into the beam. However, while the stored beam current may be significant, during the storage ring mode (i.e., not during injection or top-off), the total number of stored electrons is limited and serves as an upper limit to beam loss events. Devices called beam stops, shutters, or injection stoppers are inserted into the front end of beamlines to protect personnel and equipment from the consequences of beam losses during injection events. Obviously it is imperative to fully understand the beam loss characteristics at every stage of operation. Fig. 3.21 shows the layout of a portion of a typical synchrotron radiation facility showing the installation of some types of safety equipment used in various operational modes.

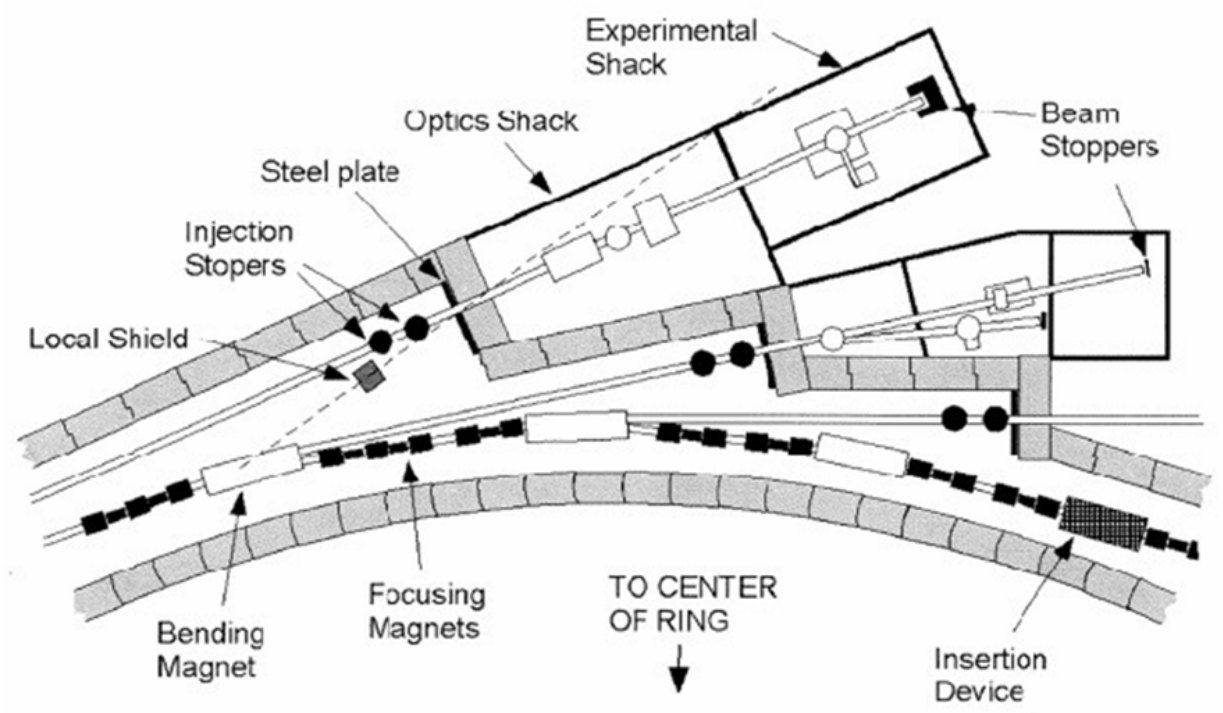

Fig. 3.21 Typical synchrotron radiation facility experimental installation. The storage ring is at the bottom of the frame. So called "ratchet-walls" separate the experimental installations from the main storage ring. [Adapted from (NC03).] 


\section{ChAPTER 3 PRompt RAdiATion Fields Due to EleCtrons}

\subsubsection{Gas Bremsstrahlung - Straight Ahead}

At these facilities, the decay of the stored beam will be dominated by scattering from the residual gas particles. Though the vacuum can be made to be very good, the path lengths of the electrons in a storage ring mode are extremely long, when huge numbers of orbits are taken into account. Also, the synchrotron radiation photons themselves can induce outgassing in certain materials that may increase residual pressures within the "vacuum". The process of beam interaction in the residual gas is obviously a "thin target" phenomenon, otherwise the electron beam could not be stored! Under some circumstances, equipment damage concerns are important. Following scattering events the electrons will spiral radially inward and be lost. Workers at various laboratories have developed computational methods using both analytical and Monte Carlo techniques to address these matters. In the discussion here, results using both methods are used as illustrations.

From Eq. (3.7) gas bremsstrahlung has a nearly $1 / k$ energy spectrum (with $k$ denoted as the photon energy to distinguish it from the electron beam energy). The spectrum extends essentially from zero up to the kinetic energy of the stored electrons. The angular distribution is highly forward-peaked having a characteristic angle (i.e., a " $1 / \mathrm{e}$ " angle) of $0.511 / E$ in radians for electron beam energy $E(\mathrm{MeV})$. The dose is approximately proportional to $E^{2.5}$ and of course the mass thickness of the air column in the ring section through which the electrons pass. It is obvious that the photons from the gas bremsstrahlung are far more energetic and hence more difficult to shield than are the synchrotron radiation photons of much lower energies (i.e. x-rays). To better understand this, Tromba and Rindi (Tr90) performed Monte Carlo calculations with the code EGS4 for the geometry shown in Fig. 3.22.
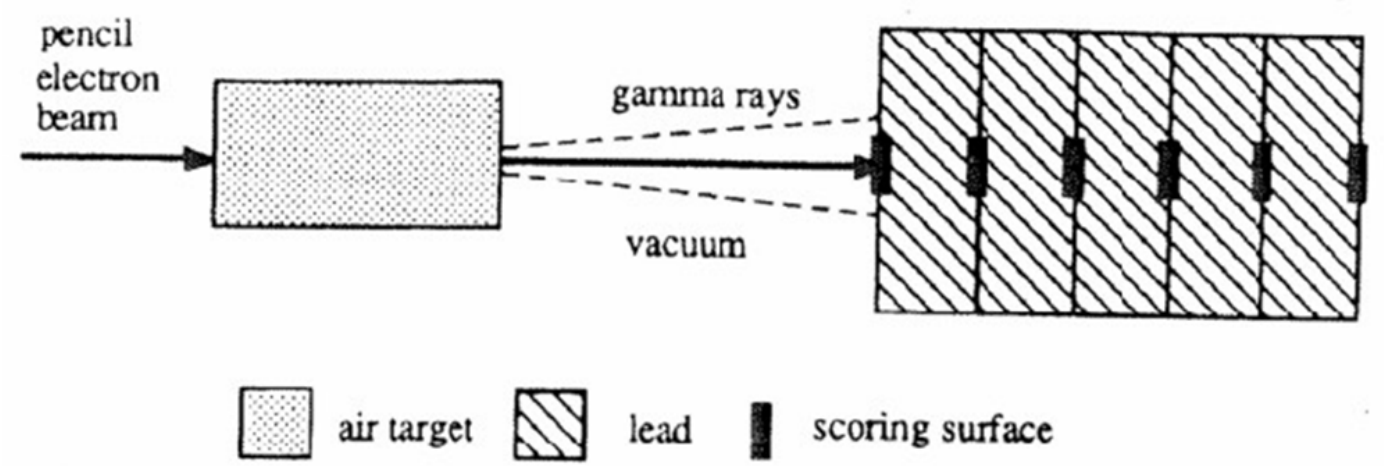

Fig. 3.22 Geometry considered by Tromba and Rindi in their Monte Carlo calculations of gas bremsstrahlung. An electron pencil beam crosses an air target. The bremsstrahlung photons are attenuated in lead. The number of photons and the relative dose are scored on a small surface (about $1.0 \mathrm{~cm}^{2}$ ), smaller than the photon-beam angular opening at different depths in the lead. [Adapted from (Tr90).]

As a result of their calculations, these authors proposed the following expression for the dose rate $d D / d t_{10}$ meters $\left(\mathrm{Gyh}^{-1}\right)$ at 10 meters "on axis" from the end of the straight section (i.e, the "air target" in Fig. 3.22): 


\section{ChAPTER 3 Prompt RAdiATION Fields Due to Electrons}

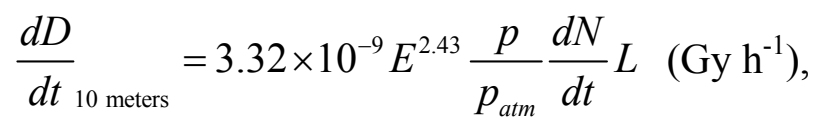

where $E$ is the electron energy $(\mathrm{GeV}), d N / d t$ is the number of electrons $\mathrm{s}^{-1}$ passing through the straight section $p / p_{\text {atm }}$ is the ratio of the residual pressure to atmosphere pressure, and $L$ is the length of the straight section in meters. It is often convenient to work with the beam current $I(\mathrm{~mA})$, and residual pressure $P$ (torr). $[1.0$ torr $=1.0 \mathrm{~mm}$ of $\mathrm{Hg}$ pressure $=1 / 760$ of a standard atmosphere $=133.3 \mathrm{~Pa}$.] Eq. (3.56) then becomes

$$
\frac{d D}{d t}_{10 \text { meters }}=2.72 \times 10^{4} E^{2.43} P I L\left(\mathrm{~Gy} \mathrm{~h}^{-1}\right) .
$$

For other distances $r(\mathrm{~m})$ measured from the center of the straight section, inside of this narrow radiation cone, one should scale this result by the inverse square factor,

$$
\frac{d D(r)}{d t}=\frac{d D}{d t} \text { 10 meters } \quad\left\{\frac{10+L / 2}{r}\right\}^{2} \text {. }
$$

Eq. (3.58) is valid as long as one is within the radiation emission cone and $r>L / 2$.

Of course one will need to calculate the thickness of shielding needed to attenuate this source of radiation to some desired level. Fortunately, as exhibited in Figs. 3.10 and 3.11, the photon mean free path is very weakly dependent upon energy over several orders of magnitude in the energy domain of interest. Tromba and Rindi found that for lead shielding, the attenuation, after some initial buildup region of a few centimeters, can be characterized as an exponential one having an attenuation coefficient of $\approx 0.6 \mathrm{~cm}^{-1}$, a parameter only very weakly dependent upon beam energy.

\subsubsection{Gas Bremsstrahlung - Secondary Photons}

Another consequence of gas bremsstrahlung is the generation of secondary photons by interactions of the gas bremsstrahlung photons with materials. This applies when the bremsstrahlung photons are incident on some sort of absorber or beam "shutter" in the absence of the beam electrons that have been deflected somewhere else by bending magnets. A useful "prescription" has been presented by Liu and Vylet (Li01) to estimate this effect. For a thin piece of material, the lateral photon dose is largest at somewhat forward angles. For more massive objects, the maximum in the lateral photon dose is at more backward angles. From Eq. (3.5), the fractional energy transferred from an electron to the photons $d E / E$ is equal to the ratio of the mass thickness $t$ of the column of residual gas to its radiation length $X_{o}$. Thus, the fractional energy or power (with units of time included) transferred to gas bremsstrahlung photons from the circulating electrons is $t / X_{o}$, where the radiation length of air from Table 1.2 is $36.66 \mathrm{~g} \mathrm{~cm}^{-2}$. One can multiply this ratio by the stored power of the electron beam to determine the bremsstrahlung power; that is, the power that is delivered to the bremsstrahlung photons. To illustrate how this 


\section{ChAPTER 3 PRompt RAdiation Fields Due to ElECTRONS}

can be used, consider an example for a $3.0 \mathrm{GeV}$ storage ring that stores $500 \mathrm{~mA}$ of electron beam current. Further assume that $L=5.0$ meters and the residual gas pressure is $1.0 \times 10^{-9}$ torr. For this, applying the atmospheric density at NTP (from Table 1.2),

$t=500 \mathrm{~cm} \times \frac{10^{-9} \text { torr } \times \text { atmosphere }}{760 \text { torr }} \frac{1.205 \mathrm{~g}}{1000 \mathrm{~cm}^{3} \text { atmosphere }}=7.928 \times 10^{-13}\left(\mathrm{~g} \mathrm{~cm}^{-2}\right)$.

Thus the fraction of the total beam power diverted into gas bremsstrahlung by interactions in the residual gas of this particular straight section is

$$
F_{\text {brem }}=\frac{t}{X_{o}}=\frac{7.928 \times 10^{-13}}{36.66}=2.162 \times 10^{-14}
$$

At this machine the stored beam power is $1.5 \times 10^{9}$ watts. Applying the result of Eq. (3.59), $3.24 \times 10^{-5}$ watts is transferred into bremsstrahlung at this particular location. When this bremsstrahlung bombards a solid object in a beamline an electromagnetic cascade is initiated. For simplicity, this "object" will be taken to be a cylinder several radiation lengths long characterized by a Molière radius $X_{m}$ as defined in Eq. (3.19). Eq. (3.34) (see also Fig. 3.14) gives the fraction of the incident bremsstrahlung beam power that escapes a thickness $R$ of this shield laterally $F_{\text {esc }}\left(0 \leq F_{\text {esc }} \leq 1\right)$;

$$
F_{\text {esc }}=\frac{U\left(R / X_{m}\right)}{E_{o}}=0.8 \exp \left[-3.45\left(R / X_{m}\right)\right]+0.2 \exp \left[-0.889\left(R / X_{m}\right)\right] .
$$

Thus, in our example $3.24 \times 10^{-5} F_{\text {esc }}$ watts will escape laterally. Making a unit conversion, this is equivalent to $2.022 \times 10^{8} F_{\text {esc }} \mathrm{MeV} \mathrm{s}^{-1}$. For these laterally-produced photons it is reasonable to take their average energy to be about $1.0 \mathrm{MeV}$. Thus, this configuration represents a finite, uniform line source of strength $S_{L}=4.044 \times 10^{5} F_{\text {esc }}$ photons cm $\mathrm{cm}^{-1}$. It has been shown by others [e.g., Jaeger et al. (Ja68)], that the flux density at a distance $a$ away from a line source length $L$ on the perpendicular bisector is

$$
\phi(a)=\frac{S_{L}}{2 \pi a} \operatorname{Tan}^{-1}\left(\frac{L}{2 a}\right)
$$

with the value of $\operatorname{Tan}^{-1}(L / 2 a)$ obtained in radians. Evaluating this at $a=1.0$ meter,

$\phi(100 \mathrm{~cm})=\frac{4.045 \times 10^{5}}{2 \pi(100 \mathrm{~cm})}\left(\frac{\text { photons }}{\mathrm{cm} \mathrm{s}}\right) F_{\text {esc }} \operatorname{Tan}^{-1}\left(\frac{500}{200}\right)=766.3 F_{\text {esc }}\left(\right.$ photons $\left.\mathrm{cm}^{-2} \mathrm{~s}^{-1}\right)$.

Multiplying by an appropriate dose per fluence value of $4 \times 10^{-6} \mu \mathrm{Sv} \mathrm{cm}^{2}$ (see Fig.1.5) gives a dose rate $d H / d t=3.07 \times 10^{-3} F_{\text {esc }} \mu \mathrm{Sv} \mathrm{s}{ }^{-1}=11.0 F_{\text {esc }} \mu \mathrm{Sv} \mathrm{h}{ }^{-1}$, a measurable value even with the extremely good vacuum postulated if $F_{\text {esc }}$ is at all significant. Liu and Vylet report that estimates of this type agree with measurement to within a factor of two or 


\section{ChAPTER 3 Prompt RAdiation Fields Due to Electrons}

three. This methodology is somewhat overly simplistic, it ignores some forward-peaking. For thick targets, the dose equivalent rate will be larger at backward angles. For small objects struck by the beam, a point source approximation may be a better choice.

Liu et al. (Li95) have performed a more sophisticated calculation of such photon dose rates using the EGS4 code for backward angles (i.e., "upstream" of the beam shutter). Their results for various materials are given in Fig. 3.23.

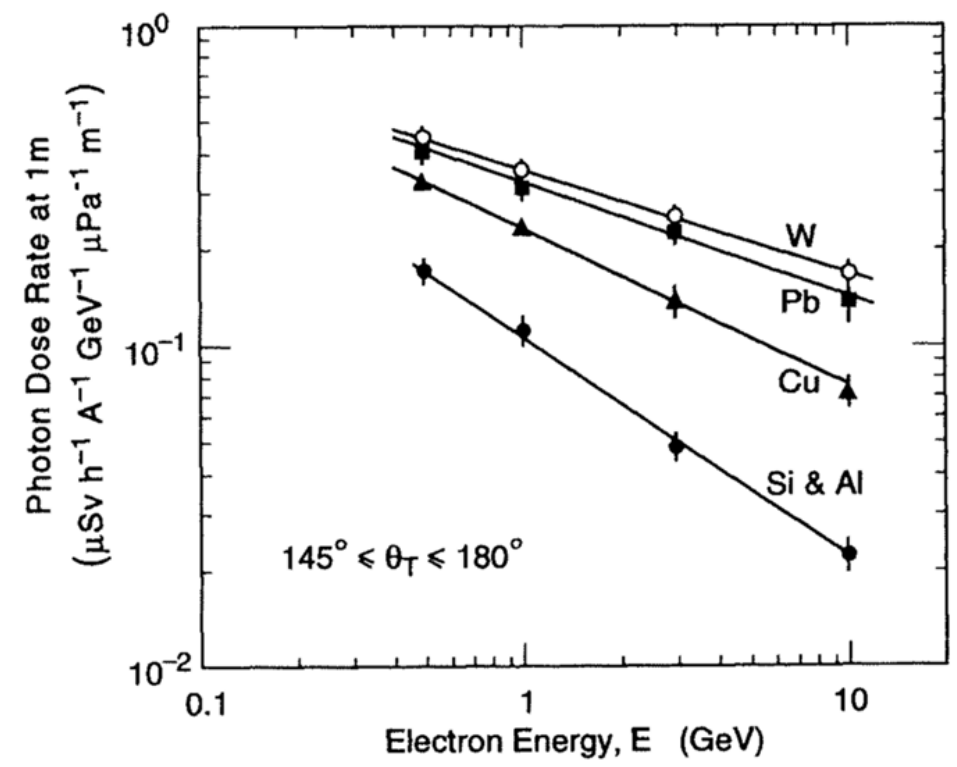

Fig. 3.23 Secondary photon dose rate at $1.0 \mathrm{~m}$ lateral to large targets of various materials as a function of electron beam energy. The values are normalized to a beam current of 1.0 Ampere, per $\mathrm{GeV}$ beam energy, and an air path of 1.0 meter with a pressure of $1 \mu \mathrm{Pa}\left(=7.50 \times 10^{-9}\right.$ torr $)$. [Adapted from (Li95).]

These curves exhibit an energy dependence due to the fact that for the higher energies, the location of the shower maximum is located deeper in the absorber. Thus, at higher energies the photons will be more attenuated as they move backwards out of the target. Liu et al. report parametrizations of the energy dependencies of $H_{s}$, the photon surface dose rate $\left(\mu \mathrm{Sv} \mathrm{h}^{-1} \mathrm{~A}^{-1} \mathrm{GeV}^{-1} \mu \mathrm{Pa}^{-1} \mathrm{~m}^{-1}\right)$ at 1 meter lateral distance from the beam line:

$$
\begin{aligned}
& H_{\mathrm{s}}=0.35 E^{-0.33} \text { (tungsten), } \\
& H_{S}=0.32 E^{-0.36} \text { (lead), } \\
& H_{S}=0.23 E^{-0.49} \text { (copper), and } \\
& H_{S}=0.11 E^{-0.69} \text { (silicon and aluminum). }
\end{aligned}
$$

\subsubsection{Gas Bremsstrahlung - Neutron Production Rates}

The prodigious production of photons can lead to correspondingly significant photoneutron production. Using a methodology similar to that employed above, Eq. (3.59) can be used to calculate the bremsstrahlung power at the end of a given straight section. These photons may then be incident on some device such as a beam shutter and produce neutrons. For example the straight section discussed above is operational, then 


\section{ChAPTER 3 Prompt RAdiATION Fields Due to Electrons}

$3.24 \times 10^{-5}$ watts goes into the bremsstrahlung at that location. Typical beam shutters might be made of tungsten. For this material Fig. 3.5 and Table 3.1 give a total photoneutron yield of $2.36 \times 10^{9}$ neutrons s ${ }^{-1} \mathrm{~W}^{-1}$, so $7.65 \times 10^{4}$ neutrons s${ }^{-1}$ will be emitted if this bremsstrahlung power is incident. Since the photoneutrons are isotropic and "point source" conditions are a good approximation, a flux density of 0.61 neutrons $\mathrm{cm}^{-2} \mathrm{~s}^{-1}$ is found at a distance of 1.0 meter without including the effects of any intervening shielding. For these giant resonance neutrons a conservative dose equivalent per fluence value is $3.2 \times 10^{-4} \mu \mathrm{Sv} \mathrm{cm}{ }^{2}$. When applied here, a dose equivalent rate of $d H / d t=1.95 \times 10^{-4}$ $\mu \mathrm{Sv} \mathrm{s}^{-1}=0.70 \mu \mathrm{Svh}^{-1}$ is calculated. Neutrons from quasi-deuteron and photopion reactions are ignored in this estimate. According to Liu and Vylet (Li01), these values are in reasonable agreement with measurement and more sophisticated calculations.

Liu et al. (Li95) have given more detailed results for neutron production by gas bremsstrahlung incident on various materials provided in Fig. 3.24. The left frame of this figure presents both the normalized neutron dose rates at 1.0 meter from the target and the neutron yield with the target as a function of target atomic number. These results are for targets with sufficient size $\left(\approx 30 X_{o}\right.$ long and $30 X_{m}$ in diameter $)$ to generate maximal neutron yields. The right frame of this figure can be used to estimate results for shorter targets made of lead.

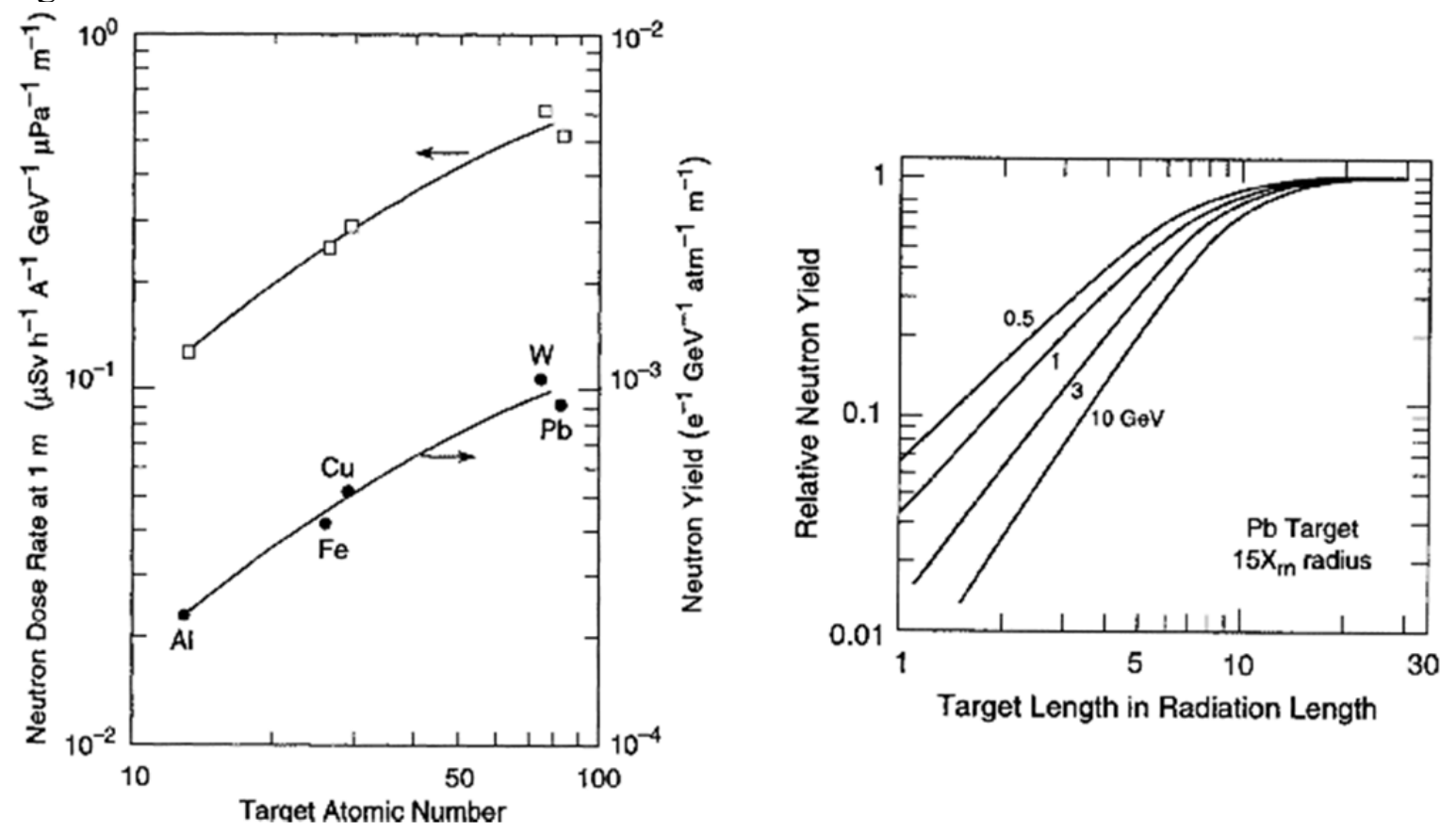

Fig. 3.24 Left frame: Neutron dose rate at 1.0 meter away from a beamline device struck by gas bremsstrahlung and the neutron yield within the device as a function of the atomic number of the device. The values are normalized to a beam current of $1.0 \mathrm{~A}$, per GeV beam energy, and an air path of 1.0 meter with a pressure of $1.0 \mu \mathrm{PA}$. Right frame: Relative neutron yield as a function of the target length in units of radiation length for a cylindrical lead target 15 Molière units in radius struck by gas bremsstrahlung for four electron beam energies. [Adapted from (Li95).] 


\section{ChAPTER 3 PRompt RAdiation Fields Due to ElECTRONS}

\subsubsection{Importance of Ray Tracing}

As a final word, given the plethora of beamlines, penetrations, and devices mounted both within a light source storage ring and associated with its beam lines and experiments, it is generally important to do careful ray tracing studies for both the ring shielding and beamline shielding design to be sure that secondary radiation from electron losses in the ring (normal and abnormal) as well as synchrotron radiation, gas bremsstrahlung "beams", and photoneutrons in beamlines are effectively prevented from reaching undesired locations. All operational modes including injection events, top-offs, and storage ring runs need to be considered. One must be sure that no "holes" are available that could result in significant radiation exposures. Fig. 3.25 shows the complexity of a typical configuration, clearly illustrating why such ray tracing should be done. It is evident that all three spatial dimensions must be considered.

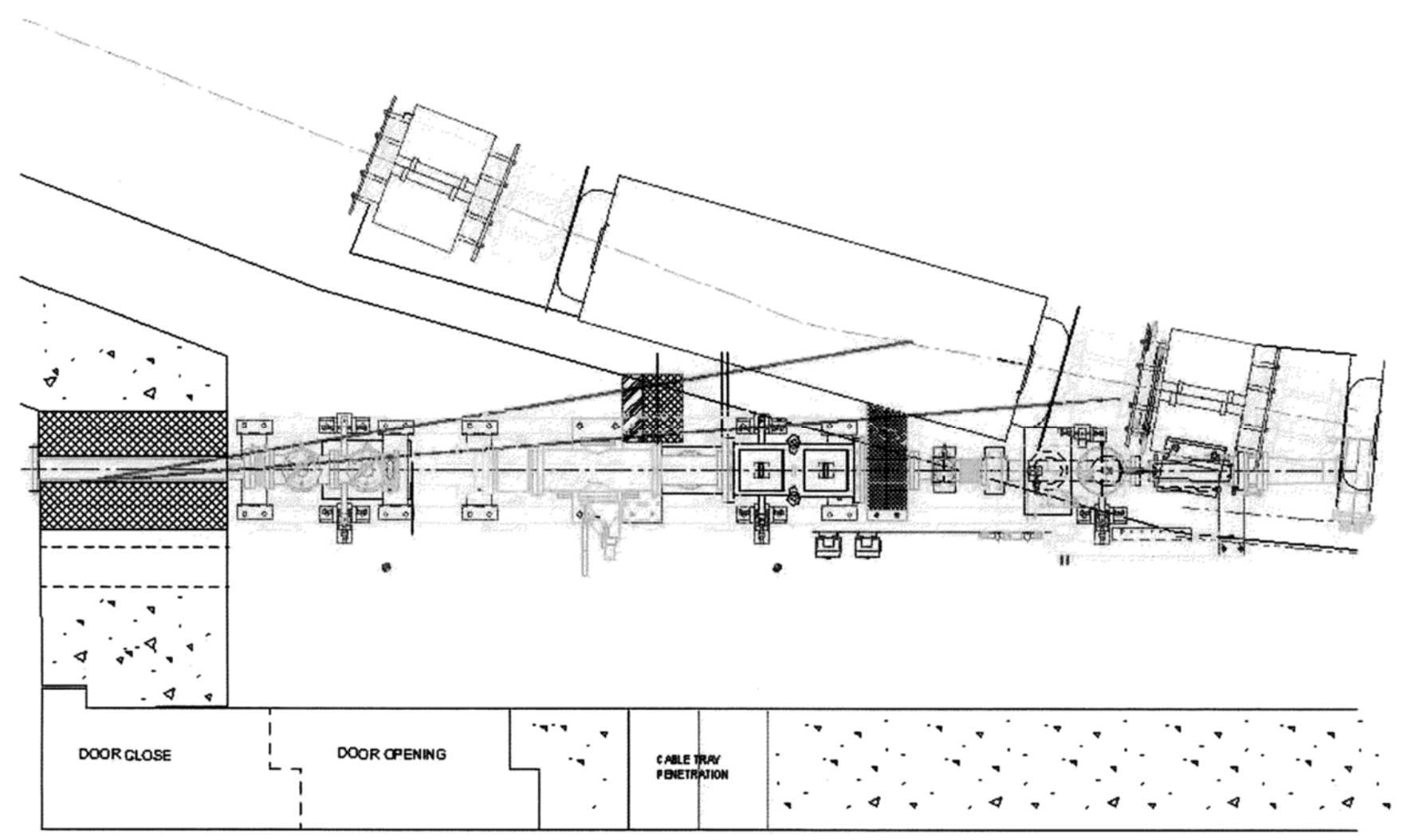

Fig. 3.25 Example of the importance of ray tracing in synchrotron radiation source experimental area. [J. Liu and V. Vylet, private communication.] 


\section{ChAPTER 3 PRompt RAdiATION Fields DUE To EleCtRons}

\section{Problems}

1. An electron accelerator has a beam profile in the form of a $2.0 \mathrm{~mm}$ diameter circle uniformly illuminated by the beam. Make a crude plot of the value of the dose equivalent rate in the beam as the energy increases from $1.0 \mathrm{MeV}$ to $10 \mathrm{GeV}$. The average beam current is 1.0 microamp $(1.0 \mu \mathrm{A})$. Assume the beam profile is unchanged during acceleration. Compare the results with Swanson's simple formula, said to be a "conservative" value. Is his formula "conservative" above $100 \mathrm{MeV}$ ? (Hint: Use Fig. 1.4.)

2. Calculate for electrons the critical energy and length of material that corresponds to the radiation length for carbon and for lead. What does this say about the effectiveness of low- $Z$ versus high- $Z$ shielding materials for electrons?

3. A $100 \mathrm{MeV}$ electron accelerator produces a $1.0 \mu \mathrm{A}$ beam incident on a high- $Z$ (thick) target. Estimate the bremsstrahlung absorbed dose rates at $\theta=0^{\circ}$ and $90^{\circ}$ at $r=2 \mathrm{~m}$ from the target using Swanson's rules of thumb. Compare the $0^{\circ}$ result with the "in-the-beam dose equivalent rate" found in Problem 1. How do the bremsstrahlung and in-beam dose rates compare?

4. Suppose the Tevatron enclosure at Fermilab is used to house an electron synchrotron. The radius of the synchrotron is $1000 \mathrm{~m}$. If the circulating beam is $10^{12}$ electrons, calculate the median energy of the synchrotron radiation photons for $\mathrm{E}_{0}=100 \mathrm{GeV}$. Also find $\theta_{c}$ of the "lobe."

5. For the accelerator of Problem 3, calculate the neutron flux density at $r=2.0 \mathrm{~m}$ from giant resonance neutrons at large angles using the values in Table 3.1 for a high-Z (tungsten) target. Also use Table 3.1 to estimate the dose equivalent rate at $r=2.0 \mathrm{~m}$. Check this result by "guessing" that the average neutron energy is between 1.0 and $10 \mathrm{MeV}$ and use the curve in Fig. 1.5. Compare this neutron dose with the bremsstrahlung dose at large angles obtained in Problem 3.

6. For a $20 \mathrm{GeV}$ electron accelerator operating at $1.0 \mathrm{~kW}$, the electron beam strikes a beam stop made of aluminum or iron. How long (in $z$ ) does the beam stop have to be to range out all of the muons for either aluminum or iron based on the mean range? Compare the dose equivalent rates at the immediate downstream ends of each material if $10 \%$ of the muons leak through due to straggling and multiple Coulomb scattering can be neglected. (Assume the production of muons from iron is approximately equal to that from Al. Recall the inverse square law.) 


\section{ChAPTER 3 PRompt RAdiATION FieldS Due to ELECTRONS}

7. In the discussion of the longitudinal development of electromagnetic showers, there are three different formulations (Rossi-Griesen, Bathow, and Van Ginneken). Using Van Ginneken's scaling method, calculate the value of the parameter $\zeta\left(\mathrm{g} \mathrm{cm}^{-2}\right)$ for $E_{O}=1000 \mathrm{MeV}, 10 \mathrm{GeV}$, and $100 \mathrm{GeV}$ for copper and lead. Determine the number of radiation lengths to which $\zeta$, corresponds for each material at each energy.

8. Compare the results of Van Ginneken for the location of the longitudinal shower maximum with Bathow's result for copper and lead at the three energies given in Problem 7 for incident electrons. Is the agreement better or worse as the energy increases?

9. A hypothetical electron accelerator operates at either $100 \mathrm{MeV}$ or $10 \mathrm{GeV}$ and delivers a beam current of $1.0 \mu \mathrm{A}$. Using Table 3.2, calculate the dose equivalent rates in both $\mathrm{Sv} \mathrm{s}^{-1}$ and $\mathrm{rem} \mathrm{h}^{-1}$ at the end of a $300 \mathrm{~cm}$ long aluminum beam stop; averaged over a $15 \mathrm{~cm}$ radius that are due to bremsstrahlung. (The beam stop is a cylinder much larger than $15 \mathrm{~cm}$ in radius.) Then assume that, in order to save space, a high- $Z$ beam stop is substituted. How long of a high- $Z$ beam stop is needed to achieve the same dose rates? (Assume lead is a suitable high- $Z$ material.) Why is the length of high- $Z$ shield different for the two energies? In this problem, assume the values in Table 3.2 are valid for energies as low as $0.1 \mathrm{GeV}$.

10. In the accelerator and beam stop of Problem 9, if the radius of the beam stop is 30 $\mathrm{cm}$, what is the maximum dose equivalent rate $\left(\mathrm{Sv} \mathrm{s}^{-1}\right.$ and $\left.\mathrm{rem} \mathrm{h}^{-1}\right)$ on the lateral surface (at contact at $r=30 \mathrm{~cm}$ ) of the beam stop for both energies, $100 \mathrm{MeV}$ and $10 \mathrm{GeV}$, and both materials? Again assume approximate validity at $100 \mathrm{MeV}$ of the results.

11. Calculate the dose equivalent rate due to neutrons outside a 1.0 meter thick concrete shield surrounding a cylindrical tunnel (inner radius 1 meter) in which is located a copper target stuck by $1.0 \mu \mathrm{A}$ beam of $100 \mathrm{GeV}$ electrons. The geometry should be assumed to be optimized for producing giant resonance photoneutrons and the calculations should be performed at $\theta=30,60$ and $90^{\circ}$ ( $\rho=2.5 \mathrm{~g} \mathrm{~cm}^{-3}$ for concrete.). Express the result as $\mathrm{Sv} \mathrm{s}^{-1}$ and $\mathrm{rem} \mathrm{h}^{-1}$. For $\theta=90^{\circ}$, use Eq. (3.36) as a check. 


\section{Chapter 4 Prompt Radiation Fields Due to Protons and Ions}

\subsection{Introduction}

In this chapter the major features of development of prompt radiation fields and the shielding of these fields as they are produced at proton and ion accelerators are addressed. Emphasis is placed on the shielding of neutrons in view of their general dominance of the radiation fields. The shielding against muons at such accelerators is also covered. Methods for utilizing the results of both semi-empirical and Monte Carlo methods in the solution of practical shielding problems are presented.

\subsection{Radiation Production by Proton Accelerators}

\subsubsection{The Direct Beam; Radiation Hazards and Nuclear Interactions}

Measured by the connection of dose with fluence, the direct beams at proton accelerators nearly always present a greater hazard than do any secondary beams since the primary beam intensities are generally larger and their cross-sectional areas smaller. Fig. 1.4 gives the dose equivalent per fluence as a function of proton energy. This quantity has a prominent transition at about $200 \mathrm{MeV}$. Below that energy the proton range in tissue is less than the typical thickness of the human body. Those protons thus range out in the body and deposit nearly all of their energy in its tissues. Above $200 \mathrm{MeV}$, the fraction of the proton's energy that escapes from the body gets larger, with less dose thus delivered.

The ionization range of a proton increases monotonically with energy. Since the mass of the proton is so much larger than that of the electron, the radiative processes of bremsstrahlung and synchrotron radiation are usually negligible at current accelerators. As will be discussed in Section 4.5, at high energies the cross sections for inelastic interactions become nearly independent of energy with approximately the values tabulated in Table 1.2. Thus, as an individual proton passes through a material medium, the probability of it participating in an inelastic nuclear reaction before it loses its remaining energy to ionization becomes significant and, as the energy increases, the dominant means by which protons are absorbed. Tesch has summarized this effect and the results are shown in Fig. 4.1 for various materials and energies (Te85).

\subsubsection{Neutrons and Other Hadrons at High Energies}

The production and behavior of neutrons at proton and ion accelerators have different characteristics as the energy of the beam particles $E_{\mathrm{o}}$ is increased. Phenomena in a sequence of domains of proton energy of approximate boundaries will be discussed.

\subsubsection{1 $E_{o}<10 \mathrm{MeV}$}

For a nuclear reaction the Q-value $Q_{v}$ is the energy released by the reactions and is defined in terms of the rest masses $m_{i}$ of the participating particles or ions as

$$
Q_{v}=\left[\left(m_{1}+m_{2}\right)-\left(m_{3}+m_{4}\right)\right] c^{2},
$$




\section{Chapter 4 Prompt Radiation Fields Due to Protons AND Ions}

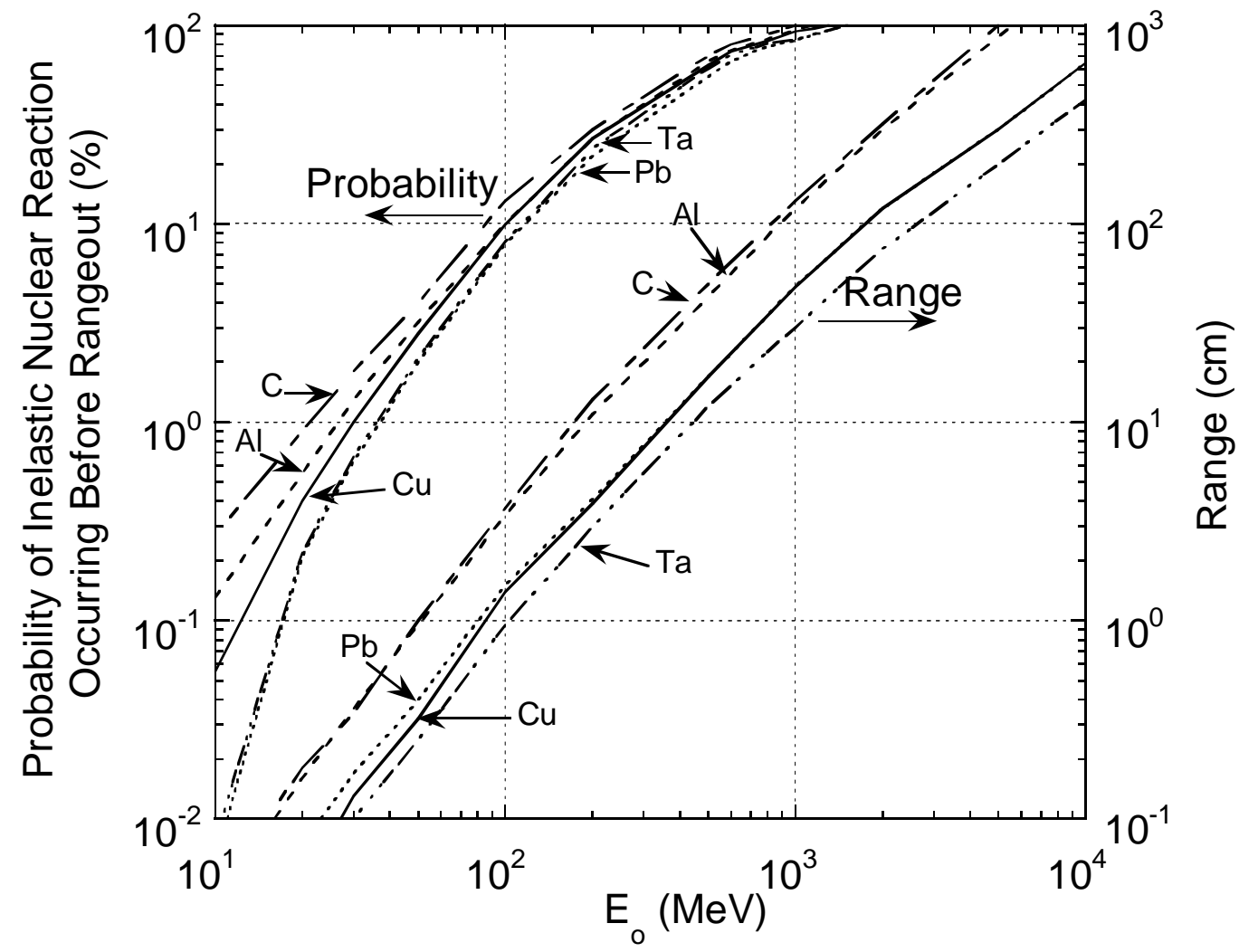

Fig. 4.1 Ionization range of protons (curves on right and right hand scale) and probability of inelastic nuclear interaction within the range (curves on left and left hand scale) for various materials [Adapted from (Te85).]

for the nuclear reaction: $m_{1}+m_{2} \rightarrow m_{3}+m_{4} \cdot{ }^{13}$ Conventionally, the projectile is represented by $m_{1}$ while generally the less massive emitted particle is represented by $m_{3}$. A value of $Q_{\nu}>0$ implies an exothermic nuclear reaction. Endothermic $\left(Q_{\nu} \leq 0\right)$ reactions are characterized by a nuclear reaction threshold energy $E_{t h}$ given by

$$
E_{t h}=\frac{m_{1}+m_{2}}{m_{2}}\left|Q_{v}\right|
$$

Below a kinetic energy of about $10 \mathrm{MeV},(\mathrm{p}, \mathrm{n})$ reactions are important for some materials because these reactions commonly have very low threshold energies $\left(E_{t h}<5 \mathrm{MeV}\right)$. For example, the reaction ${ }^{7} \mathrm{Li}(\mathrm{p}, \mathrm{n})^{7} \mathrm{Be}$ has a threshold energy of $1.9 \mathrm{MeV}$ and a reaction cross section $\sigma$ that quickly rises as a function of energy to a value of about $300 \mathrm{mb}$.

The cross sections of low energy nuclear reactions are highly reflective of the details of the structure of the target nuclei including its excited states. This results in dependencies on target material, scattering angle, and energy.

${ }^{13}$ A more compact notation commonly used for the same nuclear reaction is $m_{2}\left(m_{1}, m_{3}\right) m_{4}$, such as ${ }^{12} \mathrm{C}(\mathrm{p}, \mathrm{n}){ }^{12} \mathrm{~N}$ in place of $\mathrm{p}+{ }^{12} \mathrm{C} \rightarrow \mathrm{n}+{ }^{12} \mathrm{~N}$. 


\section{Chapter 4 Prompt RAdiation Fields Due to Protons AND Ions}

\subsubsection{2 $10<E_{o}<200 \mathrm{MeV}$}

For protons having kinetic energies of this magnitude and higher, neutrons are usually the dominant feature of the radiation fields resulting from the interactions of the protons. In this energy domain, the neutron yields are smoother functions of energy than was the case at lower energies, but are also more forward-peaked. For this domain Tesch (Te85) has summarized the total neutron yields $Y$ per incident proton for different materials as a function of energy in Fig. 4.2. In this figure the smooth curves agree with the original primary data obtained from a myriad of experiments to within about a factor of two. An important feature is that for low energies, $Y \propto E_{o}^{2}$ while for $E_{O}>1.0 \mathrm{GeV}, Y \propto E_{O}$. Especially at lower energies, many so-called evaporation neutrons are produced. These neutrons can be viewed as "boiling" off of a nucleus that has been "heated" by absorption of energy from the incident particle. They consequently have an isotropic spatial distribution. Other neutrons that are produced are cascade neutrons that result directly from individual nuclear reactions. In contrast to the evaporation neutrons, cascade neutrons are likely to have a directionality that is at least mildly "forward-peaked". In this region there is much angular distribution data available from nuclear physics research. Representative examples are given in Figs. 4.3 and 4.4 for 52 and $200 \mathrm{MeV}$ protons, respectively. Additional examples of are found in reference (NC03).

\subsubsection{3 $200 \mathrm{MeV}<E_{o}<1.0 \mathrm{GeV}$; ("Intermediate" Energy)}

In this region, many more reactions become energetically available. Also, the number of protons emitted gradually becomes approximately equal to the number of neutrons produced. In fact, at the highest energies the radiation effects of protons and neutrons are essentially identical. Both must be taken into account. Thus reliance on the values shown in Fig. 4.2 could underestimate radiation effects by as much as a factor of two. Also, at these energies, cascade neutrons become much more important than evaporation neutrons. Thus the radiation field is more sharply forward-peaked with increasing primary particle energy.

\subsubsection{4 $E_{O}>1.0 \mathrm{GeV}(" H i g h "$ Energy Region $)$}

In this domain the situation is more complex. Figs. 4.5, 4.6, 4.7, and 4.8 show representative data at $14,26,22$, and $225 \mathrm{GeV} .{ }^{14}$ These results should be regarded as thin target values. "Thin" target in this context means a target shorter than the mean free path for removal of the high energy protons. Table 4.1 summarizes common removal mean free paths based upon the nuclear collision lengths of Table 1.2.

The yield $Y$ or fluence $\Phi$ is often normalized either to the number of protons incident on a given target $N_{o}$ or to the number of interacting protons $N_{\text {int }}$. In a target of thickness $x$ and mean free path $\lambda, N_{\text {int }}=N_{o}[1-\exp (-x / \lambda)]$ so that $Y / N_{o}=\left\{Y / N_{\text {int }}\right\}[1-\exp (-x / \lambda)]$.

\footnotetext{
${ }^{14}$ Much of the experimental data presented in this and other sections related to high energy interactions were obtained using activation "threshold" detectors, a technique discussed further in Section 9.5.3.
} 


\section{Chapter 4 Prompt Radiation Fields Due to Protons ANd Ions}

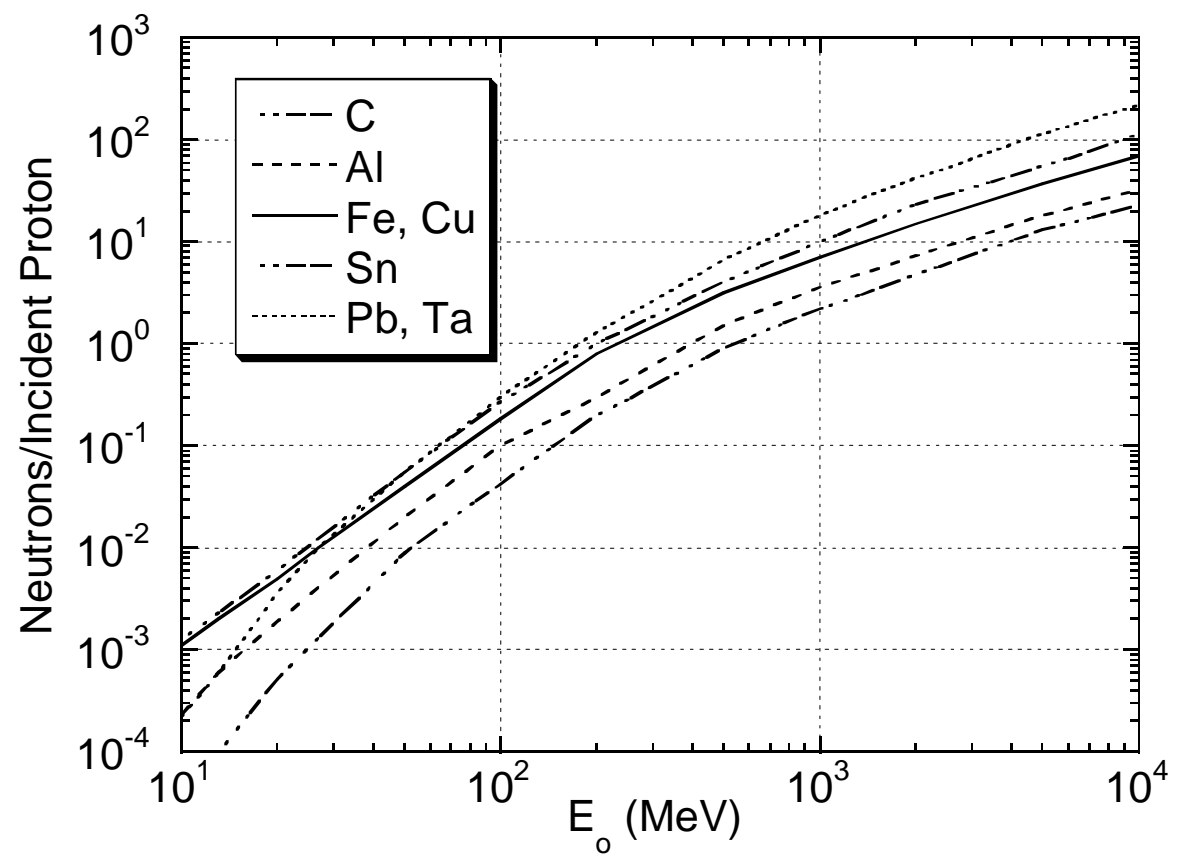

Fig. 4.2 Total neutron yield per proton for different target materials as a function of incident proton energy $E_{0}$. These values apply to relatively thick targets and include some degree of shower development. [Adapted from (Te85).]

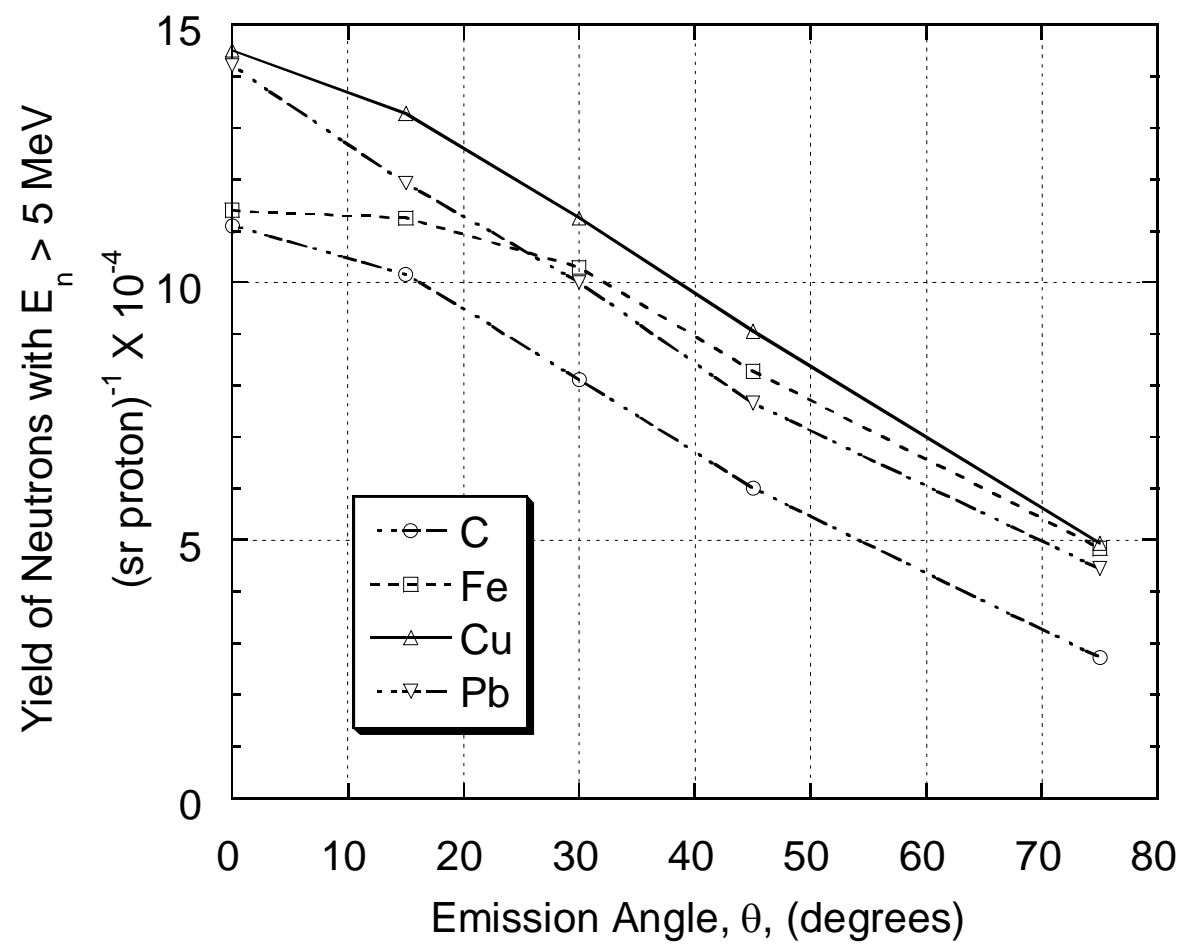

Fig. 4.3 Measured angular distributions of total neutron yield above $5.0 \mathrm{MeV}$ for carbon, iron, copper, and lead bombarded by $52 \mathrm{MeV}$ protons. The measurements were normalized at $\theta=15^{\circ}$. The curves are drawn to guide the eye. [Adapted from (Na78).] 


\section{Chapter 4 Prompt Radiation Fields Due to Protons and Ions}

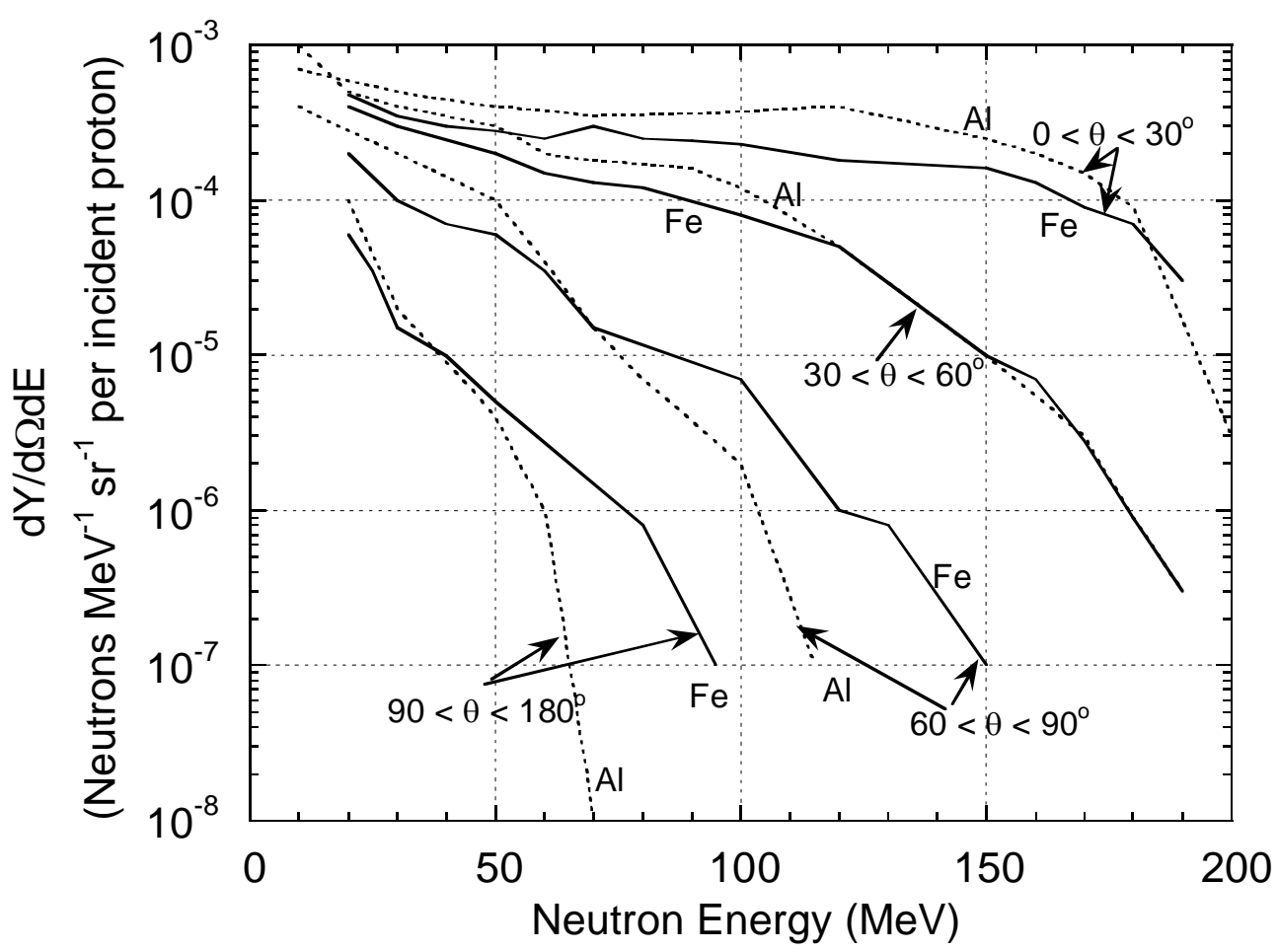

Fig. 4.4 Calculated energy spectra of neutrons emitted by iron and aluminum targets bombarded by $200 \mathrm{MeV}$ protons for four ranges of the value of $\theta$. The results for iron are from (Ha88) while those for aluminum are from (A175). [Adapted from (Ha88).]

Considerable efforts have been made to semi-empirically fit the distributions of the yields of secondary particles produced by proton interactions. These were done both to supply the needs of the particle physics community and address radiation safety issues. They began in the early days of radiation protection and the results were embodied in the continual development of Monte Carlo programs designed to calculate the properties of hadronic cascades as discussed in Section 4.6. An example of a particularly successful early model is one developed by Ranft (Ra67) expressed as the following formula for the yield of protons or neutrons of momentum $p(\mathrm{GeV} / \mathrm{c})$ :

$$
\begin{gathered}
\frac{d^{2} Y}{d \Omega d p}=\left\{\frac{A}{p_{o}}+\frac{B p}{p_{o}^{2}}\left[1+f\left(p_{o}\right)\left\{1-\frac{p_{o}}{p}\right\}\right]\right\} \times\left\{1+f\left(p_{o}\right)\left[1-\frac{p p_{o}}{m_{p}^{2}}\right]\right\} p^{2} \exp \left(-C p^{2} \theta\right) \\
\text { [protons or neutrons sr } \mathrm{sr}^{-1}(\mathrm{GeV} / \mathrm{c})^{-1} \text { per interacting proton] }
\end{gathered}
$$

where

$p_{o}$ is the primary proton momentum $(\mathrm{GeV} / \mathrm{c})$,

$m_{p}$ is the proton rest energy $\left(\mathrm{GeV} / \mathrm{c}^{2}\right)$,

$f\left(p_{0}\right)=\left\{1+\left(p_{o} / m_{p}\right)^{2}\right\}^{1 / 2}$, and

$\theta$ is the production angle (radians). 


\section{Chapter 4 Prompt Radiation Fields Due to Protons and Ions}

The parameters $A, B$, and $C$ of the Ranft model are material-dependent. Selected values are given in Table 4.2. It is commonly convenient to express yields in terms of interaction particles rather than incident particles in view of Eq. (1.4). According to Patterson and Thomas (Pa73), when Eq. (4.3) is numerically integrated above the indicated energy threshold, it describes well the experimental data presented in Figs. 4.7 and 4.8. Fig. 4.7 gives results normalized to the number of interacting particles while Fig. 4.8 present results normalized to the number of particles incident on a particular target.

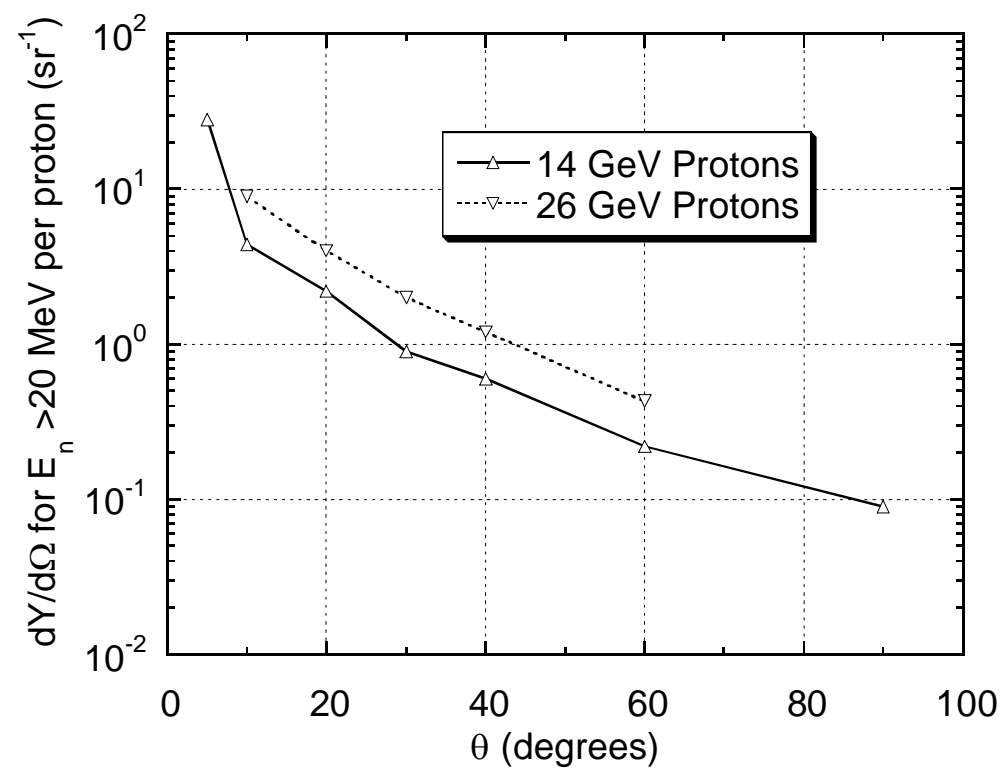

Fig. 4.5 Measurements of the angular distribution $d Y / d \Omega$ of neutrons above $20 \mathrm{MeV}$ produced by 14 and $26 \mathrm{GeV}$ protons incident on a thin beryllium target. The yield is per interacting proton. The lines are drawn to guide the eye. [Adapted from (Gi68).]

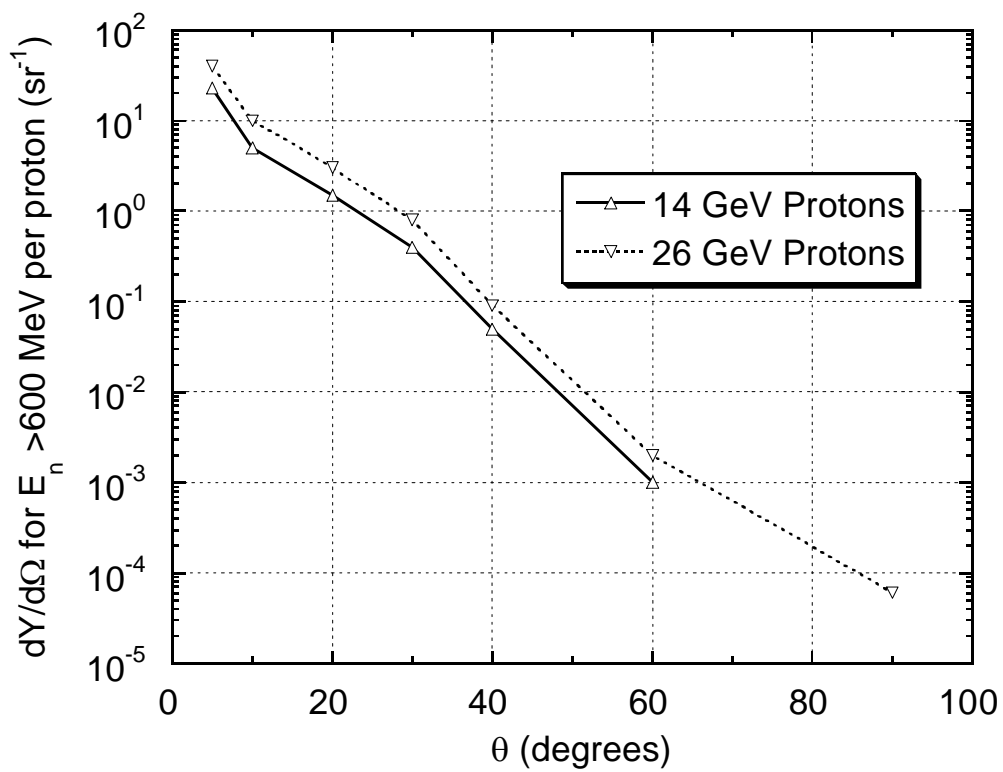

Fig. 4.6 Measurements of the angular distribution $d Y / d \Omega$ of neutrons above $600 \mathrm{MeV}$ produced by 14 and $26 \mathrm{GeV}$ protons incident on a thin beryllium target. The yield is per interacting proton. The lines are drawn to guide the eye [Adapted from (Gi68).] 


\section{Chapter 4 Prompt Radiation Fields Due to Protons ANd Ions}

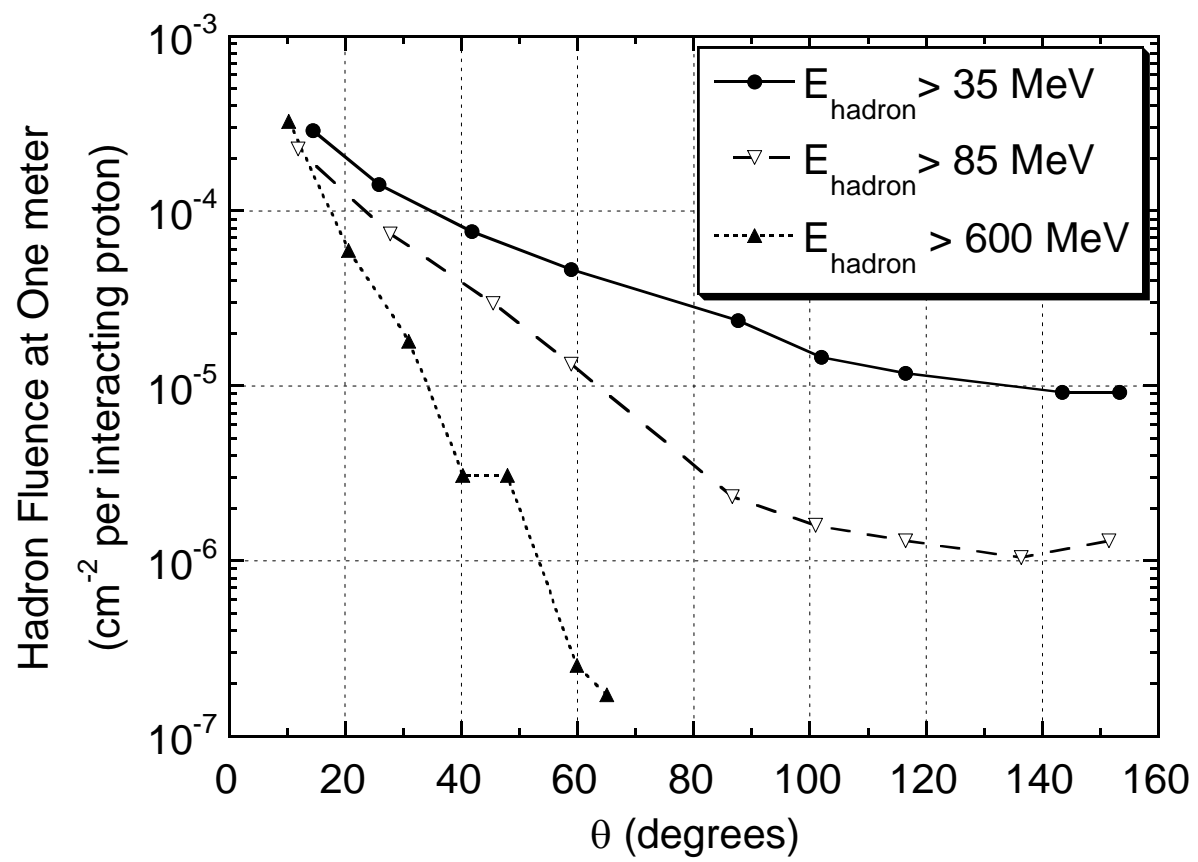

Fig. 4.7 Measured angular distributions of hadron fluence (particles $\mathrm{cm}^{-2}$ ) at 1.0 meter from a copper target bombarded by $22 \mathrm{GeV}$ protons. Several choices of hadron energy thresholds are shown. The measured fluence is normalized to the number of interacting protons. [Adapted from (Ra72).]

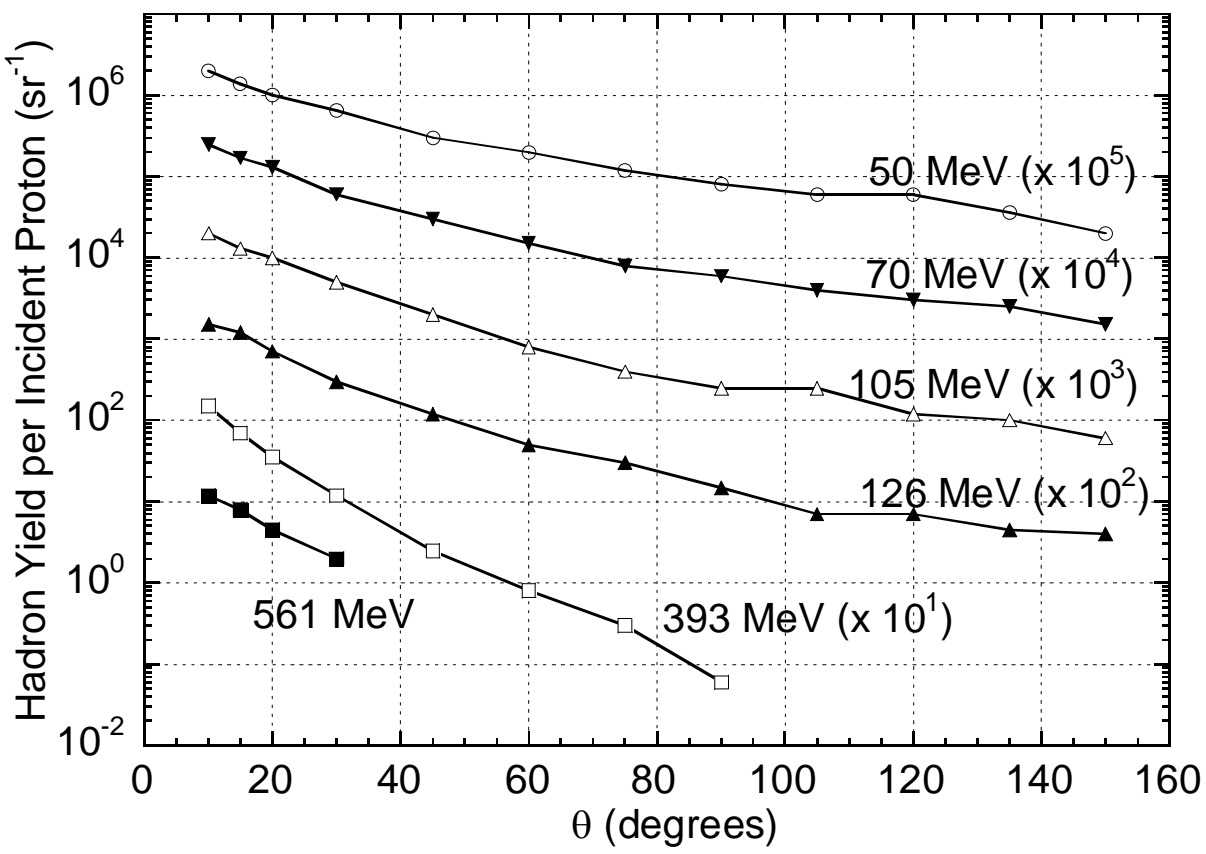

Fig. 4.8 Measurements of hadron yields above different energy thresholds as a function of production angle $\theta$ around a $15 \mathrm{~cm}$ long copper target bombarded by $225 \mathrm{GeV}$ protons. The data have been multiplied by the indicated powers of 10 prior to plotting. The lines are intended to guide the eye. The measured yield is normalized to the number of protons incident on this particular target. [Adapted from (St85).] 
Chapter 4 Prompt RAdiation Fields Due to Protons AND IoNS

Table 4.1 Summary of removal mean free paths for high energy protons. [Adapted from Table 1.2.]

\begin{tabular}{|l|c|c|c|}
\hline Material & Density & $\begin{array}{c}\text { Removal Mean } \\
\text { Free Path } \\
\text { (grams } \mathbf{~ c m}^{-2} \text { ) }\end{array}$ & $\begin{array}{c}\text { Removal Mean } \\
\text { Free Path } \\
\text { (cm) }\end{array}$ \\
\hline hydrogen gas @ STP & $9.00 \times 10^{-5}$ & 43.3 & $4.81 \times 10^{5}$ \\
\hline beryllium & 1.85 & 55.8 & 30.16 \\
\hline carbon & 2.27 & 60.2 & 26.58 \\
\hline aluminum & 2.70 & 70.6 & 26.15 \\
\hline iron & 7.87 & 82.8 & 10.52 \\
\hline copper & 8.96 & 85.6 & 9.55 \\
\hline lead & 11.35 & 116.2 & 10.24 \\
\hline uranium & 18.95 & 117.0 & 6.17 \\
\hline air @ STP & $1.29 \times 10^{-3}$ & 62.0 & $4.81 \times 10^{4}$ \\
\hline water & 1.00 & 60.1 & 60.10 \\
\hline concrete (typical) & 2.50 & 67.4 & 26.96 \\
\hline silicon dioxide (quartz) & 2.64 & 66.5 & 25.19 \\
\hline plastics (polyethylene) & 0.93 & 56.9 & 61.29 \\
\hline
\end{tabular}

Table 4.2 Material-dependent parameters to be used in Eq. (4.3). [From (Ra67).]

\begin{tabular}{|l|l|l|l|}
\hline Target & $\boldsymbol{A}$ & $\boldsymbol{B}$ & $\boldsymbol{C}$ \\
\hline $\mathrm{H}_{2}$ & 0.55 & -0.30 & 2.68 \\
\hline $\mathrm{Be}$ & 0.68 & -0.39 & 3.12 \\
\hline $\mathrm{Fe}$ & 0.92 & -0.75 & 2.90 \\
\hline $\mathrm{Pb}$ & 1.14 & -1.06 & 2.73 \\
\hline
\end{tabular}

\section{$\underline{4.2 .3 \quad \text { Sullivan's Formula }}$}

For simple radiation protection estimates, Sullivan [(Su89), (Su92)] has developed a formula for the fluence $\Phi(\theta)$ of hadrons with $E_{O}>40 \mathrm{MeV}$ that will be produced at 1.0 meter from a copper target struck by protons in the energy region $5<E_{o}<500 \mathrm{GeV}$ per interacting proton;

$$
\Phi(\theta)=\frac{1}{2\left[\theta+\left(\frac{35}{\sqrt{E_{0}}}\right)\right]^{2}} \quad\left(\mathrm{~cm}^{-2} \text { per interacting proton }\right),
$$

where $E_{o}$ is in $\mathrm{GeV}$ and $\theta$ is in degrees. At proton energies below $2 \mathrm{GeV}$, this formula also approximately accounts for the distributions of all neutrons per interacting proton. This equation is plotted in Fig. 4.9, for "lateral" $\left(\theta \approx 90^{\circ}\right)$ and "forward" $\left(\theta \approx 0^{\circ}\right)$ directions. 


\section{Chapter 4 Prompt Radiation Fields Due to Protons AND Ions}

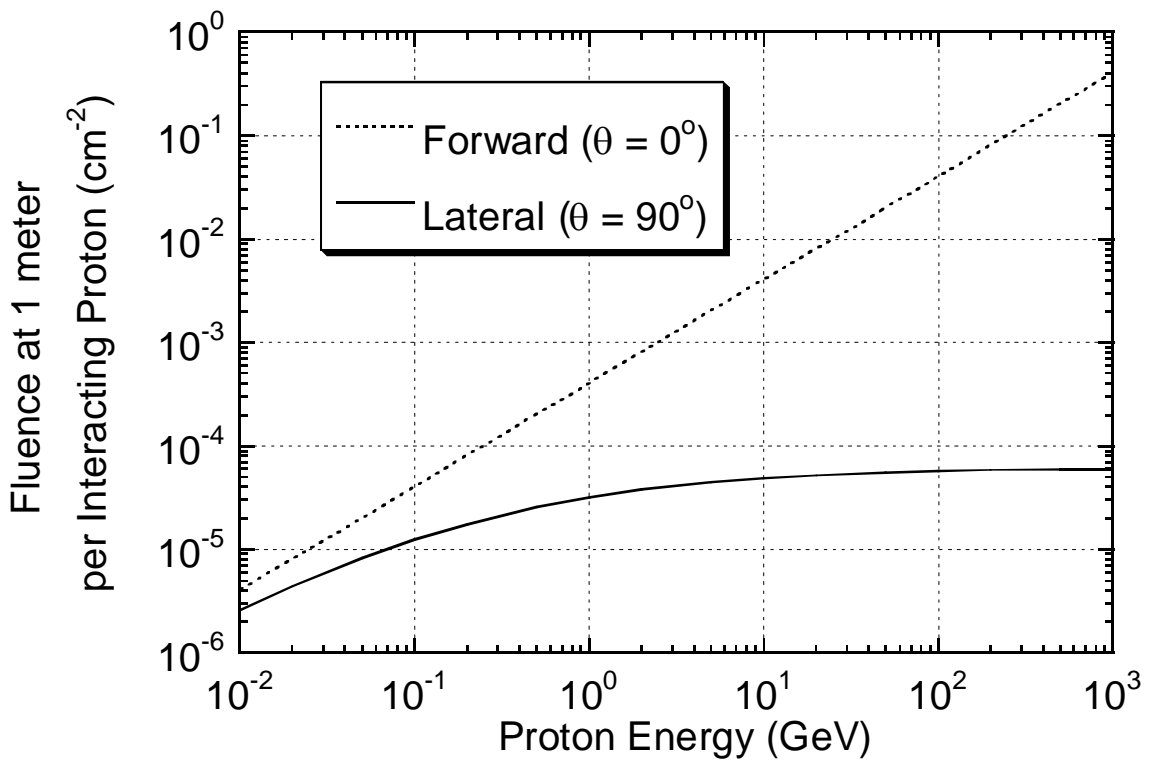

Fig. 4.9 Plot of Eq. (4.4) for two different values of $\theta$. The proton is interacting in a copper target. [Adapted from $(\mathrm{Su} 89)$.]

Of course, the dose equivalent is more directly germane to radiation protection than is the fluence. In principle, the dose equivalent can be obtained by integrating the product of the fluence and the dose equivalent or effective dose per fluence $P(E)$ over the spectrum (see Section 1.3.3);

$$
H=\int_{0}^{E_{\max }} d E P(E) \Phi(E)
$$

or by discrete summation, taking into account the "coarseness" of available data and/or calculations;

$$
H=\sum_{j=1}^{m} \Delta_{j}(E) P_{j}(E) \Phi_{j}(E)
$$

Tesch (Te85) has done this obtaining the dose equivalent at $\theta=0^{\circ}$ and 1.0 meter from a copper target bombarded by protons of various energies. The result is plotted in Fig. 4.10. Above about $1.0 \mathrm{GeV}$ the dose equivalent is approximately proportional to $E_{\mathrm{o}}$. Levine (Le72) has measured the angular distribution of absorbed dose for 8.0 and $24 \mathrm{GeV} / \mathrm{c}$ protons incident on a $\mathrm{Cu}$ target. Results are in approximate agreement with those of Tesch.

\section{$\underline{4.2 .4 \quad \text { Muons }}$}

At proton accelerators muons arise from two principal mechanisms; from pion and kaon decay and from so-called "direct" production. Production by means of pion and kaon 


\section{Chapter 4 Prompt Radiation Fields Due to Protons ANd Ions}

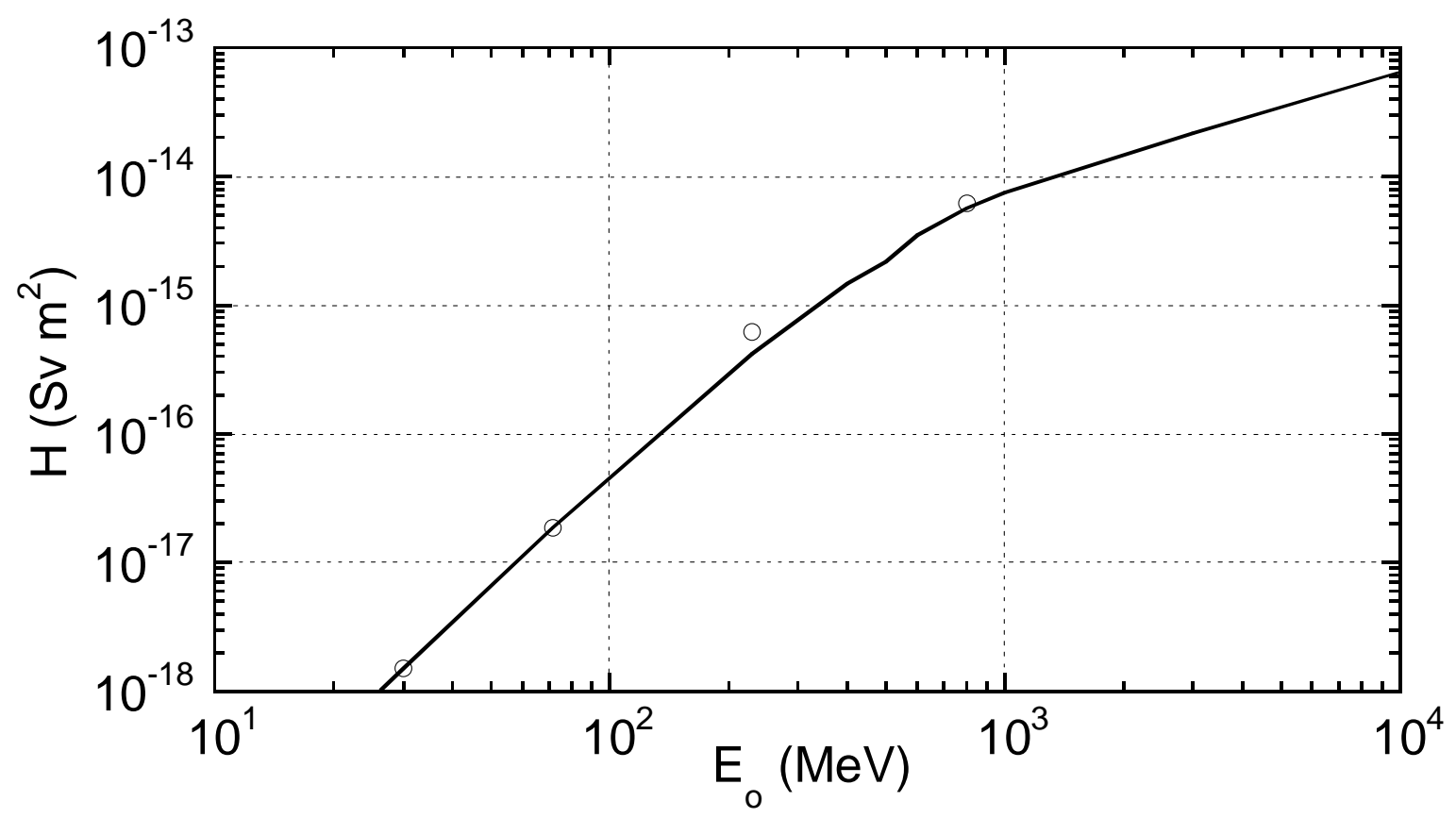

Fig. 4.10 Dose equivalent per proton due to neutrons at $\theta=90^{\circ}$ with energies higher than $8.0 \mathrm{MeV}$ at a distance of 1.0 meter from a copper target. The curve is an interpolation between the experimental measurements denoted by the open symbols. [Adapted from (Te85).]

decay proceeds as follows where masses of the parent particles, the branching fractions (the percentage of time the parent particle decays by the stated reaction), the mean-lives $\tau$, and the product of the speed of life $c$ and the mean-life $c \tau$ are also given (PDG04):

$$
\begin{gathered}
\pi^{ \pm} \rightarrow \mu^{ \pm}+v_{\mu} ; m_{\pi}=139.570 \mathrm{MeV}, \tau=2.6033 \times 10^{-8} \mathrm{~s},(99.99 \% \text { branch }), \\
c \tau=7.8045 \mathrm{~m}, \text { and } \\
K^{ \pm} \rightarrow \mu^{ \pm}+v_{\mu} ; m_{K}=493.677 \mathrm{MeV}, \tau=1.2384 \times 10^{-8} \mathrm{~s},(63.51 \% \text { branch }), \\
c \tau=3.713 \mathrm{~m} .
\end{gathered}
$$

"Direct" muon production, important only at very high energy hadron accelerators, is discussed covered in more detail in Section 4.7.3. Muon radiation fields are forwardpeaked and normally dominated by those from pion decay. Usually, Monte Carlo techniques are needed to accurately estimate muon intensities since one needs to:

- calculate the production of pions from the proton interactions,

- follow the pions until they decay or interact,

- adequately account for the range-energy relation and range straggling, and

- track the muons to the point of interest, for example, through magnetic fields. 


\section{Chapter 4 Prompt RAdiation Fields Due to Protons AND IONS}

\subsection{Primary Radiation Fields at Ion Accelerators}

Most of Section 4.2 has discussed general considerations appropriate for the primary radiation fields generated by accelerated ions as well as to protons. Here special issues found in radiation fields produced by ions other than protons are described. A comprehensive reference on this topic is that of Nakamura and Heilbronn (Na05).

\subsubsection{Light Ions (Ion Mass Number $A<5$ )}

For such ions there are exothermic reactions that should be treated as special cases. Noteworthy examples (followed by their reaction Q-values $Q_{v}$ in parentheses) are;

$$
\begin{array}{ll}
\mathrm{D}(\mathrm{d}, \mathrm{n}){ }^{3} \mathrm{He} & \left(Q_{v}=3.266 \mathrm{MeV}\right), \\
{ }^{9} \mathrm{Be}(\alpha, \mathrm{n}){ }^{12} \mathrm{C} & \left(Q_{v}=5.708 \mathrm{MeV}\right), \text { and } \\
{ }^{3} \mathrm{H}(\mathrm{d}, \mathrm{n}){ }^{4} \mathrm{He} & \left(Q_{v}=17.586 \mathrm{MeV}\right) .
\end{array}
$$

In some cases monoenergetic beams of neutrons can be produced using these or the following slightly endothermic reactions:

$$
\begin{array}{ll}
{ }^{12} \mathrm{C}(\mathrm{d}, \mathrm{n}){ }^{13} \mathrm{~N} & \left(Q_{v}=-0.281 \mathrm{MeV}\right), \\
{ }^{3} \mathrm{H}(\mathrm{p}, \mathrm{n}){ }^{3} \mathrm{He} & \left(Q_{v}=-0.764 \mathrm{MeV}\right), \text { and } \\
{ }^{7} \mathrm{Li}(\mathrm{p}, \mathrm{n}){ }^{7} \mathrm{Be} & \left(Q_{v}=-1.646 \mathrm{MeV}\right) .
\end{array}
$$

The energies of such neutrons can range from 0 to $24 \mathrm{MeV}$ for bombarding energies up to $10 \mathrm{MeV}$. In general, deuteron stripping and breakup reactions (d,n) have the highest yields because the binding energy of the deuteron is only $2.225 \mathrm{MeV}$. In effect, one gets an extra neutron essentially "for free". Furthermore, the neutrons due to deuteron stripping reactions typically have a kinetic energy of about half that of the incident deuteron if the latter has a kinetic energy that is large compared with the binding energy of the target nucleus. This phenomenon is especially pronounced at the lower energies. In the low energy region, and especially with light ions, one should carefully consider all possible reactions given the materials present in conjunction with the ions that are being accelerated. Patterson and Thomas (Pa73) have summarized total neutron yields for light ions. In general, the yields for the various light ions behave similarly to those due to protons. That is, the yield is within, typically, a factor of three of that expected for proton beams. A good measurement of neutron yields due to $40 \mathrm{MeV} \alpha$-particles has been provided by Shin et al. (Sh95). Higher energy neutron production data for 640 and 710 $\mathrm{MeV} \alpha$-particles has been provided by Cecil et al. (Ce80).

\subsubsection{Heavy Ions (Ions with $A>4$ )}

At higher energies and especially at higher masses, neutron yield and dose data and calculations are very sparse. The data are usually normalized in terms of kinetic energy per atomic mass unit, the specific energy, expressed in units of $\mathrm{MeV}$ atomic mass unit ${ }^{-1}$ 


\section{Chapter 4 Prompt Radiation Fields Due to Protons and Ions}

(energy $\mathrm{amu}^{-1}$ ) or energy nucleon ${ }^{-1}$ because of some scaling of reaction parameters with that parameter. Often the small distinction between energy $\mathrm{amu}^{-1}$ and energy nucleon ${ }^{-1}$ is ignored. Up to $20 \mathrm{MeV}$ amu $^{-1}$, Ohnesorge et al. (Oh80) have measured dose equivalent rates at 1.0 meter and $\theta=90^{\circ}$ from thick targets of iron, nickel, or copper bombarded by ${ }^{4} \mathrm{He},{ }^{12} \mathrm{C},{ }^{14} \mathrm{~N},{ }^{16} \mathrm{O}$, and ${ }^{20} \mathrm{Ne}$ beams. The dose was found to be essentially independent of ion type as a function of specific energy. At $10 \mathrm{MeV} \mathrm{amu}{ }^{-1}$, a value of $6.3 \times 10^{-18} \mathrm{~Sv}$ (incident ion) $^{-1}$ was measured while at $20 \mathrm{MeV} \mathrm{amu}^{-1}$, a value of $3.6 \times 10^{-17} \mathrm{~Sv}$ (incident ion $)^{-1}$ was found. Other data relevant to this general energy region are exemplified by those of Hubbard et al. (Hu60), Aleinikov et al. (A185), and especially Nakamura (Na85).

Tuyn et al. (Tu84) reported studies done with $86 \mathrm{MeV} \mathrm{amu}^{-1}{ }^{12} \mathrm{C}$ ions incident on $\mathrm{Fe}$ targets slightly thicker (longer) than an interaction length. The results are shown in Fig. 4.11. At a specific energy of $155 \mathrm{MeV} \mathrm{amu}^{-1}$, Britvitch et al. (Br99) have measured energy spectra and total neutron yields and angular distributions for ${ }^{4} \mathrm{He},{ }^{12} \mathrm{C}$, and ${ }^{16} \mathrm{O}$ ions stopping in a thick target of an alloy of tungsten, nickel, and copper commonly known as "Hevimet". The differential yields, $d Y / d \Omega$, were fit by the form

$$
\frac{d Y}{d \Omega}=C \exp (-\beta \theta)
$$

with the total yields being found by performing the integration,

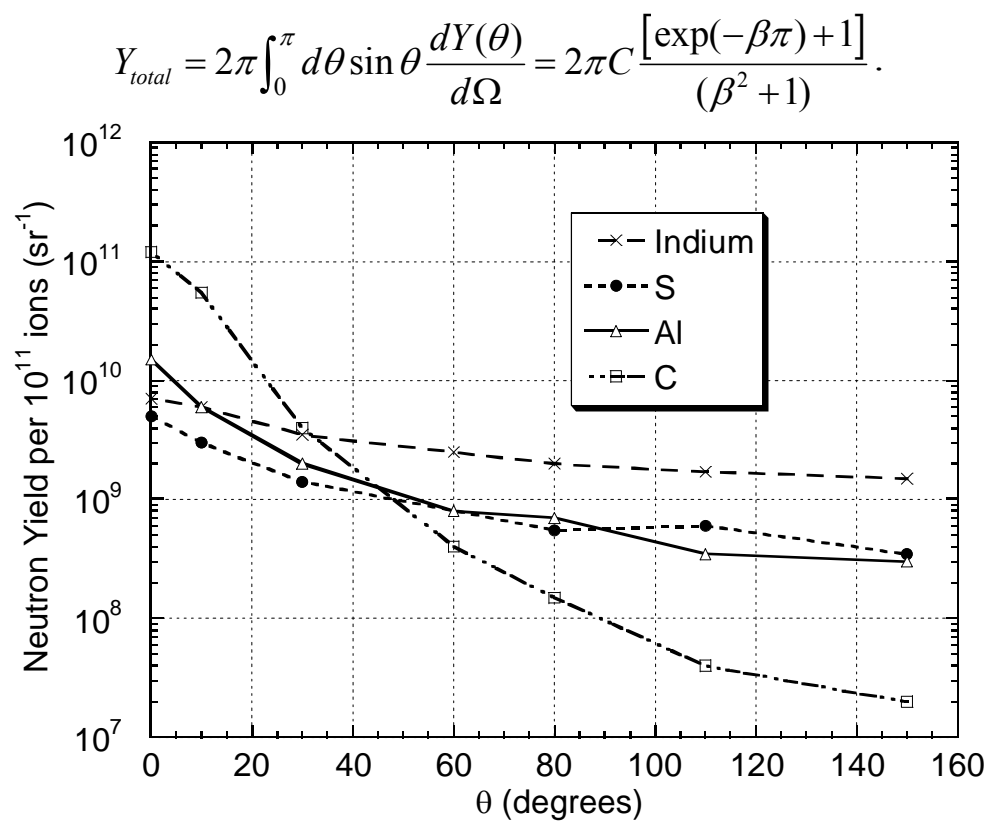

Fig. 4.11 Measured neutron yields for $86 \mathrm{MeV} a m u^{-1}{ }^{12} \mathrm{C}$ ions incident on an iron target. Activation detectors (see Section 9.5.3) with the following sensitive regions in neutron energy $E_{n}$ were used: moderated indium foils ("Indium") $\left(0.4<E_{n}<107 \mathrm{eV}\right),{ }^{32} \mathrm{~S}(\mathrm{n}, \mathrm{p})^{32} \mathrm{P}\left(E_{n}>3.0 \mathrm{MeV}\right)$, ${ }^{27} \mathrm{Al}(\mathrm{n}, \alpha){ }^{24} \mathrm{Na}\left(E_{n}>7.0 \mathrm{MeV}\right)$, and ${ }^{12} \mathrm{C}(\mathrm{n}, 2 \mathrm{n}){ }^{11} \mathrm{C}\left(E_{n}>20 \mathrm{MeV}\right)$. The lines are intended to guide the eye. [Adapted from (Tu84).] 


\section{Chapter 4 Prompt Radiation Fields Due to Protons AND Ions}

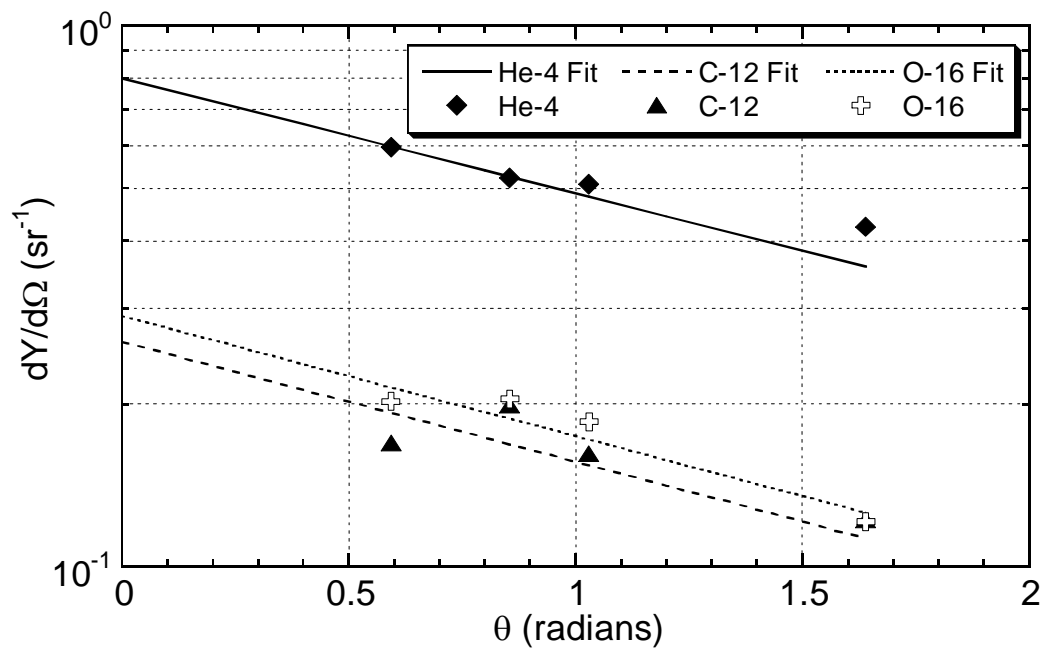

Fig. 4.12 Neutron yields per incident ion for $155 \mathrm{MeV} \mathrm{amu}^{-1}$ ions reported by Britvich et al. (Br99). The diamonds are measurements for ${ }^{4} \mathrm{He}$ which were fitted by parameters $(C$ \{neutrons (incident ion) $)^{-1}$ and $\beta\left\{\operatorname{radian}^{-1}\right\}$ ) of $(0.8,0.49)$ as defined by Eqs. (4.7) and (4.8). The results for ${ }^{12} \mathrm{C}$ are denoted by triangles and were fit by $(C, \beta)$ values of $(0.26,0.51)$. The results for ${ }^{16} \mathrm{O}$ are denoted by crosses and were fit by $(C, \beta)$ values of $(0.29,0.51)$.

The results are presented in Fig. 4.12. The total neutron yields for ${ }^{4} \mathrm{He},{ }^{12} \mathrm{C}$, and ${ }^{16} \mathrm{O}$ were found to be $4.90,1.56$, and 1.74 neutrons per incident ion, respectively. Clapier and Zaidins (C183) have surveyed a sample of ion data from 3 to $86 \mathrm{MeV} a m u^{-1}$ and offer approximations to the total neutron yields and angular distributions over that domain. They found the total yield per ion $Y$ to be given by

$$
\begin{aligned}
& \left.Y(W, Z)=C(Z) W^{\eta(Z)} \text { (neutrons } \text { ion }^{-1}\right) \quad \text { with } \\
& \eta(Z)=1.22 \sqrt{Z} \quad \text { and } \\
& C(Z)=\frac{1.96 \times 10^{-4}}{Z^{2.75}} \exp \left\{-0.475(\ln Z)^{2}\right\},
\end{aligned}
$$

where $Z$ is the atomic number of the projectile and $W$ is the specific energy $\left(\mathrm{MeV} \mathrm{amu}{ }^{-1}\right)$. They found essentially no dependence on the atomic number of the target material and assert that an average neutron energy of 6-7 MeV is appropriate. To fit the angular distribution $d Y / d \Omega$, the "form factor" $F(\theta, \xi)$ defined as follows was found to be useful:

$$
F(\theta, \xi)=\frac{1}{4 \pi}\left(\frac{1}{\ln \{1+1 / \xi\}}\right)\left(\frac{1}{\xi+\sin ^{2}(\theta / 2)}\right),
$$

where $\theta$ is defined as usual and the fitting parameter $\xi$ is related to the ratio of fluences $\Phi$ at $\theta=0$ and 90 degrees, and thus related to "forward-peakedness". In a subsequent paper, Aleinikov et al. (A185) developed the following parameterization for $\xi$ : 


\section{Chapter 4 Prompt Radiation Fields Due to Protons and Ions}

$$
\xi=\frac{\Phi\left(90^{\circ}\right)}{\Phi\left(0^{\circ}\right)-\Phi\left(90^{\circ}\right)}=\frac{1}{\Phi\left(0^{\circ}\right) / \Phi\left(90^{\circ}\right)-1} .
$$

In this scheme, $\quad \frac{d Y(\theta)}{d \Omega}=Y(W, Z) F(\theta, \xi)$.

Values of the parameters $C(Z)$ and $\eta(Z)$ for specific circumstances are given in Table 4.3.

Table 4.3 Values of the parameters $\eta(Z)$ and $C(Z)$ as expressed in Eqs. (4.9). [Adapted from (Cl83).]

\begin{tabular}{|c|l|l|l|}
\hline Atomic Number & Element & $\eta(Z)$ & $\boldsymbol{C ( Z )}$ \\
\hline 1 & hydrogen & 1.5 & $1.7 \times 10^{-4}$ \\
\hline 2 & helium & 2.6 & $3.9 \times 10^{-6}$ \\
\hline 6 & carbon & 1.7 & $2.5 \times 10^{-6}$ \\
\hline 8 & oxygen & 3.6 & $3.6 \times 10^{-7}$ \\
\hline 10 & neon & 7.0 & $2.7 \times 10^{-10}$ \\
\hline 18 & argon & 7.0 & $5.1 \times 10^{-11}$ \\
\hline 36 & krypton & 7.9 & $6.0 \times 10^{-12}$ \\
\hline 82 & lead & 11.0 & $1.7 \times 10^{-13}$ \\
\hline & \multicolumn{2}{l}{} \\
\hline
\end{tabular}

Aleinikov et al. (A185) also give a few examples of the values of their parameter $\xi ; 0.07$ for uranium incident on uranium at $9 \mathrm{MeV}$ amu $^{-1}, 0.025$ for neutrons of energy $E_{n}<20$ $\mathrm{MeV}$ produced by ${ }^{12} \mathrm{C}$ ions at $86 \mathrm{MeV}$ amu $^{-1}$ incident on iron, and $3 \times 10^{-4}$ for neutrons of energy $E_{n}>20 \mathrm{MeV}$ produced these same ions (based on an analysis of the data presented in Fig. 4.11). In principle one could use values given in Table 4.3 or the direct calculation using Eqs. (4.9b) and (4.9c) and obtain some idea of the uncertainties inherent in this fit of such a broad range of data. However, the uncertainties in this type of fit are quite large due to the functional forms that were used.

McCaslin et al. (McC85) measured the angular distribution yields of $670 \mathrm{MeV} \mathrm{amu}^{-1} \mathrm{Ne}$ and $\mathrm{Si}$ ions stopped in a copper target. The distributions for the two different projectiles were similar with fits to the data provided for the ${ }^{20} \mathrm{Ne}$ ions. For these, including all neutrons above $6.5 \mathrm{MeV}$ at a radius of one meter, McCaslin found

$$
\Phi(\theta)=372 \frac{1}{\theta} \text { neutrons } \mathrm{m}^{-2} \text { per ion for } 2^{\circ}<\theta<180^{\circ}, \theta \text { in degrees }
$$

and for the same ions, including all neutrons above $20 \mathrm{MeV}$;

$$
\begin{aligned}
& \Phi(\theta)=248 \mathrm{e}^{-0.2 \theta} \text { neutrons } \mathrm{m}^{-2} \text { per ion for } 0^{\circ}<\theta<20^{\circ}, \theta \text { in degrees, and } \\
& \Phi(\theta)=10 \mathrm{e}^{-0.038 \theta} \text { neutrons } \mathrm{m}^{-2} \text { per ion for } 20^{\circ}<\theta<120^{\circ}, \theta \text { in degrees. }
\end{aligned}
$$

The neutron yields at this high specific energy for heavy ions turn out to be quite large. By integrating the above over all angles, one finds a total yield of 74.1 neutrons per incident ion for $E_{n}>6.5 \mathrm{MeV}$ for ${ }^{20} \mathrm{Ne}$ incident ions (see Problem 4). 


\section{Chapter 4 Prompt Radiation Fields Due to Protons AND Ions}

A more complete picture of heavy ion neutron yields is clearly desirable for intermediate to high energies. The results of Kurosawa et al. (Ku99), Heilbronn et al. (He99), and Kurosawa et al. ( $\mathrm{Ku} 00)$ spanning the periodic table represent a major advance. Along with a good parameterizeration of measured neutron angular distributions not discussed in detail here, $(\mathrm{Ku} 00)$ presents a useful simple formula based upon geometrical considerations that describes total yield $Y$ of neutrons having energies above $5.0 \mathrm{MeV}$ emitted into the hemisphere $0<\theta<90^{\circ}$. This heavy ion neutron yield formula is

$$
\left.Y=\frac{1.5 \times 10^{-6}}{N_{T}^{1 / 3}} W_{P}^{2}\left(A_{P}^{1 / 3}+A_{T}^{1 / 3}\right)^{2} N_{P} \frac{A_{P}}{Z_{P}^{2}} \text { (neutrons particle }{ }^{-1}\right)
$$

where the subscripts $P$ and $T$ denote the projectile ion and the target, respectively, and $Z$, $N$, and $A$ have their usual meanings of atomic number, neutron number, and mass number. $W_{P}$ is the projectile specific kinetic energy in $\mathrm{MeV} \mathrm{amu}^{-1}$. This formula describes data generally within factors of two or three for ions from He to Xe incident ions fully stopped in targets ranging from $\mathrm{C}$ to $\mathrm{Pb}$ over the specific energy domain $100<E_{P}<800 \mathrm{MeV} \mathrm{amu}^{-1}$. Fig. 4.13 shows representative total neutron yields for heavy ions from measurements and calculations using Eq. (4.16) compared with the yields found for protons at the same specific energy.

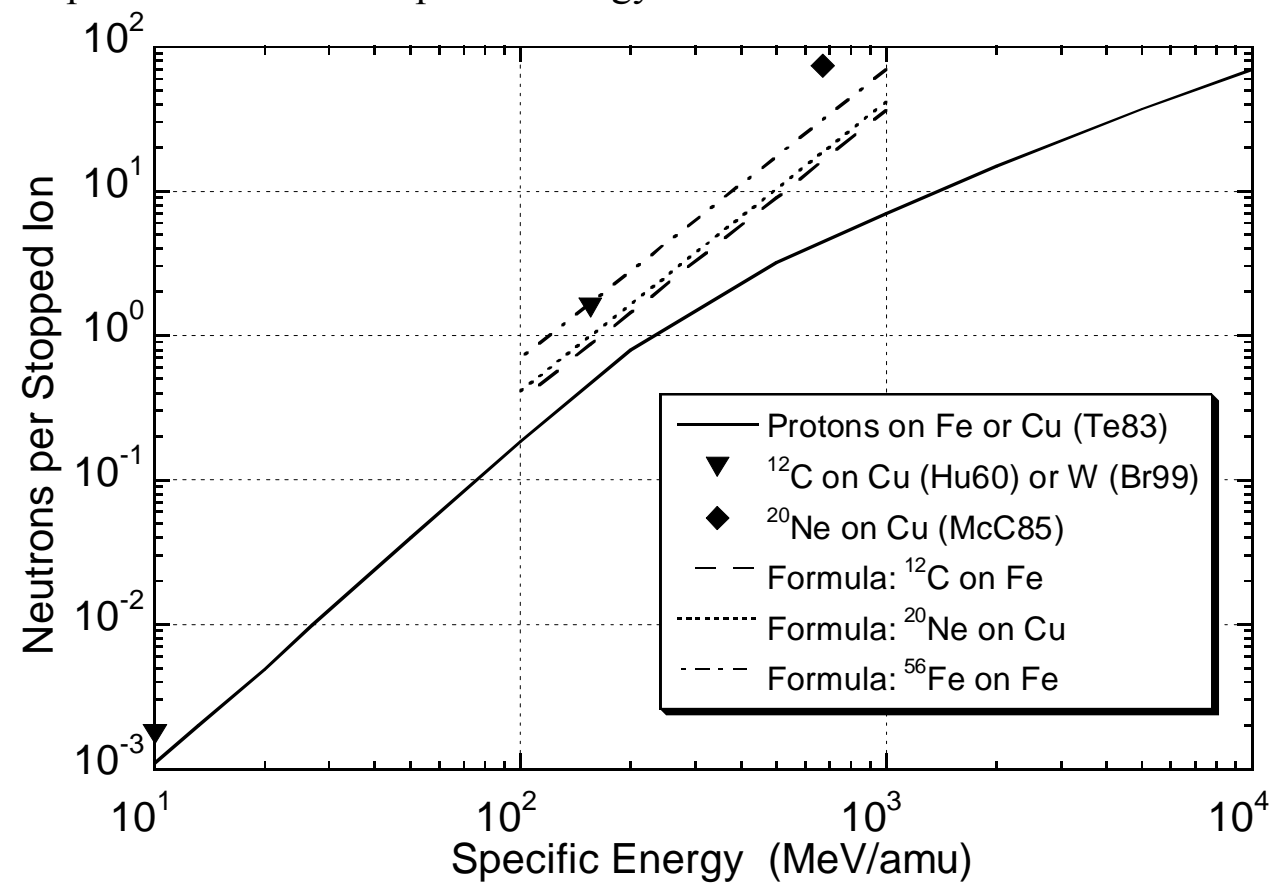

Fig. 4.13 Neutron yields as a function of specific energy for selected projectiles and targets as reported by the cited references along with results obtained using Eq. (4.16). ${ }^{15}$

\footnotetext{
${ }^{15}$ Measurements of neutron yields using heavy ions are rather difficult. Compared with protons or light ions, target thickness effects are more important due to the shorter ionization ranges in materials. Results are sometimes inconsistent. E.g. Eq. (4.16) gives a yield of only 18.6 neutrons ion ${ }^{-1}$ for the situation studied by McCaslin (McC85). The factor of four discrepancy could be due to a collection of variables such as target sizes and detector efficiencies. Escape of energy via mesons can be important at the higher energies.
} 


\section{Chapter 4 Prompt Radiation Fields Due to Protons AND Ions}

\subsection{Hadron (Neutron) Shielding for Low Energy Incident Protons $\left(E_{o}<15 \mathrm{MeV}\right)$}

Neutron shielding in this region is complicated by significant nuclear structure effects. There are many resonances associated with compound nucleus that can be excited. There are also nuclear reaction channels leading to a large number of nuclear excited states with a wide variety of nuclear structure quantum numbers and very narrow width in energy. Clark (C171) has expressed some general rules of attack on the neutron shielding problem. Clark's principles are:

- $\quad$ The shield must be sufficiently thick and the neutrons so distributed in energy that only a narrow band of the most penetrating source neutrons give any appreciable ultimate contribution to the dose outside the shield."

- "There must be sufficient hydrogen in the shield, intimately mixed or in the final shield region, to assure a very short characteristic transport length from about one $\mathrm{MeV}$ to absorption at or near thermal energy."

- $\quad$ The source energy distribution and shield material (non-hydrogenous) properties must be such as to assure a short transport distance for slowing down from the most penetrating energies to one $\mathrm{MeV}$."

An elementary method used to calculate shielding thicknesses in this energy domain is removal cross section theory. It has been found that the dose equivalent $H$ as a function of shield thickness $t$ is approximately given for these neutrons by

$$
H(t)=\Phi_{o} P G \exp \left(-\Sigma_{r} t\right),
$$

where $\Phi_{0}$ is the fluence before the shielding as measured or perhaps calculated from neutron yield information, $P$ is the "average" dose equivalent per fluence factor, $G$ is a "geometry factor", $t(\mathrm{~cm})$ is the thickness of the shield. For parallel beams, $G=1.0$ while for an isotropic point source, $G=1 / r^{2}$. $\Sigma_{r}$ is the macroscopic removal cross section;

$$
\Sigma_{r}=\frac{0.602 \sigma_{r} \rho}{A}\left(\mathrm{~cm}^{-1}\right)
$$

where $\sigma_{r}$ is the microscopic removal cross section in barns, $\rho$ is the density $\left(\mathrm{g} \mathrm{cm}^{-3}\right)$, and $A$ is the mass number. For mixtures of $n$ materials,

$$
\Sigma_{r}=\sum_{i}^{n} \frac{\rho_{i}}{\rho} \sum_{r i}
$$

where the right-most quantity is the removal cross section per unit mass of the $i^{\text {th }}$ constituent and $\rho_{i}$ is the partial density of the $i^{t h}$ material. In this formulation the overall density is equal to the sum of the partial densities. For $A>8$,

$$
\sigma_{r}=0.21 A^{-0.58} \text { (barns) }
$$




\section{Chapter 4 Prompt RAdiation Fields Due to Protons AND Ions}

for neutrons of approximately $8 \mathrm{MeV}$.

Fig. 4.14 shows the values of $\sigma_{r}$ as a function of mass number at this energy. Table 4.4 gives representative values for $\sigma_{r}$ for sample energies where this approach is applicable.

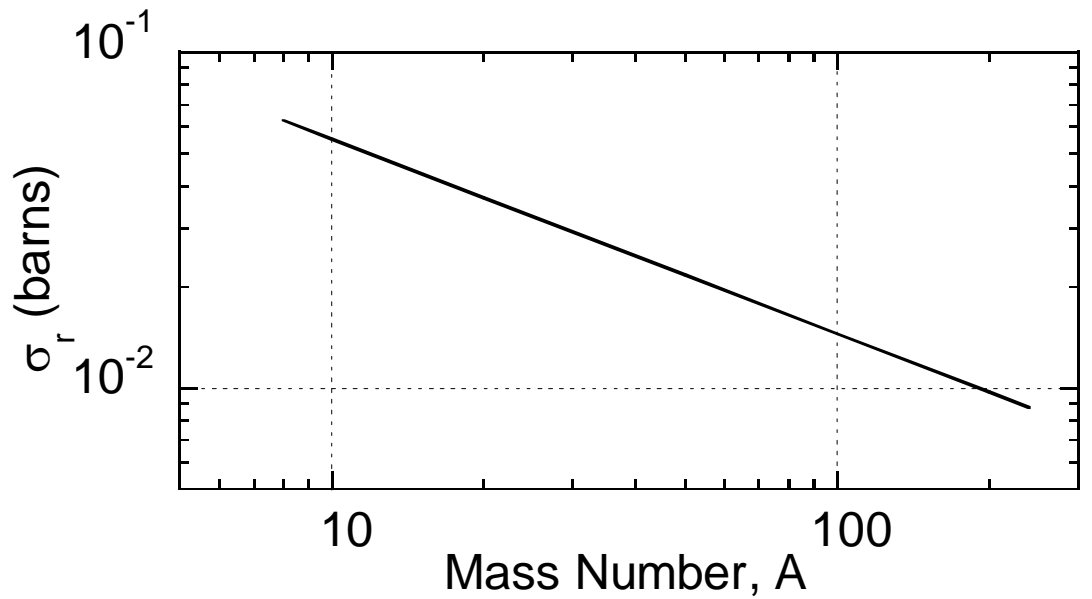

Fig. 4.14 Removal cross sections per unit atomic mass number for fission neutrons as a function of mass number at a neutron energy of $8.0 \mathrm{MeV}$. Over the range $8 \leq A \leq 240$, the values are well fit by Eq. (4.20). [Adapted from (Pa73)].

Table 4.4 Removal cross sections, $\sigma_{r}$ (barns), for low energy neutrons. The typical accuracy is quoted to be $\pm 5 \%$. [Adapted from (Pa73).]

\begin{tabular}{|l|c|c|c|c|c|c|}
\hline Element & $\mathbf{1 . 0} \mathbf{M e V}$ & $\begin{array}{l}\text { Fission } \\
\text { Spectrum }\end{array}$ & $\mathbf{2 . 9} \mathbf{M e V}$ & $\mathbf{4 . 0} \mathbf{M e V}$ & $\mathbf{6 . 7} \mathbf{~ M e V}$ & $\mathbf{1 4 . 9} \mathbf{M e V}$ \\
\hline Carbon & & 0.9 & 1.58 & 1.05 & 0.83 & 0.50 \\
\hline Aluminum & & 1.31 & & & & \\
\hline Iron & 1.1 & 1.96 & 1.94 & 1.98 & 2.26 & 1.60 \\
\hline Copper & & 2.04 & & & & \\
\hline Lead & & 3.28 & 2.70 & 3.44 & 3.77 & 2.95 \\
\hline
\end{tabular}

The use of removal cross sections describe attenuation data rather effectively despite the fact that as more shielding is penetrated, neutrons of lower energy tend to dominate the spectrum over those found in the few $\mathrm{MeV}$ region.

\subsection{Limiting Attenuation at High Energy}

The most important feature of neutron shielding at higher energy accelerators is the fact that the attenuation length becomes an approximate constant at high energy. Perhaps first noticed by Lindenbaum (Li61), as the energy increases the neutron inelastic cross sections increase rapidly until about $25 \mathrm{MeV}$ where they generally level off and then fall rapidly with energy in the region $25<E_{n}<100 \mathrm{MeV}$ to a value which becomes independent of energy, aside from a slight, gradual increase at the very highest energies. The result is 


\section{Chapter 4 Prompt RAdiation Fields Due to Protons AND Ions}

that high energy neutrons attenuate approximately exponentially with an attenuation length $\lambda_{\text {atten }}$ that is rather insensitive to energy. Thus, in units of length,

$$
\lambda_{\text {atten }}=\frac{1}{N \sigma_{\text {in }}} \quad(\mathrm{cm})
$$

where $\sigma_{\text {in }}$ is the inelastic cross section, roughly equivalent to the so-called "absorption cross section" and $N$, as before, is the number of absorber nuclei per unit volume. $\sigma_{\text {in }}$ specifically does not include elastic scattering and thus is always smaller than the total cross section. In a simple-minded approach, $\sigma_{\text {in }}$ can be taken approximately to be the geometrical cross section with the nucleon radius taken to be $1.2 \times 10^{-13} \mathrm{~cm}$. It follows (see Problem 5) that in the high energy limit, one can multiply by the density to get

$$
\rho \lambda_{\text {atten }}=36.7 A^{1 / 3}\left(\mathrm{~g} \mathrm{~cm}^{-2}\right) .
$$

Fig. 4.15 gives $\sigma_{\text {in }}$ for several materials up to a kinetic energy of $1.4 \mathrm{GeV}$. For our present purposes $\sigma_{i n}$ is essentially constant at higher energies.

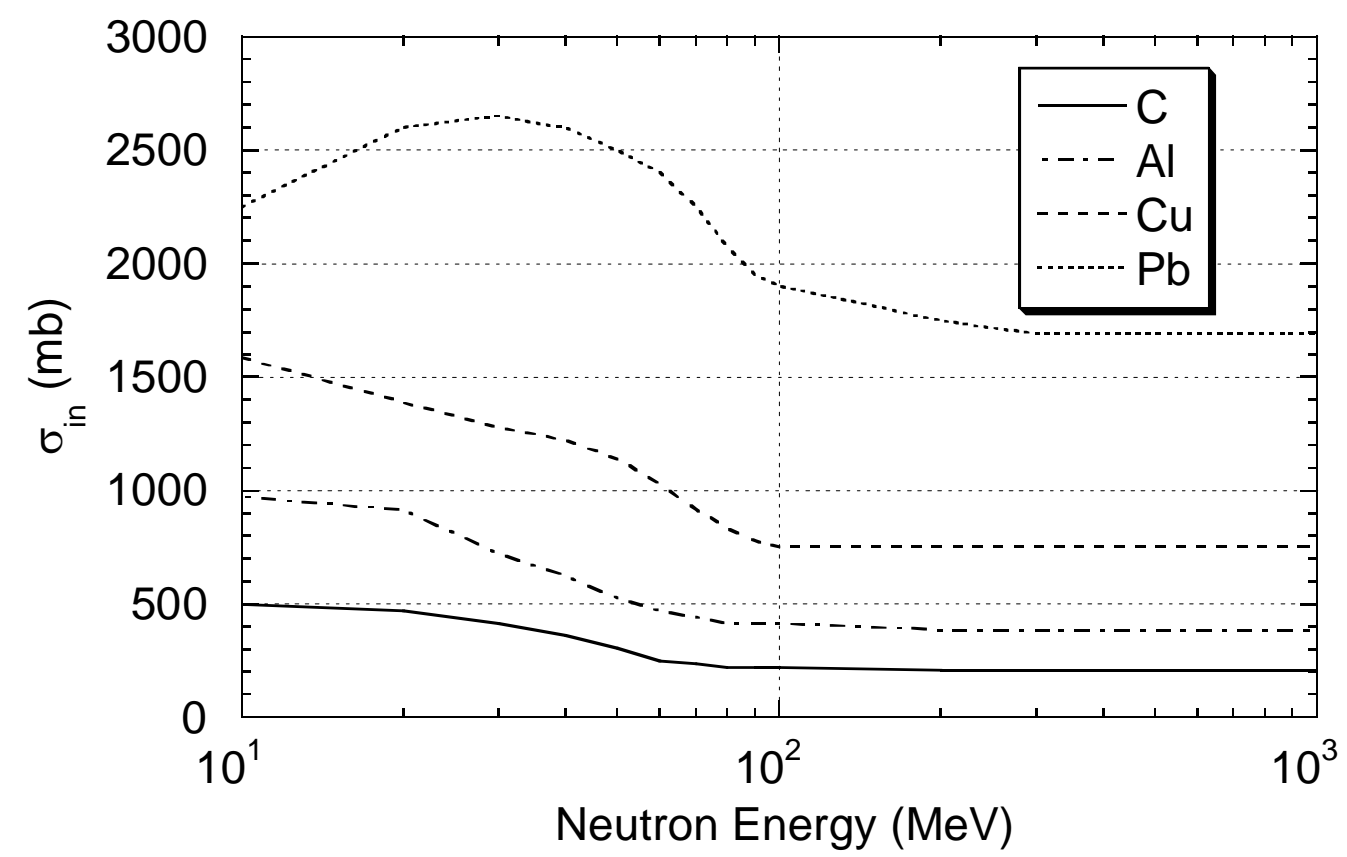

Fig. 4.15 Inelastic neutron cross sections as a function of energy in the range 10 to $1000 \mathrm{MeV}$. [Adapted from (Li61).]

The high energy asymptotes were first verified by historic cosmic ray data and are wellrepresented by

$$
\sigma_{\text {in }}=43 A^{0.69}(\mathrm{mb})
$$




\section{Chapter 4 Prompt RAdiation Fields Due to Protons AND Ions}

In the high energy limit, the interaction length $\lambda_{\text {inel }}$ is thus given by

$$
\lambda_{\text {inel }}=\frac{\rho}{N \sigma_{\text {in }}}=38.5 A^{0.31}\left(\mathrm{~g} \mathrm{~cm}^{-2}\right)
$$

Thus, this geometric approximation is reasonably accurate. Values of the high energy interaction lengths are available for many different materials and representative examples are found in Table 1.2. Fig. 4.16 shows the results for absorption cross sections based upon these values. Fassò et al. (Fa90) have provided extensive tabulations of the value of $\sigma_{\text {in }}(\mathrm{mb})$ for a variety of particles, energies, and materials in the high energy region as functions of particle momenta up to $10 \mathrm{TeV} / \mathrm{c}$.

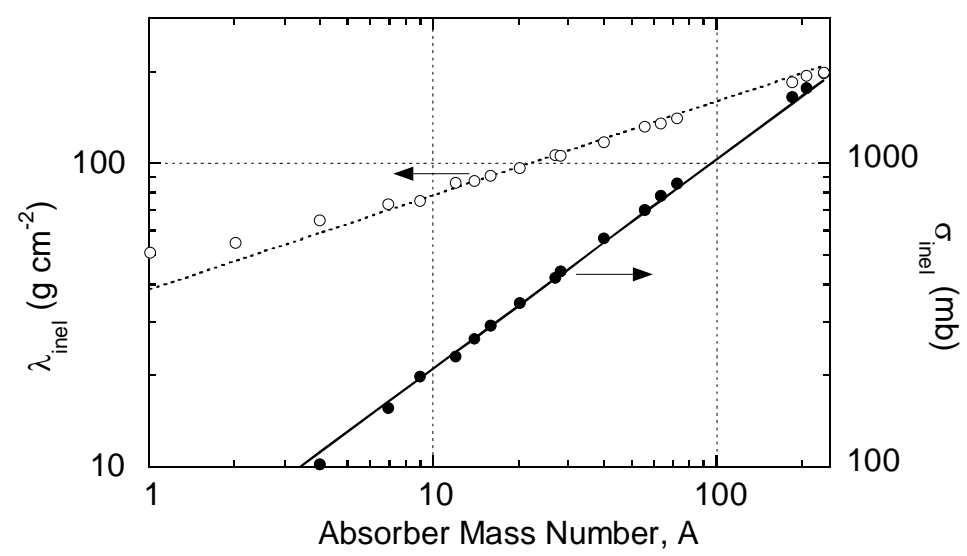

Fig. 4.16 Inelastic mean free path and cross section as a function of mass number $A$. [Adapted from (Pa73).]

The leveling off of attenuation length for concrete for a broad beam of particles as a function of particle energy is especially important due to the widespread use of this material in shielding. Fig. 4.17 gives the results for both neutrons and protons. An important feature of these results is the equivalence of the attenuation lengths for protons and neutrons at high energies. Due to the similarities of chemical composition, results for soil shielding in this energy regime can be taken to be the same as those for concrete when $\lambda$ is expressed in units of areal density, e.g. in $\mathrm{g} \mathrm{cm}^{-2}$.

\subsection{Intermediate and High Energy Shielding-The Hadronic Cascade}

\subsubsection{The Hadronic Cascade from a Conceptual Standpoint}

The hadronic cascade is initiated at proton accelerators when the beam interacts with targets, beam absorbers, and accelerator components to produce neutrons and other particles. Such cascades can also arise at electron accelerators since (see Chapter 3 ) high energy secondary hadrons can also result from electromagnetic interactions. The collision of a high energy nucleon with a nucleus produces a large number of particles; pions, kaons, and other nucleons as well as fragments of the struck nucleus. According to Thomas and Stevenson, above about $1.0 \mathrm{GeV}$ and at forward angles, the pions, protons, 


\section{ChAPTER 4 Prompt RAdiation Fields Due to Protons AND Ions}

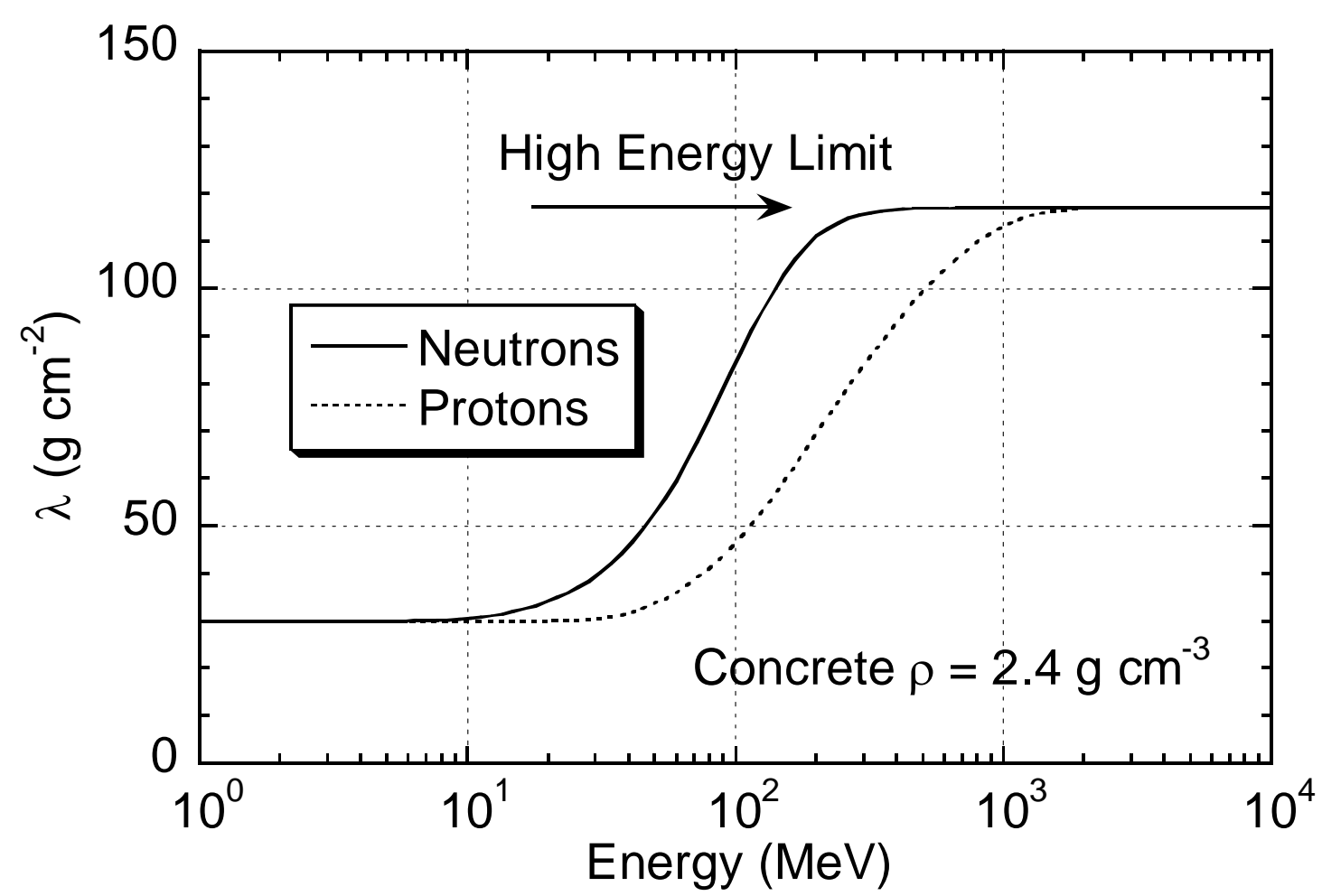

Fig. 4.17 The attenuation length $\lambda$ for a broad beam of monoenergetic neutrons and protons in concrete shielding as a function of particle kinetic energy. The high energy limit is $117 \mathrm{~g} \mathrm{~cm}^{-2}$. [Adapted from (Th88).]

and neutrons, can be nearly equal in number (Th88). The neutrons may be classified as either evaporation neutrons or cascade neutrons (see Section 4.2.2.2). Cascade neutrons are emitted by direct impact. The spectrum extends in energy up to the incident energy with diminishing probability and follows an energy dependence roughly proportional to $1 / E$. As the proton kinetic energy increases other particles, notably $\pi^{+*}, \pi^{0}$, and $\mathrm{K}^{+,}$, play roles in the cascade when their production becomes energetically possible. They are absorbed with absorption lengths comparable in magnitude to, but not identical with, those of protons. These particles also decay into muons. Because of their long ionization ranges and lack of nuclear interactions, muons provide a pathway for energy to escape the cascade. Hadrons, principally neutrons with $E_{n}>150 \mathrm{MeV}$, propagate the cascade. This is clear from the attenuation lengths shown in Figs. 4.15 and 4.17. Nucleons in the range $20<E_{n}<150 \mathrm{MeV}$ also deposit their energy predominantly by nuclear interactions but their energy gets distributed over many particles of all types energetically possible. The charged particles produced in such cascades are generally "ranged-out" by ionization in material or create yet other particles in the cascade. The role played by the energy of approximately $150 \mathrm{MeV}$ for hadronic cascade propagation is qualitatively analogous to that of the critical energy for electromagnetic cascades.

Neutral pions $\left(\pi^{0}\right)$ are produced when the kinetic energy of the incident proton significantly exceeds the $\pi^{0}$ rest energy of $135.0 \mathrm{MeV}$. The $\pi^{0}$ mean-life of $8.4 \times 10^{-17} \mathrm{~s}$ is very short so that for the $\pi^{\mathrm{O}}, c \tau=25.1 \mathrm{~nm}$. Hence, $\pi^{\mathrm{O}} \mathrm{s}$ do not travel very far at all before 


\section{Chapter 4 Prompt RAdiation Fields Due to Protons AND Ions}

decaying. The principal decay ( $99 \%$ branching ratio) is into a pair of photons emitted in opposite directions in the center of mass refence frame of the moving $\pi^{\circ}$. An energetic $\pi^{0}$ thus appears as two forward-peaked photons each with half of the $\pi^{0}$ s total energy. The decay photons from $\pi^{0}$ decay readily initiate an electromagnetic cascade along with the hadronic one. It is possible for the electromagnetic channel to feed back into the hadronic cascade because it, too, produces high energy hadrons. However this effect is generally of little importance and, for most shielding calculations, the electromagnetic component of a hadronic cascade can be ignored. The principal exception involves energy deposition calculations at forward angles (small values of $\theta$ ). In fact, at hundreds of $\mathrm{GeV}$, electromagnetic cascades dominate the energy deposition at very forward angles. This can have important ramifications for radiation damage to equipment, the heat load on cryogenic systems, and the ability of targets to survive bombardment.

In general, the neutrons are the principal drivers of the cascade because of the ionization energy loss for pions and for protons below $450 \mathrm{MeV}$, the energy at which the ionization range becomes roughly equal to the interaction length. Also, any magnetic fields that are present which can deflect and disperse charged particles present will not, of course, affect the neutrons. Furthermore, neutrons are produced in large quantities at large values of $\theta$ compared with the forward-peaked pions. These phenomena, in general, apply also to ions heavier than the proton with suitable corrections (especially at low energies) for nuclear structure effects. Scaling of proton results for heavier ions will, in general, roughly be according to the specific energy (MeV/amu). Fig. 4.18 is a schematic flow chart of the hadronic cascade process (IC78).

\subsubsection{A Simple One-Dimensional Cascade Model}

A simple one-dimensional model of the hadronic cascade was first proposed by Lindenbaum (Li61). This approach provides some "intuition" into the nature of the hadronic cascade. Fig. 4.19 defines the geometry of the Lindenbaum model. Suppose one initially has $N_{o}$ incident high energy nucleons. After an individual collision, one of them continues in its original direction at a reduced energy but with the same attenuation length $\lambda$ or will generate one or more secondary particles also with the same value of $\lambda$. The value of $\lambda$ is approximately constant due to the limiting attenuation at high energy. This process continues until a number of collisions $n$ have occurred that are sufficient to degrade the particle energies to approximately $150 \mathrm{MeV}$. Below $150 \mathrm{MeV}$ the inelastic cross sections greatly increase with decreasing particle energy (see Fig. 4.15). This additional absorption at the lower energies can be said to "remove" the particle. For the present discussion, it is assumed that $n$ is an integer, an approximation since in reality $n$

has a statistical distribution. Following these assumptions, the number $v_{1}$ that reach $z$ having made no additional collisions that produce secondary particles is

$$
v_{1}=N_{o} \exp (-z / \lambda) .
$$




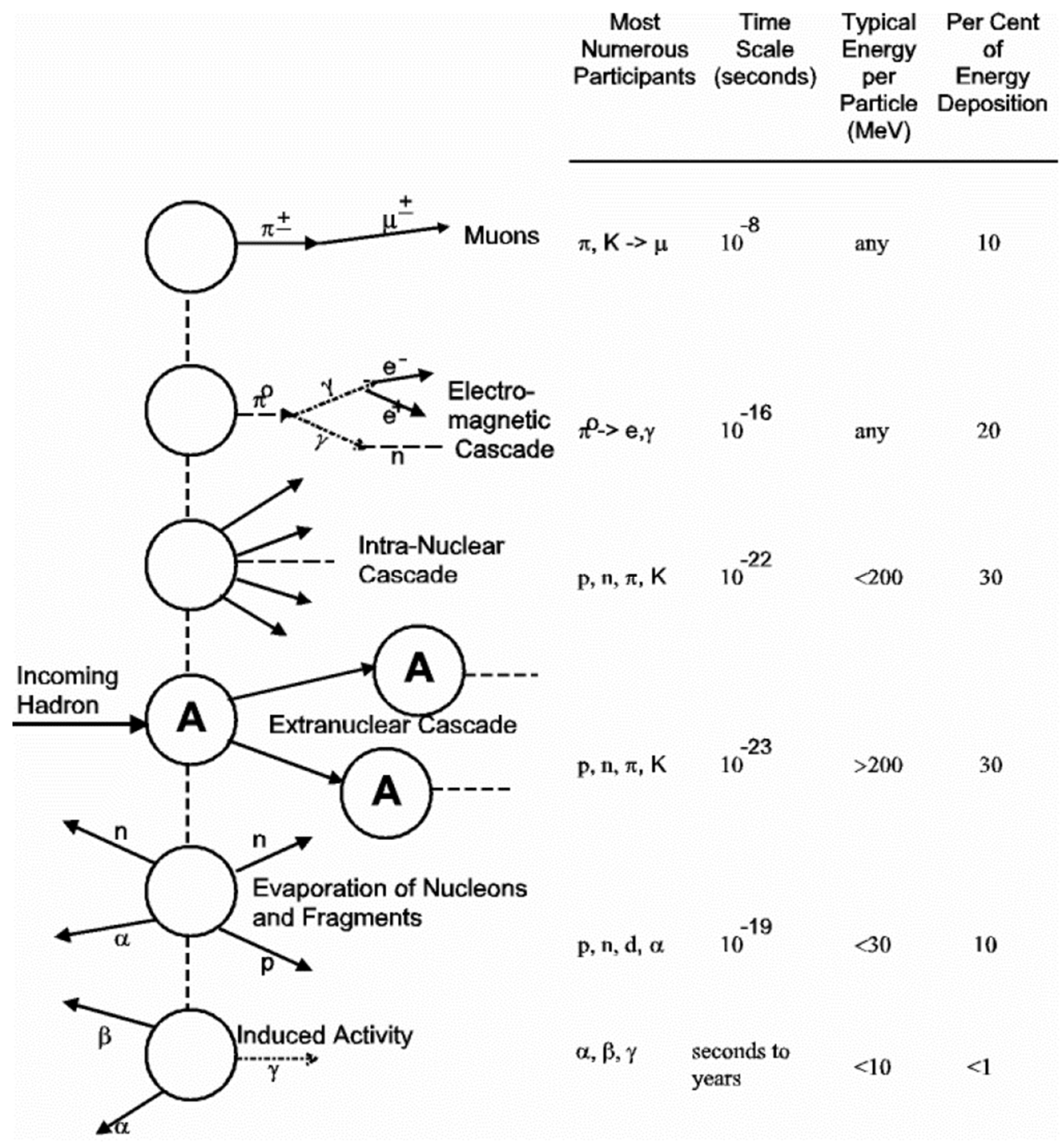

Fig. 4.18 Schematic representation of the development of the hadronic cascade and the major participants in any given path. The approximate time scales, the typical energies, and the fraction of the total energy deposition due to these participants are also shown. [Adapted from (IC78).] 


\section{Chapter 4 Prompt Radiation Fields Due to Protons AND Ions}

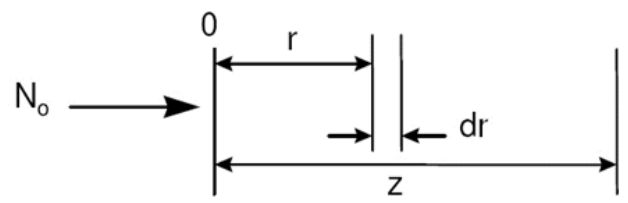

a)

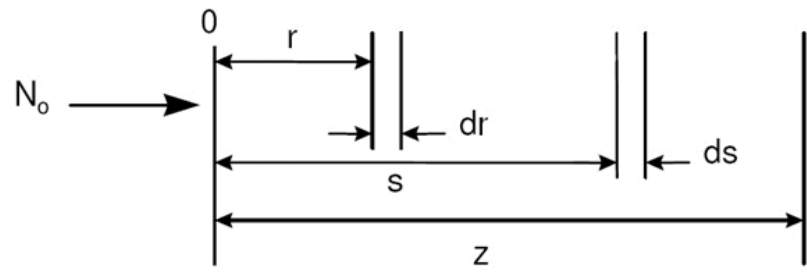

b)

Fig. 4.19 a) Single collision geometry for the Lindenbaum model. b) Two collision geometry for the Lindenbaum one-dimensional model. [Adapted from (Th88).]

Now suppose that there is one additional collision between 0 and $z$ that produces secondary particles. The number $v_{2}$ of additional particles that reach $z$ is given by the product of the number that reach elemental coordinate $d r$; the probability of subsequently reaching $z$; the probability of interacting in $d r, d r / \lambda$; and the multiplicity $m_{1}$ of particles produced in the interaction as illustrated in Fig. 4.19a. Integrating over $d r$;

$\int_{0}^{z} d r \frac{m_{1}}{\lambda}\left[N_{0} \exp (-r / \lambda)\right][\exp \{-(z-r) / \lambda\}]=\left(N_{0} m_{1} \frac{z}{\lambda}\right) \exp (-z / \lambda)=v_{2}$

Continuing, now suppose there are two additional collisions occur that produce secondary particles as in Fig. 4.19b. The number $v_{3}$ of additional particles that reach $z$ is the product of those that reach $s$ having made one particle-producing collision; the probability of subsequently reaching $z$; the multiplicity in the second interaction $m_{2}$; and the probability of interacting in $d s$;

$$
\begin{aligned}
& \int_{o}^{z} d s \frac{m_{2}}{\lambda}\left[N_{0} m_{1} \frac{s}{\lambda} \exp (-s / \lambda)\right][\exp \{-(z-s) / \lambda\}]= \\
& \left\{N_{0} m_{1} m_{2} \frac{z}{\lambda^{2}} \exp (-z / \lambda)\right\} \int_{0}^{z} s d s=\left\{N_{0} m_{1} m_{2} \frac{z^{2}}{2 \lambda^{2}} \exp (-z / \lambda)\right\}=v_{3} .
\end{aligned}
$$

Therefore, with $n$ defined as above, one can write for the total number of particles that reach $z$ :

$$
N_{n}(z)=N_{0} \beta_{n}(z / \lambda) \exp (-z / \lambda)
$$




\section{Chapter 4 Prompt RAdiation Fields Due to Protons AND Ions}

where $\beta$ is a buildup factor:

$$
\begin{aligned}
\text { for } n=1 ; & N_{1}=v_{1} \text { and } \beta_{1}=1, \\
\text { for } n=2 ; & N_{2}=v_{1}+v_{2} \text { and } \beta_{2}=1+\left(m_{1} z / \lambda\right), \text { while } \\
\text { for } n=3 ; & N_{2}=v_{1}+v_{2}+v_{3} \text { and } \beta_{3}=1+\left(m_{1} z / \lambda\right)+\left(m_{1} m_{2} z^{2} / 2 \lambda^{2}\right) . \\
\text { For arbitrary } n ; & \beta_{n}=1+\frac{m_{1} z}{\lambda}+\frac{m_{1} m_{2} z^{2}}{2 \lambda^{2}}+\ldots+\frac{1}{(n-1) !}\left(\frac{z^{n-1}}{\lambda^{n-1}}\right) \prod_{i=1}^{n-1} m_{i} .
\end{aligned}
$$

Thus, this buildup factor is a monotonically increasing function of $z$. If one makes the assumptions that the multiplicity stays the same for all interactions, $m_{1}=m_{2}=\ldots=m$, and that $n$ is large, comparison with the series expansion of the exponential function reveals that $\beta_{n}$ approximates an exponential dependence on $z$. The condition on $n$ implies that the shield must be quite thick. The general result is that the attenuation length of the cascade $\lambda_{\text {cas }}$ is somewhat larger than the value of the interaction length $\lambda$ for a single interaction. The upper frame of Fig. 4.20 is a plot of the results of this model for several values of $m$ and $n$ as a function of $z / \lambda$ while the lower frame is a plot for a specific case $(n=3$ and $m=2)$. The exponential region is not completely achieved until $z / \lambda \approx 5$. In concrete this represents a depth of approximately $600 \mathrm{~g} \mathrm{~cm}^{-2}$.or about $2.5 \mathrm{~m}$. In the lower frame, these calculations are also compared with the results of data from the experiment of Citron et al. (Ci65) with $19.2 \mathrm{GeV} / \mathrm{c}$ protons incident on an iron slab taken under experimental conditions which approximated these values of $m$ and $n$.

Analytical approaches such as this one are constructive qualitatively but have severe limitations, among which are;

- the restriction to one dimension,

- the neglect of ionization energy losses and escape of energy carried by muons,

- the neglect of elastic and multiple Coulomb scattering,

- the assumption that all secondary particles go forward,

- the assumption that multiplicities are not dependent on energy and particle type,

- the assumption that $\lambda$ is a constant for all particles at all energies, and

- the neglect of radiative and electromagnetic cascade effects.

\subsubsection{A Semiempirical Method; the Moyer Model for a Point Source}

A number of references [(Pa73), (IC78), (Fa90), (Ro76), (St82), (Th84), (McC87), (Te83), (Te85), (McC85), (Co82a), and (Co85a)] bear on the development of this model predominantly based on exponential approximation with constants fitted to measurements spanning proton beam energies from 7.4 to $800 \mathrm{GeV}$. The summary of this method presented here is largely taken from Patterson and Thomas (Pa73) and Fassò et al. (Fa90). 


\section{Chapter 4 Prompt Radiation Fields Due to Protons ANd Ions}
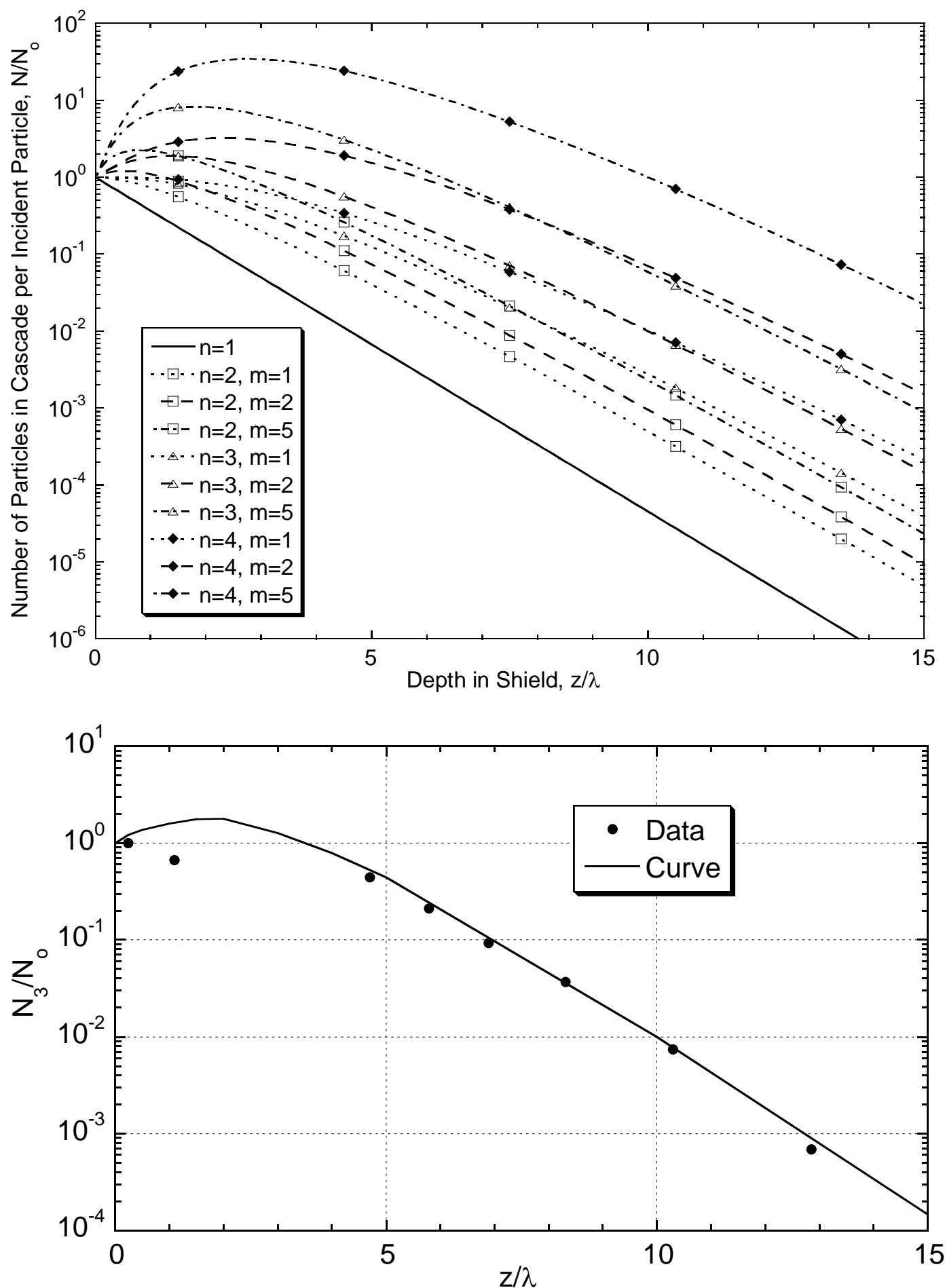

Fig. 4.20 Upper frame: Development of a one-dimensional cascade in the Lindenbaum model with $n=1$ to 4 and $m=1,2$, and 5. Lower frame: The same approximation with $n=3$ and $m=2$ from Thomas and Stevenson (Th88) labeled "Curve” compared with the laterally integrated star density in nuclear emulsions produced by a $19.2 \mathrm{GeV} / \mathrm{c}$ proton beam incident on an iron slab measured by Citron et al. (Ci65) which is labeled "Data". 


\section{Chapter 4 Prompt Radiation Fields Due to Protons and Ions}

This so-called Moyer Model was first developed by Burton J. Moyer to solve particular shielding problems related to the $6 \mathrm{GeV}$ Bevatron at the Lawrence Radiation Laboratory (now the Lawrence Berkeley National Laboratory). The model predates the development of large, fast computers and advanced Monte Carlo techniques but remains useful as means of checking more sophisticated calculations. Consider the situation shown in Fig. 4.21 for a "point" target source.

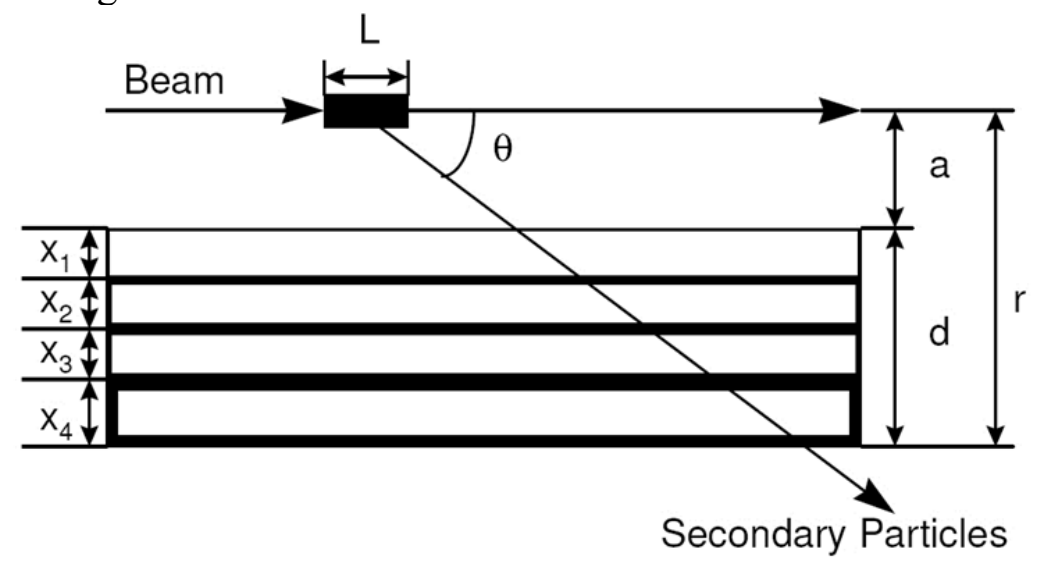

Fig. 4.21 Sketch of the geometry for the empirical Moyer Model. A beam of $N_{p}$ protons impinges on the target of length $L$. The shield materials represented by the layers $x_{i}$, could be, for example, iron, concrete, earth, and air (i.e., vacuum) respectively. $a$ is the inner radius of the tunnel. The observer is situated at a radial thickness of $d$ equal to the sum of the thicknesses of the four layers and is at a distance $r^{\prime}=r \csc \theta$ from the beam-target interaction point.

The number of neutrons $d N / d E$ which are emitted into a given element of solid angle $d \Omega$ at angle $\theta$ relative to a target struck by $N_{p}$ protons in an energy interval $E+d E$ is given for a single shield material of thickness $d$ by

$$
\frac{d N}{d E}=N_{p} B(E)\left(\frac{d^{2} Y}{d E d \Omega}\right) d \Omega \exp \left(-\frac{d \csc \theta}{\lambda(E)}\right),
$$

where $B(E)$ is a buildup factor and the exponential function accounts for the attenuation of the radiation field by shielding of thickness $d$ at the angle $\theta$. The energy-dependent interaction length is denoted by $\lambda(E) . \lambda(E)$ and $d$ must be in the same units, usually of length in meters or centimeters. The role of the double differential of the yield is obvious. In the above, the flux density at coordinates $(r, \theta)$ can be obtained at distance $r^{\prime}$ from the target by including the factor

$$
\frac{d \Omega}{d A}=\frac{1}{(a+d)^{2} \csc ^{2} \theta}=\frac{1}{r^{2} \csc ^{2} \theta}=\frac{1}{r^{\prime 2}} .
$$

The total fluence, $\Phi$, at the point where the ray emerges from the shield is given by

$$
\Phi=\frac{N_{p}}{r^{\prime 2}} \int_{E_{\min }}^{E_{\max }} d E \frac{d^{2} Y}{d E d \Omega} B(E) \exp \left(-\frac{d \csc \theta}{\lambda(E)}\right) .
$$




\section{ChAPTER 4 Prompt RAdiation Fields Due to Protons AND Ions}

The following are Moyer's simplifying assumptions:

A. $\lambda(E)=\lambda=$ constant for $E \geq 150 \mathrm{MeV}$ and $\lambda(E)=0$ for $E<150 \mathrm{MeV}$. This is a simplified rendering of the leveling-off of the inelastic cross section at high energy. Thus,

$$
\Phi\left(E_{n}>150 \mathrm{MeV}\right)=\frac{N_{p}}{r^{\prime 2}} \exp \left(-\frac{d \csc \theta}{\lambda}\right) \int_{150 \mathrm{MeV}}^{E_{\max }} d E \frac{d^{2} Y}{d E d \Omega} B(E) .
$$

B. The neutrons emitted at angle $\theta$ can be represented by a simple function $f(\theta)$ multiplied by a multiplicity factor $M\left(E_{\max }\right)$ that depends only on the incident energy, thus;

$$
\begin{array}{r}
\Phi\left(E_{n}>150 \mathrm{MeV}\right)=\frac{N_{p}}{r^{\prime 2}} \exp \left(-\frac{d \csc \theta}{\lambda}\right) M\left(E_{\max }\right) f(\theta)= \\
\frac{N_{p}}{r^{\prime 2}} \exp \left(-\frac{d \csc \theta}{\lambda}\right) g\left(E_{\max }, \theta\right)
\end{array}
$$

where $g\left(E_{\max }, \theta\right)$ is an angular distribution function that is constant for a given value of $E_{\max }$ and for a particular target.

C. The dose equivalent per fluence $P$ for neutrons is not strongly dependent on energy over a rather wide energy range near $E \approx 150 \mathrm{MeV}$ (see Fig. 1.5).

D. Thus the dose equivalent just outside of the shield due to neutrons with $E>150$ $\mathrm{MeV}$ can be taken to be $H_{150} \approx P_{150} \Phi\left(E_{n}>150 \mathrm{MeV}\right)$, where $P_{150}$ is the value of this conversion factor at $150 \mathrm{MeV}$.

The total dose equivalent, $H_{\text {equiv }}$, is then given by

$$
H_{\text {equiv }}=k H_{150} \text { where } k \geq 1 \text {. }
$$

This implicitly assumes that the low-energy neutrons are in equilibrium with those having $E>150 \mathrm{MeV}$ so that the spectrum no longer changes with depth. This is a valid assumption for a shield more than a few mean free paths thick. These assumptions lead to

$$
H_{\text {equiv }}=\frac{k P_{150} N_{p} g\left(E_{\max }, \theta\right)}{(a+d)^{2} \csc ^{2} \theta} \exp \left(-\frac{d \csc \theta}{\lambda}\right) .
$$

One can generalize the results for the geometry shown in Fig. 4.21 with multiple materials in the shield. The parameter $\zeta$, which replaces the ratio $d / \lambda$ in the argument of the exponential function in Eq. (4.36), is introduced to take care of the $n$ shielding components; 


\section{Chapter 4 Prompt RAdiAtion Fields Due to Protons AND IONS}

$$
\zeta=\sum_{i=1}^{n} \frac{x_{i}}{\lambda_{i}}
$$

where the sum is over the $n$ layers of shielding.

Moyer model parameters have been determined by experiment. Stevenson (St82) and Thomas and Thomas (Th84) have determined from global fits to data over a wide domain of energy that $f(\theta)$ is well described by

$$
f(\theta)=\exp (-\beta \theta)
$$

where $\theta$ is in radians, $\beta$ is in radians ${ }^{-1}$. For proton kinetic energies well above $E_{n}=150$ $\mathrm{MeV}, \beta \approx 2.3 \mathrm{rad}^{-1}$. Thus,

$$
\begin{gathered}
H_{\text {equiv }}=\frac{H_{o, \text { equiv }}\left(E_{p}\right) \exp (-\beta \theta) \exp (-\zeta \csc \theta)}{(r \csc \theta)^{2}} \\
\text { with } r=a+\sum_{i=1}^{n} x_{i} .
\end{gathered}
$$

The value of $H_{o \text {,equiv }}\left(E_{p}\right) \exp (-\beta \theta)$ is determined from the yield data and empirical measurements. $H_{o, \text { equiv }}\left(E_{p}\right)$ is best fit as a power law of form; $H_{o, \text { equiv }}\left(E_{p}\right) k E^{\eta}$. From such results, per incident proton;

$$
\begin{aligned}
H_{o, \text { equiv }}\left(E_{p}\right)=\left[(2.84 \pm 0.14) \times 10^{-13}\right] E_{p}(0.80 \pm 0.10) & \left(\mathrm{Sv} \mathrm{m}{ }^{2}\right) \\
& =2.84 \times 10^{-8} E_{p} 0.8\left(\mathrm{mrem} \mathrm{m}^{2}\right)=2.84 \times 10^{-4} E_{p} 0.8\left(\mathrm{mrem} \mathrm{cm}^{2}\right)
\end{aligned}
$$

with $E_{p}$ in $\mathrm{GeV}$. Using the 1990 Radiation Protection System, Cossairt has determined that to obtain effective dose, $H_{\text {eff }}$ with the Moyer model, the constant 2.84 +0.14 should be replaced with $3.98 \pm 0.20$ with all other units the same (Co13). These results are derived for relatively "thick" targets (like accelerator magnets) in tunnel configurations. Fassò et al. (Fa90), based on Monte Carlo results, gave values for "thin" targets of $k=2.0 \times 10^{-14}\left(\mathrm{~Sv} \mathrm{~m}^{2}\right)$ and $n=0.5$. A thin-walled beam pipe would be an example of a "thin" target. The variations thus reflect buildup in the shower. For thick lateral shields close to the beam where the cascade immediately becomes fully developed and selfshielding arises, $k=(6.9 \pm 0.1) \times 10^{-15}\left(\mathrm{~Sv} \mathrm{~m}^{2}\right)$ and $n=0.8$ independent of target material [(Fa90) and (St87)]. The value of $n=0.8$ for thick shields has also been rigorously discussed by Gabriel et al. (Ga94) and verified by Torres (To96).

Similarly, within the context of the Moyer model, recommended, but likely conservatively large, semi-empirical values of $\lambda$ for concrete and other materials as a function of mass number $A$ are;

concrete: $1170+20 \mathrm{~kg} \mathrm{~m}^{-2}=117 \mathrm{~g} \mathrm{~cm}^{-2}$

other materials: $428 A^{1 / 3} \mathrm{~kg} \mathrm{~m}^{-2}=42.8 A^{1 / 3} \mathrm{~g} \mathrm{~cm}^{-2}$. 


\section{Chapter 4 Prompt Radiation Fields Due to Protons AND Ions}

These values of $\lambda$ are $15-30 \%$ larger than the high energy nuclear interaction lengths listed in Table 1.2, a result consistent the discussion of buildup in connection with the results of Lindenbaum's one-dimensional approximation. These semi-empirical values of $\lambda$ are also larger than those found in realistic Monte Carlo calculations (see examples later in this chapter and in Appendix B) since they are based on a point source model, a useful construct but one that is never actually achieved with thick targets.

If one sets the partial derivative $\partial H / \partial \theta$ equal to zero, one can derive an equation for determining the value of $\theta=\theta^{\prime}$ at which the maximum dose equivalent occurs;

$$
\zeta \cos \theta^{\prime}-\beta \sin ^{2} \theta^{\prime}+2 \cos \theta^{\prime} \sin \theta^{\prime}=0 .
$$

Generally this equation can be solved by successive approximation methods. One can substitute into the above equation to get the maximum dose equivalent at a given radial depth. According to McCaslin (McC87), with $r$ in meters and over a wide range of values of $\zeta$, the following holds for dose equivalent

$$
H_{\max }=1.66 \times 10^{-14} E_{p}^{0.8} \exp (-\zeta) \frac{\zeta^{-0.245}}{r^{2}} \quad(\text { Sv per incident proton }) . \quad(4.43)^{16}
$$

For values of $\zeta>2.0$ the following is an equally accurate approximation for the maximum dose equivalent;

$$
H_{\max }=1.26 \times 10^{-14} E_{p}^{0.8} \frac{\exp (-1.023 \zeta)}{r^{2}}(\text { Sv per incident proton })
$$

\subsubsection{The Moyer Model for a Line Source}

The Moyer model can be extended to a line source. Assume a uniform source of one proton interacting per unit length. Then, the doses from the individual increments along the line source contribute to the total at any given point $P$ external to the shield. Fig. 4.22 shows the integration variables.

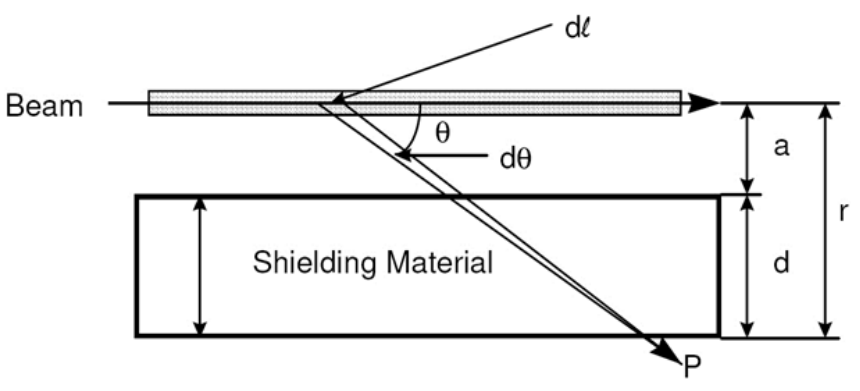

Secondary Particles

Fig. 4.22 Variables of integration of Moyer point source result needed to obtain Moyer line source results. As in Fig. 4.21, the shielding of thickness $d$ could be comprised of multiple layers of thickness $\zeta$ mean free paths.

\footnotetext{
${ }^{16}$ It has been discovered that Eq. (4.43) as transcribed in reference (Fa90) erroneously omitted the negative sign coefficient of 0.245 in this equation.
} 


\section{Chapter 4 Prompt Radiation Fields Due to Protons and Ions}

One can integrate contributions of the elements $d \ell$ of a line source at given perpendicular distance $r$ as follows. Making the change of variable of integration from the line integral to an integral over angle $\theta,\left(d \ell=r \csc ^{2} \theta d \theta\right)$;

$$
\begin{aligned}
H=H_{0}\left(E_{p}\right) \int_{-\infty}^{\infty} d \ell \frac{\exp (-\beta \theta) \exp (-\zeta \csc \theta)}{r^{2} \csc ^{2} \theta}= \\
H_{0}\left(E_{p}\right) \int_{0}^{\pi} d \theta r \csc ^{2} \theta \frac{\exp (-\beta \theta) \exp (-\zeta \csc \theta)}{r^{2} \csc ^{2} \theta}= \\
\frac{H_{0}\left(E_{p}\right)}{r} \int_{0}^{\pi} d \theta \exp (-\beta \theta) \exp (-\zeta \csc \theta)=\frac{H_{0}\left(E_{p}\right)}{r} M(\beta, \zeta) \\
\text { (per interacting proton per unit length). }
\end{aligned}
$$

The integral in the above $M(\beta, \zeta)$ is known as the Moyer integral. The values of this integral have been tabulated by Routti and Thomas (Ro76) and have been approximated by Cossairt (Co13). In view of the results found empirically for point sources, $M(2.3, \zeta)$ has obvious special significance and is tabulated extensively by, among others, Fassò et al. (Fa90). Tesch (Te83) made an important contribution in that he determined an approximation to this integral that has become known as the Tesch approximation:

$$
M_{T}(2.3, \zeta)=0.065 \exp (-1.09 \zeta)
$$

For "intermediate" values of $\zeta, M_{T}(2.3, \zeta)$ can be used instead of $M(2.3, \zeta)$ to simplify calculations. Table 4.5 gives the ratio $M_{T}(2.3, \zeta) / M(2.3, \zeta)$ as a function of $\zeta$. Of course, few so-called "line sources" are actually infinite in length. Thus, the integration can be limited to a finite angular range. Likewise, only a limited angular range (and hence length) contributes significantly to the Moyer integral. Tables 4.6 and 4.7 give angular integration limits that defines the region contributing $90 \%$ of the value of $M(2.3, \zeta)$ as a function of $\zeta$ (Table 4.6) and the distances along the $z$-axis that likewise contribute to $90 \%$ of the value of $M(2.3, \zeta)$ as a function of the radial distance and $\zeta$ (Table 4.7). These calculations were done for concrete shields. McCaslin (McC85) demonstrated that the Moyer Model approach is also effective for moderately energetic heavy ions. It has also been found that the Moyer Model approach works well even in the intermediate energy region of $200<E_{o}<1000 \mathrm{MeV}$. This may be interpreted as due to the relatively smooth dependence of neutron yield upon incident proton kinetic energy. The Moyer Model generally does not provide sufficiently accurate results at forward angles. For these situations, the Boltzmann equation must be solved usually with Monte Carlo calculations.

\subsection{The Use of Monte Carlo Shielding Codes for Hadronic Cascades}

\subsubsection{Examples of Results of Monte Carlo Calculations}

It should be obvious that validity of the Moyer Model approach is limited to simple 
Table 4.5 Values of the Ratio $M_{T}(2.3, \zeta) / M(2.3, \zeta)$ as a function of $\zeta$. [Adapted from (Fa90).]

\begin{tabular}{|c|c|c|c|}
\hline$\zeta$ & $\boldsymbol{M}_{\mathbf{T}}(\mathbf{2 . 3}, \boldsymbol{\zeta}) / \boldsymbol{M}(\mathbf{2 . 3} / \zeta)$ & $\zeta$ & $\boldsymbol{M}_{\mathbf{T}}(\mathbf{2 . 3}, \boldsymbol{\zeta}) \mathbf{M}(\mathbf{2 . 3} / \zeta)$ \\
\hline 0.2 & 0.27 & 11 & 1.02 \\
\hline 1 & 0.53 & 12 & 0.99 \\
\hline 2 & 0.75 & 13 & 0.95 \\
\hline 3 & 0.90 & 14 & 0.91 \\
\hline 4 & 1.00 & 15 & 0.86 \\
\hline 5 & 1.06 & 16 & 0.82 \\
\hline 6 & 1.09 & 17 & 0.78 \\
\hline 7 & 1.10 & 18 & 0.73 \\
\hline 8 & 1.10 & 19 & 0.69 \\
\hline 9 & 1.08 & 20 & 0.65 \\
\hline 10 & 1.06 & & \\
\hline
\end{tabular}

Table 4.6 Angular integration limits in $\theta$ (degrees) which contain $90 \%$ of the Moyer Integral $M(2.3, \zeta)$. [Adapted from (Fa90).]

\begin{tabular}{|c|c|c|c|c|c|}
\hline$\zeta$ & Lower Limit & Upper Limit & $\zeta$ & Lower Limit & Upper Limit \\
\hline 2.5 & 31.52 & 106.58 & 12 & 57.25 & 106.29 \\
\hline 3 & 24.35 & 107.15 & 13 & 58.45 & 106.04 \\
\hline 4 & 39.00 & 107.64 & 14 & 59.74 & 105.78 \\
\hline 5 & 42.67 & 107.73 & 15 & 60.66 & 105.54 \\
\hline 6 & 45.77 & 107.66 & 16 & 61.49 & 105.29 \\
\hline 7 & 48.51 & 107.48 & 17 & 62.34 & 105.04 \\
\hline 8 & 50.69 & 107.28 & 18 & 63.22 & 104.80 \\
\hline 9 & 52.7 & 107.04 & 19 & 64.08 & 104.54 \\
\hline 10 & 54.34 & 106.79 & 20 & 64.63 & 104.30 \\
\hline 11 & 56.07 & 106.54 & & & \\
\hline
\end{tabular}

Table 4.7 Distances corresponding to $\mathbf{9 0 \%}$ limits in Moyer Integrals. [Adapted from (Fa90).]

\begin{tabular}{|c|c|c|c|c|c|}
\hline $\begin{array}{c}\text { Radial } \\
\text { Distance } \\
\text { (meters) }\end{array}$ & $\begin{array}{c}\text { Thickness } \\
\text { (concrete) } \\
\text { (meters) }\end{array}$ & $\begin{array}{c}\text { Thickness } \\
\text { (concrete) } \\
\zeta\end{array}$ & $\begin{array}{c}\text { Upstream } \\
\text { Limit, } \mathbf{z}_{\mathbf{1}} \\
\text { (meters) }\end{array}$ & $\begin{array}{c}\text { Downstream } \\
\text { Limit, } \mathbf{z}_{\mathbf{2}} \\
\text { (meters) }\end{array}$ & $\begin{array}{c}\text { Total Length } \\
\mathbf{z}_{\mathbf{2}}-\mathbf{z}_{\mathbf{1}} \\
\text { (meters) }\end{array}$ \\
\hline 1.5 & 0.5 & 1.0 & -4.2 & 0.3 & 4.5 \\
\hline 2.0 & 1.0. & 2.0 & -3.7 & 0.6 & 4.3 \\
\hline 3.5 & 2.5 & 5.0 & -3.8 & 1.1 & 4.9 \\
\hline 6.0 & 5.0 & 10.0 & -4.3 & 1.8 & 6.1 \\
\hline 8.5 & 7.5 & 15.0 & -4.8 & 2.4 & 7.2 \\
\hline 11.0 & 10.0 & 20.0 & -5.2 & 2.8 & 8.0 \\
\hline
\end{tabular}

shielding configurations. Furthermore, one cannot include magnetic fields. Also, the model is not valid at forward angles and for kinetic energies lower than a few hundred $\mathrm{MeV}$. It is also incapable of handling the production of other types of particles aside from neutrons that can often be copiously produced at forward angles. Labyrinth penetrations and residual radioactivity considerations are unaddressed. Thus, the Monte Carlo 


\section{Chapter 4 Prompt Radiation Fields Due to Protons and Ions}

technique is the primary to use in such work. Appendix A describes a number of Monte Carlo programs that have been developed at various laboratories for a variety of purposes. In this section, methods of using results from such computations are reviewed.

The code HETC remains an important benchmark. A simple example of the results of a calculation performed using this code is shown in Fig. 4.23 taken from Alsmiller's results (AL75) for $200 \mathrm{MeV}$ protons incident on "thin" and "thick" aluminum targets. It is a plot of $r^{2} \mathrm{H}$ as a function of angle for several intervals of $\theta$ in a spherical concrete shield with the beam incident on a target at the center of the sphere. For higher energies, CASIM and FLUKA have also served the role as benchmark programs while MARS is undoubtedly at the time of this writing the most versatile. FLUKA and MARS model a multitude of physical effects in detail.

Representative results for solid iron and concrete cylinders bombarded by protons of various energies are provided in Figs. 4.24, 4.25, and 4.26. These values allow one to estimate the dose equivalent per incident proton at various locations and for various proton beam energies. They are also useful for obtaining a quick understanding of the effects of a beam absorber. Detailed calculations should be performed to assure adequately accurate designs.

\subsubsection{General Comments on Monte Carlo Star-to-Dose Conversions}

Several of these codes calculate the star density as their most basic output quantity. This quantity, generally denoted by $S$, is more correctly called the density of inelastic interactions $\left(\right.$ stars $\mathrm{cm}^{-3}$ ) and is relatively easy to tabulate as the calculation proceeds since only a simple counting process is involved. The term "star" comes from historic cosmic ray experiments in which the high energy interaction events, with their large multiplicities, appeared as tracks originating from a point. In a shield comprised of more than one material, the star density may change dramatically from one material boundary to the other, reflective of differing material densities and atomic numbers. A related quantity is the star fluence, denoted by $\Phi_{S}$. Star fluence is the product of the star density and the nuclear interaction length. The star fluence roughly corresponds to the fluence of hadrons having energies above that where the cross section "levels off". It is also reflective of any "artificial" thresholds in the calculation. In contrast with star density, due to the property of continuity the star fluence is conserved across material boundaries.

The dose equivalent per star density is a rather important parameter of radiation protection calculations. Perhaps the best results have been provided by Stevenson (St86). While this conversion factor is somewhat dependent upon the position in the shield, after a shield thickness sufficient to establish "equilibrium" spectra, a constant value may be used for high energy protons (i.e., $E_{\text {proton }}>1.0 \mathrm{GeV}$ ), and other hadrons, within a given material. In other words, the energy and spatial dependences are rather weak. Values for these quantities, as well as the related dose equivalent per star fluence conversion factors, are given in Table 4.8. 


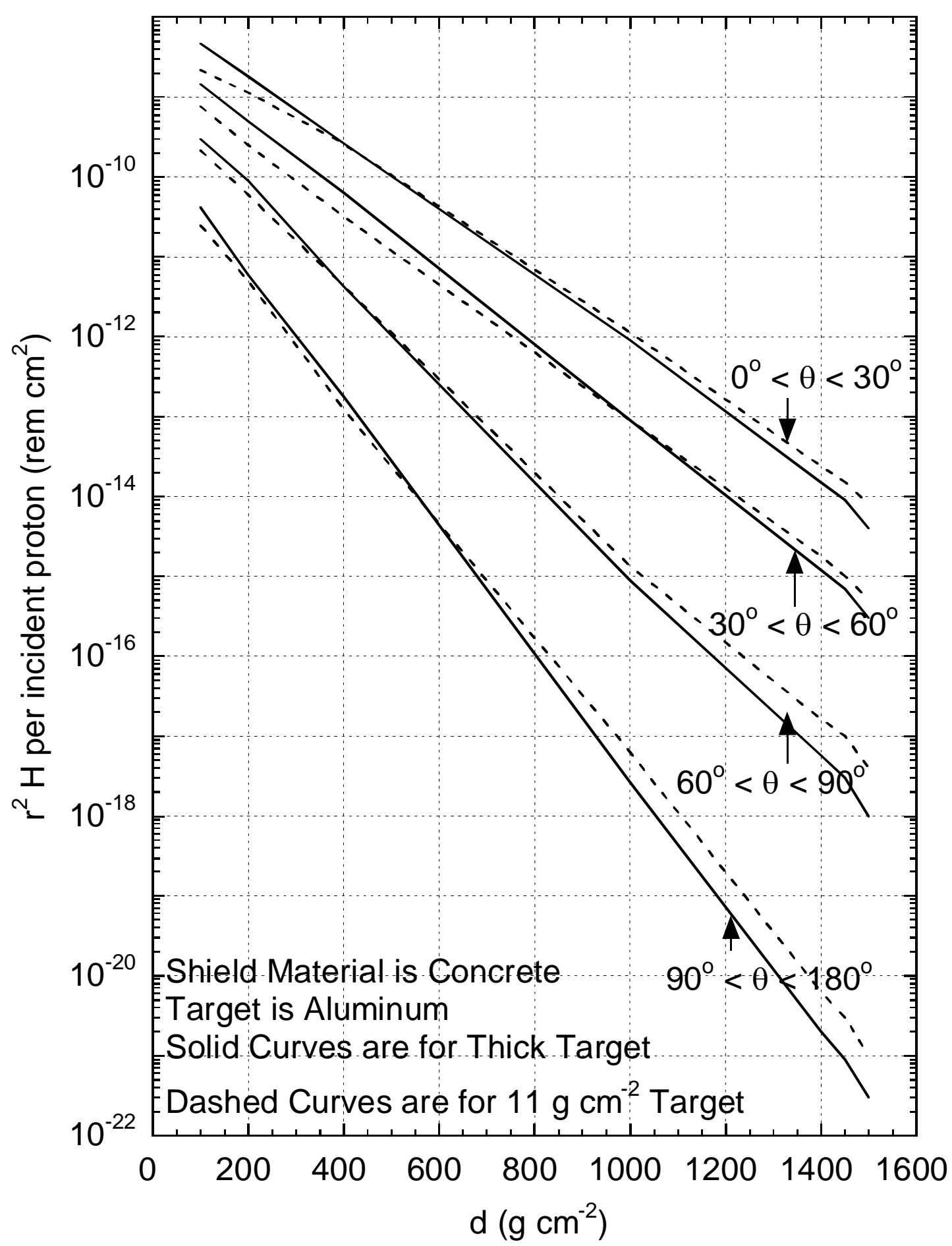

Fig. 4.23 HETC calculations of $r^{2} H$ as a function of CONCRETE shield thickness $d$ averaged over several intervals of $\theta$ for $200 \mathrm{MeV}$ protons incident on an aluminum target centered in a spherical shield. [Adapted from (A175).] 


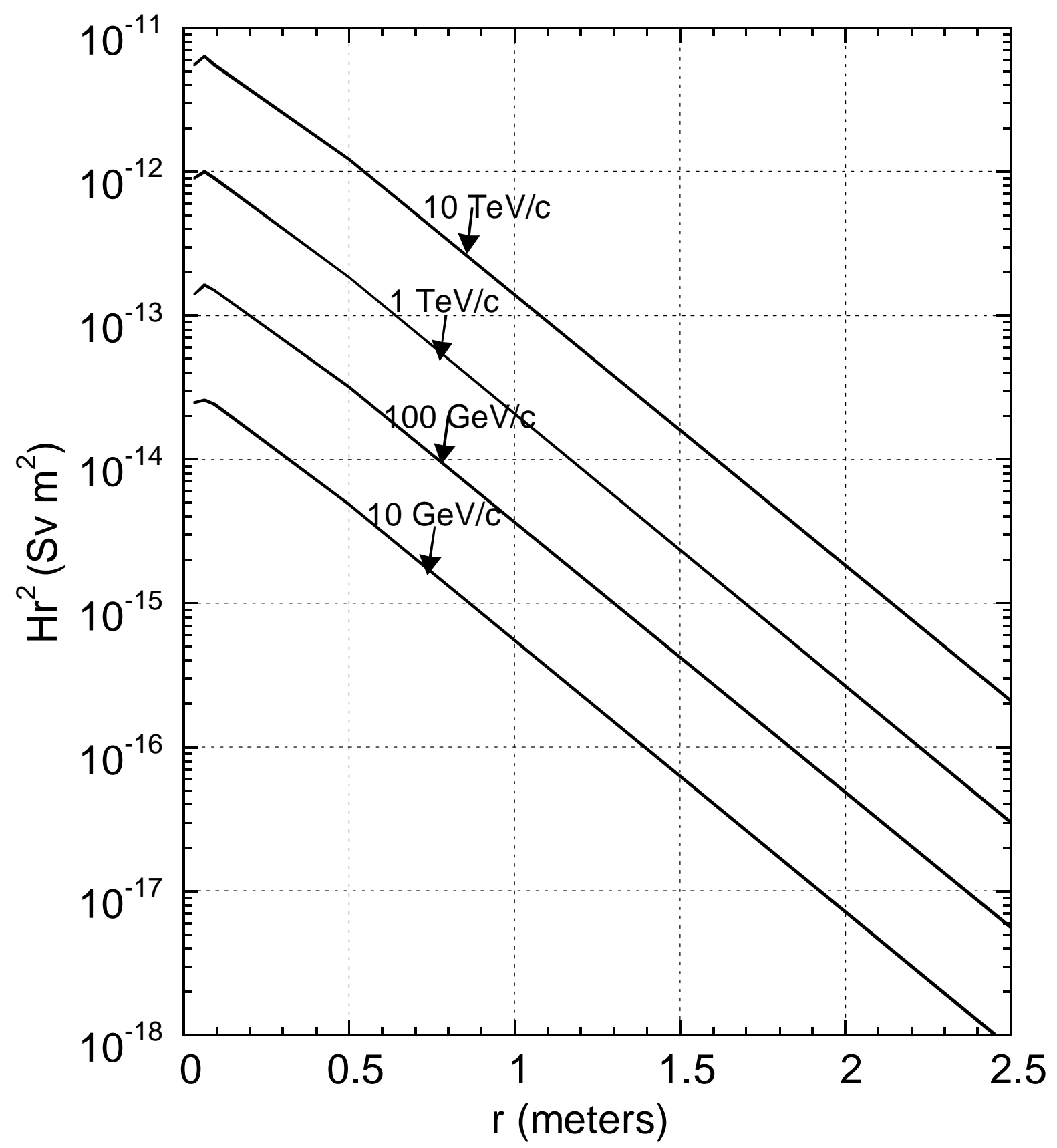

Fig. 4.24 Variation of the dose equivalent per proton at the position of the longitudinal maximum multiplied by the square of the radius $H r^{2}$ versus radius $r$ for proton-induced cascades in IRON of density $7.2 \mathrm{~g} \mathrm{~cm}^{-3}$. The coordinate $r$ is defined as in Fig. 4.22. The results are fits to calculations obtained using FLUKA and CASIM. [Adapted from (Fa90).] 


\section{Chapter 4 Prompt Radiation Fields Due to Protons and Ions}

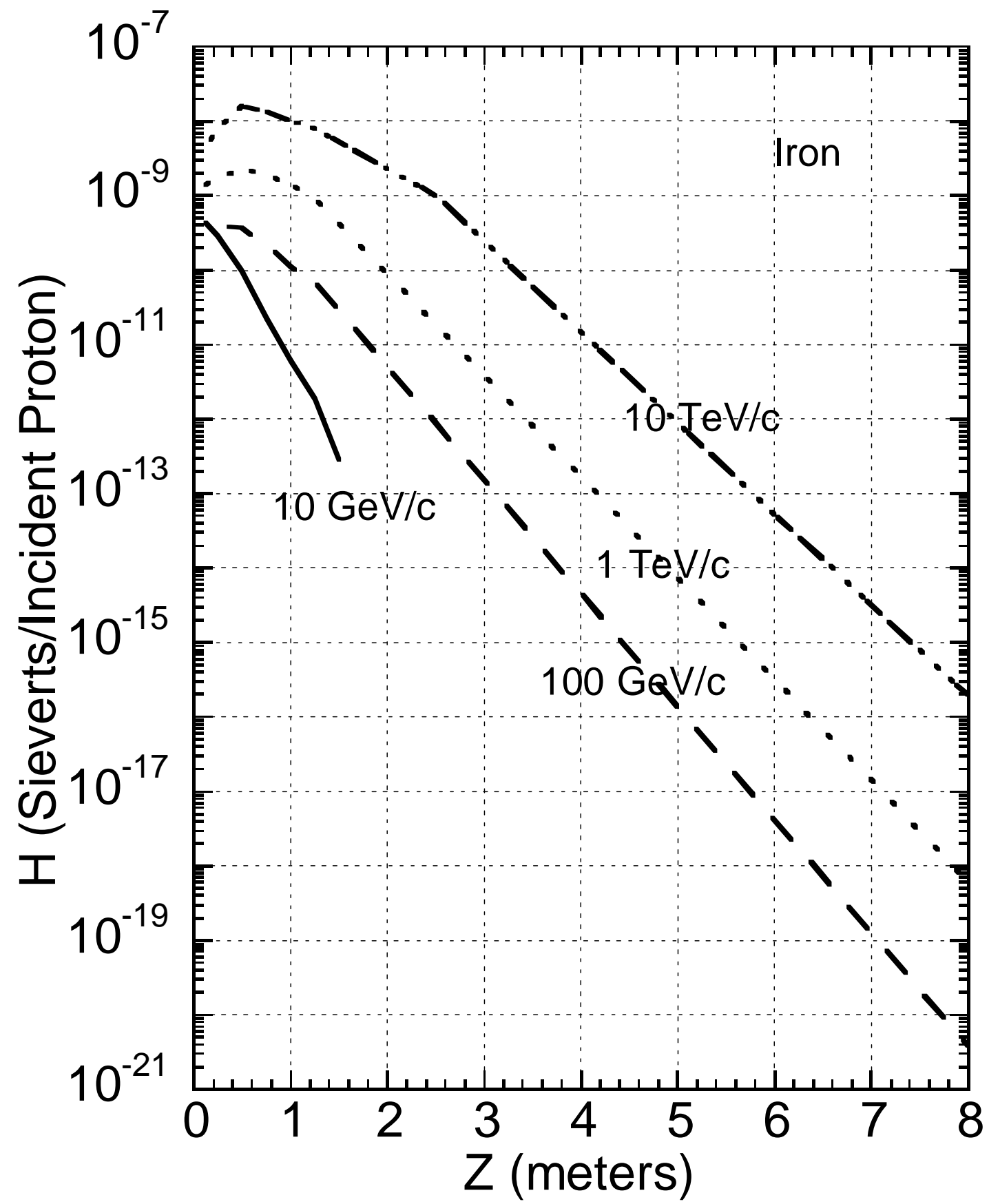

Fig. 4.25 Dose equivalent per proton $H$ on the longitudinal axis $Z$ as a function of depth $Z$ in the shield for proton-induced cascades in IRON of density $7.2 \mathrm{~g} \mathrm{~cm}^{-3}$. The curves are the result of CASIM calculations for incident proton momenta of $100 \mathrm{GeV} / \mathrm{c}, 1.0 \mathrm{TeV} / \mathrm{c}$, and $10 \mathrm{TeV} / \mathrm{c}$ and FLUKA results for $10 \mathrm{GeV} / \mathrm{c}$. [Adapted from $(\mathrm{Fa} 90)$.] 


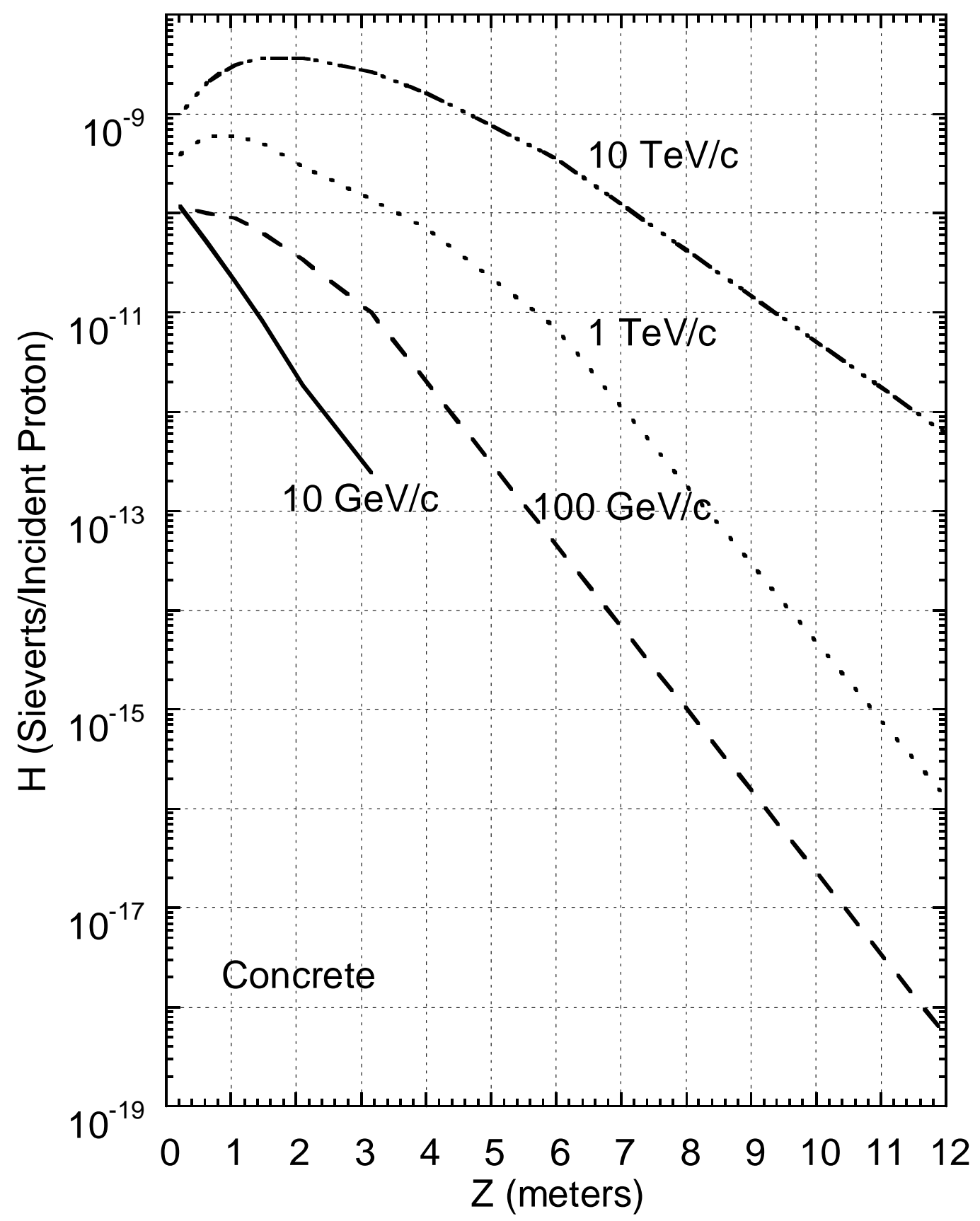

Fig. 4.26 Dose equivalent per proton $H$ on the longitudinal axis $Z$ as a function of depth $Z$ in the shield for proton-induced cascades in CONCRETE of density $2.4 \mathrm{~g} \mathrm{~cm}^{-3}$. The curves are the result of CASIM calculations for incident proton momenta of $100 \mathrm{GeV} / \mathrm{c}, 1.0 \mathrm{TeV} / \mathrm{c}$, and $10 \mathrm{TeV} / \mathrm{c}$ and FLUKA results for $10 \mathrm{GeV} / \mathrm{c}$. [Adapted from (Fa90).] 


\section{ChAPTER 4 Prompt RAdiation Fields Due to Protons AND Ions}

Table 4.8 Coefficients to convert star densities $S$ and star fluence $\Phi_{S}$ into dose equivalent. A star density is transformed into the corresponding star fluence by the relation $\Phi_{S}=S \lambda$ where $\lambda$ is the nuclear interaction length. [Adapted from (St86).]

\begin{tabular}{|c|c|c|c|c|}
\hline $\begin{array}{c}\text { Proton } \\
\text { Energy } \\
\text { (GeV) }\end{array}$ & $\begin{array}{l}\text { Absorber } \\
\text { Material }\end{array}$ & $\begin{array}{l}\text { Dose Equivalent/Star } \\
\text { Density } \\
\left(\mathrm{Sv} \mathrm{cm}^{3} \text { star }^{-1}\right)\left(\mathrm{x10}^{-8}\right)\end{array}$ & $\lambda(\mathbf{c m})$ & $\begin{array}{c}\text { Dose Equivalent/Star } \\
\text { Fluence } \\
\left(\mathrm{Sv} \mathrm{cm}^{2} \mathrm{star}^{-1}\right)\left(\mathrm{x10}^{-9}\right)\end{array}$ \\
\hline 10 & Iron $^{a}$ & $2.04+0.06$ & 17.1 & $1.19 \pm 0.04$ \\
\hline 100 & Iron $^{\mathrm{a}}$ & $2.15+0.08$ & 17.8 & $1.21 \pm 0.05$ \\
\hline 1000 & Iron $^{\mathrm{a}}$ & $2.12+0.08$ & 17.2 & $1.23+0.05$ \\
\hline Mean & Iron $^{\mathrm{a}}$ & $2.10 \pm 0.04$ & & $1.21 \pm 0.02$ \\
\hline 100 & Aluminum & $4.62+0.17$ & 38.6 & $1.20 \pm 0.04$ \\
\hline \multirow[t]{2}{*}{100} & Tungsten & $1.19 \pm 0.05$ & 9.25 & $1.29 \pm 0.05$ \\
\hline & Concrete & 4.9 & 40.0 & 1.22 \\
\hline Mean & All & & & $1.22 \pm 0.02$ \\
\hline
\end{tabular}

${ }^{\mathrm{a}}$ As discussed in detail in Section 6.3.5, iron shielding presents a unique problem due to the copious emission of low energy neutrons in shields of modest thickness. The values reported here are for relatively thin iron shields of only one or two mean free paths. If a thick iron shield is encountered that is not "finished" with at least $50 \mathrm{~cm}$, or so, of concrete as the outermost layer, one should multiply these conversion factors by a factor of approximately 5 . This table implies use of the material density values of Table 1.2.

Compilations of such calculations have been given by Van Ginneken (Va75 and Va87) Cossairt (Co82b), and Reitzner (Re12). Fassò et al. (Fa90) have also compiled a comprehensive set of Monte Carlo results. A convenient way to display these results is to provide contour plots of star density as function of longitudinal coordinate $Z$ and radial coordinate $R$ assuming cylindrical symmetry. Appendix B provides examples of results of hadronic Monte Carlo calculations that are meant to illustrate a number of situations commonly encountered. One of the salient advantages of the Monte Carlo method is the ability to handle configurations of arbitrary complexity and results for both solid cylinders and more complicated commonly encountered configurations are provided in Appendix B.

\subsubsection{Shielding Against Muons at Proton Accelerators}

The production of muons has been discussed previously in Section 4.2.4. At higher energies, there are significant complications in that muon creation mechanisms, in addition to the production of pions and kaons and their subsequent decays, are possible. However, the muons from pion decay and kaon decay generally, but not universally, represent the most important consideration in practical shielding calculations. In Monte Carlo calculations, it is straightforward to "create" muons and follow them through the shielding medium. Muon transport in material is well understood [(Co89a), (Co89b)].

The particle energy downgrades quickly in hadronic showers so the most penetrating muons must originate in the first few generations of the cascade process. These energetic muons are not distributed over a large volume of space as are the neutrons. However, geometric effects such as collimation, or deflections by magnetic fields encountered near 


\section{ChAPTER 4 Prompt RAdiAtion Fields Due to Protons AND IONS}

the point of production, can affect the muon fluence at large distances. Thus, the presence of large "empty" spaces, that is, decay paths (vacuum or air), near the point of interaction provide opportunity for the pions or kaons to decay into muons before they can be removed by nuclear interactions in solid materials. This is particularly important for the typical situation of a target used to produce secondary beams followed (downstream) by an air or vacuum gap (the space for decay into muons) and then a beam absorber. If magnetic fields are present, the muon fluence generally peaks in the bend plane. Multiple Coulomb scattering from nuclei is an important effect in muon transport.

Generally the dominant sources of muons are those due to the decay of pions and kaons. There are several important facts about such muons that are summarized below:

A. The decay lengths (mean length for $\pi$ or $\mathrm{K}$ to decay), $\Lambda$, are given by:

$\Lambda_{\pi}=55.9 p$ (meters), where $p$ is the pion momentum in $\mathrm{GeV} / \mathrm{c}$, and

$\Lambda_{\mathrm{K}}=7.51 p$ (meters), where $p$ is the kaon momentum in $\mathrm{GeV} / \mathrm{c}$.

The available decay path in conjunction with the decay length can be used to estimate the total number of muons present. For example, a beam of $10^{7} \mathrm{such}$ pions at $20 \mathrm{GeV} / \mathrm{c}$ over a distance of 50 meters will decay into $10^{7} \times[50$ meters $] /[(56 \times 20)$ meters decay length $]=4.5 \times 10^{5}$ muons. This uses the fact that the decay path (50 meters) is small compared with the mean decay length of 1120 meters. If the decay path $x$ were comparable to the decay length $\Lambda$, the final intensity would need to be multiplied by the exponential factor $\{1-\exp (x / \Lambda)\}$.

B. If $\beta \approx 1$, relativistic kinematics determines that the ratio, $k_{i}$, of the minimum momentum of the daughter muon $\left(p_{\min }\right)$ to the momentum of the parent pion or kaon $\left(p_{i}\right)$ is given by

$$
k_{i}=p_{\min } / p_{\text {parent }}=\left(m_{\mu} / m_{\text {parent }}\right)^{2} .
$$

The result is that $k_{i}$ has a value of 0.57 for muons with pion parents and 0.046 for muons with kaon parents. Thus if, say, a beam transport system restricts the momentum of pions to some minimum value, then the momentum of the decay muons has a minimum value given by the above.

C. Since in the frame of reference of the kaon or pion parent the decay is isotropic, and there is a one-to-one relationship between the muon momentum, $p$, and the angle of emission, for $p>>m_{\text {parent }}$ (in units where $c=1$ ) the momentum spectrum of the muons can be expressed as

$$
\frac{d N}{d p}=\frac{1}{p_{\text {parent }}\left(1-k_{i}\right)} .
$$




\section{Chapter 4 Prompt RAdiation Fields Due to Protons AND Ions}

This means that the spectrum of daughter muons uniformly extends from the momentum of the parent down to the minimum established in Eq. (4.47).

D. Relativistic kinematics also gives the result that in the laboratory frame of reference the maximum angle $\theta_{\max }$ between the momentum vector of the muon and that of the parent particle is given by

$$
\tan \theta_{\max }=\frac{\left(m_{\text {parent }}^{2}-m_{\mu}^{2}\right)}{2 p_{\text {parent }} m_{\mu}} .
$$

For muons originating from pion decay, $\theta_{\max }$ is at most several milliradians. However, for muons originating from, say, the decay of $5.0 \mathrm{GeV}$ kaons, $\theta_{\max }$ is a relatively large $12^{\circ}$. Thus $\pi \rightarrow \mu$ decays can be assumed to be approximately collinear while $\mathrm{K} \rightarrow \mu$ decays have significant divergence at the lower energies.

Monte Carlo calculations are needed to adequately describe the production and transport of muons because of the sensitivity to details of the geometry that determine the pion and kaon flight paths and influence the muon populations. Fassò et al. (Fa90) has presented some useful information about the yield of muons that one can use to make approximate estimates by giving calculated values of angular distributions of muon spectra with an absolute normalization from pion and kaon decays for one meter decay paths. Neither the effects of absorbers nor magnetic fields are included in these results. For other decay paths that are short compared with the decay length, one can simply scale by the length of the actual decay path in meters. The results are displayed in Fig. 4.27.

Decays of other particles can be important sources of muons at higher energies, especially those found in hadron-hadron collisions at high energy colliders. Notable are those from charm (D) and bottom (B) meson decays (Fa90). The muons from these sources are often called direct muons due to the short lifetimes and decay lengths involved. The masses of these parent particles and their mean-lives $\tau$ are (PDG04):

$$
\begin{aligned}
& m\left(\mathrm{D}^{ \pm}\right)=1869.4 \pm 0.5 \mathrm{MeV}, \tau=(1.040 \pm 0.007) \times 10^{-12} \mathrm{~s}, c \tau=311.8 \mu \mathrm{m} \text { and } \\
& m\left(\mathrm{~B}^{ \pm}\right)=5279.0 \pm 0.5 \mathrm{MeV}, \tau=\left(1.671_{ \pm}+0.018\right) \times 10^{-12} \mathrm{~s}, c \tau=501 \mu \mathrm{m} .
\end{aligned}
$$

Figs. 4.28 and 4.29 give results for muons originating from these decays in the same format as used in Fig. 4.27. The length of the decay path is irrelevant for these small values of $c \tau$.

Sullivan's approximation for muons is a method of estimating muon flux densities at proton accelerators based upon a semi-empirical fit to existing muon production data (Su92). Eq. (4.50) gives Sullivan's result for the flux density of muons per meter of decay path as a function of shield thickness found along the proton beam axis (that is, on the straight-ahead maximum of the muons); 


\section{Chapter 4 Prompt Radiation Fields Due to Protons AND Ions}

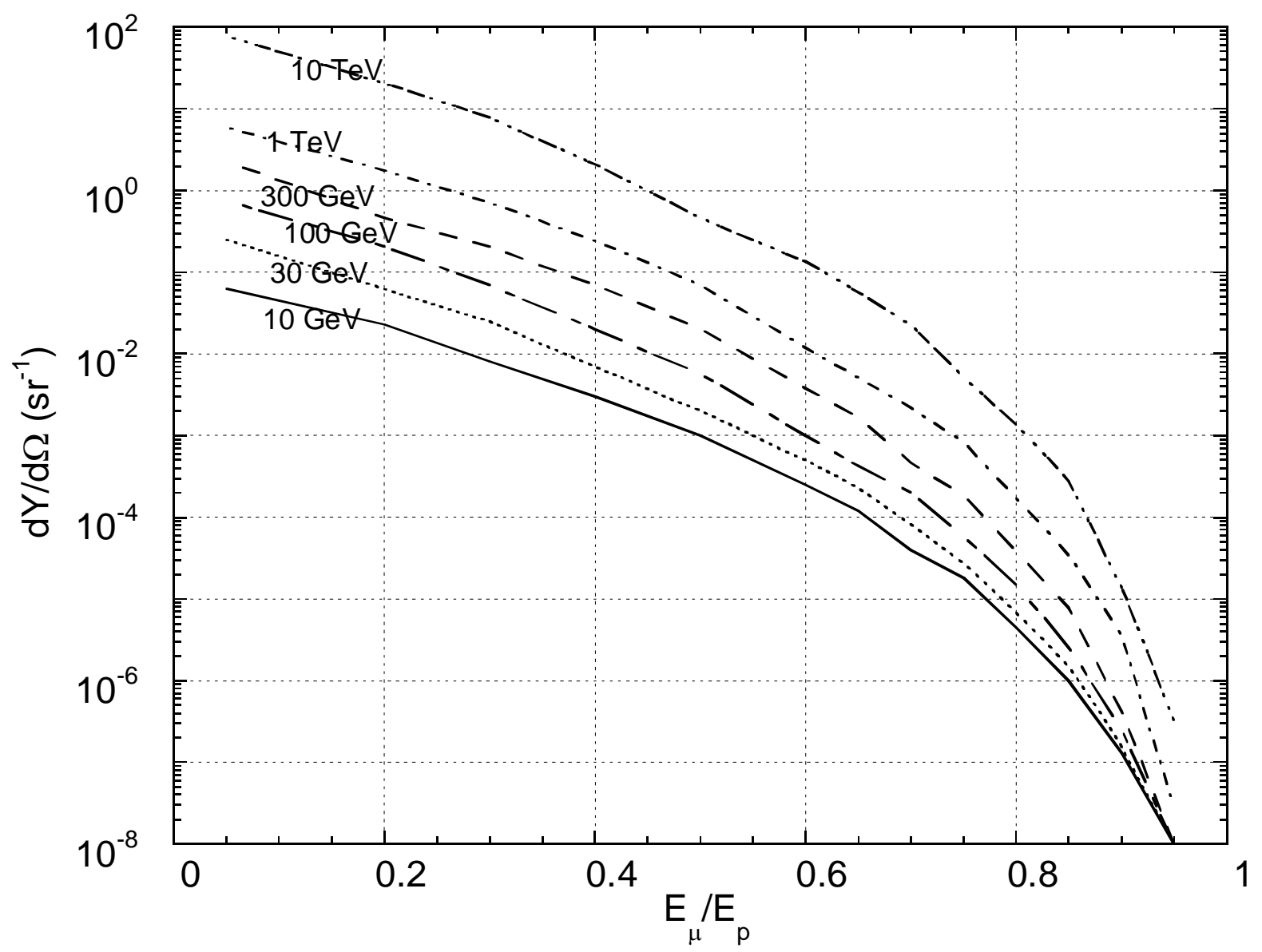

Fig. 4.27 Yield of muons from the decay of pions and kaons of both charges produced in proton-Fe collisions at several energies of the incident proton at $\theta=0$. The distance available for decay (the decay path) is taken to be 1.0 meter. The abscissa $E_{\mu} / E_{p}$ is the muon energy expressed as a fraction of the incident proton energy. The ordinate $d Y / d \Omega$ is the number of muons per unit solid angle $\left(\mathrm{sr}^{-1}\right)$ per incident proton having an energy greater than $E_{\mu} \cdot$ [Adapted from $(\mathrm{Fa} 90)$.]

$$
\Phi=0.085 \frac{E z}{Z^{2}} \exp \left\{-\frac{\alpha t}{E}\right\},
$$

where $\Phi$ is the fluence (muons $\mathrm{m}^{-2}$ ) per interacting proton, $E$ is the proton beam energy $(\mathrm{GeV}), Z$ is the distance of the point of concern to the point of production of the pions and kaons (meters), $z$ is the average path length (i.e., the decay path) of the pions and kaons in air, gases, or vacuum prior to their absorption by solids or liquids, and $\alpha$ is an effective average energy loss rate $\left(\mathrm{GeV}\right.$ meter $\left.^{-1}\right)$ for the muons in a shield of thickness $t$ (meters). Values of $\alpha$ for typical shielding materials are provided in Table 4.9. $z$ can be taken to be the actual physical length of the decay path, or according to Sullivan, for a solid beam absorber, $z$ can reasonably be taken to be 1.8 times the hadron nuclear interaction mean free path for the material comprising the beam absorber. It is obvious that the argument of the exponential in Eq. (4.50) can be expanded as the sum over the materials comprising a composite shield. Sullivan has also given a prescription for 


\section{Chapter 4 Prompt Radiation Fields Due to Protons and Ions}

calculating the full width at half maximum FWHM of the muon distribution at the boundary of such a shield:

$$
F W H M=4.6 \frac{Z}{\sqrt{E \alpha t}} \text { (meters). }
$$

Table 4.9 Values of $\alpha$ for typical shielding materials for use in Eqs. (4.50) and (4.51) according to Sullivan (Su92).

\begin{tabular}{|c|c|c|}
\hline Material & $\alpha\left(\mathrm{GeV} \mathrm{m}^{-1}\right)$ & Density, $\rho\left(\mathrm{g} \mathrm{cm}^{-3}\right)$ \\
\hline Concrete $^{\mathrm{a}}$ & 9.0 & 2.35 \\
\hline Water & 4.0 & 1.0 \\
\hline Iron & 23.0 & 7.4 \\
\hline Lead & 29.0 & 11.3 \\
\hline
\end{tabular}

${ }^{\mathrm{a}}$ The value for concrete can be used for earth if one adjusts it to the correct density.

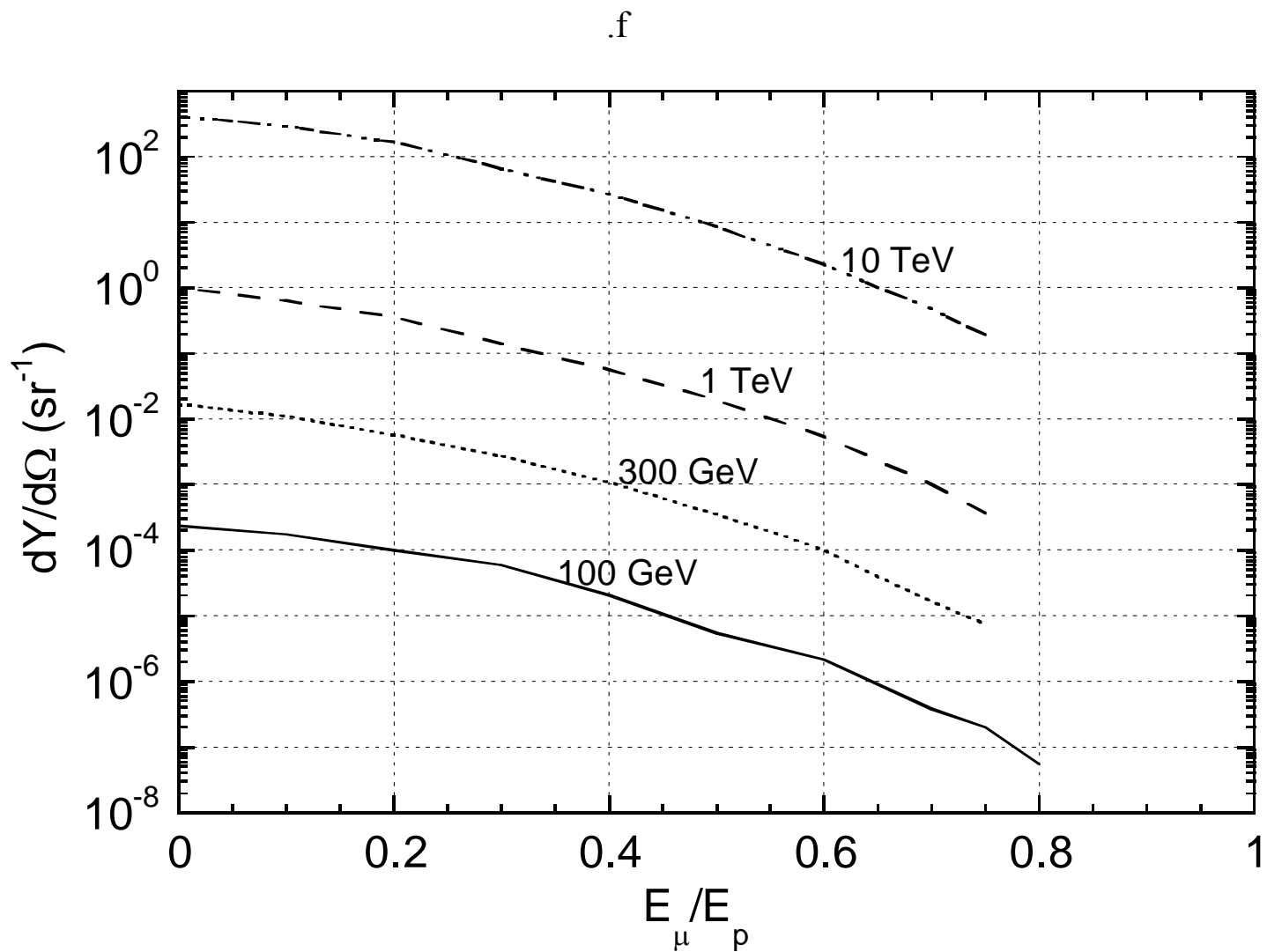

Fig. 4.28 Yield of muons from the decay of D-mesons produced in proton-proton collisions at four incident proton energies and at $\theta=0$. The abscissa $E_{\mu} / E_{p}$ is the muon energy expressed as a fraction of the incident proton energy. The ordinate $d Y / d \Omega$ is the number of muons per unit solid angle $\left(\mathrm{sr}^{-1}\right)$ per incident proton having an energy greater than $E_{\mu}$ [Adapted from (Fa90).] 


\section{Chapter 4 Prompt Radiation Fields Due to Protons and Ions}

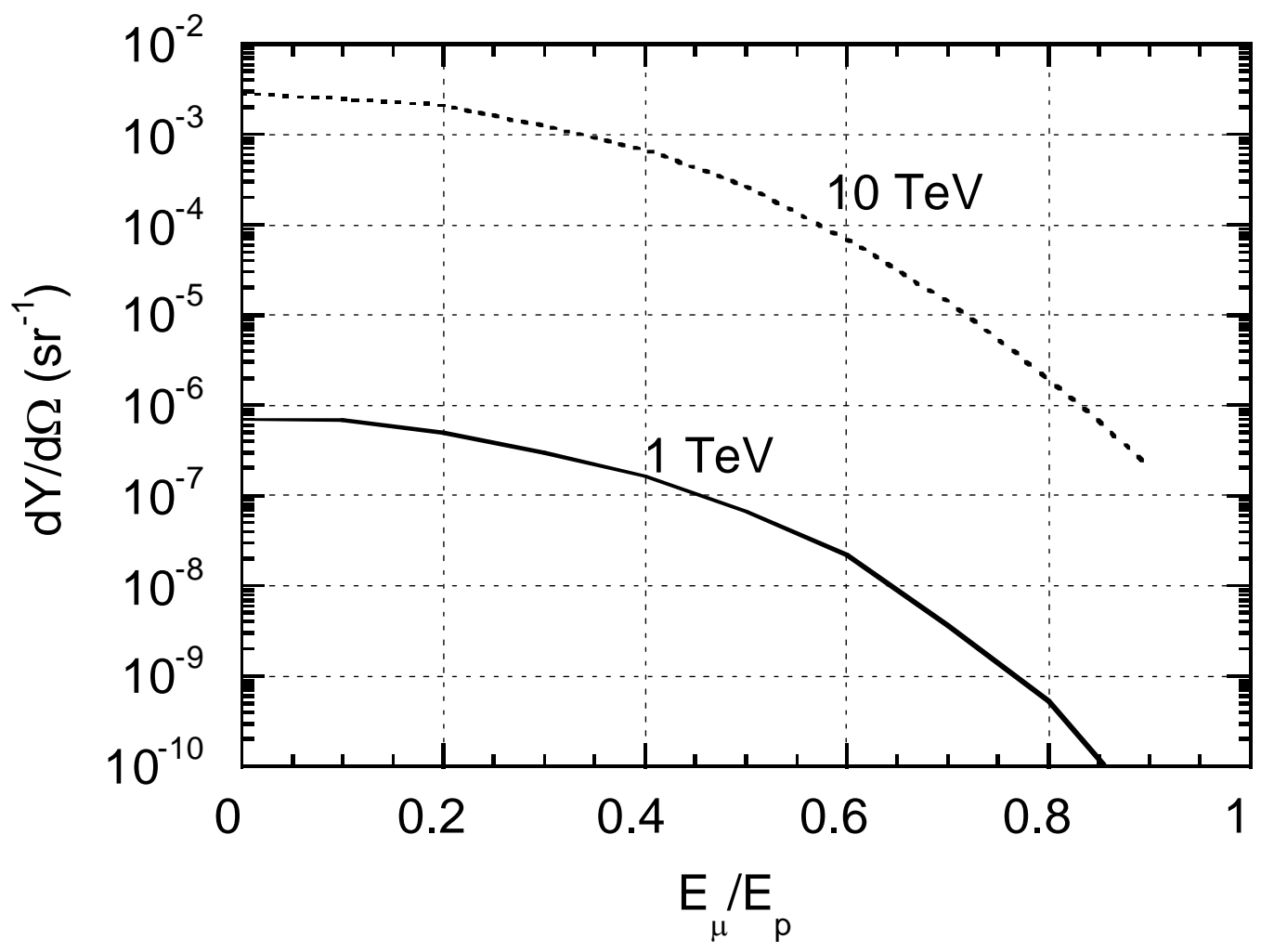

Fig. 4.29 Yield of muons from the decay of B-mesons produced in proton-proton collisions at various energies of the incident proton and at $\theta=0$. The abscissa $E_{\mu} / E_{p}$ is the muon energy expressed as a fraction of the incident proton energy. The ordinate $d Y / d \Omega$ is the number of muons per unit solid angle $\left(\mathrm{sr}^{-1}\right)$ per incident proton having an energy greater than $E_{\mu}$. [Adapted from $(\mathrm{Fa} 90)$.] 


\section{Chapter 4 Prompt RAdiation Fields Due to Protons AND Ions}

\section{Problems}

1. One can use measurement results to check Sullivan's formula, Eq. (4.4), for hadron fluence above $40 \mathrm{MeV}$ for high-energy proton interactions. Check the agreement for the 22 and $225 \mathrm{GeV} / \mathrm{c}$ data in Figs. 4.7 and 4.8 for 3 representative angles at one meter. (Ignore the fact that the formula is for hadrons $>40 \mathrm{MeV}$ while the only data provided is for hadrons with energies $>35 \mathrm{MeV}$ and $>50$ $\mathrm{MeV}$, but do not ignore the difference between normalizing to incident versus interacting protons.) (It is valid to make the comparison on yield per interacting proton since the results in Fig. 4.8 is for targets approximately one interaction length long.) Comment on the quality of the agreement.

2. Calculations can also be used to check the Tesch curve for dose equivalent at $\theta=90^{\circ}$ (Fig. 4.10). Use the $200 \mathrm{MeV}$ calculations in Fig. 4.4 to do this by crudely numerically integrating the $60^{\circ}<\theta<90^{\circ}$ yields to determine the average energy of the neutrons and the total fluence at $\theta=90^{\circ}$ and at 1.0 meter. Use the results along with dose equivalent per fluence curves to obtain the dose equivalent per proton to compare with Tesch's result. (Iron is considered equivalent to copper for this problem.)

3. A copper target at an accelerator is struck by $1.0 \mu \mathrm{A}$ of $100 \mathrm{MeV}$ protons.

a) Use Tesch's curve in Fig. 4.10 to calculate the dose equivalent rate at $2.0 \mathrm{~m}$ and $\theta=90^{\circ}$ relative to this target.

b) Compare this result with the neutron dose equivalent rate calculated in Chapter 3, Problem 5 for an electron accelerator having the same intensity and beam energy and discuss. (Scale the relevant result of Chapter 3, Problem 5 by the appropriate yield for copper versus tungsten.)

4. It is often necessary to work from fragmentary data to determine other quantities.

a) Use McCaslin's results, Eqs. (4.13, 4.14, and 4.15), and the appropriate dose equivalent fluence ${ }^{-1}$ factors to calculate the dose equivalent rate at 1.0 meter and at $\theta=30^{\circ}$ for a target struck by $10^{8} 670 \mathrm{MeV} / \mathrm{amu}{ }^{20} \mathrm{Ne}$ ions per sec. Compare this with the effective dose rate. (Hint: Use all available spectrum information.)

b) Use McCaslin's results to obtain the total yield of neutrons per ion with $E_{n}>6.5$ $\mathrm{MeV}$. Assuming the target to be iron or copper, how does this yield correspond to that due to $700 \mathrm{MeV}$ protons? Do this for both $E_{n}>6.5 \mathrm{MeV}$ and $E_{n}>20 \mathrm{MeV}$ to understand the overall composition. Hint: Integrate over the unit sphere (double integral over spherical coordinates $\theta \& \phi$ ) and convert all quantities associated with angles from degrees to radians. The following indefinite integrals are needed:

$$
\int \frac{d x \sin x}{x}=x-\frac{x^{3}}{3 \times 3 !}+\frac{x^{5}}{5 \times 5 !}-\frac{x^{7}}{7 \times 7 !}+\ldots \text { and } \int d x e^{a x} \sin b x=\frac{e^{a x}[a \sin b x-b \cos b x]}{a^{2}+b^{2}}
$$




\section{ChAPTER 4 Prompt RAdiation Fields Due to Protons AND Ions}

The elemental area on the sphere of radius $r$ is $d A=r^{2} \sin \theta d \theta d \phi$, where $\phi$ is the standard azimuthal coordinate in a spherical coordinate system.

5. It is asserted that if the assumption is made that the limiting attenuation is simply geometric, with the nucleon radius equal to $1.2 \times 10^{-13} \mathrm{~cm}$, then $\rho \lambda_{\text {atten }}=36.7 A^{1 / 3}(\mathrm{~g}$ $\mathrm{cm}^{-2}$ ). Show this to be the case using the volume of a nucleus and nucleons along with the cross section.

6. a) Use the Moyer Model to calculate the dose equivalent rate (mrem h${ }^{-1}$ ) lateral $\left(\theta=90^{\circ}\right)$ to a magnet centered in a $1.5 \mathrm{~m}$ radius tunnel. The magnet is struck by $10^{12}$ protons at $100 \mathrm{GeV}$ per second. The tunnel walls consist of $0.333 \mathrm{~m}$ concrete followed by soil having the same composition $\left[\rho\right.$ (concrete) $=2.5 \mathrm{~g} \mathrm{~cm}^{-3}$, $\rho$ (soil) $\left.=2.0 \mathrm{~g} \mathrm{~cm}^{-3}\right]$. Perform the same calculation for several thicknesses of soil out to 6 meters of soil radially. Do this for increments of 1.0 meter from 1.0 meter to 6.0 meters of soil.

b) Calculate the result if the same beam loss occurs uniformly over a string of such magnets 100 meters long in the same tunnel at the same soil thicknesses as above. Use the Tesch approximation. Approximately how many meters of beam loss does it take to cause $90 \%$ of the calculated dose equivalent rate at $6.0 \mathrm{~m}$ of lateral soil shield?

c) For the point loss in part a), at what value of $\theta$ does the maximum dose equivalent rate occur and what is its magnitude outside of 6 meters of soil shield? (Use successive approximations to solve.)

7. a) An accelerator delivers $10^{12} 120 \mathrm{GeV}$ protons per second head-on on the inner edge of a magnet located in a cylindrical tunnel centered on the beam axis. Use the results of the most relevant MARS calculation found in Appendix B to determine the approximate effective dose rate at $R=400 \mathrm{~cm}$ and compare with a result using the Moyer equation for a point loss. Both calculations should be at the location of the maximum effective dose. Assume the geometrical parameters to be identical to those in the Appendix B calculation and $\rho$ (concrete $)=2.4 \mathrm{~g} \mathrm{~cm}^{-3}$ and $\rho$ (soil) $=2.24$ $\mathrm{g} \mathrm{cm}^{-3}$. Ignore the difference between effective dose and dose equivalent for this comparison. What might explain the difference between the two results?

b) As a further comparison, consider an accelerator that delivers $10^{12} 1.0 \mathrm{TeV}$ protons per second head-on on the inner edge of a magnet. Use the CASIM calculations found in Appendix B to determine the approximate dose equivalent rate at $R=400$ centimeters and compare with a result using the Moyer equation for point loss.

Both calculations should be at the location of the maximum dose equivalent. Once again assume the geometrical parameters to be identical to those in the Appendix B calculation and $\rho$ (concrete) $=2.4 \mathrm{~g} \mathrm{~cm}^{-3}$ and $\rho$ (soil) $=2.25 \mathrm{~g} \mathrm{~cm}^{-3}$. What might explain the apparent disagreement between the two results? 


\section{Chapter 4 Prompt RAdiation Fields Due to Protons AND Ions}

c) Discuss any general observations from the results of these two calculations.

8. Using the results of Monte Carlo hadron calculations (FLUKA/CASIM) calculate, for solid shields of iron (cylinders), what longitudinal thickness of iron is needed to achieve the same hadron dose equivalent per proton on the beam axis as found at $R=50 \mathrm{~cm}$ at $10 \mathrm{GeV} / \mathrm{c}, 100 \mathrm{GeV} / \mathrm{c}, 1000 \mathrm{GeV} / \mathrm{c}$ and $10 \mathrm{TeV} / \mathrm{c}$. Use the maximum value of $H(r=50 \mathrm{~cm})$.

9. In Fig. 4.4, we have calculations of neutron energy spectra for $200 \mathrm{MeV}$ protons incident on various targets, including aluminum. In Fig. 4.23, calculations of dose equivalent values for spherical concrete shielding surrounding aluminum targets at $E_{p}=200 \mathrm{MeV}$ are given. At shielding thicknesses approaching zero and at forward angles, are the two results in "sensible" (that is, approximate, agreement)? (Hint: Integrate" crudely over the forward spectrum to obtain the fluence/proton and convert this fluence to dose equivalent.)

a) Make the comparison for zero shield thickness and in the angular range $0<\theta<30^{\circ}$.

b) Now use the shielding calculations to obtain the dose equivalent rate $\left(\mathrm{rem} \mathrm{h}^{-1}\right)$ due to a $1 \mu \mathrm{A}$ beam incident at $200 \mathrm{MeV}$ on such a thick target at a distance of $4 \mathrm{~m}$ from the target with $0,1,2, \& 3 \mathrm{~m}$ of intervening concrete shielding ( $\rho=2.5$ $\mathrm{g} \mathrm{cm}^{-3}$ ) for $\theta=15^{\circ}$ and $\theta=75^{\circ}$. (Hint: Use the center of the angular bins.)

10. Assume that a target is struck by $100 \mathrm{GeV}$ protons and that a $10 \mathrm{~m}$ long decay space exists for $\pi$ and $\mathrm{K}$ decay. Use the curves in Fig. 4.27 to crudely estimate the muon flux density and dose equivalent rates $\left(\mathrm{mrem} \mathrm{h}^{-1}\right)$ at $1.0 \mathrm{~km}$ away and at $\theta=0^{\circ}$ if $10^{12}$ protons/second are targeted in this manner with the following additional assumptions are made:

a) Assume that there is no shielding present and neglect air scattering and inscattering from the ground. (Hint: The muon yield for this decay space will scale with the length of the decay space.)

b) Assume there is 100 meters of intervening shielding of earth $\left(\rho=2 \mathrm{~g} \mathrm{~cm}^{-3}\right)$ (Hint: Use Fig. 1.9 range-energy curves to determine the mean energy of muons which will penetrate this much shielding). Neglect multiple scattering and rangestraggling.

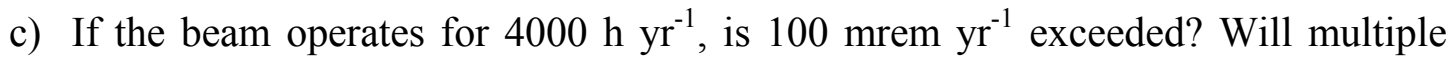
scattering increase or decrease this dose equivalent? (Answer both questions for the soil-shielded case only.)

d) Repeat Part b) of the same calculation using Sullivan's semi-empirical approach. If the disagreement between the results obtained using the two methods is large, suggest an explanation of a possible cause of the difference. 


\section{Chapter 5 Low Energy Prompt Neutron Radiation Phenomena}

\section{$5.1 \quad$ Introduction}

In this chapter two phenomena that involve low-energy particles, most importantly neutrons, are discussed; the transmission of photons and neutrons through penetrations and the control of neutron "skyshine". They must be understood at most accelerators, particularly those that operate above the energy thresholds for producing neutrons. Both phenomena exhibit behavior qualitatively independent of incident particle type and energy.

\subsection{Transmission of Photons and Neutrons Through Penetrations}

All neutron-producing accelerators need to control the transmission of photons and neutrons through penetrations since all have accessways to permit entry of personnel and equipment. Penetrations for cables, for radio-frequency (RF) waveguides, and other equipment are also present. Personnel access penetrations will typically have crosssectional dimensions of about $1.0 \mathrm{~m}$ by $2.0 \mathrm{~m}$ (door-sized) while utility ducts will generally be much smaller. Often the utility penetrations are partially filled with cables and other items as well as pipes delivering cooling water.

Penetrations are commonly called labyrinths, mazes, or even chicanes [e.g., Sullivan (Su92)]. Two general rules are advised for all penetrations of accelerator shielding:

- A particle or photon beam should not be aimed directly toward a penetration. This assures that the penetrations are transmitting primarily scattered particles.

- For any labyrinth, the sum of the wall thickness between the source and the "outside" should be equivalent to that which would be required if the labyrinth were not present. The "void" presented by the passageway does not provide any shielding laterally!

\subsubsection{Albedo Coefficients}

Before describing the details of penetration design, one should review some simple parameterizations of the scattering of photons and neutrons. These reflections can be treated through the use of reflection or albedo coefficients. Such coefficients account for the reflection of particles analogous to the diffuse reflection of visible light by various kinds of surfaces. They take into account the appropriate microscopic scattering cross sections in a macroscopic way. These have general applications beyond the design of penetrations. Figs. 5.1 and 5.2 give the albedo coefficients $\alpha_{x}$ and $\alpha_{n}$ for monoenergetic photons and neutrons, respectively, incident on flat surfaces of infinite dimensions of concrete plotted as functions of energy for various conditions of incidence. Clearly, the albedo of neutrons is typically larger and somewhat less strongly dependent on energy than is that of photons. Chilton et al. have given more detailed results for concrete and for other materials [(Ch63), (Ch64), (Ch65a), (Ch65b), and (Ch84)]. A good summary is provided in (NC03). 

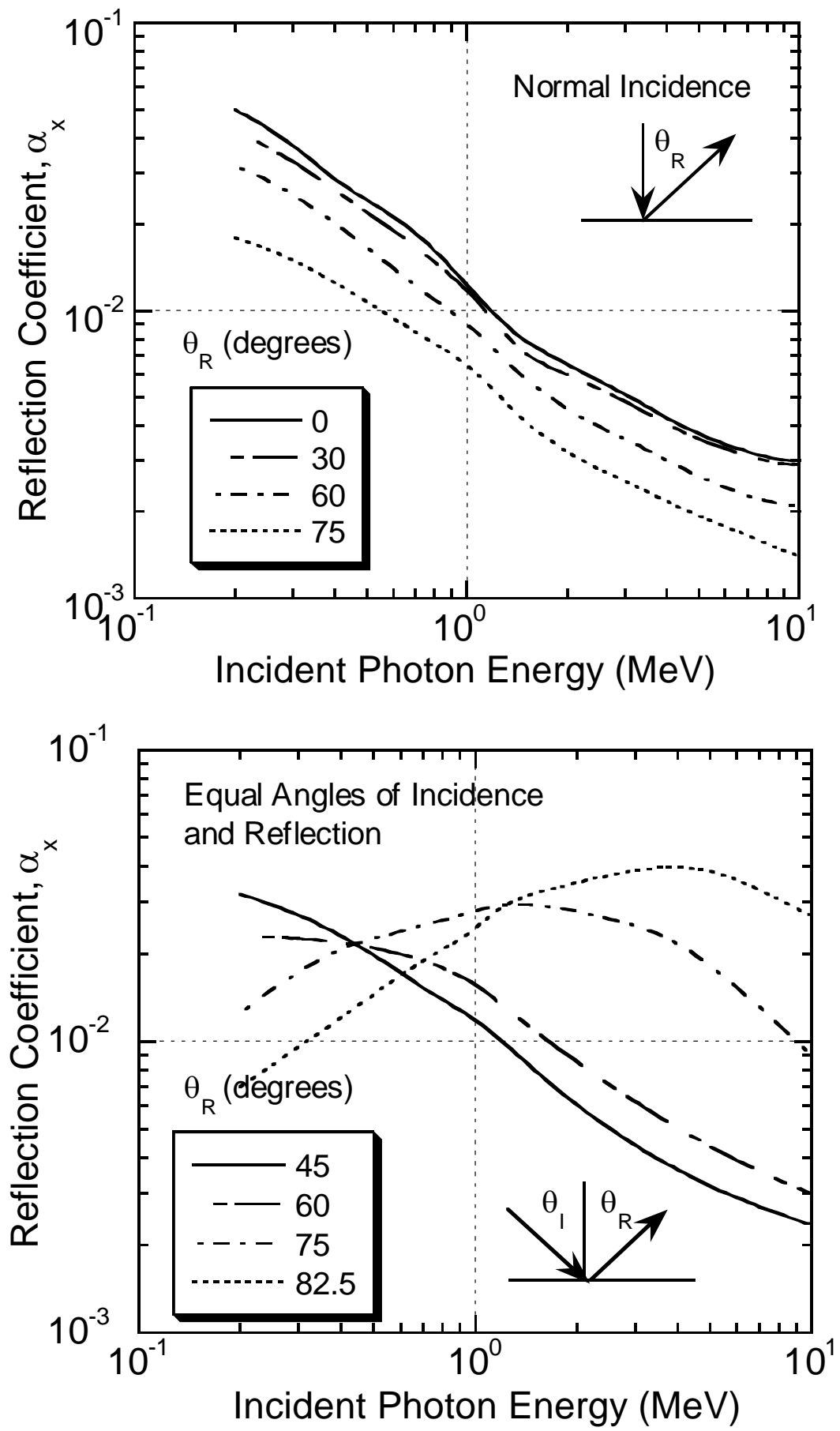

Fig. 5.1 Reflection coefficients $\alpha_{x}$ for monoenergetic PHOTONS incident on ordinary concrete as a function of incident photon energy for several angles of reflection assuming normal incidence (top frame) and for equal angles of incidence and reflection $\left(\theta_{l}=\theta_{R}\right)$ (bottom frame). For photon energies higher than $10 \mathrm{MeV}$, the use of the $10 \mathrm{MeV}$ values of $\alpha_{x}$ is expected to be conservative. [Adapted from ( $\mathrm{NC03}$ ) and references cited therein.] 

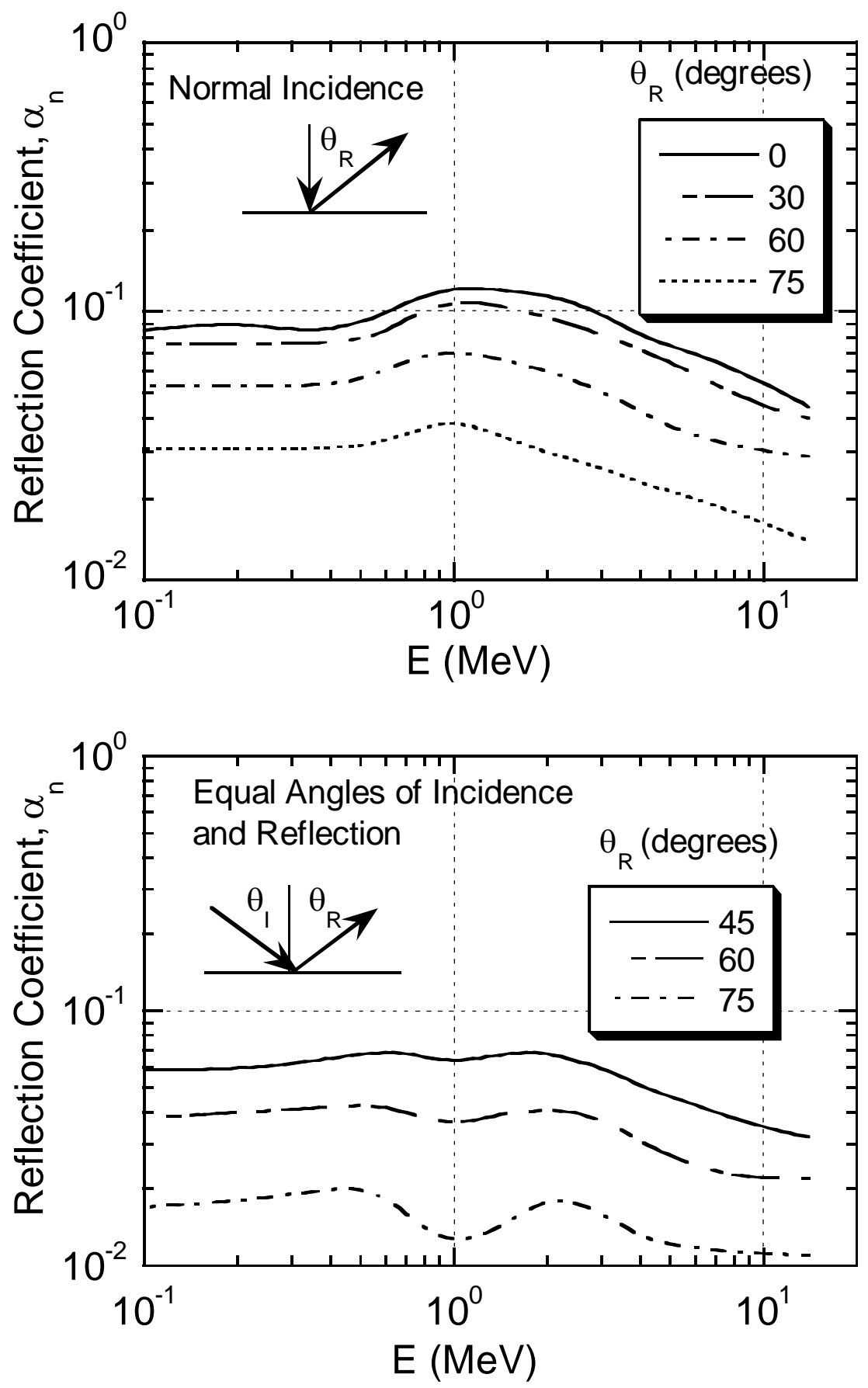

Fig. 5.2 Reflection coefficients $\alpha_{n}$ for monoenergetic NEUTRONS incident on ordinary concrete as a function of incident neutron energy for several angles of reflection assuming normal incidence (top frame) and for equal angles of incidence and reflection $\left(\theta_{1}=\theta_{R}\right)$ (bottom frame). [Adapted from ( $\mathrm{NC03}$ ) and references cited therein.] 


\section{Chapter 5 Low Energy Prompt Neutron Radiation Phenomena}

\subsubsection{Usage of Photon Albedo Coefficients}

A particular application of these coefficients in the design of labyrinths is given here as an illustration. Fig. 5.3 shows an example of a labyrinth providing access to a collimated photon source of some known dose equivalent or effective dose (or dose rate, with inclusion of units of inverse time) $H_{\mathrm{o}}$ determined at some reference distance $d_{\mathrm{o}}$. To use these coefficients correctly, knowledge of the photon energy spectrum at this location is also needed. For example, such a de facto photon "beam" can arise from the forwardpeaked photons due to the targetry of a beam from an electron accelerator. With the reflection coefficients $\alpha_{x}$, one can use the following formula to obtain a conservative estimate of the dose equivalent or effective dose (or dose rate) $H_{r j}$ after $j$ sections (not counting the initial path length to the wall $d_{i}$ ) of the maze:

$$
H_{r_{j}}=H_{0}\left(\frac{d_{0}^{2}}{d_{i}^{2}}\right)\left(\frac{\alpha_{1} A_{1}}{d_{r_{1}}^{2}}\right) \prod_{k=2}^{k=j}\left(\frac{\alpha_{k} A_{k}}{d_{r_{k}}^{2}}\right), j \geq 2 .
$$

In this formula, the coefficient $\alpha_{1}$ is selected to be representative of that expected at the initial photon energy while $A_{1}$ estimates the cross sectional area of the wall struck by the initial photons evaluated by projecting the beam profile to the wall. For $k \geq 2, A_{k}$ is the cross-sectional area of the $k^{\text {th }}$ leg of the maze. The first parenthetical factor is just inverse square propagation of the beam to the wall, the second factor models "reflection" into the first leg, and the product factor accounts for reflection into the remaining legs. For right angle, straight labyrinths such as this one, it is reasonable to use the values plotted for normal incidence $\left(\theta_{\mathrm{I}}=90^{\circ}\right)$ and $\theta_{\mathrm{R}}=75^{\circ}$. For successive legs after the first, taking the value of $\alpha_{k}$ to be that for $0.5 \mathrm{MeV}$ photons is often considered to be a conservative approach. This is because if $E_{\mathrm{o}}$ is the initial photon energy in $\mathrm{MeV}$, the energy of the scattered photon, $E_{\text {scatt }}(\mathrm{MeV})$, following Compton scattering is given by

$$
E_{\text {scatt }}=\frac{E_{0}}{1+\left(E_{0} / 0.511\right)(1-\cos \theta)} .
$$

Thus, $E_{\text {scatt }}$ has a maximum value of $0.511 \mathrm{MeV}$ after a scatter of $90^{\circ}$ for $E_{0}>>0.511$ $\mathrm{MeV}$, the rest energy of the scattered electron. If the maze is of uniform cross section $A$ and has $j$ legs, then the product in the numerator is simply $\alpha A$ raised to the $(j-1)^{\text {th }}$ power, $(\alpha A)^{j-1}$, where $\alpha=\alpha_{k}$ for all legs after the first. In the denominator, the distances are just those defined in Fig. 5.3 and, of course, represent the inverse-square law dependence. This formula is "conservative" for photon energies exceeding $10 \mathrm{MeV}$, but at the higher energies the uncertainties are larger. This approach is probably most accurate if the ratios $d_{r k} /\left(A_{k}\right)^{1 / 2}$ lie between 2.0 and $6.0(\mathrm{NC} 03)$. 


\section{Chapter 5 Low Energy Prompt Neutron Radiation Phenomena}

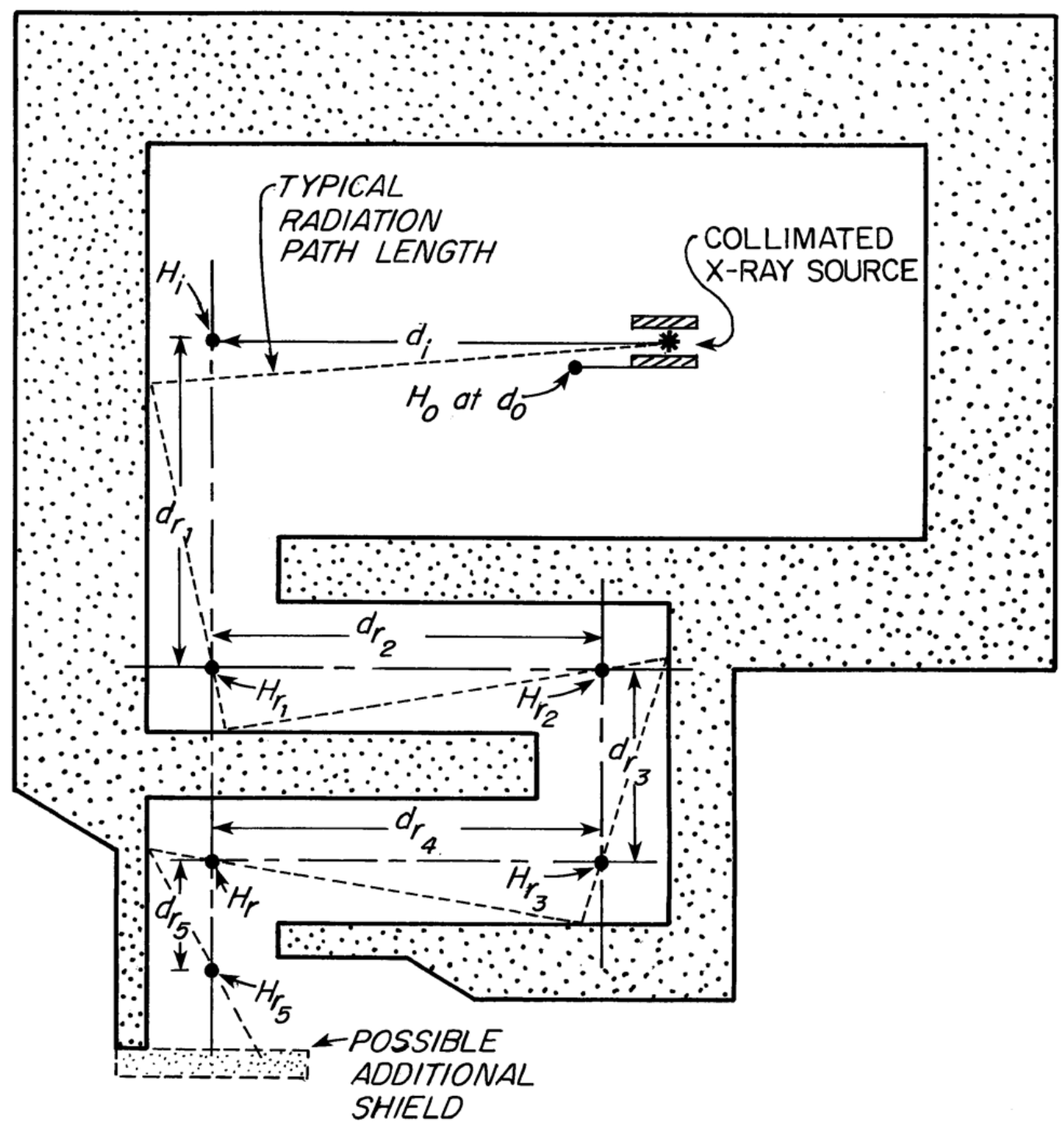

Fig. 5.3 Generalized labyrinth design illustrating successive reflections of photons from a collimated source through the maze. The source could just as well originate from an electron beam originating from the right side of the figure incident on a target located at the point in space labeled "collimated x-ray source". The various path lengths can be approximated by a sequence of centerline distances, as shown in the diagram. [Reproduced from (NC03).]

\subsubsection{Neutron Attenuation in Labyrinths-General Considerations}

Unfortunately, the more complex physics of the transport of neutrons discourages the use of a similar formula similar to that employed above using photon albedo coefficients. The radiation source, or potential radiation source for situations of concern from the standpoint of accidental beam losses, should be evaluated according to the methods described previously. Typical methods for addressing the attenuation of radiation by 


\section{Chapter 5 Low Energy Prompt Neutron Radiation Phenomena}

penetrations involve the use of the results of calculations performed using Monte Carlo codes. These can be used for both straight and curved labyrinths, but most practical experience is with the straight variety. In this section, the results of such work will be presented in order to give the reader useful information in the evaluation of such penetrations. A typical straight line personnel access labyrinth is shown in Fig. 5.4.

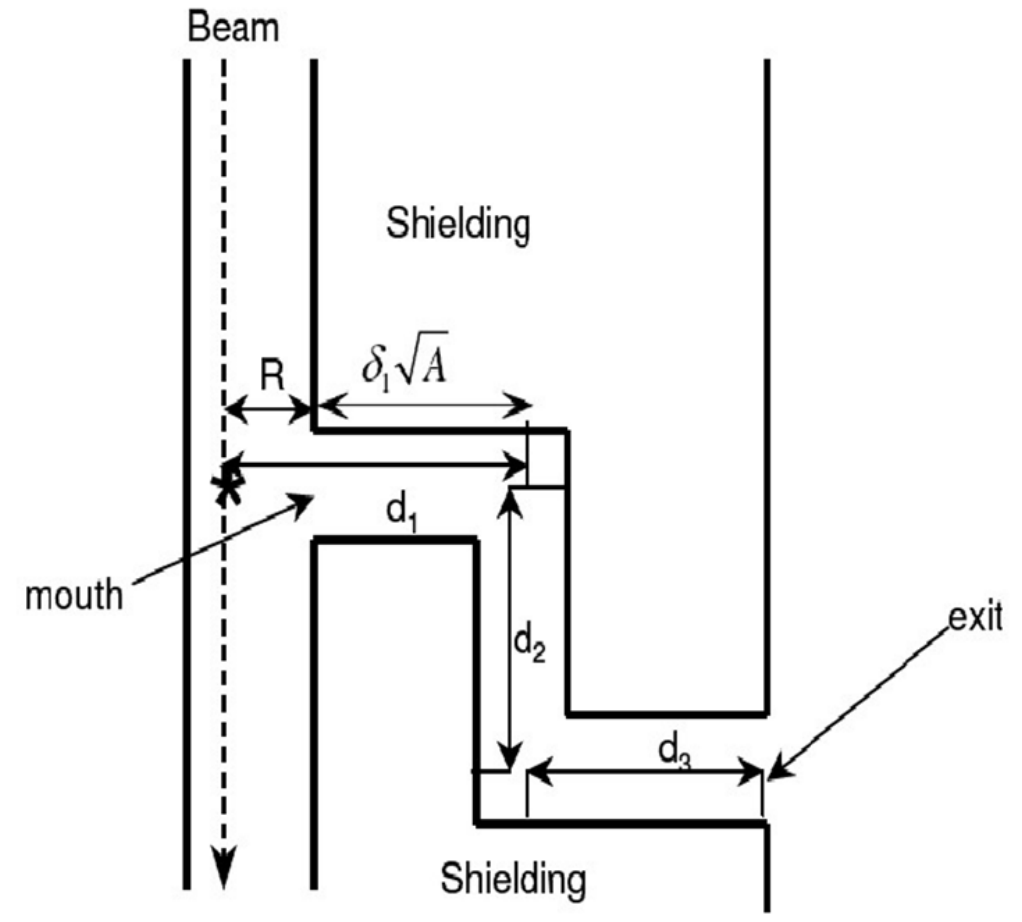

Fig. 5.4 Schematic plan view of a typical personnel access labyrinth of three "legs" at an accelerator that defines the coordinate system and terminology associated with labyrinth calculations. The $\left.{ }^{*}\right)$ denotes the location of a loss of beam at a point adjacent to the "mouth" of the labyrinth. The lengths of all legs after the first are measured between centers of turns.

An overwhelming conclusion drawn from existing body of data is that the bombarding particle energy, or even particle type, has very little effect upon the attenuation by a labyrinth viewing a source of beam loss other than the fact that the total yield of "source" neutrons increases as a function of incident energy and ion type. One can thus estimate the absorbed dose, dose equivalent, effective dose, or neutron fluence at the exit of a labyrinth by using attenuation estimates in the legs multiplied by an estimate of the neutron fluence or dose found at the entrance, or mouth, of the penetration into the beam enclosure. The validity of this factorization approximation allows attenuation measurements and calculations obtained at proton accelerators to be of general utility.

\subsubsection{Attenuation in the First Legs of Straight Penetrations}

For penetrations exposed to targets struck by hadrons, we first consider the straight penetration studied by Gilbert et al. (Gi68) who measured the transmission of a very long 


\section{Chapter 5 Low Energy Prompt Neutron Radiation Phenomena}

straight tunnel of dimensions $2.8 \mathrm{~m}$ high by $1.8 \mathrm{~m}$ wide and $100 \mathrm{~m}$ long. $14 \mathrm{GeV}$ protons were incident on a target providing a good "point source" $3.2 \mathrm{~m}$ from the tunnel mouth. The use of a set of activation detectors having different energy thresholds made it possible to obtain some information about the neutron energy spectrum as well. The measurement technique employed will be discussed in somewhat more detail in Chapter 9. An absolute normalization to beam loss was not reported. Table 5.1 gives the thresholds, or approximate sensitive domains, of nuclear reactions used in this particular measurement (see Section 9.5.3). The dosimeters used to detect photons were sensitive to gamma rays produced by the capture of neutrons in the air and in the tunnel walls.

Table 5.1 Detectors and their characteristics as used in the measurements summarized in Fig. 5.5. The sensitive energy ranges are approximate. [Adapted from (Gi68).]

\begin{tabular}{|l|l|l|}
\hline Detector & Nuclear Reaction & Energy Range (MeV) \\
\hline$\beta \gamma$ Dosimeters & photons and charged particles & all \\
\hline Gold $(\mathrm{Au})$ & $\left.{ }^{197} \mathrm{Au}(\mathrm{n}, \gamma)\right)^{198} \mathrm{Au}$ & Thermal Energies \\
\hline Aluminum $(\mathrm{Al})$ & ${ }^{27} \mathrm{Al}(\mathrm{n}, \alpha)^{24} \mathrm{Na}$ & $\mathrm{E}>6 \mathrm{MeV}$ \\
\hline Carbon $(\mathrm{C})$ & ${ }^{12} \mathrm{C}(\mathrm{n}, 2 \mathrm{n}){ }^{11} \mathrm{C}$ & $\mathrm{E}>20 \mathrm{MeV}$ \\
\hline
\end{tabular}

The results of the measurements are presented in Fig. 5.5. The "fits" to the relative response $R$ as a function of depth in the penetration $d$ shown in this figure were arbitrarily matched to the measurements at a depth of 20 meters in the tunnel and fit by an exponential attenuation multiplied by an inverse square-law dependence;

$$
R(d)=R_{20}\left(\frac{20}{d_{1}}\right)^{2} \exp \left[-d_{1} / \lambda(E)\right]
$$

where $R_{20}$ is the response measured at $d_{1}=20$ meters and $\lambda(E)$ is an energy-dependent attenuation length.

The responses as a function of depth $d_{1}$ are quite revealing. At short depths into the tunnel ( $<20 \mathrm{~m}$ long) the "attenuation" of the fast neutrons is almost entirely accounted for by inverse-square law considerations. Further into the tunnel, the responses clearly illustrate that neutrons of lower energy (i.e., as illustrated by the response for gold) attenuate more rapidly by air and wall-scattering than do the higher energy neutrons. Factoring out the inverse-square dependence for this long tunnel, the remaining attenuation is well-described by exponential absorption with effective mean free paths $\lambda$ corresponding to effective removal cross sections for the unique neutron spectral regions "seen" by the individual detectors. The $\lambda$ values determined by fitting these data are given in Table 5.2. The effective removal cross sections determined by this measurement are about a factor of 1.5 to 2.0 smaller than those that would be inferred from the known absorption cross sections of the constituents of air. This is evidence of "in-scattering" by the concrete walls since more neutrons than expected were observed at the larger distances into the tunnel. 


\section{Chapter 5 Low Energy Prompt Neutron Radiation Phenomena}

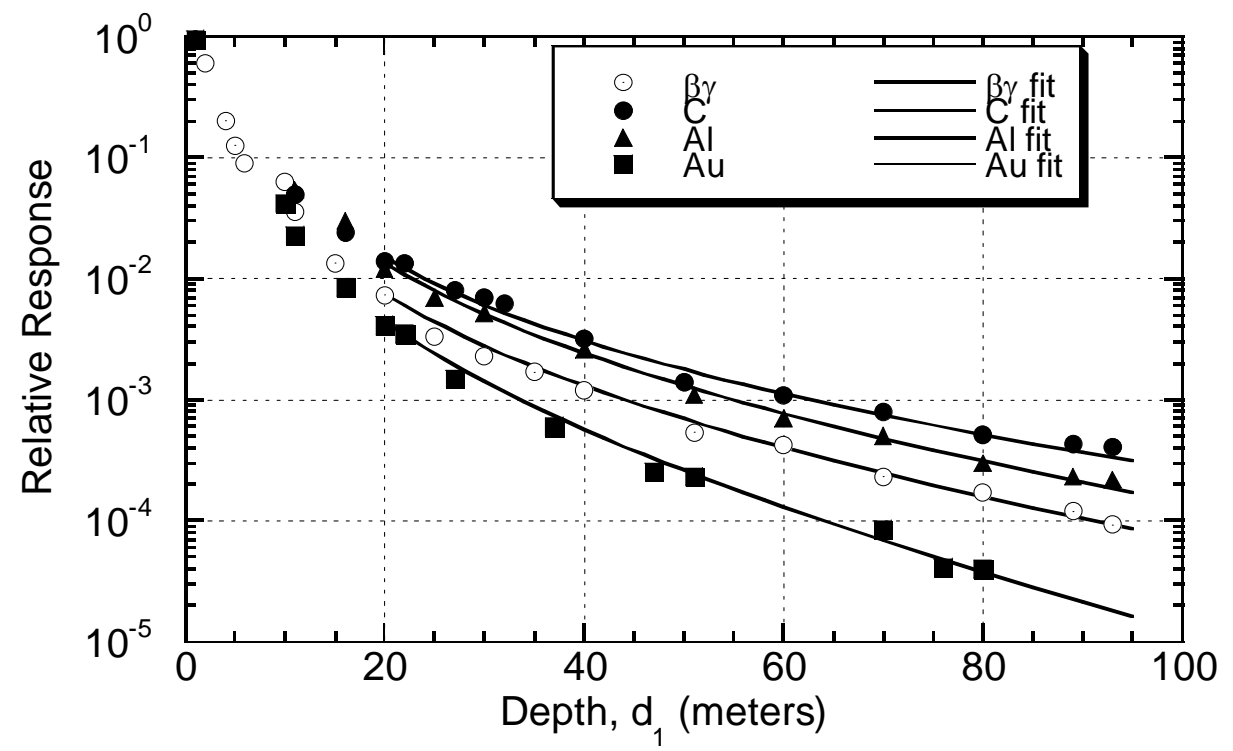

Fig. 5.5 The relative transmission of neutron flux density and gamma dose rate along a large straight tunnel described in the text. The measurement results are shown as the symbols while the solid lines represent the fits described in the text matched to the data at a depth of 20 meters. [Adapted from (Gi69).]

Table 5.2 Mean free paths and removal cross sections for tunnel transmission as exhibited by the measurements summarized in Fig. 5.5. [Adapted from (Gi69).]

\begin{tabular}{|l|c|c|}
\hline Detector & $\begin{array}{c}\lambda, \begin{array}{c}\text { Mean Free Path } \\
\text { (meters) }\end{array} \\
\text { Section (barns) }\end{array}$ & $\begin{array}{l}\text { Inferred Removal Cross } \\
\text { (mens }\end{array}$ \\
\hline$\beta \gamma$ Dosimeters & 55 & 3.3 \\
\hline Gold $(\mathrm{Au})$ & 30 & 6.2 \\
\hline Aluminum $(\mathrm{Al})$ & 60 & 3.2 \\
\hline Carbon $(\mathrm{C})$ & 100 & 1.9 \\
\hline
\end{tabular}

An important principle is the labyrinth scaling rule. The attenuation of neutrons in the legs of labyrinths generally scale with a unit length equal to the square root of its crosssectional area, provided that the height-to-width ratio does not vary greatly outside of the range 0.5 to 2.0 (Th88). Some details of the source geometry are very important in such a straight penetration. Goebel [(Go75), summarized in (Fa90)] has calculated universal attenuation curves for "first" legs of labyrinths (i.e., those sections first encountered as one moves outward from the beam). Goebel compared results from the codes SAM-CE (Co73), AMC (Ma67), and ZEUS [(D'H68), (Se71)]. Gollon and Awschalom (Go71) have generated similar curves using the ZEUS code for a variety of geometries. The three situations of point source, line source, and plane or point source off-axis for a straight tunnel displayed as universal dose attenuation curves as calculated by Goebel are given in Fig. 5.6. An off-axis point source is one not centered in front of the labyrinth mouth. For neutron radiation fields, the attenuation of a labyrinth is essentially the same for all the "dose" quantities discussed in Section $1.3 ; H_{\text {equiv }}, H_{\text {eff, }} D$, etc. 


\section{Chapter 5 Low Energy Prompt Neutron Radiation Phenomena}

The distance down the passageway is measured in units of the square root of the crosssectional area of the passageway. Clearly, neutrons from a plane or off-axis sources are more readily attenuated since the tunnel aperture provides less solid angle for acceptance.

Cossairt (Co95) found that Goebel's point source dependence in a tunnel of cross sectional area $A$ can be approximated by the following expression, where $\delta_{1}=\left(d_{1}-R\right) / A^{1 / 2}$ and $r_{o}$ is a fitting parameter;

$$
H_{1}\left(\delta_{1}\right)=\left[\frac{r_{o}}{\delta_{1}+r_{o}}\right]^{2} H_{o}(R) \text { with } r_{o}=1.4
$$

$H_{1}\left(\delta_{1}\right)$ is the dose at distance $\delta_{1}$ in the first leg as measured from the mouth of the passageway in "units" of the square root of the cross-sectional area of the first leg (see Fig. 5.4). $H_{\mathrm{o}}(R)$ is the dose at the mouth the determination of which will be discussed later. The result of this fit is included in Fig. 5.6. Over the domain of $0<\delta_{1}<9.0$ the expression fits the Goebel curve within $\pm 10 \%$, sufficiently accurate for most radiation protection purposes. The domain in $\delta_{1}$ is an appropriate one given the fact that most "personnel" labyrinths are of cross-sectional area of about $1.0 \times 2.0 \mathrm{~m}^{2}$ with a typical unit length of about 1.4 meters. A 10 "unit" long first leg is thus about $14 \mathrm{~m}$, a rather long segment.

Tesch (Te82) has developed a very simple approach to the problem of dose attenuation by multi-legged labyrinths at proton accelerators typical of personnel passageways of approximately $2.0 \mathrm{~m}^{2}$ cross section. For the first leg the expression is an inverse-square law dependence augmented by a factor of 2.0 to approximate "in-scattering";

$$
H_{1}\left(d_{1}\right)=2 H_{o}(R)\left(\frac{R}{d_{1}}\right)^{2}
$$

In Eq. (5.5), the distance into the labyrinth $d_{1}$ (defined as in Fig. 5.4), is in meters and is not scaled by the cross-sectional area of the passageway. Eq. (5.5) is valid only for personnel tunnels of approximately $2 \mathrm{~m}^{2}$ cross section.

\subsubsection{Attenuation in Second and Successive Legs of Straight Penetrations}

Stevenson and Squier reported the results of measurements in a two-legged penetration at the NIMROD synchrotron (St73). This penetration was of cross section $2.3 \times 2.3 \mathrm{~m}^{2}$ and the walls were made of concrete. The target at the mouth of the labyrinth was bombarded by $7.0 \mathrm{GeV}$ protons. Fig. 5.7 is a plot of the transmission of particle flux density along this tunnel using different nuclear reactions, again employed because of their thresholds. One can see that, proceeding from the target outward in the passageway, beyond the abrupt jump that arises as the corner hides the target from view, the fast neutron components are attenuated more readily than is the thermal one. This phenomena associated with "turning the corner" was also verified by Cossairt et al. (Co85b). 


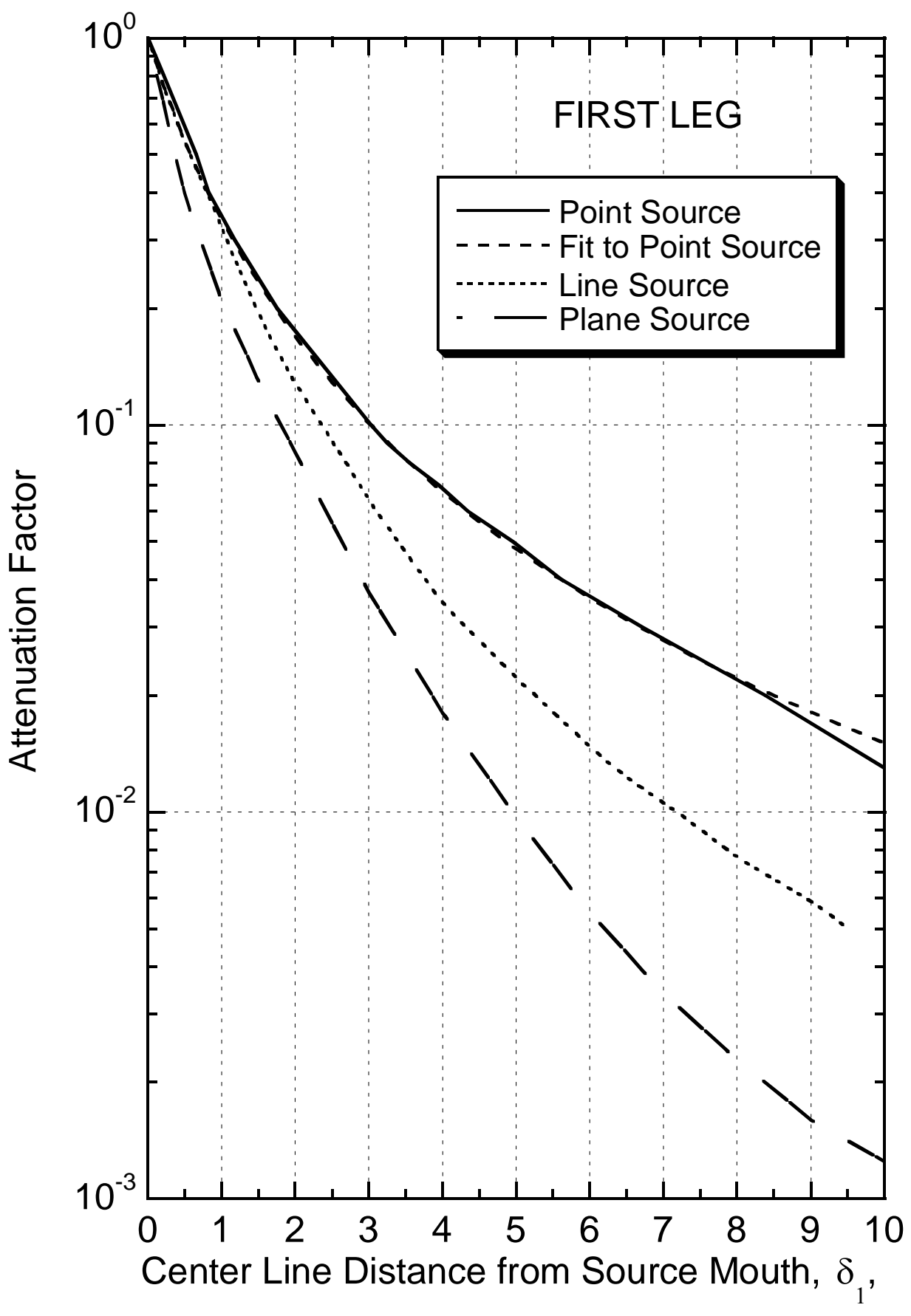

(in units of the square root of the cross section)

Fig. 5.6 Universal transmission curves for the first leg of a labyrinth as a function of normalized distance $\delta_{1}$ from the mouth. The fit for the point source curve represented by Eq. (5.4) is also included. The curve for a plane source is also suitable to use with an off-axis point source. [Adapted from (Go75) and (Co95).] 


\section{Chapter 5 Low Energy Prompt Neutron Radiation Phenomena}

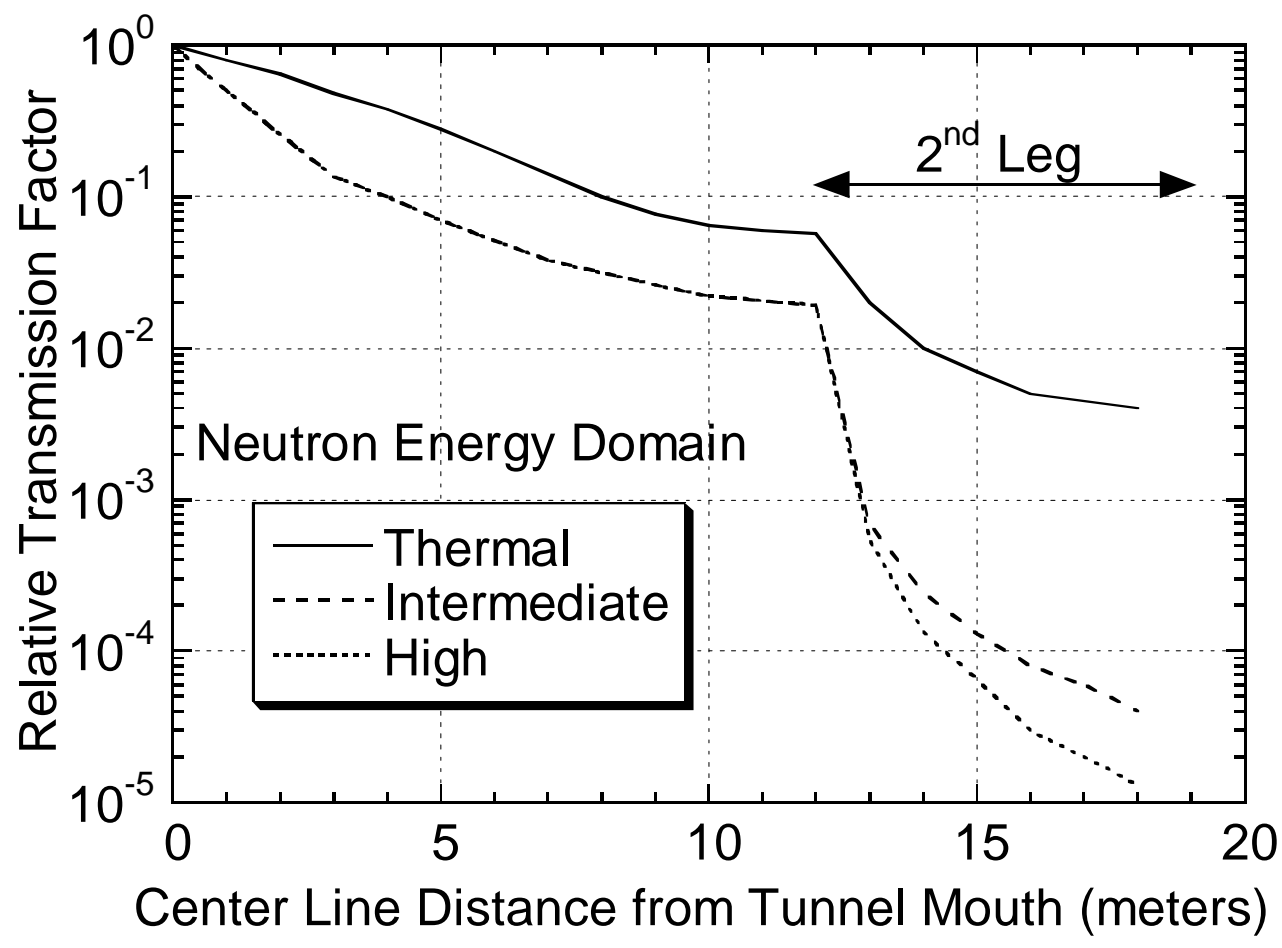

Fig. 5.7 Relative transmission of particle flux density along a two-legged labyrinth using threshold detectors. The curve labeled thermal corresponds to the measured attenuation of thermal neutrons, the curve labeled intermediate corresponds to neutrons having energies between approximately 6.0 and $25 \mathrm{MeV}$ while the curve labeled high corresponds to neutron energies above $20 \mathrm{MeV}$. [Adapted from (St73).]

Second and successive legs of such straight penetrations thus change the situation dramatically, principally by modifying the spectrum of the transmitted neutrons. Fig. 5.8 displays a universal curve for second and succeeding legs that can serve as a companion to that given for the first leg in Fig. 5.6. The distance from the center of the preceding turn normalized to the square root of the cross sectional area of the $i^{\text {th }}$ leg $A_{i}$, the "unit length", is $\delta_{i}=d_{i} / A^{1 / 2}$, for second and succeeding legs.

Cossairt (Co95) found following recursive expression to describe this curve, where $\delta_{i}$ is the distance in the $i^{\text {th }}$ leg measured in "units" of the square root of the cross-sectional area of the $i^{\text {th }}$ leg:

$$
H_{i}\left(\delta_{i}\right)=\left\{\frac{\exp \left(-\delta_{i} / a\right)+A \exp \left(-\delta_{i} / b\right)+B \exp \left(-\delta_{i} / c\right)}{1+A+B}\right\} H_{i-1}\left(\delta_{i-1}\right) i^{\text {th }} \operatorname{leg}(i>1)
$$

With the fitting parameters;

$$
\begin{array}{ll}
a=0.17, & A=0.21, \\
b=1.17, & \text { and } B=0.00147 . \\
c=5.25, &
\end{array}
$$

The results of this fit are included in Fig. 5.8. 


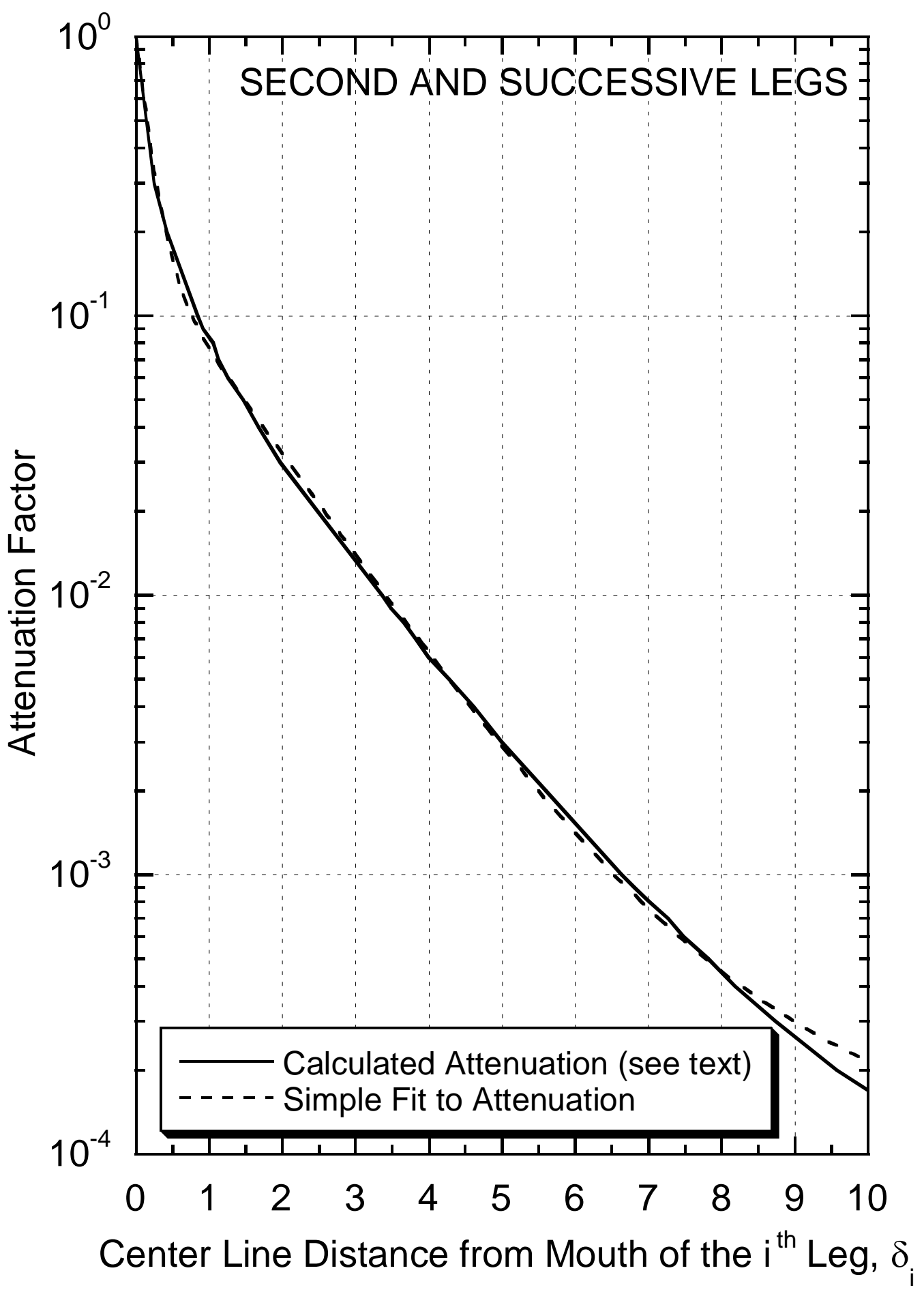

(in units of the square root of the tunnel cross section)

Fig. 5.8 Universal transmission curve for the second and subsequent legs of labyrinths as a function of normalized distance from the center of the previous turn $\delta_{i}$. The calculated attenuation (solid curve) is that reported by Goebel et al. using the code AMC. The dashed curve is the fit provided by Eq. (5.6). [Adapted from (Go75) and (Co95).] 


\section{Chapter 5 Low Energy Prompt Neutron Radiation Phenomena}

Tesch (Te82) also has developed the following formula for the transmission of the second and successive legs:

$$
H_{i}\left(d_{i}\right)=\left\{\frac{\exp \left(-d_{i} / 0.45\right)+0.022 A_{i}^{1.3} \exp \left(-d_{i} / 2.35\right)}{1+0.022 A_{i}^{1.3}}\right\} H_{i-1}\left(i^{\text {th }} \operatorname{leg}, I>1\right)
$$

Here $A_{i}$ is the cross sectional area of the $i^{\text {th }}$ leg in units of square meters. As was the case with respect to Eq. (5.5), this formula uses the distances $d_{i}$ (meters) along the labyrinth (not in units of the square root of the cross sectional area). As it was for Eq. (5.5), the results are valid for "door-sized" labyrinths with cross sectional areas of about $2.0 \mathrm{~m}^{2}$.

Fig. 5.9 shows a four-legged labyrinth providing entrance to a tunnel above a target struck by $400 \mathrm{GeV}$ protons accelerated by the Tevatron at Fermilab. Fig. 5.10 compares experimental measurements (Co85b) of absorbed dose throughout this labyrinth with several methods of calculation. As one can see, all methods of calculating the attenuation discussed here are approximately valid even for this four-legged labyrinth. Though the first leg does not have a truly "open view" of the target, it is well-described by these methods as a "first" leg. Further, these results show that behavior in legs beyond the second one is that of successive "second" legs. ${ }^{17}$

For this labyrinth, a recombination chamber technique (see Section 9.5.7) was used to measure the neutron quality factor $Q$ at two locations, one at the end of the first leg and one in the middle of the short second leg denoted by $R$ in Fig. 5.9. The values of $Q$ were determined in terms of the 1973 Radiation Protection System. The results were $Q=5.5 \pm 0.6$ (first leg) and $Q=3.4 \pm 0.1$ (second leg). This indicates a reduction of the average neutron energy in the second leg which was further verified by a measurement of the neutron energy spectrum (see Fig. 6.7) using a multisphere technique (see Section 9.5.2.1) that resulted in $Q=3.1 \pm 0.7$. This spectrum was measured at the location denoted by $S$ in Fig. 5.9. The spectrum measured in the second leg exhibited domination by thermal, or near-thermal, neutrons. It is clear that several approaches to the design of labyrinths are equally effective for practical radiation protection work.

\subsubsection{Attenuation in Curved Tunnels}

Curved tunnels are principally used to provide access for large, especially lengthy, equipment items that cannot negotiate right-angle bends. These have not been treated in nearly the same detail as have the rectilinear passageways. It appears that the attenuation is described by an exponential function having an attenuation length $\lambda$ that is only a function of the radius $R$ of the tunnel. Patterson and Thomas (Pa73) determined that

$$
\lambda=0.7 \sqrt{R}
$$

\footnotetext{
${ }^{17}$ In the labyrinth shown in Fig. 5.9, the shielding blocks were, in fact, aligned as indicated. This is contrary to good design practice as the blocks should have been overlapped to prevent "streaming". This deficiency of design may explain the excess of measurement over calculation at the end of the third leg.
} 


\section{Chapter 5 Low Energy Prompt Neutron Radiation Phenomena}
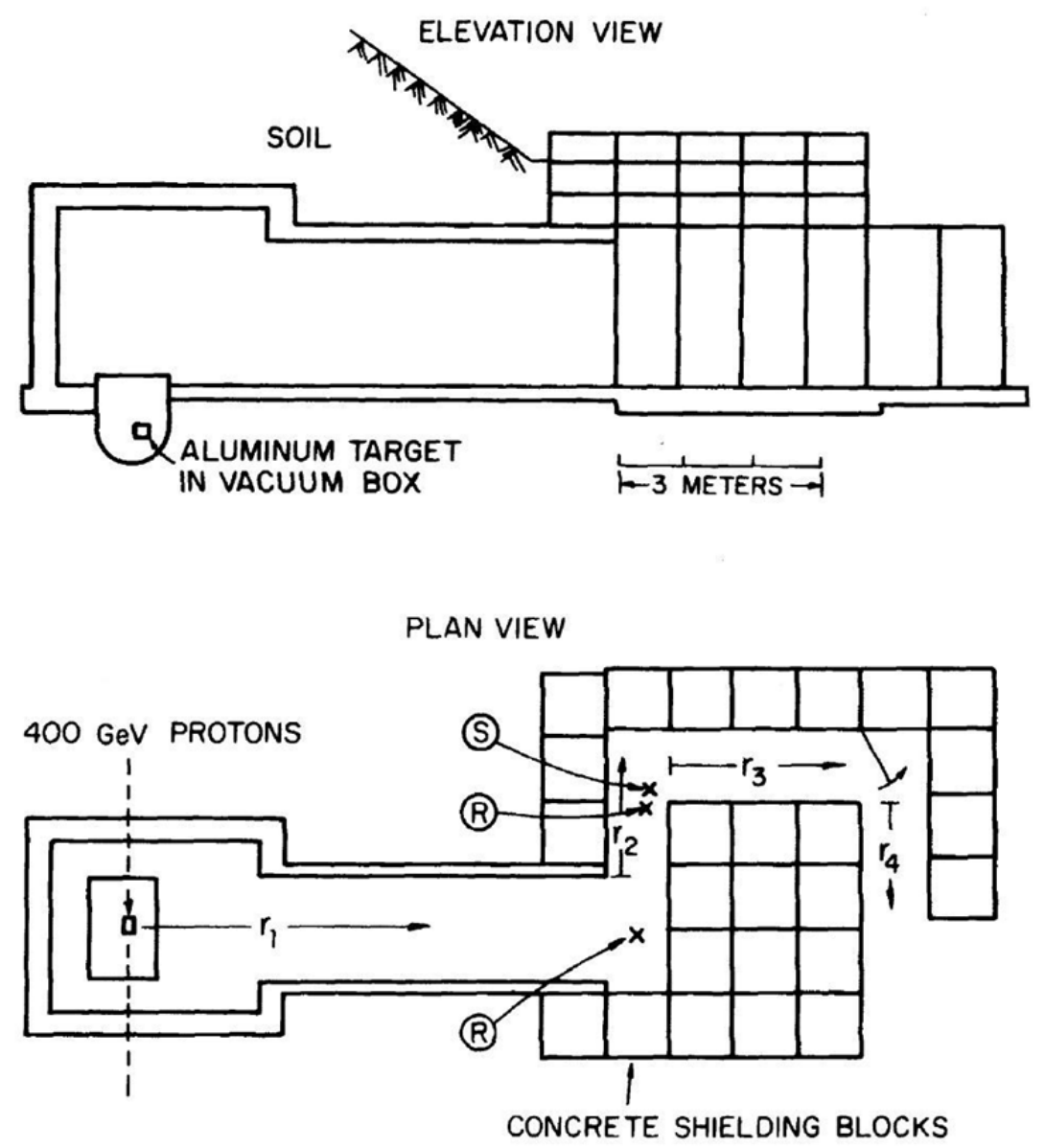

Fig. 5.9 Labyrinth enclosure in which $400 \mathrm{GeV}$ protons interacted with an aluminum target located beneath the floor as shown. The neutron energy spectrum was measured at the location denoted $\mathbf{S}$ and the quality factor of the radiation field was measured at the locations denoted R. [Taken from (Co85b).]

where $R$ is in meters and $4<R<40$ meters. Thus, the dose $H(r)$ or fluence at any circumferential distance through the tunnel, $x$, is given by

$$
H(x)=H_{o} \exp (-x / \lambda)
$$

where $x$ and $\lambda$ are expressed in mutually consistent units.

\subsubsection{Attenuation Beyond the Exit}

A question that often arises in discussion labyrinths dose rate outside of the exit of the passageway. Qualitative experience is that beyond the exit, the neutrons "disappear" rather rapidly. This phenomenon is plausibly a result of the fact that the neutron energy spectrum is heavily dominated by thermal and near-thermal neutrons in all legs after the first. Such neutrons, therefore, having suffered many scatters are not be collimated in any particular direction, being a thermalized "gas". Elwyn (E191) has studied this by 


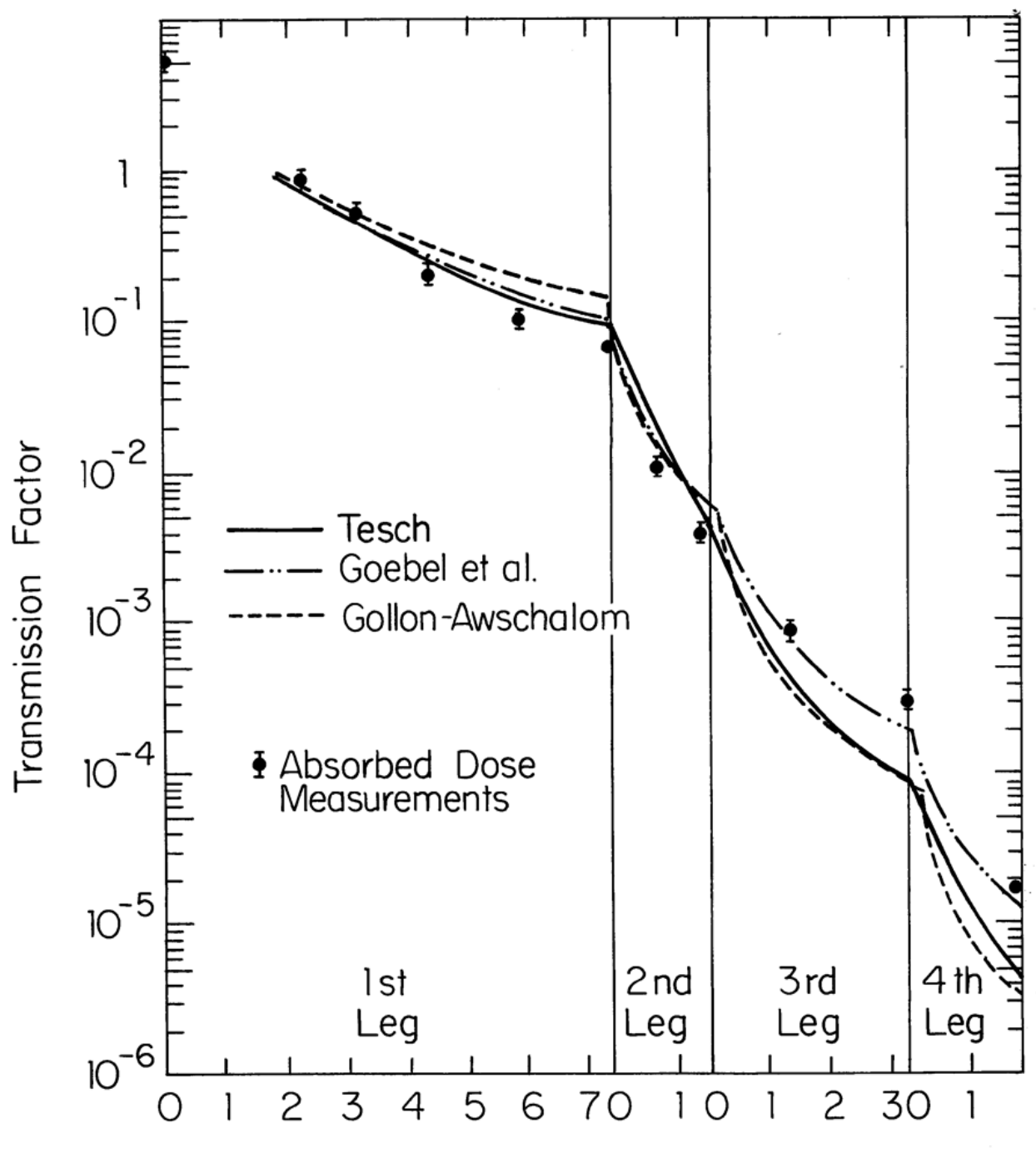

Centre-line Distance in meters

Fig. 5.10 Measurements and predictions of transmission in a tunnel at Fermilab. The results of Tesch (Te82), Goebel et al. (Go75), and Gollon and Awschalom (Go71) are compared with measurements of absorbed dose conducted at the position shown in the four-legged labyrinth displayed in Fig. 5.9. Fortuitously, the "Transmission Factor" plotted as the ordinate is also the absolute scale of the absorbed dose measurement in units of mrad per $10^{10}$ incident 400 $\mathrm{GeV}$ protons (fGy proton ${ }^{-1}$ ). [Reproduced from (Th88) and (Co85b).]

assuming that the exit of the labyrinth is a circular disk of area $A$, equivalent in area to the actual exit. Further, it is assumed that the neutrons emerge from this disk at all random directions with source fluence $S_{A}$. Fig. 5.11 illustrates the geometry. It is further assumed that there is only emission into the $2 \pi$ steradian hemisphere outside the exit. The differential flux density at $P$ on the axis of the disk is

$$
d \phi=\frac{S_{A} \cos \alpha d A}{2 \pi \rho^{2}}
$$




\section{Chapter 5 Low Energy Prompt Neutron Radiation Phenomena}

where $d A=r d r d \theta, \rho^{2}=h^{2}+r^{2}$, and angle $\alpha$ is defined in Fig. $5.11(\cos \alpha=h / \rho)$. The $\cos \alpha$ factor is present to take into account the solid angle of the source elemental area subtended at point $P$.

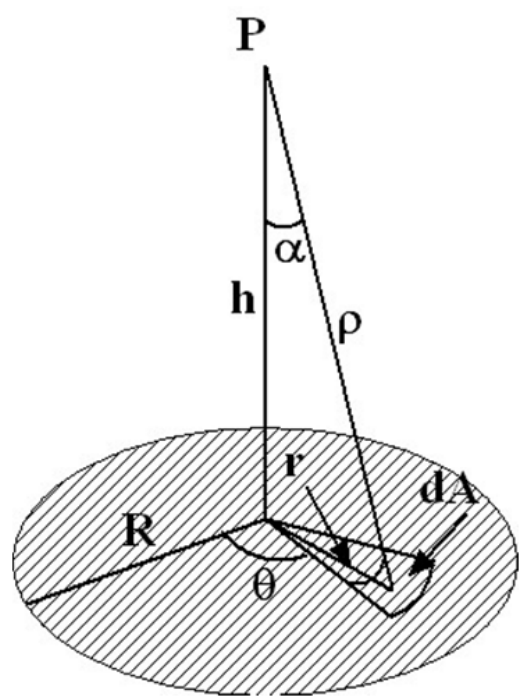

Fig. 5.11 Diagram of labyrinth exit neutron calculation. The coordinates are explained in the text.

Thus,

$$
d \phi=\frac{S_{A} h r}{2 \pi \rho^{3}} d r d \theta
$$

Integrating,

$$
\begin{array}{r}
\phi(h)=\frac{S_{A} h}{2 \pi} \int_{0}^{R} d r \int_{0}^{2 \pi} d \theta \frac{r}{\left(r^{2}+h^{2}\right)^{3 / 2}}=S_{A} h \int_{0}^{R} d r \frac{r}{\left(r^{2}+h^{2}\right)^{3 / 2}}= \\
S_{A} h\left[\frac{-1}{\sqrt{r^{2}+h^{2}}}\right]_{0}^{R}=S_{A}\left[1-\frac{1}{\sqrt{1+\left(\frac{R}{h}\right)^{2}}}\right],
\end{array}
$$

where attenuation by the air is neglected. One uses this result by approximating the area of the exit opening by the area of a disk have an equivalent area. At large distances, one can apply a "point source" approximation due to the fact that

$$
\phi(h) \approx \frac{S_{A}}{2}\left(\frac{R}{h}\right)^{2} \quad \text { for } h>>R .
$$

For $h=0, \phi(0)=S_{A}$ as expected. The dramatic decrease of fluence with distance is illustrated by the tabulation of a few values in Table 5.3. 
Table 5.3 Estimates of relative neutron flux or dose equivalent as a function of scaled distance from the exit of a labyrinth [Eq. (5.12)\}

\begin{tabular}{|c|c|}
\hline $\boldsymbol{h} / \boldsymbol{R}$ & $\boldsymbol{\phi}(\boldsymbol{h}) \boldsymbol{S}_{\boldsymbol{A}}$ \\
\hline 0.5 & 0.55 \\
\hline 1.0 & 0.29 \\
\hline 2.0 & 0.11 \\
\hline 4.0 & 0.03 \\
\hline 10.0 & 0.005 \\
\hline
\end{tabular}

\subsubsection{Determination of the Source Factor}

Generally, the dose at the mouth of a labyrinth can be obtained using Monte Carlo techniques or by directly using the information about neutron yields. For protons, approximations that use Moyer Model parameters discussed in Chapter 4 are likely to overestimate the dose at the entrance. This is because the Moyer parameters implicitly assume development of the shower (intrinsically a "buildup" mechanism, as seen in Chapter 4) in the enclosure shielding. This buildup does not happen in the passageway.

For high energy proton accelerators, a rule of thumb for the source term found to be sufficiently accurate for most personnel protection purposes has been developed by Ruffin and Moore (Ru76). It was improved by inclusion of Moyer energy scaling by Rameika (Ra91). In this model, about one fast neutron proton ${ }^{-1} \mathrm{GeV}^{-1}$ of proton beam energy is taken to be produced with an isotropic distribution. These could be considered to be evaporation neutrons, not the cascade neutrons produced with much higher multiplicity in the forward direction. These isotropically produced neutrons dominate the spectrum and determine the dose equivalent [1973 System] or effective dose [1990 System] of those that enter the labyrinth and have kinetic energies between 1.0 to 10 $\mathrm{MeV}$. From the dose equivalent per fluence factors $P(E)$ of Fig. $1.5,1.0$ rem of $1-10 \mathrm{MeV}$ neutrons represents a fluence of approximately $3 \times 10^{7} \mathrm{~cm}^{-2}$. Thus, at distance $R(\mathrm{~cm})$ from the source;

$$
H_{\text {equiv }}=\frac{E_{0}^{0.8} N_{p}}{4 \pi R^{2}\left(3 \times 10^{7}\right)}=2.65 \times 10^{-9} \frac{E_{0}^{0.8} N_{p}}{R^{2}}(\mathrm{rem}) \text {, }
$$

where $E_{\mathrm{O}}$ is in $\mathrm{GeV}$, and $N_{p}$ is the number of incident protons. The constant, $2.65 \times 10^{-9}$ $\left(\right.$ rem $\mathrm{cm}^{2}$ ), turns out to be approximately one-third the value obtained by using the Moyer source parameter along with high energy value of the Moyer angular factor at $\theta=\pi / 2$ [Eqs. (4.38) and (4.41)];

$$
\left(2.8 \times 10^{-7} \mathrm{rem} \mathrm{cm}^{2}\right) \exp (-2.3 \pi / 2)=7.6 \times 10^{-9} \mathrm{rem} \mathrm{cm}^{2} \text {. }
$$

For effective dose, $H_{\text {eff }}$, the constant $2.65 \times 10^{-9}$ should be replaced with $3.71 \times 10^{-9}$ (Co95).

To obtain the source factor for neutrons produced by electrons, the neutron yields discussed in Section 3.2.3 can be utilized. 


\section{Chapter 5 Low Energy Prompt Neutron Radiation Phenomena}

\section{$5.3 \quad$ Skyshine}

Thin roof shielding represents a serious problem that has plagued a number of accelerators such as the Cosmotron (at Brookhaven National Laboratory), the Bevatron (at the Lawrence Berkeley National Laboratory), the Fermilab experimental areas, and likely elsewhere. The phenomenon of interest, known as skyshine, is the situation in which the roof of some portion of the accelerator or an associated experimental facility is shielded more thinly than are the sides of the same enclosure that directly view the radiation source. The first attempt to calculate the skyshine radiation field was made by Lindenbaum (Li61). Fassò et al. (Fa90) give a rather complete description of the phenomena while Patterson and Thomas (Pa73), Rindi and Thomas (Ri75), Stevenson and Thomas (St84a), and Cossairt and Coulson (Co85c) present specific results. Neutron skyshine has been encountered at nearly all major accelerators. This has resulted from lack of identification of it as a design issue, the need to accommodate other constraints such as the minimization of the weight of shielding borne by the roofs of large experimental halls, or the need to repurpose existing facilities for higher energies and/or beam powers.

\section{$\underline{\text { 5.3.1 Simple Parameterizations }}$}

When addressing the skyshine question, it is often customary to plot the neutron fluence, dose equivalent, or effective dose as a function of distance from the source by multiplying it by the square of the distance from the source, i.e. as $r^{2} \Phi(r)$. Stevenson and Thomas (St84a) included plots of a number of measurements of neutron skyshine obtained at proton accelerators producing protons of energies ranging from $30 \mathrm{MeV}$ to 30 $\mathrm{GeV}$, and also at high energy electron accelerators having energies of 7.5 and $12 \mathrm{GeV}$. In general, the quantity $r^{2} \Phi(r)$ as a function of $r$ is characterized by a buildup region followed by exponential falloff. Most skyshine distributions are isotropic at ground level (i.e., independent direction with respect to the beam axis). In typical skyshine data $\lambda$, the effective attenuation length measured at ground level, has been found to vary between a minimum value of about 200 meters and much larger values which approach one kilometer. This quantity is dependent upon the energy spectrum of the neutron radiation field that is the source of the skyshine.

Patterson and Thomas (Pa73) give a formula that describes such behavior for $r$ (meters) greater than about 20 meters;

$$
\Phi(r)=\frac{a Q}{4 \pi r^{2}}\left(1-e^{-r / \mu}\right) e^{-r / \lambda} .
$$

In this equation, a value of $a=2.8$ fits data well and represents an empirical buildup factor, while $\mu$ is the corresponding buildup relaxation length. Nearly all of existing measurements are well described by taking $\mu$ to be 56 meters. $Q$ is the source strength 


\section{Chapter 5 Low Energy Prompt Neutron Radiation Phenomena}

and dimensionally must be consistent with $\Phi(r)$. Thus, for the standard meaning of $\Phi(r)$ as the fluence, $Q$ is the number of neutrons emitted by the source needed to result in that fluence. A plot of $r^{2} \Phi(r)$ for a variety of choices of the value of $\lambda$ in Eq. (5.16) is given in Fig. 5.12.

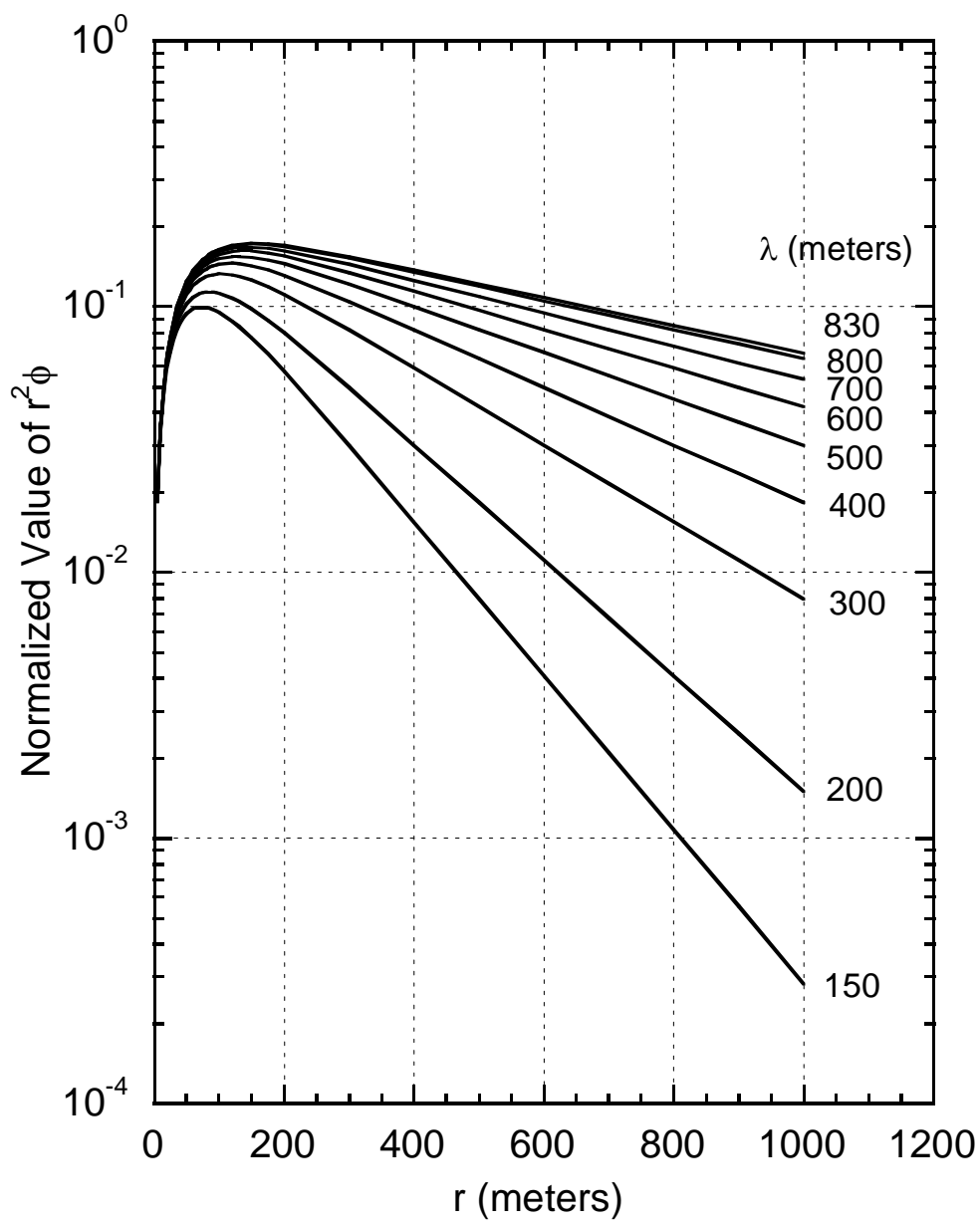

Fig. 5.12 Plot of skyshine distributions according to Eq. (5.16) for a variety of values of $\lambda$. The ordinate is the quantity $r^{2} \phi(r)$ in that equation for a value of $Q=1$.

Values of $\lambda>830$ meters are possible if very high energy neutrons $(E>>150 \mathrm{MeV})$ are

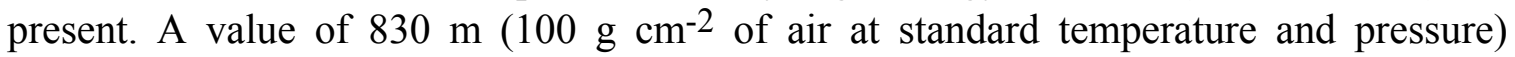
corresponds to the interaction length in air of the neutrons of approximately $100 \mathrm{MeV}$ likely to control the propagation of hadronic cascades. Thus, $\lambda$ is determined by the neutron energy spectrum present at the thinly shielded location. Larger values of $\lambda$ are more plausibly due to multiple sources or an extended source. In such circumstances, the radiation field may also not necessarily be isotropic. Cossairt and Coulson (Co85c) described an example of a non-isotropic, complex skyshine source involving high energies, an extended source, and a very thin shield that resulted in a value of $\lambda \approx 1200$ meters. 


\section{Chapter 5 Low Energy Prompt Neutron Radiation Phenomena}

The procedure, then, for using Eq. (5.16) is to do the following:

A. Estimate the total emission rate of neutrons from the source. This can be done by using information about the neutron spectrum at the source to choose an "average" energy and intensity. The dose per fluence factor at that energy can then be used in conjunction with a dose rate survey over the thinly shielded region to determine the total neutron emission rate $Q$ by numerically integrating over the area of the top of the shield.

B. Estimate the value of $\lambda$ from the neutron energy spectrum information.

C. Apply Eq. (5.16) to determine the radial dependence.

\subsubsection{A More Rigorous Treatment}

A more rigorous treatment has been reported by Stevenson and Thomas (St84a) is based on the work of Alsmiller, Barish, and Childs (Al81) and Nakamura and Kosako (Na81). These groups have independently performed extensive calculations of the neutrons emitted into cones of small vertex angle. Alsmiller, Barish, and Childs used the Discrete Ordinates Transport (DOT) Code while Nakamura and Kosako used the Monte Carlo code MORSE. For selected distances from the skyshine source, these workers have calculated the dose equivalent as a function of both the source neutron energy and the emission cone's semivertical angle (that is, the half-angle the rotation of which defines the cone into which the neutrons are emitted). The authors define this quantity, the socalled neutron importance, as the dose equivalent per emitted neutron as a function of the energy of the emitted neutron and of the distance from the source. This quantity is a measure of how "important" a given emitted neutron is in delivering radiation dose equivalent to a point on the ground located at a given distance from the skyshine source. The results of the Alsmiller calculation for an example semivertical angle of $37^{\circ}$ are plotted in Fig. 5.13. Numerical tabulations of these neutron importance functions according to (A181) for a variety of domains of semi-vertical angle are given by (NC03), and (Th88) Appendix C. The corresponding, but somewhat less-detailed, results of Nakamura and Kosako confirm the more detailed results of Alsmiller et al.

Stevenson and Thomas (St84a) were able to derive an alternative "recipe" for skyshine neutron calculations to that expressed in Eq. (5.16) by making two assumptions:

A. The neutron energy spectrum has the $1 / E$ form up to the proton energy or some other limiting upper energy and zero at higher energies. This likely overestimates the contribution of the higher energy neutrons.

B. The neutrons are solely emitted into a cone whose semivertical angle is about $35^{\circ}$ to $40^{\circ}$, values common for typical structures and approximately $37^{\circ}$. This may overestimate the doses by up to a factor of three for sources of smaller semivertical angles. 


\section{Chapter 5 Low Energy Prompt Neutron Radiation Phenomena}

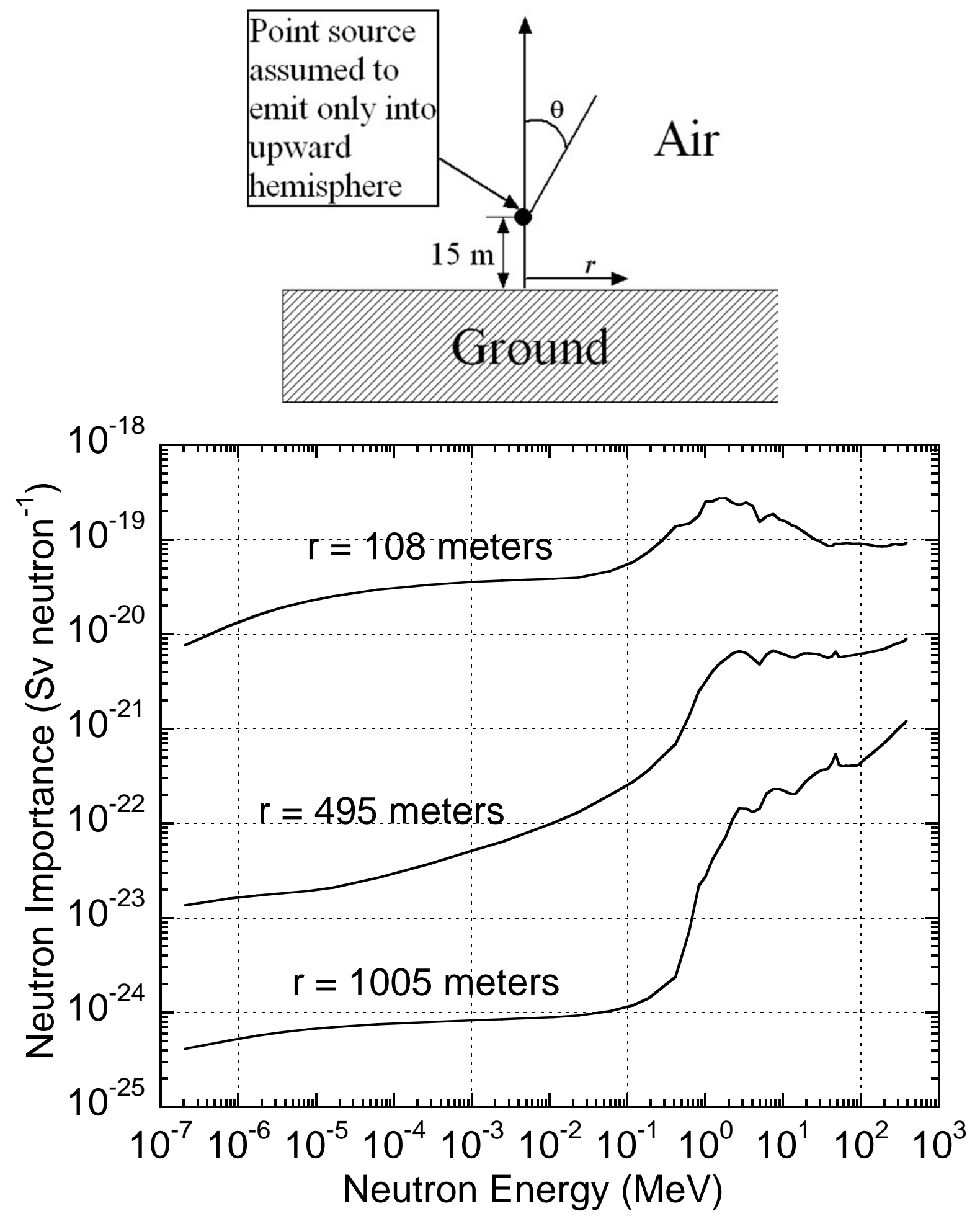

Fig. 5.13 Top frame: The definition of the semivertical cone angle used in skyshine calculations of Alsmiller et al. (A181). Bottom frame: Neutron skyshine importance functions for a semivertical cone angle of $37^{\circ}$ at three different values of the distance from a point source. [Adapted from Alsmiller et al. (A181)]. 


\section{Chapter 5 Low Energy Prompt Neutron Radiation Phenomena}

Stevenson and Thomas parameterized the skyshine phenomena as follows:

$$
\Phi(r)=\frac{Q^{\prime}}{4 \pi r^{2}} e^{-r / \lambda} .
$$

In this equation, the buildup exponential factor has been suppressed so the formula is valid only at large distances (i.e., $r>>56$ meters). In addition, the source strength denoted by $Q^{\prime}$ implicitly includes the buildup factor of 2.8 .

Further, they used the Alsmiller importance functions to estimate the value of $\lambda$ based upon the upper energy of the $1 / E$ spectrum. Fig. 5.14 displays the results of doing this for several choices of upper energies at three distances in a plot in which the inverse-square dependence is suppressed. A comparison with a measurement conducted at Brookhaven National Laboratory is also provided.

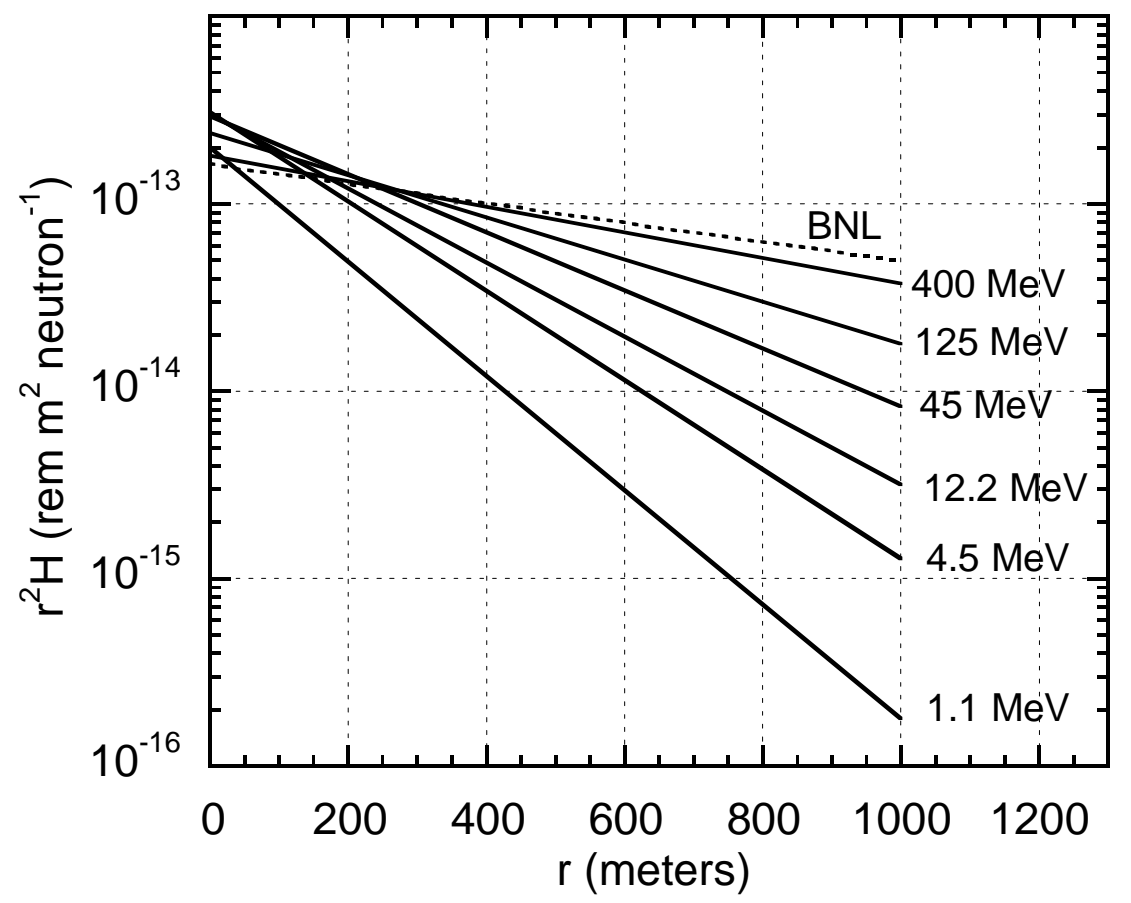

Fig. 5.14 Variation of dose equivalent with distance $r$ for $1 / E$ neutron spectra with different upper energies. The ordinate is the dose equivalent $H$ multiplied by $r^{2}$. The curve labeled "BNL" is the result of a measurement at the Brookhaven National Laboratory Alternating Gradient Synchrotron, a $30 \mathrm{GeV}$ proton accelerator. [Adapted from (St84a).]

The slopes, then, were used to obtain theoretical values of $\lambda$ as a function of upper energy that are plotted in Fig. 5.15. To determine the source term, the straight lines in Fig. 5.14 (on the semi-logarithmic plot) were extrapolated to zero and used to determine intercepts at $r=0$ ranging from $1.5 \times 10^{-15}$ to $3 \times 10^{-15} \mathrm{~Sv} \mathrm{~m}^{2}$ neutron ${ }^{-1}\left(1.5 \times 10^{-13}\right.$ to $3 \times 10^{-13} \mathrm{rem} \mathrm{m}^{2}$ neutron $^{-1}$ ). Hence, conservatively, Stevenson and Thomas found that, over a rather large range of incident proton energies spatial dependence of the dose equivalent, 


\section{Chapter 5 Low Energy Prompt Neutron Radiation Phenomena}

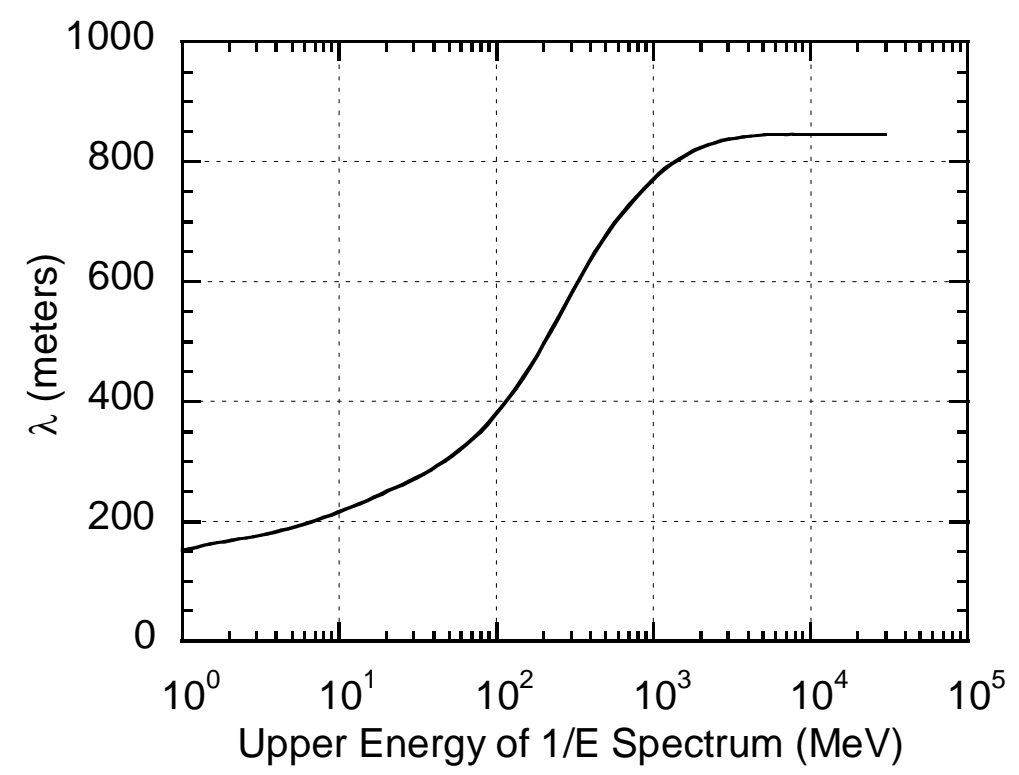

Fig. 5.15 Effective absorption length $\lambda$ as a function of upper neutron energy $E$ for $1 / E$ spectra. [Adapted from (St84a).]

$H(r)$ can be described by

$$
H(r)=\frac{3 \times 10^{-13}}{r^{2}} \mathrm{e}^{-r / \lambda}(\mathrm{rem} / \mathrm{emitted} \text { neutron, } r \text { in meters }) \text {. }
$$

One has to determine the total number of neutrons emitted. This can be done as before by measuring the integral of dose equivalent times the area over the thinly shielded location and using the reciprocal of the dose equivalent per fluence conversion factor appropriate for the neutron energy spectrum at hand to get the total number of neutrons emitted. The use of Eq. (5.18) will lead to an overestimate of neutrons for values of $r$ less than approximately 100 meters because the extrapolation ignores the observed exponential buildup of the skyshine.

Stevenson and Thomas (St84a) give a convenient table, useful for general purposes, of dose equivalent per fluence conversion factors of ICRP Publication 21 [1973 System (IC73)] integrated over such $1 / E$ spectra which is provided here as Table 5.4. ${ }^{18}$

\subsubsection{Examples of Experimental Verifications}

Measurements at Fermilab (Co85c) have confirmed the validity of these methods for a "source" involving the targetry of $400 \mathrm{GeV}$ protons. Fig. 5.16 shows two measured and fitted radial distributions made using Eq. (5.16). In Fig. 5.16 Survey 2 corresponds to a shielding configuration where the neutron energy spectrum was inferred to be of very high energy while "Survey 4" was likely to involve a much less energetic spectrum.

${ }^{18}$ Use of 1990 Radiation Protection System quantities, e.g., $H_{\text {eff }}$ instead of $H_{\text {equiv }}$, will require a small adjustment of the result of Eq. (5.18) not provided in this chapter. 


\begin{tabular}{|c|c|}
\hline $\begin{array}{c}\text { Upper Energy } \\
(\mathrm{MeV})\end{array}$ & $\begin{array}{c}\text { Spectrum Averaged Dose } \\
\text { Equivalent Conversion Factor } \\
\left(10^{-9} \mathrm{rem} \mathrm{cm}^{2} \text { neutron }{ }^{-1}\right)\end{array}$ \\
\hline 1.6 & 3.9 \\
\hline 2.5 & 4.8 \\
\hline 4.0 & 5.6 \\
\hline 6.3 & 6.4 \\
\hline 10 & 7.2 \\
\hline 16 & 7.9 \\
\hline 25 & 8.6 \\
\hline 40 & 9.4 \\
\hline 63 & 10.1 \\
\hline 100 & 10.9 \\
\hline 160 & 11.7 \\
\hline 250 & 12.5 \\
\hline 400 & 13.4 \\
\hline 630 & 14.6 \\
\hline 1000 & 16.2 \\
\hline 1600 & 18.4 \\
\hline 2500 & 21.2 \\
\hline 4000 & 25.0 \\
\hline 6300 & 30.0 \\
\hline 10000 & 36.5 \\
\hline
\end{tabular}

Survey 4 was made for the same beam and target after the concrete shield thickness around the source was greatly increased compared with that present when Survey 2 was obtained. The normalization to "COUNTS-M $\mathrm{MR}^{2} \mathrm{HR}^{-1}$ refers to an integration of an instrumental response over the surface area of the source and was approximately proportional to the emitted neutron fluence. The instrument calibration of "COUNTS/HR" made possible an estimate of the dose equivalent at $r=200$ meters for the two surveys. Based on configuration details not described in detail here, one can estimate that the spectrum of emitted neutrons of Survey 2 had an upper energy of $\approx 1.0 \mathrm{GeV}$, while the spectrum of emitted neutrons of Survey 4 had an upper energy of $\approx 100 \mathrm{MeV}$.

Using the appropriate dose equivalent per fluence conversion factor, the value of $Q$ for the Survey 2 conditions was determined experimentally to be $2.5 \times 10^{5} \mathrm{mrem} \mathrm{m}^{2} \mathrm{hr}^{-1}$. This was obtained from the measured absorbed dose surface integral of $5 \times 10^{4} \mathrm{mrad} \mathrm{m}^{2} \mathrm{hr}^{-1}$ and assumed a quality factor of 5.0. For the Survey 4 conditions, $Q$ was found to be $4.0 \times 10^{4}$ $\mathrm{mrem} \mathrm{m}^{2} \mathrm{hr}^{-1}$. Again, this was obtained from the measured absorbed dose surface integral of $8.1 \times 10^{3} \mathrm{mrad} \mathrm{m}^{2} \mathrm{hr}^{-1}$ and assumed a quality factor of 5.0. Table 5.5 makes a comparison with the prescription of (St84a) for these data. In this table $H$ is the dose equivalent in one hour at 200 meters. The prescription of Stevenson and Thomas (St84a) is used to calculate the dose equivalent in one hour at 200 meters. The agreement is well within all uncertainties involved. 


\section{Chapter 5 Low Energy Prompt Neutron Radiation Phenomena}

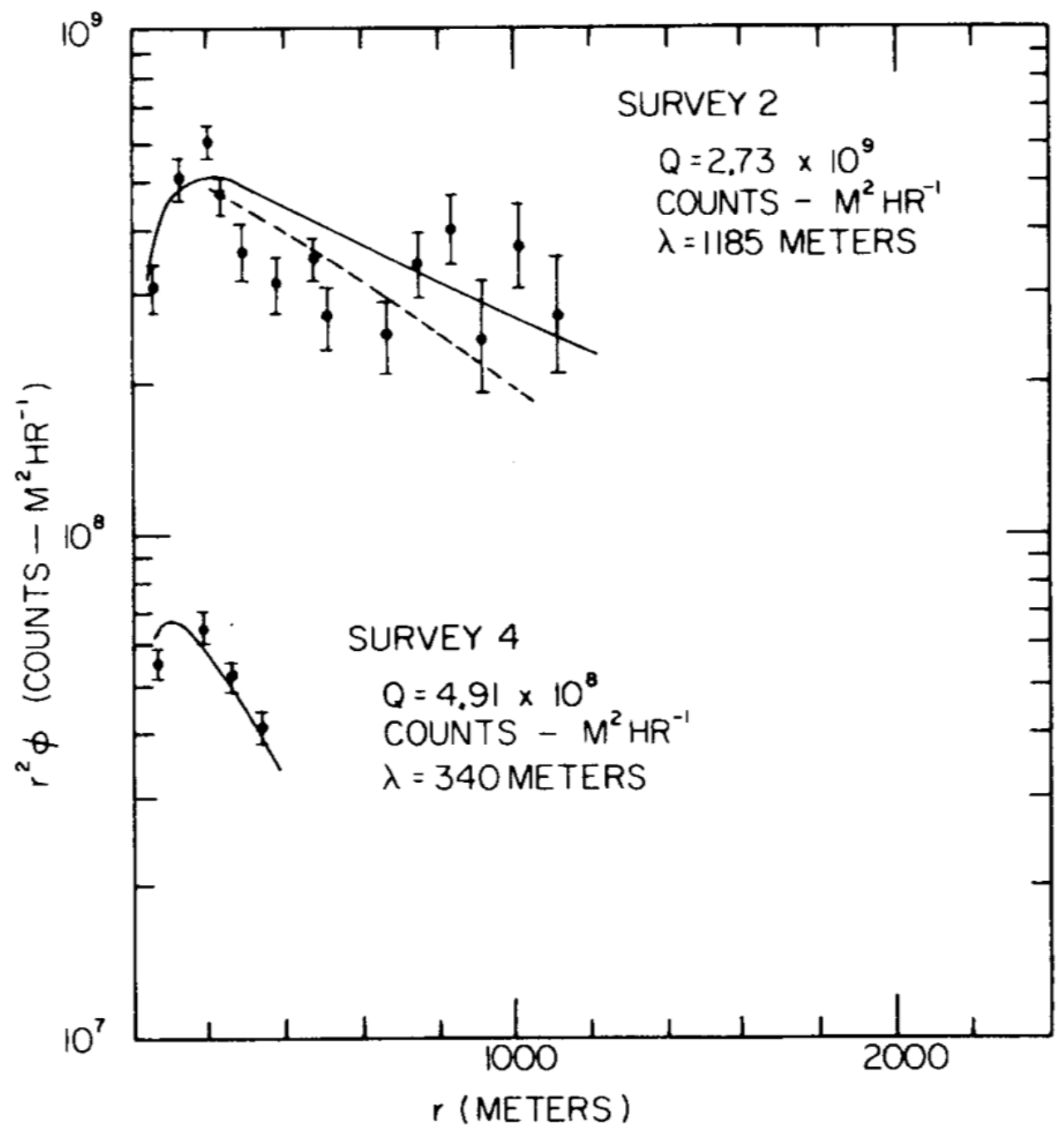

Fig. 5.16 Skyshine data from two different surveys plotted as $r^{2} \phi$ as a function of distance from the source $r$. The solid curves are from the least squares fit of Eq. (5.16) to the data points while the dashed curve is the fit if $\lambda$ is constrained to have a value of $830 \mathrm{~m}$. Error bars represent one standard deviation counting statistics. [Reproduced from (Co85c).]

Another illustration is provided by Elwyn and Cossairt (E186) in connection with neutron radiation field emerging from an iron shield that is more fully described in Section 6.3.5. Fig. 5.17 shows the measured radial dependence of neutron flux as a function of distance from that iron shield. 
Table 5.5 Comparisons of Fermilab neutron skyshine data with results of parameterizations of surveys shown in Fig. 5.16, assuming $1 / E$ spectra with inferred upper energies. The quantities are all for a one hour time period.

\begin{tabular}{|c|c|c|c|c|c|c|}
\hline Survey & $\begin{array}{c}\lambda \\
\text { (meters) } \\
\text { (Co85c) }\end{array}$ & $\begin{array}{c}E_{\max } \\
\text { (inferred) } \\
(\mathrm{MeV})\end{array}$ & $\begin{array}{c}\text { Dose } \\
\text { Equivalent per } \\
\text { Fluence } \\
\left(\text { mrem cm }{ }^{2}\right) \\
(\text { St84a) }\end{array}$ & $\begin{array}{c}Q Q \\
\text { (measured) } \\
\left(\text { (mrem m }^{2}\right) \\
(\text { Co85c) }\end{array}$ & $\begin{array}{c}H(200 \mathrm{~m}) \\
\text { (calculated) } \\
\text { (mrem) }\end{array}$ & $\begin{array}{c}H(200 \mathrm{~m}) \\
\text { (measured) } \\
(\text { mrem) } \\
(\text { Co85c) }\end{array}$ \\
\hline Survey 2 & 1200 & 1000 & $16.2 \times 10^{-6}$ & $2.5 \times 10^{5}$ & 1.0 & 1.6 \\
\hline Survey 4 & 340 & 100 & $10.9 \times 10^{-6}$ & $4.0 \times 10^{4}$ & 0.15 & 0.15 \\
\hline
\end{tabular}

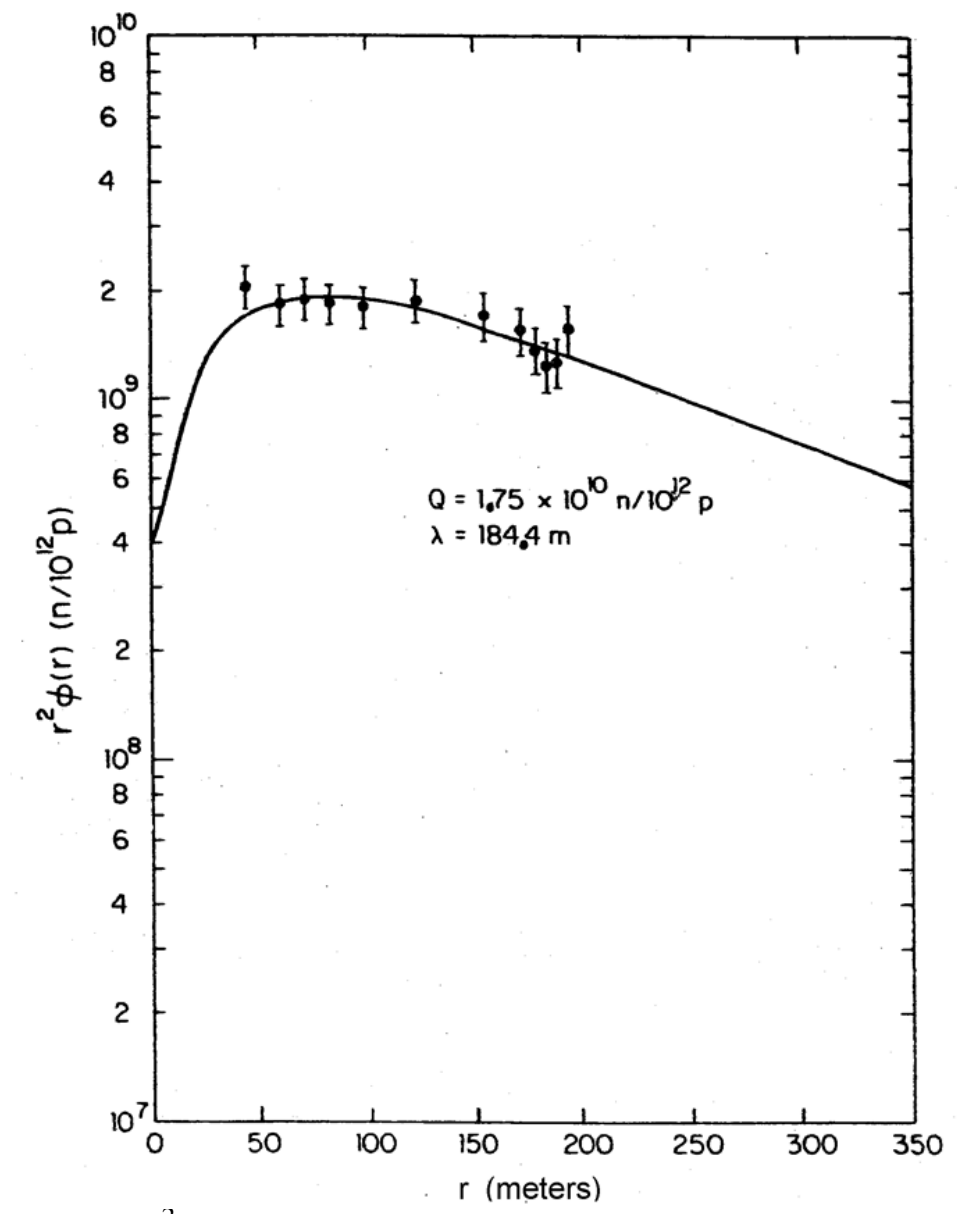

Fig. 5.17 The product of $r^{2}$ and the neutron fluence $\phi(r)$ per $10^{12}$ protons incident on a target as a function of the distance from the source $r$. The source is that described in connection with Fig. 6.8. The smooth curve is a fit to Eq. (5.16) with parameters $\lambda=184.4 \mathrm{~m}$ and $Q=1.74 \times 10^{10}$ neutrons per $10^{12}$ protons. [Reproduced from (E186).]

From other considerations pertaining to an iron shield discussed in Section 6.3.5, it is known that the radiation field is dominated by neutrons of energies near $847 \mathrm{keV}$. Using 


\section{Chapter 5 Low Energy Prompt Neutron Radiation Phenomena}

the measured data, normalized to $10^{12}$ incident protons the parameters $Q=1.75 \times 10^{10}$ and $\lambda=184.4$ meters were determined by fitting the skyshine data using Eq. (5.16). Evaluating $\Phi$ at $r=200$ meters,

$$
\Phi(200)=\frac{2.8\left(1.75 \times 10^{10}\right)}{4 \pi(200)^{2}}[1-\exp (200 / 56)] \exp (-200 / 184.4)=3.20 \times 10^{4}{\text { neutrons } \mathrm{m}^{-2}}^{.}
$$

Thus, taking the measured neutron flux at $r=200$ meters and applying a dose equivalent per fluence value of $3.0 \times 10^{-5} \mathrm{mrem} \mathrm{cm}^{-2}$ appropriate for $847 \mathrm{keV}$ neutrons (see Fig. 1.5) gives a dose equivalent per $10^{12}$ incident protons of $9.6 \times 10^{-5} \mathrm{mrem}$ at $r=200$ meters. The value of $\lambda$ that fitted the skyshine data is also consistent with the neutron energy spectrum, known to be dominated by neutrons of about $1.0 \mathrm{MeV}$ kinetic energy.

Elwyn and Cossairt also estimated a value of $(3.4 \pm 2.0) \times 10^{10}$ per $10^{12}$ incident protons for the total neutron emission of the source by performing a numerical integration over the surface area of the source, separate from the result determined using the skyshine measurement. Applying the prescription of Stevenson and Thomas (St84a) found in Eq. (5.17);

$$
\begin{aligned}
& H(200)=\frac{\left(3 \times 10^{-13}\right)\left[(3.4 \pm 2.0) \times 10^{10}\right]}{(200)^{2}} \exp \left(-\frac{200}{184.4}\right) \\
& =(8.6 \pm 5.1) \times 10^{-5} \text { mrem per } 10^{12} \text { protons }
\end{aligned}
$$

at this same location. This result is very consistent with that found using Eq. (5.19). 


\section{Chapter 5 Low Energy Prompt Neutron Radiation Phenomena}

\section{Problems}

1. A $1.0 \mu \mathrm{A} 100 \mathrm{MeV}$ electron beam is incident on an "optimized bremsstrahlung" target in a shielding configuration and labyrinth like that in Fig. 5.3. Using the facts given in Chapter 3 (Swanson's Rules of Thumb, etc.) about bremsstrahlung, calculate the dose equivalent rate at the exit of a labyrinth having two legs. Set all distances $d_{i}, d_{1}$, and $d_{2}$ to 3.0 meters. If the goal is to get the dose equivalent rate at the exit to be $<1.0 \mathrm{mrem} \mathrm{h}^{-1}$, is this a sensible design? The legs are $1.0 \times 2.0 \mathrm{~m}^{2}$ in cross section. Since no other information is available, use $\alpha=10^{-2}$ as a "conservative" value. For purposes of this problem, photons constitute the only component of radiation present. [Hint: One needs to calculate the projected diameter of the beam at the wall where the first scatter occurs. This can be done using Eq. (3.12).]

2. A $500 \mathrm{GeV}$ proton beam of $10^{11}$ protons/second strikes a magnet $2.0 \mathrm{~m}$ from the mouth of a 3-legged labyrinth. Each of the three legs is 4.0 meters long and $1.0 \times 2.0 \mathrm{~m}^{2}$ in cross section. The length of leg 1 is measured from the mouth of the labyrinth to the center of the first turn, all other lengths are measured between centers of turns. Assume the source is an on-axis "point source". Using Goebel's "universal" curves and Rameika's source term, what is the dose equivalent rate at the exit expressed in rem $\mathrm{hr}^{-1}$. How far away from the exit does the value of $d H / d t$ fall to $10 \mathrm{mrem} \mathrm{hr}^{-1}$.

3. A high energy accelerator has a section of beamline which was poorly designed. Beam losses and insufficient shielding have resulted in a region of roof $10 \mathrm{~m}$ wide and $50 \mathrm{~m}$ long where a neutron dose equivalent rate averaging $100 \mathrm{mrem} \mathrm{h}^{-1}$ (averaged over the surface of the weak shield) is found. An energy spectrum measurement indicates the spectrum shape to be approximately $1 / E$ with an upper end point of approximately $500 \mathrm{MeV}$. Calculate the dose equivalent rate due to skyshine at distance $r=50,100,200,500, \& 1000 \mathrm{~m}$ using both formulae presented here. 


\section{ChAPTER 6 SHIElding MATERIALS AND NEUTRON ENERgy SPECTRA}

\subsection{Introduction}

It is advisable to review the relevant properties of the most common materials used in radiation shielding. Also, since many shielding problems are driven by the nature of the energy spectrum of the neutrons, such spectra are discussed here in some detail. Examples of neutron energy spectra measured external to shielding at various types of accelerator facilities are presented.

\subsection{Discussion of Shielding Materials Commonly Used at Accelerators}

Given the size of many modern accelerators, economic considerations commonly dominate shielding designs; requiring the use of relatively inexpensive, but not necessarily otherwise optimum, shielding materials. In all situations good engineering practices concerning structural properties, appropriate floor loading strength, and fire protection must be taken into account to assure an acceptable level of occupational and public safety. Briefly, low atomic number materials are best used for targets, collimators, and beam stops at electron accelerators to reduce photon production. On the other hand, high atomic number materials are preferred at proton and heavy ion accelerators for these components to reduce neutron production. As discussed previously, at beam energies above about 5.0 MeV, neutrons are produced in most materials. Furthermore, some materials have superior heat transfer characteristics that enhance durability and reliability while reducing personnel exposures incurred in maintenance activities.

\section{$\underline{6.2 .1 \quad \text { Earth }}$}

Earth has many admirable qualities as a shield material besides its low cost. Notably, the water it contains enhances the effectiveness of the neutron attenuation because the mass of a proton is essentially equal to that of a neutron. This facilitates the transfer of energy from the particle to the shielding medium. Due to conservation of energy and momentum, in an elastic collision the energy $\Delta E$ that can be transferred from a neutron having kinetic energy $E_{o}$ to a target nucleus as a function of scattering angle $\theta$ is given by

$$
\frac{\Delta E}{E_{o}}=\frac{4 \frac{M}{m_{n}} \cos ^{2} \theta}{\left(1+\frac{M}{m_{n}}\right)^{2}},
$$

where $M$ is the rest mass of the recoiling nucleus and $m_{n}$ is the rest mass of the incident neutron. Thus, at small scattering angles (i.e., $\theta \approx 0$ ), nearly all of the neutron kinetic energy can be transferred to the protons in the water. For comparison, a ${ }^{12} \mathrm{C}$ nucleus is capable of absorbing only maximum of 28.4 per cent of the incident neutron energy in a single collision. The proton energy will then be dissipated in the medium by means of ionization and nuclear interactions. Ranges soil water content ( $\%$ of dry weight) for different soil types are; sand (0-10), sandy loam (5-20), loam (8-25), silty loam (10-30), 


\section{CHAPTER 6 SHIELding MATERIALS AND NEUTRON ENERgy SPECTRA}

dry loam (14-30), and clay (15-30). Earth thus includes enough high atomic number elements to be generally effective against photons. It is generally a crackless shield, not prone to neutron leakage by streaming through such voids. The total density of earth including the water content varies widely, approximately from $1.70-2.25 \mathrm{~g} \mathrm{~cm}^{-3}$, dependent upon soil type and water content. In general, sandy soils have lower values of density than do heavy clays found in glacial deposits. Extrusive volcanic soils can have very low densities. In view of this variability, the detailed characteristics of the soil found at a particular accelerator site including water content may be needed for accurate shielding design. An example of an elemental composition of dry earth is given in Table 6.1 .

Table 6.1 Example elemental composition, dryweight per cent, of a representative soil. [Adapted from (Ch84).]

\begin{tabular}{|c|c|}
\hline Element & Global Average (\%) \\
\hline $\mathrm{O}$ & 43.77 \\
\hline $\mathrm{Si}$ & 28.1 \\
\hline $\mathrm{Al}$ & 8.24 \\
\hline $\mathrm{Fe}$ & 5.09 \\
\hline $\mathrm{Mn}$ & $0.07 \pm 0.06$ \\
\hline $\mathrm{Ti}$ & $0.45 \pm 0.43$ \\
\hline $\mathrm{Ca}$ & 3.65 \\
\hline $\mathrm{Mg}$ & 2.11 \\
\hline $\mathrm{K}$ & 2.64 \\
\hline $\mathrm{Na}$ & 2.84 \\
\hline
\end{tabular}

\subsubsection{Concrete}

Concrete has obvious advantages in that it can either be poured in place permanently or cast into modular blocks in configurations having considerable structural strength. Typical steel reinforcement has essentially no effect on the shielding properties. Concrete blocks may be used to shield targets, beam stops, etc. in a manner that allows their ready access for maintenance. When concrete blocks are used, they generally should be overlapped to avoid streaming through the cracks. The concrete density, locally variable due to available ingredients in the aggregate, can be increased by adding a heavier material to the recipe to increase both the density and average atomic number. Table 6.2 due to Chilton (Ch84) gives examples of some partial densities of various concretes. These partial densities are locally variable due to their strong dependence upon the aggregate material used in the concrete mix. When shielding neutrons, the concrete water content is quite important because it accounts for almost all of the hydrogen present. Under conditions of extremely low humidity, the water content of concrete can decrease with time, to as little as $50 \%$ of the initial value over a 20 year period. Heating due to the energy deposition of the beam can also drive out the water. 
Table 6.2 Examples of partial densities of representative concretes after curing. [Adapted from (Ch84).]

\begin{tabular}{|c|c|c|c|c|}
\hline $\begin{array}{l}\text { Type: } \\
\text { Additive: } \\
\text { Density }\left(\mathrm{g} \mathrm{cm}^{-3}\right) \text { : }\end{array}$ & $\begin{array}{c}\text { Ordinary } \\
2.34\end{array}$ & $\begin{array}{c}\text { Magnetite } \\
\left(\mathrm{FeO}, \mathrm{Fe}_{2} \mathrm{O}_{3}\right) \\
3.53\end{array}$ & $\begin{array}{c}\text { Barytes } \\
\left(\mathrm{BaSO}_{4}\right) \\
3.35\end{array}$ & $\begin{array}{c}\text { Magnetite \& Fe } \\
4.64\end{array}$ \\
\hline $\mathrm{H}$ & 0.013 & 0.011 & 0.012 & 0.011 \\
\hline $\mathrm{O}$ & 1.165 & 1.168 & 1.043 & 0.638 \\
\hline $\mathrm{Si}$ & 0.737 & 0.091 & 0.035 & 0.073 \\
\hline $\mathrm{Ca}$ & 0.194 & 0.251 & 0.168 & 0.258 \\
\hline $\mathrm{Na}$ & 0.040 & & & \\
\hline $\mathrm{Mg}$ & 0.006 & 0.033 & 0.004 & 0.017 \\
\hline $\mathrm{Al}$ & 0.107 & 0.083 & 0.014 & 0.048 \\
\hline $\mathrm{S}$ & 0.003 & 0.005 & 0.361 & \\
\hline K & 0.045 & & 0.159 & \\
\hline $\mathrm{Fe}$ & 0.029 & 1.676 & & 3.512 \\
\hline $\mathrm{Ti}$ & & 0.192 & & 0.074 \\
\hline $\mathrm{Cr}$ & & 0.006 & & \\
\hline $\mathrm{Mn}$ & & 0.007 & & \\
\hline $\mathrm{V}$ & & 0.011 & & 0.003 \\
\hline $\mathrm{Ba}$ & & & 1.551 & \\
\hline
\end{tabular}

\subsubsection{Other Hydrogenous Materials}

\subsubsection{Polyethylene and Other Materials That Can Be Borated}

Polyethylene $\left(\mathrm{CH}_{2}\right)_{\mathrm{n}}$ is a very effective neutron shield because of its hydrogen content (14\% by weight) and its density $\left(\approx 0.92 \mathrm{~g} \mathrm{~cm}^{-3}\right)$ because it can attenuate so-called "fast" neutrons. Thermal neutrons can be captured through the ${ }^{1} \mathrm{H}(\mathrm{n}, \gamma)^{2} \mathrm{H}$ reaction, a process having a cross section of 0.33 barn for neutrons in thermal equilibrium at room temperature $\left(E_{n}=0.025 \mathrm{eV}\right)$. The emitted $\gamma$-ray of $2.2 \mathrm{MeV}$ energy is sometimes a problem as an additional source of radiation. The fluence of these photons can be reduced by adding boron to the polyethylene. In such borated polyethylene many of the thermal neutrons are captured with the ${ }^{10} \mathrm{~B}(\mathrm{n}, \alpha)^{7} \mathrm{Li}$ reaction. The cross section of this reaction for room temperature thermal neutrons is much larger than for hydrogen, 3837 barns. In $94 \%$ of these captures, the emitted $\alpha$-particle is accompanied by a $0.48 \mathrm{MeV} \gamma$-ray. The $\alpha$ particle is readily absorbed by ionization in the material while the $\gamma$-ray has a somewhat shorter attenuation length than does the $2.2 \mathrm{MeV} \gamma$-ray (see Fig. 3.10). Borated polyethylene is commercially available with additives of boron (up to $32 \%$ ), lithium (up to $10 \%$ ), and lead (up to $80 \%$ ) in various shapes; e.g. planes, spheres, and cylinders.

These materials can be useful if it is necessary to economize on space and also to accomplish shielding of photons and neutrons simultaneously. Pure polyethylene is flammable, but some of the commercial products available contain self-extinguishing additives. Some of these materials are available in powder form, for molding into a desired shape by the user. Besides polyethylene, boron has been added to other materials 


\section{CHAPTER 6 SHIELding MATERIALS AND NeUtron ENERgy SPECTRA}

to form effective thermal neutron shields. These include other plastics, putties, clays, glasses, and even water to accomplish specific shielding objectives. Plastic materials like polyethylene can be subject to significant radiation damage at relatively low levels of integrated absorbed dose (Fa90), with possible effects upon their structural integrity.

\subsubsection{Water, Wood, and Paraffin}

The high hydrogen content make these materials seem attractive as a shielding material. However, Water tends to rust out its containers with loss of shielding. Exposed to thermal neutrons, it also emits the $2.2 \mathrm{MeV}$ capture $\gamma$-ray from ${ }^{1} \mathrm{H}(\mathrm{n}, \gamma)^{2} \mathrm{H}$ reaction. Adding boron is difficult due to the relative insolubility of boron salts. An exception with better solubility is potassium tetraborate. Wood is approximately as effective per unit length thickness as is concrete for shielding intermediate energy neutrons. However wood is flammable and prone to rot. Chemically treated wood that is nearly completely fireproof is available, but caution is warranted about the flammability of the material over time. Treated wood can also have reduced structural strength. Paraffin historically has been used for neutron shielding. Largely spurned due to its high flammability, it has been used successfully packaged in metal containers. Various forms of paraffin have melting points in the range of $49-72^{\circ} \mathrm{C}$.

\section{$\underline{6.2 .4 \text { Iron }}$}

With its relatively high density and low cost, iron is an attractive shielding material. The density of iron varies widely, from a low of $7.0 \mathrm{~g} \mathrm{~cm}^{-3}$ for some cast irons to a high of 7.8 $\mathrm{g} \mathrm{cm}^{-3}$ for some steels. The "textbook" value of $7.87 \mathrm{~g} \mathrm{~cm}^{-3}$ in Table 1.2 is almost never attained in the bulk quantities needed for radiation shielding. Because of its nonmagnetic properties and resistance to corrosion, stainless steel is often used in accelerator components. Due to concerns about accelerator-produced radioactivity (see Chapter 7), knowledge of the elemental composition of various alloys is useful. For example, longlived ${ }^{60} \mathrm{Co}$ can be produced in stainless steel but not in pure iron. The use of steel wool to fill cracks in a large shield is undesirable due to the contamination hazard of the resulting rust. Iron has a very important deficiency as a neutron shield discussed in Section 6.3.5.

\section{$\underline{\text { 6.2.5 High Atomic Number Materials (Lead, Tungsten, and Uranium) }}$}

The materials in this category are valuable due to their high atomic number, especially when shielding photons. Lead has a high density of $11.3 \mathrm{~g} \mathrm{~cm}^{-3}$. It is resistant to corrosion. Pure lead has major drawbacks due to its poor structural characteristics and low melting point $\left(327.4{ }^{\circ} \mathrm{C}\right)$. It is usually best used when laminated to some other more structurally stable material. Some alloys of lead also perform better. Lead is available as an additive to other materials to improve the ability to shield photons. Fabric blankets containing shredded lead can be used to shield radioactivated components to minimize exposures associated with accelerator maintenance activities during shutdowns if the material is not allowed to become activated. Lead must be handled in accordance with good industrial hygiene practices due to its chemical toxicity. The chemical toxicity as 


\section{ChAPTER 6 SHIELding MATERIALS AND NeUtron ENERgy SPECTRA}

well as any potential radioactive contamination renders the use of lead wool to fill cracks undesirable. Bismuth, having a density of $9.75 \mathrm{~g} \mathrm{~cm}^{-3}$, is sometimes used as a lowertoxicity substitute for lead for shielding against photons.

Tungsten is an excellent, but relatively expensive, shielding material. Its high density $\left(19.3 \mathrm{~g} \mathrm{~cm}^{-3}\right)$ and high melting temperature $\left(3410^{\circ} \mathrm{C}\right)$ make it extremely useful as a component in photon shields, beam absorbers, and beam collimators. It is difficult to machine, so alloys such as Hevimet are commonly used. Hevimet consists of tungsten (90\%), nickel (7.5\%) and copper (2.5\%) with a typical density ranging from 16.9 to 17.2 $\mathrm{g} \mathrm{cm}^{-3}$ (Ma68). It is commercially available from several sources and in various forms exemplified by HD18 ${ }^{19}$. HD18 is $95 \%$ tungsten, $3.5 \%$ nickel, and $1.5 \%$ copper, and has a density of $18 \mathrm{~g} \mathrm{~cm}^{-3}$.

Uranium is superficially an attractive shielding material in its depleted form. In depleted uranium, the concentration of $235 \mathrm{U}$ compared with the dominant ${ }^{238} \mathrm{U}$ usually is reduced from the natural value of $0.72 \%$, usually to $\leq 0.2 \%$. Its high density $\left(19.0 \mathrm{~g} \mathrm{~cm}^{-3}\right)$ and relatively high melting point $\left(1133^{\circ} \mathrm{C}\right)$ can be useful. Uranium may not be a good choice in environments with a high neutron flux density due to its susceptibility to fission. Depleted uranium is relatively safe, but if is combined with hydrogenous materials, nuclear fission criticality should be considered for the specific material and geometric arrangement to be employed. Even in the absence of hydrogen, the possibility of criticality can exist if the material is insufficiently depleted (Bo87).

Uranium has major deficiencies as a shielding material. It has a large anisotropic thermal expansion coefficient. Also, it readily oxidizes when exposed to air, especially under conditions of high humidity. The oxide is readily removable and presents a significant internal exposure hazard. Prevention of oxidation by sealing the material with epoxy or paint meets with only limited success due eventual embrittlement and chipping caused by the intense $\beta$-radiation field at the material surface. Sealed containers filled with dry air or noble gases or liquefied noble gases such as argon appear to be the best storage solution to limit oxidation. Small chips of this element are pyrophoric, complicating machining-type processes by posing yet another safety hazard. Uranium is classified as a "nuclear material" by the U. S. Department of Energy rendering it subject to stringent accountability requirements.

\section{$\underline{\text { 6.2.6 Miscellaneous Materials (Beryllium, Aluminum, and Zirconium) }}$}

These three materials find considerable usage as accelerator components because of various properties. Beryllium, especially in the form of the oxide $\mathrm{BeO}$ with a melting point of $2530{ }^{\circ} \mathrm{C}$, is often used as a target material in intense beams because of its excellent heat transfer properties with a resultant ability to endure large values of energy deposition density. It has been used at high energy accelerators in relatively large quantities as a "filter" to enrich one particle type at the expense of another, taking

\footnotetext{
${ }^{19}$ Mi-Tech Metals, Inc., 4701 Massachusetts Ave., Indianapolis, Indiana 46218
} 


\section{CHAPTER 6 SHIELding MATERIALS AND NEUTRON ENERgy SPECTRA}

advantage of particle-specific variations in absorption cross sections. Of concern, especially when fabricating components, is the extreme chemical toxicity of the metal and its compounds. Aluminum is used in accelerator components because of its nonmagnetic properties and its resistance to corrosion. It is a poor shield against neutrons. Zirconium, having good thermal conductivity properties and a high melting point $\left(1852{ }^{\circ} \mathrm{C}\right)$, has a very small thermal neutron capture cross section. It is not a good neutron absorber but has been found to be useful in beam-handling component material.

\subsection{Neutron Energy Spectra Outside of Shields}

As has been discussed previously, at most accelerators the shielding is largely designed to attenuate neutrons emitted in all directions. Exceptions are found at forward angles at higher energy accelerators where energetic muons need to be shielded. In this section examples of neutron energy spectra found at accelerators external to shielding are presented and discussed. These examples, not intended to be a comprehensive set, illustrate the general principles.

\section{$\underline{6.3 .1 \quad \text { General Considerations }}$}

In the most simple approximation, outside of thick shields of soil or concrete that contain some hydrogen content (usually as water), accelerator neutron shields are generally to first order proportional to inverse energy. Such $\mathbf{1} / \mathbf{E}$ spectra can span energies extending from those of thermal neutrons $\left(<E_{n}>\approx 0.025 \mathrm{eV}\right)$ up to the energy of the accelerated particles. More commonly they are cut off at some upper energy of lower value. At this level of approximation, the spectrum is given by

$$
\frac{d \phi(E)}{d E}=k \frac{1}{E}
$$

where $k$ is a normalizing constant. Rohrig (Ro83) observed that it often convenient to plot such spectra as flux per logarithmic energy interval by simply plotting $E d \phi(E) / d E$;

$$
\frac{d \phi(E)}{d \ln E}=E \frac{d \phi(E)}{d E}
$$

In neutron physics, $E \phi(E)$ is the fluence per unit "lethargy" and such a plot is called a lethargy plot. Such a plot suppresses the dominant $1 / E$ dependence typically found in such spectra. Most of the example spectra discussed here are lethargy plots.

\subsubsection{Examples of Neutron Spectra Due to Incident Electrons}

Alsmiller and Barish have calculated the neutron energy spectra that arise when $400 \mathrm{MeV}$ electrons are incident on a thick copper target (A173). Predictions of the neutron yields over several ranges of production angle $\theta$ resulted from these calculations. They considered four different shielding materials; soil, concrete, ilmenite $\left(\mathrm{FeTiO}_{3}\right)$, and iron. 


\section{ChAPTER 6 SHIELding MATERIALS AND NeUtron ENERgy SPECTRA}

Calculations of neutron energy spectra and the fractional contributions to the total dose equivalent from neutrons with energies less than a given energy $E$ for the same spectrum for the angular region $0<\theta<30^{\circ}$ are presented in Fig. 6.1. These results are for a radial depth in the shield of 7.0 mean free paths of the highest energy neutrons found in this source spectrum within this angular range. Table 6.3 gives the densities and the neutron mean free paths used for the four materials. (Ilmenite is a mineral containing iron and titanim oxide; $\mathrm{FeTiO}_{3}$.)

\section{Table 6.3 Material properties used in the calculations} of Alsmiller and Barish. [Adapted from (Al73).]

\begin{tabular}{|l|c|c|}
\hline Material & $\begin{array}{c}\text { Density } \\
\left(\mathbf{g ~ c m}^{-\mathbf{3}} \mathbf{)}\right.\end{array}$ & $\begin{array}{c}\text { Mean Free Path } \\
\left(\mathbf{g ~ c m}^{-\mathbf{2}} \mathbf{)}\right.\end{array}$ \\
\hline soil & 1.8 & 103.6 \\
\hline concrete & 2.3 & 105.3 \\
\hline ilmenite & 3.8 & 120.6 \\
\hline iron & 7.8 & 138.6 \\
\hline
\end{tabular}

In Fig. 6.1, the inverse square dependence was removed to eliminate the effect of "geometrical" attenuation within the shield. It should be clear that the neutron spectrum in the iron shield is markedly different from that found in the soil and concrete shields. The characteristics of the spectra found in the ilmenite are intermediate, perhaps related to the presence of iron in this material. This behavior associated with iron shielding is found at nearly all accelerators and will be discussed in Section 6.3.5.

\subsubsection{Examples of Neutron Spectra Due to Low and Intermediate Energy Protons}

Calculations and measurements of neutron energy spectra at various depths in several different shielding due to $52 \mathrm{MeV}$ protons have been reported by Uwamino et al. (Uw82) and are presented in Fig. 6.2. Alsmiller et al. (A175) have provided predictions of neutron energy spectra averaged over specific angular intervals for $200 \mathrm{MeV}$ protons stopped in a thick water target. The results are given for large angles and are presented in Fig. 6.3.

\subsubsection{Examples of Neutron Spectra Due to High Energy Protons}

In the regime of proton energies well above $1.0 \mathrm{GeV}$ the details of the spectra are far more sensitive to geometrical considerations than they are dependent upon the incident proton energy. O'Brien carried out a calculation of a generalized neutron spectrum to be found external to a high energy proton accelerator with a proton kinetic energy greater than $0.8 \mathrm{GeV}$ (OB71). The results were compared with measurements and alternative "generic" spectrum calculations performed by Höfert and Stevenson [(Hö84a), (St84b)]. The results are provided in Fig. 6.4 for both "lateral" $\left(\theta \approx 90^{\circ}\right)$ and "forward" $(\theta \approx 0)$ angular regions. Those for forward angles also include the spectra of charged pions and protons. It is clear that at the forward angles, the fluence of hadrons at high energies is likely to be a mixture of charged particles and neutrons. 

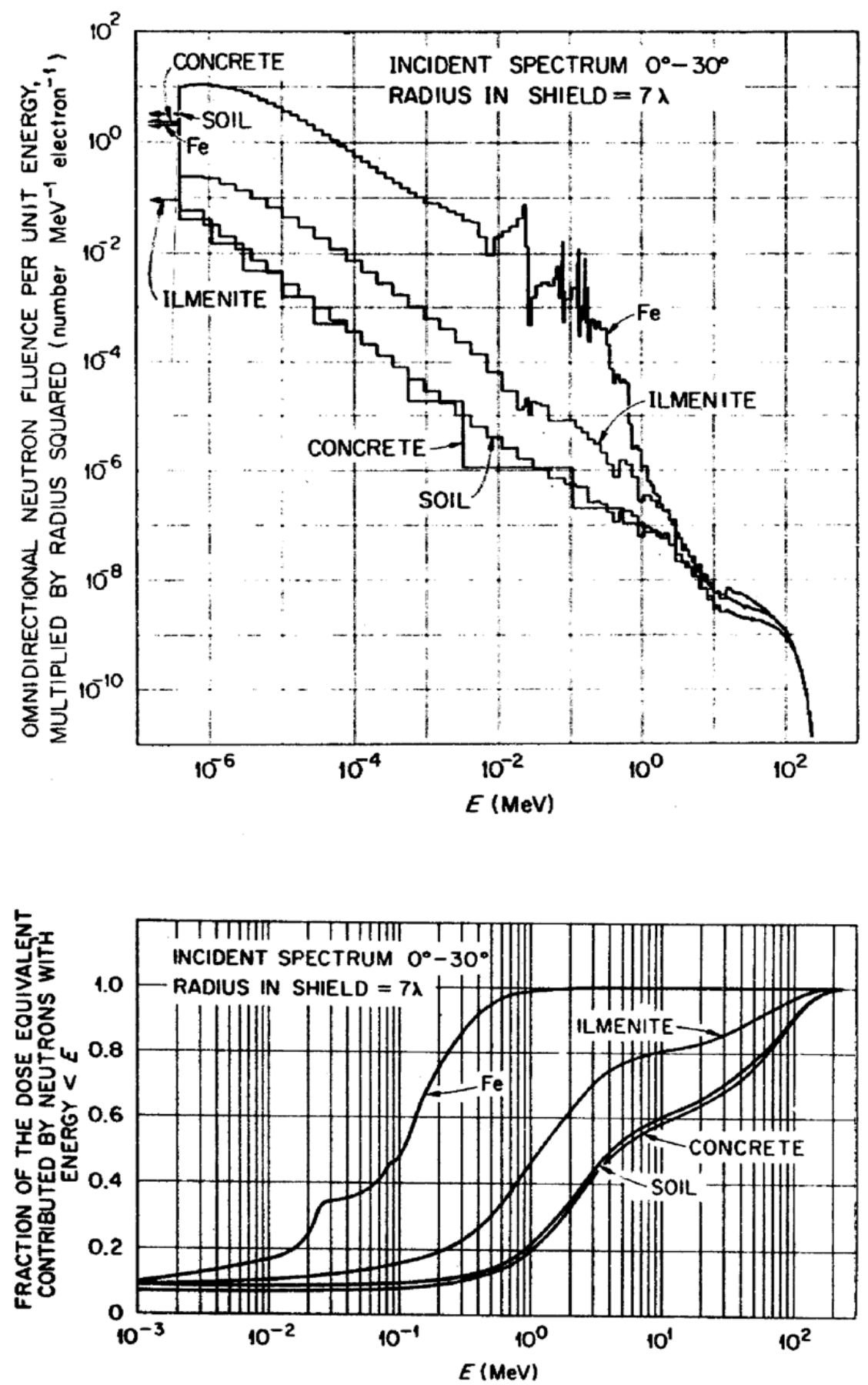

Fig. 6.1 Neutron spectral information from $400 \mathrm{MeV}$ electrons incident on a thick $\mathrm{Cu}$ target. The upper frame shows the omnidirectional neutron fluence per unit energy multiplied by the square of the radial depth in the shield as a function of energy for the various shield materials studied by Alsmiller and Barish. The lower frame shows the fractional contribution to the total dose equivalent from neutrons with energies less than $E$ as a function of $E$ for the illustrated shielding materials. [Reproduced from (A173).] 

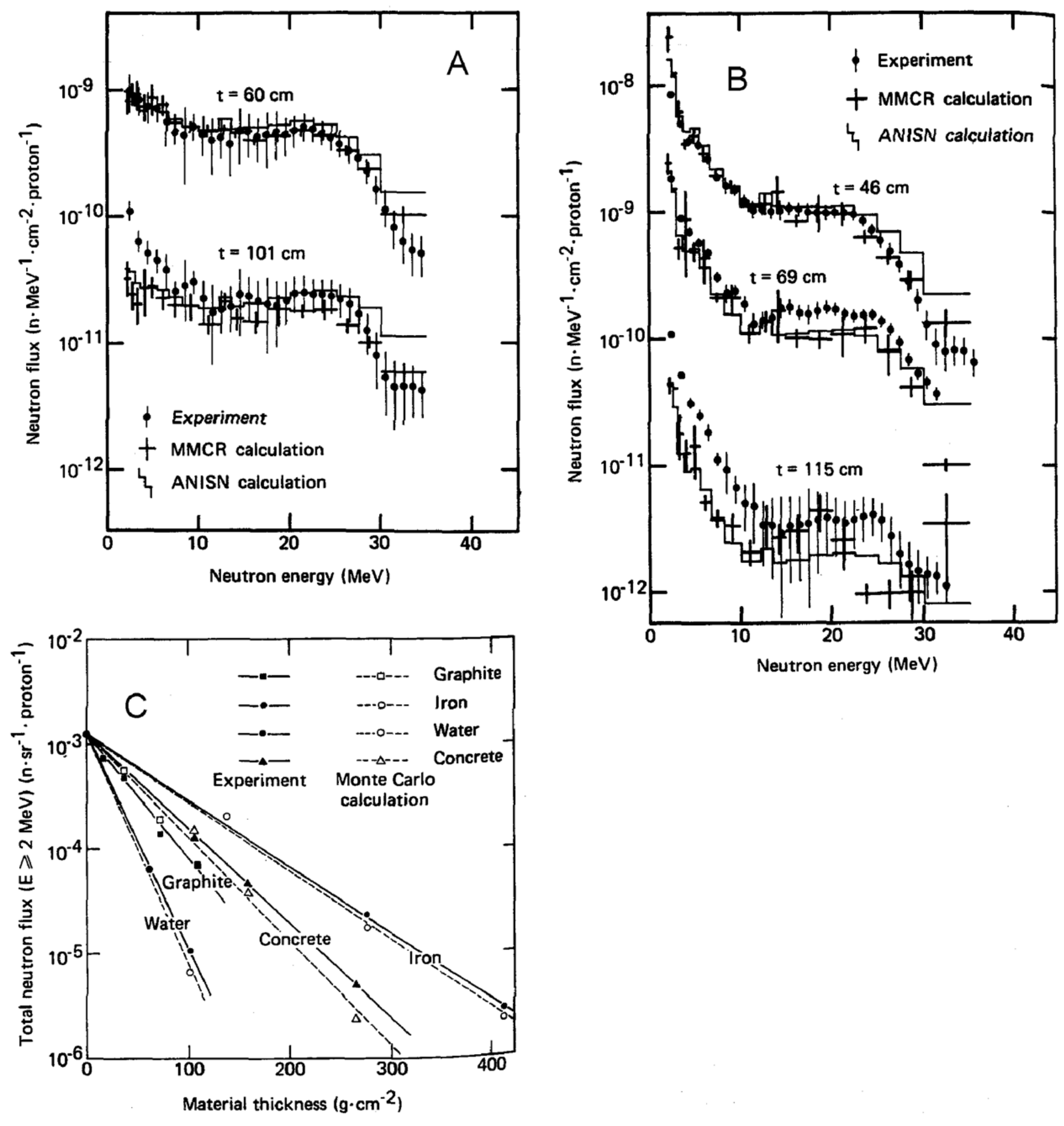

Fig. 6.2 Forward neutron energy spectra and attenuations measured and calculated by Uwamino et al. for $52 \mathrm{MeV}$ protons incident on several different shielding materials. Following a thickness $t$ the spectra were determined at $\theta=0$. Frame $\mathbf{A}$ displays spectra for water at two different values of $t$, frame $\mathbf{B}$ displays spectra for ordinary concrete at three different values of $t$, and frame $\mathbf{C}$ provides data on the attenuation profiles for various materials. [Adapted from (Uw82).] 


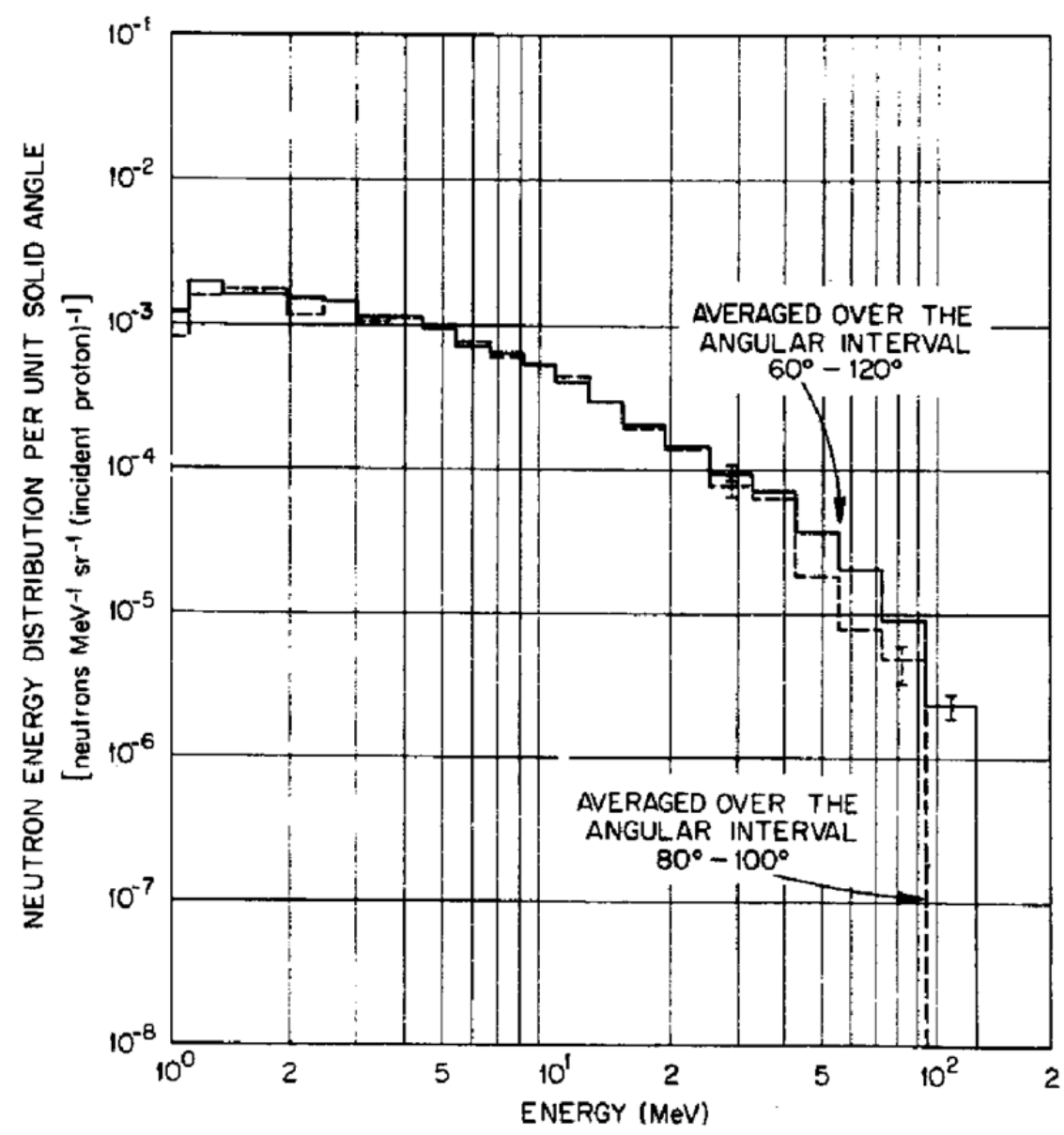

Fig. 6.3 Energy distribution of neutrons averaged over particular angular intervals, produced when $200 \mathrm{MeV}$ protons are stopped in a thick water target. The protons are incident at $\theta=0^{\circ}$. [Adapted from (A175).]

Details of the geometry can produce peaks in the spectra. Examples have been reported by various workers [(Pa73), (Th88), (E186), (McC88), (Co88)]. Such peaks are typically encountered in the few $\mathrm{MeV}$ region. Figs. 6.5, 6.6, 6.7, and 6.8 are plots of neutron spectra and sketches of the corresponding shielding geometry taken from Cossairt et al. [(Co88), republished in (Co09a)]. These spectra were obtained (i.e., "unfolded") using the Bonner sphere technique discussed in Section 9.5.2.1. In these four figures in the sketches of the configurations involved, "spheres" denote the locations of the measurements. The results are typical of the spectra found at high energy proton accelerators. Fig. 6.5 ("Debuncher Ring") is rather typical of the spectra found external to earth and concrete shields lateral to high energy proton accelerators. The neutron energy spectrum displayed in Fig. 6.6 ("Tevatron Tunnel") is particularly interesting because its shape was demonstrated to be essentially independent of proton energy over the range of 150 to $900 \mathrm{GeV}$ (McC88). Fig. 6.7 ("Labyrinth") is typical of the results obtained in the second and succeeding sections ("legs") of a labyrinth penetration. Fig. 6.8 ("Iron Leakage") is of special interest and discussed in Section 6.3.5. 


\section{CHAPTER 6 SHIELding MATERIALS AND NEUTRON ENERgy SPECTRA}
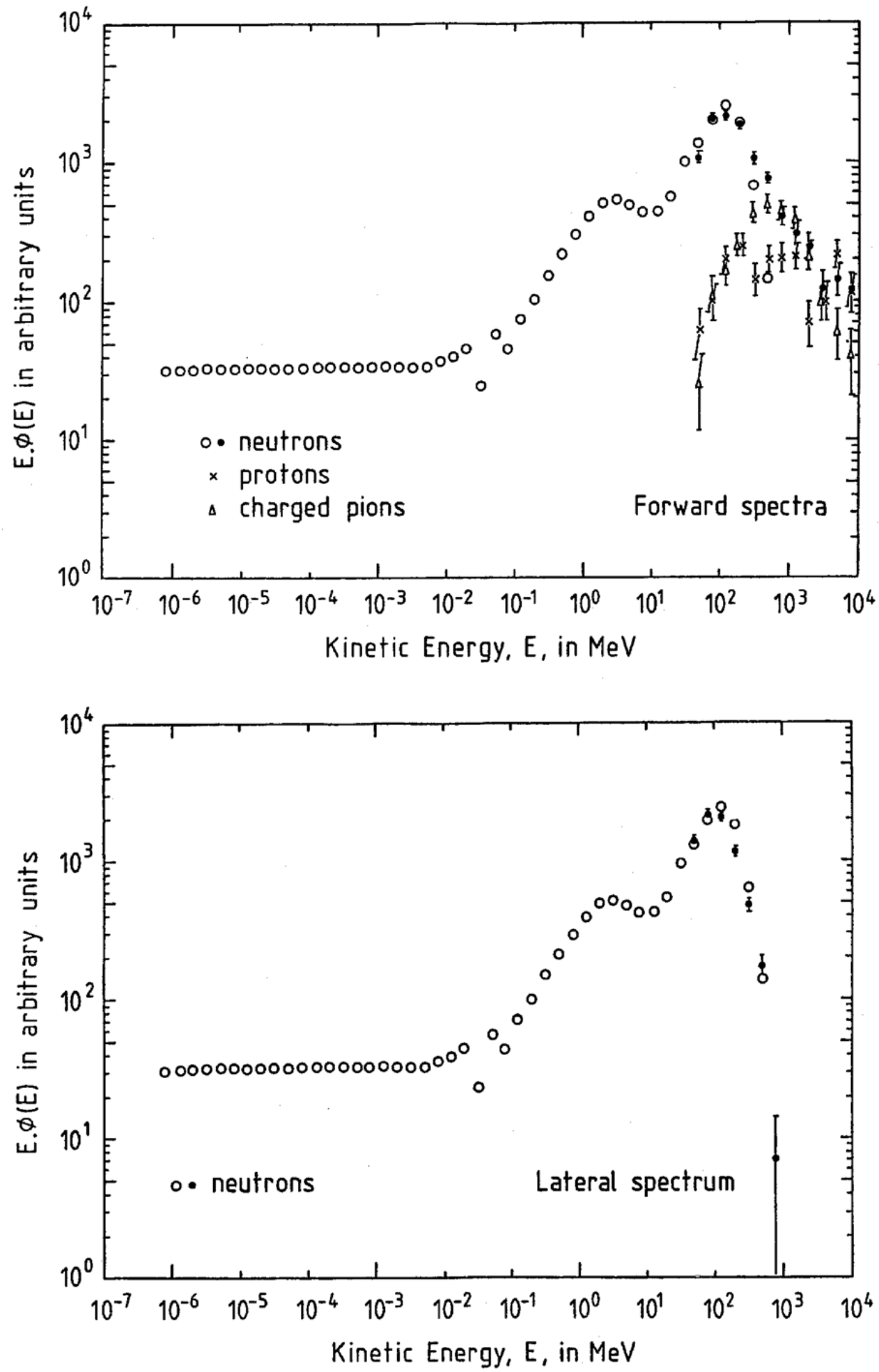

Fig. 6.4 Hadron energy spectra outside of a concrete shield at a high energy proton accelerator at forward angles (upper frame) and at large angles (lower frame). The open circles represent the calculations of O'Brien (OB71) while the other symbols represent the calculations of Höfert and Stevenson. [Adapted from (Hö84a).] 


\section{DEBUNCHER RING of $A P-10$}
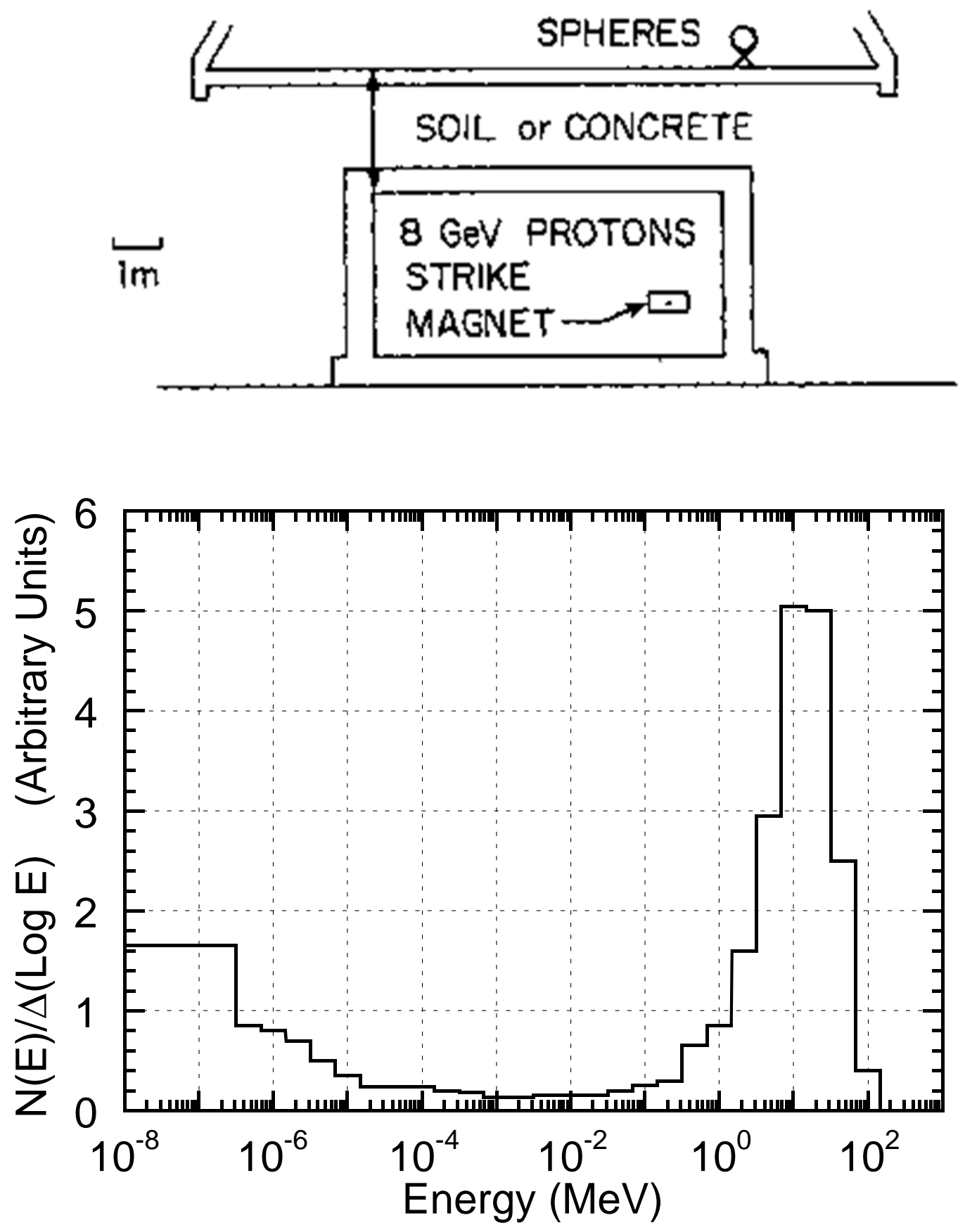

Fig. 6.5 Neutron energy spectrum (lower frame) obtained external to a beam enclosure (upper frame) in which $8 \mathrm{GeV}$ protons struck the yoke of a magnet. The location was the Fermilab Debuncher Ring. The beam was directed into the page in the cross-sectional view shown in the upper frame. The normalization of the spectrum is arbitrary. [Adapted from (Co88).] 

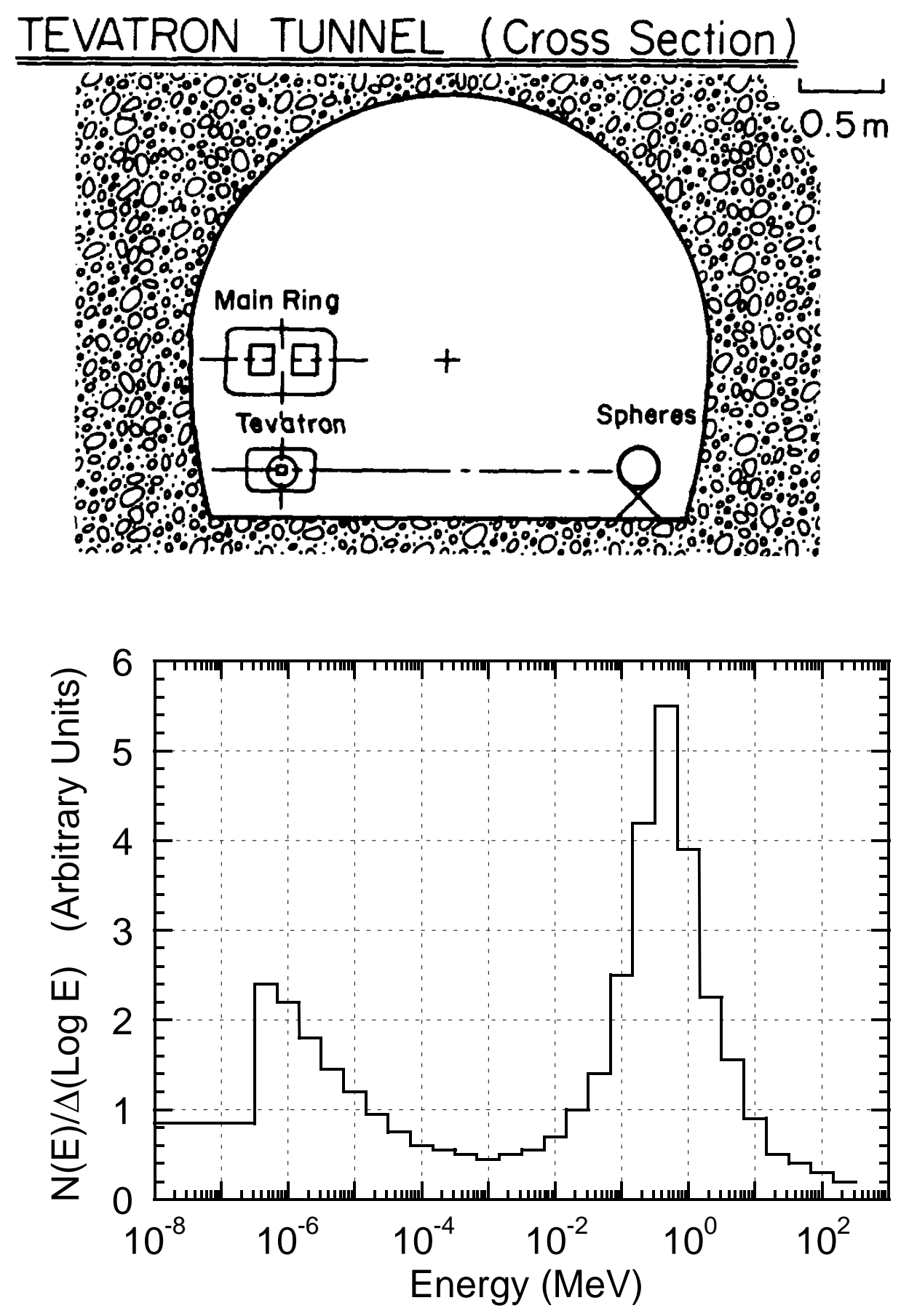

Fig. 6.6 Neutron energy spectrum (lower frame) obtained internally in a beam enclosure (upper frame) in which $800 \mathrm{GeV}$ protons interacted with residual gas in the Tevatron vacuum chamber during circulating beam conditions. The site was the Fermilab Tevatron Ring. The beam was directed into the page in the cross-sectional view shown in the upper frame. The normalization of the spectrum is arbitrary. [Adapted from (McC88).] 


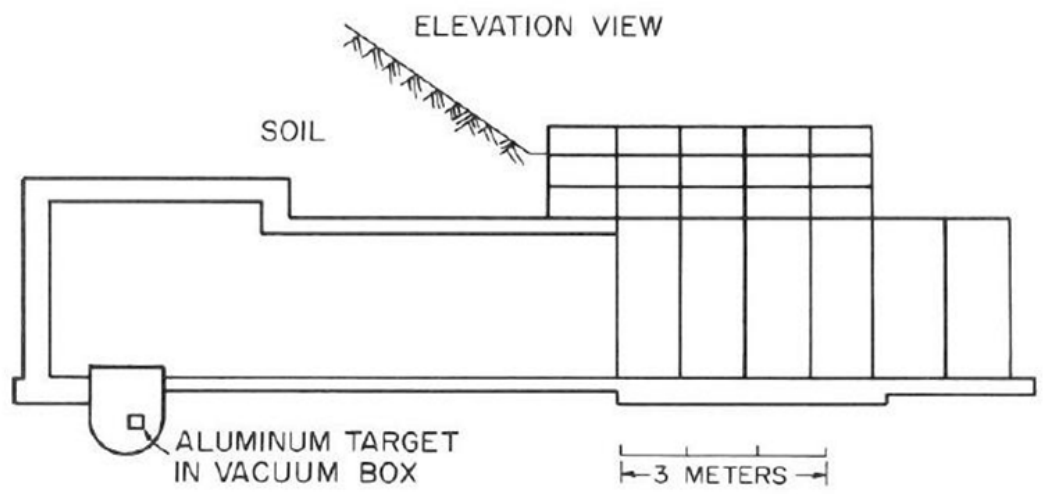

PLAN VIEW
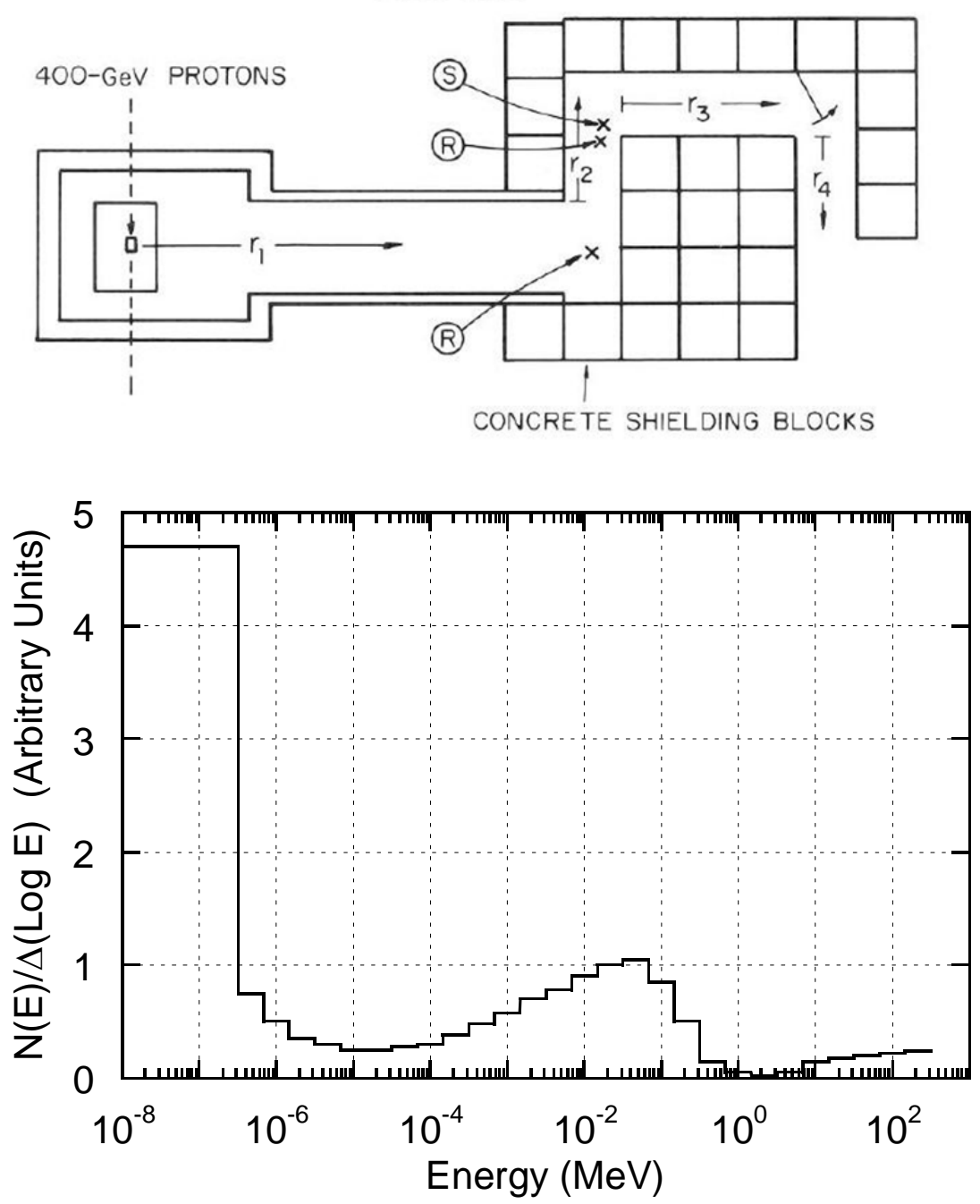

Fig. 6.7 Neutron energy spectrum (lower frame) obtained within a labyrinth enclosure (upper frame) in which $400 \mathrm{GeV}$ protons interacted with an aluminum target located beneath the floor of the enclosure shown. The spectrum was measured in the second leg at the location denoted S. A quality factor measurement was made at the locations denoted $\mathbf{R}$ (see Sections 5.2 .4 and 9.5.7). The normalization of the spectrum is arbitrary. [Adapted from (Co85b).] 


\section{Chapter 6 SHiElding MATERIALS AND NEUTRon ENERgy SPECTRA}

\subsubsection{Leakage of Low Energy Neutrons Through Iron Shielding}

One peak commonly found in such spectra is of particular importance. As discovered by Alsmiller and Barish (A173), iron has a major deficiency as a shield for fast neutrons. The dominant mechanism by which fast neutrons lose energy is inelastic scattering. At energies below the first excited state of any nucleus, inelastic scattering becomes energetically impossible and elastic scattering is left as the only removal process aside from nuclear reactions. As evident from Eq. (6.1), elastic scattering is a very inefficient mechanism for energy transfer from neutrons scattering off a much massive nucleus such as iron. It is analogous to scattering billiard balls off of bowling balls compared with the scattering of billiard balls off other billiard balls of equal mass. Similarly, the scattering of neutrons by the "free" protons in hydrogenous materials transfers energy much better than does elastic scattering of neutrons from iron nuclei. The first excited state of ${ }^{56} \mathrm{Fe}$, the dominant isotope in natural iron (92\% abundance), is at $847 \mathrm{keV}$. Thus, neutrons having kinetic energies above $847 \mathrm{keV}$ in a given spectrum will be slowed by inelastic scattering to $E_{n} \approx 847 \mathrm{keV}$. Once below that energy, since elastic scattering of neutrons from iron is a very inefficient, the process of slowing them further is inhibited. Thus an accumulation of neutrons just below the energy of this first excited state will occur. The effect is further enhanced since the radiation weighting or quality factor for neutrons as a function of energy also has its maximum value at about $500 \mathrm{keV}$ (see Fig. 1.3).

A good example of this phenomenon is provided by the geometry and spectra shown in Figs. 6.8. The effect is also seen in the spectra of Fig. 6.1. Both spectra in Fig. 6.8 were measured at about $\theta=90^{\circ}$ from a beam absorber struck by secondary particles due to 800 $\mathrm{GeV}$ proton interactions far upstream of the beam absorber (E186). The secondary particles were chiefly a multitude of hadrons of several hundred GeV. The beam absorber was shielded by the yoke of a large iron magnet as shown in Fig. 6.8a. Originally, the neutron energy spectra was measured directly adjacent to this iron shield with the result shown in Fig. 6.8b. Later, in order to reduce the intensity of the neutron radiation in an adjacent location of rather high occupancy, eight additional concrete shielding blocks square in cross section and $91.4 \mathrm{~cm}$ thick were placed between the neutron detectors and the beam absorber up to a height of about 0.5 meters above the beam line. These are shown as the cross-hatched blocks in Fig. 6.8a. The neutron energy spectrum was measured again with the result displayed in Fig. 6.8c. For the bare iron situation the normalized dose equivalent rate external to the shield was over 40 times that measured after the concrete was installed. This factor is far in excess of the approximate factor of 10 expected from simple attenuation of the equilibrium cascade neutron spectrum, indicative of both the importance of the leakage neutrons and the maximization of their quality or radiation weighting factor.

In general, an iron shield "capped" or "backed" by such a concrete shield will be an efficient use of space. Apparently, about $60 \mathrm{~cm}$ of concrete is the most efficient thickness to use for this purpose [(Yu83), (Za87)]. Such capping must obviously be done at the outside of the shield. Shielding properties of other elements with atomic mass numbers much larger than unity and roughly similar first excited state energies will be comparable. 


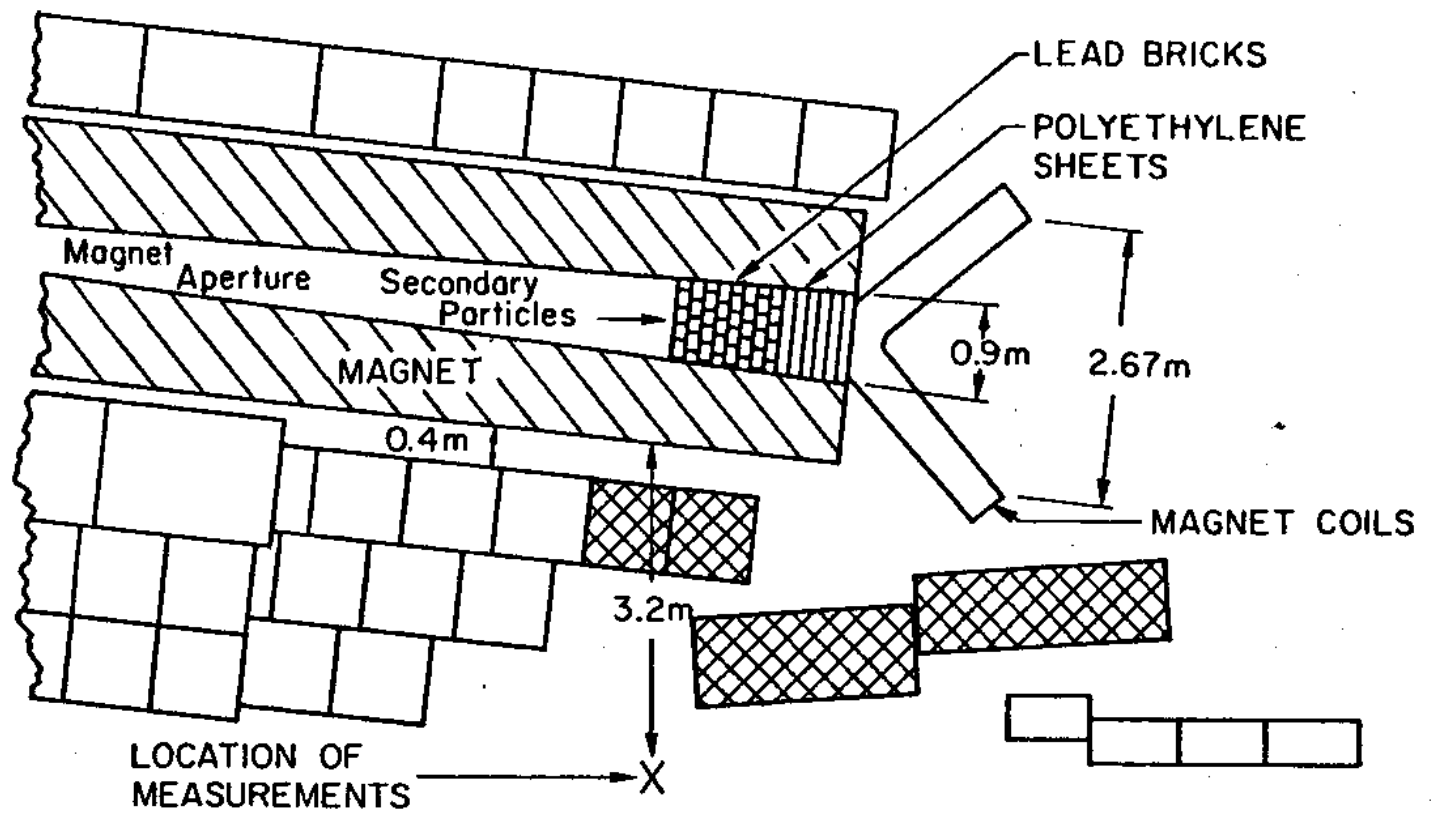

PLAN VIEW IN MAGNET MIDPLANE
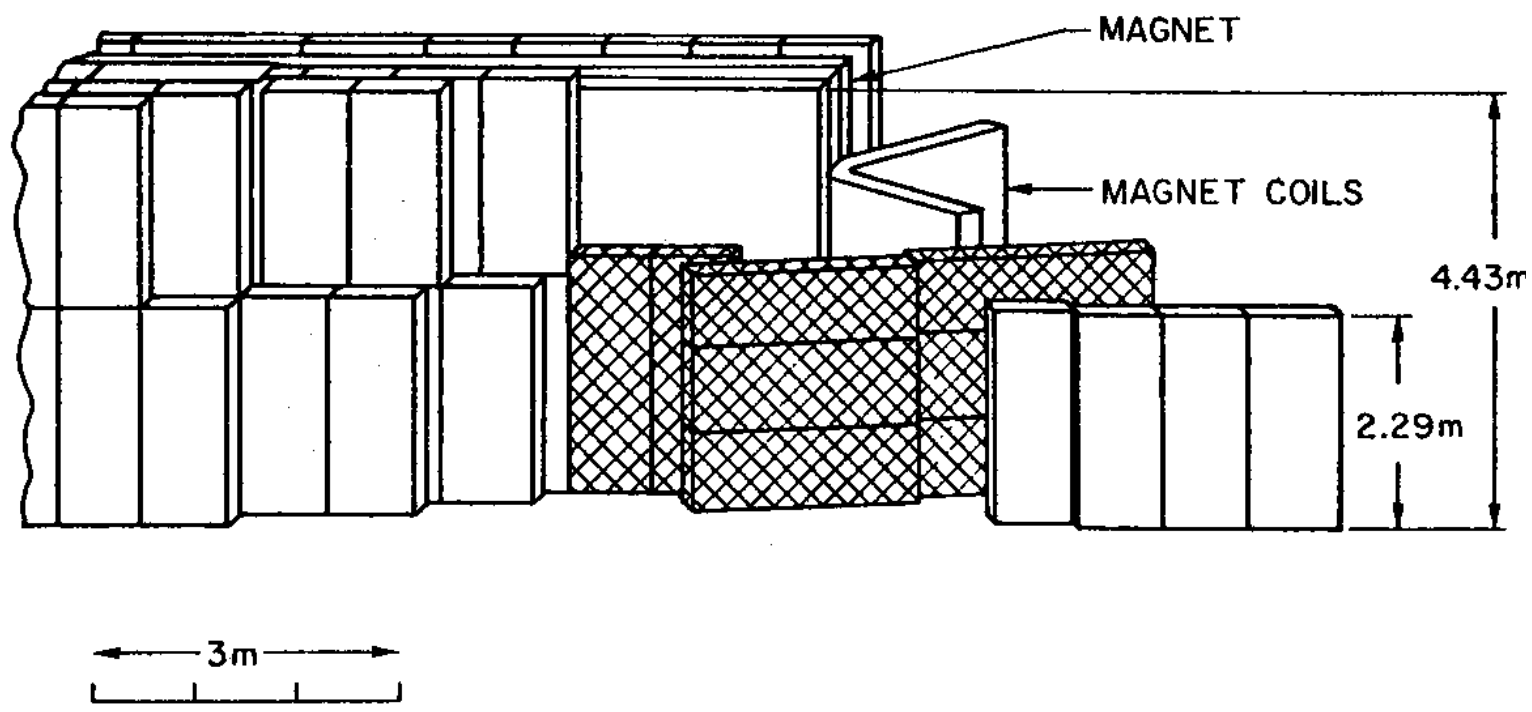

\section{SIDE VIEW}

Fig. 6.8a Situation for the measurements shown in Figs. $6.8 \mathrm{~b}$ and $6.8 \mathrm{c} .800 \mathrm{GeV}$ protons interacted with a production target and beam absorber within the gap of the magnet shown. The beam axis was horizontal, $1.8 \mathrm{~m}$ above the floor with the beam going from left to right in the figure. The target and beam absorber were far upstream (to the left) of the region shown here. Many hadrons produced by these interactions were intercepted by lead and polyethylene absorber shown in the plan view. The plain shielding blocks shown were of ordinary concrete and square in cross section. During initial operations, the cross-hatched blocks, also of ordinary concrete, were not in place between this beam absorber and the location of measurements and the result was the measured spectrum in Fig. 6.8b. Later, those blocks were added and the spectrum shown in Fig. 6.8c was measured. [Reproduced from (E186).] 


\section{CHAPTER 6 SHIELDING MATERIALS AND NEUTRON ENERgy SPECTRA}

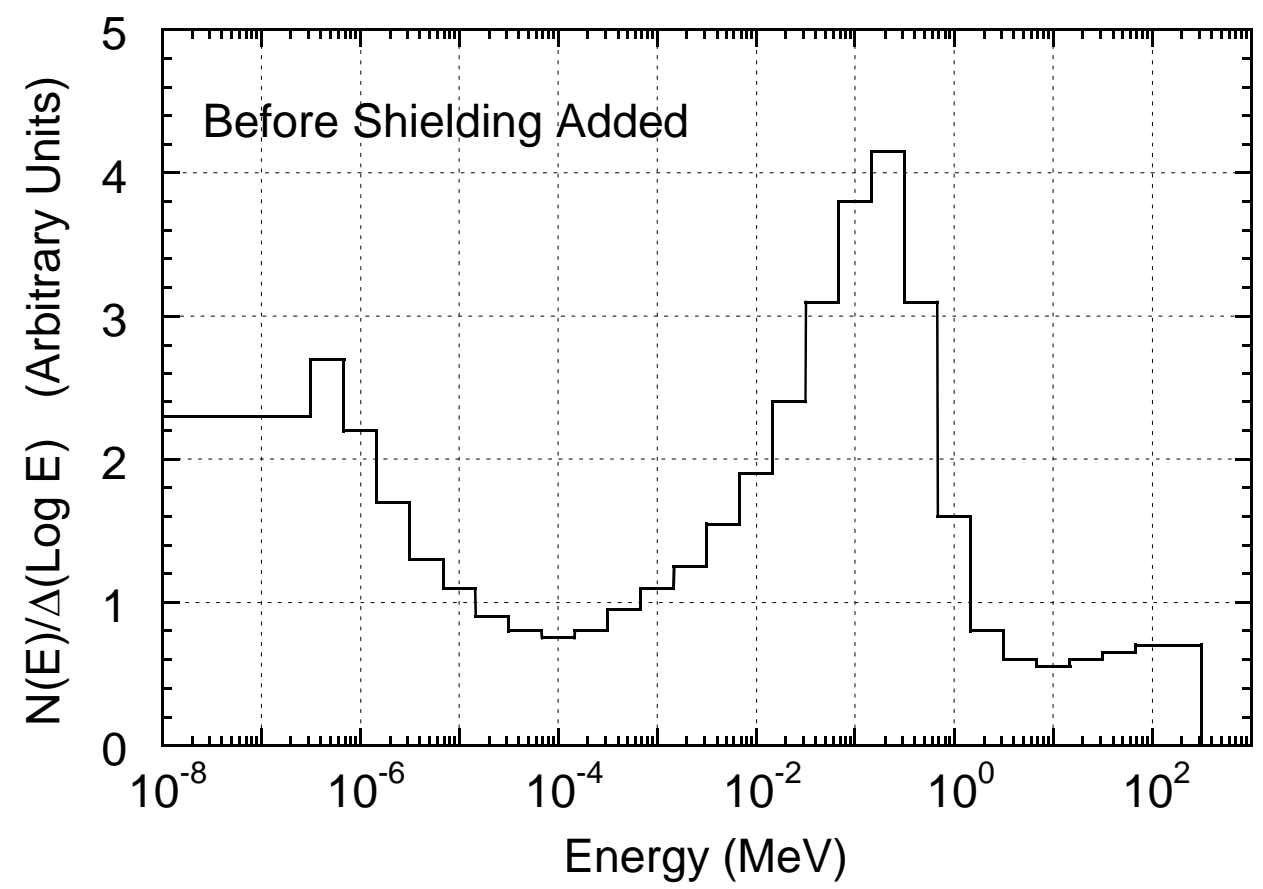

b)

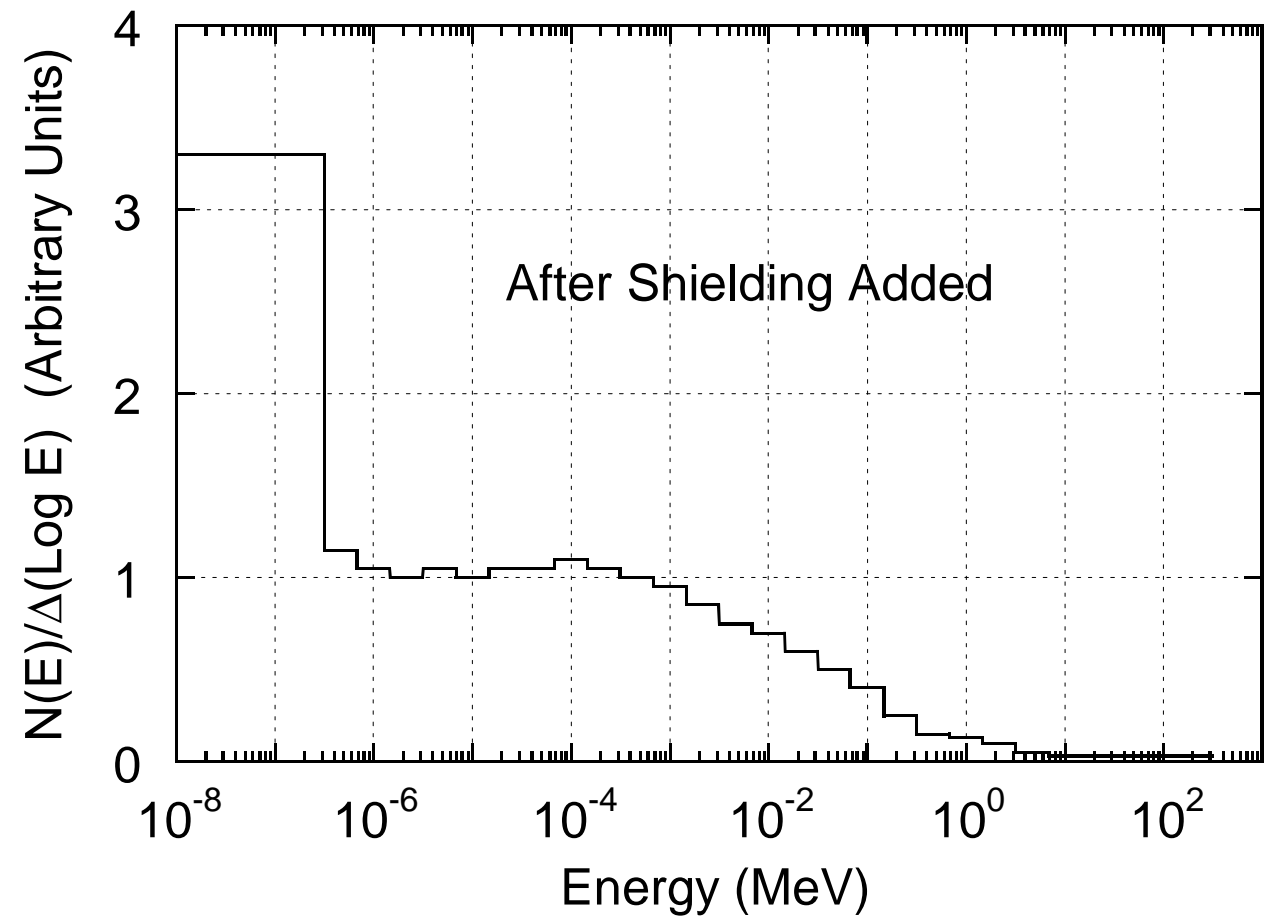

c)

Fig. 6.8 b\&c Neutron energy spectra obtained external to the shielding configuration shown in Fig. 6.8a for the two different situations described above. The normalization of the spectra is arbitrary. [Adapted from (El86).] 


\section{CHAPTER 6 SHIELding MATERIALS AND NEUTRON ENERgy SPECTRA}

\subsubsection{Neutron Spectra Due to Ions}

Measurements of neutron energy spectra due to ions remain rare. Britvich et al. (Br99) have reported results for ${ }^{12} \mathrm{C}$ ions incident on a Hevimet target at $155 \mathrm{MeV}$ nucleon ${ }^{-1}$. The spectrum was measured at $\theta=94^{\circ}$ without shielding at a distance of $121 \mathrm{~cm}$ from the target as shown in Fig. 6.9. Qualitatively similar spectra were obtained at this location with ${ }^{4} \mathrm{He}$ and ${ }^{16} \mathrm{O}$ ions at $155 \mathrm{MeV}$ nucleon ${ }^{-1}$. The spectrum of neutrons due to the ${ }^{12} \mathrm{C}$ ions was also measured at $\theta=94^{\circ}$ at a distance of $403 \mathrm{~cm}$ from the target shielded by 308 $\mathrm{g} \mathrm{cm}^{-2}$ of ordinary concrete and shown in Fig. 6.10.

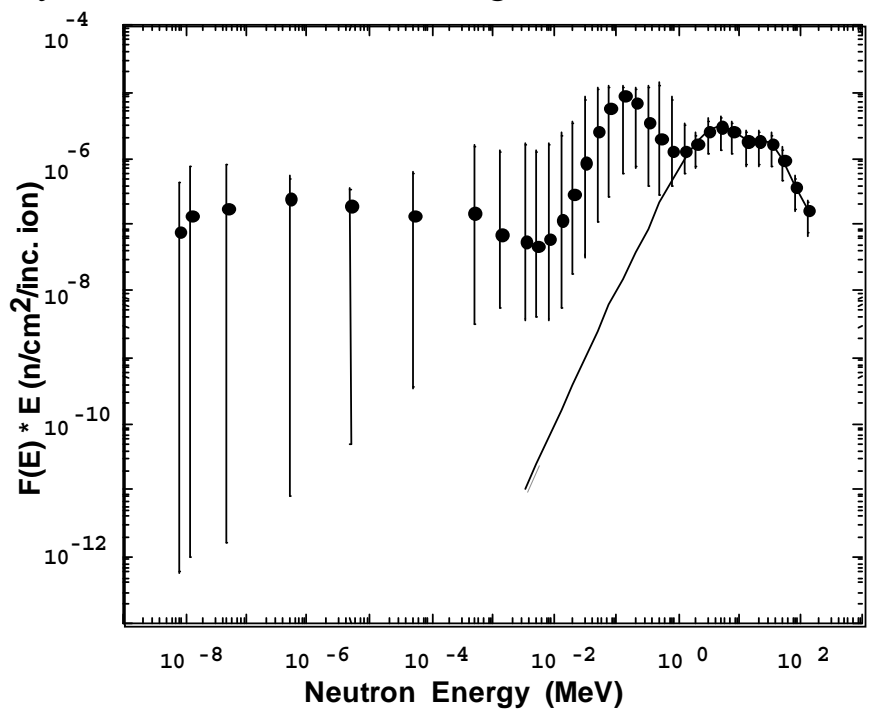

Fig. 6.9 Lethargy plot of neutron energy spectrum $F(E) \times E$ [i.e., $E \phi(E)]$ at $121 \mathrm{~cm}$ and $\theta=94^{\circ}$ from a thick Hevimet target bombarded by ${ }^{12} \mathrm{C}$ ions at $155 \mathrm{MeV}$ nucleon $^{-1}$ where $F$ denotes the normalized neutron fluence elsewhere usually denoted $\Phi$. The solid line are fits to these data as suggested by Nakamura (Na85). [Reproduced from (Br99).]

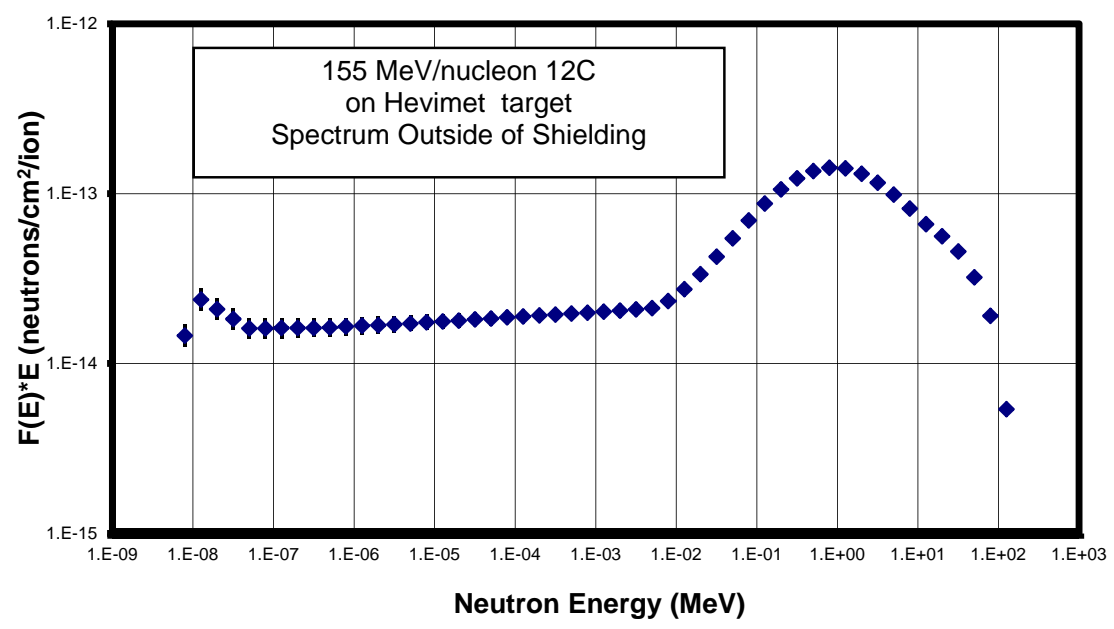

Fig. 6.10 Lethargy plot of neutron energy spectrum $F(E) \times E$ [i.e., $E \phi(E)]$ at $403 \mathrm{~cm}$ and $\theta=94^{\circ}$, and external to $128.3 \mathrm{~cm}$ of concrete shielding, from a thick Hevimet target bombarded by ${ }^{12} \mathrm{C}$ ions at $155 \mathrm{MeV}$ nucleon ${ }^{-1} . F$ denotes the normalized neutron fluence elsewhere usually denoted $\Phi$. [Reproduced from (Br99)]. 


\section{CHAPTER 6 SHIELdING MATERIALS AND NEUTRON ENERgy SPECTRA}

Intuitively, especially for ions of high atomic number, one might expect more copious production of neutrons. This matter was studied in measurements conducted by Aroura et al. (Ar97) for lead ions having a specific energy of $160 \mathrm{GeV}$ nucleon $^{-1}$. Comparisons of neutron energy spectra on top of a concrete shield surrounding a lead target were made with those obtained with $205 \mathrm{GeV}$ protons. The results are given in Fig. 6.11.
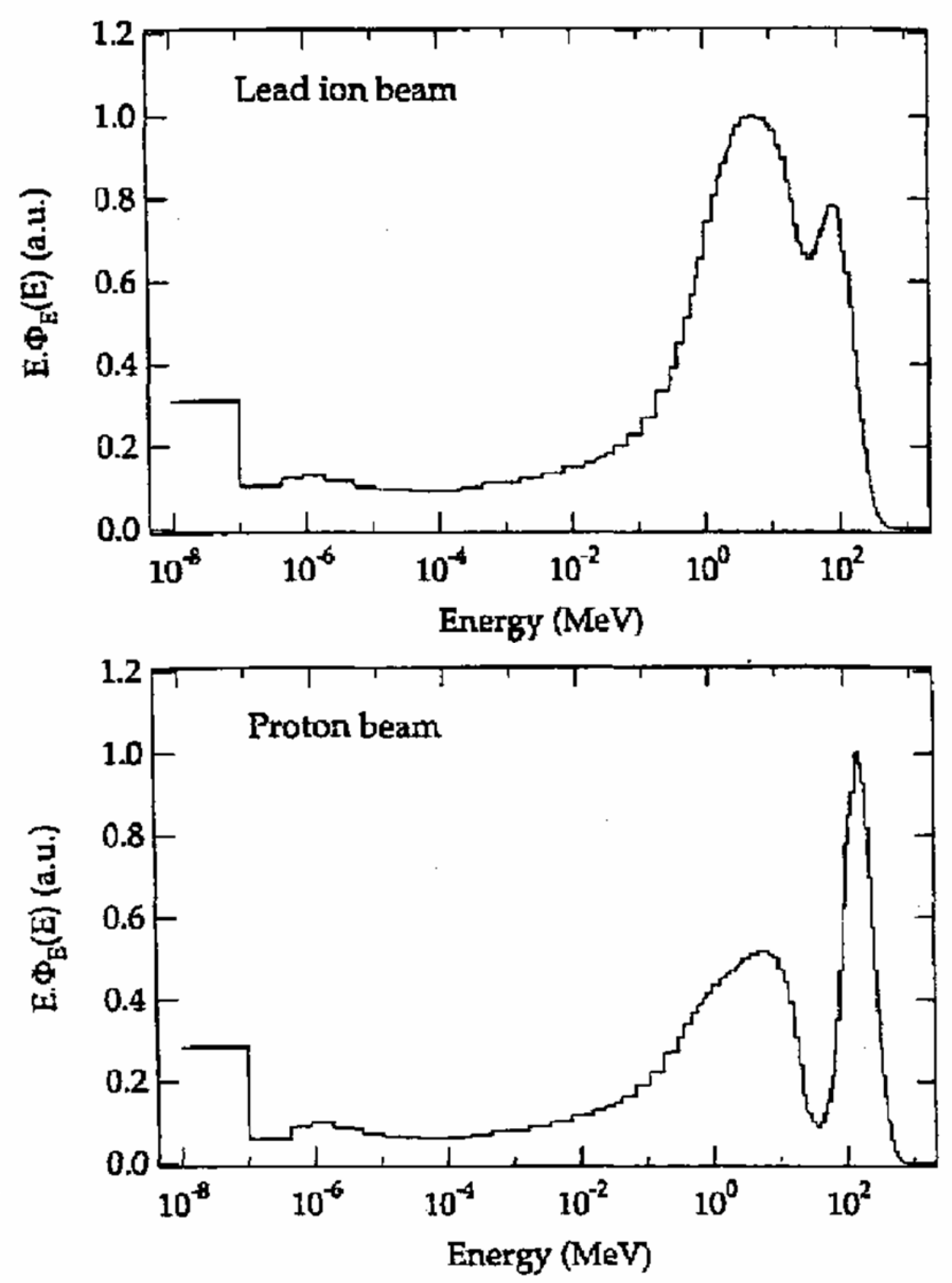

Fig. 6.11 Lethargy plots of neutron energy spectra around a lead ion beam of $160 \mathrm{GeV} \mathrm{nucleon}^{-1}$ shielded by concrete compared with that obtained with of a $205 \mathrm{GeV}$ proton beam. The units used for the ordinate are arbitrary. [Reproduced from (Ar97).] 


\subsubsection{Neutron Fluence and Dosimetry}

The distribution of neutrons in different regions of the energy spectra can be quite important both in the potential to produce induced radioactivity (see Chapters 7 and 8) and with respect to the dose equivalent or effective dose that might be received by personnel present in the prompt radiation field. As examples, Table 6.4 gives the per cent fluence determined to be in specific energy bins for the five spectra displayed in Figs. $6.5,6.6,6.7$, and 6.8. Table 6.5 gives these percentages for dose equivalent and average quality factor $\langle Q>$ for these five spectra in accordance with the 1973 Radiation Protection System.

Table 6.4 Per cent fluence in specific energy bins for selected neutron energy spectra. [Adapted from (Co88).]

\begin{tabular}{|c|c|c|c|c|c|}
\hline Energy Range & $\begin{array}{c}\text { Fig. 6.5 } \\
\text { Debuncher }\end{array}$ & $\begin{array}{c}\text { Fig. 6.6 } \\
\text { Tevatron }\end{array}$ & $\begin{array}{c}\text { Fig. 6.7 } \\
\text { Labyrinth }\end{array}$ & $\begin{array}{c}\text { Fig. 6.8b } \\
\text { Iron (Bare) }\end{array}$ & $\begin{array}{c}\text { Fig. 6.8c } \\
\text { Iron (Shielded) }\end{array}$ \\
\hline$<1.5 \mathrm{eV}$ & 31.5 & 19.5 & 71 & 28 & 55 \\
$0.0015-100 \mathrm{keV}$ & 12.5 & 36 & 24 & 46 & 43 \\
$0.1-2 \mathrm{MeV}$ & 8.5 & 36 & 2.0 & 17.5 & 2.0 \\
$2-25 \mathrm{MeV}$ & 40.5 & 7.0 & 1.0 & 4.5 & 0.1 \\
$>25 \mathrm{MeV}$ & 7.0 & 1.5 & 1.5 & 4.0 & 0.0 \\
\hline
\end{tabular}

Table 6.5 Per cent of dose equivalent $H_{\text {equiv }},(1973$ System) in specific energy bins for sample spectra along with average quality factor $\langle Q\rangle$. [Adapted from (Co88).]

\begin{tabular}{|c|c|c|c|c|c|}
\hline Energy Range & $\begin{array}{c}\text { Fig. 6.5 } \\
\text { Debuncher }\end{array}$ & $\begin{array}{c}\text { Fig. 6.6 } \\
\text { Tevatron }\end{array}$ & $\begin{array}{c}\text { Fig. 6.7 } \\
\text { Labyrinth }\end{array}$ & $\begin{array}{c}\text { Fig. 6.8b } \\
\text { Iron (Bare) }\end{array}$ & $\begin{array}{c}\text { Fig. 6.8c } \\
\text { Iron (Shielded) }\end{array}$ \\
\hline$<1.5 \mathrm{eV}$ & 1.5 & 2.0 & 32 & 4.0 & 41.5 \\
$0.0015-100 \mathrm{keV}$ & 0.5 & 6.0 & 16 & 11.5 & 37 \\
$0.1-2 \mathrm{MeV}$ & 9.0 & 58.5 & 9.0 & 35 & 17 \\
$2-25 \mathrm{MeV}$ & 75 & 26 & 13 & 24 & 3.5 \\
$>25 \mathrm{MeV}$ & 14 & 7.5 & 30 & 25 & 1.0 \\
\hline$<Q>(\mathrm{NC} 71)$ & 5.8 & 6.9 & 3.1 & 5.4 & 2.5 \\
\hline
\end{tabular}

Using the 1990 Radiation Protection System gives different values (Co09a). Table 6.6 gives the percentages of effective dose in these same energy bins along with average effective quality factors (i.e., radiation weighting factors) using the ICRP Report 60 (IC91) and ICRP Report 103 (IC07) prescriptions.

Table 6.6 Per cent of effective dose $H_{\text {eff }}(1990$ System) in specific energy bins for sample spectra along with average quality factor $<Q>$. [Adapted from (Co09a).]

\begin{tabular}{|c|c|c|c|c|c|}
\hline Energy Range & $\begin{array}{c}\text { Fig. 6.5 } \\
\text { Debuncher }\end{array}$ & $\begin{array}{c}\text { Fig. 6.6 } \\
\text { Tevatron }\end{array}$ & $\begin{array}{c}\text { Fig. 6.7 } \\
\text { Labyrinth }\end{array}$ & $\begin{array}{c}\text { Fig. 6.8b } \\
\text { Iron (Bare) }\end{array}$ & $\begin{array}{c}\text { Fig. 6.8c } \\
\text { Iron (Shielded) }\end{array}$ \\
\hline$<1.5 \mathrm{eV}$ & 1.0 & 1.8 & 22 & 3.9 & 31 \\
$0.0015-100 \mathrm{keV}$ & 0.6 & 4.6 & 12 & 9.1 & 30 \\
$0.1-2 \mathrm{MeV}$ & 5.1 & 45 & 9.0 & 31 & 16 \\
$2-25 \mathrm{MeV}$ & 63 & 35 & 15 & 22 & 12 \\
$>25 \mathrm{MeV}$ & 30.3 & 13.6 & 42 & 34 & 11 \\
\hline$<Q>$ (IC91) & 7.7 & 11 & 6.1 & 8.2 & 6.4 \\
$<Q>$ (IC07) & 7.5 & 9.5 & 4.2 & 6.6 & 3.6 \\
\hline
\end{tabular}




\section{CHAPTER 6 SHIELDING MATERIALS AND NEUTRON ENERgy SPECTRA}

Fig. 6.12 is a plot of cumulative values of the same quantities calculated for $1000 \mathrm{GeV}$ protons incident on the face of a thick cylindrical concrete shield. As determined by Van Ginneken and Awschalom (Va75), the dependence upon incident proton energy of the distributions of fluence and dose equivalent is slight.

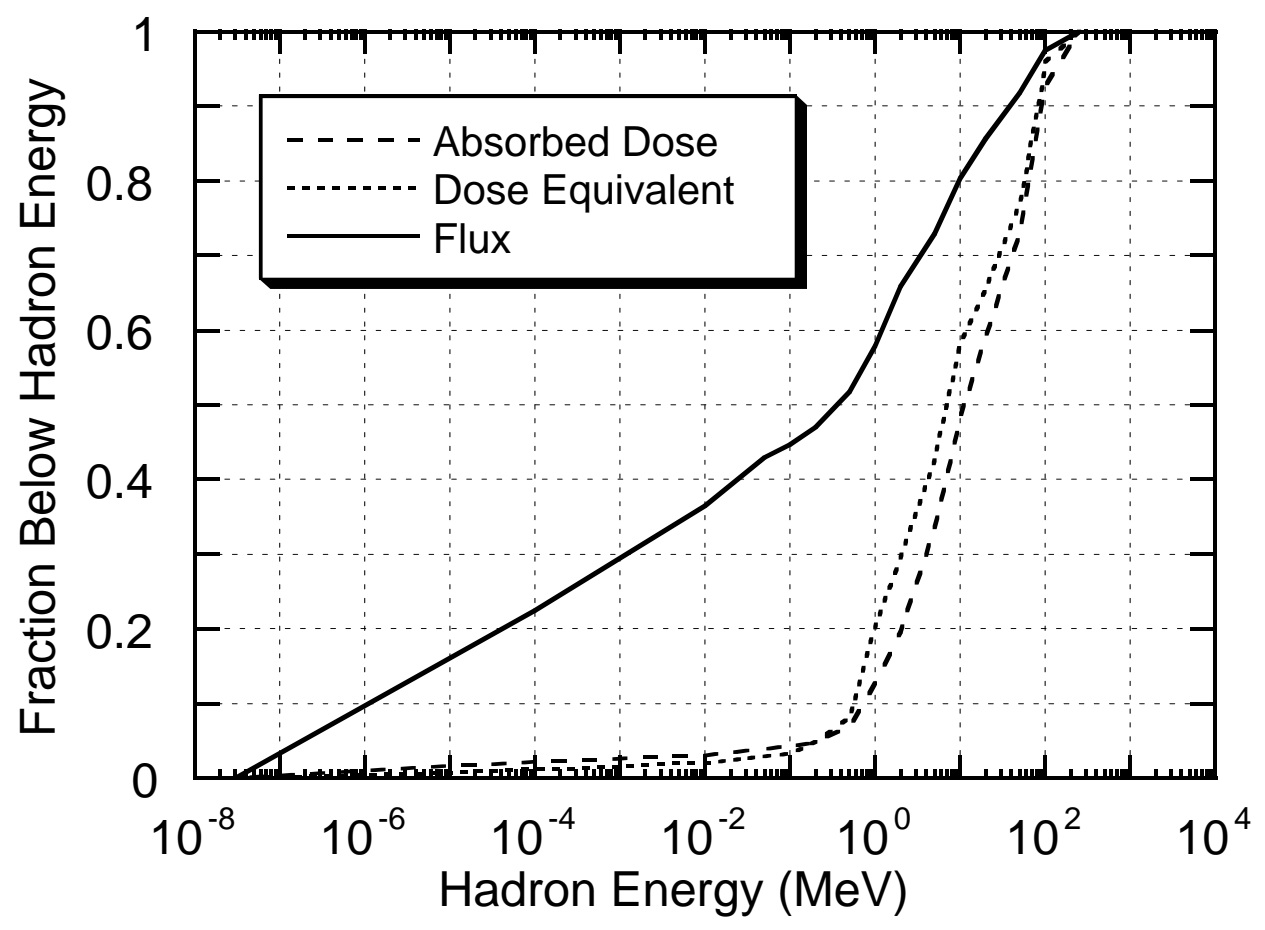

Fig. 6.12 Fraction of the omnidirectional flux, entrance absorbed dose, and maximum dose equivalent (1973 System) below a given hadron kinetic energy as a function of hadron energy for the region between zero and $450 \mathrm{~cm}$ depth and between $300 \mathrm{~cm}$ and $750 \mathrm{~cm}$ radius calculated for $1000 \mathrm{GeV} / \mathrm{c}$ protons incident on the face of a solid concrete cylinder. [Adapted from (Va75).] 


\section{CHAPTER 7 INDUCED RADIOACTIVITY IN ACCELERATOR COMPONENTS}

\subsection{Introduction}

In this chapter the production of induced radioactivity at accelerators is described. The discussion begins with a review of the basic principles of the production of radioactivity. It proceeds with a discussion of the activation of accelerator components. Generalizations are included that may be used for practical health physics applications.

\subsection{Fundamental Principles of Induced Radioactivity}

In principle, induced radioactivity can be produced at nearly all accelerators above all but the most minimal energies. When the accelerated beam particles interact with a nucleus, the resultant nuclear reactions can convert it into a different nuclide, one that may or may not be radioactive. The activity of a given radionuclide refers to the number of atoms that decay per unit time. The customary unit of activity is the Curie $(\mathrm{Ci})$ and its submultiples. One Curie was historically defined to be the activity of one gram of natural radium. It is precisely defined to be $3.7 \times 10^{10}$ decays per second. The SI unit of activity is the Becquerel (Bq); 1.0 decay per second, with multiples such as Gbq commonly used. Also important is the specific activity, the activity per unit volume (e.g., $\mathrm{Bq} \mathrm{cm} \mathrm{cm}^{-3}$ ) or the activity per unit mass (e.g., $\mathrm{Bq} \mathrm{g}^{-1}$ ). Attention needs to be paid to the units used!

Radioactive decay is a random process characterized by a mean-life (units of time) denoted by $\tau$, and its reciprocal the decay constant (units of inverse time) ${ }^{20} \lambda$, with $\lambda=1 / \tau$. If a total of $N_{\text {tot }}(t)$ atoms of a radionuclide are present at time $t$, the total activity $A_{\text {tot }}(t)$ is determined from the random nature of radioactive decay to be

$$
A_{t o t}(t)=-\frac{d N_{t o t}(t)}{d t}=\frac{1}{\tau} N_{t o t}(t)=\lambda N_{t o t}(t)
$$

If at time $t=0, N_{\text {tot }}(0)$ atoms are present, then this simple differential equation has the solution at some later time $t=T$ [with $T>0$ ];

$$
A_{t o t}(T)=\lambda N_{t o t}(0) \exp (-\lambda T)=A_{t o t}(0) \exp (-\lambda T)
$$

Usually, the time required to decay to half of the original activity, the half-life $t_{1 / 2}$ is tabulated and is related to the mean-life by the following:

$$
\tau=\frac{1}{\ln 2} t_{1 / 2}=\frac{1}{0.693} t_{1 / 2}=1.442 t_{1 / 2}
$$

In this text values of half-lives listed are from the National Nuclear Data Center (Tu05).

\footnotetext{
${ }^{20}$ Care needs to be taken with respect to the usage of the symbol $\lambda$. In the literature and in this text it is, in different discussions, used for both the attenuation length and for the decay constant.
} 


\section{CHAPTER 7 INDUCED RADIOACTIVITY IN ACCELERATOR COMPONENTS}

The most simple activation situation at accelerators is that of the steady irradiation of some material by a spatially uniform flux density of particles that begins at time $t=0$ and continues at a constant rate for an irradiation period that ends at $t=t_{i}$. This is followed by a decay period called the cooling time that is denoted $t_{c}$, a period of time that begins at $t=t_{i}$ and ends at $t=t_{i}+t_{c}$. For this simple situation, self-absorption of the hadrons by the target is ignored, as is the fact that a spectrum of particles of different types or energies might be incident. Thus the process of producing the radioactivity is characterized by a single average cross section $\sigma$. In the more generalized situations the value of this cross section must be obtained from averaging over the energy spectra of the particles incident.

The number of atoms of the radionuclide of interest per unit volume will thus be governed by the following equation during the period of the irradiation:

$$
\frac{d n(t)}{d t}=-\lambda n(t)+N \sigma \phi
$$

where $n(t)$ is the number density of atoms of the radionuclide of interest at time $t\left(\mathrm{~cm}^{-3}\right)$, $N$ is the number density of "target" atoms $\left(\mathrm{cm}^{-3}\right), \sigma$ is the production cross section $\left(\mathrm{cm}^{2}\right)$ and $\phi$ is the flux density $\left(\mathrm{cm}^{-2} \mathrm{sec}^{-1}\right)$ of incident particles. On the right hand side of Eq. (7.4), the first term represents the loss of radionuclides through decay during the irradiation while the second term is the gain of radionuclides through the production reaction under consideration. The equation has the following solution for $0<t<t_{i}$ :

$$
n(t)=\frac{N \sigma \phi}{\lambda}\left(1-\mathrm{e}^{-\lambda t}\right)
$$

Thus the specific activity induced in the material as a function of time during the irradiation is given by $a(t)=\lambda n(t)$, hence

$$
a(t)=N \sigma \phi\left(1-\mathrm{e}^{-\lambda t}\right)\left(\mathrm{Bq} \mathrm{cm}^{-3}\right) \text { for } 0<t<t_{i} .
$$

To obtain specific activity in units of Curies $\mathrm{cm}^{-3}$, one must simply divide the result by the conversion factor $3.7 \times 10^{10} \mathrm{~Bq} \mathrm{Ci}^{-1}$. At the instant of completion of the irradiation $\left(t=t_{i}\right)$, the specific activity is

$$
a\left(t_{i}\right)=N \sigma \phi\left\{1-\exp \left(-\lambda t_{i}\right)\right\}\left(\mathrm{Bq} \mathrm{cm}^{-3}\right),
$$

so that the specific activity as a function of time is characterized by a buildup from zero to the saturation concentration value of $N \sigma \phi$ for infinitely long irradiations. After the irradiation has ceased $\left(t>t_{i}\right)$, the specific activity as a function of the cooling time $t_{c}$ will obviously decay exponentially and be given by the activation equation;

$$
a\left(t_{c}\right)=N \sigma \phi\left\{1-\exp \left(-\lambda t_{i}\right)\right\}\left\{\exp \left(-\lambda t_{c}\right)\right\}\left(\mathrm{Bq} / \mathrm{cm}^{3}\right)
$$




\section{CHAPTER 7 INDUCED RADIOACTIVITY IN ACCELERATOR COMPONENTS}

where $t_{c}$ is the cooling time and

$$
t_{c}=t-t_{i}
$$

To obtain total activities where uniform flux densities of particles of constant energy are incident on a homogeneous target, one can simply multiply by the volume of the target. In more complex cases numerical integrations are needed.

For $\gamma$-ray emitters typical of those emitted by accelerator-produced radionuclides in the range of from about $100 \mathrm{keV}$ to $10 \mathrm{MeV}$, many textbooks in health physics demonstrate that the absorbed dose rate $d D / d t\left(\mathrm{rad} \mathrm{h}^{-1}\right)$ at a distance $r$ (meters) from a point source is approximately given in terms of the source strength $S(\mathrm{Ci})$ and the photon energies present $E_{\gamma i}(\mathrm{MeV})$ by

$$
\frac{d D}{d t}=0.4 \frac{S}{r^{2}} \sum_{i} E_{\gamma i} .
$$

The summation is over all $\gamma$-rays present, including appropriate branching fractions if multiple photons are emitted in the course of decay. If $d D / d t$ is desired as an approximate absorbed dose rate in Gy $\mathrm{h}^{-1}$ at a distance $r$ (meters) from a source strength $S$ in gigaBecquerels $^{21}(\mathrm{GBq})$, the constant 0.4 is replaced with $1.081 \times 10^{-4}$. For non-point sources, an appropriate spatial integration must be performed.

\subsection{Activation of Components at Electron Accelerators}

\subsubsection{General Phenomena}

At electron accelerators, as was described in Chapter 3, the direct interactions of electrons in material results in the copious production of photons. Through various nuclear reaction channels, these photons then proceed to produce charged particles and neutrons that then interact further with material to produce radioactivity. In general, if the facility is properly shielded against prompt radiation, the radioactivity hazard will be confined to accelerator components and the interior of the accelerator enclosure. This is consistent with the experience at most accelerators. Usually most of the radiation exposure incurred by the workers is due to maintenance activities on radioactivated components, handling and moving of activated items, radiation surveys, and radioactive waste handling rather than to exposure to the prompt radiation fields. An understanding of the production of radionuclides can help reduce personnel exposures through the selection of more appropriate machine component materials and the optimization of decay ("cool-down") times recommended after the beam has been turned off. Some familiarity with the relevant cross sections is extremely useful. "Global" data (i.e., data spanning the periodic table and a large domain of energies) have been compiled by Barbier (Ba69). The results are given Figs. 7.1, 7.2, and 7.3.

\footnotetext{
${ }^{21} \mathrm{GBq}\left(10^{9} \mathrm{~Bq}\right)$ or $\mathrm{MBq}\left(10^{6} \mathrm{~Bq}\right)$ are often better units of activity for practical work than is the tiny $\mathrm{Bq}$.
} 

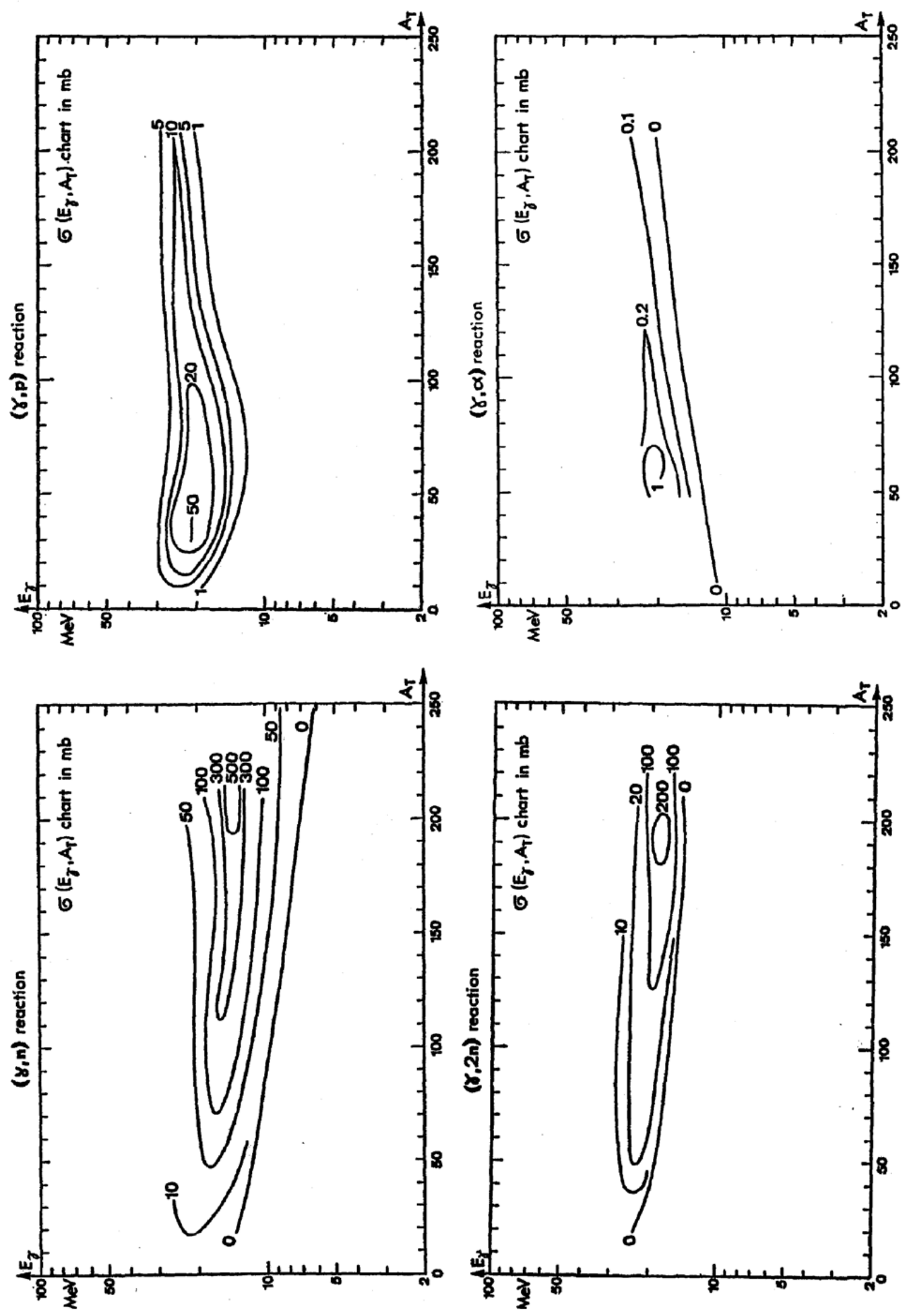

Fig. 7.1 Contours of equal cross section for photon-induced nuclear reactions $(\gamma, n),(\gamma, p),(\gamma, 2 n)$, and $(\gamma, \alpha)$ as a function of photon energy $E_{\gamma}$ and target material mass number $A_{T}$. The contour labeled " 0 " indicates the approximate boundary of the region of insignificant cross sections. The results have been smoothed in these plots. [Adapted from (Ba69).] 


\section{CHAPTER 7 INDUCED RADIOACTIVITY IN ACCELERATOR COMPONENTS}
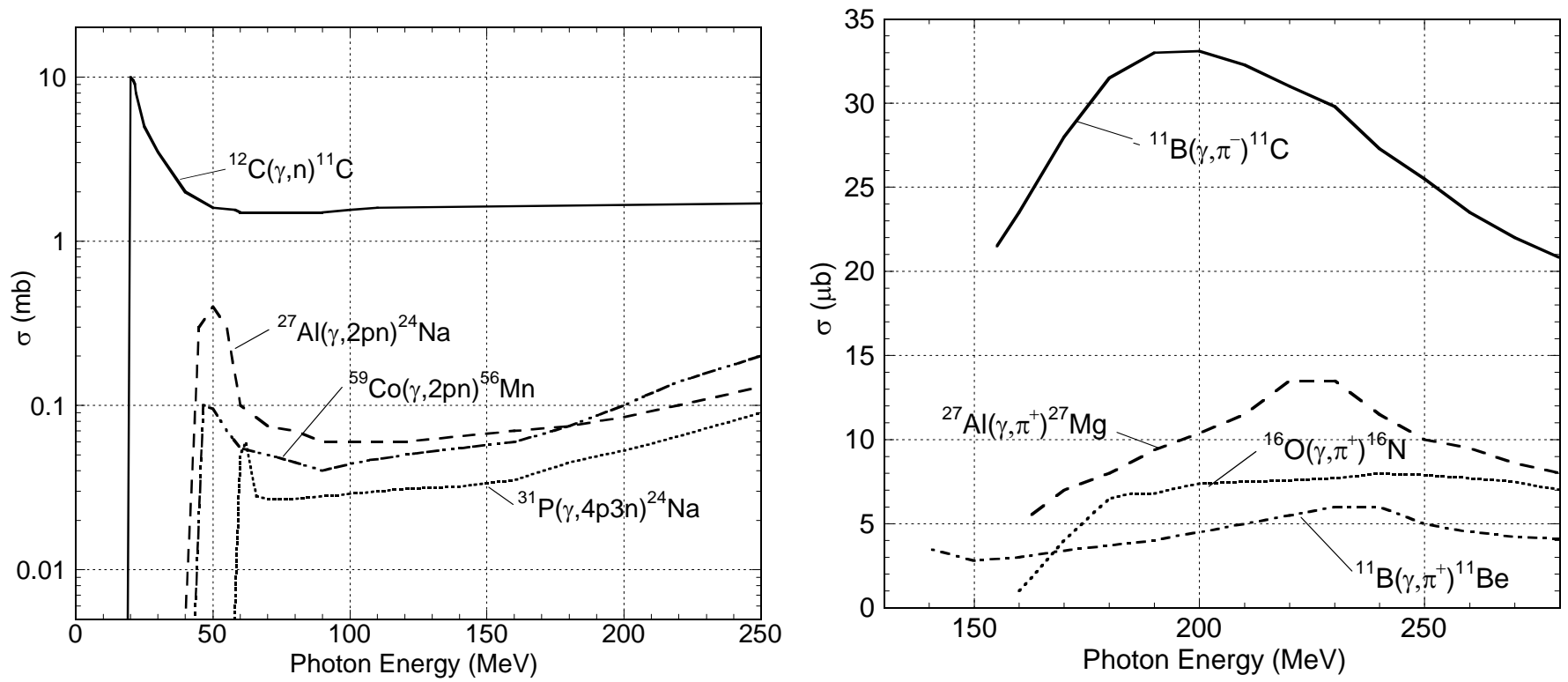

Fig. 7.2 Excitation functions for important photon-induced reactions (left frame) and for photo-pion reactions (right frame) at intermediate energies. [Adapted from (Ba69).]
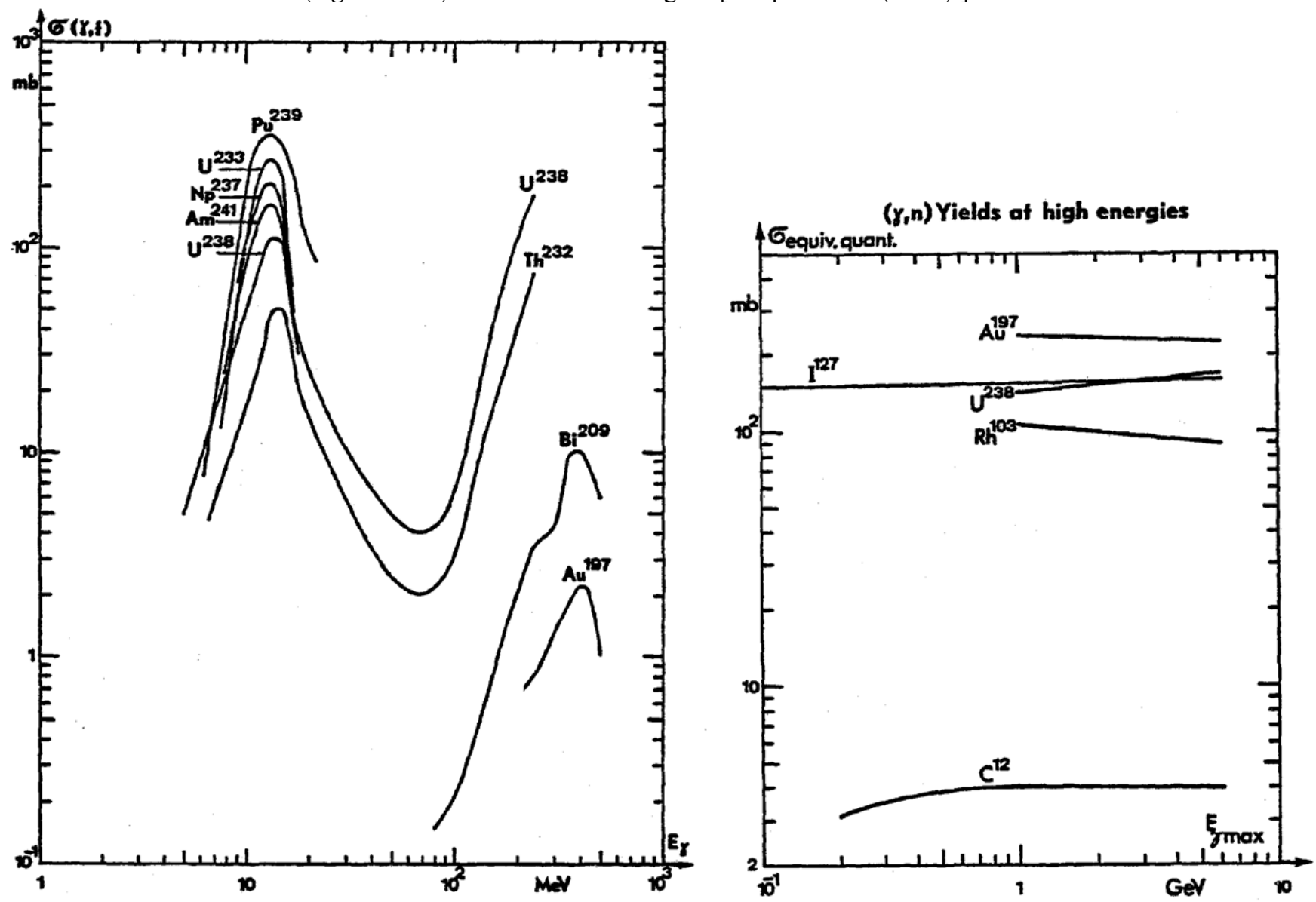

Fig. 7.3 Excitation functions for photofission $(\gamma, \mathrm{f})$ and photoneutron $(\gamma, \mathrm{n})$ reactions at intermediate and high energies for photons incident on a variety of materials. [Adapted from (Ba69).] 


\section{CHAPTER 7 INDUCED RADIOACTIVITY IN ACCELERATOR COMPONENTS}

Fig. 7.1 presents data in the form of contour plots of the cross sections $\sigma\left(E_{\gamma}, A_{T}\right)$ where $\mathrm{E}_{\gamma}$ is the photon energy and $A_{T}$ is the atomic mass number of the target material. Figs. 7.2 and 7.3 provide the cross sections for specific processes as a function of energy. These figures are intended to illustrate the importance of various processes at different energies. Specific data from the literature should be obtained for precise, accurate calculations.

\subsubsection{Results for Electrons at Low Energies}

Data such as those presented in Section 7.3.1 form the basis of detailed activation calculations. Swanson (Sw79a) utilized the methodology of Approximation B of the analytical shower theory of Rossi and Griesen (Ro41), discussed in Section 3.4, to estimate saturation activities rates in various materials. Since the energy domain below about $35 \mathrm{MeV}$ is characterized by rapidly varying cross sections, Swanson provided energy-dependent results. Here only reactions of the type $(\gamma, n),(\gamma, p),(\gamma, n p)$, and $(\gamma, 2 n)$ were considered. Other reactions were ignored due to higher energy thresholds and small cross sections. Swanson points out that the dependence of the induced activity as a function of energy above a given nuclear reaction threshold will generally follow that of the neutron yields (see Fig. 3.5). In Swanson's calculations, the material in question absorbs all of the beam power and has been irradiated for an infinite time with no cooldown $\left[t_{i}=\infty, t_{c}=0\right.$ in Eq. (7.8)]. Thus, so-called saturation activities are calculated, normalized to the incident electron beam power $(\mathrm{kW})$. Results of these calculations, taking into account the natural isotopic abundances and assuming energies well above the reaction thresholds, are given in Table 7.1. The results are likely accurate to about $\pm 30 \%$. At these low energies, the distribution of the radioactivity can often be approximated as a point source for calculating the residual absorbed dose rates using, for example, Eq. (7.10), taking the summation over all of the $\gamma$-ray emitters presented at a given time.

Table 7.1 also provides the specific gamma-ray constant $\Gamma$ for each tabulated radionuclide. These constants connect activity with the absorbed dose rates at a distance of one meter from a point source of a given activity, accounting for all the photons emitted by the decaying radionuclide and including those emitted secondarily such as internal bremsstrahlung and annihilation radiation due to $\beta^{+}$emission manifested in the form of a pair of $0.511 \mathrm{MeV}$ photons. For point sources, absorbed dose rates at other distances can be calculated by incorporating the inverse square law. In this context, absorbed dose rate is loosely connected with a somewhat obsolete unit of measure, the exposure rate expressed in Roentgens $h^{-1}\left(\mathrm{R} \mathrm{h}^{-1}\right)$. Exposure rate measures the liberation of ions in air by photons and is only defined for photon radiation fields. $1.0 \mathrm{R} \mathrm{h}^{-1}$ is the hourly liberation of one electrostatic unit of charge $\mathrm{cm}^{-3}$ of air at STP $\left[1.0 \mathrm{R}=2.58 \times 10^{-4}\right.$ Coulomb kg-1 . An exposure rate of $1.0 \mathrm{R} \mathrm{h}^{-1}$ is approximately equal to $0.95 \mathrm{rad} \mathrm{h}^{-1}$ of absorbed dose rate in tissue placed in the radiation field under consideration.

\subsubsection{Results for Electrons at High Energies}

For higher energy electrons, more reaction channels become available but the energy 


\section{CHAPTER 7 INDUCED RADIOACTIVITY IN ACCELERATOR COMPONENTS}

dependence is diminished. Swanson has also performed calculations of the production of radionuclides in this energy domain and the results are provided here in Table 7.2 (Sw79a). The results are valid to within an approximate factor of two for any beam energy $E_{o}$ that is somewhat above the nuclide production threshold. Saturation activities

Table 7.1 Estimations of saturation activities and saturation absorbed dose rates at one meter for electrons of energy $E_{o}$ incident on various target materials of naturally-occurring isotopic abundances normalized to the beam power. [Adapted from Swanson (1979a).]

\begin{tabular}{|c|c|c|c|c|c|c|c|}
\hline \multirow[t]{2}{*}{$\begin{array}{l}\text { Target } \\
\text { Material }\end{array}$} & \multirow[t]{2}{*}{ Nuclide } & \multirow[t]{2}{*}{ Half-life $^{d}$} & \multirow[t]{2}{*}{$\begin{array}{c}\text { Threshold } \\
\text { (MeV) }\end{array}$} & \multicolumn{2}{|c|}{$\begin{array}{c}\text { Specific gamma ray constant, } \\
\Gamma\end{array}$} & \multicolumn{2}{|c|}{$\begin{array}{l}\text { Saturation activity per } \\
\text { unit beam power }\end{array}$} \\
\hline & & & & $\begin{array}{c}{\left[\left(\mathrm{mGy} \mathrm{h}^{-1}\right)\right.} \\
\left.\mathbf{x}\left(\mathrm{GBq} \mathrm{m}^{-2}\right)^{-1}\right]\end{array}$ & $\begin{array}{l}{\left[\left(\operatorname{rad~h}^{-1}\right)\right.} \\
\left.x\left(\operatorname{Ci~m}^{-2}\right)^{-1}\right]\end{array}$ & $\left(G^{\prime} q k^{-1}\right)$ & $\left(\mathrm{Ci} \mathrm{kW}^{-1}\right)$ \\
\hline $\mathrm{Al}$ & ${ }^{24} \mathrm{Na}$ & $\begin{array}{r}14.95 \mathrm{~h} \\
6.34 \mathrm{~s} \\
\end{array}$ & $\begin{array}{l}23.7 \\
13.0\end{array}$ & $\begin{array}{l}0.48 \\
0.16\end{array}$ & $\begin{array}{l}1.8 \\
0.59\end{array}$ & $\begin{array}{l}1.1 \\
330\end{array}$ & $\begin{array}{l}0.03 \\
8.8\end{array}$ \\
\hline $\mathrm{Fe}$ & $\begin{array}{l}{ }^{54} \mathrm{Mn} \\
{ }^{56} \mathrm{Mn} \\
{ }^{53} \mathrm{Fe}\end{array}$ & $\begin{array}{r}312.2 \mathrm{~d} \\
2.58 \mathrm{~h} \\
8.51 \mathrm{~min} \\
\end{array}$ & $\begin{array}{l}20.4 \\
10.6 \\
13.6 \\
\end{array}$ & $\begin{array}{l}0.32 \\
0.23 \\
0.18 \\
\end{array}$ & $\begin{array}{l}1.2 \\
0.86 \\
0.67\end{array}$ & $\begin{array}{c}22.0 \\
1.18 \\
27.0\end{array}$ & $\begin{array}{l}0.59 \\
0.032 \\
0.74\end{array}$ \\
\hline $\mathrm{Ni}$ & $\begin{array}{l}{ }^{56} \mathrm{Ni} \\
{ }^{56} \mathrm{Co}^{\mathrm{a}} \\
{ }^{57} \mathrm{Ni} \\
{ }^{57} \mathrm{Co}^{\mathrm{a}}\end{array}$ & $\begin{array}{r}6.08 \mathrm{~d} \\
77.23 \mathrm{~d} \\
35.6 \mathrm{~h} \\
217.78 \mathrm{~d}\end{array}$ & $\begin{array}{l}22.5 \\
12.2\end{array}$ & $\begin{array}{l}0.43 \\
0.62 \\
0.34 \\
0.35\end{array}$ & $\begin{array}{l}1.6 \\
2.3 \\
1.4 \\
1.3\end{array}$ & $\begin{array}{r}2.6^{\mathrm{b}} \\
155^{\mathrm{b}}\end{array}$ & $\begin{array}{l}0.07^{\mathrm{b}} \\
4.2^{\mathrm{b}}\end{array}$ \\
\hline $\mathrm{Cu}$ & $\begin{array}{l}{ }^{61} \mathrm{Cu} \\
{ }^{62} \mathrm{Cu} \\
{ }^{64} \mathrm{Cu}\end{array}$ & $\begin{array}{c}3.33 \mathrm{~h} \\
9.67 \mathrm{~min} \\
12.7 \mathrm{~h}\end{array}$ & $\begin{array}{c}19.7 \\
10.8 \\
9.91\end{array}$ & $\begin{array}{l}0.19 \\
0.16 \\
0.10\end{array}$ & $\begin{array}{l}0.71 \\
0.60 \\
0.38\end{array}$ & $\begin{array}{l}32.2 \\
407 \\
185\end{array}$ & $\begin{array}{c}0.87 \\
11 \\
5.0\end{array}$ \\
\hline $\mathrm{W}$ & $\begin{array}{l}{ }^{182 \mathrm{~m}} \mathrm{Ta} \\
{ }^{182} \mathrm{Ta}^{\mathrm{a}} \\
{ }^{183} \mathrm{Ta} \\
{ }^{181} \mathrm{~W} \\
{ }^{185 \mathrm{~m}} \mathrm{~W} \\
{ }^{185} \mathrm{~W}^{\mathrm{a}}\end{array}$ & $\begin{array}{c}15.84 \mathrm{~min} \\
114.4 \mathrm{~d} \\
5.1 \mathrm{~d} \\
121.2 \mathrm{~d} \\
1.67 \mathrm{~min} \\
75.1 \mathrm{~d}\end{array}$ & $\begin{array}{l}7.15 \\
\\
7.71 \\
7.99 \\
7.27\end{array}$ & $\begin{array}{r}0.04 \\
0.16 \\
0.04 \\
0.02 \\
0.05 \\
\text { no } \gamma \text {-ray }\end{array}$ & $\begin{array}{c}0.15 \\
0.61 \\
0.15 \\
0.09 \\
0.18 \\
\text { no } \gamma \text {-ray }\end{array}$ & $\begin{array}{l}13.3 \\
23.3 \\
340 \\
\\
300^{\mathrm{b}}\end{array}$ & $\begin{array}{l}0.36 \\
0.63 \\
9.1 \\
\\
8.1^{\mathrm{b}}\end{array}$ \\
\hline $\mathrm{Au}$ & $\begin{array}{l}{ }^{195 m} \mathrm{Au} \\
{ }^{195} \mathrm{Au}^{\mathrm{a}} \\
{ }^{196 m} \mathrm{Au} \\
{ }^{196} \mathrm{Au}^{\mathrm{a}}\end{array}$ & $\begin{array}{c}30.5 \mathrm{~s} \\
186.1 \mathrm{~d} \\
9.6 \mathrm{~h} \\
6.17 \mathrm{~d}\end{array}$ & $\begin{array}{r}14.8 \\
8.07\end{array}$ & $\begin{array}{l}0.04 \\
0.02 \\
0.03 \\
0.08\end{array}$ & $\begin{array}{l}0.16 \\
0.07 \\
0.12 \\
0.30\end{array}$ & $\begin{array}{r}204^{\mathrm{b}} \\
1520^{\mathrm{b}}\end{array}$ & $\begin{array}{r}5.5 \\
41^{\mathrm{b}}\end{array}$ \\
\hline $\mathrm{Pb}$ & $\begin{array}{l}{ }^{203} \mathrm{~Pb} \\
{ }^{204 \mathrm{~m}} \mathrm{~Pb}\end{array}$ & $\begin{array}{l}6.21 \mathrm{~s} \\
1.14 \mathrm{~h}\end{array}$ & $\begin{array}{c}8.38 \\
14.8 \\
\end{array}$ & $\begin{array}{l}0.05 \\
0.32\end{array}$ & $\begin{array}{l}0.19 \\
1.20\end{array}$ & $\begin{array}{l}17.4 \\
44\end{array}$ & $\begin{array}{l}0.47 \\
1.2\end{array}$ \\
\hline
\end{tabular}

${ }^{\mathrm{a}}$ This radionuclide is the progeny of the radionuclide above it.

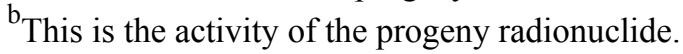

"The term "no $\gamma$-rays" is applied to radionuclides having no, or very rare, emission of photons in their decay.

${ }^{\mathrm{d}}$ More recent values of half-lives are found in (Tu05).

and absorbed dose rates normalized to the beam power are provided. This quantity is useful because of the dominance, and lack of energy dependence, of the photoneutron production process, as was discussed in Section 3.2. The electrons are assumed to be totally absorbed in the material and no self-shielding effects are taken into account. The distribution of radioactivity within the material is not taken into account. Saturation conditions imply $t_{i}=\infty, t_{\mathrm{c}}=0$ in Eq. (7.8). 


\section{CHAPTER 7 INDUCED RADIOACTIVITY IN ACCELERATOR COMPONENTS}

Table 7.2 Estimations of saturation activities and absorbed rates at one meter in various materials, assuming "point source” conditions for high energy electrons. Results have been summed over the naturally-occurring isotopic composition of the materials. Radionuclides contributing $<0.1\left(\mathrm{mGy} \mathrm{h}^{-1}\right)\left(\mathrm{kW} \mathrm{m}^{-2}\right)^{-1}$ or with $t_{1 / 2}<1.0$ minute have been excluded as have products of thermal neutron capture reactions. [Adapted from Swanson (1979a).]

Material: Natural Aluminum

Produced Radionuclide

\begin{tabular}{|l|c|c|}
\hline & Half-life $^{\mathbf{c}}$ & $\begin{array}{c}\text { Threshold } \\
(\mathbf{M e V})\end{array}$ \\
\hline${ }^{7} \mathrm{Be}$ & $53.22 \mathrm{~d}$ & 33.0 \\
\hline${ }^{11} \mathrm{C}$ & $20.33 \mathrm{~min}$ & 33.5 \\
\hline${ }^{15} \mathrm{O}$ & $2.04 \mathrm{~min}$ & 33.4 \\
\hline${ }^{18} \mathrm{~F}$ & $1.83 \mathrm{~h}$ & 34.4 \\
\hline${ }^{22} \mathrm{Na}$ & $2.60 \mathrm{y}$ & 22.5 \\
\hline${ }^{24} \mathrm{Na}$ & $14.95 \mathrm{~h}$ & 23.7 \\
\hline${ }^{26 \mathrm{~m}} \mathrm{Al}$ & $6.34 \mathrm{~s}$ & 13.0 \\
\hline
\end{tabular}

\section{Saturation Activity per Unit Beam Power $\left(\mathrm{GBq} \mathrm{kW}^{-1}\right) \quad\left(\mathrm{Ci} \mathrm{kW}^{-1}\right)$}

\begin{tabular}{|c|c|} 
& \\
\hline 4.8 & 0.13 \\
1.9 & 0.051 \\
\hline 2.5 & 0.07 \\
\hline 5.2 & 0.14 \\
\hline 9.2 & 0.25 \\
\hline 10.4 & 0.28 \\
\hline 321 & 8.8
\end{tabular}

Saturation Absorbed Dose per Unit Beam Power ${ }^{\mathrm{a}}$ $\left(\mathrm{mGy} \mathrm{h}^{-1}\right) \mathbf{x} \quad\left(\operatorname{rad~h}^{-1}\right) \mathbf{x}$ $\left(\mathbf{k W ~ m}^{-2}\right)^{-1} \quad\left(\mathrm{~kW} \mathrm{~m}^{-2}\right)^{-1}$

\section{Material: Natural Iron}

\begin{tabular}{|l|c|c|c|c|c|l|}
\hline${ }^{46} \mathrm{Sc}$ & $83.79 \mathrm{~d}$ & 37.4 & 7.4 & 0.2 & 2.0 & 0.2 \\
\hline${ }^{48} \mathrm{~V}$ & $15.97 \mathrm{~d}$ & 25.9 & 15.0 & 0.4 & 8.0 & 0.8 \\
\hline${ }^{51} \mathrm{Cr}$ & $27.70 \mathrm{~d}$ & 19.7 & 15.0 & 0.4 & 3.0 & 0.3 \\
\hline${ }^{52} \mathrm{Mn}$ & $5.59 \mathrm{~h}$ & 20.9 & 1.5 & 0.04 & 0.4 & 0.04 \\
\hline${ }^{52 \mathrm{~m}} \mathrm{Mn}$ & $21.1 \mathrm{~min}$ & 20.9 & 1.3 & 0.036 & 0.23 & 0.023 \\
\hline${ }^{54} \mathrm{Mn}$ & $312.1 \mathrm{~d}$ & 20.4 & 22.0 & 0.59 & 7.0 & 0.7 \\
\hline${ }^{56} \mathrm{Mn}$ & $2.58 \mathrm{~h}$ & 10.6 & 1.1 & 0.03 & 0.3 & 0.03 \\
\hline${ }^{52} \mathrm{Fe}$ & $8.28 \mathrm{~h}$ & 24.1 & 2.2 & 0.06 & 0.4 & 0.04 \\
\hline${ }^{53} \mathrm{Fe}$ & $8.51 \mathrm{~min}$ & 13.6 & 27.4 & 0.74 & 4.9 & 0.49 \\
\hline${ }^{55} \mathrm{Fe}$ & $2.74 \mathrm{y}$ & 11.2 & 490 & 13.3 & 90 & 9 \\
\hline
\end{tabular}

Material: Natural Copper

\begin{tabular}{|l|c|c|c|c|c|c|}
\hline${ }^{58 m} \mathrm{Co}$ & $9.04 \mathrm{~h}$ & 41.8 & 24.4 & 0.66 & 4.0 & 0.4 \\
\hline${ }^{58} \mathrm{Co}$ & $70.9 \mathrm{~d}$ & 41.8 & 24.4 & 0.66 & 2.0 & 0.2 \\
\hline${ }^{60} \mathrm{Co}$ & $5.27 \mathrm{y}$ & 18.9 & 24.0 & 0.65 & 8.0 & 0.8 \\
\hline${ }^{63} \mathrm{Ni}$ & $100 \mathrm{y}$ & 17.1 & 16.6 & 0.45 & no $\gamma$-rays & no $\gamma$-rays \\
\hline${ }^{61} \mathrm{Cu}$ & $3.33 \mathrm{~h}$ & 19.7 & 32.2 & 0.87 & 6.0 & 0.6 \\
\hline${ }^{62} \mathrm{Cu}$ & $9.64 \mathrm{~min}$ & 10.8 & 407 & 11 & 65 & 6.5 \\
\hline${ }^{64} \mathrm{Cu}$ & $12.7 \mathrm{~h}$ & 9.9 & 185 & 5 & 19 & 1.9 \\
\hline
\end{tabular}

Material: Natural Tungsten

\begin{tabular}{|l|c|c|c|c|c|c|}
\hline${ }^{182 \mathrm{~m}} \mathrm{Ta}$ & $15.84 \mathrm{~min}$ & 7.15 & 13.3 & 0.36 & 0.3 & 0.03 \\
\hline${ }^{182} \mathrm{Ta}$ & $114.4 \mathrm{~d}$ & 7.15 & 13.3 & 0.36 & 1.1 & 0.11 \\
\hline${ }^{183} \mathrm{Ta}$ & $5.1 \mathrm{~d}$ & 7371 & 22.9 & 0.62 & 0.9 & 0.09 \\
\hline${ }^{184} \mathrm{Ta}$ & $8.7 \mathrm{~h}$ & 14.9 & 1.78 & 0.048 & 0.4 & 0.04 \\
\hline${ }^{185} \mathrm{Ta}$ & $49.4 \mathrm{~min}$ & 8.39 & 20.7 & 0.56 & 0.6 & 0.06 \\
\hline${ }^{181} \mathrm{~W}$ & $121.2 \mathrm{~d}$ & 8.00 & 330 & 8.9 & 8.0 & 0.8 \\
\hline${ }^{185 \mathrm{~m}} \mathrm{~W}$ & $1.67 \mathrm{~min}$ & 7.27 & 300 & 8.1 & 7.3 & 0.73 \\
\hline${ }^{185} \mathrm{~W}$ & $75.1 \mathrm{~d}$ & 7.27 & 300 & 8.1 & no $\gamma$-rays & no $\gamma$-rays \\
\hline
\end{tabular}

${ }^{\mathrm{a}}$ The term "no $\gamma$-rays" is applied to radionuclides having no, or very rare, emission of photons in their decay. 
Table 7.2-continued

\begin{tabular}{|c|c|c|c|c|c|c|}
\hline \multicolumn{7}{|c|}{ Material: Natural Tungsten } \\
\hline \multicolumn{3}{|c|}{ Produced Radionuclide } & \multicolumn{2}{|c|}{$\begin{array}{c}\text { Saturation Activity } \\
\text { per Unit Beam Power }\end{array}$} & \multicolumn{2}{|c|}{$\begin{array}{c}\text { Saturation Absorbed Dose } \\
\text { per Unit Beam Power }\end{array}$} \\
\hline \multicolumn{7}{|c|}{ Material: Natural Lead } \\
\hline${ }^{204} \mathrm{Tl}$ & $3.78 \mathrm{y}$ & 14.83 & 0.92 & 0.025 & no $\gamma$-rays & no $\gamma$-rays \\
\hline${ }^{206} \mathrm{Tl}$ & $4.20 \mathrm{~min}$ & 7.46 & 37 & 1.0 & no $\gamma$-rays & no $\gamma$-rays \\
\hline${ }^{207 \mathrm{~m}} \mathrm{Tl}$ & $1.33 \mathrm{~s}$ & 8.04 & 93 & 2.5 & 9.1 & 0.91 \\
\hline${ }^{207} \mathrm{Tl}$ & $4.77 \mathrm{~min}$ & 8.04 & 93 & 2.5 & no $\gamma$-rays & no $\gamma$-rays \\
\hline${ }^{202 \mathrm{~m}} \mathrm{~Pb}$ & $3.53 \mathrm{~h}$ & 15.3 & 2.2 & 0.06 & 0.3 & 0.03 \\
\hline${ }^{202} \mathrm{~Pb}$ & $5.25 \times 10^{4} y$ & 15.3 & 2.2 & 0.06 & no $\gamma$-rays & no $\gamma$-rays \\
\hline${ }^{203 m} \mathrm{~Pb}$ & $6.21 \mathrm{~s}$ & 8.38 & 31 & 0.83 & 1.3 & 0.13 \\
\hline${ }^{203} \mathrm{~Pb}$ & $2.16 \mathrm{~d}$ & 8.38 & 31 & 0.83 & 0.7 & 0.07 \\
\hline${ }^{204 \mathrm{~m}} \mathrm{~Pb}$ & $1.14 \mathrm{~h}$ & 14.8 & 89 & 2.4 & 14 & 1.4 \\
\hline \multicolumn{7}{|c|}{ Material: Typical Ordinary Concrete ${ }^{b}$} \\
\hline${ }^{15} \mathrm{O}$ & $2.04 \mathrm{~min}$ & 15.7 & 96 & 2.6 & 15 & 1.5 \\
\hline${ }^{22} \mathrm{Na}$ & $2.60 \mathrm{y}$ & 12.4 & 3.7 & 0.1 & 1.2 & 0.12 \\
\hline${ }^{27} \mathrm{Si}$ & 4.16 & 17.2 & 74 & 2.0 & 12 & 1.2 \\
\hline${ }^{38} \mathrm{~K}$ & $7.64 \mathrm{~min}$ & 13.1 & 3.7 & 0.1 & 1.5 & 0.15 \\
\hline
\end{tabular}

a The term"no $\gamma$-rays" is applied to radionuclides having no, or very rare, emission of photons in their decay. ${ }^{\mathrm{b}}$ By weight per cent, the isotopic composition of concrete was taken to be: ${ }^{12} \mathrm{C}(0.10),{ }^{16} \mathrm{O}(53.0),{ }^{23} \mathrm{Na}(1.6)$,

${ }^{24} \mathrm{Mg}(0.16),{ }^{27} \mathrm{Al}(3.4),{ }^{28} \mathrm{Si}(31.0),{ }^{39} \mathrm{~K}(1.2),{ }^{54} \mathrm{Fe}(0.08)$, all others (9.5).

${ }^{\mathrm{c}}$ More recent values of half-lives are found in (Tu05).

Cooling curves have been published by Barbier (Ba69) for high energy electrons incident on various materials for an infinite irradiation at the rate of one electron per second. The results are given in Fig. 7.4 again per $\mathrm{MeV}$ of incident electron energy, for an infinite irradiation time $t_{i}$. In this figure, results are given for the absorbed dose rates $\left(\mathrm{mGy} \mathrm{hr} \mathrm{hr}^{-1}\right)$ per electron $\mathrm{s}^{-1}$ assuming the applicability of point source conditions. As discussed in Chapter 3, the lack of strong energy dependence and the simplicity of the photoneutron spectra make possible these rather uncomplicated results.

\subsection{Activation of Components at Proton and Ion Accelerators}

\subsubsection{General Phenomena}

Protons having energies above about $10 \mathrm{MeV}$, or sometimes less, will produce radioactivity upon interacting with matter. This will also occur for other ions above a specific energy of about $10 \mathrm{MeV}$ nucleon ${ }^{-1}$. In some special cases radioactivity can be produced at much lower energies due to exothermic nuclear reactions that either produce radionuclides directly or emit neutrons capable of inducing radioactivity through their secondary interactions. As with electron accelerators, if a given accelerator is properly designed with respect to the shielding against prompt radiation and has proper access controls to avoid direct beam-on exposure to people, the induced radioactivity is very likely to be the dominant source of occupational radiation exposure. 


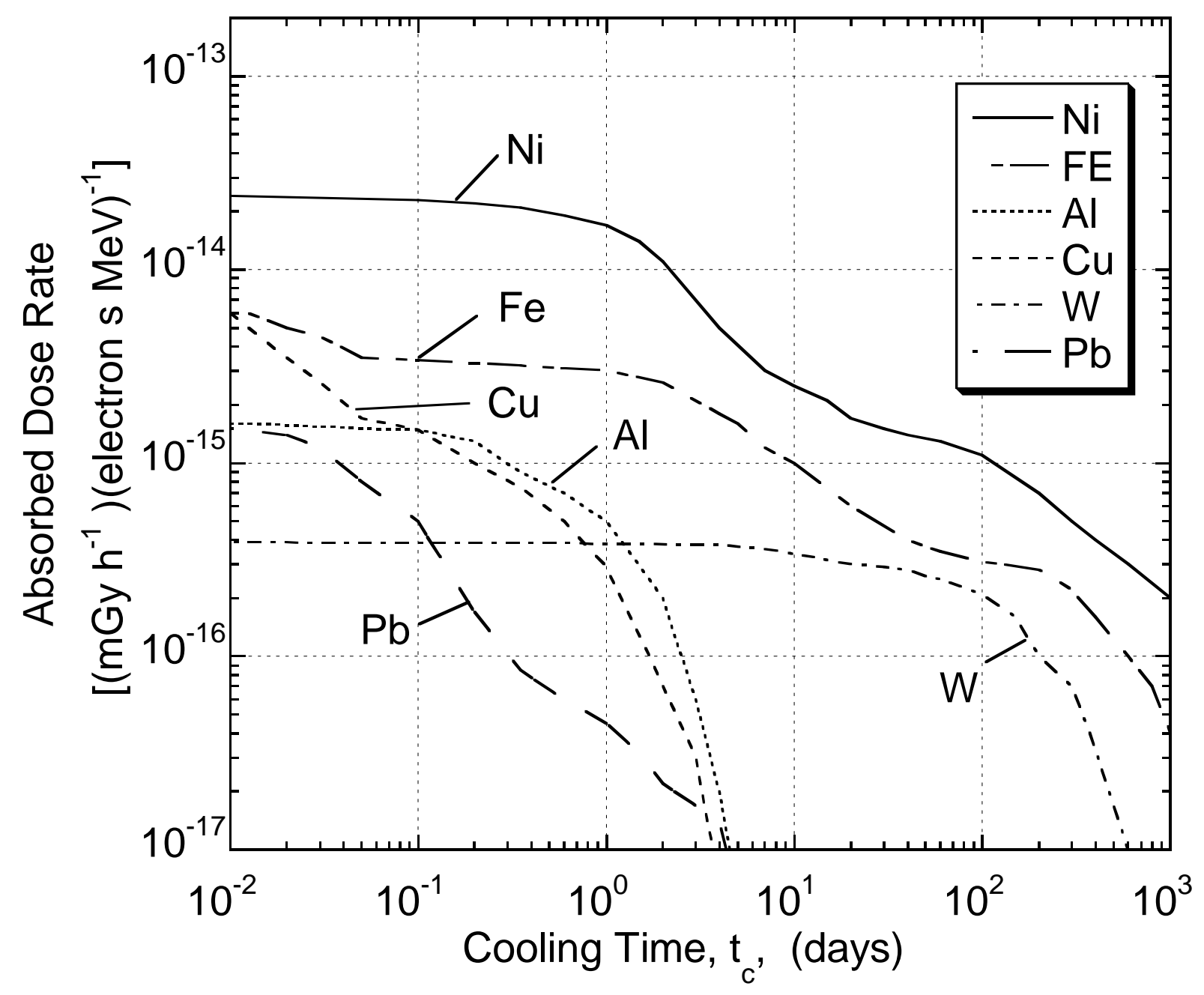

Fig. 7.4 Examples of total photon absorbed dose rates due to radioactive nuclei produced in large targets of various materials irradiated by an electron current of 1.0 electron $\sec ^{-1}$ per $\mathrm{MeV}$ of incident electron energy as a function of time since the cessation of the irradiation. The irradiation was assumed to have occurred for an infinitely long period of time. The absorbed dose rates are those found at one meter from a point source containing all of the radioactive nuclei produced. [Adapted from Barbier (1969).]

For the lower incident energies, perhaps below about $30 \mathrm{MeV}$, one is first concerned with production of radionuclides by such processes as $(\mathrm{p}, \gamma)$ and single- and multi-nucleon transfer reactions. While the details of the total cross sections for such reactions are complex, the systematics and approximate energy dependencies are globally well understood. In general, one has endothermic nuclear reactions that have a threshold $E_{t h}$ 


\section{CHAPTER 7 INDUCED RADIOACTIVITY IN ACCELERATOR COMPONENTS}

below which the process is forbidden by conservation of energy. For nuclear reactions induced by ions $E_{t h}$ is related to the reaction $Q$-value [see Eq. (4.1)] $Q_{v}$ by

$$
E_{t h}=\frac{m+M}{M}\left|Q_{v}\right|
$$

where $Q_{v}$ is negative in an endothermic reaction that thus has a positive value of $E_{t h}$. In this equation, $m$ is the mass of the incident projectile while $M$ is the mass of the target atom, assumed to stationary in the laboratory frame of reference. The treatise by Barbier (Ba69) has addressed activation by many types of particles. As was the case with electrons, some of these results are in the form of contour plots of the cross sections $\sigma\left(E_{\gamma}, A_{T}\right)$ where $E_{\gamma}$ is the photon energy and $A_{T}$ is the mass number of the target material. These data are intended to convey the general idea of the importance of various processes at different energies. Examples of other data have also been provided concerning specific reaction processes at a variety of energies. These results are provided in Figs. 7.5, 7.6, 7.7, 7.8, 7.9, and 7.10. Some of the results for the light elements (Fig. 7.9) are especially important for environmental radiation considerations (see Chapter 8) while those for iron and copper targets (Fig. 7.10) are of great importance due to the ubiquitous presence of those elements in accelerator components.

Thick target yields of radionuclides for materials having a range of atomic numbers have been systematically studied by Cohen for a number of nuclear processes spanning the periodic table (Co78). Fig. 7.11 is a representative plot of the general features of such excitation functions of such nuclear reactions. Specific processes may vary considerably from this behavior since "resonances" at specific nuclear excited states have been ignored. Table 7.3 lists a variety of such nuclear reactions along with the range of values of energy above threshold at which the radioactivity production rate has risen to $0.1 \%$ of the saturation value and also the range of saturation values for the production of radioactivity. It is assumed that the target thickness comfortably exceeds the range of the incident ion and that the irradiation period greatly exceeds the half-life of the radionuclide of interest. For shorter bombarding periods $t_{i}$ one needs to multiply by the factor $\left[1-\exp \left(-\lambda t_{i}\right)\right]$. Over the energy range of these curves, the importance of activation by secondary particles is small compared to that encountered at higher energies. 

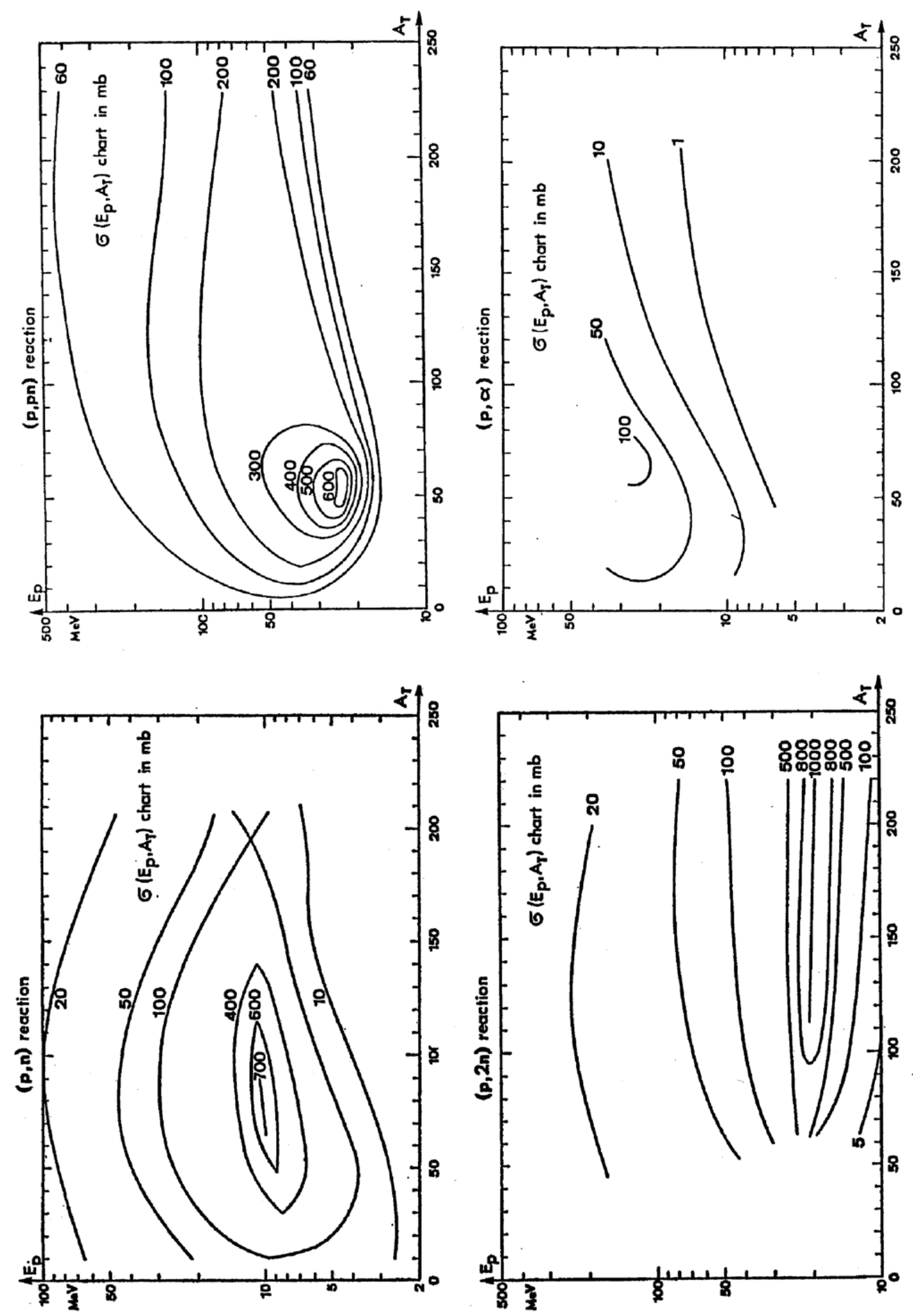

Fig. 7.5 Contours of equal cross section for proton-induced nuclear reactions $(\mathrm{p}, \mathrm{n}),(\mathrm{p}, 2 \mathrm{n}),(\mathrm{p}, \mathrm{pn})$, and $(\mathrm{p}, \alpha)$ as a function of particle energy $E_{p}$ and target material mass number $A_{T}$. The results have been smoothed in these plots. [Adapted from (Ba69).] 

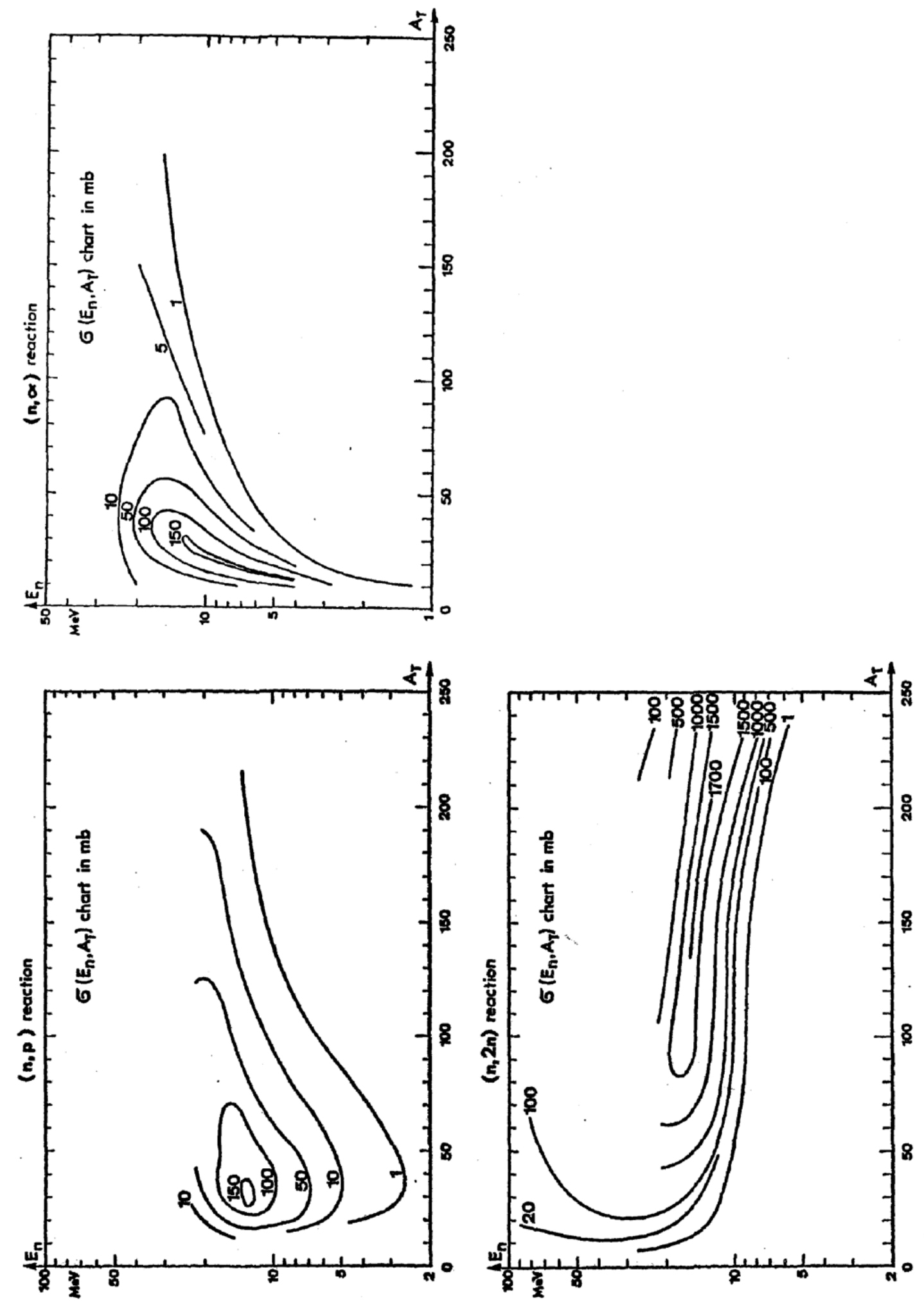

Fig. 7.6 Contours of equal cross section for neutron-induced nuclear reactions $(n, p),(n, 2 n)$, and $(n, \alpha)$ as a function of particle energy $E_{n}$ and target material mass number $A_{T}$. The results have been smoothed in these plots. [Adapted from (Ba69).] 

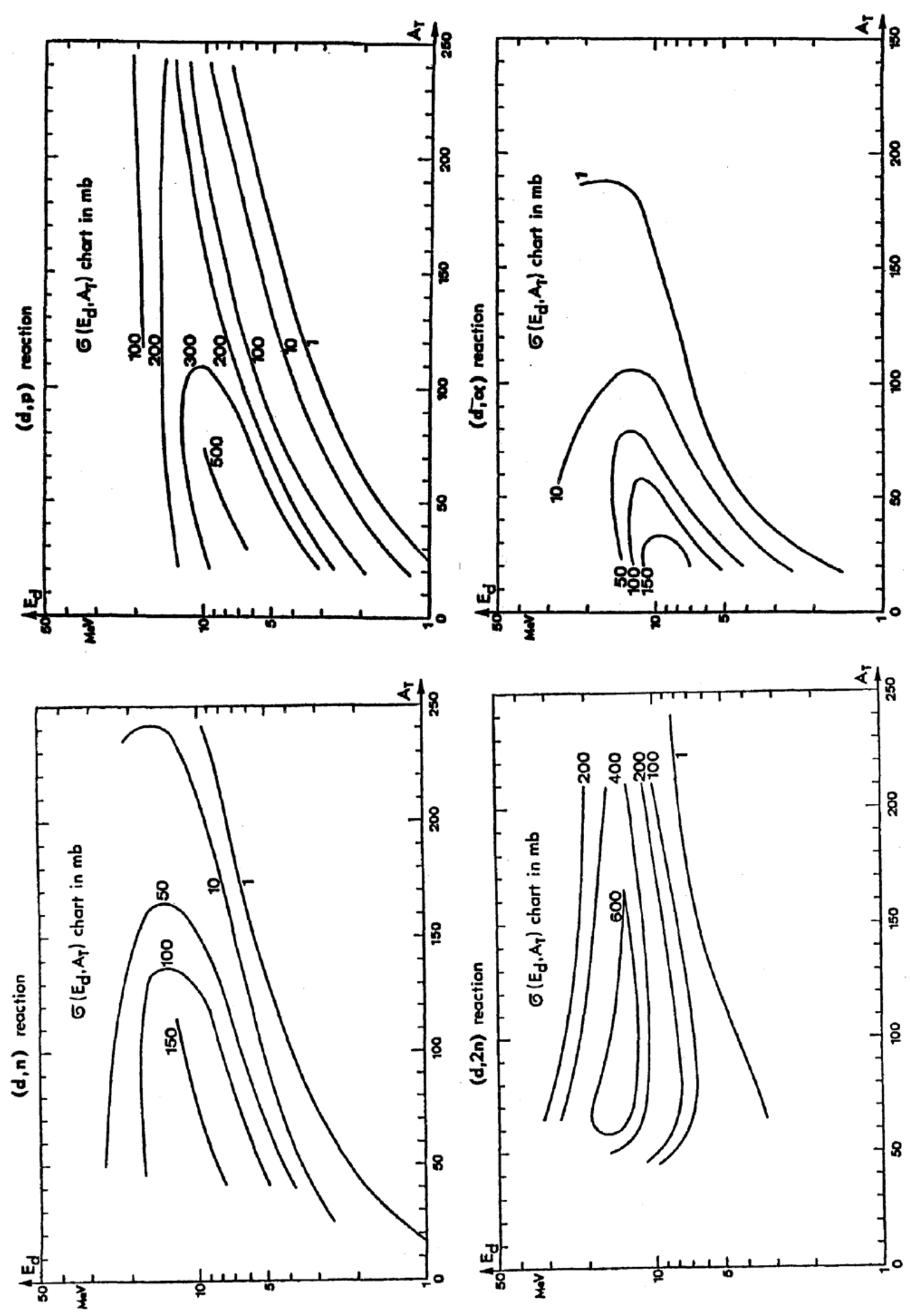

Fig. 7.7

Contours of equal cross section for deuteron-induced nuclear reactions $(\mathrm{d}, \mathrm{n}),(\mathrm{d}, 2 \mathrm{n}),(\mathrm{d}, \mathrm{p})$, and $(\mathrm{d}, \alpha)$ as a function of particle energy $E_{d}$ and target material mass number $A_{T}$. The results have been smoothed in these plots. [Adapted from (Ba69).] 

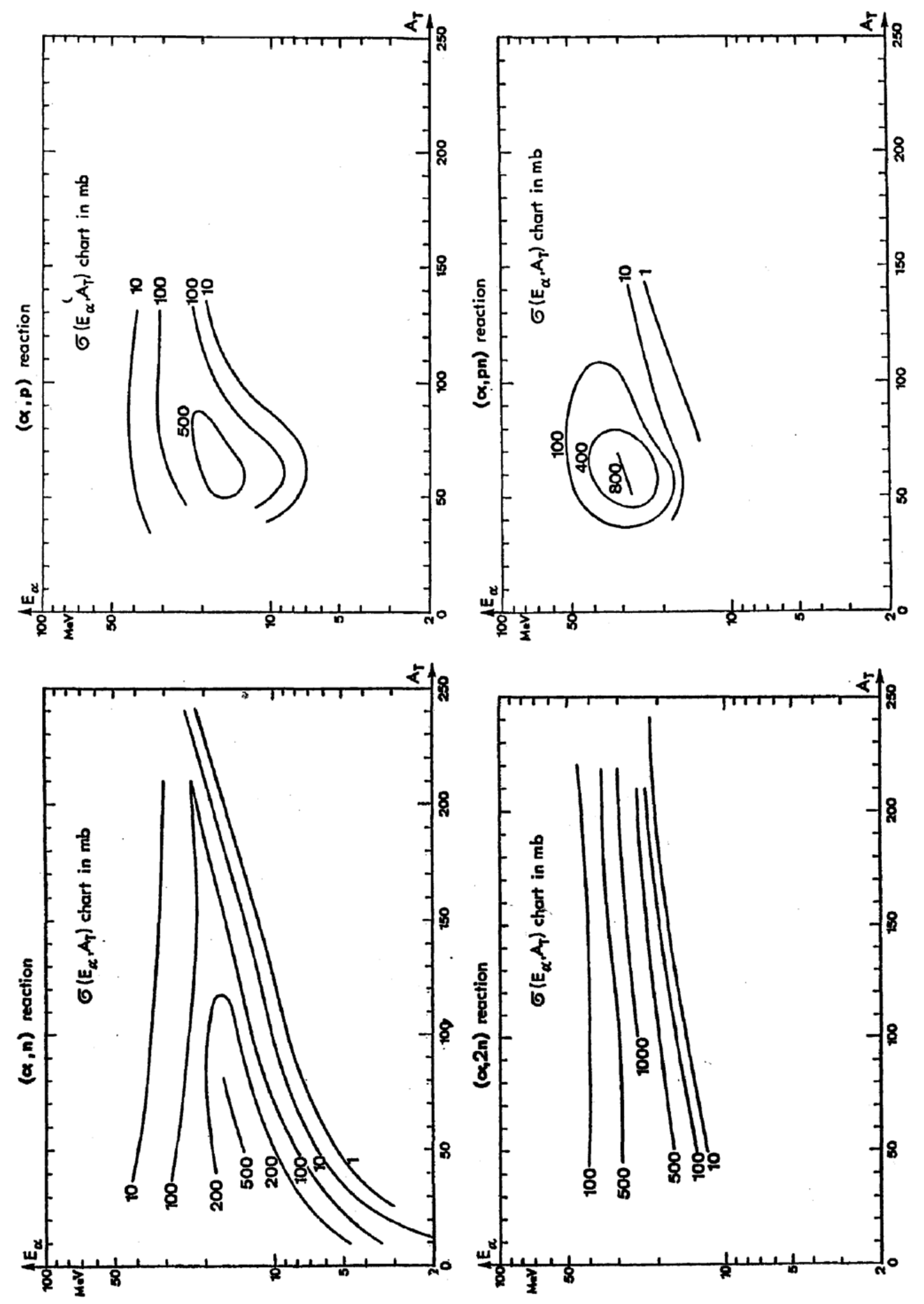

Fig. 7.8 Contours of equal cross section for $\alpha$-particle-induced nuclear reactions $(\alpha, n),(\alpha, 2 n),(\alpha, p)$, and $(\alpha, \mathrm{pn})$ as a function of particle energy $E_{\alpha}$ and target material mass number $A_{T}$. The results have been smoothed in these plots. [Adapted from (Ba69).] 

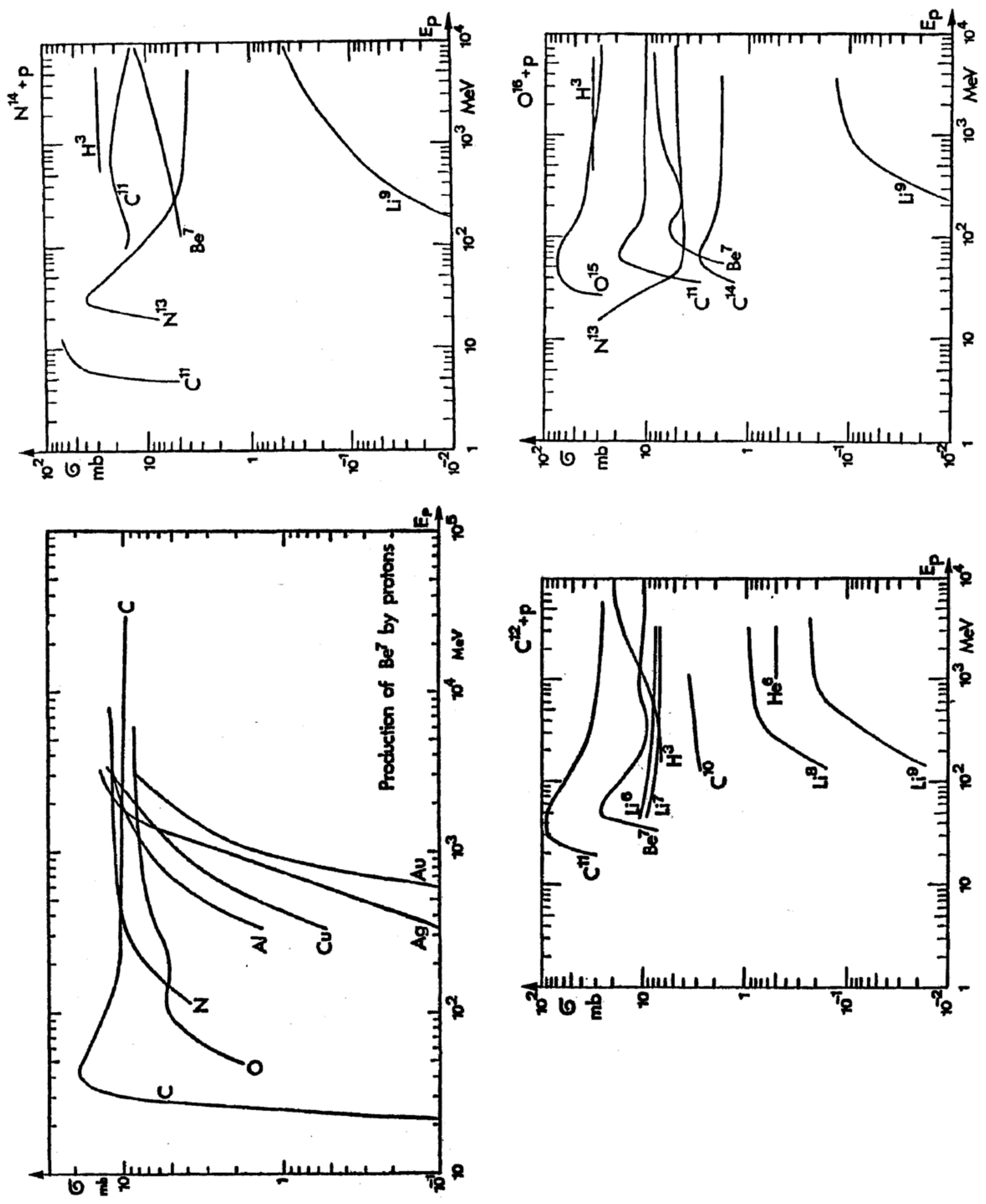

Fig. 7.9 Excitation functions for the production of various radionuclides by protons incident on some low atomic number targets. [Reproduced from (Ba69).] 


\section{CHAPTER 7 INDUCED RADIOACTIVITY IN ACCELERATOR COMPONENTS}
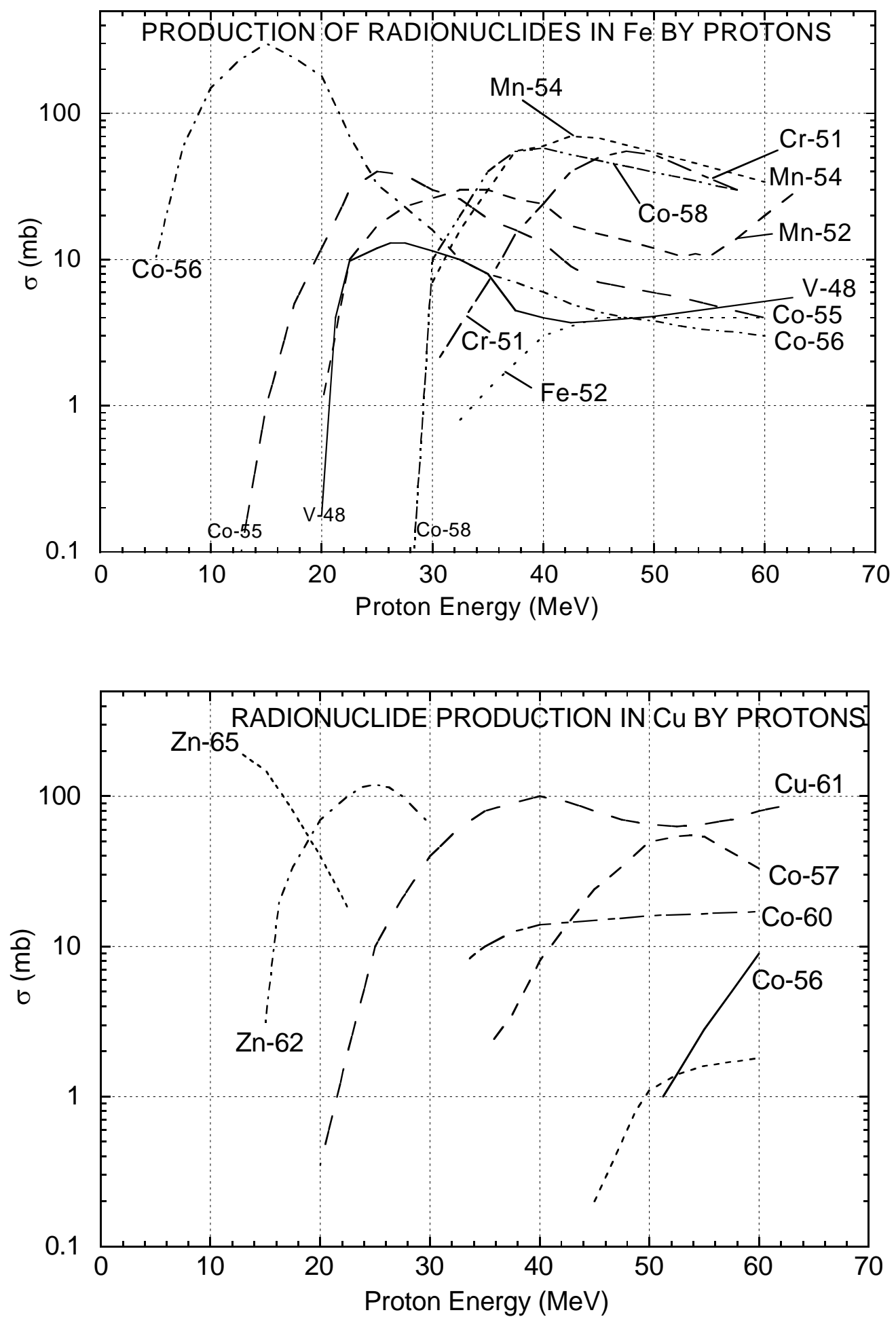

Fig. 7.10 Excitation functions for the production of various radionuclides by protons incident on iron and copper targets. [Adapted from (Ba69).] 


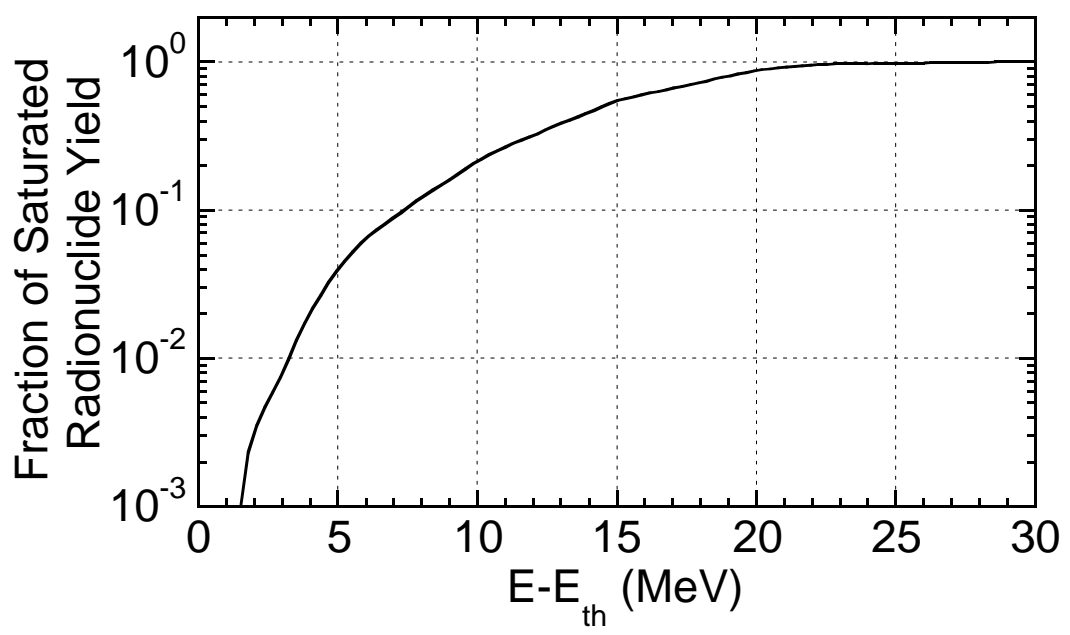

Fig. 7.11 Typical behavior of radionuclide production by $(\mathrm{p}, \gamma)$ or few-nucleon transfer reactions for energies not far above the reaction threshold $E_{t h}$. This behavior is typical of the nuclear reactions tabulated in Table 7.3. For detailed calculations, data related to specific reactions on particular target materials should be used. [Adapted from (Co78).]

Table 7.3 Tabulation of generalized parameters for the production of radionuclides by means of low energy nuclear reactions which span the periodic table. The ranges of energies are listed at which the production yields are at approximately $0.1 \%$ of the tabulated saturation values. The "low/high" values for the saturated activity are also given. [Adapted from (Co78).]

\begin{tabular}{|c|c|c|c|c|c|c|c|c|c|}
\hline Reaction & $\begin{array}{c}0.1 \% \\
\text { Yield- } \\
\text { low } \\
\left(\mathrm{E}-\mathrm{E}_{\text {th }}\right) \\
(\mathrm{MeV})\end{array}$ & $\begin{array}{l}0.1 \% \\
\text { Yield- } \\
\text { high } \\
\left(\mathrm{E}-\mathrm{E}_{\mathrm{th}}\right) \\
(\mathrm{MeV})\end{array}$ & $\begin{array}{l}\text { Sat. } \\
\text { Yield- } \\
\text { low } \\
(\mu \mathrm{Ci} / \\
\mu \mathrm{A})\end{array}$ & $\begin{array}{c}\text { Sat. } \\
\text { Yield- } \\
\text { high } \\
(\mu \mathrm{Ci} / \\
\mu \mathrm{A})\end{array}$ & Reaction & $\begin{array}{c}0.1 \% \\
\text { Yield- } \\
\text { low } \\
\left(\mathrm{E}-\mathrm{E}_{\mathrm{th}}\right) \\
(\mathrm{MeV})\end{array}$ & $\begin{array}{l}0.1 \% \\
\text { Yield- } \\
\text { high } \\
\left(\mathrm{E}-\mathrm{E}_{\mathrm{th}}\right) \\
(\mathrm{MeV})\end{array}$ & $\begin{array}{c}\text { Sat. } \\
\text { Yield- } \\
\text { low } \\
(\mu \mathrm{Ci} / \\
\mu \mathrm{A})\end{array}$ & $\begin{array}{c}\text { Sat. } \\
\text { Yield- } \\
\text { high } \\
(\mu \mathrm{Ci} / \\
\mu \mathrm{A})\end{array}$ \\
\hline$(\mathrm{p}, \gamma)$ & 4 & 9 & $3 \times 10^{2}$ & $10^{3}$ & $\left({ }^{3} \mathrm{He}, \gamma\right)$ & 4 & 6 & 1 & 2 \\
\hline$(\mathrm{p}, \mathrm{n})$ & 0 & 6 & $3 \times 10^{5}$ & $8 \times 10^{5}$ & $\left({ }^{3} \mathrm{He}, \mathrm{n}\right)$ & 3 & 12 & $10^{2}$ & $3 \times 10^{2}$ \\
\hline$(p, 2 n)$ & 1 & 4 & $3 \times 10^{5}$ & $10^{6}$ & $\left({ }^{3} \mathrm{He}, 2 \mathrm{n}\right)$ & 2 & 7 & $3 \times 10^{2}$ & $4 \times 10^{3}$ \\
\hline$(p, 3 n)$ & 1 & 6 & $3 \times 10^{5}$ & $10^{6}$ & $\left({ }^{3} \mathrm{He}, 3 n\right)$ & 2 & 5 & $2 \times 10^{3}$ & $3 \times 10^{4}$ \\
\hline$(p, 4 n)$ & 5 & 8 & $2 \times 10^{5}$ & $10^{6}$ & $\left({ }^{3} \mathrm{He}, 2 \mathrm{p}\right)$ & 4 & 12 & $2 \times 10^{2}$ & $10^{4}$ \\
\hline$(p, 5 n)$ & 5 & 10 & $10^{5}$ & $2 \times 10^{6}$ & $\left({ }^{3} \mathrm{He}, \alpha\right)$ & 6 & 14 & $2 \times 10^{2}$ & $10^{3}$ \\
\hline (p,pn) & 2 & 5 & $2 \times 10^{5}$ & $2 \times 10^{6}$ & $\left({ }^{3} \mathrm{He}, \mathrm{p} 3 \mathrm{n}\right)$ & 10 & 15 & $10^{4}$ & $4 \times 10^{5}$ \\
\hline$(\mathrm{p}, \mathrm{p} 2 \mathrm{n})$ & 3 & 8 & $3 \times 10^{5}$ & $2 \times 10^{6}$ & $(\alpha, \gamma)$ & 10 & 13 & 3 & 20 \\
\hline$(\mathrm{d}, \gamma)$ & 5 & 7 & 30 & 100 & $(\alpha, n)$ & 1 & 9 & $3 \times 10^{2}$ & $10^{4}$ \\
\hline$(\mathrm{d}, \mathrm{n})$ & 2 & 7 & $4 \times 10^{3}$ & $3 \times 10^{5}$ & $(\alpha, 2 n)$ & 1 & 4 & $5 \times 10^{3}$ & $4 \times 10^{4}$ \\
\hline$(d, 2 n)$ & 2 & 5 & $2 \times 10^{5}$ & $6 \times 10^{6}$ & $(\alpha, 3 n)$ & 1 & 6 & $3 \times 10^{3}$ & $7 \times 10^{5}$ \\
\hline$(d, 3 n)$ & 1 & 4 & $3 \times 10^{5}$ & $10^{6}$ & $(\alpha, 4 n)$ & 5 & 8 & $3 \times 10^{3}$ & $4 \times 10^{4}$ \\
\hline$(d, 4 n)$ & 4 & 8 & $2 \times 10^{5}$ & $6 \times 10^{5}$ & $(\alpha, 5 n)$ & 5 & 8 & $10^{4}$ & $3 \times 10^{5}$ \\
\hline$(d, 5 n)$ & 6 & 10 & $10^{5}$ & $10^{6}$ & $(\alpha, p)$ & 5 & 8 & $6 \times 10^{2}$ & $2 \times 10^{4}$ \\
\hline$(\mathrm{d}, \mathrm{p})$ & 2 & 7 & $4 \times 10^{4}$ & $3 \times 10^{5}$ & $(\alpha, p n)$ & 3 & 12 & $3 \times 10^{3}$ & $8 \times 10^{4}$ \\
\hline$(\mathrm{d}, \mathrm{p} 2 \mathrm{n})$ & 2 & 10 & $10^{5}$ & $2 \times 10^{6}$ & $(\alpha, \mathrm{p} 2 \mathrm{n})$ & 5 & 15 & $3 \times 10^{3}$ & $7 \times 10^{4}$ \\
\hline$(\mathrm{d}, \mathrm{p} 3 \mathrm{n})$ & 8 & 15 & $10^{5}$ & $2 \times 10^{6}$ & $(\alpha, p 3 n)$ & 7 & 15 & $10^{4}$ & $3 \times 10^{4}$ \\
\hline$(d, 2 p)$ & 5 & 15 & $3 \times 10^{3}$ & $4 \times 10^{4}$ & $(\alpha, 2 p)$ & 5 & 10 & $10^{2}$ & $3 \times 10^{3}$ \\
\hline$(\mathrm{d}, \alpha)$ & 4 & 7 & $10^{4}$ & $3 \times 10^{4}$ & $(\alpha, \alpha n)$ & 6 & 16 & $3 \times 10^{3}$ & $3 \times 10^{4}$ \\
\hline$(\mathrm{d}, \alpha \mathrm{n})$ & 5 & 15 & $2 \times 10^{4}$ & $10^{5}$ & & & & & \\
\hline
\end{tabular}




\section{CHAPTER 7 INDUCED RADIOACTIVITY IN ACCELERATOR COMPONENTS}

\subsubsection{Methods of Systematizing Activation Due to High Energy Hadrons}

For proton and ion accelerators of higher energy, the neglect of secondary reactions and the restriction to few- and multi-nucleon transfer reactions can be a serious deficiency in the accuracy of estimation of induced radioactivity because of the rise in importance of such processes as spallation. Below a kinetic energy of about $40 \mathrm{MeV}$ only few-nucleon transfer reactions are generally prolific. The variety of radionuclides that can be produced increases with increasing bombarding energy because more nuclear reaction thresholds are exceeded. As a general rule, at high energies $\left(E_{O} \approx 1 \mathrm{GeV}\right.$ or greater), one must consider that all radionuclides in the periodic table that have mass numbers less than that of the material exposed to the flux of hadrons may be produced. Of course, many of these are of little significance due to short half-lives and small production cross sections.

Table 7.4 gives a list radionuclides typically encountered at high energy proton accelerators and their half-lives. In this table only nuclides with half-lives between 10 minutes and 5 years are listed. Also, all "pure" $\beta^{-}$(electron) emitters are ignored. Pure $\beta^{-}$ emitters are those radionuclides that emit no $\gamma$-rays in their decays. They generally present minimal external exposure hazards at accelerators as compared with $\gamma$-ray emitters in routine maintenance activities since the radionuclides are produced throughout the materials comprising accelerator components, with resultant self-shielding of most of the electrons compared with the less effective shielding of the more penetrating $\gamma$-rays. In contrast, $\beta^{+}$(positron) emitters are included in this table due to the generation of the pairs of $0.511 \mathrm{MeV}$ photons that result from annihilation of the positrons with electrons in matter. Approximate thresholds and high energy cross sections for production of these radionuclides by protons are also provided. Cross sections for production of radionuclides with mass numbers far below that of the target are usually less and sometimes much less than those listed here.

A systematic way of addressing the great multiplicity of radionuclides produced in accelerator components by high energy particles is highly desirable since it is often not practical to handle them all separately. Global properties of the distribution of radionuclides are found to be useful. Sullivan and Overton (Su65) have treated this problem in an elegant manner restated here. The initial starting point is a modification of Eq. (7.8) describing the dose rate as a function of irradiation and cooling times $t_{i}$ and $t_{c}$

$$
\delta\left(t_{i}, t_{c}\right)=G \phi\left[1-\exp \left(-\lambda t_{i}\right)\right] \exp \left(-\lambda t_{c}\right)
$$

where $\delta\left(t_{i}, t_{c}\right)$ is the absorbed dose rate, $\phi$ is the flux density, and $G$ is a collection of many contributing factors including the production cross section, the energy of the beam, the types of secondary particles produced, the isotopic composition of the irradiated component, the geometric configuration, the energy of the $\gamma$-rays produced, and the attenuation coefficients for the $\gamma$-rays produced. 
Table 7.4 Summary of radionuclides commonly identified in materials irradiated around accelerators. Approximate cross sections for their production at the high energy limit and approximate thresholds are given for selected radionuclides. [Adapted from (NC03) and (Ba69) with half-lives from (Tu05).]

\begin{tabular}{|c|c|c|c|c|}
\hline $\begin{array}{l}\text { Target } \\
\text { Material }\end{array}$ & Radionuclides & $\begin{array}{c}\text { Approximate } \\
\text { Threshold (MeV) }\end{array}$ & Half-life & $\begin{array}{l}\text { Production Cross Section } \\
\text { (High Energy Limit) (mb) }\end{array}$ \\
\hline Plastics \& Oils & $\begin{array}{l}{ }^{3} \mathrm{H} \\
{ }^{7} \mathrm{Be} \\
{ }^{11} \mathrm{C} \\
\end{array}$ & $\begin{array}{r}11 \\
2 \\
20 \\
\end{array}$ & $\begin{array}{c}12.32 \mathrm{y} \\
53.24 \mathrm{~d} \\
20.33 \mathrm{~min} \\
\end{array}$ & $\begin{array}{l}10 \\
10 \\
20\end{array}$ \\
\hline $\mathrm{Al}$, Concrete & $\begin{array}{c}\text { As above, plus } \\
{ }^{18} \mathrm{~F} \\
{ }^{22} \mathrm{Na} \\
{ }^{24} \mathrm{Na} \\
\end{array}$ & $\begin{array}{r}40 \\
30 \\
5\end{array}$ & $\begin{array}{l}1.83 \mathrm{~h} \\
2.60 \mathrm{y} \\
15.00 \mathrm{~h}\end{array}$ & $\begin{array}{r}6 \\
10 \\
10\end{array}$ \\
\hline $\mathrm{Fe}$ & $\begin{array}{c}\text { As above, plus } \\
{ }^{42} \mathrm{~K} \\
{ }^{43} \mathrm{~K} \\
{ }^{44} \mathrm{Sc} \\
{ }^{44 m} \mathrm{Sc} \\
{ }^{46} \mathrm{Sc} \\
{ }^{47} \mathrm{Sc} \\
{ }^{48} \mathrm{Sc} \\
{ }^{48} \mathrm{~V} \\
{ }^{51} \mathrm{Cr} \\
{ }^{52} \mathrm{Mn} \\
{ }^{52} \mathrm{Mn} \\
{ }^{54} \mathrm{Mn} \\
{ }^{52} \mathrm{Fe} \\
{ }^{55} \mathrm{Fe} \\
{ }^{59} \mathrm{Fe} \\
{ }^{56} \mathrm{Co} \\
{ }^{57} \mathrm{Co} \\
{ }^{58} \mathrm{Co} \\
\end{array}$ & $\begin{array}{r}20 \\
30 \\
20 \\
\\
30 \\
30 \\
\\
5 \\
30 \\
30 \\
\end{array}$ & $\begin{array}{c}12.32 \mathrm{~h} \\
22.3 \mathrm{~h} \\
3.97 \mathrm{~h} \\
2.44 \mathrm{~d} \\
83.8 \mathrm{~d} \\
3.35 \mathrm{~d} \\
1.82 \mathrm{~d} \\
15.97 \mathrm{~d} \\
27.7 \mathrm{~d} \\
5.59 \mathrm{~d} \\
21.1 \mathrm{~min} \\
312.1 \mathrm{~d} \\
8.28 \mathrm{~h} \\
2.74 \mathrm{y} \\
44.5 \mathrm{~d} \\
77.2 \mathrm{~d} \\
271.7 \mathrm{~d} \\
70.9 \mathrm{~d} \\
\end{array}$ & $\begin{array}{r}6 \\
30 \\
30 \\
\\
30 \\
4 \\
\\
\\
30 \\
30 \\
25 \\
\end{array}$ \\
\hline $\mathrm{Cu}$ & $\begin{array}{c}\text { As above, plus } \\
{ }^{57} \mathrm{Ni} \\
{ }^{65} \mathrm{Ni} \\
{ }^{60} \mathrm{Co} \\
{ }^{60} \mathrm{Cu} \\
{ }^{61} \mathrm{Cu} \\
{ }^{62} \mathrm{Cu} \\
{ }^{64} \mathrm{Cu} \\
{ }^{62} \mathrm{Zn} \\
{ }^{65} \mathrm{Zn} \\
\end{array}$ & $\begin{array}{l}40 \\
30 \\
20\end{array}$ & $\begin{array}{c}35.6 \mathrm{~h} \\
2.52 \mathrm{~h} \\
5.27 \mathrm{y} \\
23.7 \mathrm{~min} \\
3.33 \mathrm{~h} \\
9.67 \mathrm{~min} \\
12.70 \mathrm{~h} \\
9.19 \mathrm{~h} \\
243.7 \mathrm{~d} \\
\end{array}$ & $\begin{array}{r}2 \\
15 \\
100 \\
\\
60 \\
100 \\
\end{array}$ \\
\hline
\end{tabular}

If the number of radionuclides produced by the irradiation which have decay constants in the interval between $\lambda$ and $\lambda+d \lambda$ is represented by the differential $d m$, then the corresponding increment in absorbed dose rate due to them $d \delta\left(t_{i}, t_{c}\right)$ is given by

$$
d \delta\left(t_{i}, t_{c}\right)=d m G \phi\left[1-\exp \left(-\lambda t_{i}\right)\right] \exp \left(-\lambda t_{c}\right) .
$$




\section{CHAPTER 7 INDUCED RADIOACTIVITY IN ACCELERATOR COMPONENTS}

If it is assumed that the value of $G$ is independent of $\lambda$, or its dependence on $\lambda$ is small compared to other factors, then one can integrate, ${ }^{22}$

$$
\delta\left(t_{i}, t_{c}\right)=G \phi \int_{\lambda_{0}}^{\infty} d \lambda \frac{d m}{d \lambda}\left[1-\exp \left(-\lambda t_{i}\right)\right] \exp \left(-\lambda t_{c}\right)
$$

Here $\lambda_{o}$ is the shortest decay constant, corresponding to the longest mean-life, to be considered. For a given material of atomic mass number $A$, a study of the chart of the nuclides [e.g., (Tu05), (Se81)] reveals that there is a finite number of radionuclides within a reasonable domain of half-lives that can be produced in that material (i.e., with mass number $<A$ ). Fig 7.12 is a plot of the number of radionuclides as a function of halflife $t_{1 / 2}$ that have half-lives less than $t_{1 / 2}$ for several choices of the upper limit on atomic mass number $A$ of the target material. This corresponds to the distribution half-lives of radionuclides that could be produced in a target of mass number $A$ irradiated by high energy hadrons. Sullivan and Overton observed that the cumulative distributions are welldescribed over the approximate domain $10^{-3}<t_{1 / 2}<10^{3}$ days by

$$
N\left(t_{1 / 2}\right)=a+b \ln \left(t_{1 / 2}\right)
$$

where $N\left(t_{1 / 2}\right)$ is the number of radionuclides with half-lives less than the value of $t_{1 / 2}$ and $a$ and $b$ are fitting parameters. Because of the one-to-one correspondence between values of $t_{1 / 2}, \tau$, and $\lambda$ in this Sullivan-Overton approximation one can just as well write

$$
m(\lambda)=a+b \ln \lambda
$$

where $m(\lambda)$ is the number of radionuclides with decay constants greater than $\lambda$ for the material of concern. Thus,

$$
\frac{d m(\lambda)}{d \lambda}=\frac{b}{\lambda}
$$

Substituting into Eq. (7.14), one gets

$$
\begin{aligned}
& \delta\left(t_{i}, t_{c}\right)=G b \phi \int_{\lambda_{0}}^{\infty} \frac{d \lambda}{\lambda}\left[1-\exp \left(-\lambda t_{i}\right)\right] \exp \left(-\lambda t_{c}\right)= \\
& G b \phi\left\{\int_{\lambda_{0}}^{\infty} \frac{d \lambda}{\lambda} \exp \left(-\lambda t_{c}\right)-\int_{\lambda_{0}}^{\infty} \frac{d \lambda}{\lambda} \exp \left[-\lambda\left(t_{i}+t_{c}\right)\right]\right\} .
\end{aligned}
$$

The changes of variables $\alpha=\lambda t_{c}$ [first term] and $\alpha^{\prime}=\lambda\left(t_{i}+t_{c}\right)$ [second term] are helpful;

$$
\delta\left(t_{i}, t_{c}\right)=G b \phi\left\{\int_{\lambda_{o} t_{c}}^{\infty} d \alpha \frac{e^{-\alpha}}{\alpha}-\int_{\lambda_{o}\left(t_{i}+t_{c}\right)}^{\infty} d \alpha^{\prime} \frac{e^{-\alpha^{\prime}}}{\alpha^{\prime}}\right\}
$$

\footnotetext{
${ }^{22}$ Taking this step implies the assumption that, on average, the radionuclide production cross sections under consideration are independent of both the half-lives and the particle energies. Somewhat remarkably, this approximation is a sufficiently accurate one for the present purpose.
} 


\section{CHAPTER 7 INDUCED RADIOACTIVITY IN ACCELERATOR COMPONENTS}
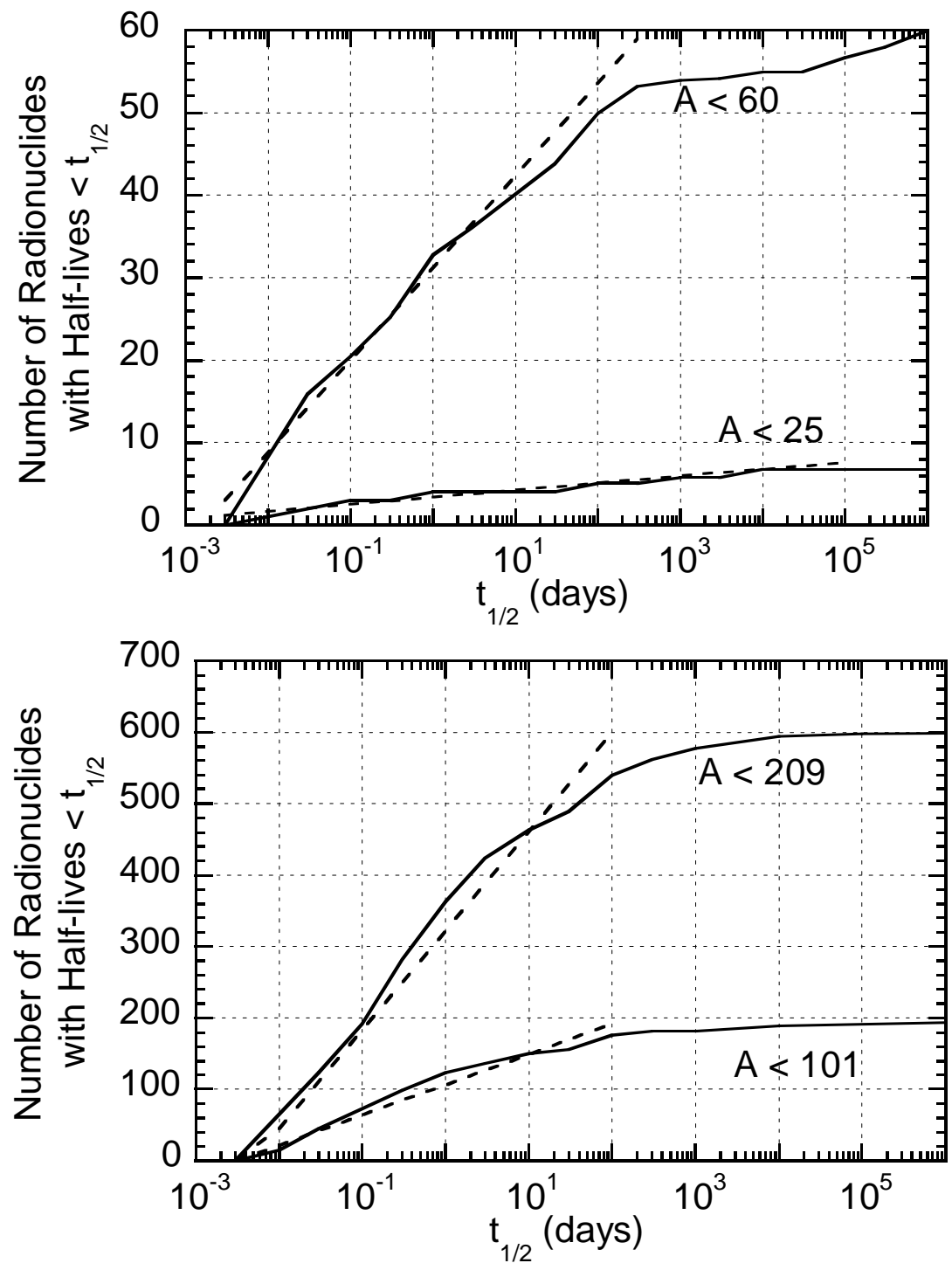

Fig. 7.12 Total number of radionuclides having half-lives up to a given half-life $t_{1 / 2}$ as a function of $t_{1 / 2}$ for target mass numbers $A$ less than those indicated. [Adapted from (Ba69).]

Recognizing that the integrands are identical and simplifying by rearranging the limits of integration,

$$
\delta\left(t_{i}, t_{c}\right)=G b \phi \int_{\lambda_{o} t_{c}}^{\lambda_{o}\left(t_{i}+t_{c}\right)} d \alpha \frac{e^{-\alpha}}{\alpha}
$$

The integration results in a series expansion found in standard tables of integrals;

$$
\int_{x_{1}}^{x_{2}} \frac{e^{a x} d x}{x}=\left[\ln x+\frac{a x}{1 !}+\frac{a^{2} x^{2}}{2 \times 2 !}+\frac{a^{3} x^{3}}{3 \times 3 !}+\ldots\right]_{x_{1}}^{x_{2}} .
$$




\section{CHAPTER 7 INDUCED RADIOACTIVITY IN ACCELERATOR COMPONENTS}

Substituting,

$$
\int_{\lambda_{o} t_{c}}^{\lambda_{o}\left(t_{i}+t_{c}\right)} \frac{e^{-\alpha} d \alpha}{\alpha}=\left[\ln \alpha-\alpha+\frac{\alpha^{2}}{4}-\frac{\alpha^{3}}{18}+\ldots\right]_{\lambda_{o} t_{c}}^{\lambda_{o}\left(t_{i}+t_{c}\right)} .
$$

Evaluating, one obtains

$$
\delta\left(t_{i}, t_{c}\right)=G b \phi\left[\ln \left(\frac{t_{i}+t_{c}}{t_{c}}\right)-\lambda_{0} t_{i}+\ldots\right] .
$$

Since $\lambda_{o}$ approaches zero (corresponding to large mean-lives), the following is obtained:

$$
\delta\left(t_{i}, t_{c}\right) \approx B \phi \ln \left(\frac{t_{i}+t_{c}}{t_{c}}\right)
$$

where several constants are merged in the new parameter $B$.

\subsubsection{Gollon's Rules of Thumb}

Gollon has further elaborated on these principles in stating four very useful "rules of thumb" for high energy hadron accelerators at which the extranuclear hadron cascade process produces the major fraction of the induced activity (Go76).

Rule 1: $\quad$ This is equivalent to Eq. (7.10), repeated here for convenience;

$$
\frac{d D}{d t}=0.4 \frac{S}{r^{2}} \sum_{i} E_{\gamma i}
$$

where the summation is over all $\gamma$-rays present, including appropriate branching fractions if more than one photon is emitted per decay. [See comment about alternative units related to Eq. (7.10).]

Rule 2: In many materials, about $50 \%$ of the nuclear interactions produce a nuclide with a half-life longer than a few minutes. Further, about $50 \%$ of these have a half-life longer than one day. Thus, approximately $25 \%$ of the nuclear interactions (e.g., the "stars" discussed in Section 4.7.2) produce a radionuclide having a half-life exceeding approximately one day.

Rule 3: $\quad$ For most common shielding materials, the approximate dose rate $d D / d t$ due to a constant irradiation is [see Eq. (7.24)] given by

$$
\frac{d D}{d t}=B \phi \ln \left(\frac{t_{i}+t_{c}}{t_{c}}\right)
$$




\section{CHAPTER 7 INDUCED RADIOACTIVITY IN ACCELERATOR COMPONENTS}

In the above, the geometry and material-dependent factor $B$ can often be determined empirically, or estimated by using Rule 2, while $\phi$ is the incident flux density. This expression also works for intermediate energy heavy ion beams, for example at $86 \mathrm{MeV}$ nucleon $^{-1}$ (Tu84).

Rule 4: In a hadronic cascade, each proton or neutron produces about four inelastic interactions for each $\mathrm{GeV}$ of energy.

Some examples can illustrate the use of these rules of thumb. As one illustration, in a short target of $1 / 10$ of an interaction length, approximately $10 \%$ of an incident beam of $10^{11}$ protons s ${ }^{-1}$ will interact. Assume this has been occurring for several months (long enough to reach saturation production for many radionuclides) at this constant rate. Using Rule 2 in conjunction with the above rate, one determines that the decay rate after one day of the shutdown is $2.5 \times 10^{9} \mathrm{~Bq}(68 \mathrm{mCi})$. If each of these decays produces a $1.0 \mathrm{MeV}$ $\gamma$-ray, then Rule 1 gives an absorbed dose rate of $27 \mathrm{mrad} \mathrm{h}^{-1}\left(\approx 0.27 \mathrm{mGy} \mathrm{h}^{-1}\right)$ at one meter away.

Rule 3 can be used in such a calculation to predict the absorbed dose rate from a point source at some future time after beam shutdown. Furthermore, this rule is not restricted to "point" sources but can be used for larger ones, with suitable adjustments to the geometry factors. Sometimes one can estimate the product $B \phi$ or use a measurement of the exposure or absorbed dose rate early in a shutdown period to determine it empirically in order to predict the "cooldown" for later times using Eq. (7.26) as a tool in planning radiological work. Rule 3 also clearly works for extended shields irradiated by secondary particles from a well-developed cascade.

Rule 4 can be used to crudely estimate the activation of a beam absorber by incident high energy particles when it is coupled with Rule 2. For example, a beam of $10^{12} 400 \mathrm{GeV}$ protons $\mathrm{s}^{-1}(=0.16 \mu \mathrm{A}$ or $64 \mathrm{~kW})$ produces a total of $4 \times 400 \times 10^{12}$ stars $^{-1}$ in a beam absorber. If $25 \%$ of these produce a radionuclide with a half-life $>1.0$ day (Rule 2), then the total amount of the moderately long-lived radioactivity (at saturation) is

$$
\frac{(0.25 \text { atoms } / \mathrm{star})\left(1.6 \times 10^{15} \mathrm{stars} / \mathrm{sec}\right)}{3.7 \times 10^{10} \mathrm{sec}^{-1} \mathrm{Ci}^{-1}}=10.8 \mathrm{kCi} \text {. }
$$

At a sufficiently large distance (say 10 meters), Rule 1 could be used to calculate an absorbed dose rate from a point source assuming all decays are $1.0 \mathrm{MeV} \gamma$-rays;

$$
\frac{d D}{d t} 0.4(1.0 \mathrm{MeV})\left[\frac{1.08 \times 10^{4} \mathrm{Ci}}{10^{2} \mathrm{~m}^{2}}\right]=43 \mathrm{rads}^{-1} .
$$




\section{CHAPTER 7 INDUCED RADIOACTIVITY IN ACCELERATOR COMPONENTS}

\subsubsection{The Barbier Danger Parameter}

A valuable quantity used to quantify the absorbed dose rate $d D / d t$ at the surface of a thick target is the danger parameter $\mathbf{D}$ as developed by Barbier (Ba69) for a thick object irradiated by beam having a uniform flux density $\phi$. If this source of radioactivity subtends solid angle $\Omega$ at the point of concern, then

$$
\frac{d D}{d t}=\frac{\Omega}{4 \pi} \varphi \mathbf{D}
$$

At contact with a semi-infinite slab of uniformly irradiated material, the fractional solid angle factor $(\Omega / 4 \pi)$ has the intuitively obvious value of $1 / 2$. The danger parameter has the physical interpretation as the absorbed dose rate found inside a cavity of arbitrary form embedded in an infinite volume of a material which has been uniformly irradiated by a unit flux density $\left(1.0\right.$ particle $\left.\mathrm{s}^{-1} \mathrm{~cm}^{-2}\right)$. Fig. 7.13 gives representative examples of plots of D for several elements and a few compounds. These curves thus can be used to predict cooling of various components around accelerators. Gollon (Go76) has also provided "cooling curves" for iron struck by high energy protons. These are given in Fig. 7.14 and include both calculations by Armstrong and Alsmiller (Ar69a) and empirical measurements at the Brookhaven National Laboratory AGS, the Fermilab Main Ring Accelerator, and the Fermilab Neutrino Experimental Area target station.

Of course, situations arise where the determination of $\phi$ in the danger parameter equation is not at all simple. For example, one can have activation in a large object where the hadronic cascade is contributing numerous hadrons at a variety of energies from a multitude of directions. Fortunately, important features of activation phenomena have little or no correlation with energy. The chief of these is evidenced by the excitation functions of various reactions. As seen in Figs. 7.9, 7.10, and 7.11, the cross sections rise just above the threshold and then, in the region of tens of $\mathrm{MeV}$ above the threshold, a leveling-off occurs. In general the cross sections for production of radionuclides by neutrons and protons (and even other ions and particles) do not differ greatly from each other (i.e., within one to two orders of magnitude) at the higher energies.

\subsubsection{The Utilization of Monte Carlo Star Densities in Activation Calculations}

The "leveling-off" of the cross section as a function of energy has some very important implications. A prominent one is the fact that for estimating activation, one can perform approximate calculations without performing integration over energy if one has some reasonable estimate of the hadron flux density above the reaction threshold of interest. An average effective cross section can then be used. Another feature of these excitation functions is the fact that the leveling off occurs in the region from a few 10's to a few 100 's of MeV, precisely where relatively fast Monte Carlo hadron shielding calculations are available from several different codes (e.g., CASIM, FLUKA, HETC, and MARS). 


\section{CHAPTER 7 INDUCED RADIOACTIVITY IN ACCELERATOR COMPONENTS}
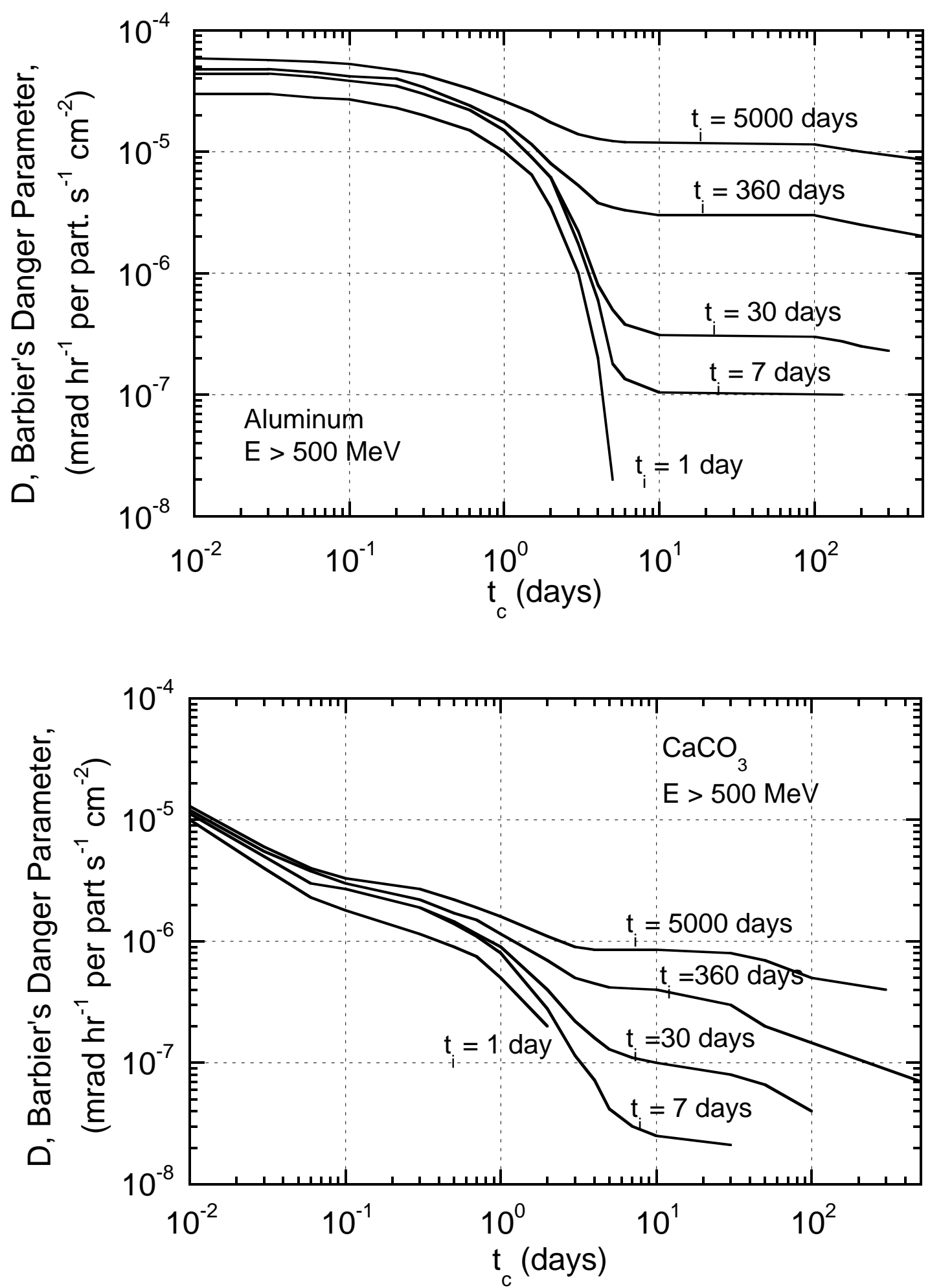

Fig. 7.13 Values of the Barbier danger parameter $\mathbf{D}$ for selected materials at a proton irradiation energy of $500 \mathrm{MeV}$. [Adapted from (Ba69).] 

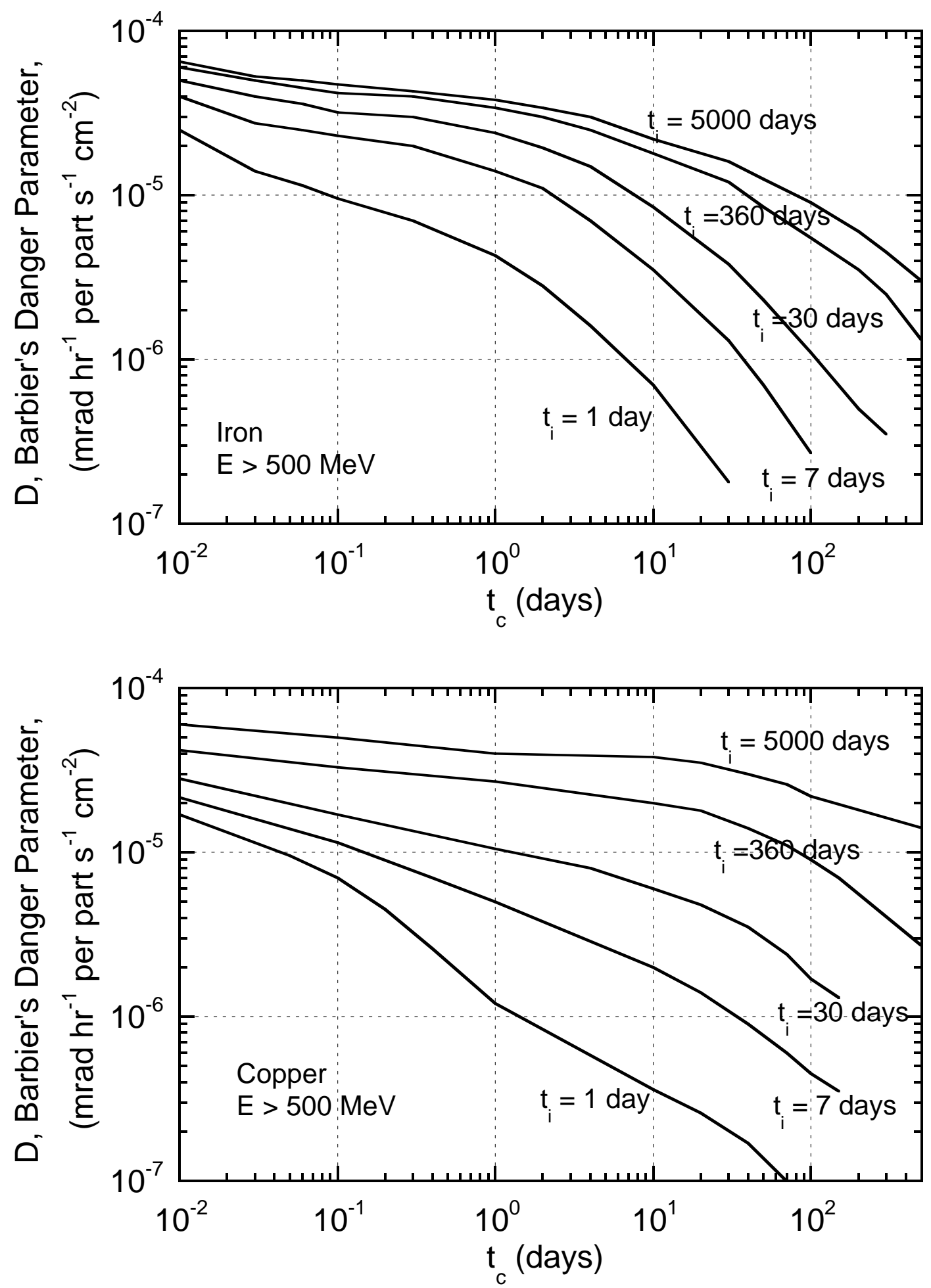

Fig. 7.13-continued. 


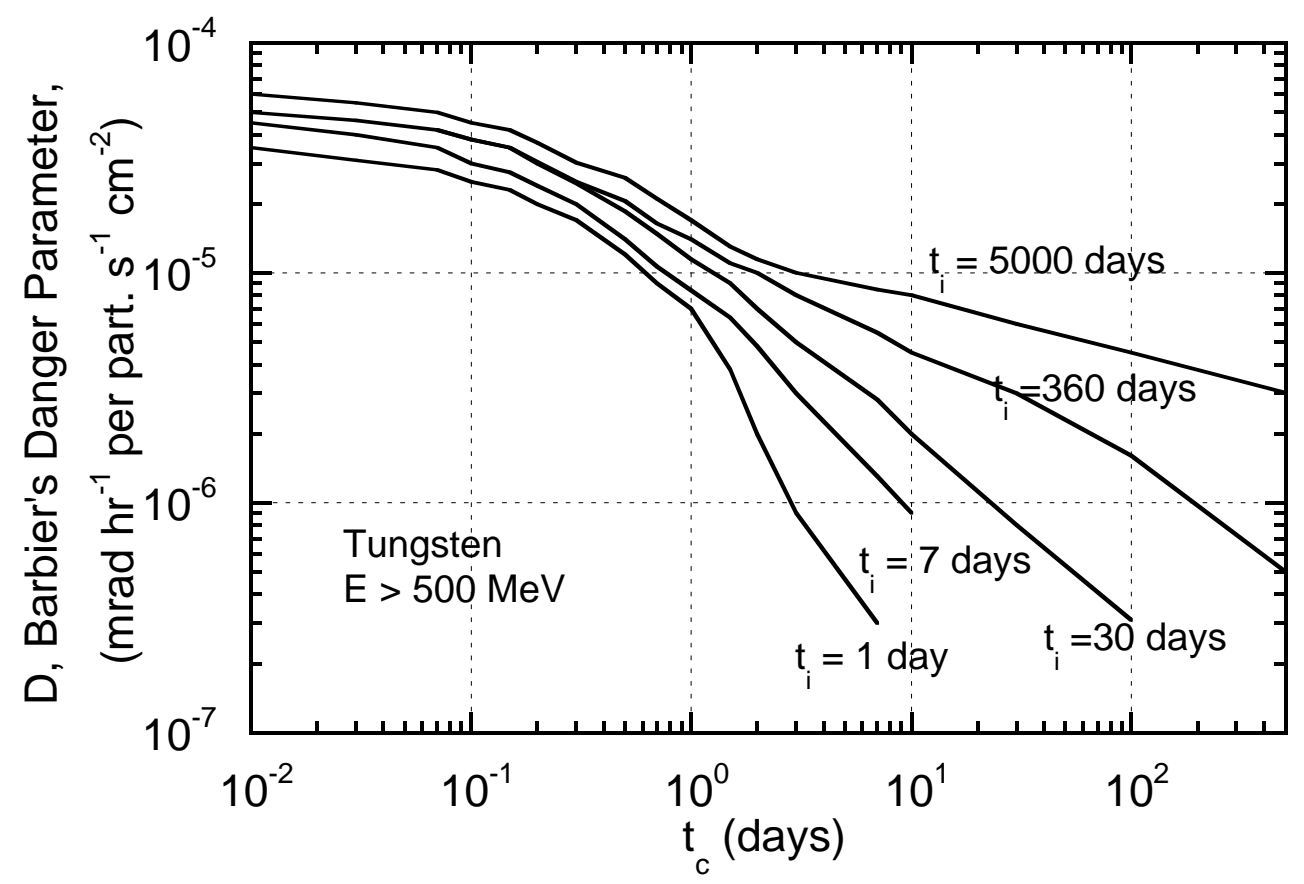

Fig. 7.13-continued.

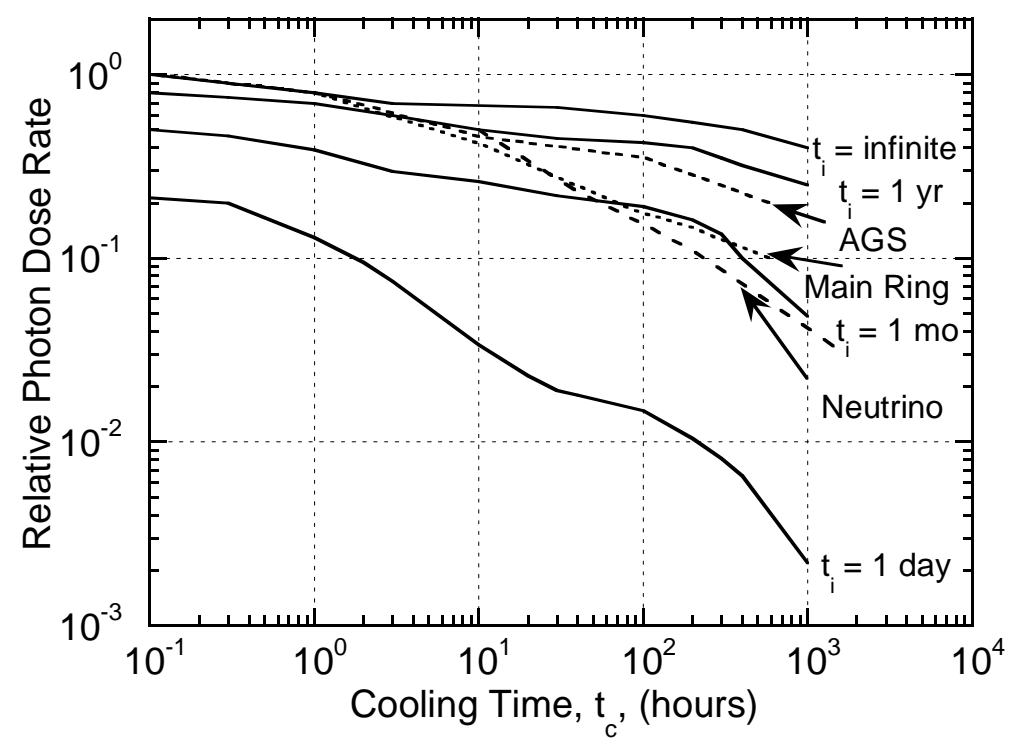

Fig. 7.14 Cooling curves for various irradiation times for iron struck by high energy protons as calculated by Armstrong and Alsmiller (Ar69a). Also shown are the results of measurements. The one labeled "Main Ring", is the measured average cooling curve for the Fermilab Main Ring synchrotron after its initial three years of operation at energies of 200 or $400 \mathrm{GeV}$. The curve labeled "Neutrino" is for a neutrino target station at Fermilab after eight months of operation at $400 \mathrm{GeV}$. The curve labeled "AGS" is for an extraction splitter in use for many years at the Brookhaven Alternating Gradient Synchrotron at energies up to $30 \mathrm{GeV}$. [Adapted from (Go76).] 


\section{CHAPTER 7 INDUCED RADIOACTIVITY IN ACCELERATOR COMPONENTS}

It is often possible to relate the flux density of high energy hadrons (i.e., those with energies above the leveling off) to the star density $S$ calculated from such Monte Carlo calculations through the relationship

$$
\phi(\vec{r})=\frac{\lambda}{\rho} \frac{d S(\vec{r})}{d t},
$$

where $\phi(\vec{r})$, the flux density $\left(\mathrm{cm}^{-2} \mathrm{~s}^{-1}\right)$ at position vector at $\vec{r}$, is related to the rate of star density production $d S(\vec{r}) / d t\left(\right.$ stars $\left.\mathrm{cm}^{-3} \mathrm{~s}^{-1}\right)$ at the same location ${ }^{23} \cdot \rho\left(\mathrm{g} \mathrm{cm}^{-3}\right)$ is the density and $\lambda\left(\mathrm{g} \mathrm{cm}^{-2}\right)$ is the interaction length ${ }^{24}$. The value of $\phi(\vec{r})$ so determined could, in principle, be substituted into Eq. (7.29) for calculating absorbed dose rate due to residual activity using the Barbier danger parameter $\mathbf{D}$, if one makes suitable adjustments in the solid angle. These considerations are implicit in the following equation

$$
\frac{d D(\vec{r})}{d t}=\frac{\Omega}{4 \pi} \frac{d S(\vec{r})}{d t} \omega\left(t_{i}, t_{c}\right)
$$

where the so-called "omega factor" ( $\omega$-factor) $\omega\left(t_{i}, t_{c}\right)$ related to the Barbier danger parameter $\mathbf{D}$ has been introduced. The choice of the low-energy cutoff used in the Monte Carlo calculation can be important. For a lower momentum cutoff of $300 \mathrm{MeV} / \mathrm{c}$ (equivalent to a kinetic energy of about $47 \mathrm{MeV}$ for nucleons) using CASIM, Gollon (Go76) determined

$$
\begin{aligned}
& \omega(\infty, 0)=9 \times 10^{-6} \mathrm{rad} \mathrm{h}^{-1} /\left(\mathrm{star} \mathrm{cm}^{-3} \mathrm{~s}^{-1}\right)=9 \times 10^{-8} \mathrm{~Gy} \mathrm{~h}^{-1} /\left(\mathrm{star} \mathrm{cm}^{-3} \mathrm{~s}^{-1}\right) \\
& \text { (infinite irradiation, zero cooling time), and } \\
& \begin{array}{l}
\omega(30 \mathrm{~d}, 1 \mathrm{~d})=2.5 \times 10^{-6} \mathrm{rad} \mathrm{h}^{-1} /\left(\mathrm{star} \mathrm{cm}^{-3} \mathrm{~s}^{-1}\right)=2.5 \times 10^{-8} \mathrm{~Gy} \mathrm{~h}^{-1} /\left(\mathrm{star} \mathrm{cm}^{-3} \mathrm{~s}^{-1}\right) \\
(30 \text { days irradiation, } 1 \text { day cooling time }) .
\end{array}
\end{aligned}
$$

This was for an "equilibrium" spectrum in a well-developed hadronic cascade. Estimates of $\omega$-factor values for other choices of values of $t_{i}$ and $t_{c}$ for irradiated iron can be made by scaling results obtained by Armstrong and Alsmiller (Ar69a) and Gabriel and Santoro (Ga73) as done, again for irradiated iron, by Cossairt (Co98). The condition $t_{i}=30 \mathrm{~d}$, $t_{c}=1.0 \mathrm{~d}$ has become a generally accepted standard condition for further calculations.

Stevenson (St01) has provided similar $\omega$-factors for a variety of materials common at accelerators as provided in Table 7.5. Such $\omega$-factors for residual activation of thin objects; those with linear dimensions less than a fraction of a nuclear interaction length, have been obtained by Mokhov et al. (Mo06) using the MARS code. As explained by these authors, the lower energy (or momentum) cutoff is especially important because

\footnotetext{
${ }^{23}$ This flux density is the delivery rate of the "star fluence" discussed in Section 4.7.2.

24 Once again, the adherence to traditional notation requires that care must be taken not to confuse interaction length with activity constant since they both are customarily denoted by the same symbol, $\lambda$.
} 


\section{CHAPTER 7 INDUCED RADIOACTIVITY IN ACCELERATOR COMPONENTS}

of the importance of contributions from spallation reactions in the $20-50 \mathrm{MeV}$ domain. Detailed calculations for three different energy groups are provided in Fig. 7.15.

Table $7.5 \omega$-Factors for converting star density production rate to residual dose rate for $t_{i}=30 \mathrm{~d}, t_{c}=1.0$ $d$ in various materials [Taken from (St01).]

\begin{tabular}{|c|c|}
\hline Material & $\begin{array}{c}\omega \text {-Factor } \\
\left(\mathrm{Gy} \mathrm{h}^{-1}\right) /\left(\operatorname{star} \mathrm{cm}^{-3} \mathrm{~s}^{-1}\right)\end{array}$ \\
\hline Iron & $1.0 \times 10^{-8}$ \\
\hline Copper & $1.0 \times 10^{-8}$ \\
\hline Stainless steel & $1.3 \times 10^{-8}$ \\
\hline Aluminum & $2.0 \times 10^{-9}$ \\
\hline Lead & $1.5 \times 10^{-8}$ \\
\hline Tungsten & $1.1 \times 10^{-8}$ \\
\hline Normal concrete & $3.0 \times 10^{-9}$ \\
\hline Marble & $6.0 \times 10^{-10}$ \\
\hline
\end{tabular}

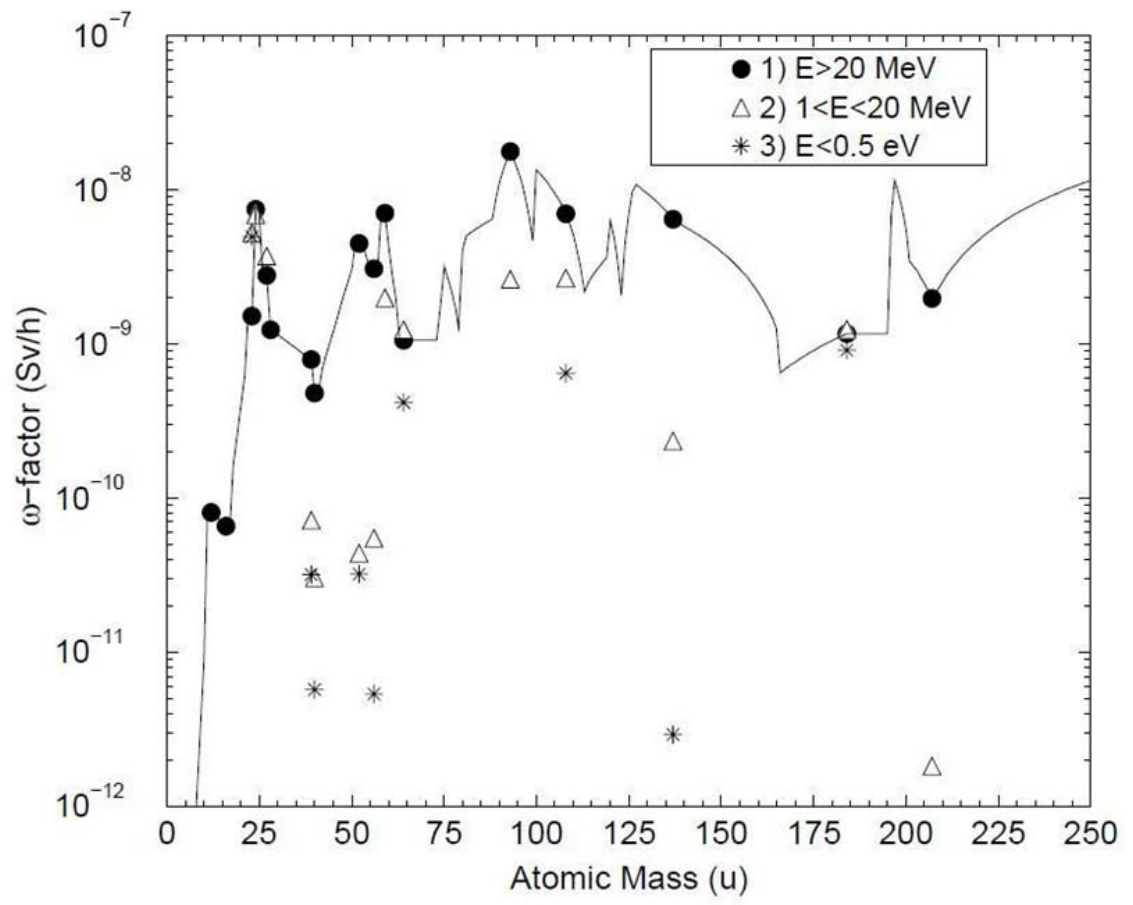

Fig. 7.15 $\omega$-factor dependence on mass of a target nucleus for three energy groups with $t_{i}=30 \mathrm{~d}, t_{c}=1.0 \mathrm{~d}$ determined using the MARS code. Normalization is per star $\mathrm{cm}^{-3} \mathrm{~s}^{-1}$ for $E>20 \mathrm{MeV}$, and per neutron $\mathrm{cm}^{-2} \mathrm{~s}^{-1}$ for the lower energy groups. The symbols represent the result using FLUKA of a previous study (Hu03) and the curve is a interpolation of the results of that study and those of an earlier one for the high energy group (Hu98). [Reproduced from (Mo06).]

Huhtinen has performed related calculations [(Hu98),(Hu03)]. Given the obvious variability, these results should be used with some degree of caution. They can readily be used to predict the relative "cooling" rates of various components around accelerators with a fair degree of accuracy. Their use in the precise prediction of absolute absorbed 


\section{CHAPTER 7 INDUCED RADIOACTIVITY IN ACCELERATOR COMPONENTS}

dose rates near activated accelerator components requires additional care. To do this, the geometric configuration should be simple and well-defined, the flux density of thermal neutrons should be a small component of the prompt radiation field, and the activation of other materials in proximity such as the enclosure walls should be taken into account. Cracks through the shielding materials can sometimes result in higher dose rates that are difficult to model. The interactions of thermal neutrons in concrete shielding can make a significant contribution to the dose equivalent rate. This phenomenon has been discussed by Armstrong and Barish (Ar69b) and by Cossairt (Co96) and is summarized in Section 7.4.4. A good example of a detailed treatment of a specific situation incorporating the neutron energy spectrum, large gaps between shielding components, and comparisons of calculations with measurements is that of Rakhno et al. (Ra01).

Gollon derived a simple relationship between dose rates involving cooling times different from "standard" ones for which values of $\mathbf{D}$ and $\omega$ are available. As before, the dose rate after irradiation time $t_{i}$ and cooldown time $t_{c}$ is

$$
\delta\left(t_{i}, t_{c}\right)=\sum_{\mu} \mathrm{A}_{\mu}\left[1-\exp \left(-\lambda_{\mu} t_{i}\right)\right] \exp \left(-\lambda_{\mu} t_{c}\right),
$$

where the summation over index $\mu$ includes all relevant radionuclides with the product of flux density and geometry factors being absorbed (and allowed to vary with radionuclide) in the quantity $A_{\mu}$.

Rearranging, Gollon obtained

$$
\begin{gathered}
\delta\left(t_{i}, t_{c}\right)=\sum_{\mu} \mathrm{A}_{\mu}\left[\exp \left\{-\lambda_{\mu} t_{c}\right\}-\exp \left\{-\lambda_{\mu}\left(t_{i}+t_{c}\right)\right\}\right]= \\
\delta\left(\infty, t_{c}\right)-\delta\left(\infty, t_{i}+t_{c}\right) .
\end{gathered}
$$

Thus, the infinite irradiation curve can be used to determine any other combination of the times $t_{i}$ and $t_{c}$. In fact, this formula may be used also with empirical results such as, for example, radiation survey data, in order to predict future radiological conditions.

A reliable method for connecting the production of "stars" in material (i.e., as calculated by a Monte Carlo code) to the production of atoms of some radionuclide is by the ratios of cross sections. Thus, at some point in space $\vec{r}$ the rate of production of atoms $\mathrm{cm}^{-3}$ $n_{i}(\vec{r})$ of some radionuclide $i$ is approximately given by

$$
\frac{d n_{i}(\vec{r})}{d t} \approx \frac{\sigma_{i}}{\sigma_{i n}} \frac{d S(\vec{r})}{d t}=\frac{\Sigma_{i}}{\sum_{i n}} \frac{d S(\vec{r})}{d t},
$$

where one essentially scales the star density production rate (e.g., stars $\mathrm{cm}^{-3} \mathrm{~s}^{-1}$ ) by the 


\section{CHAPTER 7 INDUCED RADIOACTIVITY IN ACCELERATOR COMPONENTS}

ratio of the production (reaction) cross section for the nuclide of interest $\sigma_{i}$ to the total inelastic cross section $\sigma_{i n}$ or, equivalently, by the ratio of the macroscopic cross sections $\left(\Sigma_{\mathrm{i}} / \Sigma_{\mathrm{in}}\right)$. The phenomena will obey the usual activation equation. The reason this is approximate is due to the familiar concerns about constancy of cross sections with energy, the lack of perfect "matching" of effective reaction thresholds, etc.

\subsubsection{Uniform Irradiation of the Walls of an Accelerator Enclosure}

Somewhat special considerations may apply to the concrete shielding surrounding accelerators. As was seen before in Table 6.2, ordinary concrete typically contains a partial density of about $0.04 \mathrm{~g} \mathrm{~cm}^{-3}$ of sodium. This "typical" value varies a great deal due to the variety of minerals that might be present in local concrete. The significance of this seemingly small additive is that the naturally dominant isotope present is ${ }^{23} \mathrm{Na}$. This nucleus has the relatively large thermal neutron capture cross section of $535 \mathrm{mb}$. Patterson $(\mathrm{Pa} 58)$ determined that average thermal neutron flux density $\phi_{t h}$ in a concrete room is approximately given as follows:

$$
\phi_{t h}=\frac{1.25 N_{f}}{S}\left(\mathrm{~cm}^{-2} \mathrm{~s}^{-1}\right)
$$

where $N_{f}$ is the total number rate of production of fast neutrons in the enclosure and $S$ is the inside surface area of the enclosure $\left(\mathrm{cm}^{2}\right)$. Thus, a substantial flux density of thermal neutrons can be present in an accelerator room and this flux can produce significant amount of ${ }^{24} \mathrm{Na}$ with its 15 -hour half-life. In view of Eq. (7.10), the pair of relatively energetic photons emitted in the decay of ${ }^{24} \mathrm{Na}(1.37$ and $2.75 \mathrm{MeV})$ increases the residual dose rate. Furthermore, while the dose due to activated components falls off radially with distance, if absorption by the air is not significant the photon flux density due to activation of the walls of an empty room uniformly irradiated by such thermal neutrons is a constant independent of position within the enclosure. Thus, the absorbed dose rate due to the walls anywhere inside the enclosure will be equal to the absorbed dose rate at the wall. This has been explicitly demonstrated for cylinders by Armstrong and Barish (Ar69b) and is also true for the interior of all mathematically "well-behaved" closed surfaces (Co96). This fact can readily be demonstrated by analogy to the Gauss Law in electrostatics $^{25}$ as follows by examining the situation in Fig. 7.16.

Consider a simple, closed surface that emits an omnidirectional flux density of some particle $\phi_{O}$ (e.g., particles $\mathrm{cm}^{-2} \mathrm{~s}^{-1}$ ) that is constant over the surface. One wants to calculate the flux density at some arbitrary point in space $P$ within the surface. $P$ is located at radius vector $\vec{r}$. Consider further the contributions of the particles emitted by some elemental area $d \vec{A}$ at $P$ where $d \vec{A}$ is perpendicular to the surface at coordinate vector $\vec{r}$. The solid angle subtended at $P$ by $d \vec{A}$ is

\footnotetext{
${ }^{25}$ The Gauss law of electrostatics has been treated elsewhere; e.g., Jackson (Ja75) and Konopinski (Ko81).
} 


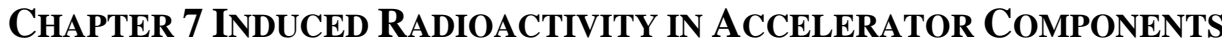

$$
d \Omega=\frac{d \vec{A} \bullet \hat{n}}{\left|\vec{r}^{\prime}-\vec{r}\right|^{2}},
$$

where the unit vector $\hat{n}$ is given by

$$
\hat{n}=\frac{\vec{r}^{\prime}-\vec{r}}{\left|\vec{r}^{\prime}-\vec{r}\right|} .
$$

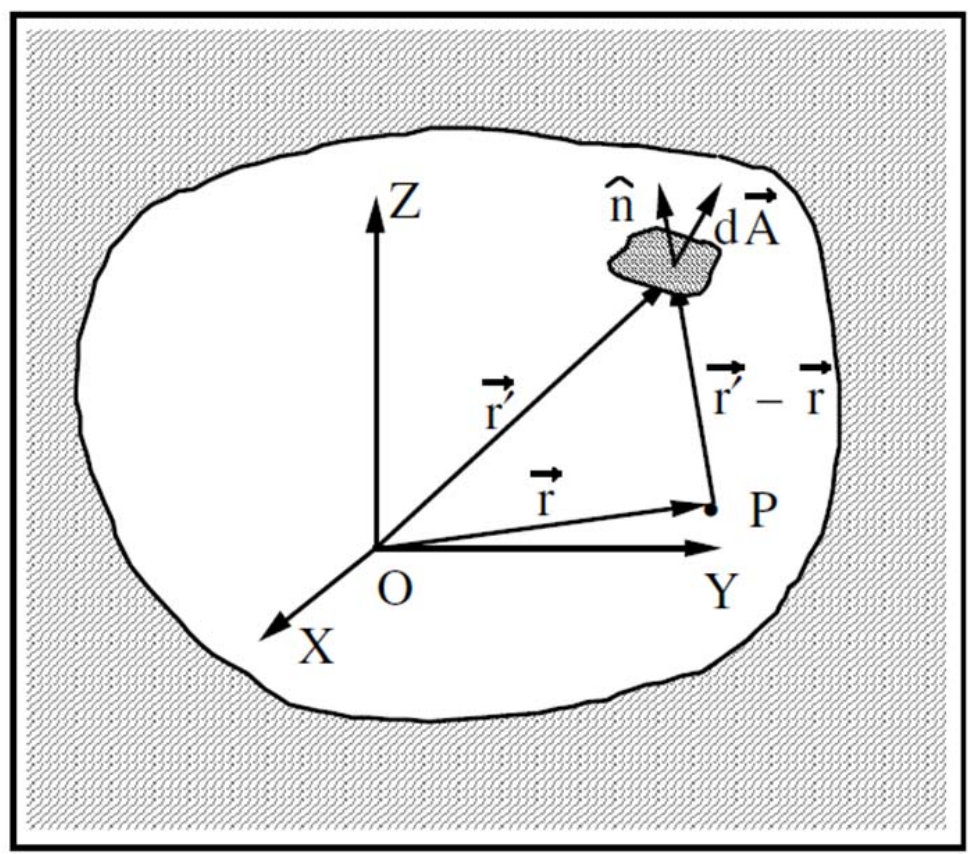

Fig. 7.16 Geometry for deriving the relationship between a surface of uniform emission and the flux density at any point within it. [Reproduced from (Co96).]

But the increment of fluence at point $P$ due to elemental area $d \vec{A}$ is given by

$$
d \phi=\frac{\phi_{o}}{4 \pi} \frac{d \vec{A} \bullet \hat{n}}{\left|\vec{r}^{\prime}-\vec{r}\right|^{2}} .
$$

Thus,

$$
d \phi=\frac{\phi_{o}}{4 \pi} d \Omega \text { and } \int_{4 \pi} \frac{\phi_{o}}{4 \pi} d \Omega=\phi_{o} .
$$

In some situations it is important to minimize the amount of sodium in the concrete ingredients in order to reduce exposures to individuals conducting maintenance on the accelerator. In fact, the phenomena described above has been noticed at accelerators and sometimes leads to "disappointment" in how little gamma-ray exposure rates are reduced when activated accelerator components are removed from enclosures with equally activated walls. For example, Armstrong and Barish (Ar69b) have calculated residual 


\section{CHAPTER 7 INDUCED RADIOACTIVITY IN ACCELERATOR COMPONENTS}

dose rates inside a cylindrical accelerator tunnel due to both the magnets and the concrete walls for $3.0 \mathrm{GeV}$ protons incident on iron. These authors also included some other reactions due to higher energy neutrons, such as spallation, that are capable of also producing ${ }^{24} \mathrm{Na}$ from common ingredients of concrete. The results are shown in Fig. 7.17 for the surface at the tunnel wall.

The discussion of the production of radioactivity continues in Chapter 8 with specific emphasis on environmental radiation protection.

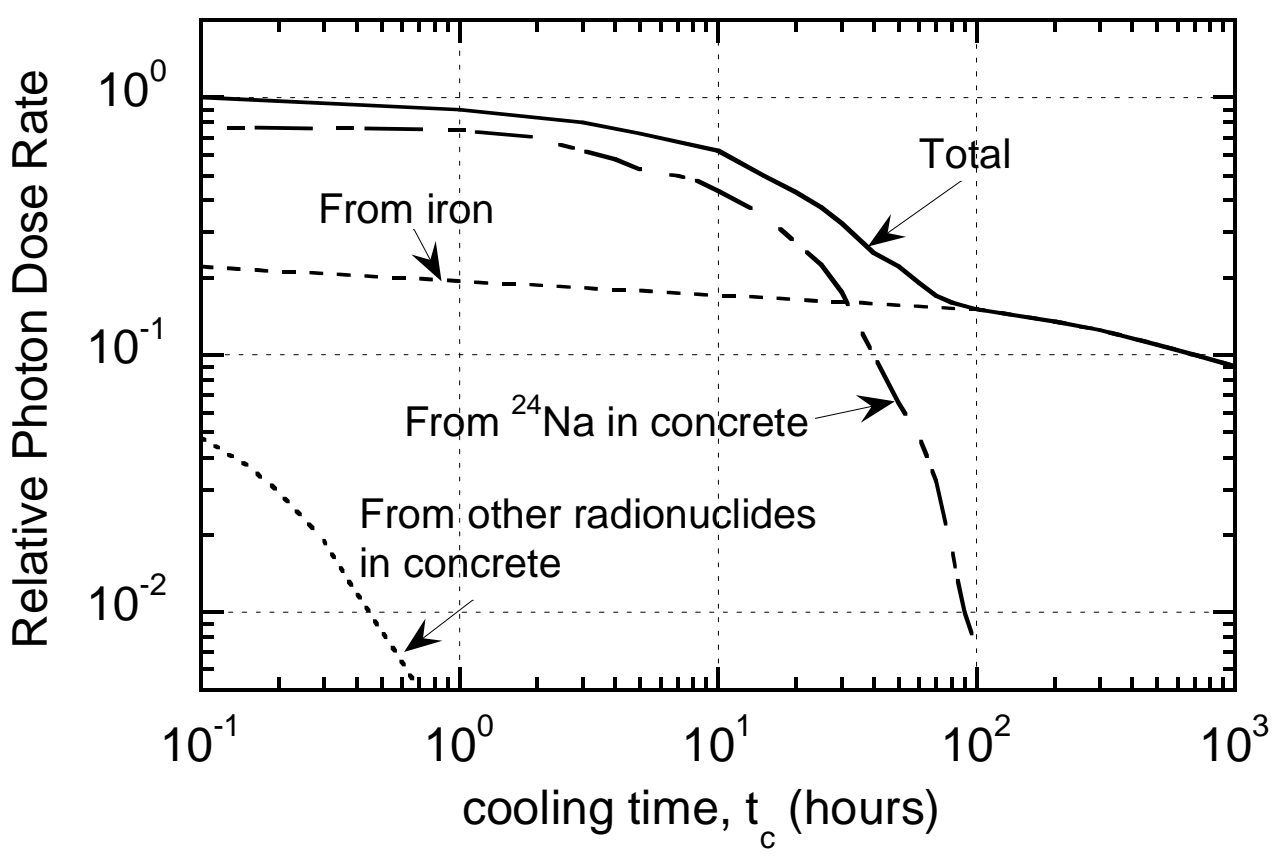

Fig. 7.17 Photon dose rate at surface of tunnel wall after infinite irradiation time for concrete containing $1.0 \%$ sodium by weight. [Adapted from (Ar69b).] 


\section{CHAPTER 7 INDUCED RADIOACTIVITY IN ACCELERATOR COMPONENTS}

\section{Problems}

1. A $1.0 \mathrm{~mA}$ beam of $30 \mathrm{MeV}$ electrons is absorbed by an aluminum target. Calculate the saturation activities of all major radionuclides produced in the target assuming sufficient kinetic energy to avoid threshold effects such as those illustrated in Fig. 3.5. Assuming no self-absorption and an infinitely long irradiation period, what will the absorbed dose rate at a distance of 2.0 meters away immediately after beam shutdown, and then 1.0 hour later? The target can be assumed to be a point source for this estimate.

2. A copper beam stop has been bombarded with high energy hadrons for 30 days and exhibits a dose rate of $100 \mathrm{mrem} \mathrm{hr}^{-1}$ at 1.0 meter away 1.0 day after the beam is turned off. Maintenance work needs to be scheduled in the vicinity within the next six months. Using both Gollon's Rule No. 3 and the Barbier Danger parameter curves, predict the cooling curve and determine when the dose rate is less than a $20 \mathrm{mrem} \mathrm{hr}^{-1}$ maintenance work criteria. Make a table of dose rate versus cooling time in days for both methods. How well do the two methods agree? (Hint: Use the initial value of the dose rate to scale values of $\mathbf{D}$.)

3. A $100 \mathrm{GeV}$ beam $\left(10^{12}\right.$ protons $\left.^{-1}\right)$ strikes the center of a large solid iron cylinder $30 \mathrm{~cm}$ in radius for 30 days. Use the star density curves from the Appendix B and the " $\omega$ " factors calculated by Gollon to estimate the residual dose rate after 1.0 day cooldown at contact with the side of the cylinder in the "hottest" spot. Using Gollon's Rule 3, how long must the repair crew wait to service this item in a contact radiation field of absorbed dose rate $<10 \mathrm{rad} \mathrm{hr}^{-1}$ ?

4. A copper target is bombarded with high energy protons such that 10 stars per incident proton are produced. If the incident beam is $10^{11}$ protons $\mathrm{s}^{-1}$, what is the specific activity (average) of ${ }^{54} \mathrm{Mn}$ that is produced after two years of operation? ${ }^{54} \mathrm{Mn}$ has a high energy spallation production cross section of about $20 \mathrm{mb}$ in $\mathrm{Cu}$. The target is a cylinder, $10 \mathrm{~cm}$ radius by $15 \mathrm{~cm}$ long. The half-life of ${ }^{54} \mathrm{Mn}$ is 312 days. Express the answer in both $\mathrm{Bq}^{-3}$ and $\mathrm{Ci} \mathrm{cm}^{-3}$. (Hint: This problem is best handled if the calculation is done at saturation and then corrected for the noninfinite irradiation time. Also, one needs to use the inelastic cross section, given for example, in Chapter 4.) 


\subsection{Introduction}

Chapter 7 provided the tools needed to address the subject of induced radioactivity. In this chapter, the discussion of induced radioactivity at accelerators goes on to address its production and propagation in environmental media such as air, soil, rock, and water. Aspects pertinent to both occupational radiation safety and environmental protection are covered. Also included is introductory material on meteorology and hydrogeology related to the propagation of this radioactivity.

\subsection{Airborne Radioactivity}

\section{$\underline{8.2 .1 \quad \text { Production }}$}

Thomas and Stevenson (Th88) and Swanson and Thomas (Sw90) reviewed the topic of the production of radioactivity in air. The principal source of radioactivity in air at accelerators results from the interaction of primary and secondary particles directly with the constituent target nuclei in the air in accelerator enclosures. Activated dust and gaseous emission from activated liquids are usually of much less importance. Table 8.1 gives the abundances and number densities of atoms $N_{j}$ of the most common stable nuclides found in the atmosphere using the elemental volumetric abundances of the U.S. Standard Atmosphere (Li00) with the isotopic abundances of Tuli (Tu05).

Table 8.1 Atomic number densities of the most prominent elements and stable nuclides in the atmosphere at sea level at STP $\left(273.15{ }^{\circ} \mathrm{K}, 760 \mathrm{~mm} \mathrm{Hg}\right)$

\begin{tabular}{|l|c|c|l|}
\hline $\begin{array}{l}\text { Element or } \\
\text { Isotope }\end{array}$ & $\begin{array}{l}\text { \% By } \\
\text { Volume }\end{array}$ & $\begin{array}{l}\text { \% Isotopic } \\
\text { Abundance (Tu05) }\end{array}$ & $\begin{array}{l}\boldsymbol{N}_{\boldsymbol{j}} \text { (Atoms } \\
\mathbf{c m}^{-3} \text { ) }\end{array}$ \\
\hline Nitrogen $\left(\mathbf{N}_{2}\right)$ & $\mathbf{7 8 . 0 8 4}$ & & $\mathbf{4 . 1 9 5 9 \times 1 0 ^ { 1 9 }}$ \\
\hline${ }^{14} \mathrm{~N}$ & & 99.636 & $4.1806 \times 10^{19}$ \\
\hline${ }^{15} \mathrm{~N}$ & & 0.364 & $1.5273 \times 10^{17}$ \\
\hline Oxygen $\left(\mathbf{O}_{2}\right)$ & $\mathbf{2 0 . 9 4 7 6}$ & & $\mathbf{1 . 1 2 5 6 \times 1 0 ^ { 1 9 }}$ \\
\hline${ }^{16} \mathrm{O}$ & & 99.757 & $1.1229 \times 10^{19}$ \\
\hline${ }^{17} \mathrm{O}$ & & 0.038 & $4.2774 \times 10^{15}$ \\
\hline${ }^{18} \mathrm{O}$ & & 0.205 & $2.3075 \times 10^{16}$ \\
\hline Argon $(\mathbf{A r})$ & $\mathbf{0 . 9 3 4}$ & & $\mathbf{2 . 5 0 9 4 \times 1 0 ^ { 1 7 }}$ \\
\hline${ }^{36} \mathrm{Ar}$ & & 0.3336 & $8.3715 \times 10^{14}$ \\
\hline${ }^{38} \mathrm{Ar}$ & & 0.0629 & $1.5784 \times 10^{14}$ \\
\hline${ }^{40} \mathrm{Ar}$ & & 99.6035 & $2.4995 \times 10^{17}$ \\
\hline
\end{tabular}

Patterson and Thomas (Pa73), have expanded the activation equation, Eq. (7.8), to obtain the total specific activity $S\left(\mathrm{~Bq} \mathrm{~cm}^{-3}\right)$ of an enclosed volume of radioactive air;

$S=C \sum_{i}\left\{\sum_{j} N_{j} \bar{\sigma}_{i j \gamma} \phi_{\gamma}+\sum_{j} N_{j} \bar{\sigma}_{i j T H} \phi_{T H}+\sum_{j} N_{j} \bar{\sigma}_{i j H E} \phi_{H E}\right\}\left\{1-\exp \left(-\lambda_{i} t_{i r r a d}\right)\right\} \exp \left(-\lambda_{i} t_{c}\right)$

where $\phi_{\gamma}, \phi_{T H}$, and $\phi_{H E}$, represent the average photon, thermal neutron, and high energy particle flux densities, respectively. To avoid confusion, in this equation $t_{\text {irrad }}$ is the irradiation time while $t_{c}$ represents the decay time. The $\bar{\sigma}_{i j k}$ values are the corresponding 


\section{Chapter 8 Induced RAdioactivity in EnVironmental Media}

cross sections averaged with the flux density over energy,

$$
\bar{\sigma}_{i j k}=\left[\int_{E_{\min }}^{E_{\max }} d E \sigma_{i j k}(E) \phi_{k}(E)\right] / \int_{E_{\min }}^{E_{\max }} d E \phi_{k}(E),
$$

where the limits of integration correspond to the three broad phenomenological energy domains in the summation. The constant $C$ is the conversion to specific activity, equal to

unity for activity in $\mathrm{Bq} \mathrm{cm}^{-3}$ if the units of length implicit in the quantities in Eq. (8.1) are in $\mathrm{cm}$. The outer sum over index $i$ includes all possible radionuclides produced and the sum over the index $j$ is over the parent nuclei found in air. The flux densities are, without further information, the average over some relevant spatial volume.

Table 8.2 lists the radionuclides commonly produced from the principle constituents in air along with the reaction mechanisms associated with their production and an estimate of the average production cross section. The large cross sections for $(n, \gamma)$ and $(n, p)$ reactions are for captures of neutrons of thermal energies $\left(\left\langle E_{n}\right\rangle \approx 0.025 \mathrm{eV}\right)$ while the remaining cross sections are generally the saturation cross sections found in the region above approximately a few 10 's of $\mathrm{MeV}$. The photon-induced reactions $(\gamma, \mathrm{n})$ are present at virtually all accelerators and at most energies. The corresponding cross sections will, of course, be energy-dependent especially at energies just above the reaction thresholds.

\subsubsection{Accounting for Ventilation}

Adjustments for the presence of ventilation can be quite conveniently made for a given radionuclide by using an effective decay constant $\lambda^{\prime}$ that includes the physical decay constant $\lambda$ along with a ventilation term $r$;

$$
\lambda^{\prime}=\lambda+r, \quad \text { where } r=\frac{D}{V},
$$

where $D$ is the ventilation rate in air volume per unit time and $V$ is the enclosure volume. Thus $r$ is the number of air changes per unit time. The applicable differential equation, an extension of Eq. (7.4) with ventilation included, is

$$
\frac{d n^{\prime}}{d t}=-\lambda n^{\prime}(t)-r n^{\prime}(t)+N \sigma \phi=-\lambda^{\prime} n^{\prime}(t)+N \sigma \phi .
$$

After an irradiation time, $t_{i}$, with no initial activation, the solution [see Eq. (7.5)] is

$$
n^{\prime}\left(t_{i}\right)=\frac{N \sigma \phi}{\lambda+r}\left\{1-\exp \left[-(\lambda+r) t_{i}\right]\right\}
$$

So the specific activity including ventilation mixing $a^{\prime}\left(t_{i}\right)$ is given by 


$$
a^{\prime}\left(t_{i}\right)=\lambda n^{\prime}\left(t_{i}\right)=\frac{\lambda N \sigma \phi}{\lambda+r}\left\{1-\exp \left[-(\lambda+r) t_{i}\right]\right\}
$$

But $N \sigma \phi$ is just the saturation concentration $a_{\text {sat }}$ without mixing [see Eq. (7.7)]. Hence, with ventilation mixing the saturation concentration $a_{s a t}^{\prime}$ is

$$
a_{\text {sat }}^{\prime}=\frac{\lambda a_{\text {sat }}}{\lambda+r} \text {. }
$$

Table 8.2 Radionuclides with half-lives greater than 1.0 minute that can be produced in air at accelerators. [Adapted from (Sw90), with values of half-lives

\begin{tabular}{|c|c|c|c|c|c|}
\hline Radionuclide & Half-life & Emission & $\begin{array}{c}\text { Parent } \\
\text { Element }\end{array}$ & $\begin{array}{l}\text { Production } \\
\text { Mechanism }\end{array}$ & $\begin{array}{l}\text { High Energy } \\
\text { Production Cross } \\
\text { Section (mb) }\end{array}$ \\
\hline${ }^{3} \mathrm{H}$ & $12.32 \mathrm{y}$ & $\beta^{-}$ & $\begin{array}{l}\mathrm{N} \\
\mathrm{O}\end{array}$ & $\begin{array}{l}\text { Spallation } \\
\text { Spallation }\end{array}$ & $\begin{array}{l}30 \\
30\end{array}$ \\
\hline${ }^{7} \mathrm{Be}$ & $53.22 \mathrm{~d}$ & $\gamma, \mathrm{e}^{-}$capture & $\begin{array}{c}\mathrm{N} \\
\mathrm{O} \\
\mathrm{Ar}\end{array}$ & $\begin{array}{l}\text { Spallation } \\
\text { Spallation } \\
\text { Spallation }\end{array}$ & $\begin{array}{l}10 \\
5.0 \\
0.6\end{array}$ \\
\hline${ }^{11} \mathrm{C}$ & $20.33 \mathrm{~min}$ & $\beta^{+}$ & $\begin{array}{l}\mathrm{N} \\
\mathrm{O} \\
\mathrm{Ar}\end{array}$ & $\begin{array}{l}\text { Spallation } \\
\text { Spallation } \\
\text { Spallation }\end{array}$ & $\begin{array}{c}10 \\
5 \\
0.7\end{array}$ \\
\hline${ }^{14} \mathrm{C}$ & $5700 y$ & $\beta^{-}$ & $\mathrm{N}$ & $\left(\mathrm{n}_{\text {thermal }}, \mathrm{p}\right)$ & 1640 \\
\hline${ }^{13} \mathrm{~N}$ & $9.96 \mathrm{~min}$ & $\beta^{+}$ & $\begin{array}{l}\mathrm{N} \\
\mathrm{N} \\
\mathrm{O} \\
\mathrm{Ar}\end{array}$ & $\begin{array}{l}\text { Spallation } \\
\quad(\gamma, \mathrm{n}) \\
\text { Spallation } \\
\text { Spallation }\end{array}$ & $\begin{array}{c}10 \\
10 \\
9 \\
0.8\end{array}$ \\
\hline${ }^{14} \mathrm{O}$ & $1.18 \mathrm{~min}$ & $\beta^{+}, \gamma$ & $\begin{array}{l}\mathrm{O} \\
\mathrm{Ar}\end{array}$ & $\begin{array}{l}\text { Spallation } \\
\text { Spallation }\end{array}$ & $\begin{array}{c}1.0 \\
0.06\end{array}$ \\
\hline${ }^{15} \mathrm{O}$ & $2.04 \mathrm{~min}$ & $\beta^{+}$ & $\begin{array}{c}\mathrm{O} \\
\mathrm{O} \\
\mathrm{Ar}\end{array}$ & $\begin{array}{c}\text { Spallation } \\
(\gamma, \mathrm{n}) \\
\text { Spallation }\end{array}$ & $\begin{array}{l}40 \\
10\end{array}$ \\
\hline${ }^{18} \mathrm{~F}$ & $1.83 \mathrm{~h}$ & $\beta^{+}$, & $\mathrm{Ar}$ & Spallation & 6.0 \\
\hline${ }^{24} \mathrm{Ne}$ & $3.38 \mathrm{~min}$ & $\beta^{-}, \gamma$ & $\mathrm{Ar}$ & Spallation & 0.12 \\
\hline${ }^{22} \mathrm{Na}$ & $2.603 \mathrm{y}$ & $\beta^{+}, \gamma$ & $\mathrm{Ar}$ & Spallation & 10 \\
\hline${ }^{24} \mathrm{Na}$ & $14.95 \mathrm{~h}$ & $\beta^{-}$ & $\mathrm{Ar}$ & Spallation & 7.0 \\
\hline${ }^{27} \mathrm{Mg}$ & $9.46 \mathrm{~min}$ & $\beta^{-}, \gamma$ & $\mathrm{Ar}$ & Spallation & 2.5 \\
\hline${ }^{28} \mathrm{Mg}$ & $20.92 \mathrm{~h}$ & $\beta^{-}, \gamma$ & $\mathrm{Ar}$ & Spallation & 0.4 \\
\hline${ }^{28} \mathrm{Al}$ & $2.24 \mathrm{~min}$ & $\beta^{-}, \gamma$ & $\mathrm{Ar}$ & Spallation & 13 \\
\hline${ }^{29} \mathrm{Al}$ & $6.56 \mathrm{~min}$ & $\beta^{-}, \gamma$ & $\mathrm{Ar}$ & Spallation & 4.0 \\
\hline${ }^{31} \mathrm{Si}$ & $2.62 \mathrm{~h}$ & $\beta^{-}, \gamma$ & $\mathrm{Ar}$ & Spallation & 6.0 \\
\hline${ }^{30} \mathrm{P}$ & $2.50 \mathrm{~min}$ & $\beta^{+}, \gamma$ & $\mathrm{Ar}$ & Spallation & 4.4 \\
\hline${ }^{32} \mathrm{P}$ & $14.26 \mathrm{~d}$ & $\beta^{-}$ & $\mathrm{Ar}$ & Spallation & 25 \\
\hline${ }^{33} \mathrm{P}$ & $25.34 \mathrm{~d}$ & $\beta^{-}$ & $\mathrm{Ar}$ & Spallation & 9 \\
\hline${ }^{35} \mathrm{~S}$ & $87.51 \mathrm{~d}$ & $\beta^{-}$ & $\mathrm{Ar}$ & Spallation & 23 \\
\hline${ }^{34 \mathrm{~m}} \mathrm{Cl}$ & $32.0 \mathrm{~min}$ & $\beta^{-}, \gamma$ & $\mathrm{Ar}$ & Spallation & 0.6 \\
\hline${ }^{38} \mathrm{Cl}$ & $37.24 \mathrm{~min}$ & $\beta^{-}, \gamma$ & $\mathrm{Ar}$ & $(\gamma, p n)$ & 4.0 \\
\hline${ }^{39} \mathrm{Cl}$ & $55.6 \mathrm{~min}$ & $\beta^{-}, \gamma$ & $\mathrm{Ar}$ & $(\gamma, p)$ & 7.0 \\
\hline${ }^{41} \mathrm{Ar}$ & $1.83 \mathrm{~h}$ & $\beta^{-}, \gamma$ & $\mathrm{Ar}$ & $\left(\mathrm{n}_{\text {thermal }}, \gamma\right)$ & 660 \\
\hline
\end{tabular}
from (Tu05).] 


\section{Chapter 8 Induced RAdioActivity in EnVironmental Media}

For reasons not related directly to radiation protection, particle beams at low energy accelerators must be contained in continuous vacuum systems. This serves to greatly minimize the activation of air at such facilities. In contrast, at high energy accelerators, it is quite common to have air gaps at certain interface points to accommodate devices associated with beam targetry or beamline diagnostics that render continuous vacuum impractical. Such air gaps are only found in external beam lines and possibly in linear accelerators. The beam in a circular accelerator or storage ring is, of necessity, contained in continuous vacuum since any air gaps, if traversed multiple times by the beam particles, would result in an unacceptable rate of beam loss by interaction with the air. At higher energy accelerators the large multiplicity of secondary particles produced as a part of cascade processes, either electromagnetic or hadronic, can produce airborne radioactivity external to the beamline vacuum.

If the accelerator enclosures were completely sealed, there would be no releases to the outside world and the hazard of these airborne radionuclides would be entirely restricted to those who might have to enter the enclosures. This would, however, allow the longerlived radionuclides to build up according to Eq. (8.1). Also, ventilation is generally needed to provide cooling of components and fresh breathing air for workers. Typically, the average residence time of air in accelerator enclosures is limited to a range of between approximately 30 minutes and not much longer than 1.0 hour $^{26}$. Thus, in equilibrium the airborne radionuclides in the accelerator environment will have half-lives only up to the order of one hour. The residence time of the air in conjunction with the cross sections determines the radionuclides of importance.

\subsubsection{Propagation of Airborne Radionuclides in the Environment}

The other consideration concerning airborne radioactivity is that associated with the dose delivered to members of the general public when radionuclides are released to the atmosphere external to the accelerator enclosure. The U. S. Environmental Protection Agency (EPA) has placed an annual limit of 10 mrem on dose equivalent, as defined by the 1973 Radiation Protection System, to members of the general public due to the operations of DOE facilities, comparable to limits applied to other facilities by U. S. and U. S. state regulations. EPA has also specified the methods for measuring such releases (CFR89). An annual dose equivalent of such small value is usually difficult or impossible to measure. Thus the standard practice is to measure the activity released and then use calculational models to estimate the maximum dose equivalent that actual members of the public could receive. The regulations prescribe the specific computer codes that must be used to perform these calculations through the use of a Gaussian plume model that combines input data on the release of radioactivity with meteorological information. The details of such computer modeling will not be described here. Examples of such plume models are given in textbooks, a short synopsis is given here.

\footnotetext{
${ }^{26}$ At some facilities releases of airborne radionuclides to the outdoors are minimized by greatly restricting the release rate of air during accelerator operations. When personnel accesses are made subsequent to operations, the ventilation rate must then be increased to levels consistent with good industrial hygiene practice.
} 


\section{Chapter 8 Induced RAdioActivity in EnVironMental Media}

\subsubsection{Propagation of Airborne Radioactivity-Tall Stacks}

Concentrations of radionuclides at distant locations can be estimated analytically using the so-called Sutton's equation. Due to the nature of accelerator operations, only "steady-state" conditions are considered since transient accidental releases from accelerators are unlikely. A good description that applies to rather tall $(>25 \mathrm{~m})$ release points, commonly called stacks, has been presented by Cember (Ce69). More details were provided by Slade (S168). The dispersion is mainly characterized by dilution of the radionuclides and their eventual return to ground level breathing zones. The meteorological conditions of major importance to this topic are illustrated in Fig. 8.1. Relevant stability classes have been developed that describe the various possibilities. Descriptions of these stability classes are given in Table 8.3.
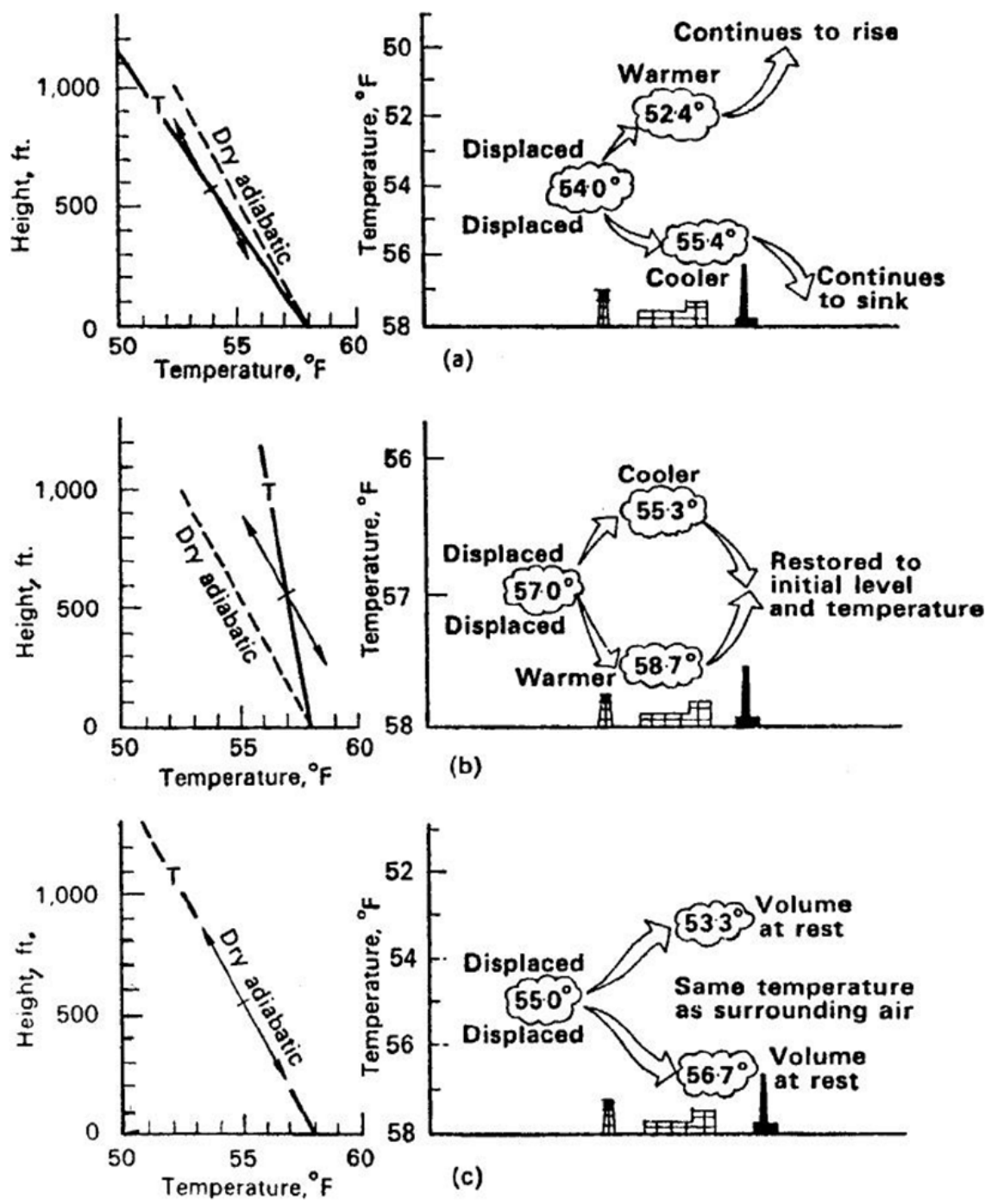

Fig. 8.1 Effect of atmospheric temperature gradient or lapse rate on a displaced volume of air for various conditions: a) Unstable lapse rate; b) Stable lapse rate; c) Neutral lapse rate [Reproduced from (S168).] 


\section{Chapter 8 IndUCEd RAdioACTIVITy in ENVIRONMENTAL MEdia}

Table 8.3 Atmospheric stability classes for use with Sutton's equation. [Adapted from (Sl68).]

\begin{tabular}{|l|l|}
\hline Superadiabatic & $\begin{array}{l}\text { If the rate of decrease of temperature with elevation is greater than that found in } \\
\text { adiabatic conditions, an unstable condition results which promotes the vertical } \\
\text { dispersion, and hence dilution. A rising parcel does not cool fast enough due to its } \\
\text { expansion and therefore remains warmer and continues to rise. Likewise, a falling } \\
\text { parcel continues to fall. }\end{array}$ \\
\hline Stable & $\begin{array}{l}\text { No heat is gained or lost by a parcel of air that rises and expands adiabatically with } \\
\text { falling temperature. The adiabatic cooling with rise normally corresponds to a gradient } \\
\text { of about } 1.0^{\circ} \mathrm{C} 100 \mathrm{~m}^{-1}\left(5.4{ }^{\circ} \mathrm{F} 1000 \mathrm{ft}^{-1}\right) \text { for dry air and about } 0.6^{\circ} \mathrm{C} 100 \mathrm{~m}^{-1}\left(3.5^{\circ} \mathrm{F}\right. \\
\left.1000 \mathrm{ft}^{-1}\right) \text { for moist air. If the atmospheric temperature gradient is less than adiabatic, } \\
\text { but still negative, stability is achieved because a rising parcel cools faster than its } \\
\text { surroundings and then tends to sink. A sinking parcel is warmer than its surroundings } \\
\text { and thus is less dense and tends to rise. This restricts the width of the plume and } \\
\text { decreases dilution. }\end{array}$ \\
\hline Inversion & $\begin{array}{l}\text { If the temperature gradient is such that the temperature increases with height, then an } \\
\text { inversion occurs. Rising effluent from a stack becomes much denser than its } \\
\text { surroundings and thus sinks. The effluent is thus more limited in its ascent and this } \\
\text { limits dilution. }\end{array}$ \\
\hline
\end{tabular}

Sutton's equation, as adapted by Cember (Ce69), for determining the concentration of a short-lived radionuclide released by a tall stack at ground level is

$$
\bar{c}(x, y)=\frac{2 Q}{\pi C^{2} \bar{u} x^{2-n}} \exp \left\{-\frac{\lambda}{\bar{u}} \sqrt{x^{2}+y^{2}}\right\} \exp \left\{-\frac{h^{2}+y^{2}}{C^{2} x^{2-n}}\right\}
$$

where the exponential involving the decay constant $\lambda$ conservatively allows for radioactive decay in transit for a particular radionuclide. The rest of the quantities are as follows:

- $(x, y, z)$ are Cartesian coordinates to the point of measurement from the foot of the stack (meters) where $x$ is along the centerline of the plume as determined by the wind direction (downwind), $y$ is the transverse coordinate, and $z$ is the vertical coordinate;

- $\bar{c}(x, y)$ is the average concentration (activity $\left.\mathrm{m}^{-3}\right)$ at ground level $(z=0)$;

- $Q$ is the emission rate of (activity $\mathrm{s}^{-1}$ );

- $\bar{u}$ is the mean wind speed $\left(\mathrm{m} \mathrm{s}^{-1}\right)$;

- $\quad C$ is the virtual diffusion constant in lateral and vertical directions (Table 8.4);

- $\quad n$ is a dimensionless parameter related to the atmospheric conditions (Table 8.4); and

- $\quad h$ is the effective chimney height (if the gas has significant emission velocity) determined from the actual chimney height $h_{a}$ by

$$
h=h_{a}+d\left(\frac{v}{\bar{u}}\right)^{1.4}\left(1+\frac{\Delta T}{T}\right)
$$

In Eq. (8.9) $d$ is the outlet diameter (meters), $v$ is the exit velocity of the gas (meters s${ }^{-1}$ ), and $\Delta T / T$ is the difference between the temperature of the gas and the ambient outdoor 


\section{Chapter 8 InduCED RAdioActivity in EnViRonMental MEdia}

temperature divided by the absolute temperature of the gas $T$. Table 8.4 gives values for certain parameters to be used in Eq. (8.8).

Table 8.4 Diffusion $\left(C^{2}\right)$ and Stability $(n)$ parameters for Cember's version of Sutton's Equation, Eq. (8.8). [Adapted from (Ce69).]

\begin{tabular}{|l|l|c|c|c|c|}
\hline Lapse Rate & $\boldsymbol{n}$ & \multicolumn{4}{|c|}{$\boldsymbol{C}^{\mathbf{2}}$} \\
& & \multicolumn{4}{|c|}{ Effective Chimney Height $\boldsymbol{h}$ (meters) } \\
& & $\mathbf{2 5}$ & $\mathbf{5 0}$ & $\mathbf{7 5}$ & $\mathbf{1 0 0}$ \\
\hline Superadiabatic & 0.20 & 0.043 & 0.030 & 0.024 & 0.015 \\
\hline Stable & 0.25 & 0.014 & 0.010 & 0.008 & 0.005 \\
\hline Moderate Inversion & 0.33 & 0.006 & 0.004 & 0.003 & 0.002 \\
\hline Large Inversion & 0.5 & 0.004 & 0.003 & 0.002 & 0.001 \\
\hline
\end{tabular}

\subsubsection{Propagation of Airborne Radioactivity-Short Stacks}

The above representation of Sutton's equation is a useful one where tall stacks are involved. However, at typical accelerator facilities it is uncommon for stacks to be very tall. Again for purposes of this discussion, only steady state conditions are treated here. For such calculations, the concentration as a function of coordinates $\bar{c}(x, y, z)$ defined as for the tall stacks, is given by a somewhat different formulation of Sutton's equation which uses the same coordinate system (S168);

$\bar{c}(x, y, z)=\frac{Q}{2 \pi \sigma_{y} \sigma_{z} \bar{u}}\left\{\exp \left[-\frac{\lambda}{\bar{u}} \sqrt{x^{2}+y^{2}}\right]\right\}\left\{\exp \left(-\frac{y^{2}}{2 \sigma_{y}^{2}}\right)\right\}\left\{\exp \left[-\frac{(z-h)^{2}}{2 \sigma_{z}^{2}}\right]+\exp \left[-\frac{(z+h)^{2}}{2 \sigma_{z}^{2}}\right]\right\}$.

For the common situation of interest where the receptor location of concern is at ground level $(z=0)$, this simplifies to

$$
\bar{c}(x, y, 0)=\frac{Q}{\pi \sigma_{y} \sigma_{z} \bar{u}}\left\{\exp \left[-\frac{\lambda}{\bar{u}} \sqrt{x^{2}+y^{2}}\right]\right\}\left\{\exp \left[-\left(\frac{y^{2}}{2 \sigma_{y}^{2}}+\frac{h^{2}}{2 \sigma_{z}^{2}}\right)\right]\right\}
$$

where the presence of the ground as a "barrier" to the air is taken into account. In these equations, the quantity $h$ is effective stack height above the ground in meters and $\sigma_{\mathrm{y}}(x)$ and $\sigma_{\mathrm{z}}(x)$, implicit functions of downwind coordinate $x$, are dispersion coefficients that have units of length (meters). All other quantities are the same as given above for tall stacks. These variables are, of course, determined from the meteorological conditions. In Eqs. (8.10) and (8.11), the exponential involving the decay constant $\lambda$ again conservatively allows for radioactive decay in transit for a particular radionuclide. Table 8.5 gives an alternative scheme for classifying the meteorological conditions. The classification may then be used with the curves in Figs. 8.2 and 8.3 to determine the values of $\sigma_{y}$ and $\sigma_{z}$ as a function of the coordinate $x$ for use in Eqs. (8.10) and (8.11). 


\section{Chapter 8 Induced RAdioActivity in EnVironMental Media}

Table 8.5 Relation of turbulence types to weather conditions. [Adapted from (Sl68).]

\begin{tabular}{|c|c|c|c|c|c|}
\hline \multicolumn{3}{|c|}{$\begin{array}{l}\text { A-Extremely unstable conditions } \\
\text { B-Moderately unstable conditions } \\
\text { C-Slightly unstable conditions }\end{array}$} & \multicolumn{3}{|c|}{$\begin{array}{l}\text { E-Slightly stable conditions } \\
\text { F-Moderately stable conditions }\end{array}$} \\
\hline \multirow{2}{*}{$\begin{array}{l}\text { Surface Wind } \\
\text { Speed } \\
\left(\mathrm{m} \mathrm{s}^{-1}\right)\end{array}$} & \multicolumn{3}{|c|}{ Daytime Insolation } & \multicolumn{2}{|c|}{ Nightime Conditions } \\
\hline & Strong & Moderate & Slight & $\begin{array}{l}\text { Thin overcast or } \\
\geq 4 / 8 \text { cloudiness }\end{array}$ & $\leq 3 / 8$ cloudiness \\
\hline$<2.0$ & $\mathrm{~A}$ & A-B & $\mathrm{B}$ & & \\
\hline 2.0 & A-B & $\mathrm{B}$ & $\mathrm{C}$ & $\mathrm{E}$ & $\mathrm{F}$ \\
\hline 4.0 & $\mathrm{~B}$ & $\mathrm{~B}-\mathrm{C}$ & $\mathrm{C}$ & $\mathrm{D}$ & $\mathrm{E}$ \\
\hline 6.0 & $\mathrm{C}$ & C-D & $\mathrm{D}$ & $\mathrm{D}$ & $\mathrm{D}$ \\
\hline$>6.0$ & $\mathrm{C}$ & $\mathrm{D}$ & $\mathrm{D}$ & $\mathrm{D}$ & $\mathrm{D}$ \\
\hline
\end{tabular}

${ }^{\mathrm{a}}$ Applicable to heavy overcast, day or night

${ }^{b}$ The degree of cloudiness is defined as that fraction of the sky above the local apparent horizon which is covered by clouds.

Airborne radioactivity releases can be minimized by

- limiting the ventilation rates during operations when people are not present in the enclosure (see footnote, this chapter),

- delaying the actual emissions by requiring long pathways to the ventilation "stacks", and

- minimizing air gaps in the beam.

\subsubsection{Radiation Protection Standards for Airborne Radioactivity}

The airborne radioactivity hazard must be assessed for workers exposed to airborne radionuclides at accelerators. Since the principal radionuclides are of relative short halflife, the hazard is commonly due to the "immersion" in a "cloud" of external dose rather than to a gaseous ingestion hazard such as is common in operations involving the processing of long-lived radioactive materials. The latter can lead to internal radiation exposures. Regulatory authorities, guided by recommendations of the International Commission on Radiation Protection (ICRP) have established Derived Air Concentrations (DACs) for radiation workers. These regulatory standards have replaced the Maximum Permissible Concentrations (MPCs) formerly employed for this purpose published by the ICRP (IC59) and NCRP (NC59). The value of 1.0 DAC is based upon the receipt of 5000 mrem $(50 \mathrm{mSv})$ of dose equivalent (CFR93) (1973 Radiation Protection System) or effective dose (CFR07) (1990 Radiation Protection System) by a person who is employed an entire working year $\left(\approx 2000 \mathrm{hrs}\right.$, or $\left.40 \mathrm{hrs} \mathrm{wk}^{-1} 52 \mathrm{wk} \mathrm{yr}^{-1}\right)$ breathing air at that concentration ${ }^{27}$. Similarly, values of Derived Concentration Standards (DCSs) have been issued (DOE11) that would result in the receipt of 100 mrem of effective dose (1990 Radiation Protection System) by a member of the public breathing air full time $\left(168 \mathrm{~h} \mathrm{wk}^{-1}\right)$ at $1.0 \mathrm{DCS}$ for one year. The DCS values replace the

\footnotetext{
${ }^{27}$ It should be noted that in some countries, DAC values are now referenced to an annual dose of 2000 mrem $(20 \mathrm{mSv})$ rather than $5000 \mathrm{mrem}(50 \mathrm{mSv})$ and thus have smaller values.
} 


\section{Chapter 8 InduCED RADIOACTIVITY IN ENVIRONMENTAL Media}

Derived Concentration Guides (DCGs) that correlated with the receipt of 100 mrem dose equivalent [1973 Radiation Protection System (DOE90)] by a member of the public who spends an entire year breathing such air full time.

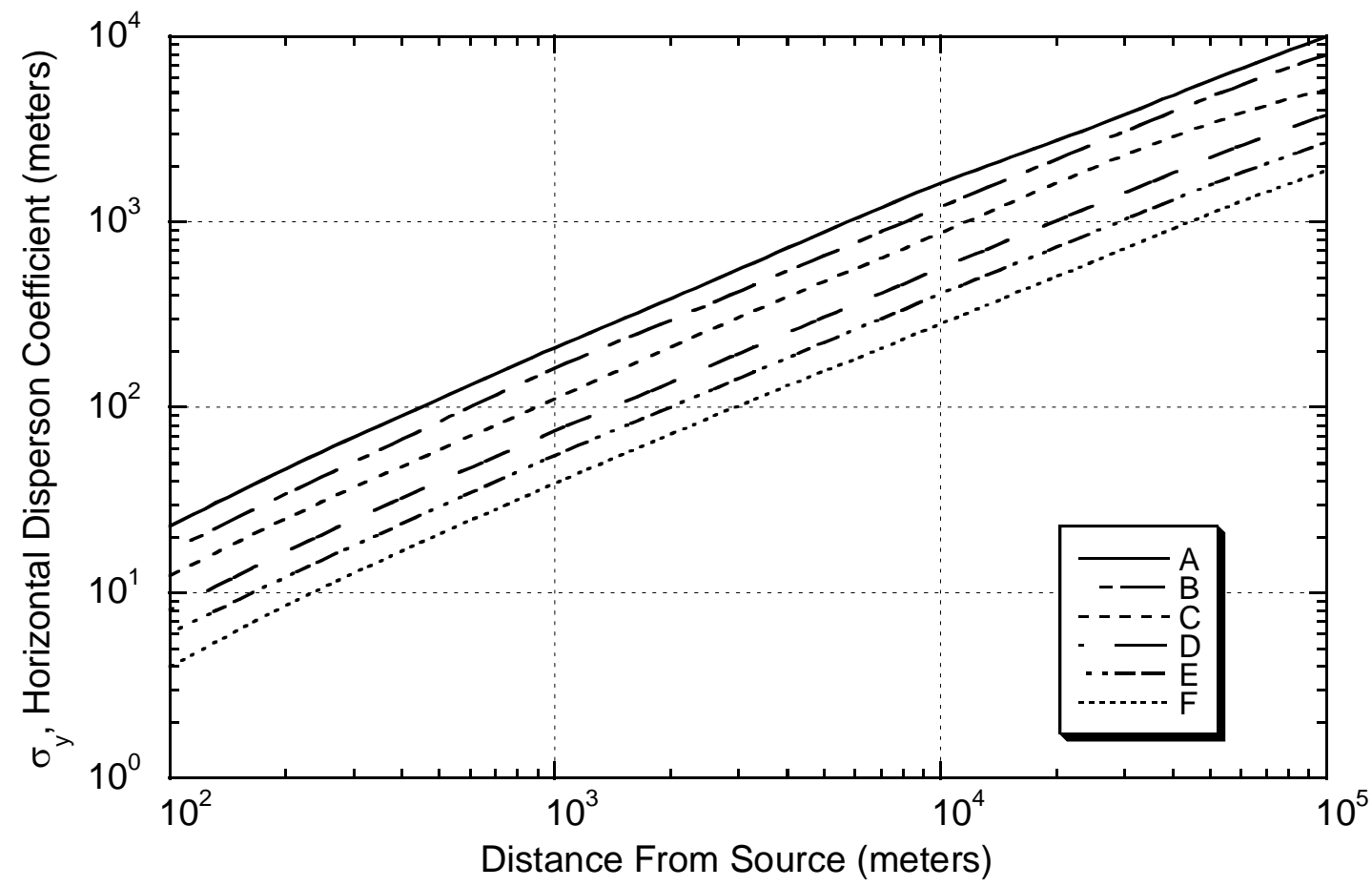

Fig. 8.2 Horizontal diffusion constants $\sigma_{y}$ as a function of downwind distance $x$ from the source for turbulence types defined in Table 8.5 for use in Eqs. (8.10) and (8.11) [Adapted from (S168).]

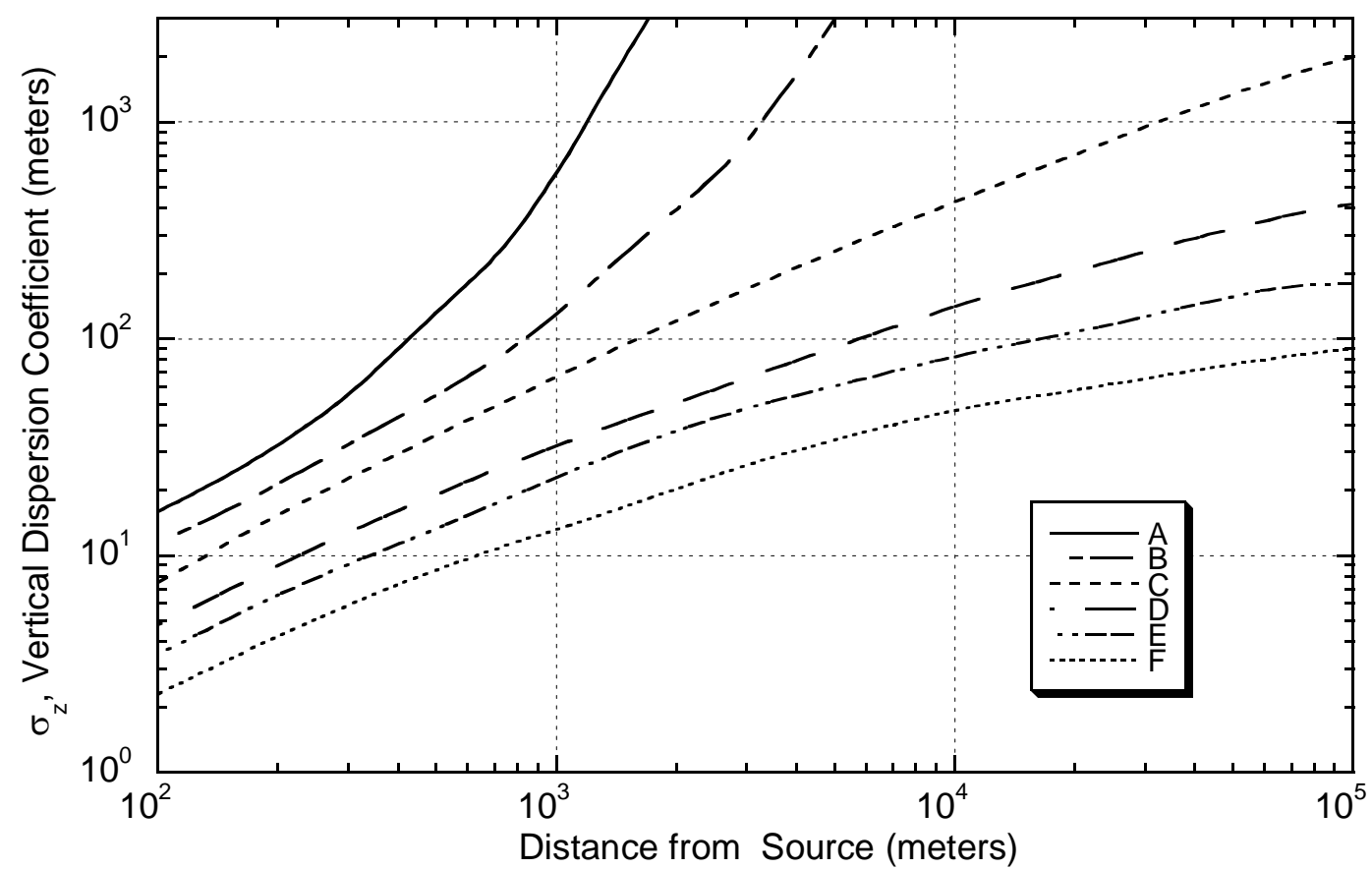

Fig. 8.3 Vertical diffusion constants $\sigma_{z}$ as a function of downwind distance $x$ from the source for turbulence types defined in Table 8.5 for use in Eqs. (8.10) and (8.11) [Adapted from (S168).] 


\section{Chapter 8 InduCEd RAdioactivity in EnVironmental Media}

Table 8.6 gives representative values of these circumstance-dependent maximum concentrations $C_{\max }$ for accelerator-produced radionuclides in air taken from U. S. Department of Energy requirements documents for workers [(CFR93),(CFR07)] and for members of the public [(DOE90), (DOE11)] for the superseded 1973 Radiation Protection System and the 1990 Radiation Protection System, respectively.

For some radionuclides commonly found at accelerators, DOE requirements documents give two sets of values under both Radiation Protection Systems, one for air inhaled into the lungs and the other for immersion in an infinite cloud of $\gamma$-emitting radionuclides. Immersion values are also usually the most limiting (i.e., smallest) ${ }^{28}$. An infinite cloud is appropriate for outdoor applications. However, for occupational exposures, the sizes of the "clouds" are not likely to be infinite but will be determined by the dimensions of the accelerator enclosures. Höfert calculated limiting concentrations for immersions clouds of various diameters (Hö69) using the methodology of (IC59). While Höfert's calculations are connected with the obsolete MPCs values, they remain of importance because they display the relative sensitivity to the dimensions of the immersion cloud. Table 8.6 gives Höfert's results for clouds of several radii.

Mixtures of radionuclides are commonly encountered. To account for the presence of multiple radionuclides, the set of individual radionuclide concentrations in the air $C_{i}$ must satisfy the following inequality:

$$
\sum_{i} \frac{C_{i}}{C_{\max , i}} \leq 1,
$$

where $C_{\max , i}$ is the regulatory standard for the $i^{\text {th }}$ radionuclide, dependent upon the circumstances of the exposure.

\section{$\underline{\text { 8.2.5 Production of Airborne Radionuclides at Electron Accelerators }}$}

At electron accelerators, significant air activation will not occur without bremsstrahlung because the nuclear cross sections of electrons are about two orders of magnitude smaller than those of photons (Sw79a) ${ }^{29}$. This airborne radioactivity is generally short-lived and the concentrations, as shall be seen in what follows, are usually quickly reduced to levels where the absorbed dose rates $\left(\operatorname{rads} \mathrm{h}^{-1}\right.$ ) are small compared to those due to the accelerator components. This result is because the radiation length of air is so much longer than that of any solid material (see Table 1.2).

\footnotetext{
${ }^{28}$ Some references and regulatory documents use the term submersion for this exposure scenario.1

29 The reverse is true for toxic gas production originating from chemical, rather than nuclear, transformations and whose reaction rate is closely proportional to the integral absorbed dose to the air. Such dose is generally higher if the primary electron beam does not strike a target to produce bremsstrahlung but rather is directly delivered to air. The production of such toxic gases, most notably ozone $\left(\mathrm{O}_{3}\right)$ is beyond the scope of this text but has been addressed by Swanson (Sw79a).
} 
Table 8.6 Airborne concentration limits for radiation workers and the general population. These represent maximum concentrations for radionuclide $i, C_{m a x, i}$, depending upon the circumstances of exposure (see text). ${ }^{30}$

\begin{tabular}{|c|c|c|c|c|c|c|c|c|c|c|c|c|}
\hline \multicolumn{13}{|c|}{ Radiation Worker Derived Air Concentrations (DACs) } \\
\hline & \multicolumn{4}{|c|}{$\begin{array}{c}\text { Inhaled Air Exposure } \\
{\left[50 \mathrm{mSv} \mathrm{y}^{-1}\left(40 \text { h }^{-1} \text { week }^{-1}\right)\right]}\end{array}$} & \multicolumn{8}{|c|}{$\begin{array}{c}\text { Immersion Exposure } \\
{\left[50 \mathrm{mSv} \mathrm{y}^{-1}\left(40 \text { h }^{-1} \text { week }^{-1}\right)\right]}\end{array}$} \\
\hline & \multirow{3}{*}{\multicolumn{2}{|c|}{$\begin{array}{l}\text { U. S. DOE } \\
1973 \text { System }^{\text {a }}\end{array}$}} & \multirow{3}{*}{\multicolumn{2}{|c|}{$\begin{array}{c}\text { U. S. DOE } \\
1990 \text { System }^{\text {b }}\end{array}$}} & \multirow{2}{*}{\multicolumn{4}{|c|}{ U. S. DOE Infinite Radius Cloud }} & \multicolumn{4}{|c|}{$\begin{array}{l}\text { Results of Höfert for } \\
\text { Immersion Clouds of } \\
\text { Selected Radiic }\end{array}$} \\
\hline & & & & & & & & & & $\mathbf{R}(\mathbf{n}$ & ters) & \\
\hline & & & & & \multicolumn{2}{|c|}{1973 System $^{\mathrm{a}}$} & \multicolumn{2}{|c|}{1990 System ${ }^{b}$} & 1.0 & 2.0 & 4.0 & $\infty$ \\
\hline & $\left(\mu \mathrm{Ci} \mathrm{m^{-3 } )}\right.$ & $\left(\mathrm{Bq} \mathrm{m}^{-3}\right)$ & $\left(\mu \mathrm{Ci} \mathrm{m} \mathbf{m}^{-3}\right)$ & $\left(\mathrm{Bq} \mathrm{m}^{-3}\right)$ & $\left(\mu \mathrm{Ci} \mathrm{m^{-3 } )}\right.$ & $\left(\mathrm{Bq} \mathrm{m}^{-3}\right)$ & $\left(\mu \mathrm{Ci} \mathrm{m^{-3 } )}\right.$ & $\left(\mathrm{Bq} \mathrm{m}^{-3}\right)$ & \multicolumn{4}{|c|}{$\left(\mu \mathbf{C i ~ m ^ { - 3 } )}\right.$} \\
\hline${ }^{3} \mathrm{H}$ & 20.0 & $7.4 \times 10^{5}$ & 20.0 & $7.4 \times 10^{5}$ & $* * *$ & $* * *$ & $* * *$ & $* * *$ & $* * *$ & $* * *$ & $* * *$ & *** \\
\hline${ }^{7} \mathrm{Be}$ & 8.0 & $3.0 \times 10^{5}$ & 10.0 & $3.7 \times 10^{5}$ & $* * *$ & $* * *$ & $* * *$ & $* * *$ & $* * *$ & $* * *$ & $* * *$ & $* * *$ \\
\hline${ }^{11} \mathrm{C}$ & 200 & $7.4 \times 10^{6}$ & 200 & $7.4 \times 10^{6}$ & 4.0 & $1.5 \times 10^{5}$ & 6.0 & $2.2 \times 10^{5}$ & 7.8 & 6.6 & 6.2 & 2.6 \\
\hline${ }^{13} \mathrm{~N}$ & $* * *$ & $* * *$ & $* * *$ & $* * *$ & 4.0 & $1.5 \times 10^{5}$ & 6.0 & $2.2 \times 10^{5}$ & 6.9 & 5.3 & 4.7 & 2.3 \\
\hline $15 \mathrm{O}$ & $* * *$ & $* * *$ & *** & $* * *$ & 4.0 & $1.5 \times 10^{5}$ & 6.0 & $2.2 \times 10^{5}$ & 6.4 & 4.3 & 3.5 & 2.0 \\
\hline${ }^{22} \mathrm{Na}$ & 0.3 & $1.1 \times 10^{4}$ & 0.2 & $7.4 \times 10^{3}$ & $* * *$ & *** & $* * *$ & $* * *$ & $* * *$ & & $* * *$ & $* * *$ \\
\hline${ }^{24} \mathrm{Na}$ & 2.0 & $7.4 \times 10^{4}$ & 0.4 & $1.5 \times 10^{4}$ & 0.9 & $3.3 \times 10^{4}$ & $* * *$ & $* * *$ & $* * *$ & & $* * *$ & $* * *$ \\
\hline${ }^{41} \mathrm{Ar}$ & *** & $* * *$ & $* * *$ & $* * *$ & 3.0 & $1.1 \times 10^{5}$ & 3.0 & $1.1 \times 10^{5}$ & 7.9 & 6.0 & 5.4 & 2.0 \\
\hline
\end{tabular}

\begin{tabular}{|c|c|c|c|c|c|c|c|c|}
\hline & \multicolumn{4}{|c|}{$\begin{array}{l}\text { U. S. DOE General Population Derived } \\
\text { Concentration Guides (DCGs) for Air } \\
1973 \text { System }^{\text {d }}\end{array}$} & \multicolumn{4}{|c|}{$\begin{array}{c}\text { U. S. DOE General Population Derived } \\
\text { Concentration Standards (DCSs) for Air } \\
1990 \text { System }^{\mathrm{e}}\end{array}$} \\
\hline & \multicolumn{2}{|c|}{$\begin{array}{c}\text { Inhaled Air Exposure } \\
{\left[1.0 \mathrm{mSv}^{2} \text { year }^{-1}\right.} \\
\left.\left(168 \mathrm{~h} \mathrm{week}^{-1}\right)\right]\end{array}$} & \multicolumn{2}{|c|}{$\begin{array}{c}\text { Immersion Exposure } \\
{\left[1.0 \mathrm{mSv}_{\text {year }}^{-1}\right.} \\
\left.\left(168 \mathrm{~h} \mathrm{week}^{-1}\right)\right]\end{array}$} & \multicolumn{2}{|c|}{$\begin{array}{c}\text { Inhaled Air Exposure } \\
{\left[1.0 \mathrm{mSv}_{\text {year }}^{-1}\right.} \\
\left.\left(168 \mathrm{~h}_{\text {week }}^{-1}\right)\right]\end{array}$} & \multicolumn{2}{|c|}{$\begin{array}{c}\text { Immersion Exposure } \\
{\left[1.0 \mathrm{mSv}_{\text {year }}^{-1}\right.} \\
\left.\left(168 \mathrm{~h}^{-1} \text { week }^{-1}\right)\right]\end{array}$} \\
\hline & $\left(\mu \mathrm{Ci} \mathrm{m^{-3 } )}\right.$ & $\left(\mathrm{Bq} \mathrm{m}^{-3}\right)$ & $\left(\mu \mathrm{Ci} \mathrm{m^{-3 } )}\right.$ & $\left(\mathrm{Bq} \mathrm{m}^{-3}\right)$ & $\left(\mu \mathrm{Ci} \mathrm{m^{-3 } )}\right.$ & $\left(\mathrm{Bq} \mathrm{m}^{-3}\right)$ & $\left.(\mu \mathrm{Ci} \mathrm{m})^{-3}\right)$ & $\left(\mathrm{Bq} \mathrm{m}^{-3}\right)$ \\
\hline${ }^{3} \mathrm{H}$ & 0.1 & $3.7 \times 10^{3}$ & $* * *$ & $* * *$ & 0.21 & $7.8 \times 10^{3}$ & $* * *$ & $* * *$ \\
\hline${ }^{7} \mathrm{Be}$ & 0.04 & $1.5 \times 10^{3}$ & *** & $* * *$ & 0.064 & $2.4 \times 10^{3}$ & $* * *$ & $* * *$ \\
\hline${ }^{11} \mathrm{C}$ & 1.0 & $3.7 \times 10^{4}$ & 0.02 & $7.4 \times 10^{2}$ & $* * *$ & $* * *$ & 0.019 & $7 \times 10^{2}$ \\
\hline${ }^{13} \mathrm{~N}$ & $* * *$ & $* * *$ & 0.02 & $7.4 \times 10^{2}$ & $* * *$ & $* * *$ & 0.019 & $7 \times 10^{2}$ \\
\hline${ }^{15} \mathrm{O}$ & $* * *$ & $* * *$ & 0.02 & $7.4 \times 10^{2}$ & $* * *$ & $* * *$ & 0.019 & $7 \times 10^{2}$ \\
\hline${ }^{22} \mathrm{Na}$ & 0.001 & 37.0 & $* * *$ & $* * *$ & $1.3 \times 10^{-4}$ & 4.8 & $* * *$ & $* * *$ \\
\hline${ }^{24} \mathrm{Na}$ & 0.01 & $3.7 \times 10^{2}$ & 0.004 & $1.5 \times 10^{2}$ & $7.0 \times 10^{-3}$ & $2.6 \times 10^{2}$ & $4.1 \times 10^{-3}$ & $1.5 \times 10^{2}$ \\
\hline${ }^{41} \mathrm{Ar}$ & $* * *$ & $* * *$ & 0.01 & $3.7 \times 10^{2}$ & $* * *$ & $* * *$ & 0.014 & $5.2 \times 10^{2}$ \\
\hline & & $\begin{array}{l}{ }^{\mathrm{a}} \text { Values t } \\
{ }^{\mathrm{b}} \text { Values ta } \\
{ }^{\mathrm{c}} \text { Values ta } \\
{ }^{\mathrm{d}} \text { Values ta } \\
{ }^{\mathrm{e}} \text { Values to }\end{array}$ & $\begin{array}{l}\text { om (CFR } \\
\text { om (CFR) } \\
\text { om (Hö69 } \\
\text { om (DOE } \\
\text { om (DOE }\end{array}$ & nended ir & 2011 & & & \\
\hline
\end{tabular}

${ }^{30}$ The values in Table 8.6 are, essentially, "worst case" values from the references cited. The user must take care to apply the specific values promulgated by the regulatory authority having jurisdiction. Furthermore, given the primacy of "customary" rather than SI units in U. S. regulations, the customary value is taken to be limiting quantity. Where choices were offered in types of exposure, the most restrictive value was taken. 


\section{CHAPTER 8 InduCED RAdioACTIVITy In ENVIRONMENTAL MEdiA}

Swanson (Sw79a) has calculated the saturation activities produced in air normalized to the electron beam power with the results provided in Table 8.7. The results of these calculations are normalized to unit path length and to beam power. To use them to determine the volume specific activity (e.g., $\mathrm{Ci} \mathrm{cm}^{-3}$ ) in a given accelerator enclosure, one must multiply the tabulated values by the available bremsstrahlung path length ${ }^{31}$ and divide by the enclosure volume. The results found in this table were calculated in a manner completely analogous to those given in Table 7.2 for other materials. For energies close to the threshold of an individual reaction, the rise of activity with beam energy $E_{o}$ (see Section 7.3.2 and Fig. 3.5) must be considered. ${ }^{41} \mathrm{Ar}$ is produced in the thermal neutron capture $(n, \gamma)$ reaction most copiously where there are high fluences of moderated neutrons present, typically near water-cooled targets and in concrete enclosures. ${ }^{3} \mathrm{H},{ }^{14} \mathrm{C}$, and ${ }^{7} \mathrm{Be}$ are too long-lived to be at levels anywhere near saturation and usually do not merit further consideration.

Table 8.7 Saturation activities per unit path length and per unit beam power produced in air by an electron beam normalized to the beam power. [Adapted from (Sw79a) with half-lives from (Tu05).]

\begin{tabular}{|c|c|c|c|c|c|c|}
\hline \multicolumn{2}{|c|}{$\begin{array}{c}\text { Produced } \\
\text { Radionuclide }\end{array}$} & \multicolumn{3}{|c|}{ Parent Stable Nuclide } & \multicolumn{2}{|c|}{$\begin{array}{l}\text { Saturation Activity per Unit } \\
\text { Length and Beam Power }\end{array}$} \\
\hline Nuclide & Half-life & Nuclide & $\begin{array}{l}\text { Reaction } \\
\text { Type }\end{array}$ & $\begin{array}{c}\text { Threshold } \\
\text { (MeV) }\end{array}$ & $\left(\mathrm{MBq} \mathrm{m}^{-1} \mathrm{~kW}^{-1}\right)$ & $\left(\mu \mathrm{Ci} \mathrm{m^{-1 }} \mathrm{kW}^{-1}\right)$ \\
\hline${ }^{3} \mathrm{H}$ & $12.32 \mathrm{y}$ & ${ }^{14} \mathrm{~N}$ & $\left(\gamma,{ }^{3} \mathrm{H}\right)$ & 22.7 & \multirow[b]{2}{*}{$(5.2)$} & \multirow[b]{2}{*}{ (140) } \\
\hline & & ${ }^{16} \mathrm{O}$ & $\left(\gamma,{ }^{3} \mathrm{H}\right)$ & 25.0 & & \\
\hline${ }^{7} \mathrm{Be}$ & 53.24 & ${ }^{14} \mathrm{~N}$ & $(\gamma, \mathrm{sp})^{\mathrm{b}}$ & 27.8 & \multirow[b]{2}{*}{$(1.1)$} & \multirow[b]{2}{*}{ (30) } \\
\hline & & ${ }^{16} \mathrm{O}$ & $(\gamma, \mathrm{sp})^{\mathrm{b}}$ & 31.9 & & \\
\hline${ }^{11} \mathrm{C}$ & $20.33 \mathrm{~min}$ & ${ }^{12} \mathrm{C}$ & $(\gamma, n)$ & 18.7 & \multirow[b]{3}{*}{ (11) } & \multirow[b]{3}{*}{$(300)$} \\
\hline & & ${ }^{14} \mathrm{~N}$ & $(\gamma, \mathrm{sp})^{\mathrm{b}}$ & 22.7 & & \\
\hline & & ${ }^{16} \mathrm{O}$ & $(\gamma, \mathrm{sp})^{\mathrm{b}}$ & 25.9 & & \\
\hline${ }^{13} \mathrm{~N}$ & $9.96 \mathrm{~min}$ & ${ }^{14} \mathrm{~N}$ & $(\gamma, n)$ & 10.6 & 520 & $1.4 \times 10^{4}$ \\
\hline${ }^{15} \mathrm{O}$ & $2.04 \mathrm{~min}$ & ${ }^{16} \mathrm{O}$ & $(\gamma, n)$ & 15.7 & 56 & $1.5 \times 10^{3}$ \\
\hline${ }^{16} \mathrm{~N}$ & $7.13 \mathrm{~s}$ & ${ }^{18} \mathrm{O}$ & $(\gamma, \mathrm{np})$ & 21.8 & $(0.02)$ & $(0.5)$ \\
\hline${ }^{38} \mathrm{Cl}$ & $37.24 \mathrm{~min}$ & ${ }^{40} \mathrm{Ar}$ & $(\gamma, \mathrm{np})$ & 20.6 & 0.22 & 6 \\
\hline${ }^{39} \mathrm{Cl}$ & $56.2 \mathrm{~min}$ & ${ }^{40} \mathrm{Ar}$ & $(\gamma, p)$ & 12.5 & 1.5 & 40 \\
\hline${ }^{41} \mathrm{Ar}$ & $1.83 \mathrm{~h}$ & ${ }^{40} \mathrm{Ar}$ & $(\mathrm{n}, \gamma)^{\mathrm{c}}$ & - & variable & variable \\
\hline
\end{tabular}

${ }^{a}$ Normalized per bremsstrahlung pathlength in air $(\mathrm{m})$ and electron beam power $(\mathrm{kW})$ incident on a high$\mathrm{Z}$ target, summed over individual contributing reactions. Values in parentheses are rough estimates.

${ }^{\mathrm{b}}$ Spallation reaction

${ }^{\mathrm{c}}$ Thermal neutron capture reaction that where high neutron fluences are moderated by water or concrete shielding.

After calculating the production rates, one can then apply the general methodology presented in this chapter determine the concentrations within the accelerator enclosure and to estimate the effective dose equivalent rates at offsite locations as well as the status of compliance with applicable regulations.

\footnotetext{
31 This path length would either be set by the physical dimensions of the room or, for a large room, by the attenuation length of the bremsstrahlung radiation in air.
} 


\section{CHAPTER 8 INDUCED RADIOACTIVITy IN ENVIRONMENTAL MEDiA}

\subsubsection{Production of Airborne Radionuclides at Proton Accelerators}

At proton accelerators, the excitation functions of the possible nuclear reactions listed in Table 8.2 exemplified by those shown in Fig. 7.9 become important. In general, the positron emitters ${ }^{11} \mathrm{C},{ }^{13} \mathrm{~N}$, and ${ }^{15} \mathrm{O}$ along with ${ }^{41} \mathrm{Ar}$ (produced by thermal neutron capture), are the nuclides most frequently seen. Work at Fermilab described by Butala et al. (Bu89) and Vaziri et al. [(Va93), (Va96)] has also confirmed these identifications and, additionally, detected ${ }^{38} \mathrm{Cl}$ and ${ }^{39} \mathrm{Cl}$. The determination of the relative contributions of the various positron emitters present must principally be done by fitting measured decay curves with a sum of exponential functions, each term of which represents one of the possible radionuclides present. This is a result of the fact that their $\gamma$-ray spectra are all dominated by $0.511 \mathrm{MeV}$ photons from positron annihilation. The results of analyses of such decay curves have been discussed in various references [(Th88), (Sw90), (Bu89), (Va93), and (Va96)]. In addition, the production of ${ }^{3} \mathrm{H}$ in the molecular form HTO and its impact should be evaluated.

It was concluded by Butala et al. that the geometry of target stations significantly can affect the composition (Bu89). For example, high intensity targets immediately surrounded with large volumes of iron and concrete (in contact with the iron) produced much less ${ }^{41} \mathrm{Ar}$ than did other targets where the bulk iron shield was located in a open room with a layer of air between the iron and the concrete. Presumably, the open space provided opportunity for the large flux of low energy neutrons expected external to a pure iron shield (see Section 6.3.5) to "thermalize" and thus enhance the production of ${ }^{41} \mathrm{Ar}$ in the air space. The large cross section for the ${ }^{40} \operatorname{Ar}(n, \gamma){ }^{41} \mathrm{Ar}$ reaction at thermal neutron energies $\left(\sigma_{t h}=660 \mathrm{mb}\right)$ also may possibly have provided the photons necessary to enhance the $(\gamma, \mathrm{p})$ and $(\gamma, \mathrm{pn})$ reactions required to produce significant quantities of ${ }^{39} \mathrm{Cl}$ and ${ }^{38} \mathrm{Cl}$, respectively. Some typical percentages of the various radionuclides, by activity concentration, released from high energy proton accelerators are given in Table 8.8.

Table 8.8 Examples of measured radionuclide compositions of typical airborne releases at proton accelerators.

\begin{tabular}{|l|c|c|c|c|c|c|}
\hline \multirow{2}{*}{ Situation } & \multicolumn{7}{|c|}{ Radionuclides (Activity \%) } \\
\cline { 2 - 7 } & ${ }^{\mathbf{1 1}} \mathbf{C}$ & ${ }^{\mathbf{1 3}} \mathbf{N}$ & ${ }^{\mathbf{1 5}} \mathbf{O}$ & ${ }^{\mathbf{3 8}} \mathbf{C l}$ & ${ }^{\mathbf{3 9}} \mathbf{C l}$ & ${ }^{\mathbf{4 1}} \mathbf{A r}$ \\
\hline CERN (Th88) 28 GeV protons & 31.0 & 47.0 & 8.0 & & & 14.0 \\
\hline Fermilab (Bu89) 800 GeV protons & & & & & & \\
\hline (no gap between iron and concrete walls) & 46.0 & 19.0 & 35.0 & & & \\
\hline$\quad$ gap between iron and concrete walls) & 42.0 & 14.0 & 0.0 & 0.0 & 10.0 & 34.0 \\
\hline Fermilab (Va93) 120 GeV protons & 58.5 & 37.9 & & 1.0 & 1.1 & 1.5 \\
\hline Fermilab (Va96) 120 GeV protons & 64.6 & 30.5 & & & & 5.0 \\
\hline
\end{tabular}

After calculating the production rates, one can then apply the general methodology presented in this chapter to estimate the effective dose equivalent rates as well as the status of compliance with applicable regulations 


\section{ChAPTER 8 InduCED RAdioActivity in EnViRonMental Media}

\subsection{Water and Geological Media Activation}

At accelerators appropriate measures need to be taken to assure protection of groundwater resources from contamination with radionuclides. Radioactivity can be produced in soil or rock and in the water it contains. Radioactivity produced in water can also be a matter of concern for occupational workers. In practice, it is not always a simple matter to separate these two areas of concern. One can, in principle, initiate calculations of water activation at accelerators by starting from "first principles" using Eq. (7.8).

\subsubsection{Water Activation at Electron Accelerators}

As before, questions of radioactivation are generally less complex at electron accelerators. As was done for atmospheric activation, Swanson (Sw79a) has provided the results of calculations to address the production of radionuclides in water at electron accelerators. Such activation will principally occur in water used to cool magnets and beam absorbers. The water become radioactive waste. The results are, again, in the form of saturation activities normalized to the electron beam power absorbed in the water volume. These are given for infinite irradiation periods with no time allowed for decay. The results are given in Table 8.9 and include specific gamma-ray constants $\Gamma_{i}$ useful for calculating absorbed dose rates near point sources. From these results, it is clear that, aside from short-lived positron emitters, only ${ }^{3} \mathrm{H}$ and ${ }^{7} \mathrm{Be}$ are of importance. Activity concentrations can be obtained by assuming rapid mixing of the saturated activity in the available volume of water. Table 8.9 gives the results due to interactions with the ${ }^{16} \mathrm{O}$ found in water. In principle ${ }^{3} \mathrm{H}$ could be produced from the hydrogen in water by means of two sequential thermal neutron capture reactions; ${ }^{1} \mathrm{H}(\mathrm{n}, \gamma)^{2} \mathrm{H}$ followed by ${ }^{2} \mathrm{H}(\mathrm{n}, \gamma)^{3} \mathrm{H}$. However this two-step process is of limited importance since the cross section for the first reaction is 0.33 barn while that for the second is 0.52 millibarn.

In practice, due to the compactness of the shielding at electron accelerators compared with that found at proton and ion accelerators, soil activation is generally negligible except perhaps in the vicinity of beam absorbers.

Table 8.9 Saturation activities per unit beam power produced in ${ }^{16} \mathrm{O}$ by an electron beam normalized to the beam power. [Adapted from (Sw79a) with half-lives from (Tu05).]

\begin{tabular}{|c|c|c|c|c|c|c|c|}
\hline \multicolumn{2}{|c|}{$\begin{array}{c}\text { Produced } \\
\text { Radionuclide }\end{array}$} & \multicolumn{2}{|c|}{$\begin{array}{c}\text { Reaction } \\
\text { Parameters }\end{array}$} & \multicolumn{2}{|c|}{$\begin{array}{c}\text { Specific Gamma Ray } \\
\text { Constant } \Gamma\end{array}$} & \multicolumn{2}{|c|}{$\begin{array}{c}\text { Saturation Activity } \\
\text { per Unit Beam Power }\end{array}$} \\
\hline & Half-life & Reaction & $\begin{array}{c}\text { Threshold } \\
\text { (MeV) }\end{array}$ & $\begin{array}{c}{\left[\left(\mathrm{mGy} \mathrm{h}^{-1}\right)\right.} \\
\left.\mathbf{x}\left(\mathrm{GBq} \mathrm{m}^{-2}\right)^{-1}\right]\end{array}$ & $\begin{array}{l}{\left[\left(\operatorname{rad~h}^{-1}\right) x\right.} \\
\left.\left(\mathrm{Ci} \mathrm{m}^{-2}\right)^{-1}\right]\end{array}$ & $\left(\mathrm{GBq}_{\mathrm{kW}} \mathrm{K}^{-1}\right)$ & $\left(\mathrm{Ci} \mathrm{kW}^{-1}\right)$ \\
\hline${ }^{3} \mathrm{H}^{\mathrm{a}}$ & $12.32 \mathrm{y}$ & $\left(\gamma,{ }^{3} \mathrm{H}\right)$ & 25.0 & - & - & 7.4 & 0.2 \\
\hline${ }^{7} \mathrm{Be}$ & $53.24 \mathrm{~d}$ & $(\gamma, 5 \mathrm{n} 4 \mathrm{p})$ & 31.9 & 0.008 & 0.03 & 1.5 & 0.04 \\
\hline${ }^{10} \mathrm{C}$ & $19.31 \mathrm{~s}$ & $(\gamma, 4 \mathrm{n} 2 \mathrm{p})$ & 38.1 & 0.29 & 1.06 & 3.7 & 0.1 \\
\hline${ }^{11} \mathrm{C}$ & $20.33 \mathrm{~min}$ & $(\gamma, 3 \mathrm{n} 2 \mathrm{p})$ & 25.9 & 0.17 & 0.62 & 14.8 & 0.4 \\
\hline${ }^{13} \mathrm{~N}$ & $9.96 \mathrm{~min}$ & $(\gamma, 2 \mathrm{np})$ & 25.0 & 0.17 & 0.62 & 3.7 & 0.1 \\
\hline${ }^{14} \mathrm{O}$ & $1.18 \mathrm{~min}$ & $(\gamma, 2 n)$ & 28.9 & 0.45 & 1.7 & 3.7 & 0.1 \\
\hline${ }^{15} \mathrm{O}$ & $2.04 \mathrm{~min}$ & $(\gamma, n)$ & 15.7 & 0.17 & 0.62 & 330 & 9 \\
\hline
\end{tabular}

${ }^{\mathrm{a}}$ Does not present an external radiation hazard. 


\section{Chapter 8 InduCEd RAdioactivity in EnVironmental Media}

\subsubsection{Water and Geological Media Activation at Proton Accelerators}

\subsubsection{Water Activation at Proton Accelerators}

At proton and ion accelerators, as with electron accelerators, radioactivity can be produced directly in water as a result of both proton and neutron interactions. Values for some of the relevant cross sections were given in Chapter 7. Equipped with knowledge of the beam energy and information about the energy spectra of neutrons that are present, one can proceed to calculate the activity produced. In general, the most important radionuclides, as is the situation with electron accelerators, result from the interactions of the hadrons with the oxygen present in the water. As before, the production of ${ }^{3} \mathrm{H}$ from the hydrogen present in the water is possible, but is rendered to be insignificant due to the small cross sections of both of the thermal neutron capture reactions required to occur sequentially. For such calculations, the production of ${ }^{3} \mathrm{H}$ in water from target atoms other than hydrogen is of special importance. Konobeyev and Korovin (Ko93) have developed a method of globally fitting the existing cross section data on the production of ${ }^{3} \mathrm{H}$ due to neutron interactions with a variety of target elements found in soils with the results shown in Fig. 8.4. The results for protons are similar.

\subsubsection{Geological Media Activation}

While calculating the production of radionuclides in soil, and in the water it contains, directly from known cross sections has an appeal due to its simplicity, in practice such calculations have been done more frequently by analyzing data obtained using irradiated samples. The work of Borak et al. (Bo72) is of singular importance in this regard. Borak et al. measured the radioactivity produced in soil by high energy hadrons by radiochemical analysis of soil samples irradiated near high energy synchrotrons; the 12 $\mathrm{GeV}$ Argonne ZGS and the $28 \mathrm{GeV}$ Brookhaven AGS. The radionuclides ${ }^{3} \mathrm{H},{ }^{7} \mathrm{Be},{ }^{22} \mathrm{Na}$, ${ }^{45} \mathrm{Ca},{ }^{46} \mathrm{Sc},{ }^{48} \mathrm{~V},{ }^{51} \mathrm{Cr},{ }^{54} \mathrm{Mn},{ }^{55} \mathrm{Fe},{ }^{59} \mathrm{Fe}$, and ${ }^{60} \mathrm{Co}$ were identified. Experiments were then performed to determine which radionuclides, and what fractions of them, could be leached by water. This study determined macroscopic production cross sections and ion velocities relative to ground water flow in soil. Of these nuclides only ${ }^{3} \mathrm{H},{ }^{22} \mathrm{Na},{ }^{45} \mathrm{Ca}$, and ${ }^{54} \mathrm{Mn}$ were observed in leach waters. The ${ }^{3} \mathrm{H}$ was assumed to be all leachable and was measured by driving it out of the sample by baking. Radionuclides with half-lives exceeding 15 days were the only ones considered. The results were based upon the elemental composition of soil given in Table 8.10.

Borak et al. measured specific activities at saturation $A_{i}\left(\mathrm{~Bq} \mathrm{~g}^{-1}\right)$ which are related to the microscopic cross sections by means of the following equation:

$$
A_{i}=\phi \sum_{j} n_{j} \sigma_{i j}
$$




\section{Chapter 8 InduCEd RAdioactivity in EnVironmental Media}

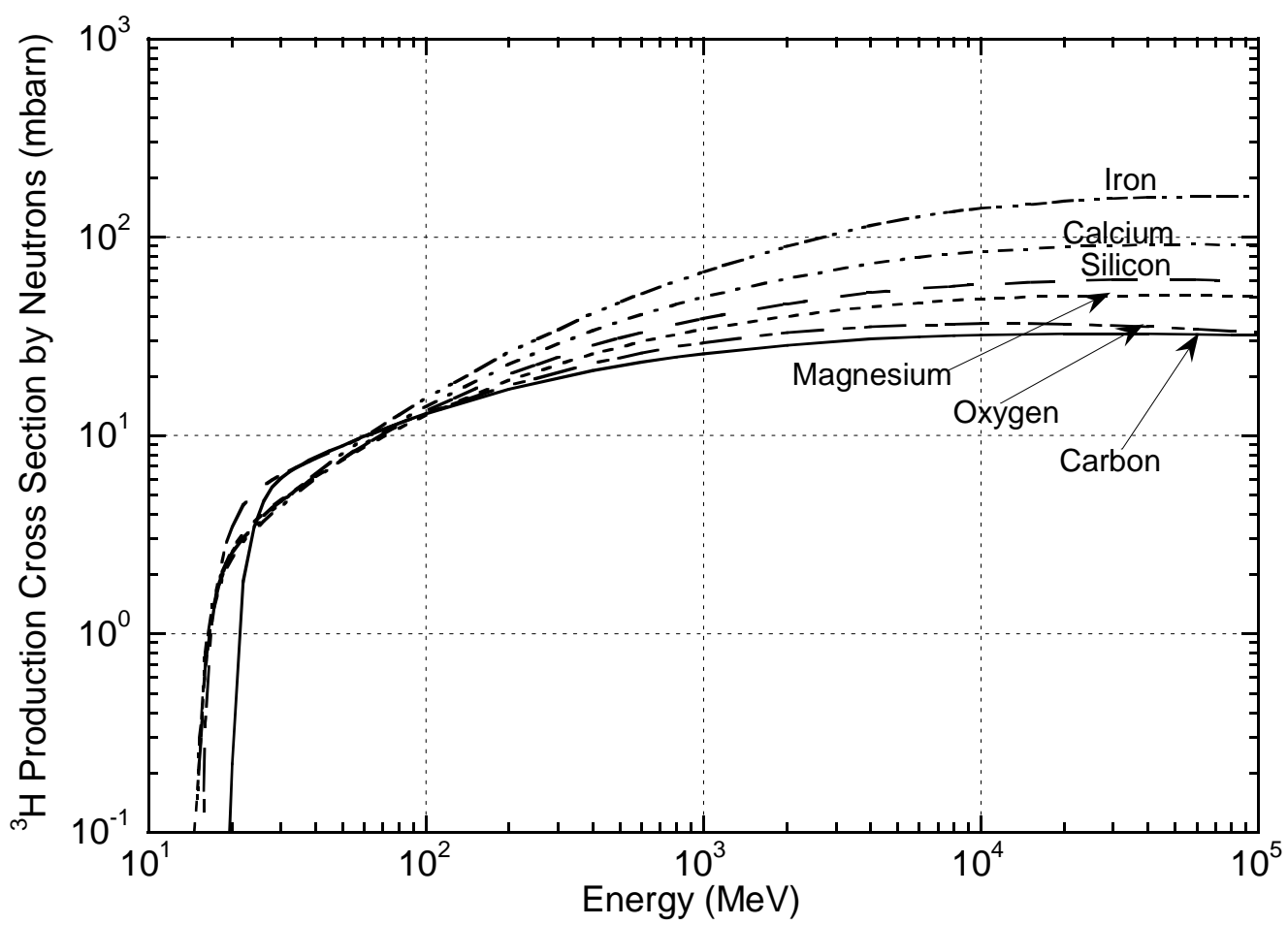

Fig. 8.4 Cross sections for the production of ${ }^{3} \mathrm{H}$ due to neutron bombardment of materials commonly found in soil and rock as a function of neutron energy. The calculations have been performed following the method of Konobeyev and Korovin (Ko93). Results for aluminum are quite similar to those found for silicon and the results for sodium are quite similar to those found for magnesium.

Table 8.10 Composition of soils typical of the Fermilab site.

[Adapted from (Bo72).]

\begin{tabular}{|l|c|c|}
\hline \multicolumn{3}{|c|}{$\begin{array}{c}\text { Elemental Composition of Soil } \\
\text { Element } \\
\text { Z, Atomic Number }\end{array}$} \\
\hline \% by Weight \\
\hline Silicon & 14 & 14.47 \\
\hline Iluminum & 13 & 2.44 \\
\hline Calcium & 26 & 1.11 \\
\hline Magnesium & 20 & 7 \\
\hline Carbon & 12 & 3.79 \\
\hline Sodium & 6 & 5.12 \\
\hline Potassium & 11 & 0.34 \\
\hline Oxygen & 19 & 0.814 \\
\hline
\end{tabular}

${ }^{\mathrm{a}}$ The mean moisture percentage was $13.15 \pm 4.45 \%$ and the mean $\mathrm{pH}$ was $7.6 \pm 0.1$.

where $\phi$ is the flux density $\left(\mathrm{cm}^{-2} \mathrm{~s}^{-1}\right), n_{j}$ is the number density of target nuclei of the $j^{t h}$ nuclide $\left(\mathrm{g}^{-1}\right)$ of the soil sample, and $\sigma_{i j}\left(\mathrm{~cm}^{2}\right)$ is the effective cross section for the transformation from target nucleus $j$ to radionuclide $i$. The summation in Eq. (8.13) is taken over the soil constituents. Borak et al. were able to directly measure these 


\section{ChAPTER 8 InduCED RAdioActivity in EnViRonMental Media}

summations; the total macroscopic cross sections summed over the soil constituents for each radionuclides of interest. Table 8.11 gives the results of the measurements of the macroscopic cross sections, denoted $\Sigma\left(\mathrm{cm}^{2} \mathrm{~g}^{-1}\right)$, for each of the radionuclides identified in the various types of soils analyzed.

Table 8.11 Macroscopic cross section for soil normalized to unit flux of hadrons with kinetic energies greater than $30 \mathrm{MeV}$. [Adapted from (Bo72).]

\begin{tabular}{|c|c|c|c|c|}
\hline Nuclide & $\begin{array}{l}\text { Glacial Till } \\
\Sigma\left(\mathrm{cm}^{2} \mathrm{~g}^{-1}\right)\end{array}$ & $\begin{array}{c}\text { Gray Sandy Clay } \\
\sum\left(\mathrm{cm}^{2} \mathrm{~g}^{-1}\right)\end{array}$ & $\begin{array}{c}\text { Red Sandy Clay } \\
\sum\left(\mathrm{cm}^{2} \mathrm{~g}^{-1}\right)\end{array}$ & $\begin{array}{c}\text { Gray Clay } \\
\Sigma\left(\mathrm{cm}^{2} \mathrm{~g}^{-1}\right)\end{array}$ \\
\hline${ }^{7} \mathrm{Be}$ & $2.9 \times 10^{-4}$ & $3.7 \times 10^{-4}$ & $3.2 \times 10^{-4}$ & $2.7 \times 10^{-4}$ \\
\hline${ }^{51} \mathrm{Cr}$ & $1.7 \times 10^{-5}$ & $3.7 \times 10^{-5}$ & $2.8 \times 10^{-5}$ & $3.1 \times 10^{-5}$ \\
\hline${ }^{22} \mathrm{Na}$ & $2.1 \times 10^{-4}$ & $2.3 \times 10^{-4}$ & $2.0 \times 10^{-4}$ & $1.6 \times 10^{-4}$ \\
\hline${ }^{54} \mathrm{Mn}$ & $5.9 \times 10^{-5}$ & $4.1 \times 10^{-5}$ & $3.5 \times 10^{-5}$ & $3.7 \times 10^{-5}$ \\
\hline${ }^{46} \mathrm{Sc}$ & $3.0 \times 10^{-5}$ & $1.3 \times 10^{-5}$ & $9.6 \times 10^{-6}$ & $1.1 \times 10^{-5}$ \\
\hline${ }^{48} \mathrm{~V}$ & $4.1 \times 10^{-6}$ & $1.1 \times 10^{-5}$ & $6.7 \times 10^{-6}$ & $7.4 \times 10^{-6}$ \\
\hline${ }^{55} \mathrm{Fe}$ & $9.3 \times 10^{-5}$ & $1.2 \times 10^{-4}$ & $7.0 \times 10^{-5}$ & $2.1 \times 10^{-4}$ \\
\hline${ }^{59} \mathrm{Fe}$ & $3.2 \times 10^{-6}$ & $1.7 \times 10^{-6}$ & $1.3 \times 10^{-6}$ & $1.6 \times 10^{-6}$ \\
\hline${ }^{60} \mathrm{Co}$ & $3.3 \times 10^{-5}$ & $1.4 \times 10^{-5}$ & $1.1 \times 10^{-5}$ & $1.3 \times 10^{-5}$ \\
\hline${ }^{45} \mathrm{Ca}$ & $1.6 \times 10^{-4}$ & $2.0 \times 10^{-5}$ & $3.0 \times 10^{-5}$ & $1.6 \times 10^{-5}$ \\
\hline${ }^{3} \mathrm{H}$ & $8.2 \times 10^{-4}$ & $1.1 \times 10^{-3}$ & $3.3 \times 10^{-4}$ & $5.2 \times 10^{-4}$ \\
\hline${ }^{3} \mathrm{H}^{*}$ & $5.9 \times 10^{-3}$ & $5.9 \times 10^{-3}$ & $4.1 \times 10^{-3}$ & $4.4 \times 10^{-3}$ \\
\hline
\end{tabular}

*Cross sections per gram of water in soil.

Borak et al. also obtained data related to the leachabilities of the various elements from the soils studied. Leachability measures the ability of water to remove a given radionuclide from the soil material. It is not related to nuclear properties but rather is related to chemical properties and processes. A qualitative summary of the results of Borak et al. as follows:

- ${ }^{3} \mathrm{H}$ : The leaching process was able to collect all the tritium as measured by a bake-out process. The average value of the macroscopic cross section in soil was found to be $5.1 \times 10^{-3} \mathrm{~cm}^{2} \mathrm{~g}^{-1}$ of water. An important conclusion is that the tritium will migrate with the same velocity as any other water in the soil.

- $22 \mathrm{Na}$ : Typically $10-20 \%$ of this nuclide was found to be leachable. On average, it appeared that the migration velocity of this nuclide is approximately $40 \%$ of that of water through the soil due to ion exchange processes.

- ${ }^{45} \mathrm{Ca}$ : At most 5\% of this nuclide was leached from the soil. The migration velocity was determined to be extremely small.

- ${ }^{54} \mathrm{Mn}$ : At most $2 \%$ of this nuclide was leached from the soil. It was determined that this nuclide will not migrate significant distances.

Thus, based upon leachability considerations, ${ }^{3} \mathrm{H}$ and ${ }^{22} \mathrm{Na}$ are the most important leachable radionuclides that can be produced in environmental media such as soil. 


\section{Chapter 8 Induced RAdioactivity in EnVironmental Media}

One can thus calculate the quantities of radionuclides that might pose a risk to groundwater in the environs of an accelerator. This can be done by using the cross sections directly, or as demonstrated by Gollon (Go78) for high energy protons, by performing, for example, Monte Carlo calculations in which the total stars (i.e. total inelastic nuclear interactions above some threshold) produced in some volume of earth shielding is determined. ${ }^{32}$ As in Eq. (7.35), the total number of atoms $K_{i}$ of the $i^{\text {th }}$ nuclide that can be produced per star in that same volume is given by

$$
K_{i}=\frac{\sum_{i}}{\sum_{i n}}
$$

where $\Sigma_{i}$ is, as above, the macroscopic cross section $\left(\mathrm{cm}^{2} \mathrm{~g}^{-1}\right)$ for the $i^{\text {th }}$ radionuclide and $\Sigma_{i n}$ is the total macroscopic inelastic cross section $\left(\mathrm{cm}^{2} \mathrm{~g}^{-1}\right)$ for soil. Gollon inferred a value of $\Sigma_{i n}=1.1 \times 10^{-2} \mathrm{~cm}^{2} \mathrm{~g}^{-1}$ for typical Fermilab soil from the results of Borak et al.

Gollon used the following values of $K_{i}$ for ${ }^{3} \mathrm{H}$ and ${ }^{22} \mathrm{Na}$, respectively, as selected from Borak's paper for soils peculiar to Fermilab (glacial till):

$$
\begin{aligned}
& K_{3}=\frac{8.2 \times 10^{-4}}{1.1 \times 10^{-2}}=0.075, \text { and } \\
& K_{22}=\frac{2.1 \times 10^{-4}}{1.1 \times 10^{-2}}=0.020 .
\end{aligned}
$$

One can then calculate the total number of atoms of radionuclides produced during some time interval in some volume by simply multiplying these factors by the number of stars (or inelastic interactions) in the same volume. The number of atoms then can be converted to activity using the decay constant. The above values of $K_{i}$ are applicable to soils such as those found at Fermilab. For other soil compositions one may need to use cross sections for producing the radionuclides of interest in various target elements and integrate over the energy spectrum of incident hadrons. Fig. 8.5 gives cross sections for producing ${ }^{22} \mathrm{Na}$ by interactions of hadrons with the various elements comprising soil due to Van Ginneken (Va71). This figure is a companion to Fig 8.4.

\section{$\underline{8.3 .3 \quad \text { Regulatory Standards }}$}

The quantity of ultimate concern, of course, is the resultant concentration in water. The water could be an actual or potential drinking water resource that might well be subject to specific regulatory requirements. The regulations may differ between different governing

\footnotetext{
32 Some Monte Carlo codes of more recent development can now calculate these quantities directly from the energy-dependent production cross sections. However, given the limited energy dependence at high energies, working with the total stars remains worthwhile as a means to achieve results rapidly, or as a "quality check" on the more complex computations.
} 


\section{Chapter 8 Induced RAdioActivity in EnVironmental Media}

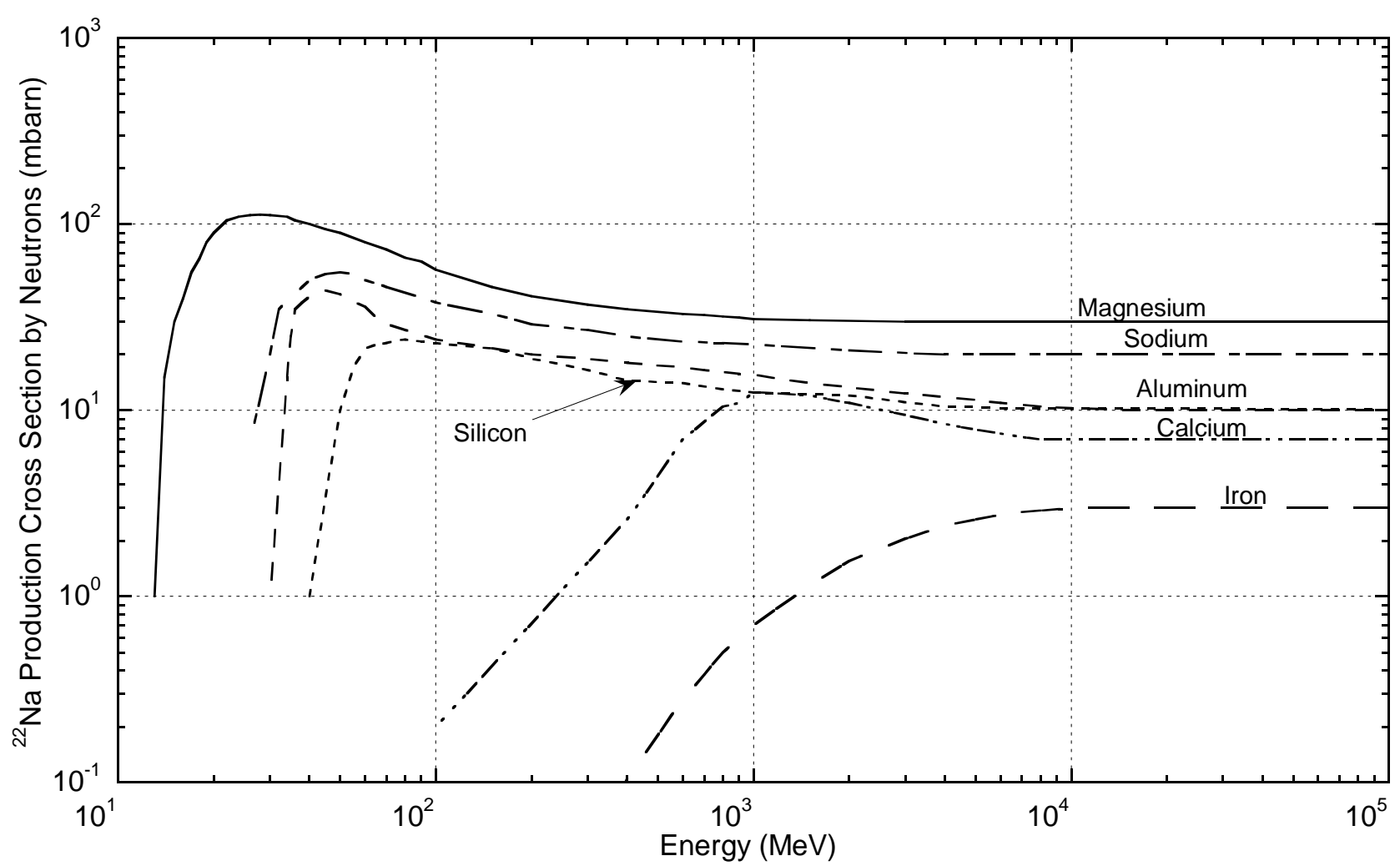

Fig. 8.5 Cross sections for the production of ${ }^{22} \mathrm{Na}$ due to neutron bombardment of materials commonly found in soil and rock as a function of neutron energy. Results for potassium are quite similar to those found for calcium. [Adapted from (Va71).]

jurisdictions. The requirements, generally not developed for application to the operations of particle accelerators, need to be understood by facility management personnel. The standards generally differ for drinking water supplies and surface water discharges. The allowable concentrations for surface waters may be larger due to the likelihood that such discharges will most certainly be diluted significantly prior to the consumption by individuals. However, in some jurisdictions this may not be the case. For public drinking water supplies, the U. S. Environmental Protection Agency [(CFR76), reaffirmed in (CFR00)] limits such concentrations to those that would produce an annual dose equivalent of $4.0 \mathrm{mrem}(40 \mu \mathrm{Sv})$ and specifically gives a limit of $20 \mathrm{pCi} \mathrm{cm}{ }^{-3}$ for tritium based on the methodologies established in 1959 [(IC59), (NC59)]. An explicit limit for ${ }^{22} \mathrm{Na}$ is not specified by USEPA. For surface water discharges, the U. S. Department of Energy (DOE11) specified Derived Concentration Standards (DCSs), values of concentrations which would result in members of the public each receiving $100 \mathrm{mrem}$ in a year should they use such water for their household needs. Using the DOE DCSs one obtains a value of $76 \mathrm{pCi} \mathrm{cm}{ }^{-3}$ for ${ }^{3} \mathrm{H}$ and $0.4 \mathrm{pCi} \mathrm{cm}^{-3}$ for ${ }^{22} \mathrm{Na}$ in drinking water to correspond to an annual effective dose of 4.0 mrem. However, EPA's explicit limit for ${ }^{3} \mathrm{H}$ in drinking water is considered as legally preeminent. Table 8.12 lists the concentration limits $C_{\max , i}$. 


\section{Chapter 8 Induced RAdioActivity in EnVironMental Media}

For purposes of this discussion, surface water discharges include those to streams, ponds, etc. while drinking water standards apply to water that could potentially end up in a source of drinking water such as a public, or even private, well. Local jurisdictions can, and in some cases have, applied drinking water standards to all discharges.

Table 8.12 Concentration Limits for ${ }^{3} \mathrm{H}$ and ${ }^{22} \mathrm{Na}$ in surface water discharges and in drinking water. [Half-lives from (Tu05).]

\begin{tabular}{|c|c|c|c|}
\hline \multirow{2}{*}{ Radionuclide } & Half-Life (years) & \multicolumn{2}{|c|}{ Concentration Limit $\boldsymbol{C}_{\mathbf{m a x}, \boldsymbol{i}}\left(\mathbf{p C i} \mathbf{~ c m}^{\mathbf{- 3}}\right)$} \\
\cline { 3 - 4 } & & Surface Water & Drinking Water \\
\hline${ }^{3} \mathrm{H}$ & 12.32 & $1900^{\mathrm{a}}$ & $20^{\mathrm{b}}$ \\
\hline${ }^{22} \mathrm{Na}$ & 2.603 & $10^{\mathrm{a}}$ & $0.4^{\mathrm{a}}$ \\
\hline
\end{tabular}

${ }^{\mathrm{a}}$ Value taken from (DOE11)

${ }^{\mathrm{b}}$ Value taken from [(CFR76), (CFR00)]. A value of $76 \mathrm{pCi} \mathrm{cm}^{-3}$ is implied by (DOE11).

In exact analogy with the situation found with airborne radioactivity (Eq. 8.12), to account for the presence of multiple radionuclides, the set of radionuclide concentrations in the water $C_{i}$ must satisfy the following inequality, where $C_{\max , i}$ is the regulatory standard for the $i^{\text {th }}$ radionuclide for the particular circumstances of exposure:

$$
\sum_{i} \frac{C_{i}}{C_{\max , i}} \leq 1
$$

\subsubsection{The Propagation of Radionuclides Through Geological Media}

The methods for calculating these concentrations in actual environmental media will vary with the regulatory authority and the "conservatism" of the institution. The most conservative assumption is to assume that saturation concentration values of production are reached. This is equivalent to assuming that the accelerator will operate "forever" in a static configuration and that the water in its vicinity never moves. This assumption is an extremely unrealistic one as it is questionable that the "motionless" water in such a medium actually comprises a potential source of useable drinking water. For an irradiation over a finite period of time, the activity concentration $C_{i}$ of radionuclide $i$ in leaching water under such conditions can be calculated by means of following formula:

$$
C_{i}=\frac{N_{p} K_{i} L_{i} S_{\text {ave }}}{1.17 \times 10^{6} \rho w_{i}}\left\{1-\exp \left(-t_{\text {irrad }} / \tau_{i}\right)\right\} \exp \left(-t_{c} / \tau_{i}\right)\left(\mathrm{pCi} \mathrm{cm}^{-3}\right),
$$

where,

$N_{p}$ is the number if incident particles delivered per year,

$K_{i}$ is as above,

$L_{i}$ is the fraction of the radionuclide of interest that is leachable,

$S_{a v e}$ is the average star density $\left(\right.$ stars $\mathrm{cm}^{-3}$ ) in the volume of interest per incident particle,

$\rho$ is the density of the medium $\left(\mathrm{g} \mathrm{cm}^{-3}\right)$, 


\section{CHAPTER 8 INDUCED RADIOACTIVITY IN ENVIRONMENTAL MEDia}

$w_{i}$ is the mass (grams) of water per unit mass (grams) of medium required to leach some specified fraction of the leachable radioactivity and is thus linked to the value of $L_{i}$,

$t_{\text {irrad }}$ is the irradiation time,

$t_{c}$ is the "cooling" time once the irradiation is suspended, and

$\tau_{i}$ is the mean-life of the $i^{\text {th }}$ radionuclide.

The constant in the denominator contains the unit conversions needed to yield results in pCi $\mathrm{cm}^{-3}$. For a given medium, the ratio $L_{i} / w_{i}$ should be determined by measurements specific to the local media. An important quantity is the effective porosity $p$ that represents the volume fraction of the material that is available to water movement. It is given by $^{33}$

$$
p=\rho w_{i}
$$

The effective porosity is essentially equal to the pore volume of the material for soils. For consolidated materials (i.e., rock) it does not include sealed pores through which movement does not occur. This provides a means by which "worst case" estimates may be made. For realistic estimates water movement must be taken into account.

At Fermilab, a simple model allowing for some movement and further dilution of water was employed for many years (Go78). In this single resident model, so-named for reasons soon to be obvious, the vertical migration of water was, conservatively, assumed to be $2.2 \mathrm{~m} \mathrm{yr}^{-1}$. In the standard clays present at Fermilab, this velocity is likely conservative (i.e., large) by at least an order of magnitude. Its use crudely allowed for the presence of cracks and fissures through which more rapid propagation of water might be possible. The tritium vertical velocity was taken to have this value while the results of Borak et al. (Bo72) were used to obtain a value of about $1.0 \mathrm{~m} \mathrm{yr}^{-1}$ for ${ }^{22} \mathrm{Na}$. Only the leachable fraction of the ${ }^{22} \mathrm{Na}$ is included. The procedure then allowed for decay during the downward migration of the total inventory of radionuclides produced in one year, integrated over the entire volume of the irradiated material, to the highest aquifer below the location of the irradiation. At that point, it was assumed that the radionuclides were rapidly transported horizontally to a shallow well where it was presumed that the flow of water collecting the radionuclides is entirely used by a single user who consumes a volume of 150 liters day ${ }^{-1}$. This value, a minimal one, was taken from results achieved by municipalities that have needed to ration public water consumption during conditions of severe drought. Thus the annual production transported vertically with radioactive decay included, was diluted into the $5.5 \times 10^{7} \mathrm{~cm}^{3} \mathrm{yr}^{-1}$ that this represents. This simple model is generally conservative but it does, in fact, neglect that fact that the water movement may not be uniform from year-to-year. It also did not take advantage of the fact that the radionuclides are initially distributed over a considerable volume as they are produced.

It is clear that better methods are warranted and a better model has been developed for use at Fermilab (Ma93). The concentration model now in use at Fermilab calculates the

${ }^{33}$ Unit analysis of Eq. (8.18): $p=\rho w_{i} \rightarrow \frac{\mathrm{gm}(\text { rock })}{\mathrm{cm}^{3}(\text { rock })} \frac{\mathrm{gm}(\text { water })}{\mathrm{gm}(\text { rock })}=\frac{\mathrm{gm}(\text { water })}{\mathrm{cm}^{3}(\text { rock })}=\frac{\mathrm{cm}^{3}(\text { water })}{\mathrm{cm}^{3}(\text { rock })}$ 


\section{ChAPTER 8 InduCED RAdioActivity in EnViRonMental Media}

production of the radionuclides of concern in accordance with Eq. (8.17). Variations of this approach in use elsewhere. The result provides an initial concentration that is available for further migration, decay, and dilution. The concentration subsequent to migration is then calculated by using up-to-date modeling techniques to calculate the reduction in the concentration due to dilution, diffusion, and radioactive decay. At the point of concern, usually the location of an aquifer producing water suitable for consumption as a supply of drinking water, the concentrations calculated are then substituted into Eq. (8.16) in order to determine if a shielding design is adequate.

To do these calculations properly requires a detailed knowledge of the media involved. Some principles will be given here but many details are left to the references [(Fe88), (Ba98), and (An07)]. In situations where a definite potential gradient, often called the hydraulic gradient $d h / d x$ is applied to water in a medium, the rate of flow is said to be advective. Under such conditions and in situations where only one dimensional coordinate is important, the average linear velocity (or seepage velocity) $v$ is given by the application of Darcy's Law as (Fe88);

$$
v=\frac{K}{p} \frac{d h}{d x}
$$

where the effective porosity $p$ is defined as above. More complicated situations involving two and three dimensions are addressable using the mathematical language of vector calculus. The derivative is the gradient of the hydraulic head in the material. $K$ in this equation represents the hydraulic conductivity. This quantity is a function of the material and its moisture content. All of the factors in this equation can, and generally should, be determined empirically for the medium and location under consideration. Typical values of $K$ are given in Table 8.13 and have been given by Batu (Ba98).

Table 8.13 Examples of typical values of hydraulic conductivity. [Adapted from (Ba98).]

\begin{tabular}{|c|c|c|}
\hline Group & Porous Materials & $\begin{array}{c}\text { Range of } K \text { values } \\
\left(\mathrm{cm} \mathrm{s}^{-1}\right)\end{array}$ \\
\hline \multirow[t]{3}{*}{ Igneous Rocks } & Weathered granite & $(3.3-52) \times 10^{-4}$ \\
\hline & Weathered gabbro & $(0.5-3.8) \times 10^{-4}$ \\
\hline & Basalt & $(0.2-4250) \times 10^{-6}$ \\
\hline \multirow[t]{10}{*}{ Sedimentary Materials } & Sandstone (fine) & $(0.5-2250) \times 10^{-6}$ \\
\hline & Siltstone & $(0.1-142) \times 10^{-8}$ \\
\hline & Sand (fine) & $(0.2-189) \times 10^{-4}$ \\
\hline & Sand (medium) & $(0.9-567) \times 10^{-4}$ \\
\hline & Sand (coarse) & $(0.9-6610) \times 10^{-4}$ \\
\hline & Limestone and dolomite & $(0.4-2000) \times 10^{-7}$ \\
\hline & Karst limestone & $(1-20000) \times 10^{-4}$ \\
\hline & Gravel & $(0.3-31.2) \times 10^{-1}$ \\
\hline & Silt & $(0.09-7090) \times 10^{-7}$ \\
\hline & Clay & $0.1-47) \times 10^{-8}$ \\
\hline Metamorphic Rocks & Schist & $(0.002-1130) \times 10^{-6}$ \\
\hline
\end{tabular}




\section{Chapter 8 Induced RAdioActivity in EnVironMental Media}

Darcy's Law can then be used to determine the rate of migration of a contaminant, in this case, radioactivity, from one point to another. During the time of migration, the concentration would be decreased by radioactive decay and dilution of the plume while possibly being increased by any ongoing radioactivation. One often encounters the problem of calculating the concentration of radionuclides at some location as a function of time during, or after, a period of irradiation comparable to the mean-lives of the radionuclides of concerns. At a given location in such a medium, denoted by the coordinate $x$, one needs to solve the following continuity equation, an extension of Eq. (7.4), for situations where the velocity of water movement $v$ can be thought of as slowly varying or a constant over time and some volume of space:

$$
\frac{\partial C_{i}}{\partial t}=\frac{L_{i}}{w_{i}^{\prime}} Q_{i}(x, t)-v \frac{\partial C_{i}}{\partial x}-\lambda_{i} C_{i}(x, t),
$$

where all variables are as in Eq. (8.17) with the refinements that $\lambda_{i}$ is the decay constant of the $i^{\text {th }}$ radionuclide, $x$ is the spatial coordinate, $t$ is the time, $w_{i}^{\prime}$ is the water content of the media per unit volume of media. The quantity $Q_{i}(x, t)$ represents the production of the $i^{\text {th }}$ radionuclide and is equivalent to the factor $N_{p} S_{\text {ave }} /\left(1.17 \times 10^{6} \rho\right)$ in Eq. (8.17). It includes any time-dependence in the delivery of beam. The middle term in the left-hand side of the equation takes care of movement from a point of one concentration to another at the seepage velocity $v$. As seen elsewhere in this text, one can commonly describe the spatial dependence of the production factor in a thick shield as an exponential function;

$$
Q_{i}(x, t)=Q_{o i}(t) \exp (-\xi x)
$$

Mokhov (Mo97) has solved this equation for the typical initial conditions of $C_{i}(x, 0)=0$ and $x \geq 0, t \geq 0$. In general,

$$
C_{i}(x, t)=\frac{L_{i}}{w_{i}} \int_{0}^{t} d t^{\prime} Q_{i}\left(x-v t^{\prime}, t^{\prime}\right) \exp \left(-\lambda t^{\prime}\right),
$$

and for an exponential spatial dependence as in Eq. (8.21) this becomes:

$$
C_{i}(x, t)=Q_{o i}(t) \frac{L_{i}}{w_{i}} \frac{1}{\eta_{i}} \exp (-\xi x)\left[\exp \left(\eta_{i} \tau\right)-1\right]
$$

with

$$
\begin{aligned}
& \eta_{i}=\xi v-\lambda_{i}, \\
& \tau=t \text { for } t<x / v, \text { and } \\
& \tau=x / v \text { for } \mathrm{t} \geq x / v .
\end{aligned}
$$




\section{CHAPTER 8 INDUCED RADIOACTIVITY IN ENVIRONMENTAL MEDiA}

$C_{i}(x, t)$ has a maximum at $x_{i, \max }$ given by

$$
x_{i, \max }=-\frac{v}{\lambda_{i}} \frac{\ln \left(\frac{\xi v}{\lambda_{i}}\right)}{1-\frac{\xi v}{\lambda_{i}}} .
$$

In using these results, one must take care that the algebraic signs of the coordinates $x$ relative to that of $v$ are properly taken into account. In situations where the seepage velocity is extremely slow, diffusion becomes the dominant mechanism for water flow and dilution. Mathematically, a second partial derivative with respect to the spatial coordinate is added to Eq. (8.20). Examples are provided by Fetter (Fe88). Computer software has been written to address this topic such as that of Sudicky et al. (Su88).

As a further example of methodologies that can be employed in solving such problems, Jackson (Ja87) has estimated the dilution for a shallow uncased well in an aquifer a distance $r$ from a beam loss point also in the aquifer. The loss point was assumed to be within the drawdown zone of the well. This was performed for a simple geology that involved a single uniform stratum of earth above some level of impervious stratum. Fig. 8.6 shows the situation described by this model. Here, a given well is modeled by using the profile of the depth of water $h(r)$ as a function of $r$. The value of $h(r)$ is determined by the depth of a test well at radius $r$ from the well under consideration and represents the hydraulic potential. The well is assumed to supply a volume $Q$ of water per day. The flux of water is determined by the gradient relation, equivalent to Darcy's Law;

$$
S_{r}=k \frac{d h(r)}{d r}
$$

where $S_{r}$ is the inward flux at radius $r$ and $k$ is a constant with dimensions of volume per unit time per unit area and is characteristic of the soil. Conservation of water yields the steady-state equation;

$$
Q=2 \pi r h(r) S_{r}=2 \pi r k h \frac{d h}{d r}=\pi k \frac{d\left(h^{2}\right)}{d(\ln r)} .
$$

The quantity $2 \pi r h \frac{d h}{d r}$ corresponds to the rate of change of volume of the cylindrical shell of height $h$ (i.e., the hydraulic head) with respect to $r$. This equation has the solution;

$$
Q \ln \left(\frac{r}{r_{o}}\right)=\pi k\left\{h^{2}(r)-h_{o}^{2}\right\}
$$

where $r_{O}$ is the radius of the well and $h_{O}$ is the height of water above the impervious stratum at the well. If $H$ is the depth of the impervious layer below the water table in a 
asymptotic region unperturbed by any wells, the radius of influence $R$ of the well can be defined by the relation;

$$
\ln \frac{R}{r_{o}}=\frac{\pi k\left\{H^{2}-h_{o}^{2}\right\}}{Q} .
$$

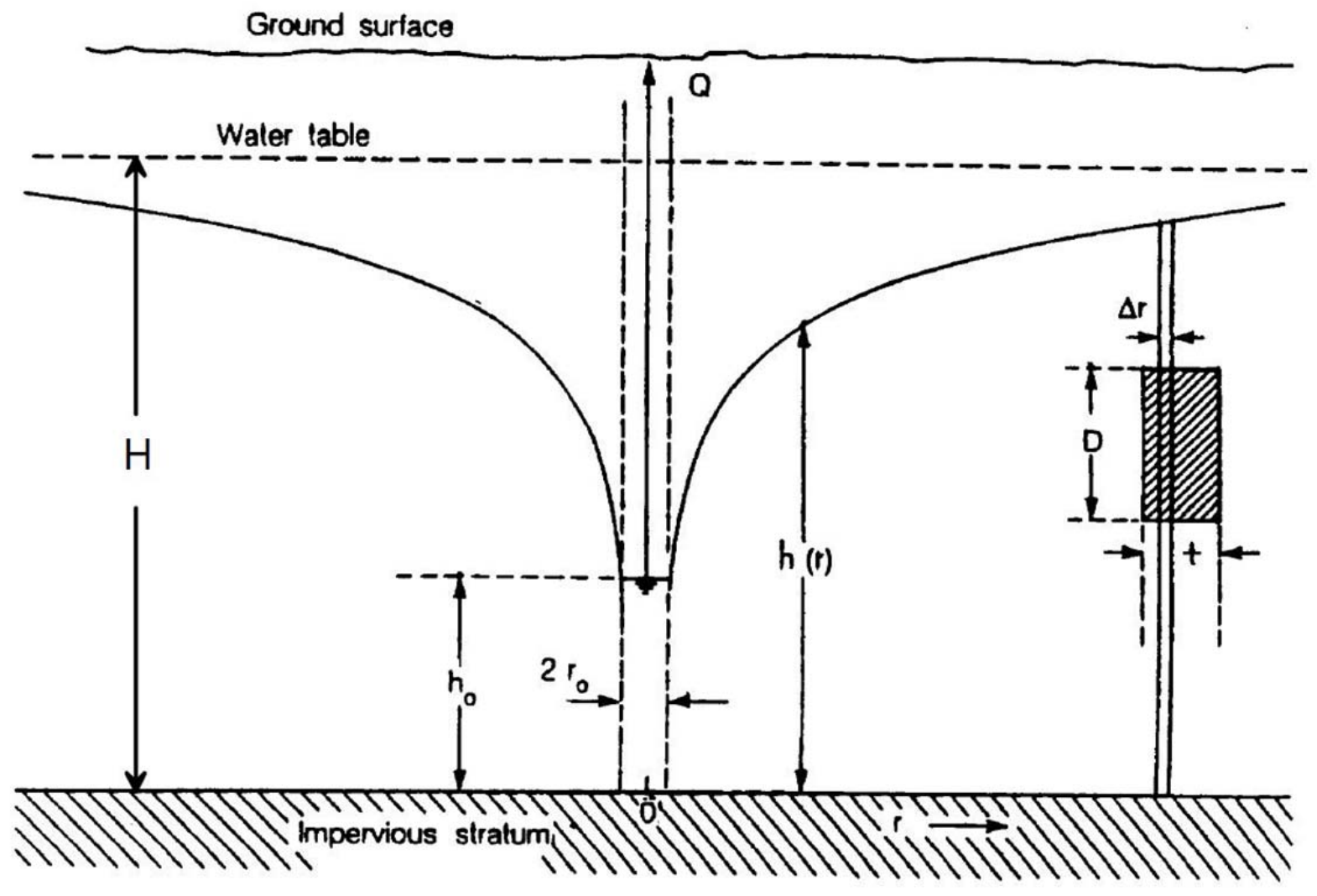

Fig. 8.6 Hydrogeological model of a shallow well in proximity to an accelerator tunnel where a beam loss occurs. The radioactivated region is represented in cross section by the cross-hatched rectangle to the right. $h$ represents the elevation of the water table above the impervious stratum as a function of $r$ while the water table is a distance $H$ above the impervious stratum where the water table is not perturbed by wells. [Adapted from (Ja87).]

However, the detailed solution is not necessary. Suppose that there is a well a distance $r$ away from the region of deposition of radioactivity near an accelerator. One also assumes that the activation zone lies below the water table and that the deposition region lies within the radius of influence of the well. This assumption leads to higher concentrations than would be obtained if the activation zone were totally, or partially, above the water table. The amount of activity drawn into the well is determined by the rate of pumping $Q$ and the necessary total flow through a cylinder of radius $r$ and height $h(r)$ as we have seen. Let $\Delta V$ be the volume of soil yielding $Q$ gallons of water. The cylindrical shell providing this amount of water will be of radial thickness $\Delta r$, where $\Delta V=2 \pi r h(r) \Delta r$. 


\section{ChAPTER 8 IndUCEd RAdioActivity in ENVIRONMENTAL MEdiA}

The fraction $F$ of the volume of activity included in this shell can be said to be given by:

$$
F=\frac{\Delta r}{t}=\frac{2 \pi r h \Delta r}{2 \pi r h t}=\frac{\Delta V}{2 \pi r h t}
$$

provided that $\Delta r<t$.

If the activated region contains leachable activity $A$ (either total activity or that of a particular radionuclide of interest), the corresponding specific activity $a$ in water drawn from the well is thus given by

$$
a=F \frac{A}{Q}=F \frac{A}{p \Delta V}=\left[\frac{\Delta V}{2 \pi r h t}\right] \frac{1}{p}\left[\frac{1}{\Delta V}\right] A=\frac{1}{2 \pi r t D} \frac{f}{p} A,
$$

where $f=D / h$ is the fraction of the total height of the cylindrical shell occupied by the activated region and $p$ is the effective porosity of the soil. The pumping volume $Q$ is implicit in $f$. Porosity values vary considerably but in general are in the range of

$$
0.2<p<0.35
$$

Thus, this formula may be used to obtain an estimate of the specific activity as a function of distance from the well, although it is perhaps not too useful for applications to beam losses far from the well. By definition, $f \leq 1$ and the lower value of porosity can be used to obtain upper limit estimates of the concentration. It must be emphasized that this model depends upon uniformity of water conduction by the strata. The presence of cracks, voids, so-called "sand lenses", or more complex geological strata can, of course, provide much more rapid movement that is not well-described by this simple model. 


\section{ChAPTER 8 IndUCEd RAdioActivity in EnVironMENTAL MEdia}

\section{Problems}

1. A $20 \mathrm{~m}$ long air gap has a beam of $10^{12} \mathrm{~s}^{-1}$ of high energy protons passing through it. First, calculate the production rate of ${ }^{11} \mathrm{C}$ in the gap at equilibrium if one approximates air in the gap by nitrogen and assumes $\sigma\left({ }^{11} \mathrm{C}\right)=10 \mathrm{mb}$. Take the density to be that of air, not nitrogen, at NTP. Assume that there are no significant losses of beam by interaction after checking to see that this assumption is, in fact, true. Table 1.2 contains helpful information.

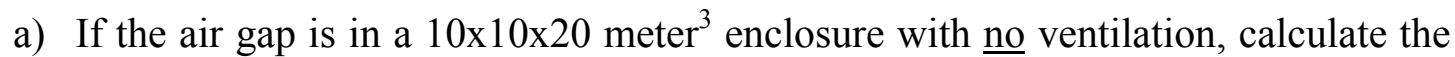
equilibrium concentration of ${ }^{11} \mathrm{C}$ in the room (in units of $\mu \mathrm{Ci} \mathrm{m}{ }^{-3}$ ) assuming extremely rapid mixing (i.e., no time allowed for decay while mixing occurs) of the enclosed air. Compare the concentration with the most restrictive derived air concentration (DAC) value for workers under the 1990 System in Table 8.6 and calculate, using simple scaling, the dose equivalent to a worker who spends full time in this room. Comment on the effect of the finite room dimensions. (This is a purely hypothetical scenario due to the much larger hazards of the intense direct beam!)

b) Calculate the concentration if two air changes $\mathrm{hr}^{-1}$ are provided.

c) Assume the exhaust of the ventilation described in part "b" is through a $10 \mathrm{~cm}$ radius stack $25 \mathrm{~m}$ tall. Calculate the air speed in the stack, and the emission rate in $\mathrm{Ci} \mathrm{s}^{-1}$. Then using Cember's version of Sutton's equation for tall stacks to estimate the concentration directly downwind at ground level, and hence the effective dose (1990 System) $1.0 \mathrm{~km}$ away with stable meteorological conditions and an average wind speed of $10 \mathrm{~km} \mathrm{hr}^{-1}$.

d) Perform the same calculation requested in "c" using the more general version of Sutton's equation appropriate to short stacks and assume the stack height to be 3.0 meters. All other conditions of the problems are the same as in "c".

2. In soil conditions similar to those at Fermilab, a volume of soil around a beam absorber approximately $10 \mathrm{~m}$ wide by $10 \mathrm{~m}$ high by $20 \mathrm{~m}$ long is the scene of a star production rate (averaged over the year) of 0.02 stars proton $^{-1}$ at a beam intensity of $10^{12}$ protons $\mathrm{s}^{-1}$.

a) Calculate the annual production of ${ }^{3} \mathrm{H}\left(t_{1 / 2}=12.3\right.$ years), the saturated activity (in $\mathrm{Bq} \& \mathrm{Ci}$ ), and the average saturated specific activity in the above volume's water (assume 10\% water content by volume).

b) Use the older Fermilab single residence model to calculate the concentration at the nearest well. Assume the activation region (beam loss point) is $50 \mathrm{~m}$ above the aquifer and the usual migration velocities. 


\section{Chapter 8 Induced RAdioActivity in EnVironmental Media}

c) "Conservatively" apply the "Jackson Model" to estimate the concentration at a well 100 meters distant from the center of the activation region.

3. The method of accounting for ventilation presented in Section 8.2.2 can readily be generalized to include other mechanisms which "remove" airborne radionuclides such as absorption, filtration, etc. Assume that an arbitrary total number " $j$ " of such mechanisms are present and that the irradiation has gone on sufficiently long to have come to equilibrium between the production of radionuclides and all modes of removal. Following termination of the irradiation, determine the fraction of the total activity that is removed from the air volume by each of the " $j$ " mechanisms. It is safe to assume that all the atoms of the radionuclide produced are removed by one of the processes. The solution of this problem has some importance for the more long-lived radionuclides for it leads to a method of estimating the total activity expected to be found on, say, filter media. 


\section{ChAPTER 9 RAdiation Protection Instrumentation at ACCELERATORS}

\section{$9.1 \quad$ Introduction}

In this chapter instruments and dosimeters currently used in the environment of particle accelerators to measure and characterize the radiation fields are discussed. The emphasis is on instrumentation that addresses those aspects of accelerator radiation fields that pose special problems perhaps somewhat unique to this branch of radiation protection. Thomas and Stevenson (Th88) and Swanson and Thomas (Sw90) also discuss these matters. Cember (Ce69) covers the basics of radiation measurement instrumentation while Knoll (Kn79) has written the most comprehensive treatise on the subject. Virtually all particle detection techniques that have been devised by physicists have to some degree been employed in radiation measurements at accelerators. Often, the specialized instruments used to characterize the accelerator radiation fields are found to be of value to the researcher in the understanding of experiment "backgrounds".

\subsection{Counting Statistics}

Many of the detection techniques employed to measure radiation fields are dependent upon the counting of individual events such as the passage of charged particles through some medium or the decay of some particle or radionuclide. Cember (Ce69) has given a good summary of counting statistics that is largely repeated here. Radioactive decays are randomly occurring events having a sampling distribution that is correctly described by the binomial distribution given by the following expansion:

$$
(p+q)^{n}=p^{n}+n p^{n-1} q+\frac{n(n-1)}{2 !} p^{n-2} q^{2}+\frac{n(n-1)(n-2)}{3 !} p^{n-3} q^{3}+\ldots+
$$

where $p$ is the mean probability for occurrence of an event, $q$ is the mean probability of non-occurrence of the event $(p+q=1)$, and $n$ is the number of chances of occurrence. The probability of exactly $n$ events occurring is given by the first $1^{\text {st }}$ term, the probability of $(n-1)$ events is given by the $2^{\text {nd }}$ term, etc. For example, in the throwing of a dice, the probability of throwing a " 1 " is $1 / 6$ while that of throwing a " 1 " 3 times in a row $(n=3)$ is

$$
p^{n}=(1 / 6)^{3}=1 / 216
$$

In three throws, the probabilities of throwing 2 "ones", 1 "one" and zero "ones" are given by the $2^{\text {nd }}, 3^{\text {rd }}$, and $4^{\text {th }}$ terms; $15 / 216,75 / 216$, and $125 / 216$, respectively.

This distribution becomes essentially equivalent to the normal or Gaussian distribution when $n$ has a value of about 30 or larger. The Gaussian distribution is as follows:

$$
p(n)=\frac{1}{\sigma \sqrt{2 \pi}} \exp \left[-(n-\bar{n})^{2} /\left(2 \sigma^{2}\right)\right]
$$

where $p(n)$ is the probability of finding exactly $n, \bar{n}$ is the mean value, and $\sigma$ in this context is the standard deviation and not a reaction cross section. 
Radioactive decays or particle reactions usually can be characterized as highly improbable, i.e. "rare", events. For such events, the binomial distribution approaches the Poisson distribution where the probability of obtaining $n$ events if the mean value is $\bar{n}$ is given by

$$
p(n)=\frac{(\bar{n})^{n} \mathrm{e}^{-\bar{n}}}{n !}
$$

For example consider $10^{-3} \mu \mathrm{Ci}\left(10^{-9} \mathrm{Ci}, 37 \mathrm{~Bq}\right)$ of activity so $\bar{n}=37$ decays $\mathrm{s}^{-1}$. The probability of exactly observing this number of events in any one second is

$$
p(37)=\frac{(37)^{37} \mathrm{e}^{-37}}{37 !}
$$

Thus $p(37)=0.066$. As in the case of the normal distribution, $68 \%$ of the events would lie within one standard deviation of the mean, $96 \%$ of the events would lie within two standard deviations of the mean, etc. For Poisson statistics the standard deviation is given by

$$
\sigma=\sqrt{n}
$$

with the relative error $\sigma / n$ thus given by $-\sqrt{n} / n$.

Often, when dealing with instrumentation, the counting rate is involved. For this quantity the following holds:

$$
r \pm \sigma_{r}=\frac{n}{t} \pm \frac{\sqrt{n}}{t}
$$

where $r$ is the counting rate per unit time, $\sigma_{r}$ is its standard deviation, and $t$ is the counting time during which the rate is measured. The quantity $t$ could even be the integration time constant of an instrument. It follows that

$$
\sigma_{r}=\frac{\sqrt{n}}{t}=\sqrt{\frac{n}{t} \cdot \frac{1}{t}}=\sqrt{\frac{r}{t}}
$$

Usually, counts due to various background radiations are present and must be dealt with. The standard deviation of the net counting rate is

$$
\sigma_{n}=\sqrt{\sigma_{g}^{2}+\sigma_{b g}^{2}}=\sqrt{\frac{r_{g}}{t_{g}}+\frac{r_{b g}}{t_{b g}}}
$$

where the subscripts $g$ refer to the measurement of the gross counting rate while the 


\section{Chapter 9 Radiation Protection Instrumentation at ACCELERAtors}

subscripts $b g$ refer to the measurement of the background counting rate. The time durations of the measurements of the rates $r_{g}$, and $r_{b g}$ are $t_{g}$ and $t_{b g}$, respectively.

Another quantity that often is important is the resolving time or dead time, of an instrument. This is the time that the detector, following an event, is incapable of measuring a second event while it is processing the first. It is a function of both electronic characteristics and the physical process inherent in the detection mechanism. It can be measured by exposure to two different sources of radiation where the instrument has a measured background rate of $R_{b g}$ and responds to first source alone with a rate $R_{l}$ and to the second source alone with a rate $R_{2}$ where both $R_{1}$ and $R_{2}$ include the background. When exposed to both sources simultaneously, the measured rate is $R_{12}$. According to Cember (Ce69), the resolving time $\tau$ is given by;

$$
\tau=\frac{R_{1}+R_{2}-R_{12}-R_{b g}}{R_{12}^{2}-R_{1}^{2}-R_{2}^{2}} .
$$

However, it is often easier to determine $\tau$ from the physical properties of the detection mechanism or from the electronic time constants of the measurement circuitry. With a finite resolving time $\tau$ and a measured counting rate is $R_{m}$, the "true" counting rate $R$ that would be observed with a perfect instrument having $\tau=0$ is given by

$$
R=\frac{R_{m}}{1-R_{m} \tau}
$$

Knoll (Kn79) gives a more detailed discussion of this topic.

\subsection{Special Considerations for Accelerator Environments}

There are several important features of accelerator radiation fields that merit attention in choosing instrumentation or measurement techniques that should be discussed here.

\subsubsection{Large Range of Flux Densities, Absorbed Dose Rates, etc.}

The dynamic range of quantities to be measured encountered at accelerators can extend from fractional mrem $\mathrm{yr}^{-1}$ encountered in environmental monitoring to large values of absorbed dose of up to megarads $\left(10^{6} \mathrm{rads}\right)$ of concern for radiation damage. ${ }^{34}$

9.3.2 Possible Large Instantaneous Values of Flux Densities, Absorbed Dose Rates, etc.

Certain accelerators such as linacs, rapid cycle synchrotrons, and "single-turn" extracted

\footnotetext{
${ }^{34}$ It is customary to quantify radiation fields in terms of absorbed dose, rather than dose equivalent, at levels above those encountered in routine personnel protection $(\approx 1-5 \mathrm{rad})$.
} 


\section{Chapter 9 RAdiation Protection Instrumentation at ACCELERAtors}

beams from synchrotrons can have very low average intensities but extremely high instantaneous rates. Such circumstances arise when the duty factor, the fraction or percentage of the time the beam is actually present due to the operational characteristics of the accelerator, is small. Thus, the dead time considerations must be taken into account or the apparent measured values of radiological quantities such as flux densities or dose rates can be misleadingly low. Some instruments can be completely paralyzed by high instantaneous rates and read "zero" in a high radiation field, a potentially dangerous situation. Thus the dead time needs to be understood.

\subsubsection{Large Energy Domain of Neutron Radiation Fields}

At any given accelerator capable of producing neutrons, the properties of nuclear interactions make it highly probable that neutrons will be present at all energies from thermal $\left(<E_{n}>\approx 0.025 \mathrm{eV}\right)$ up to nearly the energy of the beam. As will be seen, the methods of detection of neutrons vary considerably over this energy domain. Thus the choice of instrumentation is crucial to the success of the measurement. For no other particle type is the energy range of the particles encountered in the accelerator environment so large nor are the types of effective detection techniques so diverse.

\subsubsection{Presence of Mixed Radiation Fields}

At accelerators, one has to consider that any given radiation field external to shielding is likely to be comprised of a mixture of photons, neutrons, and at high energies and especially at forward angles, muons and even a multitude of other particles. In proximity to the beam, the multiplicity of particle types present can be quite large. Furthermore virtually all neutron fields contain at least some photon component, often due to the capture of thermal neutrons by means of $(n, \gamma)$ reactions. Furthermore, muon radiation fields near proton and ion accelerators commonly contain some neutron component. Thus the choice of instrumentation is somewhat dependent upon what component of the radiation field needs to be characterized.

\subsubsection{Directional Sensitivity}

Certain instruments intrinsically exhibit directional sensitivity. This feature can be either beneficial or detrimental, depending upon the situation. In all instances, it must be understood. It can lead to underestimates in radiation fields where all particles are not monodirectional. Directional sensitivity can actually be useful in certain circumstances to identify sources of unwanted radiation.

9.3.6 Sensitivity to Features of the Accelerator Environment Other than Ionizing Radiation

While the focus of this discussion is on ionizing radiation, other features must be taken 


\section{Chapter 9 Radiation Protection Instrumentation at ACCELERAtors}

into account. The most prominent of these is the presence of radiofrequency radiation (RF) that can perturb instruments that can act, sometimes rather effectively, as "antennas". Environmental effects such as temperature and humidity can also be important. In addition, one must use caution when attempting radiation measurements in the presence of magnetic fields. Induced eddy currents might be interpreted as radiation. Instruments may become magnetized and meter movements may be damaged or "paralyzed". Also, devices based on photomultiplier tubes commonly read "zero" in static magnetic fields of even moderate strength because of severe deflections of the low energy electrons within the tubes.

\subsection{Standard Instruments and Dosimeters}

This section will review instruments and dosimeters. Some of these are commonly available from commercial sources. Such commercial instruments should be used with care at accelerator facilities to be sure that their properties are adequate for usage in the particular radiological and physical environment at hand.

\subsubsection{Ionization Chambers}

A basic type of instrument commonly used at accelerators to measure absorbed dose rates is the ionization chamber. Such ion chambers rely on the collection of charge liberated by particles passing through a gas. Some detectors used in physics research now employ liquids, both room temperature and cryogenic, for the ionization medium.

For ion chambers a beneficial result from atomic physics is that the energy loss per ion pair $W$ is nearly a constant over a number of materials and rather independent of type of charged particle as exhibited by Table 9.1.

Table 9.1 Values of the energy deposition per ion pair $W$ for different materials for fast electrons and $\alpha$-particles ${ }^{\mathrm{a}}$. [Adapted from (Kn79).]

\begin{tabular}{|c|c|c|}
\hline & \multicolumn{2}{|c|}{$W($ eV/ion pair) } \\
Material & Fast Electrons & $\alpha$-particles \\
\hline $\mathrm{Ar}$ & 27.0 & 25.9 \\
\hline $\mathrm{He}$ & 32.5 & 31.7 \\
\hline $\mathrm{H}_{2}$ & 38.0 & 37.0 \\
\hline $\mathrm{N}_{2}$ & 35.8 & 36.0 \\
\hline $\mathrm{Air}$ & 35.0 & 35.2 \\
\hline $\mathrm{O}_{2}$ & 32.2 & 32.2 \\
\hline $\mathrm{CH}_{4}$ & 30.2 & 29.0 \\
\hline
\end{tabular}

${ }^{\mathrm{a}}$ The original data was obtained from Curran (Cu55).

Thus in a gas with a certain value of $W$ (eV/ion pair), a charged particle depositing a certain amount of energy $\varepsilon(\mathrm{MeV})$ will liberate an electrical charge $Q_{\text {elect }}$ (Coulombs), according to

$$
Q_{\text {elect }}=\frac{1.602 \times 10^{-13} \varepsilon}{W} \text {. }
$$




\section{Chapter 9 Radiation Protection Instrumentation at ACCELERAtors}

The charge $Q_{\text {elect }}$ is collected by electrodes biased at some voltage $V$. The collected charge generates a small change in the voltage $\Delta V$ (volts), in accord with the relation,

$$
\Delta V=\frac{\Delta Q_{\text {elect }}}{C}
$$

where $C$ is the capacitance of the total circuit (including that of the chamber) in units of Farads. For typical chambers $C$ is of the order of $10^{-10}$ Farads. The measured signal originates with $\Delta V$. Knoll $(\mathrm{Kn} 79)$ gives many details about the size and form of the electrical signals that can be measured. Such chambers can be operated either in a current mode (also called "DC" [i.e., direct current] or ratemeter mode) or in an integration mode in which the charge is collected (integrated) over some time period, then digitized into pulses that represent some increment of absorbed dose or dose equivalent. In the ion chamber mode of operation the applied voltage is sufficiently small so that gas multiplication (charge amplification) does not occur. In the most simpleminded approach, one might believe that for measurements in photon fields one could fill such a chamber with gases that "mimic" tissue and, with suitable calibration, convert the charge collected into absorbed dose. Such tissue equivalent materials range from complex mixtures to simple hydrocarbons, depending upon the accuracy of the representation of biological tissue that is desired. However, since ion chamber gases are in general much less dense than tissue, one must also capture the energy of the secondary electrons, which in the region of a few $\mathrm{MeV}$ have ranges of several meters in such gaseous material. It is thus necessary to use compensation techniques in which the solid material of the walls is chosen because of properties that match those of the gas. This condition can be readily achieved by the use of any material having an atomic number close to that of the gas, an approximation sufficiently accurate for most practical purposes. Thus, aluminum and especially plastics are reasonably equivalent to tissue and air, at least for use in photon radiation fields. Such walls should be of sufficient thickness to establish electronic equilibrium, where the flux of secondary electrons leaving the inner surface of the wall is independent of the thickness. Table 9.2 gives the wall thickness needed to establish electronic equilibrium for photons of various energies.

The measurement of absorbed dose is accomplished by application of the Bragg-Gray principle that states that the absorbed dose $D_{m}$ in a given material can be deduced (with suitable unit conversions) from the ionization produced in a small gas-filled cavity within that material as follows:

$$
D_{m}=W S_{m} P
$$

where $W$ is the average energy loss per ion pair in the gas and $P$ is the number of ion pairs per units mass formed. $S_{m}$ is the ratio of the mass stopping power (i.e., the energy loss per unit density in units of, say, $\mathrm{MeV} \mathrm{g}^{-1} \mathrm{~cm}^{2}$ ) of the material of interest to that of the chamber gas. For $D_{m}$ to be in grays $\left(\mathrm{J} \mathrm{kg}^{-1}\right), W$ must be expressed in Joules per ion pair and $P$ in ion pairs per $\mathrm{kg}$. 
Table 9.2 Thickness of ionization chamber walls required for establishment of electronic equilibrium ${ }^{\mathrm{a}}$. [Adapted from (Kn79).]

\begin{tabular}{|c|c|}
\hline Photon Energy (MeV) & Thickness \\
\hline 0.02 & $\left(\mathbf{g ~ c m}^{-2} \mathbf{)}\right.$ \\
\hline 0.05 & 0.0008 \\
\hline 0.1 & 0.0042 \\
\hline 0.2 & 0.014 \\
\hline 0.5 & 0.044 \\
\hline 1 & 0.17 \\
\hline 2 & 0.43 \\
\hline 5 & 0.96 \\
\hline 10 & 2.5 \\
\hline
\end{tabular}

${ }^{\mathrm{a}}$ From (IC71).

${ }^{\mathrm{b}}$ The thicknesses quoted are based on the range of electrons in water. The values will be substantially correct for tissue-equivalent ionization chamber walls and also for air. Half of the above thickness will give an ionization current within a few per cent of its equilibrium value.

For accelerator radiation fields that contain neutrons, or mixtures of neutrons with muons and photons, one is commonly able to use an ideal ion chamber to measure the absorbed dose $D$ and determine the dose equivalent $H_{\text {equiv }}$ or effective dose $H_{\text {eff }}$ by using the average quality factor $Q$ or radiation weighting factor $w_{R}$ as follows [see also Eq. (1.6)]:

$$
H_{\text {equiv }}=Q D \text { or } H_{e f f}=w_{R} \mathrm{D}
$$

Ion chambers with tissue equivalent walls have been used in this manner at many accelerators. The value of $Q$ or $w_{R}$ has to be determined by some other means such as those described in this chapter; usually as a separate measurement. Awschalom described the initial use of such instruments, called chipmunks, at Fermilab (Aw72). Krueger and Larson have discussed their more recent evolution $(\mathrm{Kr} 02)$. These ion chambers, now updated several times, are commercially produced and read out with Fermilab-designed electronics. The current chamber has a net volume of 3.4 liters. The $1.3 \mathrm{~mm}$ thick outer wall of aluminum is lined with a $3.2 \mathrm{~mm}$ thick layer of phenolic. They are filled with propane gas at about 1.0 atmosphere (absolute), and contain an electrometer encased in a sealed container. Several versions of the instrument, including the higher dose rate version called the scarecrow, have been studied by Freeman and Krueger (Fr84) with properties given in Table 9.3.

Typically chambers of this general type are calibrated using photons and have a typical "quality factor" built in to the electronics. Such chambers are available either as linepowered fixed monitors or as hand-held survey instruments. The use of such instruments at accelerators must be done with the assurance that the instrument will respond correctly to the radiation field present. Neutron radiation fields are generally considered to be the most difficult in which to do this successfully. Höfert and Raffnsøe at CERN reported measurements of the response of various instruments, including tissue equivalent ion chambers (Hö80). They were able to test such chambers, along with others (see below), 
in neutron radiation fields having measured neutron energies ranging from thermal to 280 $\mathrm{MeV}$. Table 9.4 provides the results. The neutron fields originated from reactor and radioactive sources, except that at $280 \mathrm{MeV}$, a neutron beam from the $600 \mathrm{MeV}$ CERN Synchrocyclotron was used.

Table 9.3 Descriptions of ionization chambers used at Fermilab. The instruments designated "new" were produced after 1980 while those designated "old" were produced earlier. [Adapted from (Fr84).]

\begin{tabular}{|c|c|}
\hline "Old" Chipmunk & $\begin{array}{l}\text { A high-pressure gas-filled ionization chamber designed by Fermilab } \\
\text { and built by LND, Inc. with } 4 \mathrm{~mm} \text { thick walls of tissue-equivalent } \\
\text { plastic. The fill gas is } 10 \text { atmospheres of ethane. The chamber is } \\
\text { enclosed in a protective box that contains a sensitive electrometer and } \\
\text { associated electronics to measure the current output and convert it to } \\
\text { the dose equivalent rate. Switch-selectable quality factors of } 1,2.5 \text {, or } \\
5 \text { are available. The instrument is equipped with a visible dose } \\
\text { equivalent ratemeter and audible alarms. It provides a remote readout } \\
\text { and capability for interface with radiation safety interlock systems. }\end{array}$ \\
\hline "New" Chipmunk & $\begin{array}{l}\text { These instruments are similar to the Old Chipmunk except for the use } \\
\text { of phenolic-lined ionization chamber, filled with propane gas at } \\
\text { atmosphere pressure and an electrometer encased in a sealed } \\
\text { container. The reduced gas pressure was chosen for safety and the } \\
\text { sealed container was provided to improve reliability over a larger } \\
\text { range of temperature and humidity. The ion chambers were supplied } \\
\text { by HPI, Inc. The latest versions of this instrument also allow for the } \\
\text { selection of a quality factor of } 10 \text {. }\end{array}$ \\
\hline "Old" Scarecrow & $\begin{array}{l}\text { A high-pressure ionization chamber with bare stainless steel walls } \\
\text { filled with } 10 \text { atmospheres of ethane gas. The instrument is otherwise } \\
\text { similar to the Old Chipmunk but with a fixed quality factor of } 4 \text { and } \\
\text { capability to measure dose equivalent rates } 100 \text { times higher (up to } 10 \\
\text { rem } \mathrm{h}^{-1} \text { ). A visible ratemeter, audible alarm, and remote readout } \\
\text { capability are present as is the provision for interface to radiation } \\
\text { safety interlocks. }\end{array}$ \\
\hline "New" Scarecrow & $\begin{array}{l}\text { The electronics and functionality is similar to that of the Old } \\
\text { Scarecrow, but the ion chamber of the New Chipmunk is used. }\end{array}$ \\
\hline
\end{tabular}

Table 9.4 Absorbed dose response and measurement errors for tissue equivalent ion chambers as a function of neutron energy. [Adapted from (Hö80).]

\begin{tabular}{|c|c|c|}
\hline $\begin{array}{c}\text { Neutron Energy } \\
(\mathrm{MeV})\end{array}$ & $\begin{array}{c}\text { Absorbed Dose Response } \\
\left(10^{5} \text { Coulombs } \mathrm{Gy}^{-1}\right)\end{array}$ & $\begin{array}{c}\text { Error } \\
(\%)\end{array}$ \\
\hline Thermal & 0.446 & 9.8 \\
\hline 0.0245 & 0.404 & 12.1 \\
\hline 0.1 & 0.622 & 6.1 \\
\hline 0.25 & 0.806 & 7.1 \\
\hline 0.57 & 0.885 & 5.4 \\
\hline 1.0 & 0.885 & 5.4 \\
\hline 2.5 & 0.993 & 6.1 \\
\hline 5.0 & 1.179 & 5.2 \\
\hline 15.5 & 1.370 & 5.2 \\
\hline 19.0 & 1.664 & 12.1 \\
\hline 280.0 & 0.389 & 10.1 \\
\hline
\end{tabular}




\section{Chapter 9 RAdiation Protection Instrumentation at ACCELERAtors}

The performance is reasonably independent of energy in the energy region that typically dominates the dose equivalent (approximately up to about 5-10 MeV).

Measurements have been conducted at Fermilab indicate that absorbed dose measured in muon radiation fields is adequately understood using the $\gamma$-ray calibration of such instruments (Co87). These tests involved comparison with direct measurements of the muon fluence using counter-telescope techniques (see Section 9.5.8) and typically are in agreement within about $10 \%$ for the Fermilab-built instruments described previously. This is not surprising since muons at high energies behave essentially as minimum ionizing particles with ionization energy losses quite similar to that of electrons.

Practical problems encountered with such ion chambers are mostly those due to radiofrequency interference, pulsed radiation fields, and environmental factors such as temperature and humidity extremes. Cossairt and Elwyn (Co87) determined that airfilled, self-reading pocket ion chambers of the type commonly issued to personnel to allow real-time monitoring of exposure to $\gamma$-rays, performed very well in muon radiation fields, measuring absorbed doses to within about $\pm 15 \%$. This is due to the fact that the ratio of muon stopping power in tissue to that in air for energies between 1.0 and 800 $\mathrm{GeV}$ is $1.07 \pm 0.05$ (St83).

\subsubsection{Geiger-Müller Detectors}

These instruments, among the oldest developed for the detection of radiation, are in conspicuous use at particle accelerators primarily with respect to detection and measurement of induced activation and removable induced activity (contamination). In some instances such instruments can be used to identify prompt radiation fields. They are very rugged and remarkably insensitive to environmental effects such as temperature and humidity. However, the typical dead time of $100 \mu \mathrm{sec}$ in prompt radiation fields at an accelerator or so renders them to be generally useless in those fields.

\subsubsection{Thermoluminescent Dosimeters (TLDs)}

Swanson and Thomas (Sw90) and Knoll (Kn79) have provided discussions of the properties of TLDs. These dosimeters are an attractive alternative to photographic film particularly to monitor personnel exposures in $\beta$ and $\gamma$ radiation fields. They have also been found to be useful in measuring neutron radiation fields when used as a pair of ${ }^{6} \mathrm{LiF}$ and ${ }^{7} \mathrm{LiF}$ TLDs crystals in the same dosimeter. This exploits the fact that the reaction ${ }^{6} \mathrm{Li}(\mathrm{n}, \alpha)^{3} \mathrm{H}$ has a large thermal neutron capture cross section of 940 barns (see Section 9.5.1.2) in contrast to the much small thermal neutron capture cross section of 0.037 barns for ${ }^{7} \mathrm{Li}(\mathrm{n}, \gamma)^{8} \mathrm{Li}$. Since a TLD containing either lithium isotope has a nearly identical efficiency for detecting photon or muon radiation, measurement of the response of the two detectors can be used to determine the dose due to thermal neutrons in the presence 


\section{Chapter 9 Radiation Protection Instrumentation at ACCELERAtors}

of photons or muons. These reactions provide tools to use in the detection of fast neutrons if moderation is supplied, as will be discussed later.

TLDs operate on the principal that some of the radiation liberated by the ionizing particle is "trapped" in band gaps in the crystal lattice. The process is well described by Knoll (Kn79). In particular, ionization elevates electrons from the valence to the conduction band where they are then captured by a "trapping center". At room temperatures, there is only a small probability per unit time that such "trapped" electrons will escape back to the conduction band from the valence band. Thus exposure to radiation continuously populates the traps. "Holes" are similarly trapped in the valence band. When readout of the dose is desired, the crystal is heated and this thermally excites the electrons and holes out of the traps. This process is accompanied by the emission of light that can then be measured as a so-called glow curve. A number of other materials can function as TLDs; notably $\mathrm{CaSO}_{4}: \mathrm{Mn}, \mathrm{CaF}_{2}$, and $\mathrm{CaF}_{2}: \mathrm{Mn}$. These materials have properties that can be optimized for particular applications. $\mathrm{CaF}_{2}: \mathrm{Mn}$ is particularly useful for environmental monitoring purposes, where extraordinarily high sensitivity is required. The large numbers of trapped electrons and holes per unit of dose permits sensitivity to absorbed doses as small as $2 \times 10^{-5}$ rads. $\mathrm{LiF}$ "fades" over time to a lesser degree than most of the other materials at room temperature and its average atomic number is very close to that of tissue, so it is particularly useful for personnel dosimetry.

TLDs can give valid results for fields as high as 100 rads. Higher doses can be measured under certain conditions if one takes care to use crystals calibrated in the intense radiation fields since linearity of the response breaks down in the high dose region. These devices exhibit superlinearity. TLDs are not generally susceptible to dose rate problems. However the readout process is intrinsically "destructive" and usually cannot be repeated.

\subsubsection{Nuclear Track Emulsions}

This discussion is summarized from that of Swanson and Thomas (Sw90). For many years thin $(\approx 25 \mu \mathrm{m})$ emulsions have been used for personal dosimetry in fast neutron fields. The technique is based upon detection of tracks left by proton recoils in the film. The energy range for which these dosimeters are effective is from roughly 0.5 to $25 \mathrm{MeV}$ because below that range the tracks are too short to be read out, while above it there are too few tracks because the (n,p) cross section (elastic scattering, mostly) decreases with energy. However, this energy range is the one that often results in significant neutron dose equivalents at accelerators. The singular important problem with NTA is that the latent image fades and leads to underestimates of the dose equivalent. The fading time can be a short as two weeks. Extreme efforts to keep out the moisture, and experience in dry climates give some indication that this problem can be overcome.

Höfert (Hö84b) and Greenhouse et al. (Gr87) have summarized experience with this dosimeter at accelerators. The dose equivalent range from about $10 \mathrm{mrem}$ to a few hundred mrem is that for which this dosimeter can be expected to perform acceptably. 


\section{Chapter 9 RAdiation Protection Instrumentation at ACCELERAtors}

Any technique based upon track formation is likely independent of dose rate effects.

\subsubsection{Track Etch Dosimeter}

Swanson and Thomas (Sw90) have discussed the use of such dosimeters. In these detectors, the passage of a charged particle through a dielectric material will result in a trail of damaged molecules in the material. These tracks can be made visible upon etching in a strong acid or base solution. The tracks will be etched at a faster rate than the undamaged portions of the material. As with nuclear emulsions, there is a minimum detectable track length that sets a threshold of about $0.5 \mathrm{MeV}$ on the neutron detection. Such detectors have been reviewed extensively by Griffith and Tommasino (Gr90). Mica, Lexan, and other materials are suitable for this purpose and electronic methods of readout are available. Repeated readouts of the processed tracks are feasible.

\subsubsection{CR-39 Dosimeters}

Swanson and Thomas (Sw90) have provided a discussion of applications of such dosimeters at accelerators. This material, also a "track detector", has largely replaced nuclear track emulsion as a neutron dosimeter. It is a casting resin also used in eyeglass lenses and therefore transparent. It is the most sensitive of the track detectors and registers recoil protons up to $15 \mathrm{MeV}$ and down to about $0.1 \mathrm{MeV}$. It is processed either chemically or electrochemically. Repeated readouts of the processed tracks are feasible. The lower limit of detection appears to be superior to that of nuclear emulsions. In this energy domain the sensitivity is adequate, about $7 \times 10^{3}$ tracks $\mathrm{cm}^{-2} \mathrm{rem}^{-1}$, but may be as much as a factor of two lower in high energy spectra. Fading appears to be insignificant. However, natural radon gas can contribute to background readings and the angle of particle incidence is important.

\subsubsection{Bubble Detectors}

The use of these detectors at accelerators has also been discussed by Swanson and Thomas (Sw90). The bubble damage polymer detector is an innovative dosimeter that is akin in some ways to a classic bubble chamber in that a liquid whose normal boiling point is below room temperature is kept under pressure. When the pressure is released bubbles form along the path of a charged particle that has traversed it. To enhance the effect, superheated droplets of a volatile liquid are dispersed in a gelatinous medium. There are two types of these detectors that have been developed; one type by Apfel (Ap79) and the other type by Ing (In84). The polymer or gel is supplied in a clear vial. When a neutron interacts in the sensitive material, a bubble is created that expands to optically visible dimensions and can thus be counted. There is no angular dependence but temperature effects must be considered. The Ing detector was reported to exhibit a constant response over the range $15<T<35^{\circ} \mathrm{C}$. The material can be tailored to match a chosen neutron energy threshold that can be as low as $10 \mathrm{keV}$ or less. Indeed, sets have 


\section{Chapter 9 RAdiation Protection Instrumentation at ACCELERAtors}

been prepared with arbitrary thresholds of $0.010,0.100,0.500,1.0,3.0$, and $10 \mathrm{MeV}$. The range of sensitivity can be adjusted to be between 1.0 and 30 bubbles per mrem, or larger, in a volume of about $4.0 \mathrm{~cm}^{3}$ and the physical mechanism is not readily sensitive to dose rate effects. Disadvantages include a high unit cost, and the fact that once the vial is opened it is only good for limited periods of time of dose integration. The materials have been successfully used at accelerator facilities. These detectors could not be expected to give accurate results in high dose rates.

One can see that no single commercial instrument "solves all problems" simultaneously, especially for neutron fields. The practitioner is encouraged to utilize a variety of instruments, including some of the special techniques discussed below to fully understand the radiation fields.

\subsection{Specialized Detectors}

\subsubsection{Thermal Neutron Detectors}

Although thermal neutrons are not commonly the major source of neutron dose equivalent at particle accelerators, they are of considerable importance in accelerator radiation protection because of the ability to moderate the fast neutrons into thermal ones, as we shall see later. Furthermore, because some of the most prominent thermal neutron detectors rely upon radioactivation (by neutron capture) as the detection mechanism, they have the advantage that the response is entirely independent of dose rate effects and hence free of dead time effects. An excellent discussion, summarized here, on thermal neutron detectors is given by Knoll (Kn79).

At the outset, there are some general features concerning thermal neutrons that need to be recalled. The kinetic energy $E$ of thermal neutrons have the familiar relationship as a function of temperature given by the Maxwell-Boltzmann distribution:

$$
f(E)=\frac{2}{\sqrt{\pi}(k T)^{3 / 2}} E^{1 / 2} \exp \left\{-\frac{E}{k T}\right\},
$$

where $f(E)$ is the fraction of particles, in this case neutrons, of energy $E$ per unit energy interval, $k$ is the Boltzmann constant (see Table 1.2), and $T$ is the absolute temperature of the gas. The most probable energy $E_{m p}$ is given by

$$
E_{m p}=k T
$$

while the average energy at any given temperature $\langle E>$ is

$$
\langle E\rangle=\frac{3}{2} k T
$$




\section{Chapter 9 RAdiation Protection Instrumentation at ACCELERAtors}

At room temperature $T=293{ }^{\circ} \mathrm{K}$, so that the most probable energy is $0.025 \mathrm{eV}$. Normally, thermal neutron cross sections are tabulated for this value of kinetic energy. Since thermal neutrons are decidedly nonrelativistic, the most probable velocity $v_{m p}$ at $T=293$ $\mathrm{oK}$ is determined from

$$
E_{m p}=\frac{1}{2} m v_{m p}^{2}=k T, \text { so that } v_{m p}=2200 \mathrm{~m} \mathrm{~s}^{-1} \text {. }
$$

As the neutron energy increases above the thermal value (up to about $1.0 \mathrm{keV}$ ), unless there are resonances present in the cross section, the absorption cross section $\sigma$ has been found to be approximately described by the relation

$$
\sigma \propto \frac{1}{\sqrt{E}} \propto \frac{1}{v}
$$

that is known as the $1 / v$ law. Thus, within the limits of validity of the $1 / v$ law, one can scale from the tabulated thermal neutron cross section $\sigma_{t h}$ as follows:

$$
\sigma(E)=\sigma_{t h} \sqrt{\frac{E_{m p}}{E}} .
$$

Several different nuclear reactions that are initiated by thermal neutrons are used as the basis of detectors. They all involve particular target nuclei and thus the detector materials sometimes depend upon isotopically separated materials to enhance the effectiveness.

\subsubsection{Boron-10}

The ${ }^{10} \mathrm{~B}(\mathrm{n}, \alpha)^{7} \mathrm{Li}$ reaction is exothermic, having a $Q$-value [see Eq. (4.1)] $Q_{v}=2.792 \mathrm{MeV}$, and leads either to the ground state of ${ }^{7} \mathrm{Li}$ or its first excited state at $0.482 \mathrm{MeV}$. The latter occurs for about $94 \%$ of the time when thermal neutrons are incident. Thus, for the dominant transition to the excited state, the reaction imparts about $2.31 \mathrm{MeV}$ to the reaction products. This energy is much larger than the kinetic energy of the incoming thermal neutron. Since energy and momenta must be conserved, for the dominant excited state branch the kinetic energy of the alpha particle $E(\alpha)=1.47 \mathrm{MeV}$ and, accordingly, $E\left({ }^{7} \mathrm{Li}\right)=0.84 \mathrm{MeV}$. This is because the following must hold:

$$
E\left({ }^{7} \mathrm{Li}\right)+E(\alpha)=2.31 \mathrm{MeV},
$$

due to energy conservation for the excited state branch, if one neglects the very small kinetic energy of the incident thermal neutron. Also,

$$
\sqrt{2 m\left({ }^{7} \mathrm{Li}\right) E\left({ }^{7} \mathrm{Li}\right)}=\sqrt{2 m(\alpha) E(\alpha)} .
$$




\section{ChAPTER 9 RAdiation Protection Instrumentation at ACCELERATORS}

holds due to conservation of momentum since the two reaction products emerge in opposite directions. The very small momentum of the thermal neutron is ignored and one recalls that, nonrelativistically, $p^{2}=2 m E$, where $m$ denotes the rest mass of the particle.

The excited state subsequently decays by emission of a photon. For this reaction, at 0.025 $\mathrm{eV} \sigma_{t h}=3837$ barns (Se81). The relatively large natural abundance of ${ }^{10} \mathrm{~B}$ is $19.8 \%$ compared with $80.2 \%$ for the other stable isotope, ${ }^{11} \mathrm{~B}(\mathrm{Tu} 05)$. The large natural abundance of ${ }^{10} \mathrm{~B}$ makes this reaction very favorable for thermal neutron detection. In addition, material enriched in ${ }^{10} \mathrm{~B}$ is readily available. Also the reaction products, and thus their deposited energies, being of short ionization range are contained in detectors of reasonable size. Fig. 9.1 gives the cross sections as a function of neutron energy for this and other thermal capture reactions discussed in this chapter. It is useful that the boron-10 reaction has a rather featureless cross section and obeys the $1 / v$ law quite well even up to an energy of approximately $0.5 \mathrm{MeV}$.

This reaction has been used principally in $\mathrm{BF}_{3}$ gas in proportional tubes. Proportional counters are somewhat similar in concept to ionization chambers except that the applied electric fields are of sufficient strength to accelerate the initial electrons liberated by the ionization to energies above the thresholds for liberating additional secondary electrons. In typical gases at one atmosphere, this threshold is of the order $10^{6}$ volts $\mathrm{m}^{-1}$. Under proper conditions, the number of electrons generated in this process can be kept proportional to the energy loss but the number of electrons released (and hence the size of the signal) can be amplified by a effective gain of a factor of many thousands. In proportional chambers, the region in which these secondary electrons are released is kept small compared to the chamber volume. If the voltage is raised beyond these conditions, then proportionality is lost and the counter enters the Geiger-Mueller mode. Knoll (Kn79) has given a detailed exposition on proportional chambers and the gas multiplication process. $\mathrm{BF}_{3}$ is the best of the boron-containing gases as a proportional counter gas because of its "good" properties as a counter gas and also because of the high concentration of boron in the gas molecule. Typical BF 3 tubes are biased at 2000 to 3000 volts with gas ionization multiplications ranging from about 100 to 500. An enriched $(96 \%) \mathrm{BF}_{3}$ tube can have an absolute detection efficiency of $91 \%$ at $0.025 \mathrm{eV}$ dropping to $3.8 \%$ at $100 \mathrm{eV}$ for neutrons incident upon it. Alternatives with somewhat better gas properties (and cleaner signals) have been achieved by using boron-lined chambers with other gases that have better properties in proportional chambers.

\subsubsection{Lithium-6}

The reaction of interest is ${ }^{6} \mathrm{Li}(\mathrm{n}, \alpha)^{3} \mathrm{H}$. For this reaction $Q_{\nu}=4.78 \mathrm{MeV}$. The process leads only to the ground state of ${ }^{3} \mathrm{H}$. As discussed in connection with the ${ }^{10} \mathrm{~B}(\mathrm{n}, \alpha)^{7} \mathrm{Li}$ reaction, conservation of energy and momentum can be shown to determine the result that $E\left({ }^{3} \mathrm{H}\right)=2.73 \mathrm{MeV}$ and $E(\alpha)=2.05 \mathrm{MeV}$. For incident thermal neutrons $\sigma_{t h}=941$ barns (Se81). The natural isotopic abundance of ${ }^{6} \mathrm{Li}$ is about 7.6\% (Tu05). Fig. 9.1 includes 


\section{ChAPTER 9 RADiATion Protection InSTRUMENTATION AT ACCELERATORS}

the cross section of this reaction as function of neutron kinetic energy. The cross section exhibits a significant resonance at about $3 \times 10^{5} \mathrm{eV}$. The apparent disadvantage of the "small" thermal cross section is offset by the higher $Q$-value and resultant larger signals.

For use in gas counters, no fill gas containing lithium having suitable properties analogous to those of $\mathrm{BF}_{3}$ has been found. Instead, ${ }^{6} \mathrm{Li}$ has been successfully added to scintillators. With the addition of a small amount $(<0.1 \%$ of the total atoms) of europium to $\mathrm{LiI}$ as $\mathrm{LiI}(\mathrm{Eu})$, the light output is as much as $35 \%$ of that of a comparable size $\mathrm{NaI}(\mathrm{Tl})$ crystal. Such scintillators have a decay time of approximately $0.3 \mu$ s. Of course, ${ }^{6} \mathrm{LiF}$ is in prominent use as a TLD and employs the same nuclear reaction. The TLD can be used in high dose rates, since no instantaneous readout is involved.

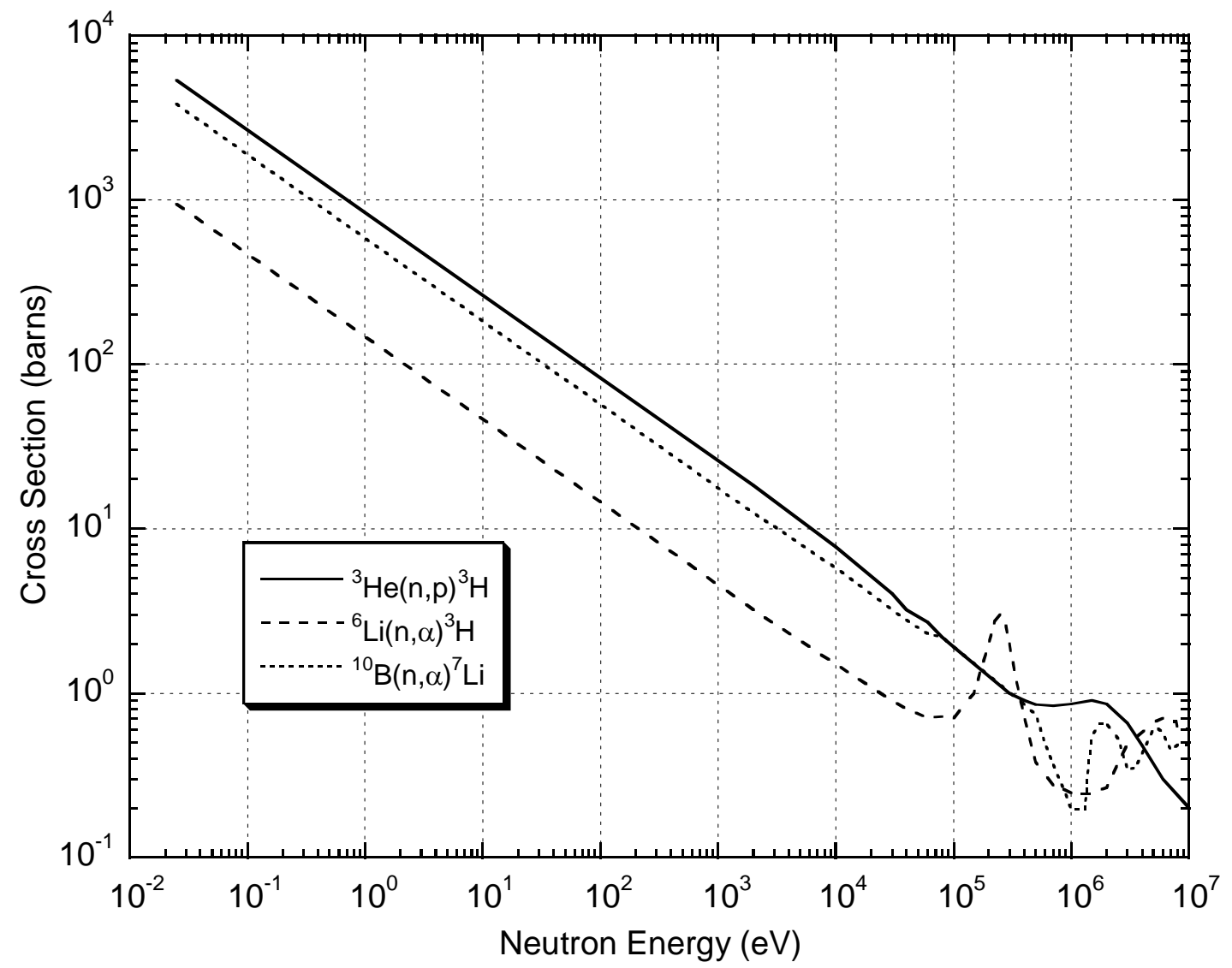

Fig. 9.1 Cross sections versus neutron energy for some reactions of interest in neutron detection. [Adapted from (Kn79).]

\subsubsection{Helium-3}

This nuclide, gaseous at room temperature, is used through the reaction ${ }^{3} \mathrm{He}(\mathrm{n}, \mathrm{p})^{3} \mathrm{H}$. The $Q_{v}=0.765 \mathrm{MeV}$ so that, as for the other reactions, $E(\mathrm{p})=0.574 \mathrm{MeV}$ and $E\left({ }^{3} \mathrm{H}\right)=0.191$ $\mathrm{MeV}$ for incident thermal neutrons. For this reaction $\sigma_{t h}=5327$ barns (Se81). Although this isotope of helium can be used directly as a detector gas, it has the disadvantage that 


\section{Chapter 9 RAdiation Protection Instrumentation at ACCELERAtors}

the natural abundance is only $0.000134 \%$ (Tu05), rendering enriched ${ }^{3} \mathrm{He}$ to be costly. There is also currently a global shortage of ${ }^{3} \mathrm{He}$. Also some of the energy can escape the sensitive volume of a detector of reasonable size because of the relatively long range of the emitted proton. Again, the cross section as a function of energy is given in Fig. 9.1. As seen, the cross section is quite "well-behaved" as a function of energy. ${ }^{3} \mathrm{He}$ is a reasonable gas for proportional chambers; however no compounds are available since it is a noble gas. In sufficient purity it will work as an acceptable proportional counter gas. Because a proton is the reaction product instead of the short range $\alpha$-particle, "wall effects" (i.e., effects in which some energy escapes the counting gas volume) may be somewhat more severe than for $\mathrm{BF}_{3}$. However, proportional chambers filled with ${ }^{3} \mathrm{He}$ can be operated at much higher pressures than are possible with $\mathrm{BF}_{3}$ and can thus have enhanced detection efficiency.

\subsubsection{Cadmium}

This discussion would be incomplete without discussing cadmium. Averaged over the naturally-occurring isotopes of cadmium, the thermal neutron capture reaction of form ${ }^{\mathrm{A}} \mathrm{Cd}(\mathrm{n}, \gamma){ }^{\mathrm{A}+1} \mathrm{Cd}$ has a cross section $\sigma_{t h}=2450$ barns (Se81). More spectacularly, the reaction ${ }^{113} \mathrm{Cd}(\mathrm{n}, \gamma){ }^{114} \mathrm{Cd}$ has a value of $\sigma_{t}=19910$ barns $(\mathrm{Se} 81) .{ }^{113} \mathrm{Cd}$ has a natural abundance of $12.2 \%$ (Tu05). Thus, even without using enriched material, the thermal neutron cross section is large. This element is not used directly in the detector medium. Rather, it is used to shield other detectors from thermal neutrons because the large cross section results in the absorption of essentially all neutrons with energies less than about $0.4 \mathrm{eV}$. Hence, one can do measurements with and without the cadmium inside of some moderator (see Section 9.5.2) and thus identify the thermal component.

\subsubsection{Silver}

Awschalom et al. were able to use thermal neutron capture on silver as a basis of a moderated detector (Aw72). As it occurs in nature, silver has two stable isotopes which both capture thermal neutrons via the $(\mathrm{n}, \gamma)$ process; ${ }^{107} \mathrm{Ag}(51.8 \%$ natural abundance, $\sigma_{t h}=40$ barns) and ${ }^{109} \mathrm{Ag}\left(48.2 \%\right.$ natural abundance, $\sigma_{t h}=93.5$ barns) [(Tu05),(Se81)]. The average value of $\sigma_{t h}$ is 63.6 barns for naturally-occurring silver (Se81). While the cross sections are not as large as those of some of the other reactions discussed, the material is readily available and enrichment is not needed. The detector which utilized these capture reactions was a moderated one (see below) in which the output of a GeigerMueller tube wrapped with silver that sensed the capture $\gamma$-rays was compared with an identical tube wrapped with tin (average mass number=118.7). Tin has an average value of $\sigma_{t h}=0.63$ barns (Se81) and is thus comparatively insensitive to thermal neutrons. The tin-wrapped tube was then used to subtract background due to muons, photons, etc.

\subsubsection{Moderated Neutron Detectors}

As seen above, many neutron reactions tend to have much smaller cross sections in the 


\section{Chapter 9 Radiation Protection Instrumentation at ACCELERAtors}

$\mathrm{MeV}$ region than they have in the "thermal" region. Historically, shortly after the discovery of the neutron, it was observed that surrounding a thermal neutron detector with hydrogenous material enhances detection rates exhibited by a "bare" thermal neutron detector placed in the same radiation field. The reason this occurs with hydrogenous materials is that discussed in connection with Eq. (6.1), repeated here for convenience. The fraction $\Delta E$ of the incident energy $E_{O}$ that can be transferred to the target nucleus after a collision where the target nucleus recoils at angle $\theta$ is given by

$$
\frac{\Delta E}{E_{o}}=\frac{4 \frac{M}{m_{n}} \cos ^{2} \theta}{\left(1+\frac{M}{m_{n}}\right)^{2}}
$$

where $M$ is the mass of the target nucleus and $m_{n}$ is the neutron mass. The energy that can be transferred in the reaction is maximized in the head-on collision $(\theta=0)$ and has its maximum value (1.0) when $M \approx m_{n}$ (i.e., for hydrogen). Even for a nucleus as light as ${ }^{12} \mathrm{C}$, the quantity $\left(\Delta E / E_{O}\right)_{\max }$ has a value of only 0.28 .

One might naively expect the detection efficiency to improve with the thickness of the moderator. However as the moderator thickness increases, the probability that a given moderated neutron will actually ever reach the detector decreases. Fig. 9.2 illustrates these tradeoffs. In general, the optimum thickness will for moderators such as polyethylene range from a few centimeters for $\mathrm{keV}$ neutrons to several tens of centimeters for $\mathrm{MeV}$ neutrons. Furthermore, for any given thickness, the overall counting efficiency as a function of energy will peak at some energy related to the thickness.

\subsubsection{Spherical Moderators, Bonner Spheres, and Related Detectors}

Bramblett, Ewing, and Bonner employed spherical moderators to obtain low resolution neutron spectra (Br60) using a method that has become known as the Bonner sphere technique. In this technique moderating spheres of different diameters surrounding a thermal neutron detector of some type are placed in a given radiation field. The normalized relative (or absolute) responses are indicative of the neutron energy spectra. As one might expect, the determination of the efficiency of each sphere as a function of energy is a rather complicated matter. Such response functions have been calculated, using techniques such as the Monte Carlo method, by a number of authors over the years since this method was invented. Hertel and Davidson (He85) have calculated the response functions for spheres that possess the "standard" set of diameters. Other response functions, perhaps more accurate in neutron fields of higher energies, have been reported by Awschalom and Sanna (Aw85). The response functions are dependent upon detector size as well as upon moderator thickness and density. The density is typically $0.95 \mathrm{~g} \mathrm{~cm}^{-3}$ for polyethylene. The results of Awschalom and Sanna are given in Fig. 9.3 for cylindrical $\mathrm{LiI}(\mathrm{Eu})$ detectors of lengths equal to their diameters which are each 1.27 


\section{Chapter 9 RAdiation Protection Instrumentation at ACCElerators}

$\mathrm{cm}$ (0.5 inch) in polyethylene spheres of this density. Intuitively, larger detectors readily give a higher efficiency response in accordance with the size of their sensitive volumes.

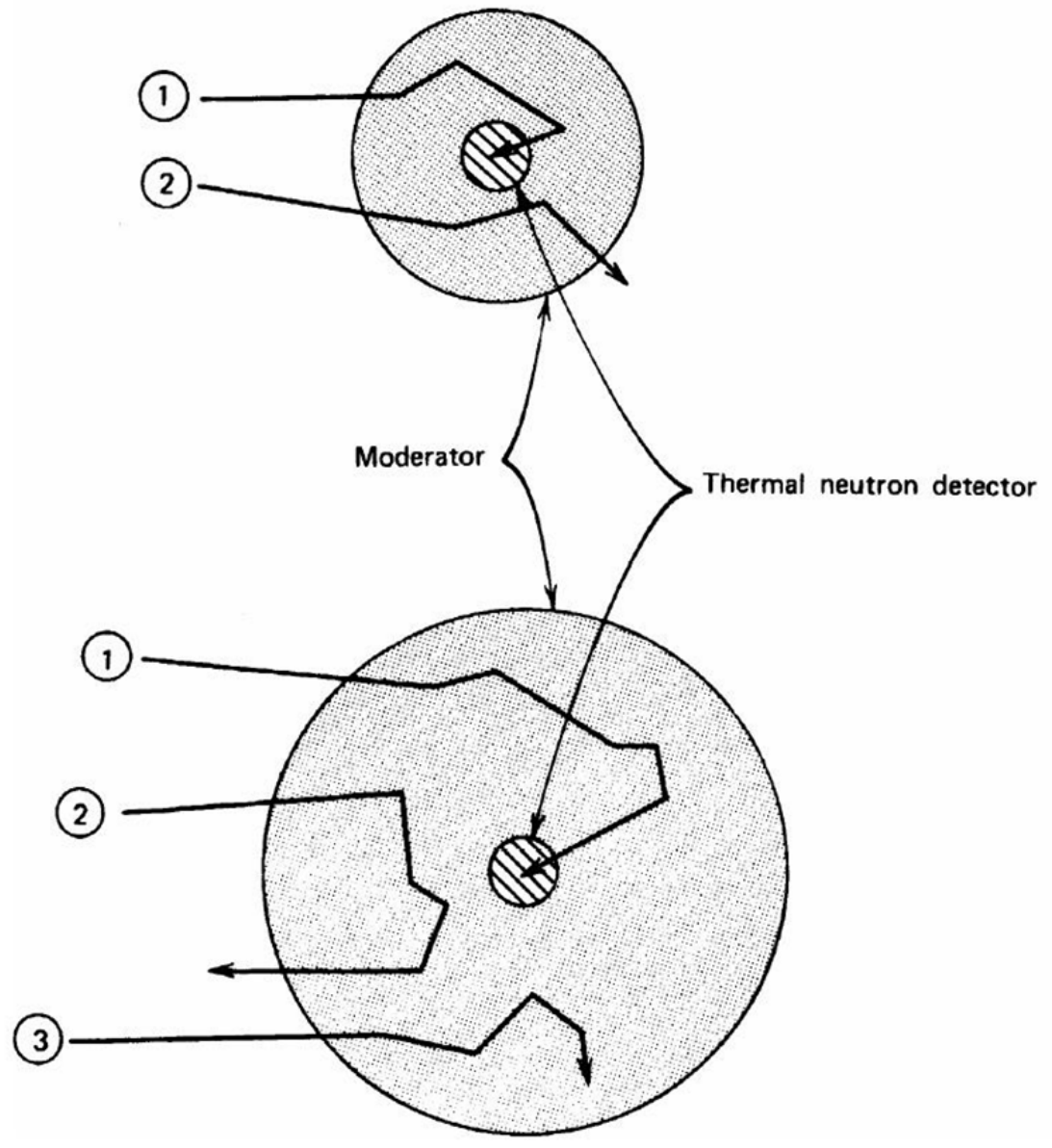

Fig. 9.2 Schematic representation of neutron tracks in moderated detectors. The small thermal neutron detector at the center is shown surrounded by two different thicknesses of moderator material. The track labeled $\mathbf{1}$ represents incident fast neutrons that are successfully moderated and detected. The track labeled 2 represents those neutrons partially or fully moderated, but escape without reaching the detector. Track 3 represents those neutrons that are parasitically captured by the moderator. Larger moderators will tend to enhance process 3 while reducing process 2. [Reproduced from (Kn79).]

Most of the efficiency calculations have been made for ${ }^{6} \mathrm{LiI}(\mathrm{Eu})$ scintillators, but also can be used for ${ }^{6} \mathrm{LiF}$ TLD dosimeters. They cannot, in general, be used for other thermal neutron capture reactions used to detect thermal neutrons as the neutron cross sections needed for the calculation of the responses will differ. There are other sets of response functions extant. Experimental verifications of the details of these response functions are rare because of the difficulty of the measurements. Kosako et al. (Ko85) have successfully verified some of the important response functions using a neutron time-offlight technique in the especially difficult $\mathrm{keV}$ energy region of neutron energy. A Bonner sphere determination of the neutron spectrum is comprised of a set of measurements of 


\section{Chapter 9 RAdiation Protection Instrumentation at ACCELERATORS}

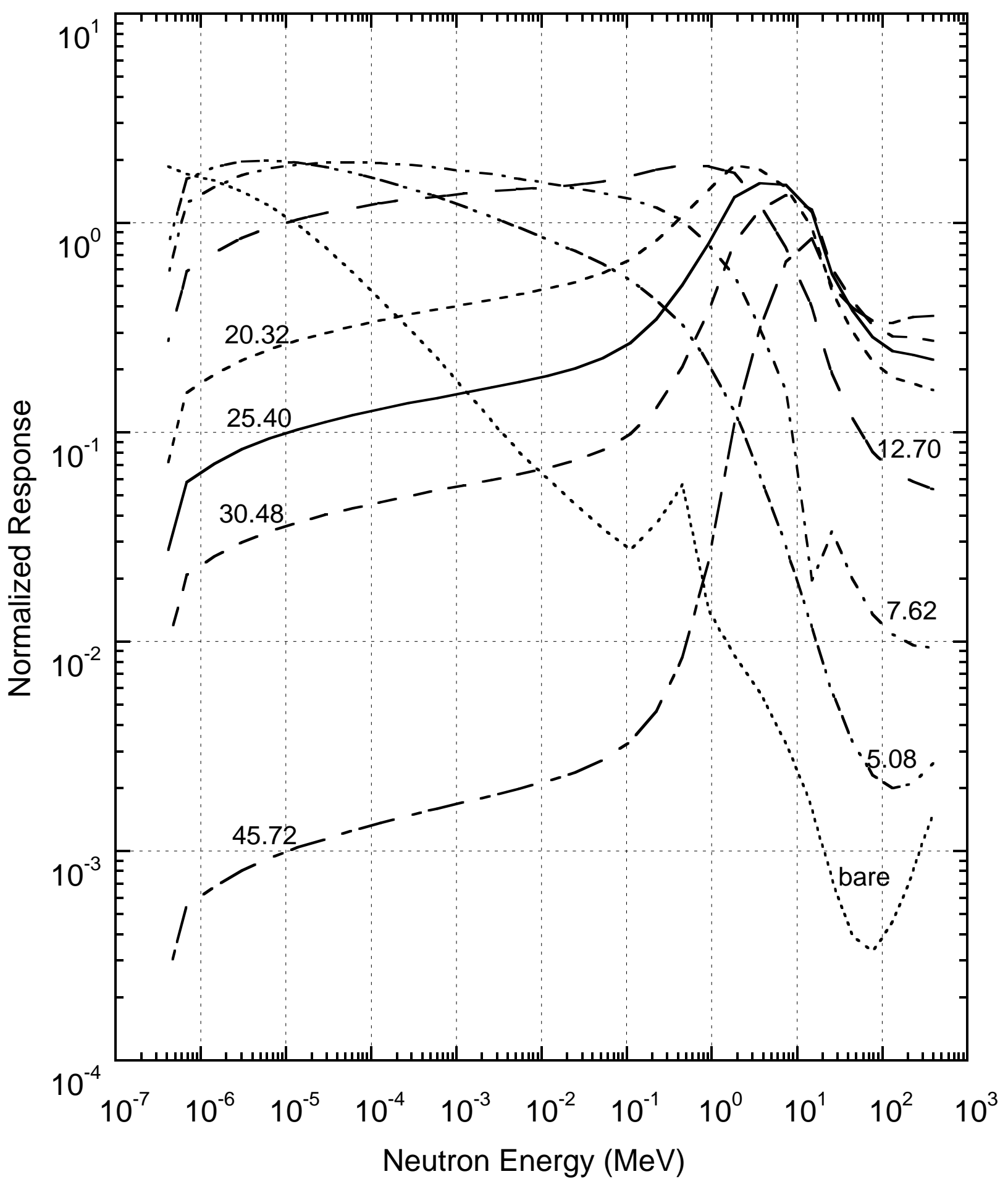

Fig. 9.3 The calculated responses for the bare $1.27 \mathrm{~cm}$ diameter by $1.27 \mathrm{~cm}$ long cylindrical LiI detector and for the same detector inside 5.08, 7.62, 12.7, 20.32, 25.4, 30.48, 38.1, and 45.72 $\mathrm{cm}$ diameter spheres as a function of neutron energy. The detector is a cylinder having a length equal to its diameter. The "normalization" is that needed to provide the correct responses according to Eq. (9.25). [Adapted from (Aw85), which contains tables of numerical values of these functions.] 


\section{Chapter 9 RAdiation Protection Instrumentation at ACCELERAtors}

the responses $r$ for the different spheres of radius $C_{r}$ where $r$ has the discrete values based on the available set. Such responses ideally are given by

$$
C_{r}=\int_{0}^{\infty} d E \frac{d N}{d E} R_{r}(E)
$$

where $d N / d E$ is the differential neutron flux density (the neutron spectrum) and $R_{r}(E)$ is the energy-dependent response function for the sphere of radius $r$. One measures $C_{r}$ and knows $R_{r}(E)$ with the objective of determining $d N / d E$ by unfolding the spectrum. In practice, one works with a discrete approximation to the integral;

$$
C_{r}=\sum_{i} \frac{d N}{d E_{i}} R_{r}\left(E_{i}\right) \Delta E_{i}
$$

where the index $i$ labels each member of the set of energy groups used. The unfolding procedure is a difficult mathematical problem that, unfortunately, suffers from being underdetermined and mathematically ill-conditioned. One has as many "unknowns" as one has energy groups, with typically only 8 or 9 measurements to determine the response. It is common for 31 energy groups to be used in an attempt to achieve "reasonable" energy resolution in the results.

Prominent computer codes in use at accelerators include BUNKI (Lo84), LOUHI (Ro80), and SWIFT (OB81). The first uses an iterative recursion method and the second uses a least squares fitting procedure with user-controlled constraints. One essentially starts with an "educated guess" at the spectrum and iterates to fit the responses. As we have seen, a $1 / E$ spectrum is a good starting point for an accelerator spectrum. SWIFT is based upon a somewhat different principle; it is a Monte Carlo program that makes no a priori assumptions on the spectrum and can thus provide a "reality check" on results using the other two. It has the disadvantage in that it is known to sometimes produce nonphysical peaks in the unfolded spectrum. In general, the codes agree best with each other for those properties that are determined by integrating over the spectrum such as the average quality factor, total fluence, and total absorbed dose and dose equivalent. Typical spectra obtained from such unfolding procedures have been reported at a number of laboratories. Fermilab results have been summarized by Cossairt et al. (Co88) and revisited by Cossairt and Vaziri (Co09a). Results are generally similar to those obtained at other laboratories. Further discussion of examples of neutron spectrum measurements is given in Chapter 6.

It is sometimes important to verify the reasonableness of the unfolded spectrum. Comparisons can be made with known spectra from radioactive sources such $\mathrm{PuBe}$ or $\mathrm{AmBe}$ and such comparisons have been made (e.g., Co88). The normalized responses $C_{r}$ can be directly used to check the qualitative "reasonableness" of the unfolded spectrum. For example, this was done for measurement in the labyrinth discussed in connection 


\section{Chapter 9 RAdiation Protection Instrumentation at ACCElerators}

with Fig. 6.7 and for the iron leakage measurements described in connection with Fig. 6.8. The results are shown in Fig. 9.4 and Fig. 9.5. In Fig. 9.4 the labyrinth responses are compared with the sphere responses for a pure thermal neutron spectrum. The enhanced responses for the intermediate-sized spheres correlate with the somewhat more energetic unfolded neutron spectrum. For the iron leakage spectrum (Fig. 9.5), one can see evidence for the "softening" of the spectrum after the concrete was added.

In the use of ${ }^{6} \mathrm{LiI}(\mathrm{Eu})$ scintillators for such detectors in mixed fields, there are situations in which the signals from photons and/or muons can overwhelm the neutron signal. Awschalom and Coulson (Aw73) developed a technique in which the ${ }^{6} \mathrm{LiI}(\mathrm{Eu})$ is surrounded by plastic scintillator. The physical configuration of such a phoswich detector, and a typical pulse height spectrum obtained by use of this detector in a long exposure to environmental neutrons are given in Figs. 9.6 and 9.7. The same detector

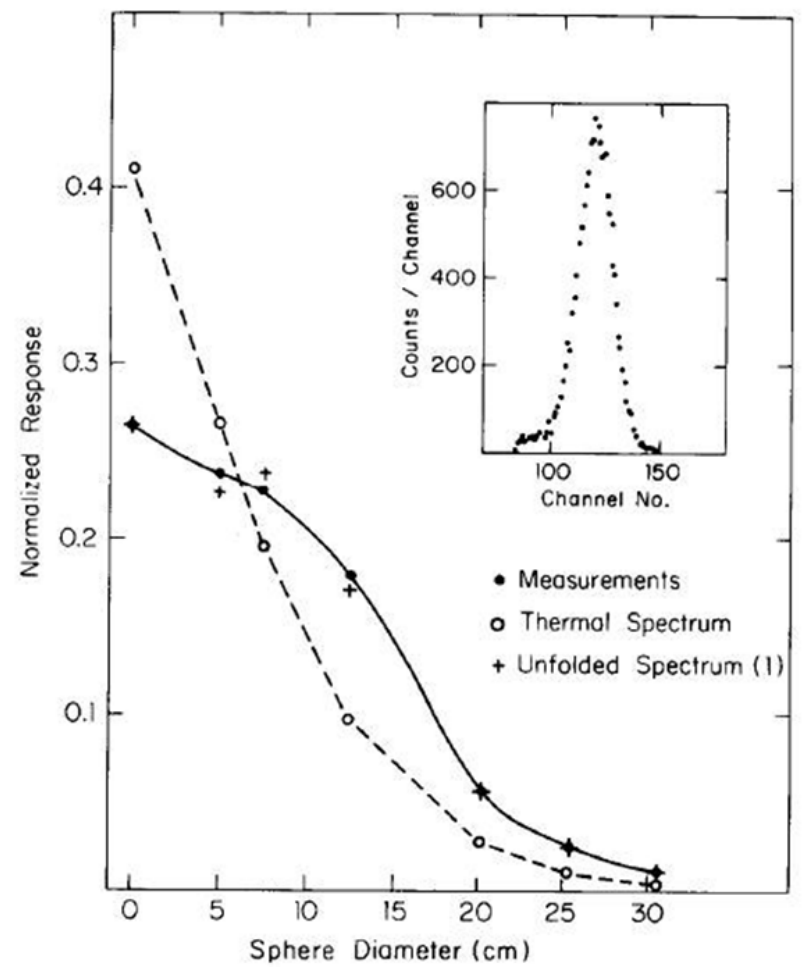

Fig. 9.4 Normalized response from the detector as a function of spherical moderator diameter. The solid circles are the measurements within the second leg of the labyrinth shown in Fig. 6.7. The open circles represent calculated results assuming a purely thermal spectrum while the crosses are the results for the neutron energy spectrum unfolded using the program SWIFT. The solid and dashed curves are drawn to guide the eye. The inset shows a typical gated spectrum of the pulse heights in the ${ }^{6} \mathrm{LiI}(\mathrm{Eu})$ phoswich detector described in the text. [Reproduced from (Co85b).]

was used to produce the pulse-height spectrum shown in the inset in Fig. 9.4. In this technique, a "fast" discriminator is set to respond to the 2-3 nanosecond decay time of the plastic scintillation signal while a "slow" discriminator is set to respond to the $1.4 \mu \mathrm{sec}$ 


\section{Chapter 9 RAdiation Protection Instrumentation at ACCElerators}

decay time of the crystal. Selecting the slow counts not accompanied by fast counts clearly gives superior discrimination against non-neutron events from environmental radiation (e.g., cosmic ray muons) which produces coincident pulses in both the crystal and the plastic scintillator (see Fig. 9.7).

In performing Bonner sphere measurements in neutron fields that are suspected of being spatially nonuniform in space, it may be necessary to measure $C_{r}$ over the set of spheres individually because arranging them in an array may result not only in undesired "crosstalk" between the moderators but also in the need to make corrections for the nonuniformities of the radiation field.

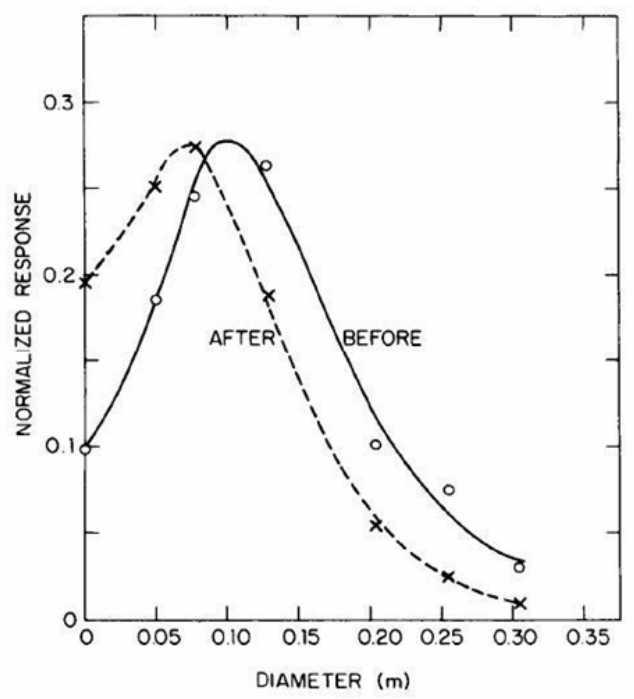

Fig. 9.5 Normalized detector response as a function of spherical moderator diameter for the situation described in Fig. 6.8. The open circles are the measurements before, and the $\mathrm{X}$ s are the measurements after the placement of the additional concrete shielding. [Reproduced from (E186).]

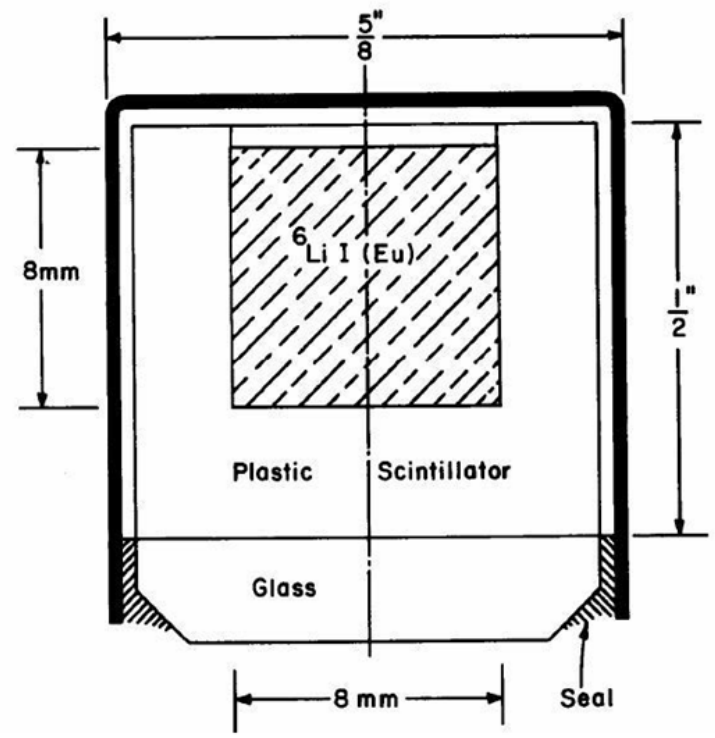

Fig. 9.6 Cross section of $8 \mathrm{~mm} \times 8 \mathrm{~mm}$ cylindrical phoswich. [Reproduced from (Aw73).] 


\section{Chapter 9 Radiation Protection Instrumentation at ACCElerators}

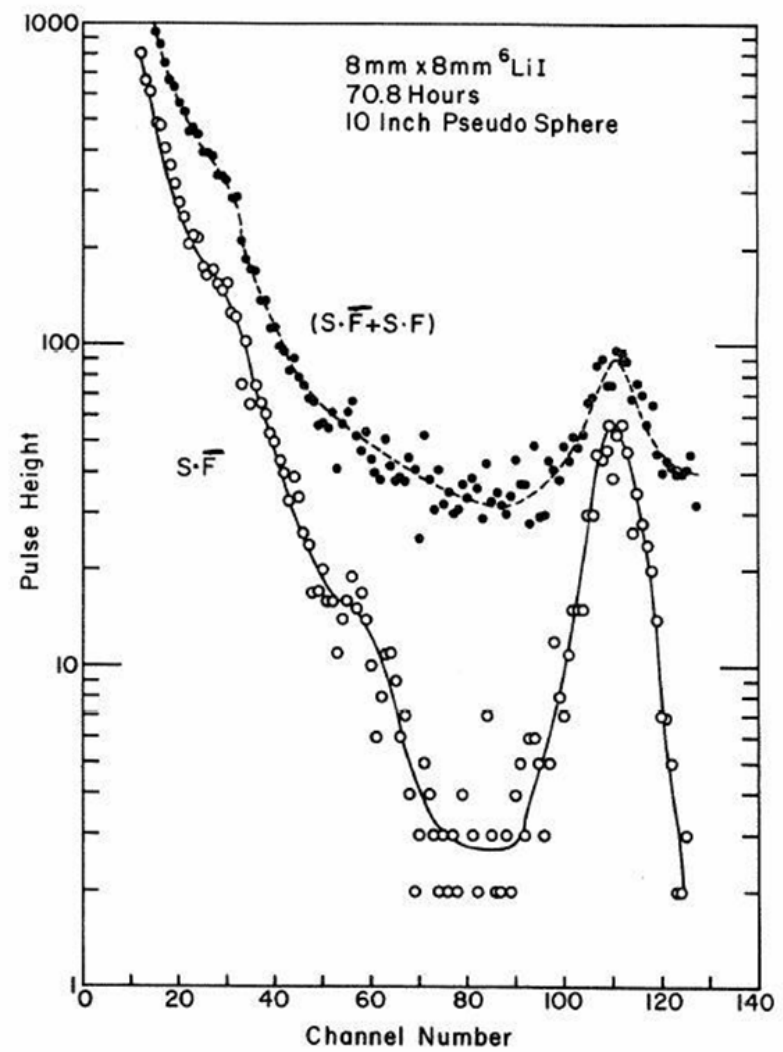

Fig. 9.7 Pulse height spectra obtained using the phoswich shown in Fig. 9.6 in a natural background radiation field. The upper curve (filled circles) is a spectrum of all slow pulses (slow without fast and slow with fast). The lower curve (open circles) is a spectrum of slow pulses not accompanied by fast pulses, interpreted to be due to neutrons. [Reproduced from (Aw73).]

Since accelerator neutron fields are often quite similar to each other, it was noticed that the choice of a single moderator size might well offer the opportunity to construct a remmeter. Such an instrument uses a given sphere response function particularly well matched to energy dependence of the dose per fluence factor $P$. The standard example of this is the Andersson-Braun detector equipped with a $\mathrm{BF}_{3}$ detector (An62). This technique was reviewed by Thomas and Stevenson (Th88). Generally the $25.4 \mathrm{~cm}$ (10 inch) diameter polyethylene sphere is used because its response curve provides the best match to the dose equivalent per fluence function. Höfert and Raffnsøe (Hö80) have measured the dose equivalent response of such an instrument as a function of neutron energy with the results given in Table 9.5. Commercial versions of this instrument usually operate in the proportional counter mode, a choice that renders them somewhat suspect in accelerator fields with high instantaneous dose rates due to small duty factors. A similar detector using ${ }^{6} \mathrm{LiI}(\mathrm{Eu})$ as the detector has been developed by Hankins (Ha62). Its response is shown in Fig. 9.8 compared with the "Inverse of the Radiation Protection Guide (RPG)" curve that embodies the relative dose equivalent delivered per neutron as a function of neutron energy. In the $\mathrm{keV}$ region, comparisons are difficult and there is some evidence that this detector over responds considerably. However, the matching was 


\section{Chapter 9 Radiation Protection Instrumentation at ACCELERAtors}

verified at thermal neutron energies. Leake (Le68) developed an alternative detector of this type. In this detector a ${ }^{3} \mathrm{He}$ proportional counter is used in a 20.8 diameter sphere to reduce background due to photons along with a cadmium filter against thermal neutrons. It is claimed that this detector is effective in photon fields as intense as $20 \mathrm{rads} \mathrm{h}^{-1}$. This type of instrument may seriously underestimate neutron dose rates above $10 \mathrm{MeV}$. The impact of the transition to the 1990 System on these results merits further study.

Table 9.5 Dose equivalent response and measurement errors for a $25.4 \mathrm{~cm}$ diameter polyethylene moderating sphere as a function of neutron energy. [Adapted from (Hö80)]

\begin{tabular}{|c|c|c|}
\hline $\begin{array}{c}\text { Neutron Energy } \\
(\mathbf{M e V})\end{array}$ & $\begin{array}{c}\text { Dose Equivalent Response } \\
\left(\mathbf{1 0} \mathbf{C}_{\mathbf{C}} \mathbf{C o u l o m b s ~ S v}^{-\mathbf{1}}\right)\end{array}$ & $\begin{array}{c}\text { Error } \\
\mathbf{( \% )}\end{array}$ \\
\hline Thermal & 0.349 & 10.0 \\
\hline 0.0245 & 3.209 & 12.1 \\
\hline 0.1 & 1.335 & 6.8 \\
\hline 0.25 & 1.082 & 6.1 \\
\hline 0.57 & 0.923 & 5.2 \\
\hline 1.0 & 0.745 & 5.2 \\
\hline 2.5 & 0.784 & 6.1 \\
\hline 5.0 & 0.653 & 5.2 \\
\hline 15.5 & 0.348 & 5.2 \\
\hline 19.0 & 0.445 & 12.2 \\
\hline 280.0 & 0.157 & 10.1 \\
\hline
\end{tabular}

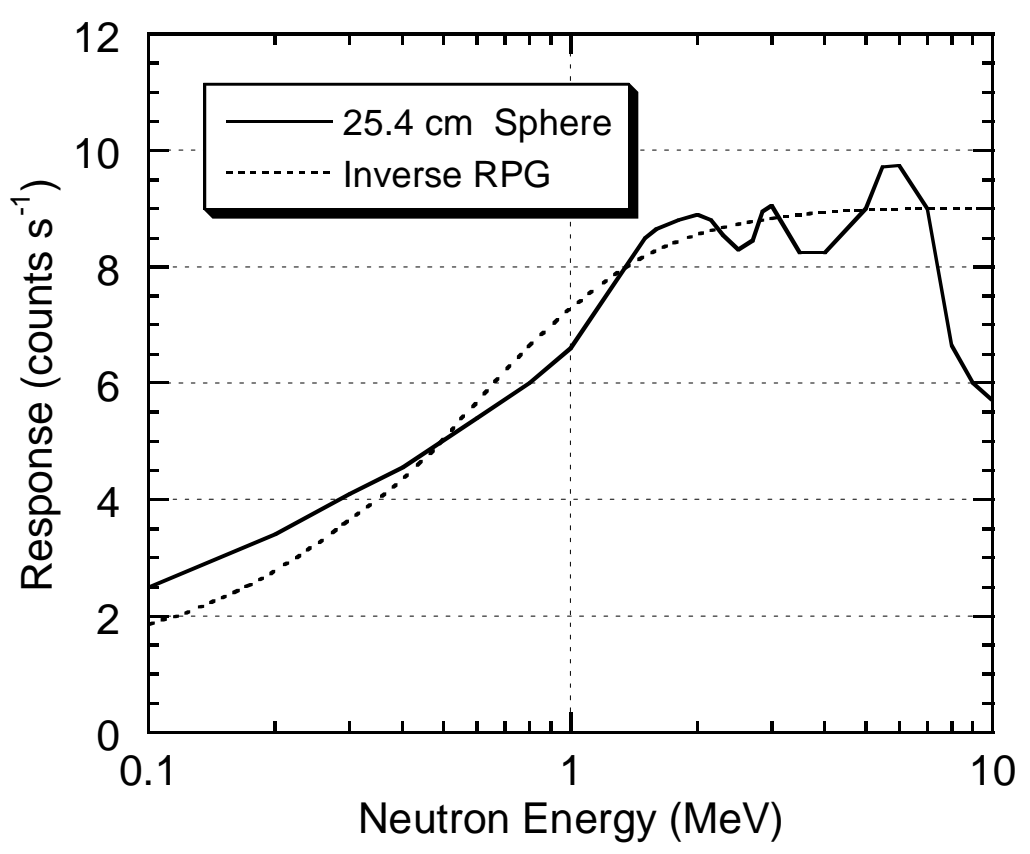

Fig. 9.8 Sensitivity of detector comprised of a $25.4 \mathrm{~cm}(10 \mathrm{in}$.$) diameter moderating sphere$ surrounding a $4 \times 4 \mathrm{~mm}^{2}$ cylindrical LiI scintillator in counts $\mathrm{s}^{-1}$ at $40 \mathrm{~cm}$ distance from a source of $10^{6}$ neutrons $\mathrm{s}^{-1}$. Also shown is the relative dose equivalent per neutron labeled as "Inverse RPG". At thermal energies, the response was measured to be 0.227 compared with a value of 0.225 for the "Inverse RPG" curve (see text). [Adapted from (Ha62).] 


\section{Chapter 9 Radiation Protection Instrumentation at ACCElerators}

It is not necessary for radiation protection purposes, that a "spherical" moderator be an exact sphere. Awschalom et al. (Aw72) measured the responses of three polyethylene moderators; a sphere, an octagon of revolution (a "pseudosphere"), and a cylinder. The sphere had a diameter of $25.4 \mathrm{~cm}$ and the dimensions of the other moderators were chosen to have the same volume as that of the sphere. The results of the measurements of Awschalom et al. are shown in Fig. 9.9. The "polar axis" is defined by the light pipe used to read out the scintillator placed in the center of each moderator. It was found that the alternative moderators have a response only mildly distinguishable from that of the sphere as a function of polar angle. Such pseudospheres and cylinders are desirable because they are cheaper to machine than are spheres. They can also be set on a flat surface without rolling about.

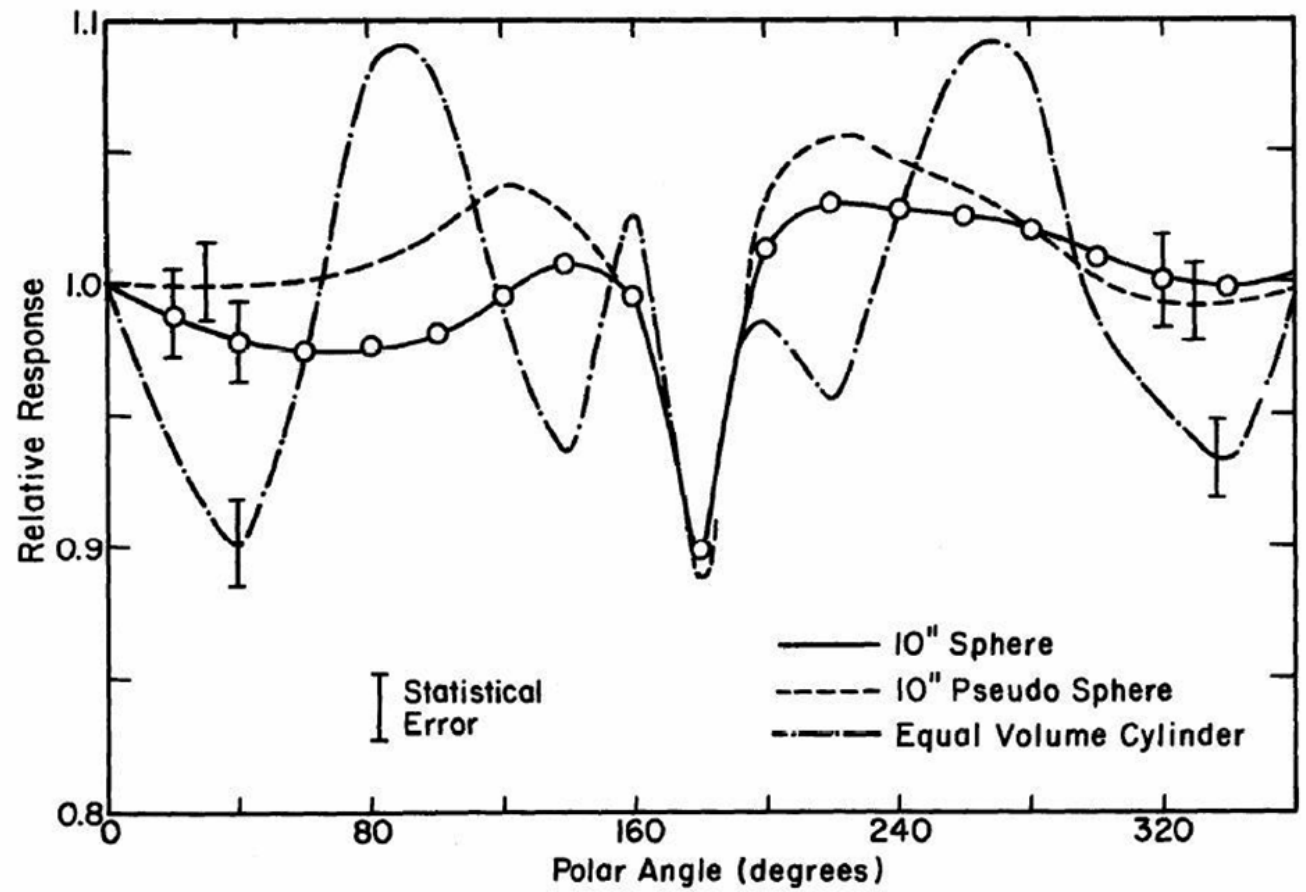

Fig. 9.9 Relative neutron detection efficiency of three different moderators with a $4 \times 4 \mathrm{~mm}^{2}$ cylindrical ${ }^{6} \mathrm{LiI}(\mathrm{Eu})$ detector at the center. The efficiencies are plotted as a function of the polar angle. The polar angle is measured from the axis of the light pipe. [Adapted from (Aw72).]

\subsubsection{Long Counters}

Another type of moderated neutron detector that has been used extensively is the long counter. The idea is to adjust the configuration of moderators around some thermal neutron detector in such a manner as to assure that the detection efficiency is approximately independent of energy over as "long" of an energy domain as practical. It has been found empirically that the best detector is a cylinder of moderating materials surrounding a thermal neutron detector (also cylindrical) on the axis. Since a cylindrical detector is desired, the $\mathrm{BF}_{3}$ proportional counter is the most popular. One end of the cylinder "views" the neutron source for best results. Hanson and McKibben (Ha47) were the pioneers of the technique. An improved version, which has rather widespread use, is 


\section{Chapter 9 Radiation Protection Instrumentation at ACCELERATORS}

that developed by DePangher and Nichols (DeP66). Fig. 9.10 shows the layout of this detector. The length and diameter are both approximately $41 \mathrm{~cm}$ and the mass is about 45 $\mathrm{kg}$. It is designed and calibrated for use with the neutrons incident on the "front" face.

Perhaps the best calibration data on this device is that of Slaughter and Rueppel (S177). They used filtered beams from a reactor $\left(E_{n} \approx \mathrm{keV}\right)$ as well as monoenergetic neutron beams from $(\mathrm{p}, \mathrm{n})$ and $(\mathrm{d}, \mathrm{n})$ reactions at accelerators to cover the energy range from 10 $\mathrm{keV}$ to $19 \mathrm{MeV}$. An average of about 3.5 counts $\mathrm{cm}^{2}$ neutron ${ }^{-1}$ sensitivity was reported over this energy domain, with deviations of from 5 to 30 per cent from absolute independence of neutron energy. A similar detector has been used to conduct studies of skyshine at Fermilab [(Co85c), (E186)]. The large peak in the pulse-height spectrum of the $\mathrm{BF}_{3}$ tube from thermal neutron capture $\left(Q_{v}=2.79 \mathrm{MeV}\right)$ renders the detector essentially insensitive, with the application of simple pulse-height discriminator, to all other radiations. Knoll (Kn79) summarizes results with long counters of alternative designs that achieve better uniformity and higher levels of sensitivity.

\subsubsection{Activation Detectors}

As we have seen, certain nuclear reactions have relatively sharp thresholds which can be used to determine portions of a hadron spectrum that exceed it since the "leveling off" of the cross sections are generally well-behaved. In addition to information on reaction thresholds provided in Chapter 7, where referral was made to activation threshold techniques, Table 9.6 summarizes some of the useful reactions along with some pertinent information about threshold detectors that have been found to be useful in practical work. Some of these reactions will be discussed further below. Thomas and Stevenson [(Th85), (Th88)] provide a list of other reactions that might have useful thresholds.

Table 9.6 Important characteristics of various activation detector nuclear reactions.

\begin{tabular}{|c|c|c|c|c|c|c|c|}
\hline Detector & Reaction & $\begin{array}{l}\text { Energy } \\
\text { Range } \\
(\mathrm{MeV})\end{array}$ & $\begin{array}{c}\text { Half-life } \\
\text { (Tu05) }\end{array}$ & $\begin{array}{c}\text { Typical } \\
\text { Detector Size }\end{array}$ & $\begin{array}{c}\text { Cross } \\
\text { Section- } \\
\text { Peak (mb) }\end{array}$ & $\begin{array}{c}\text { Cross } \\
\text { Section-High } \\
\text { Energy }(\mathbf{m b}) \\
\end{array}$ & $\begin{array}{l}\text { Particle } \\
\text { Detected }\end{array}$ \\
\hline sulfur & ${ }^{32} \mathrm{~S}(\mathrm{n}, \mathrm{p}){ }^{32} \mathrm{P}$ & $>3$ & $14.26 \mathrm{~d}$ & $4 \mathrm{~g}$ disk & $500^{\mathrm{a}}$ & $10^{\mathrm{a}}$ & $\beta^{-}$ \\
\hline aluminum & ${ }^{27} \mathrm{Al}(\mathrm{n}, \alpha)^{24} \mathrm{Na}$ & $>6$ & $15.00 \mathrm{~h}$ & $16-6600 \mathrm{~g}$ & $11^{\mathrm{b}}$ & $9^{b}$ & $\gamma$ \\
\hline aluminum & $\left.{ }^{27} \mathrm{Al}(\mathrm{n}, \mathrm{x})\right)^{22} \mathrm{Na}$ & $>25$ & $2.603 \mathrm{y}$ & $17 \mathrm{~g}$ & $30^{\mathrm{b}}$ & $10^{\mathrm{b}}$ & $\gamma$ \\
\hline $\begin{array}{l}\text { plastic } \\
\text { scintillator }\end{array}$ & ${ }^{12} \mathrm{C} \rightarrow{ }^{11} \mathrm{C}$ & $>20$ & $20.33 \mathrm{~min}$ & $13-2700 \mathrm{~g}$ & $90^{\mathrm{b}}$ & $30^{\mathrm{b}}$ & $\beta^{+}, \gamma$ \\
\hline $\begin{array}{l}\text { plastic } \\
\text { scintillator }\end{array}$ & ${ }^{12} \mathrm{C} \rightarrow{ }^{7} \mathrm{Be}$ & $>30$ & $53.24 d$ & $17 \mathrm{~g}$ & $18^{\mathrm{b}}$ & $10^{\mathrm{b}}$ & $\gamma$ \\
\hline mercury & ${ }^{198} \mathrm{Hg} \rightarrow{ }^{149} \mathrm{~Tb}$ & $>600$ & $4.12 \mathrm{~h}$ & up to $500 \mathrm{~g}$ & $2^{b}$ & $1^{\mathrm{b}}$ & $\alpha, \gamma$ \\
\hline gold & ${ }^{197} \mathrm{Au} \rightarrow{ }^{149} \mathrm{~Tb}$ & $>600$ & $4.12 \mathrm{~h}$ & $0.5 \mathrm{~g}$ & $1.6^{\mathrm{b}}$ & $0.7^{\mathrm{b}}$ & $\alpha, \gamma$ \\
\hline copper & $\mathrm{Cu} \rightarrow{ }^{24} \mathrm{Na}$ & $>600$ & $15.00 \mathrm{~h}$ & $580 \mathrm{~g}$ & $4^{c}$ & $3.9^{c}$ & $\gamma$ \\
\hline copper & $\mathrm{Cu} \rightarrow{ }^{52} \mathrm{Mn}$ & $>70$ & $5.59 \mathrm{~d}$ & $580 \mathrm{~g}$ & $5^{\mathrm{c}}$ & $4.6^{\mathrm{c}}$ & $\gamma$ \\
\hline copper & $\mathrm{Cu} \rightarrow{ }^{54} \mathrm{Mn}$ & $>80$ & $312.1 \mathrm{~d}$ & $580 \mathrm{~g}$ & $11^{\mathrm{c}}$ & $11^{\mathrm{c}}$ & $\gamma$ \\
\hline
\end{tabular}

aswanson and Thomas (Sw90)

bBarbier (Ba69)

c Baker et al. (Ba84 and Ba91). 


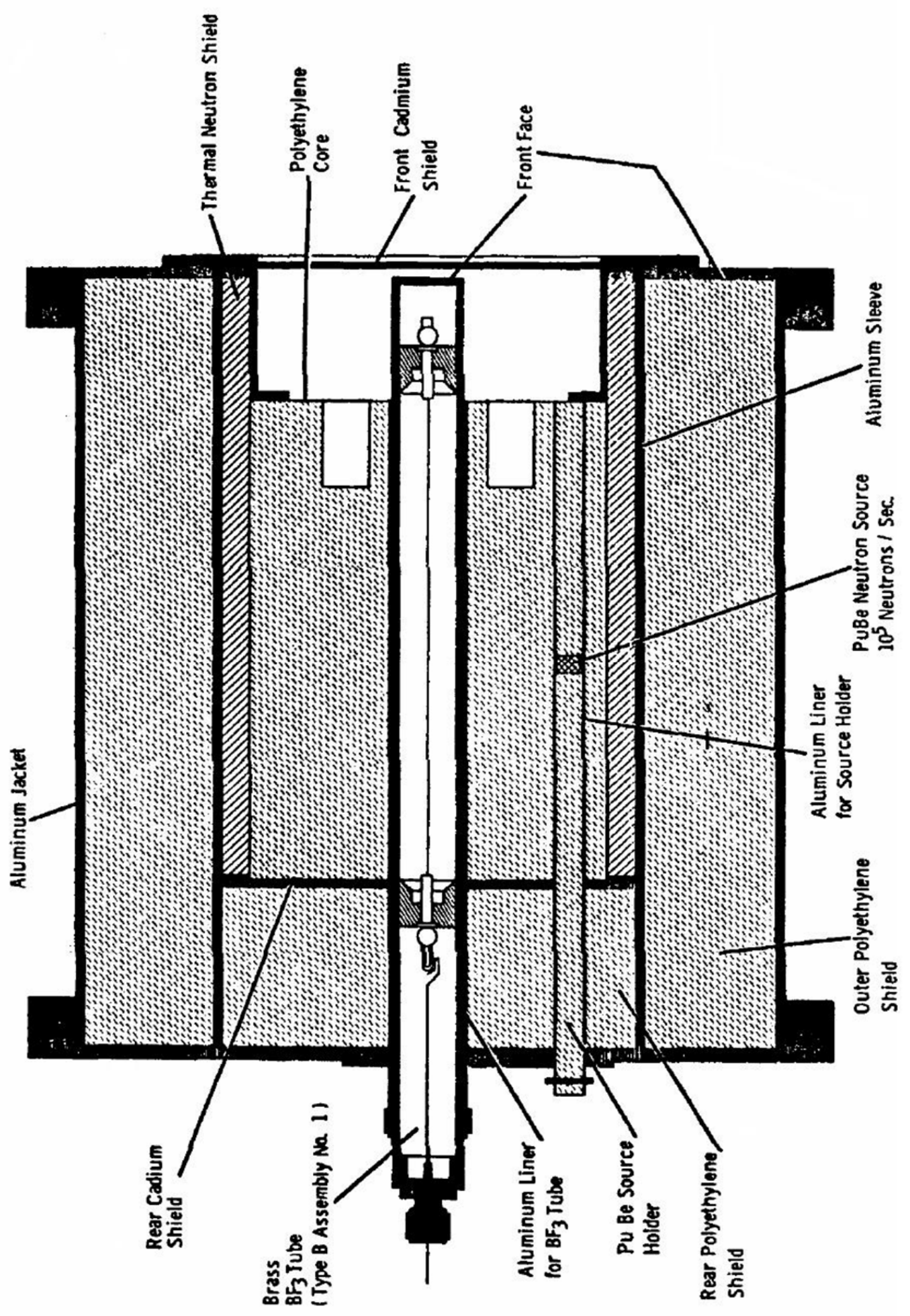

Fig. 9.10 Schematic diagram of a DePangher Long Counter. The version shown contains an optional built-in PuBe neutron source. The source would not be desirable in an instrument to be used in radiation fields near natural background. The dimensions and mass of this instrument are given in the text. [Reproduced from (DeP66).] 


\section{Chapter 9 RAdiation Protection Instrumentation at ACCELERAtors}

The family of reactions which produce ${ }^{11} \mathrm{C}$ from ${ }^{12} \mathrm{C}$ are of special interest because of the fact that plastic scintillators can themselves become activated by hadrons (especially neutrons and protons) exceeding $20 \mathrm{MeV}$. This technique was first developed by McCaslin (McC60). The cross sections for the production of ${ }^{11} \mathrm{C}$, as initiated by several different types of incident particles, are shown in Fig. 9.11. Stevenson (St84b) has determined that a value of $28 \mathrm{fSv} \mathrm{m}^{2}$ is an appropriate factor to apply to the conversion of the measured fluence of neutrons with $E_{n}>20 \mathrm{MeV}$ to the associated dose equivalent, assuming a typical accelerator spectrum found within thick shields of earth or concrete. Such measurements can be useful to determine the contribution of the high energy $(E>20$ $\mathrm{MeV}$ ) neutrons to the total neutron dose equivalent.

Moritz (Mo89) found that the use of NE102A scintillators activated by the reaction ${ }^{12} \mathrm{C}(\mathrm{n}, 2 \mathrm{n}){ }^{11} \mathrm{C}$ can be included as an additional high energy detector in a Bonner sphere measurement in order to extend the energy range. Following Stevenson, Moritz used an average cross section of $22 \mathrm{mb}$ for the ${ }^{12} \mathrm{C}(\mathrm{n}, 2 \mathrm{n}){ }^{11} \mathrm{C}$ reaction. NE102A, a common and typical plastic scintillator, has a carbon content of $4.92 \times 10^{22}$ atoms $\mathrm{g}^{-1}$ and a density of $1.032 \mathrm{~g} \mathrm{~cm}^{-3}(\mathrm{Kn} 79)$. Moritz used a cylindrical detector $5.0 \mathrm{~cm}$ in diameter by $5.0 \mathrm{~cm}$ long achieving an efficiency of $93 \%$ in detecting the $0.511 \mathrm{MeV}$ annihilation $\gamma$-rays produced as a result of the ${ }^{11} \mathrm{C}$ decay. In effect, the addition of this reaction reduced the degeneracy of the spectrum unfolding process using the code LOUHI.

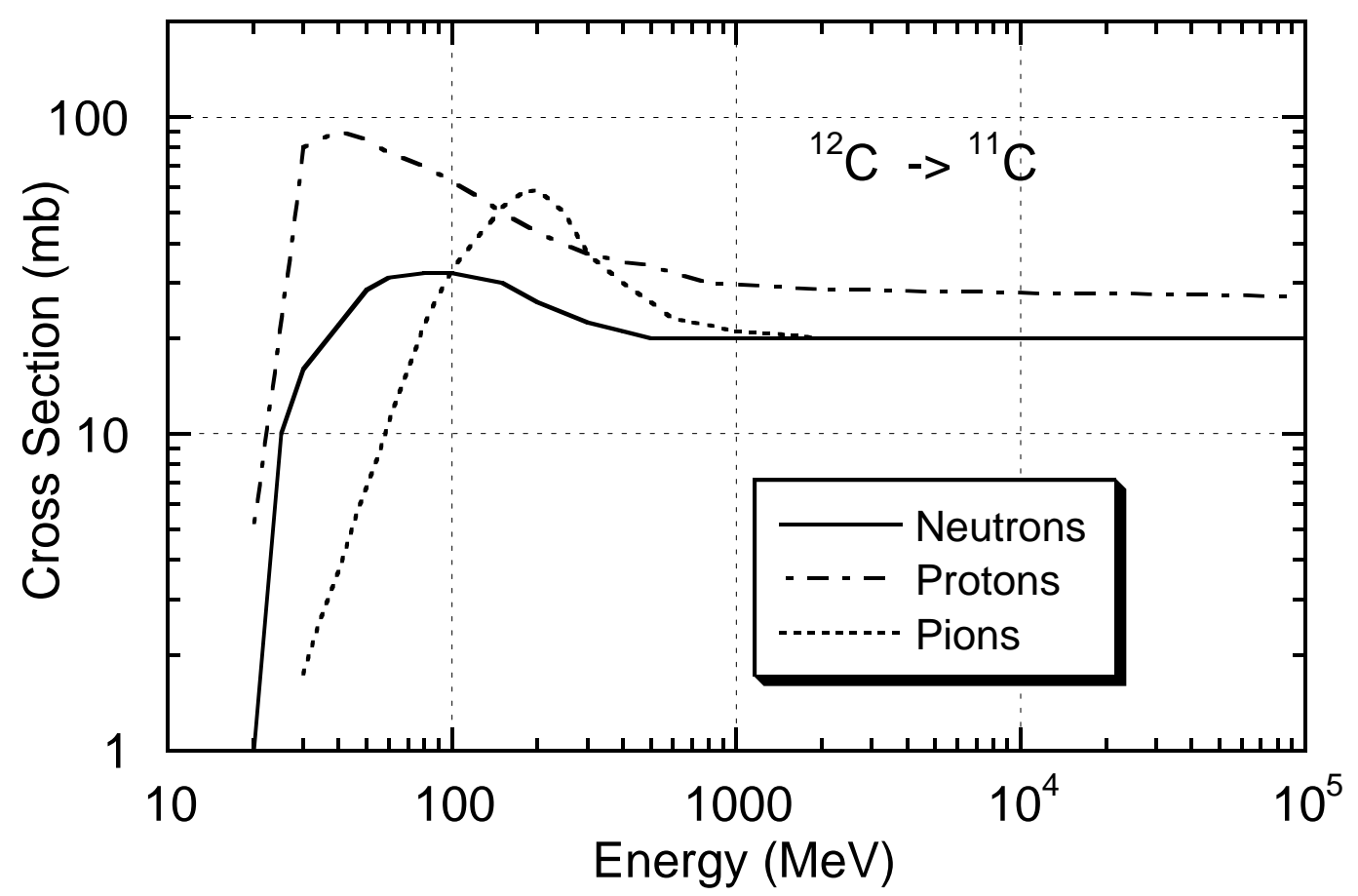

Fig. 9.11 Excitation functions for the reactions ${ }^{12} \mathrm{C} \rightarrow{ }^{11} \mathrm{C}$ induced by neutrons, pions, and protons. The arithmetic mean of the positive and negative pions cross sections is shown as the pion curve. [Adapted from (Sw90).] 


\section{Chapter 9 RAdiation Protection Instrumentation at ACCELERAtors}

Fig. 9.12 provides the excitation functions of some other useful reactions with very high thresholds. The $\mathrm{Hg} \rightarrow{ }^{149} \mathrm{~Tb}$ reaction is a suitable monitor for very high energy particles and is commonly used as a beam calibrator. However, three additional reactions involving copper targets useful for this purpose have been found by Baker et al. [(Ba84), (Ba91)]. These are more convenient because the half-lives of the radionuclide produced are longer than that of $4.1 \mathrm{hr}$ for ${ }^{149} \mathrm{~Tb}$. The cross sections have been measured for energies from 30 to $800 \mathrm{GeV}$ and included in Table 9.6.

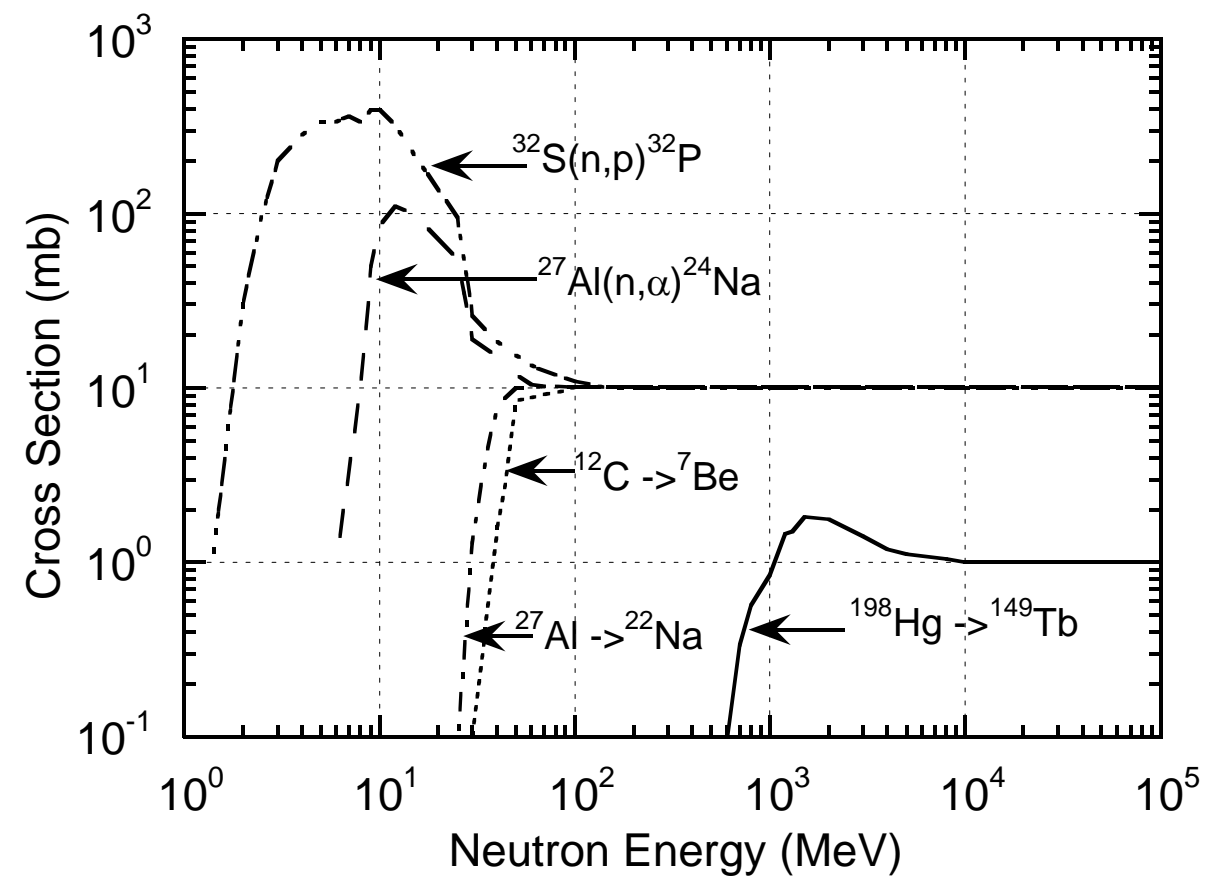

Fig. 9.12 Excitation functions of several threshold reactions. [Adapted from (Th88).]

\subsubsection{Fission Counters}

${ }^{233} \mathrm{U},{ }^{235} \mathrm{U}$, and ${ }^{239} \mathrm{Pu}$ all are well-known to be fissionable. The $Q$-values are very large (approximately $200 \mathrm{MeV}$ ) so that huge output signals are possible. For higher energy "fast" neutrons, fission reactions become possible for other, lighter elements such as bismuth. The cross sections for fast neutrons of these reactions are shown in Fig. 9.13. Fission reactions have been exploited as neutron (or hadron) detectors at accelerators. The fission of ${ }^{209} \mathrm{Bi}$ is especially interesting since the reaction has a threshold of about 50 $\mathrm{MeV}$ and also exhibits strong evidence that the neutron and proton-induced fission cross sections are approximately equal. Bismuth has been used in ionization chambers where the large energy deposited by the fission fragments gives a clear "signature" of this process, indeed recording the presence of hadrons above this relatively high energy threshold. Thus, akin to the use of ${ }^{11} \mathrm{C}$, it can provide further information about high energy neutrons and resolve ambiguities in the unfolding of spectra from Bonner sphere data. McCaslin et.al. have presented results obtained using this process (McC68). 


\section{Chapter 9 RAdiation Protection Instrumentation at ACCELERATORS}

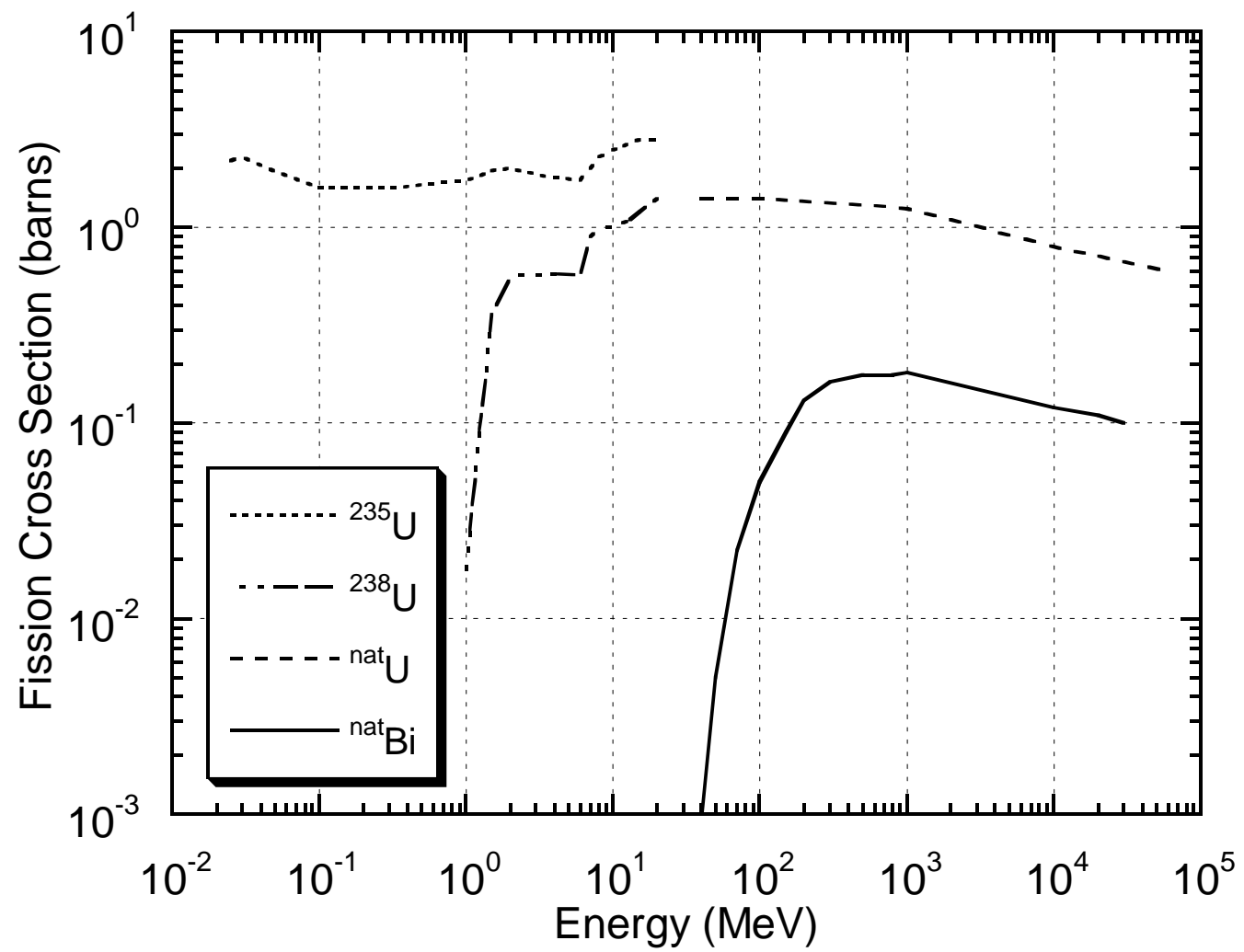

Fig. 9.13 Fission cross sections of some common target nuclides used in fission chambers for fast neutrons. The cross sections for fission of ${ }^{235} \mathrm{U}$ are much larger at lower energies not shown. [Adapted partially from (Kn79) and from (Sw90).]

\subsubsection{Proton Recoil Counters}

Knoll (Kn79) describes a variety of techniques for detecting neutrons based upon measuring the energy of recoil particles. The ${ }^{3} \mathrm{He}(\mathrm{n}, \mathrm{p})^{3} \mathrm{H}$ reaction has a reasonable cross section even into the $\mathrm{MeV}$ region but suffers from competition with $(\mathrm{n}, \mathrm{d})$ processes and elastic scattering. Elastic scattering of neutrons in which the energy of the recoil particle is measured and correlated with the neutron energy has received a great deal of attention. The most obvious recoil particle is the proton because hydrogenous detector materials (e.g., plastic scintillator) are readily available and the proton can receive the most energy in the recoil process per Eq. (9.24). Detector designers have been able to exploit the fact that scattering from hydrogen in the region $E_{n}<10 \mathrm{MeV}$ is isotropic in the center of mass frame. Knoll has shown that the probability $P\left(E_{r}\right)$ of creating a recoil proton having energy $E_{r}$ is also independent of angle in the laboratory frame within this energy domain (Kn79). Thus the recoil energy is only a function of the incident neutron energy. However, complexities enter the picture because in scintillators carbon is present along with the hydrogen and can also contribute recoil protons. Furthermore, the magnitude of the cross section is a function of neutron energy as is the efficiency of neutron detection 


\section{Chapter 9 Radiation Protection Instrumentation at ACCELERAtors}

in the scintillator. These effects, along with that of finite pulse height resolution, can lead to the need to use unfolding techniques in which the pulse height, indicative of the energy of the recoil proton, is correlated with the average neutron energy which could produce such a pulse. The technique has exhibited some promise in measuring the energy spectra of neutron radiation fields. A good summary is that of Griffith and Thorngate (Gr85) who were able to determine neutron energy spectra in the region between 2.0 and $20 \mathrm{MeV}$.

\subsubsection{TEPCs and LET Spectrometry}

In mixed radiation field dosimetry, a useful technique implemented commercially is the tissue-equivalent proportional chamber (TEPC) sometimes referred to as the "Rossi counter" after its inventor, H. Rossi (Ro55). The technique has been described by Brackenbush et al. (Br78). In this instrument tissue equivalent walls are employed to apply the Bragg-Gray principle. In such chambers, the pressure is maintained at low values, only a few torr ( 1.0 torr $=1.0 \mathrm{~mm}$ of $\mathrm{Hg}=133.3$ pascals $)$, thus the energy deposited is kept small. The energy so deposited will be equal to the linear energy transfer of the particle multiplied by the path length. At these low pressures, the gas-filled cavity has the same energy loss as does a sphere of tissue of diameter about $1.0 \mu \mathrm{m}$-hence an "equivalent diameter of $1.0 \mu \mathrm{m}$ ". In principle, determining the absorbed dose from events in such chambers is a straightforward unit conversion from a measured pulse height spectrum (calibrated in energy) to absorbed dose (in tissue);

$$
D(\mathrm{rad})=1.602 \times 10^{-8} \frac{C}{\rho V} \sum_{i=i_{\min }}^{i=i_{\max }} i N(i),
$$

where the summation is over channels $i\left(i_{\min } \leq i \leq i_{\max }\right.$, see below) corresponding to the radiation type of interest, $V$ is the sensitive volume $\left(\mathrm{cm}^{3}\right), \rho$ is the density $\left(\mathrm{g} \mathrm{cm}^{-3}\right), C$ converts the channel number to energy in $\mathrm{MeV}$, and $N(i)$ is the number of counts in channel number $i$. In most such instruments $C$ is independent of channel number.

In such chambers, the transition between photon and neutron-induced events occurs at a

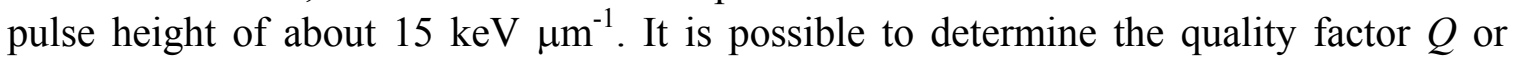
radiation weighting factor $w_{\mathrm{R}}$ from a single TEPC measurement. Under the conditions stated above and using a formula derived by Rossi (Ro68), one can unfold from the pulse height spectrum the distribution of absorbed dose as a function of LET, $D(L)$. The formula is complicated by the fact that one must average over mean chord lengths in the chamber. Such a distribution is used to calculate quality factor or radiation weighting factor, and hence the dose equivalent (1973 System) or effective dose (1990 System). The advent of microprocessors has made portable versions of such instruments. Fig. 9.14 shows a typical pulse height spectrum. In higher energy fields, dose distributions due to other particles with the same characteristic shapes but larger pulse sizes appear as the ${ }^{2} \mathrm{H}$, ${ }^{3} \mathrm{H},{ }^{3} \mathrm{He},{ }^{4} \mathrm{He}$ and even ${ }^{7} \mathrm{Li}$ "drop points". This obviously adds complexity to the unfolding procedures in the determination of LET spectra. A good discussion of the application of this technique is given by Vasilik et al. (Va85). 


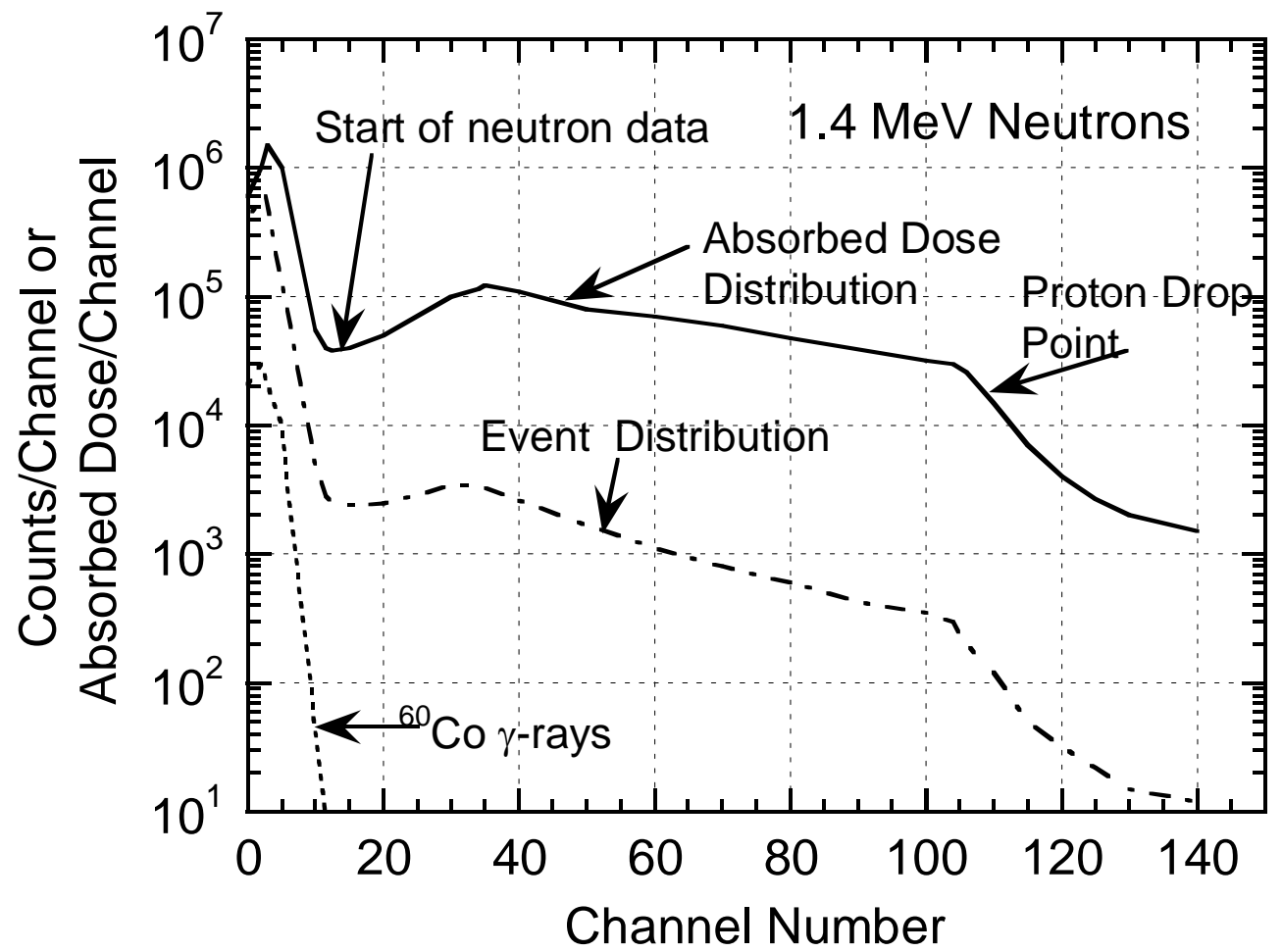

Fig. 9.14 Pulse-height spectra from a tissue-equivalent proportional counter exposed to $1.4 \mathrm{MeV}$ neutrons and ${ }^{60} \mathrm{Co} \gamma$-rays. [Adapted from (Br78).]

\subsubsection{The Recombination Chamber Technique}

An adaptation of the ion chamber that has shown considerable potential for usefulness as a dose equivalent meter in a mixed field of radiation is based on the exploitation of recombination phenomena in such chambers. As charged particles interact in such a chamber the gas is ionized. The electrodes will collect only those ions that do not recombine before they reach the cathode. The extent of such columnar recombination is dependent upon the average distance between the ions as well as upon the applied voltage. The biasing voltage sets the speed at which the ions migrate to the cathode. For a given voltage, a chamber should exhibit more severe recombination for the radiations having high LET (e.g., neutrons, heavy ions, etc.) than for those having low LET (electrons, photons, and muons). In the high LET situation, the slow moving positive ions are surrounded by a higher density of electrons than they would be in under conditions of low LET. Zielcyznski (Zi62) did the initial work on this topic.

Baarli and Sullivan reported similar results over a somewhat larger range of values of quality factor $Q(2<Q<20$ as defined by the 1973 Radiation Protection System) and further refined the technique (Ba65). It turns out that the current $i$ (or charge if integrated over time) measured in a given radiation field is related to the applied voltage $V$ by the following approximate expression:

$$
i=k V^{n} .
$$




\section{ChAPTER 9 RADiATion Protection InSTRUMENTATION AT ACCELERATORS}

The parameter $n$ is approximately proportional to the quality factor $Q$ and $k$ is a constant proportional to the intensity of the radiation field.

Using a special ion chamber engineered for this purpose, Cossairt et al. measured the correlation of $n$ with $Q$ (1973 Radiation System) or $n$ with $w_{R}$ (1990 Radiation System) (Co09b). The response of the chamber as described by Eq. (9.28) was measured in each member of a set of mixed fields staged with combinations of $\gamma$-rays from ${ }^{60} \mathrm{Co}$ and neutrons and $\gamma$-rays from a ${ }^{238} \mathrm{PuBe}$ source to expose the chamber to different average quality factors $Q$ (1973 Dosimetry System) or radiation weighting factors $w_{R}(1990$ Dosimetry System). The results are shown in Fig. 9.15.
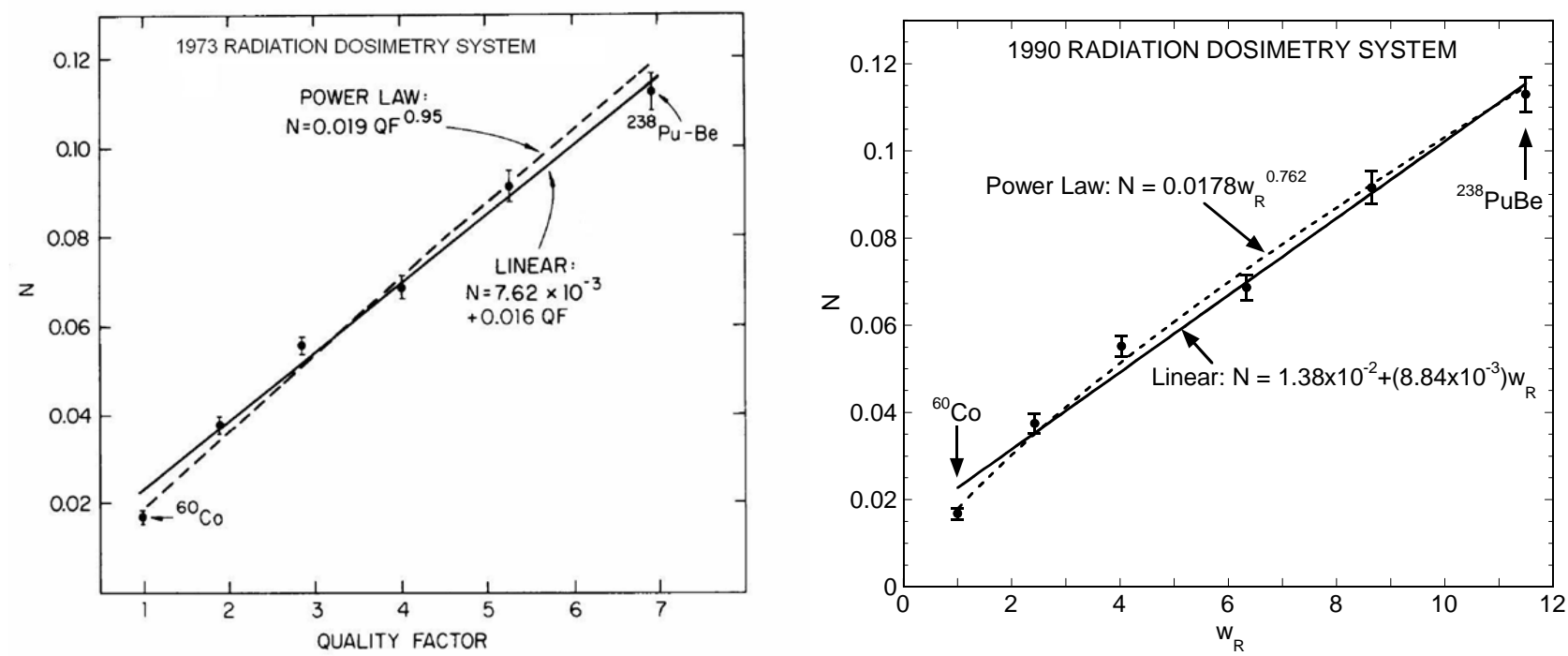

Fig. 9.15 Response of a recombination chamber as a function of quality factor $Q$ obtained in mixed fields using radioactive sources for the 1973 System in the left frame and 1990 System in the right panel. [Reproduced from (Co09b).]

Adequate fits are provided by linear and power law formulations. The results for the 1993 System were

$$
\begin{aligned}
& n=0.00762+0.016 Q, \\
& \text { and } \\
& n=0.019 Q^{0.95} .
\end{aligned}
$$

$(9.29 b)$

The results for the 1990 System were

$$
\begin{aligned}
& n=1.38 \times 10^{-2}+\left(8.84 \times 10^{-3}\right) w_{R}, \\
& \text { and } \\
& n=0.017 w_{R}^{0.762} .
\end{aligned}
$$

Patterson and Thomas $(\mathrm{Pa} 73)$ presented similar results over a somewhat larger range of $Q$ $(2<Q<20)$ under the 1973 System. Typically, the response of such a chamber is measured as a function of applied voltage for the special chamber provided for that purpose 


\section{Chapter 9 RAdiation Protection Instrumentation at ACCElerators}

over some voltage range, say, $20 \leq V \leq 1200$ volts. In fields that are not steady with time, the response must be normalized against some other measured quantity that accurately is proportional to the intensity of the radiation field. The method of least squares is then applied to determine $n$ by taking advantage of the fact that Eq. (9.28) can be rewritten as

$$
\ln i=\ln k+n \ln V .
$$

In typical measurement, such a log-log fit to the data is of moderately good quality. From the value of $n$ determined by such a fit, the quality or radiation factor can then be determined by using equations like those above determined for a particular recombination chamber. Fig. 9.16 shows the response measured in a field known to be dominated by high energy muons $(Q=1.0)$. Data taken in the iron leakage spectrum described in connection with Fig. 6.8 are shown in Fig. 9.17. Measurements of this type have been used to check the quality factors (1973 System) obtained in the unfolding of Bonner sphere data. Table 9.7 illustrates the typical agreement between these entirely different techniques for diverse radiation fields.

Zel'chinskij and Zharnovetskij (Ze67) proposed using two chambers placed in the radiation field of interest; one operated at a low voltage and other at a high voltage. The differences in responses read out by the two chambers would then be proportional to the dose equivalent rate. It turns out that measuring differences in ion chamber currents found in practical chambers is difficult due to the small currents and electrical leakage problems associated with electrical feed-throughs and cable connectors.

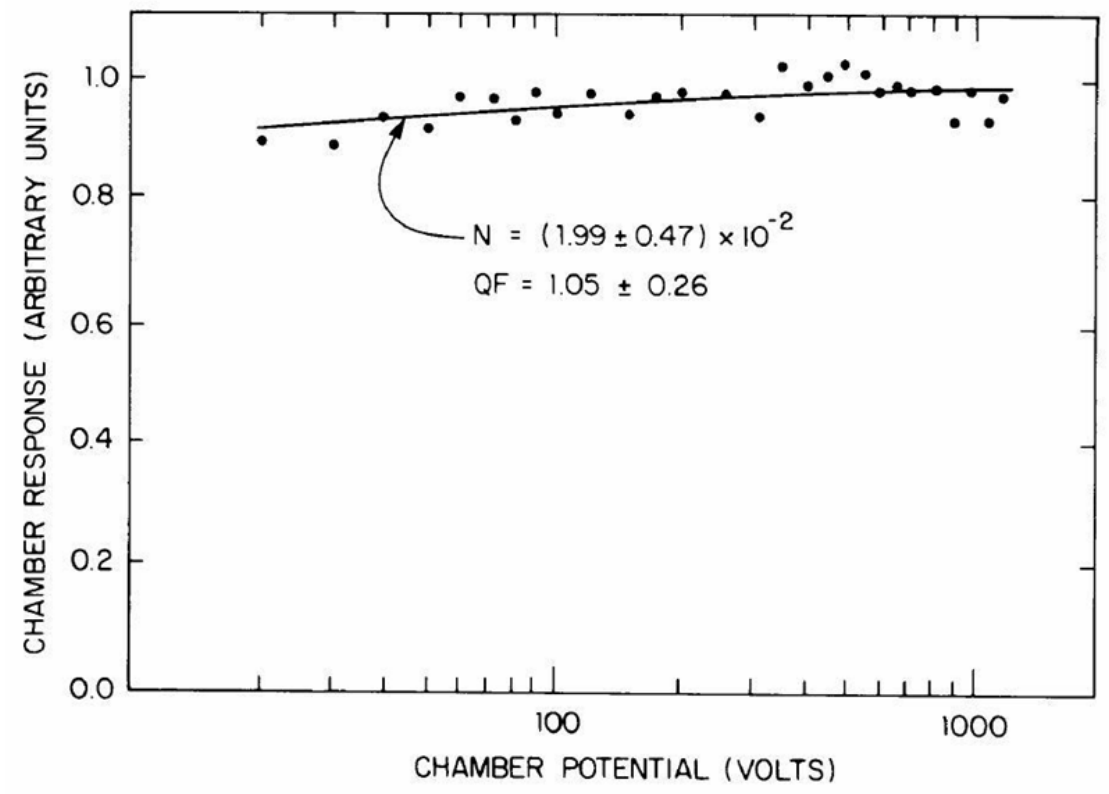

Fig. 9.16 Recombination chamber response as a function of chamber potential in a radiation field nearly completely consisting of high energy muons. "QF" is the quality factor as defined by the 1973 Radiation Protection System. [Reproduced from (Co87).] 


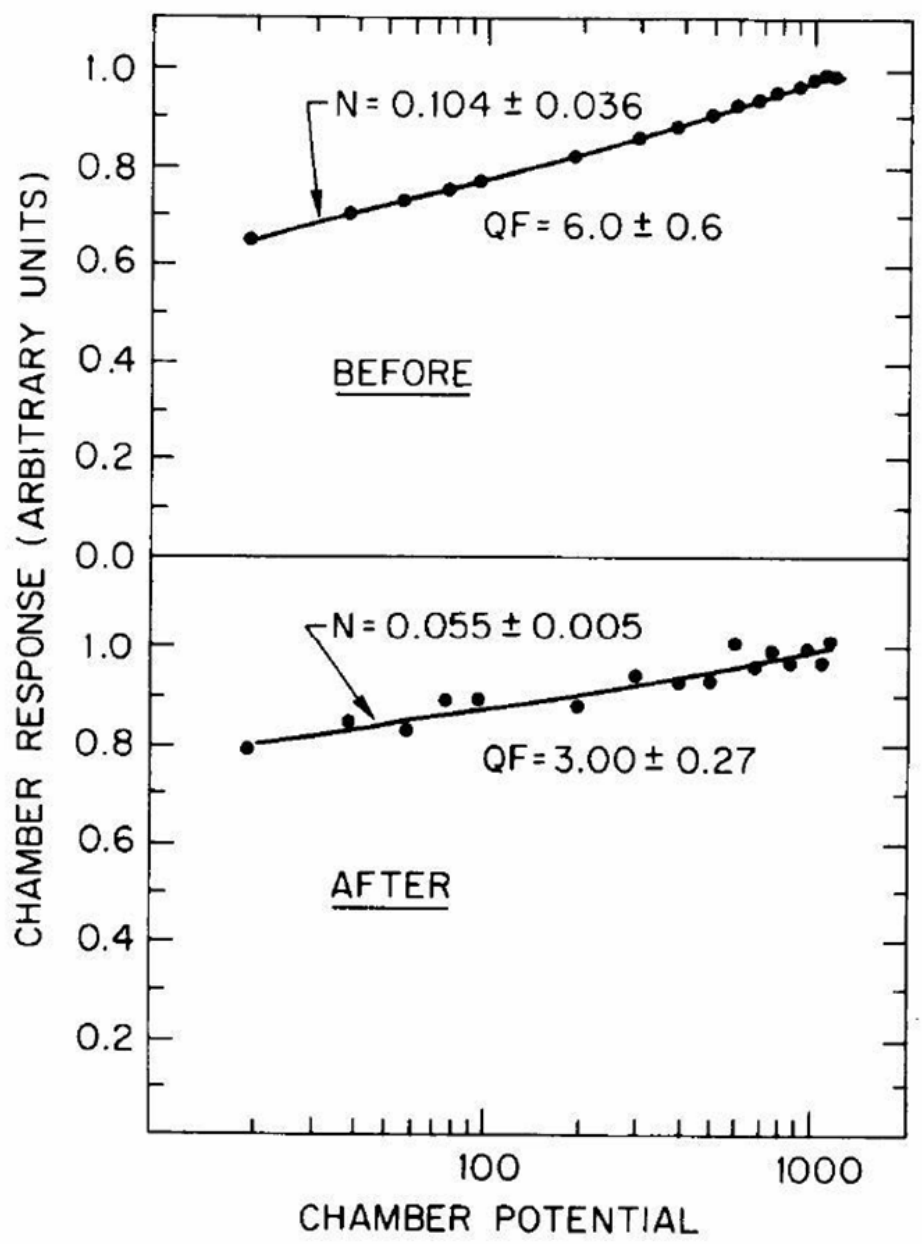

(VOLTS)

Fig. 9.17 Recombination chamber response functions measured both before (top) and after (bottom) the placement of additional shielding in the radiation field described in Fig. 6.8. Values of "QF" is the quality factor as defined by the 1973 Radiation Protection System. [Reproduced from (E186).]

Table 9.7 Average quality factors (1973 Radiation Protection System) obtained for various neutron energy spectra measurements at Fermilab. [Adapted from (Co88).]

\begin{tabular}{|l|c|c|}
\hline \multirow{2}{*}{ Description of Radiation Field } & \multicolumn{2}{|c|}{ Technique } \\
\cline { 2 - 3 } & Unfolding & Recombination \\
\hline Mixed field of neutrons and muons (Co 87) & $1.4 \pm 0.2$ & $1.1 \pm 0.3$ \\
\hline $\begin{array}{c}\text { Iron leakage spectra before shielding was added (Fig. } \\
\text { 6.8b) (E186) }\end{array}$ & $5.4 \pm 0.2$ & $6.0 \pm 0.6$ \\
\hline $\begin{array}{c}\text { Iron leakage spectra after shielding was added (Fig. } \\
\text { 6.8c) (E186) }\end{array}$ & $2.5 \pm 0.3$ & $3.0 \pm 0.3$ \\
\hline Spectrum in a labyrinth (Fig. 6.7) (Co85b) & $3.1 \pm 0.7$ & $3.4 \pm 0.1$ \\
\hline
\end{tabular}

Höfert and Raffnsøe (Hö80) have measured the dose equivalent response of such an instrument as a function of neutron energy and obtained the results in Table 9.8. 
Table 9.8 Dose equivalent response and measurement errors for recombination chamber as a function of neutron energy. [Adapted from (Hö80).]

\begin{tabular}{|c|c|c|}
\hline $\begin{array}{c}\text { Neutron Energy } \\
(\mathbf{M e V})\end{array}$ & $\begin{array}{c}\text { Dose Equivalent Response } \\
\left.\mathbf{( 1 0}^{\mathbf{5}} \mathbf{\text { Coulombs Sv }} \mathbf{-}\right)\end{array}$ & $\begin{array}{c}\text { Error } \\
\mathbf{( \% )}\end{array}$ \\
\hline thermal & 0.830 & 10.0 \\
\hline 0.0245 & 2.579 & 12.1 \\
\hline 0.1 & 1.451 & 6.2 \\
\hline 0.25 & 1.585 & 6.1 \\
\hline 0.57 & 1.215 & 5.2 \\
\hline 1.0 & 1.215 & 5.2 \\
\hline 2.5 & 1.112 & 6.1 \\
\hline 5.0 & 0.840 & 5.2 \\
\hline 15.5 & 0.728 & 5.2 \\
\hline 19.0 & 0.998 & 12.1 \\
\hline 280.0 & 0.782 & 10.1 \\
\hline
\end{tabular}

\subsubsection{Counter Telescopes}

Since the dose equivalent per fluence for muons varies so little over a wide range (see Fig. 1.4), scintillation telescopes provide an attractive method for assessing pure muon radiation fields. At suitable distances and at forward angles, muons will dominate the radiation fields and the result is that little or no discrimination against other particles is necessary.

At Fermilab a pair of $20.32 \mathrm{~cm}$ by $20.32 \mathrm{~cm}$ by $0.635 \mathrm{~cm}$ thick plastic scintillators has been used routinely (Co83). The separation distance between these "paddles" provides moderate directional sensitivity when a coincidence is required between the two scintillator paddles in a relatively parallel beam of muons. An aluminum plate, $2.54 \mathrm{~cm}$ thick, is employed in the gap between the two scintillators to reduce false coincidences due to recoil electrons (so-called " $\delta$-rays") produced in collisions occurring in the first scintillator that might reach the second if the aluminum were absent. These plates are mounted in an all-terrain vehicle, called the Mobile Environmental Radiation Laboratory (MERL), and are powered by an on-board electrical generator. (The MERL is also used for neutron measurements with a DePangher long counter and other instruments.) A microwave telemetry system provides gating pulses and proton beam intensity information so that normalized beam-on and beam-off (background) measurements can be taken simultaneously. The paddles were chosen to provide sufficient sensitivity to obtain statistical errors at the $20 \%$ level in remote locations receiving annual dose equivalents in the fractional mrem range in a scan lasting an hour or two. In such a scan, the detectors are moved across a region of elevated muon flux density, stopping at several locations to acquire data. In these detectors, a muon beam perpendicular to the detectors yields $1.72 \times 10^{5}$ counts minute $\mathrm{e}^{-1}$ per $\mathrm{mrem} \mathrm{h}^{-1}$ (or $1.03 \times 10^{7}$ counts $\mathrm{mrem}^{-1}$ ). The normal singles background (i.e., the background of an individual scintillator not counted in coincidence with the other member of the pair) due to cosmic rays at Fermilab is approximately 400 counts per minute. 


\section{Chapter 9 Radiation Protection Instrumentation at ACCELERAtors}

Smaller, more portable systems can be useful in conducting muon surveys. Fermilab has built such a system, called a muon finder, consisting of a pair of small plastic scintillators mounted in a compact package which is battery powered and can be carried by one person. It is read out by scalers and can record both singles and coincidence rates. The ratio of the two can be used to "find" unknown muon sources; hence the name of the detector. Also, the separation distance can be adjusted to enhance or reduce the directional sensitivity. This system has been described by Vaziri, et al. (Va04).

The parameters of this system are given in Table 9.9. Of course, the use of such scintillators, especially in the "singles" mode, in mixed fields of muons and neutrons requires that one must be aware of the fact that the plastic scintillators have nonzero detection efficiency for the neutrons. Vylet has used the values of total cross sections to calculate the neutron detection efficiency of the detectors described above for neutrons over a range of energies (Vy91). The results are given in Fig. 9.18. In this figure, effects due to the first and successive collisions (labeled "Total") as well as those due to just the first collisions (labeled " 1 st") with hydrogen atoms are given. The total efficiencies at the upper end of the energy region measured were an efficiency of 0.058 for the MERL paddles and 0.0235 for the muon finders.

\section{Table 9.9 Parameters of the "muon finder" used at Fermilab.}

Scintillator diameter

Scintillator thickness

Scintillator area

Scintillator spacing

Half-angle cone of sensitivity

Dose calibration (muons $\perp$ detectors)

Dose rate calibration (muons $\perp$ detectors)
$2.1 \mathrm{~cm}$

$0.635 \mathrm{~cm}$

$3.6 \mathrm{~cm}^{2}$

0.5 to $8.9 \mathrm{~cm}$

0.9 to 0.2 radians (51 to $11.5 \mathrm{deg}$. half-angle)

90 muons/ $\mu$ rem

25 muons/sec per mrem/hour

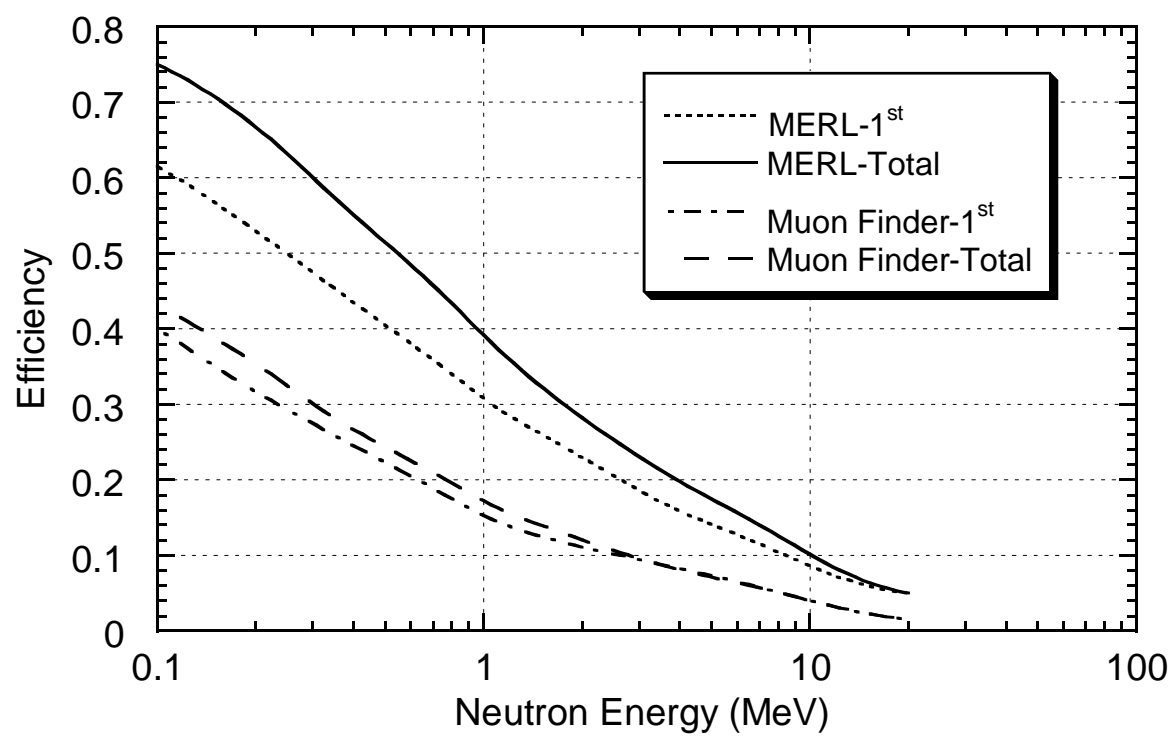

Fig. 9.18 Calculated neutron efficiencies of scintillation counters used in the "singles" mode at Fermilab as a function of neutron energy as described in the text. [Adapted from (Vy91).] 


\section{Chapter 9 RAdiation Protection InStrumentation at ACCELERATORS}

\section{Problems}

1. A cylindrical ion chamber is $5.0 \mathrm{~cm}$ in radius and $20 \mathrm{~cm}$ long. It is filled with methane $\left(\mathrm{CH}_{4}\right)$ at 1.0 atmosphere absolute pressure. It is bombarded by a uniform flux density of high energy (minimum-ionizing) muons incident perpendicularly to one of the ends. One can safely make the assumption that the passage of the muons through the entire length of the chamber represents insignificant degradation of the muon energy or direction. The dose equivalent rate in the radiation field is $0.1 \mathrm{mrem}_{\text {hour }}^{-1}$.

a) Calculate the electric current that will be drawn from this chamber that represents the "signal" to be measured and correlated with the dose equivalent rate. One could use Table 1.2 to obtain values of $(d E / d x)_{\min }$ and to obtain the density of $\mathrm{CH}_{4}$.

b) If the charge liberated in the chamber is collected (i.e., integrated electronically) for 1.0 seconds and the chamber and circuit represent a capacitance of $10^{-10}$ Farads, calculate the size of the signal pulse in volts if one neglects any "pulseshaping" of the readout electronics.

2. Consider the detector based on the $25.4 \mathrm{~cm}$ moderating sphere for which the corresponding response curve is displayed in Fig. 9.8.

a) Calculate the approximate absolute intrinsic detection efficiency for neutrons. This is to be done for the $2.0<E_{n}<8.0 \mathrm{MeV}$ energy domain and the sharp peaks in the detector response curve are to be ignored (i.e., averaged out). In this problem, $100 \%$ efficiency is defined to be 1.0 count generated for every neutron that strikes the sphere. Assume the incident neutrons to be aimed at the detector originating from a "point" source" despite the fact that this is not quite true.

b) Since the LiI detector only responds to thermal neutrons, calculate the efficiency with which the moderator transforms fast neutrons incident upon it into thermal neutrons present at the LiI. For this calculation, neglect any "dopants" in the LiI, assume that the $\mathrm{Li}$ is "natural" lithium with respect to isotopic abundance and use the fact that the atomic weight of iodine is 127 . The density of LiI is $3.5 \mathrm{~g} \mathrm{~cm}^{-3}$. Assume that the detector is $100 \%$ efficient in detecting thermal neutron captures within its volume.

3. $\mathrm{A} \mathrm{BF}_{3}$ proportional chamber is used in a DePangher long counter. This detector, when placed in a certain neutron field that is known to be dominated by neutrons of approximately $5.0 \mathrm{MeV}$ kinetic energy, has a response due to neutrons of 1.0 count $\min ^{-1}$. The detector sensitivity is that discussed in the text. The counter operates at 1.0 atmosphere absolute pressure, the atomic weight of boron is 10.8 while the atomic weight of fluorine is 19 . At STP the density of $\mathrm{BF}_{3}$ is $2.99 \mathrm{~g}$ liter $^{-1}$. 


\section{Chapter 9 Radiation Protection Instrumentation at ACCELERAtors}

a) What is the dose equivalent rate of this radiation field?

b) If the radiation field persists full time, is this detector sufficiently sensitive to detect a dose rate of 10 mrem year $^{-1}$ ?

c) In this radiation field, high energy minimum ionizing muons pass through this detector, including the proportional counter. The largest muon signals in the proportional counter will obviously result when the muons pass lengthwise through the tube. If the tube is $40 \mathrm{~cm}$ long, what will be the size of the largest muon-induced signal relative to the neutron-induced signal? Is it likely that a simple discriminator circuit can be used to eliminate the muon-induced signals? It is quite permissible to estimate the value of $(d E / d x)_{\min }$ by roughly interpolating among the values tabulated in Table 1.2.

4. One needs to understand the sensitivity of the technique of using the nuclear reaction ${ }^{12} \mathrm{C}(\mathrm{n}, 2 \mathrm{n}){ }^{11} \mathrm{C}$ in plastic scintillator to measure dose equivalent rate external to thick concrete or earth shielding near a high energy accelerator. The detector discussed in the text used by Moritz has a sensitive volume of approximately $100 \mathrm{~cm}^{3}$ (a $5.0 \mathrm{~cm}$ diameter by $5.0 \mathrm{~cm}$ long cylinder). From Knoll (Kn79) the NE102A scintillator, has a density of $1.032 \mathrm{~g} \mathrm{~cm}^{-3}$. This detector is nearly $100 \%$ efficient at sensing the $0.511 \mathrm{MeV}$ annihilation photons produced in the course of the ${ }^{11} \mathrm{C}$ decay.

a) This detector is irradiated in a particular radiation field external to such accelerator shielding. The irradiation, which is steady in time, is of sufficient length in time to result in saturation of the production of ${ }^{11} \mathrm{C}$ in the scintillator. After the beam is turned off, the detector records a rate of 10 counts per minute (including appropriate decay-correction to the instant of beam shutdown). Calculate the flux density of neutrons with $E_{n}>20 \mathrm{MeV}$ during the irradiation and use the result along with Stevenson's conclusion concerning the conversion from the flux density of neutrons with $E_{n}>20 \mathrm{MeV}$ to dose equivalent to determine the dose equivalent rate.

b) Assuming this count rate is the smallest that can be reliably detected, how much smaller in volume can the detector be for it to barely be sensitive to a dose equivalent rate of $2.0 \mathrm{mrem}_{\text {hour }}{ }^{-1}$ ? 


\section{ApPendix A Summary Descriptions of Commonly USED MonTe Carlo Codes}

This appendix provides brief summary descriptions of some of the more prominent Monte Carlo codes used at modern particle accelerators. The reader should be cautioned that most of these codes are being constantly improved and updated. The wisest practice in using them is to consult with the authors of the codes directly to obtain detailed, current information.

\section{CASIM}

A. Van Ginneken developed this "Cascade Simulation" program (Va75). It was designed to simulate the average behavior of hadrons in the region 10 to $1000 \mathrm{GeV}$ and was extended to $20 \mathrm{TeV}$ (Va87). It used inclusive production distributions directly in order to obtain the particles to follow. The particle production algorithm was based upon the Hagedorn-Ranft thermodynamic model. Only one or two high energy particles were created in each simulated collision and these carried a weight related to the probability of their production and the energy carried with them. Path length stretching and particle splitting were used. Electromagnetic showers resulting from $\pi^{\mathrm{o}}$ production were calculated using the companion code AEGIS. The user generally wrote a FORTRAN subroutine to describe the geometry of interest with "logical" (i.e., "IF", etc.) statements used to deduce the material or magnetic field in which a particle being tracked was found at a given "time" in the calculation from the particle's spatial and directional coordinates. Magnetic fields could readily be included. A muon version called CASIMU (later MUSIM) was written (Va87). The accuracy of the hadron version was been verified for energies up to $800 \mathrm{GeV}$ [(Aw75), (Aw76), (Co82a), (Co85a)]. The muon version was verified for energies up to $800 \mathrm{GeV}$ for production and transport of muons in real-life, complicated shields [(Co89a), (Co89b)]. Normally, CASIM was not set up to follow particles with momenta less than $300 \mathrm{MeV} / \mathrm{c}$ (corresponding to a kinetic energy of 47 $\mathrm{MeV}$ for nucleons). All low energy phenomena then was obtained by matching energy spectra and fluence at this energy with results of codes capable of tracking lower energy particles (e.g., HETC, FLUKA, and MARS). Direct calculations of radioactivation were not available. These were severe limitations along with the tedious method for specifying the geometry. At Fermilab, CASIM was been replaced by MARS as the code of choice. Results obtained using CASIM still serve as benchmarks.

\section{EGS}

EGS, the "Electron Gamma Shower" code is a powerful code for calculating electromagnetic cascades. A recent version is EGS5. A complete description of this code system has been written by Nelson et al. [(Ne85), (Ne90)]. This program provides a Monte Carlo analysis of electron and photon scattering including shower generation. In its standard usage, it does not calculate hadron or muon production directly. The lower limit of its validity is about $10 \mathrm{keV}$ while the upper limit of its validity is at least $1.0 \mathrm{TeV}$. Possible target materials span the periodic table. As the electron encounters target atoms, it is scattered randomly to mimic the known mechanisms of electron scattering. When secondary particles arise, they are loaded into a stack from which EGS4 selects sequentially the lowest energy particle and then traces out its further path until it leaves the target or until its energy falls below a selected cut-off value. The final kinematical 


\section{ApPendix A Summary Descriptions of Commonly USED MonTe Carlo Codes}

and charge properties of all the particles are noted and summed for all particles in the shower concluding with a "history" of all of them. Improvements with the code are continuously being made. The code is a fundamental tool at many laboratories that have electron accelerators. The code has been found to be extremely useful in applications in medicine and also in modeling the performance of high energy physics apparatus. The EGS code system is available from the High Energy Research Organization of Japan (KEK). The official EGS5 website is: http://rcwww.kek.jp/research/egs/egs5.html. (Accessed October 29, 2013).

\section{FLUKA}

FLUKA is an integrated, versatile multi-particle Monte Carlo program, capable of handling a wide variety of radiation transport problems. Its energy range extends from one $\mathrm{keV}$ (for neutrons, thermal energies) to thousands of TeV. FLUKA can simulate with a similar level of accuracy the propagation of hadronic and electromagnetic cascades, cosmic muons, slowing-down neutrons and synchrotron radiation in the keV region. An original treatment of multiple Coulomb scattering allows the code to handle accurately some challenging problems such as electron backscattering and energy deposition in thin layers. In a fully analog mode, FLUKA can be used in detector studies to predict fluctuations, coincidences and anti-coincidences. On the other hand, a rich supply of biasing options makes it well suited for studies of rare events, deep penetration and shielding in general. This code originated as high-energy particle transport code developed by a CERN-Helsinki-Leipzig collaboration led by J. Ranft as discussed by Aarnio et al. (Aa86). More recently, it has been completely rewritten and extended to low energies as discussed by Fassò et al. (Fa93). It handles more than 30 different particles, including neutrons from thermal energies to about $20 \mathrm{TeV}$ and photons from $1.0 \mathrm{keV}$ to thousands of $\mathrm{TeV}$. Several biasing techniques are available. Recoil protons and protons from $\mathrm{N}(\mathrm{n}, \mathrm{p})$ reactions are transported explicitly. The official FLUKA website is at: http://www.fluka.org/fluka.php (Accessed October 29, 2013).

\section{HETC and LAHET}

This code, developed over many years under the leadership of R. G. Alsmiller at the Oak Ridge National Laboratory, is considered by some to be the benchmark hadron shielding code of choice. It has been upgraded many times and can, in suitably augmented versions, follow particles from the $20 \mathrm{TeV}$ region down to thermal energies. It is an extremely flexible code but has the important disadvantage that the individual events are written to mass storage. It is the responsibility of the user to write a program to analyze the results. In terms of CPU-time HETC is also relatively slow so that calculations to be done should be carefully selected. It is seen to be preferable to use selected HETC runs to calibrate other faster, but less accurate codes. It has been described by Armstrong (Ar80) and Gabriel (Ga85). It includes the same event generator used for FLUKA. The Los Alamos Energy Transport Code (LAHET) variant of this code developed at the Los Alamos National Laboratory (LANL) has been described by Prael and Lichtenstein (Pr89). It is available from the Los Alamos National Laboratory. This variant permits the 


\section{ApPendix A Summary Descriptions of Commonly USED MonTe Carlo Codes}

transport of neutrons, photons, and light nuclei up to ${ }^{4} \mathrm{He}$ and employs the geometric capabilities of the MCNP code and has been merged with more recent evolutions of the MCNP family of codes. A somewhat dated website with more information about LAHET is at: http://www.oecd-nea.org/tools/abstract/detail/ccc-0696/. (Accessed October 29, 2013).

\section{MARS}

The MARS Monte Carlo code system has been under continuous development over a number of years by N. Mokhov et al. [(Ka89), (Mo95), (Kr97), (Mo04)]. Early results were compared by Mokhov and Cossairt (Mo86) with those obtained using then-current versions of CASIM and FLUKA with good agreement. The code allows exclusive and fast inclusive simulation of three-dimensional hadronic and electromagnetic cascades for shielding, accelerator, and detector components in the energy range from a fraction of an electron volt up to $100 \mathrm{TeV}$. The current version MARS15 uses the phenomenological model for inclusive hadron- and photon-nucleus interactions for $\mathrm{E}>5.0 \mathrm{GeV}$ and exclusive cascade-exciton model at $1.0 \mathrm{MeV}<\mathrm{E}<5.0 \mathrm{GeV}$. By using the Los Alamos Quark-Gluon String Model (LAQGSM) event generator (Gu01), full theoretically consistent modeling of exclusive distributions of secondary particles, spallation, fission, and fragmentation products for hadron and heavy-ion beams of any energy can be done. MARS15 includes photo- and electro-production of hadrons and muons, advanced algorithms for the 3-body decays, precise particle tracking in magnetic fields, synchrotron radiation by electrons and muons, extended histogramming capabilities and improved material description and computational performance. Along with direct energy deposition calculations, a set of dose conversion per fluence factors for all particles including neutrinos is incorporated. The code includes links to the MCNP4C code for neutron and photon transport below $20 \mathrm{MeV}$, to the ANSYS code for thermal and stress analyses, and to the MAD and STRUCT codes for lattice description for multi-turn particle tracking in large synchrotrons and collider rings. It can also be linked to EGS. The geometry module allows the use of a set of the pre-defined shapes with the MARS extended geometry input deck or with the ROOT geometry package (http://root.cern.ch/drupal/content/users-guide, accessed October 29, 2013), arbitrary user-defined 3-D descriptions, or direct use of MCNP and FLUKA geometry input desks. The code is provided with a user-friendly graphical-use interface for geometry. A parallelized version of the code can run in a multi-processor mode. The developments were induced by numerous challenging applications; Fermilab accelerator, detector and shielding upgrades, Large Hadron Collider machine and detector studies, muon and electron-positron colliders etc; as well as by a continuous desire to increase code reliability, flexibility and user friendliness. This code is continously updated and improved and can be obtained from Fermilab. It is likely the most advanced high energy code available. The official MARS website is at: http://www-ap.fnal.gov/MARS/. (Accessed October 29, 2013). 


\section{MCNP}

MCNP is a general-purpose Monte Carlo N-particle code that can be used for neutron, photon, electron, or coupled neutron/photon/electron transport, including the capability to handle situations involving nuclear criticality. This code has been developed at the Los Alamos National Laboratory and is well documented in LANL reports (e.g., Br97). The code treats an arbitrary three-dimensional configuration of materials in geometric cells bounded by first- and second-degree surfaces and fourth-degree elliptical tori. The neutron energy regime is from $10^{-11} \mathrm{MeV}$ to $150 \mathrm{MeV}$, and the photon and electron energy regimes are from $1.0 \mathrm{keV}$ to $1000 \mathrm{MeV}$. For neutrons, all reactions given in a particular cross section evaluation (such as ENDF/B-VI cross section database) are accounted for. Thermal neutrons are described by both the free gas and thermal particle scattering models. For photons, the code takes account of incoherent and coherent scattering, the possibility of fluorescent emission after photoelectric absorption, absorption in pair production with local emission of annihilation radiation, and bremsstrahlung. A continuous slowing down model is used for electron transport that includes positrons, x-rays, and bremsstrahlung but does not include external or selfinduced fields. Important standard features that make MCNP very versatile and easy to use include a powerful general source, criticality source, and surface source; both geometry and output tally plotters; a rich collection of variance reduction techniques; a flexible tally structure (including a pulse-height tally); and an extensive collection of cross-section data. The official MCNP website is at: http://mcnp.lanl.gov/. (Accessed October 29, 2013). 


\section{APPENDiX B EXAMPLES OF RESUlts OF STAR DENSITY CALCUlations}

As was discussed in Section 4.7.2, a convenient way to exhibit the "raw" output of Monte Carlo high energy hadronic cascade calculations is in the form of contour plots of star density as a function of longitudinal coordinate $Z$ and radial coordinate $R$. This appendix contains representative samples of such plots obtained using CASIM [(Va75), (Va87), and $(\mathrm{Co} 82 \mathrm{~b})]$ and MARS (Re12). This collection is illustrative in nature; it is not intended to address all situations. The main text refers to more complete compilations of calculations. Individual calculations should be done for definitive results.

Four sets of examples are provided here:

Figs. B1.a-B1.d present results obtained using CASIM for protons incident along the axis of a solid CONCRETE cylinder perpendicular to one face of the cylinder. The concrete is of "standard" composition" and is taken to have a density of $2.4 \mathrm{~g} \mathrm{~cm}^{-3}$.

Figs. B2.a-B2.d present results obtained using CASIM for protons incident along the axis of a solid IRON cylinder perpendicular to one face of the cylinder. The iron is assumed to have a density of $7.87 \mathrm{~g} \mathrm{~cm}^{-3}$.

Figs. B3.a-B3.d present results obtained using CASIM for $1.0 \mathrm{TeV}$ protons incident on some examples of beamline components in enclosures or soil shields. For the components, the standard densities found in Table 1.2 were used. The density of concrete was taken to be $2.4 \mathrm{~g} \mathrm{~cm}^{-3}$ and the density of soil was taken to be $2.25 \mathrm{~g} \mathrm{~cm}^{-3}$. Beam pipes interiors were assumed to be at vacuum. The captions describe the details of the beam loss scenarios used in the calculations.

Figs. B4.a-B4.c present much more refined up-to-date calculations obtained using MARS for $120 \mathrm{GeV}$ protons incident on some examples of beamline components in enclosures or soil shields. For these calculations the concrete density was assumed to be $2.4 \mathrm{~g} \mathrm{~cm}^{-3}$ while the soil density was taken to be $2.24 \mathrm{~g} \mathrm{~cm}^{-3}$. The point losses were simulated by positioning the centroid of the proton beam 1 standard deviation $(1 \sigma)$ from the inner surface of the chosen target aperture in a Gaussian distribution at an incident angle of zero. While a small non-zero incident angle would be more realistic, very small grazing angles on the order of $\sin \mathrm{q} \leq 10^{-4}$ or less will produce only minimal increases $(<20 \%)$ in the total dose. Larger grazing angles could potentially increase the dose by a factor of six or more. 


\section{APPENDiX B EXAMPLES OF RESUlts OF STAR DENSITY CALCUlations}

\section{B.1 Results for Solid CONCRETE Cylinders}

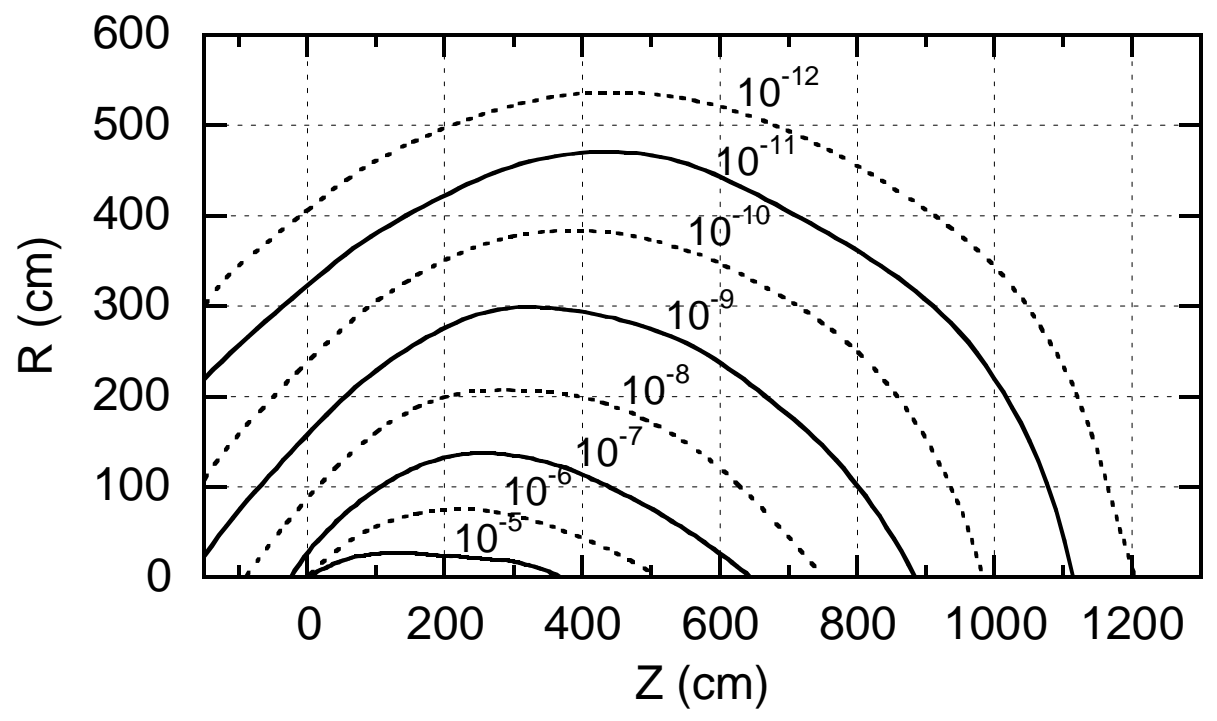

Fig. B1.a Monte Carlo results using CASIM for $30 \mathrm{GeV} / \mathrm{c}$ protons incident on a CONCRETE cylinder. Contours of equal star density $\left(\right.$ stars $\mathrm{cm}^{-3}$ ) per incident proton are plotted. The beam of $0.3 \times 0.3 \mathrm{~cm}^{2}$ cross section is centered on the cylinder axis and starts to interact at zero depth. The star density includes only those due to hadrons above $0.3 \mathrm{GeV} / \mathrm{c}$ momentum. Contours of higher star density are not shown for clarity while those of lower star density are not included due to statistical uncertainty. [Adapted from (Va75).]

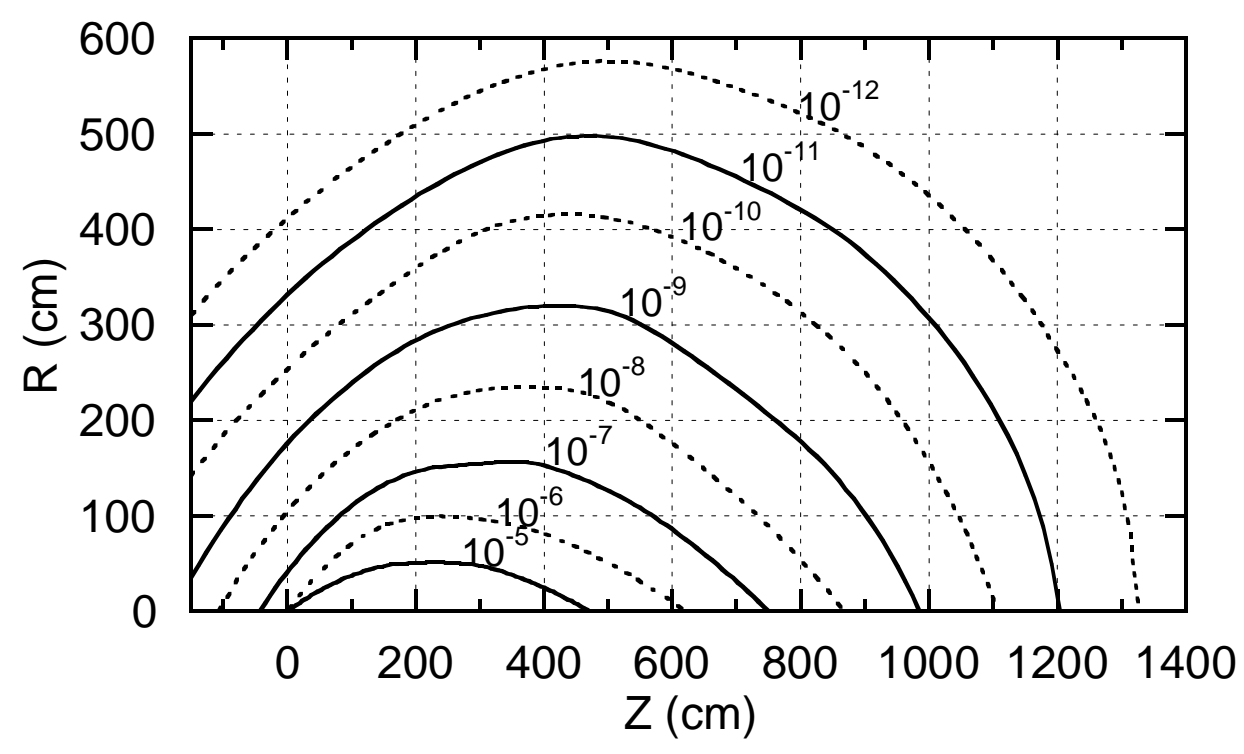

Fig. B1.b Monte Carlo results using CASIM for $100 \mathrm{GeV} / \mathrm{c}$ protons incident on a CONCRETE cylinder. Contours of equal star density $\left(\right.$ stars $\mathrm{cm}^{-3}$ ) per incident proton are plotted. The beam of $0.3 \times 0.3 \mathrm{~cm}^{2}$ cross section is centered on the cylinder axis and starts to interact at zero depth. The star density includes only those due to hadrons above $0.3 \mathrm{GeV} / \mathrm{c}$ momentum. Contours of higher star density are not shown for clarity while those of lower star density are not included due to statistical uncertainty. [Adapted from (Va75).] 


\section{APPENDiX B EXAMPLES OF RESUlts OF STAR DENSITY CALCUlations}

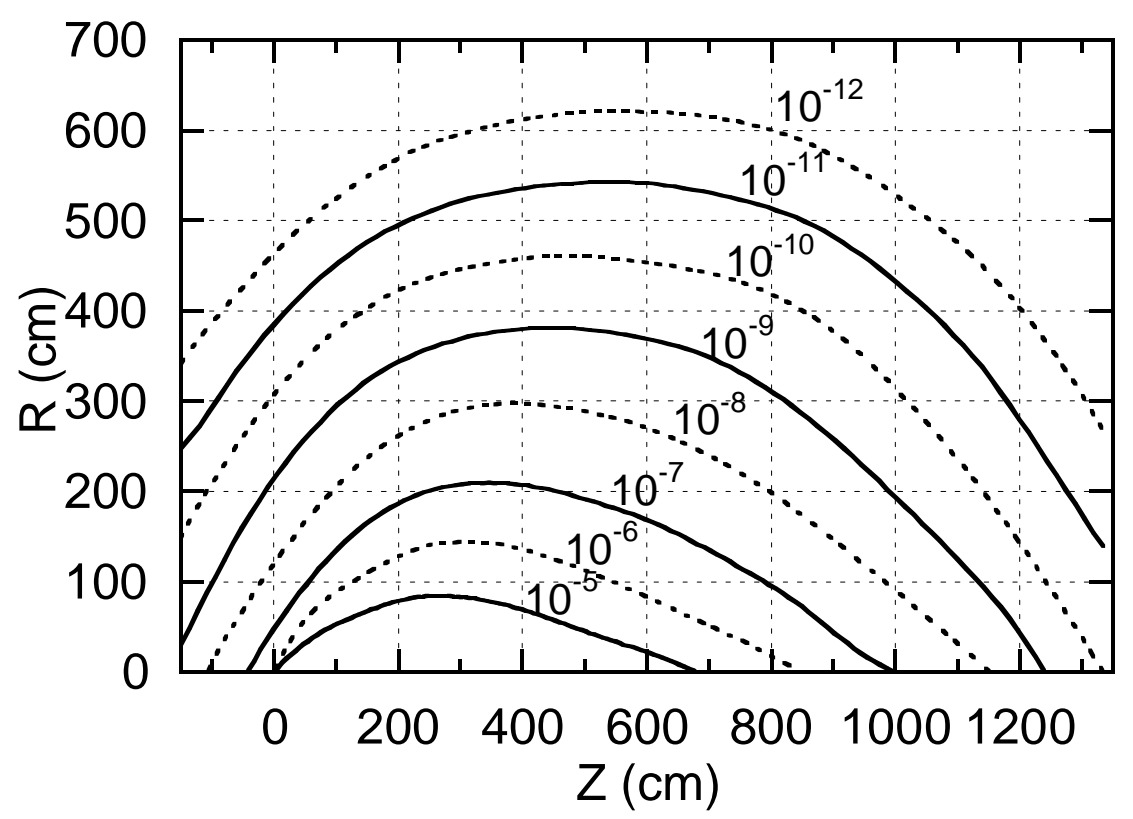

Fig. B1.c Monte Carlo results using CASIM for $1.0 \mathrm{TeV} / \mathrm{c}$ protons incident on a CONCRETE cylinder. Contours of equal star density $\left(\right.$ stars $\mathrm{cm}^{-3}$ ) per incident proton are plotted. The beam of $0.3 \times 0.3 \mathrm{~cm}^{2}$ cross section is centered on the cylinder axis and starts to interact at zero depth. The star density includes only those due to hadrons above $0.3 \mathrm{GeV} / \mathrm{c}$ momentum. Contours of higher star density are not shown for clarity while those of lower star density are not included due to statistical uncertainty. [Adapted from (Va75).]

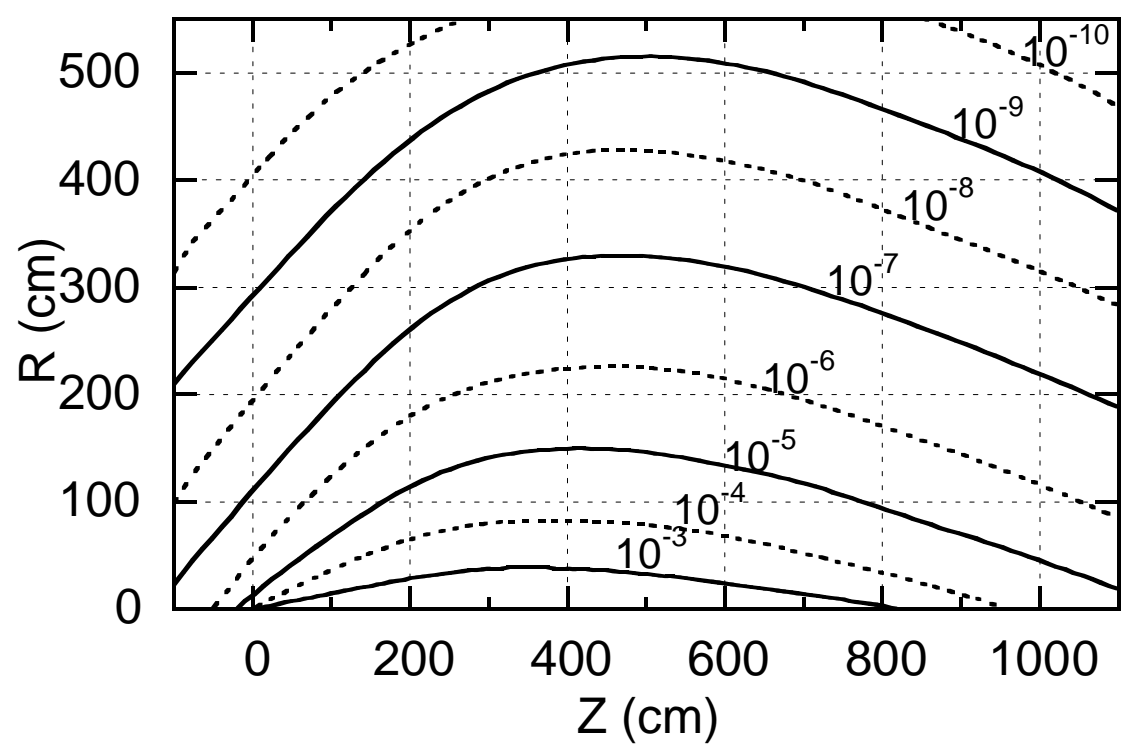

Fig. B1.d Monte Carlo results using CASIM for $10 \mathrm{TeV} / \mathrm{c}$ protons incident on a CONCRETE cylinder. Contours of equal star density (stars $\mathrm{cm}^{-3}$ ) per incident proton are plotted. The beam of $0.3 \times 0.3 \mathrm{~cm}^{2}$ cross section is centered on the cylinder axis and starts to interact at zero depth. The star density includes only those due to hadrons above $0.3 \mathrm{GeV} / \mathrm{c}$ momentum. Contours of higher star density are not shown for clarity while those of lower star density are not included due to statistical uncertainty. [Adapted from (Va87).] 


\section{B.2 Results for Solid IRON Cylinders}

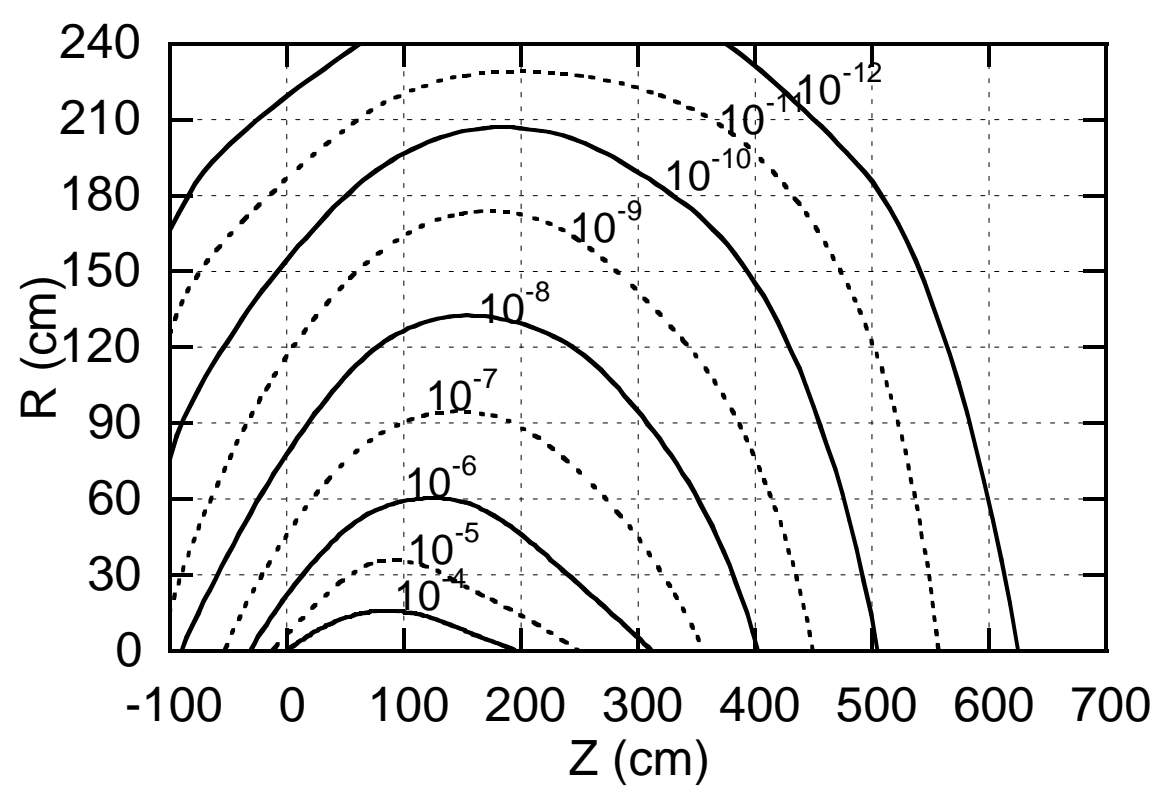

Fig. B2.a Monte Carlo results using CASIM for $30 \mathrm{GeV} / \mathrm{c}$ protons incident on an IRON cylinder. Contours of equal star density (stars $\mathrm{cm}^{-3}$ ) per incident proton are plotted. The beam of $0.3 \times 0.3 \mathrm{~cm}^{2}$ cross section is centered on the cylinder axis and starts to interact at zero depth. The star density includes only those due to hadrons above $0.3 \mathrm{GeV} / \mathrm{c}$ momentum. Contours of higher star density are not shown for clarity while those of lower star density are not included due to statistical uncertainty. [Adapted from (Va75).]

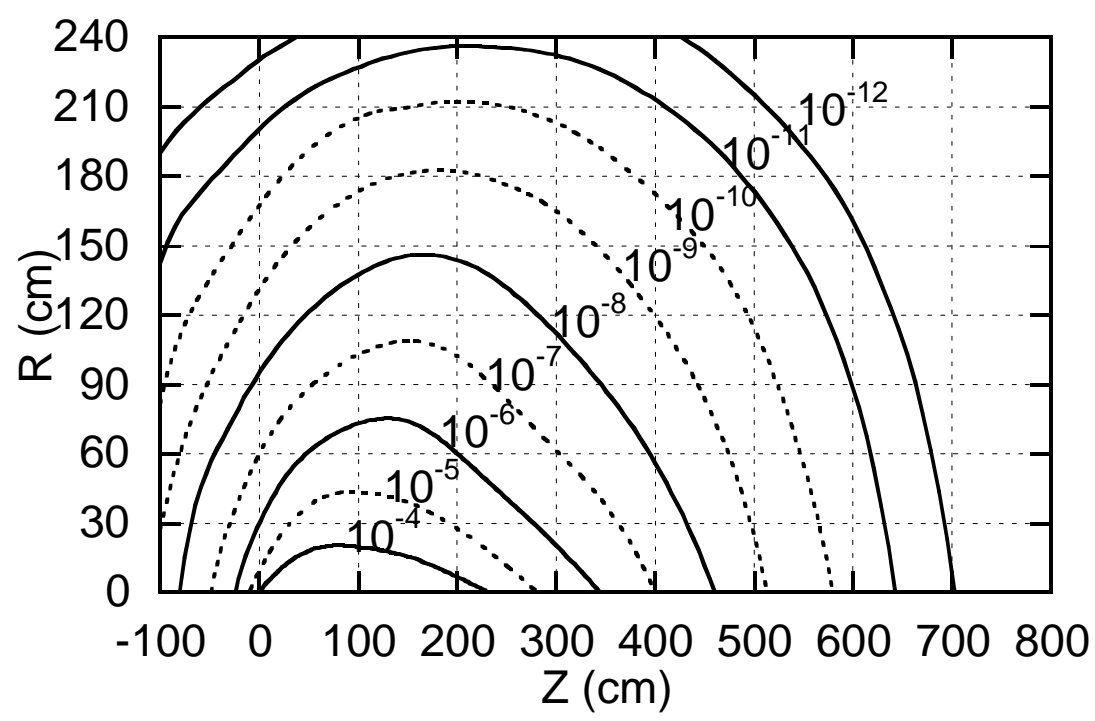

Fig. B2.b Monte Carlo results using CASIM for $100 \mathrm{GeV} / \mathrm{c}$ protons incident on an IRON cylinder. Contours of equal star density $\left(\right.$ stars $\left.\mathrm{cm}^{-3}\right)$ per incident proton are plotted. The beam of $0.3 \times 0.3 \mathrm{~cm}^{2}$ cross section is centered on the cylinder axis and starts to interact at zero depth. The star density includes only those due to hadrons above $0.3 \mathrm{GeV} / \mathrm{c}$ momentum. Contours of higher star density are not shown for clarity while those of lower star density are not included due to statistical uncertainty. [Adapted from (Va75).] 


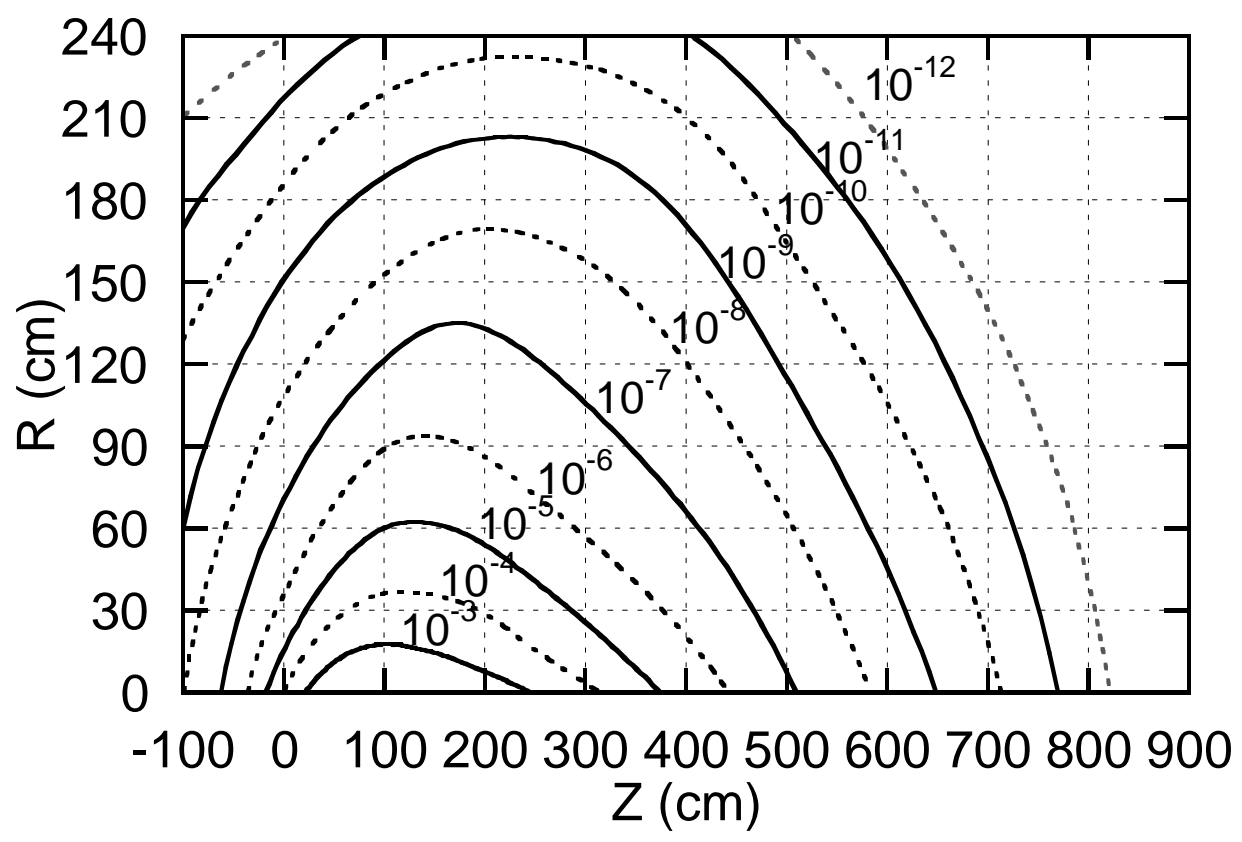

Fig. B2.c Monte Carlo results using CASIM for $1.0 \mathrm{TeV} / \mathrm{c}$ protons incident on an IRON cylinder. Contours of equal star density (stars $\mathrm{cm}^{-3}$ ) per incident proton are plotted. The beam of $0.3 \times 0.3 \mathrm{~cm}^{2}$ cross section is centered on the cylinder axis and starts to interact at zero depth. The star density includes only those due to hadrons above $0.3 \mathrm{GeV} / \mathrm{c}$ momentum. Contours of higher star density are not shown for clarity while those of lower star density are not included due to statistical uncertainty. [Adapted from (Va75).]

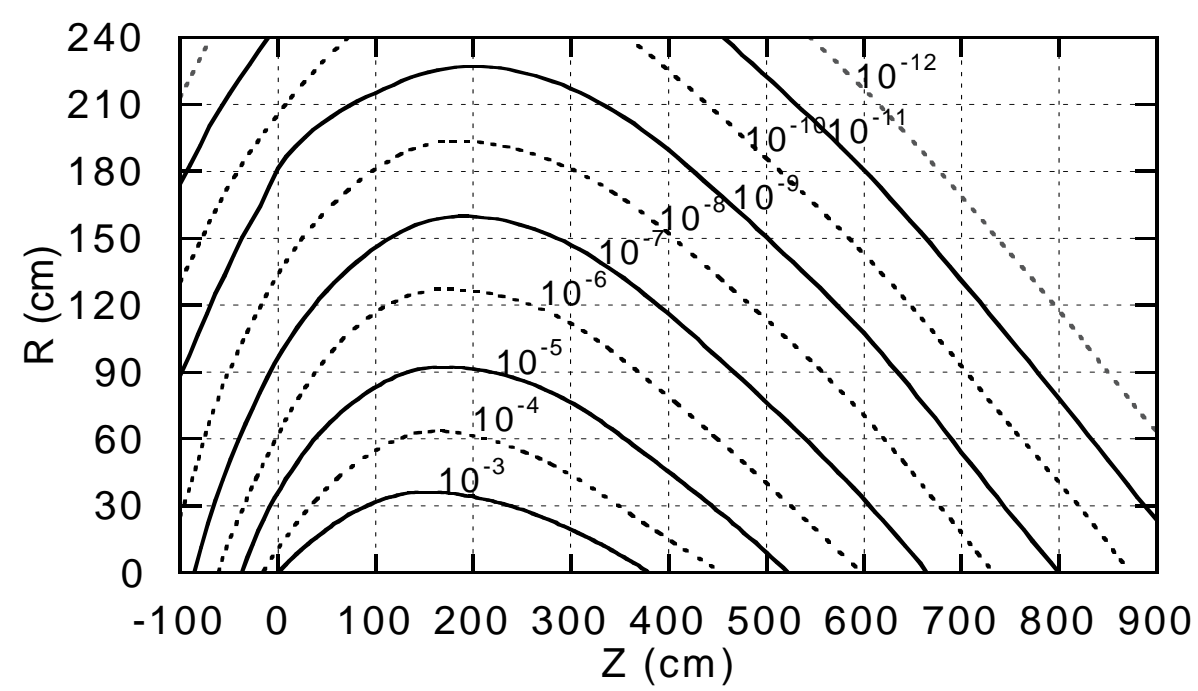

Fig. B2.d Monte Carlo results using CASIM for $10 \mathrm{TeV} / \mathrm{c}$ protons incident on an IRON cylinder. Contours of equal star density $\left(\right.$ stars $\mathrm{cm}^{-3}$ ) per incident proton are plotted. The beam of $0.3 \times 0.3 \mathrm{~cm}^{2}$ cross section is centered on the cylinder axis and starts to interact at zero depth. The star density includes only those due to hadrons above $0.3 \mathrm{GeV} / \mathrm{c}$ momentum. Contours of higher star density are not shown for clarity while those of lower star density are not included due to statistical uncertainty. [Adapted from (Va87).] 


\section{B.3 Results for 1.0 TeV Protons Incident on Magnets and Pipes}

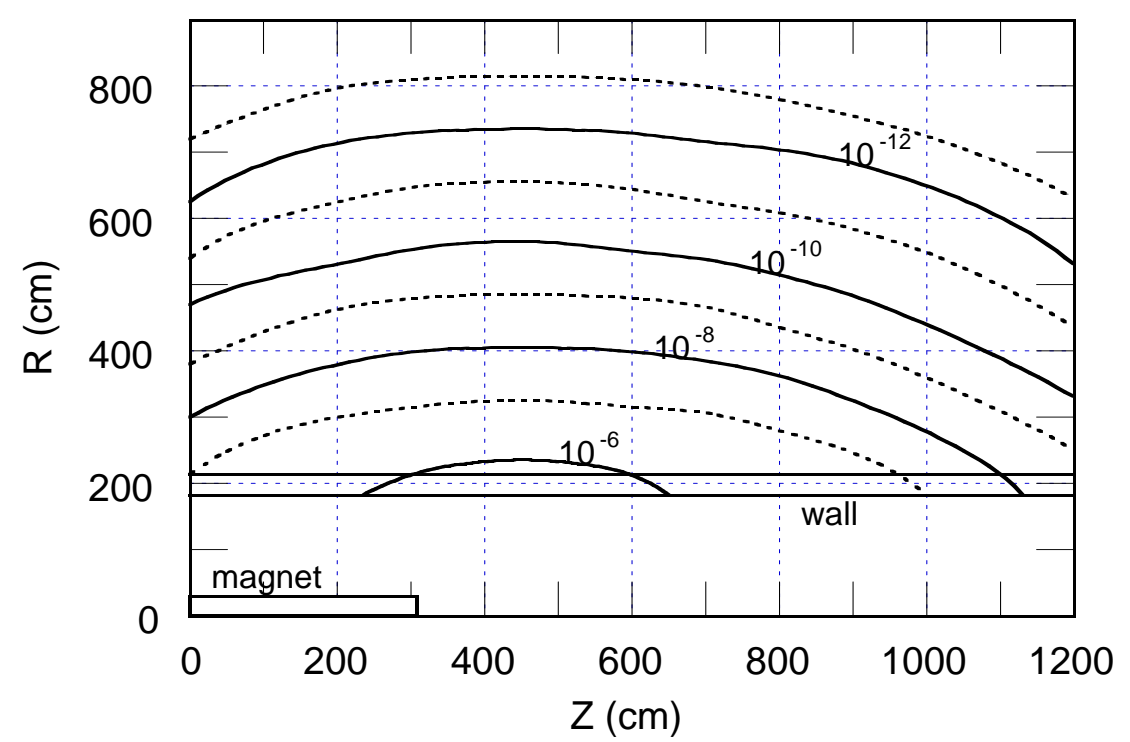

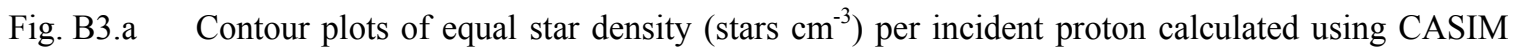
for a $1.0 \mathrm{TeV}$ proton beam incident "head on" on the inner edge of one of the pole pieces one standard deviation of beam width deep. The cross section of the magnet was rectangular with an aperture of $3.8 \times 12.7 \mathrm{~cm}^{2}$ and outer dimensions of $31.8 \times 40.6 \mathrm{~cm}^{2}$. No magnetic fields were included in the model. The results were averaged over azimuth and the magnet was centered in a cylindrical tunnel $182 \mathrm{~cm}$ in radius. The concrete wall was $30.48 \mathrm{~cm}$ thick and was surrounded by soil. [Adapted from (Co82b).]

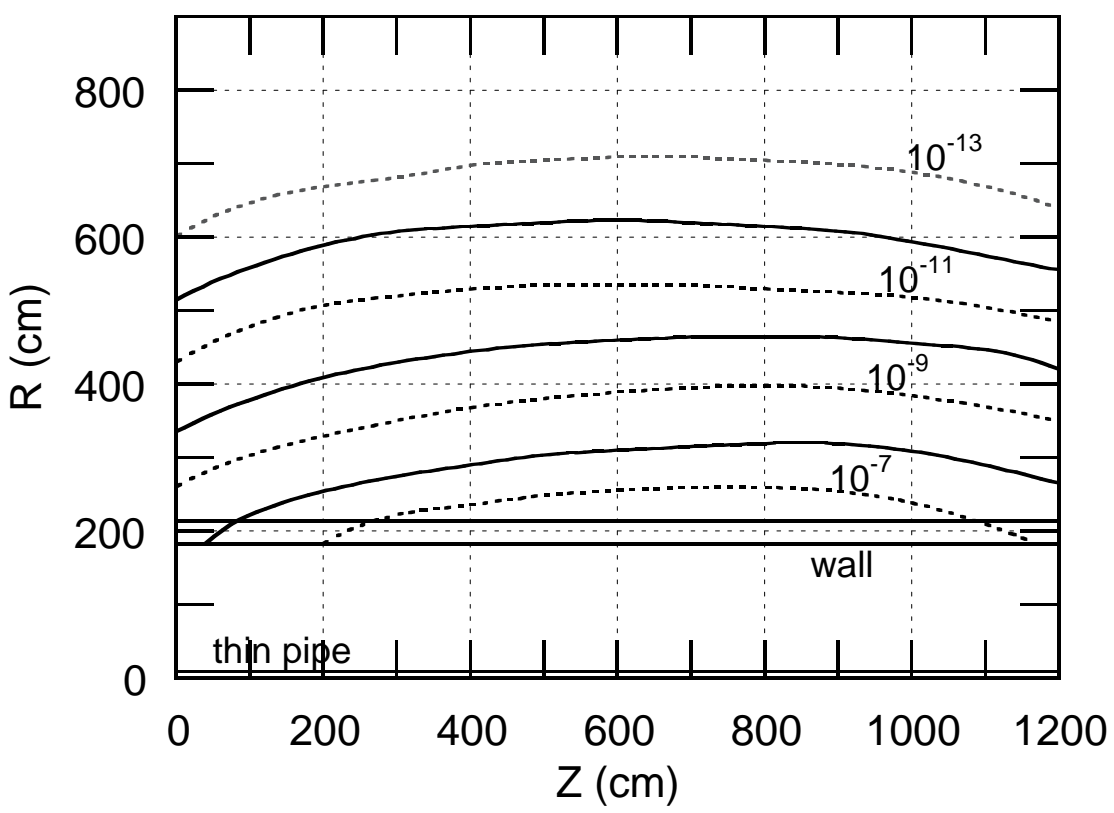

Fig. B3.b Contour plots of equal star density $\left(\right.$ stars $\left.\mathrm{cm}^{-3}\right)$ per incident proton calculated using CASIM for a $1.0 \mathrm{TeV}$ proton beam incident "head on" on a thin cylindrical aluminum pipe of 10.16 $\mathrm{cm}$ outside diameter with $0.318 \mathrm{~cm}$ thick walls. The results were averaged over azimuth and the pipe was centered in a cylindrical tunnel $182 \mathrm{~cm}$ in radius. The concrete wall was 30.48 $\mathrm{cm}$ thick and was surrounded by soil. [Adapted from (Co82b).] 


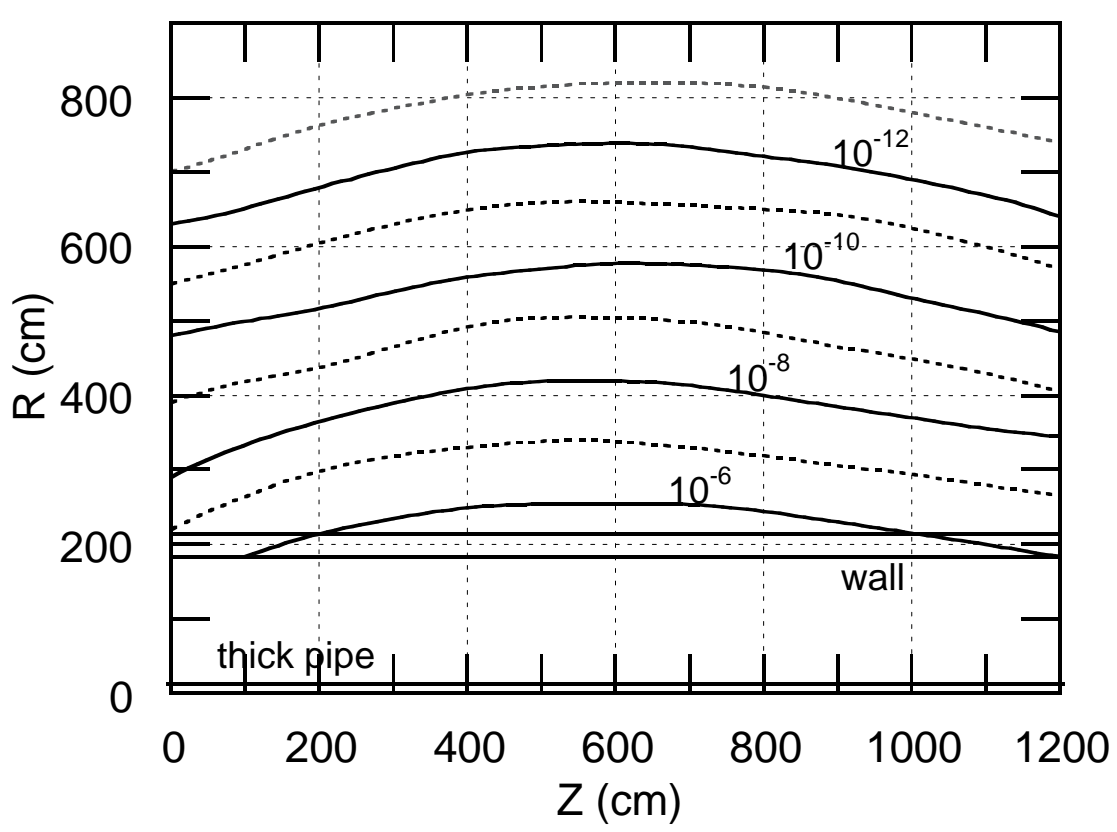

Fig. B3.c Contour plots of equal star density $\left(\right.$ stars $\left.\mathrm{cm}^{-3}\right)$ per incident proton calculated using CASIM for a $1.0 \mathrm{TeV}$ proton beam incident "head on" on a thick cylindrical iron pipe of $30.48 \mathrm{~cm}$ outside diameter with $1.27 \mathrm{~cm}$ thick walls. The results were averaged over azimuth and the pipe was centered in a cylindrical tunnel $182 \mathrm{~cm}$ in radius. The concrete wall was $30.48 \mathrm{~cm}$ thick and was surrounded by soil. [Adapted from (Co82b).]

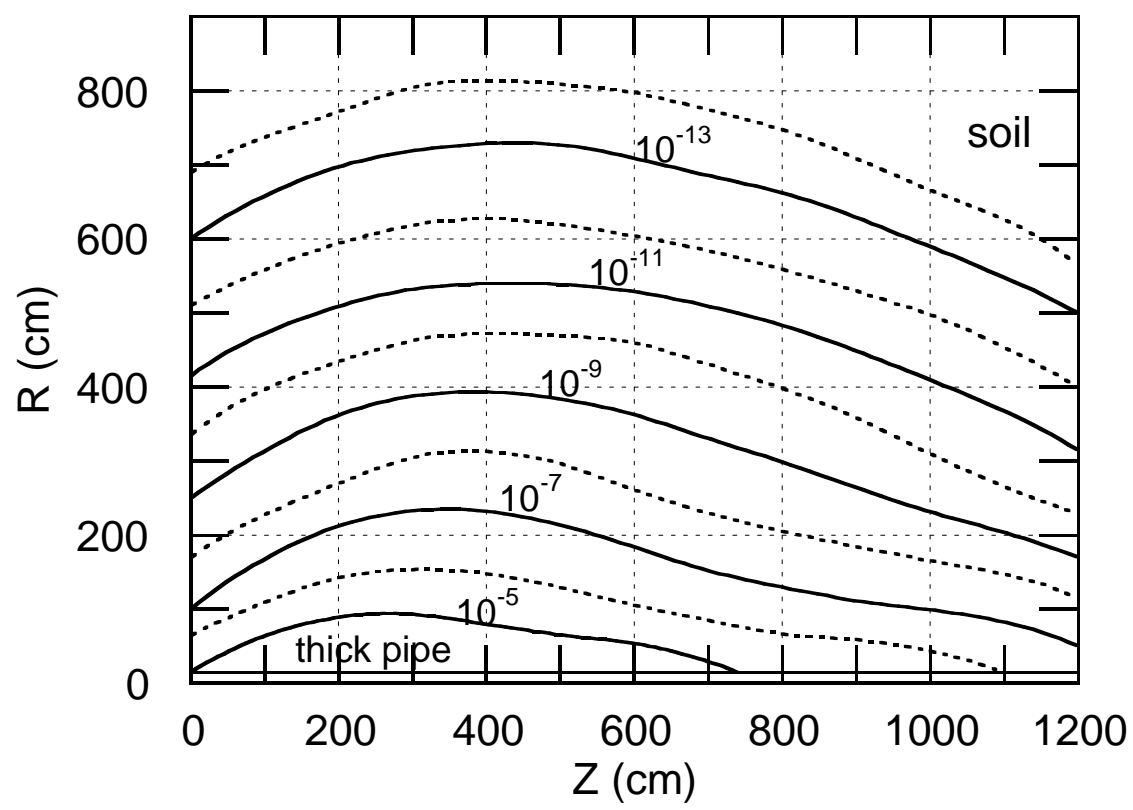

Fig. B3.d Contour plots of equal star density $\left(\right.$ stars $\left.\mathrm{cm}^{-3}\right)$ per incident proton calculated using CASIM for a $1.0 \mathrm{TeV}$ proton beam incident "head on" on a thick cylindrical iron pipe of $30.48 \mathrm{~cm}$ outside diameter with $1.27 \mathrm{~cm}$ thick walls. The pipe was surrounded by soil. [Adapted from (Co82b).] 


\section{B.4 Results for $120 \mathrm{GeV}$ Protons Incident on Magnets and Pipes}

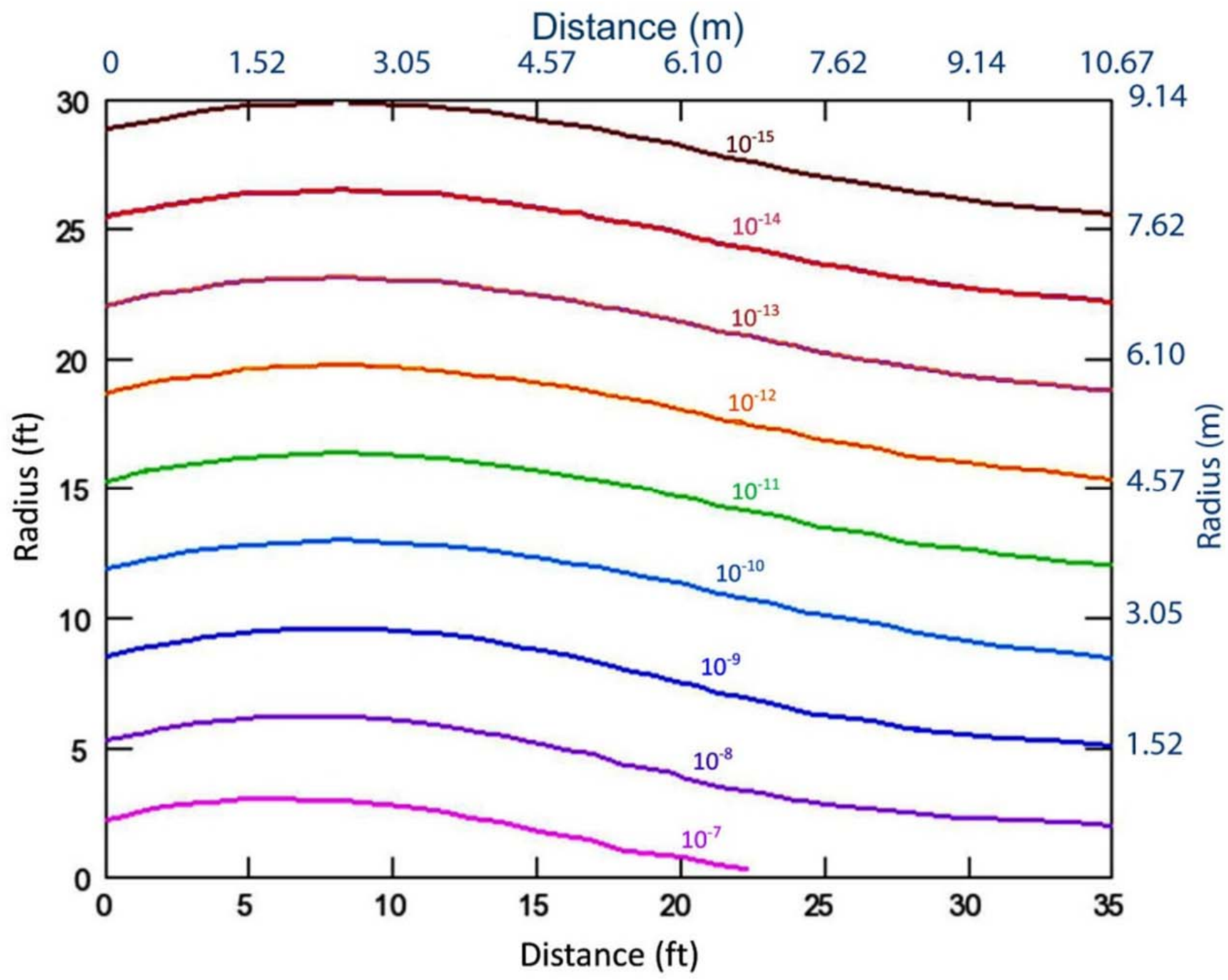

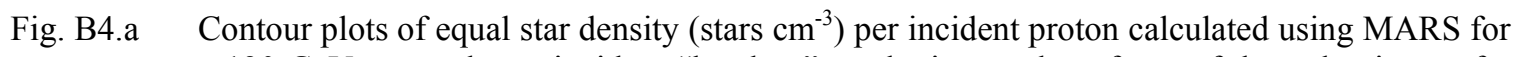
a $120 \mathrm{GeV}$ proton beam incident "head on" on the inner edge of one of the pole pieces of a magnet one standard deviation of beam width deep. The results are given as a function of distance along the beam direction and radius. The point loss was modeled to occur at a longitudinal distance of zero in the figure. The cross section of the magnet was rectangular with an aperture of $3.8 \times 12.7 \mathrm{~cm}^{2}$ and outer dimensions of $31.8 \times 40.6 \mathrm{~cm}^{2}$. No magnetic fields were included in the model. The results were averaged over azimuth and the magnet was centered in a cylindrical tunnel $100 \mathrm{~cm}$ in radius. The concrete wall was $30.48 \mathrm{~cm}$ thick and was surrounded by soil. [Adapted from (Re12).] 
APPENDix B EXAMPles of Results of Star Density Calculations

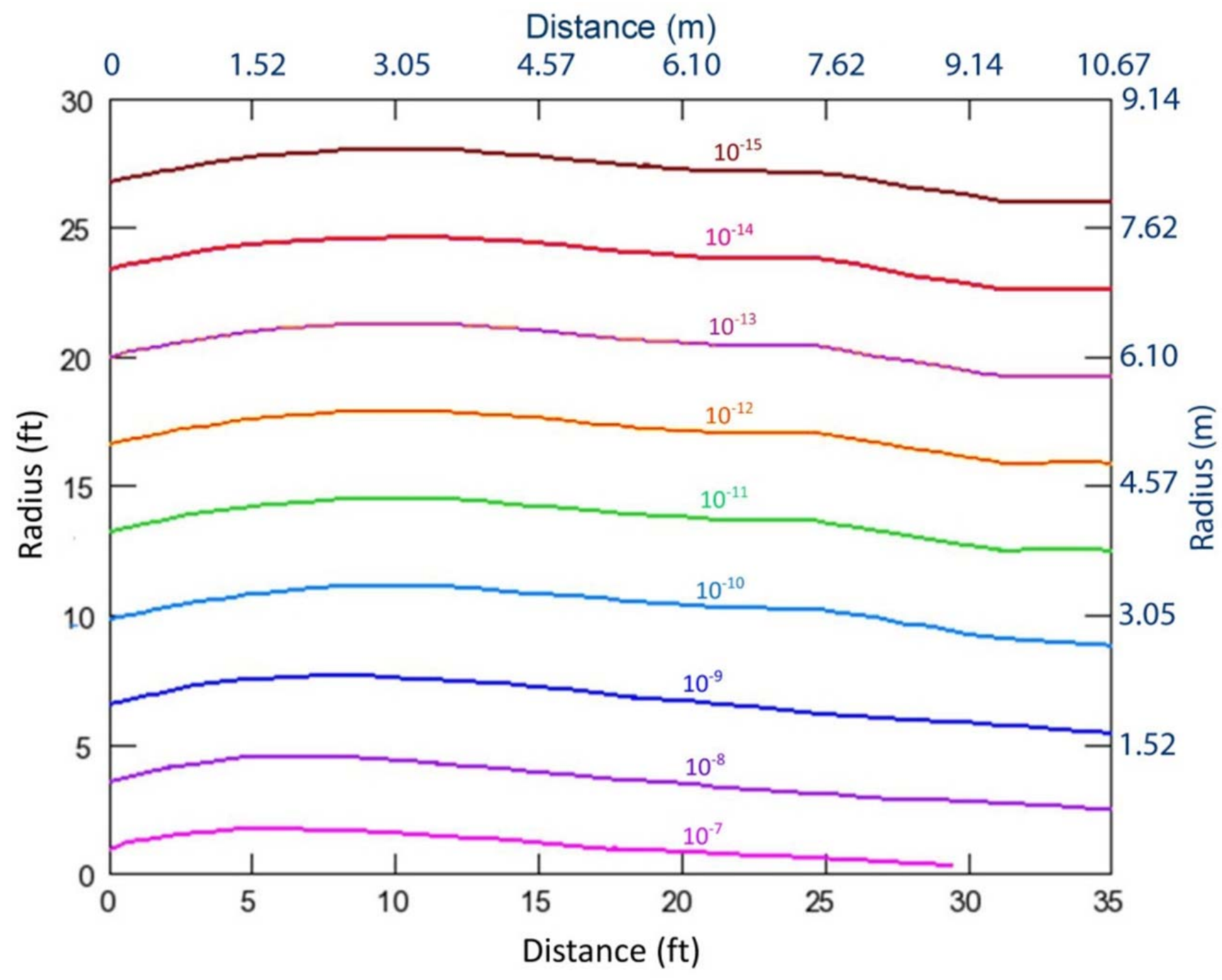

Fig. B4.b Contour plots of equal star density $\left(\right.$ stars $\left.\mathrm{cm}^{-3}\right)$ per incident proton in soil calculated using MARS for a $120 \mathrm{GeV}$ proton beam incident "head on" on a thin cylindrical aluminum pipe of $10.16 \mathrm{~cm}$ outside diameter with $0.318 \mathrm{~cm}$ thick walls. The results are given as a function of distance along the beam direction and radius. The point loss was modeled to occur at a longitudinal distance of zero in the figure. The results were averaged over azimuth and the pipe was centered in a cylindrical tunnel $100 \mathrm{~cm}$ in radius. The concrete wall was $30.48 \mathrm{~cm}$ thick and was surrounded by soil. [Adapted from $(\operatorname{Re} 12)$.] 
APPENDix B EXAMPLES OF RESUltS OF STAR DENSITY CALCULATIONS

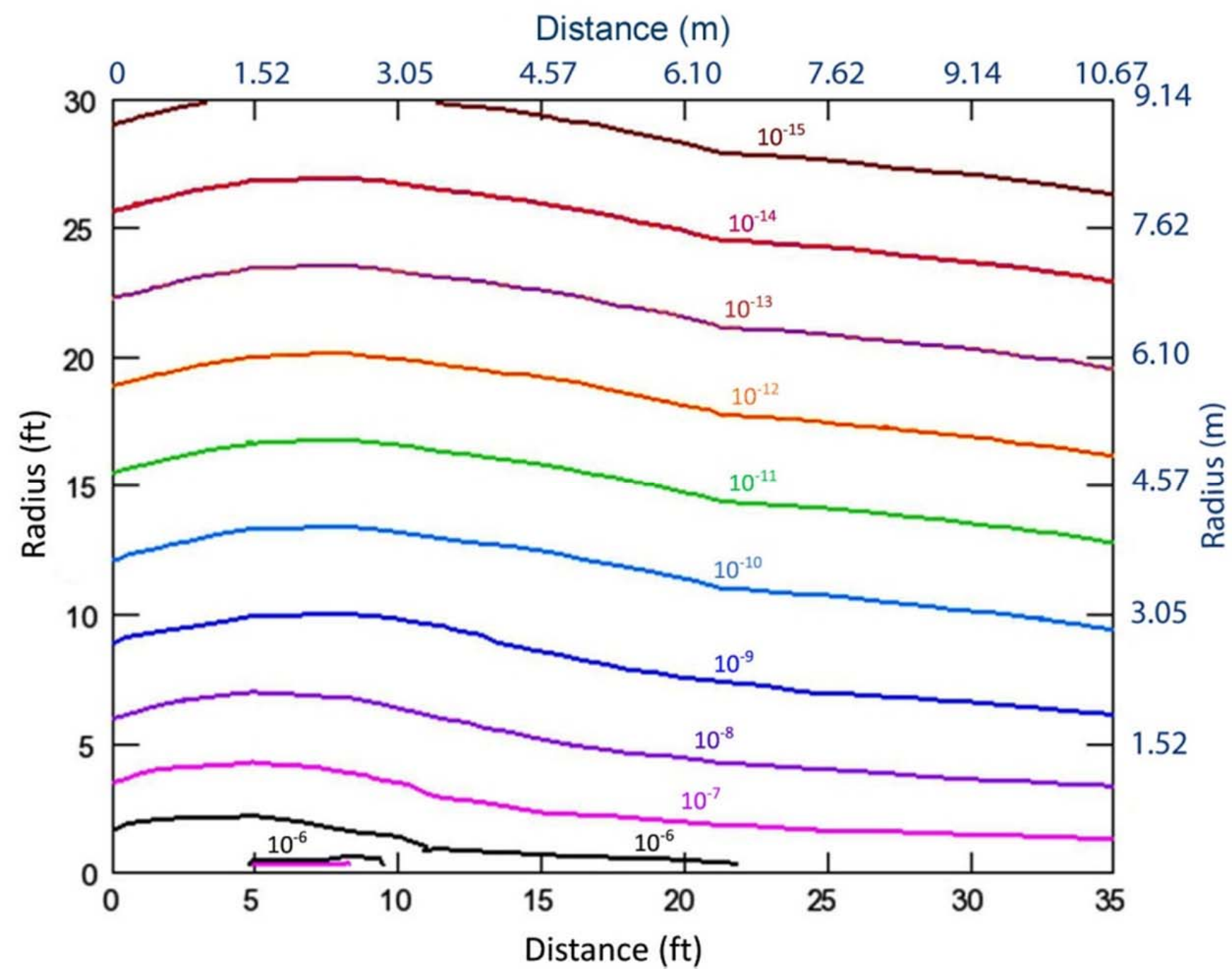

Fig. B4.c Contour plots of equal star density $\left(\right.$ stars $\left.\mathrm{cm}^{-3}\right)$ per incident proton calculated using MARS for a $120 \mathrm{GeV}$ proton beam incident "head on" on a thick cylindrical iron pipe of $30.48 \mathrm{~cm}$ outside diameter with $1.27 \mathrm{~cm}$ thick walls. The results are given as a function of distance along the beam direction and radius. The point loss was modeled to occur at a longitudinal distance of zero in the figure. The pipe was surrounded by soil. The lower star densities at very small radii result from build-up processes. These are not seen in the representative star density distributions for the other scenarios as the build-up processes take place in the concrete enclosure walls and are not included in these distributions in soil. [Adapted from (Re12).] 


\section{REFERENCES}

(Aa86) P. A. Aarnio, A. Fassò, H. J. Moehring, J. Ranft, G. R. Stevenson, "FLUKA86 users guide", CERN Divisional Report TIS-RP/168 (European Organization for Nuclear Research, 1986).

(A173) R. G. Alsmiller, Jr, and J. Barish, "Shielding against the neutrons produced when 400 $\mathrm{MeV}$ electrons are incident on a thick copper target", Part. Accel. $\underline{5}$ (1973) 155-159.

(A175) R. G. Alsmiller, R. T. Santoro, and J. Barish, "Shielding calculations for a $200 \mathrm{MeV}$ proton accelerator and comparisons with experimental data", Part. Accel. $\underline{7}$ (1975) 1-7.

(A181) R. G. Alsmiller, J. Barish, and R. L. Childs, "Skyshine at neutron energies $<400 \mathrm{MeV}$ ", Part. Accel. 11 (1981) 131-141.

(A185) V. E. Aleinikov, A. P. Cherevatenko, F. B. Clapier, and V. I. Tsovbun, "Neutron radiation field due to $6.6 \mathrm{MeV} / \mathrm{amu}{ }^{58} \mathrm{Ni}$ ions bombarding a thick $\mathrm{Cu}$ target", Rad. Prot. Dos. 11 (1985) 245-248.

(An62) I. O. Andersson and J. Braun, "A neutron rem counter", Nucleonik $\underline{6}$ (1963) 237 and I. O. Andersson and J. Braun, "A neutron rem counter with uniform sensitivity from 0.025 $\mathrm{MeV}$ to $10 \mathrm{MeV}$ ", Neutron dosimetry, (Proceedings of a Symposium held at Harwell, 1962) vol. 2, p.87.

(An07) M. P. Anderson, Introducing groundwater physics, Physics Today (May 2007) 42-47.

(Ap79) R. E. Apfel, "The superheated drop detector", Nucl. Instr. and Meth. 162 (1979) 603-608.

(Ar69a) T. W. Armstrong and R. G. Alsmiller, Jr., "Calculation of the residual photon dose rate around high energy proton accelerators", Nucl. Sci. Eng. $\underline{38}$ (1969) 53-62.

(Ar69b) T. W. Armstrong and J. Barish, "Calculation of the residual photon dose rate due to the activation of concrete by neutrons from a 3-GeV proton beam in iron", in Proceedings of the second international conference on accelerator radiation dosimetry and experience, (Stanford, CA 1969), CONF-691101, National Technical Information Service, pp. 70-91. See also Nucl. Sci. and Eng. $\underline{38}$ (1969) 265-270.

(Ar80) T. W. Armstrong, "The HETC hadronic cascade code", in Computer techniques in radiation transport and dosimetry, W. R. Nelson and T. M. Jenkins, editors (Plenum Press, New York and London 1980), pp. 373-375.

(Ar97) A. Aroua, T. Buchillier, M. Gracescu, and M. Höfert, "Neutron measurements around a high-energy lead ion beam at CERN", Rad. Prot. Dos. 70 (1997) 437-440.

(Aw72) M. Awschalom, "Bonner spheres and tissue equivalent chambers for extensive radiation area monitoring around a $1 / 2 \mathrm{TeV}$ proton synchrotron", in Proceedings of IAEA Symposium on neutron monitoring for radiation protection purposes (Vienna, 1972, vol 1, p. 297). The details of the measurements are discussed in M. Awschalom, T. Borak, and H. Howe, "A study of spherical, pseudospherical, and cylindrical moderators for a neutron dose equivalent rate meter", Fermilab Report TM-291 (Fermi National Accelerator Laboratory, July 1971).

(Aw73) M. Awschalom and L. Coulson, "A new technique in environmental neutron spectroscopy", in Proceedings of the third international conference of the International Radiation Protection Association, Washington, DC (U. S. Atomic Energy Commission, Technical Information Center, Oak Ridge Tennessee, CONF 730907-P2, 1973). 


\section{REFERENCES}

(Aw75) M. Awschalom, P. J. Gollon, C. Moore, and A. Van Ginneken, "Energy deposition in thick targets by high energy protons: measurement and calculation", Nucl. Instr. and Meth. 131 (1975) 235-241.

(Aw76) M. Awschalom, S. Baker, C. Moore, A. Van Ginneken, K. Goebel, and J. Ranft, "Measurements and calculations of cascades produced by $300 \mathrm{GeV}$ protons incident on a target inside a magnet", Nucl. Instr. And Meth. 138 (1976) 521-531.

(Aw85) M. Awschalom and R. S. Sanna, "Applications of Bonner sphere detectors in neutron field dosimetry", Rad. Prot. Dos. 10 (1985) 89-101.

(Ba65) J. Baarli and A. H. Sullivan, "Radiation dosimetry for protection purposes near high energy particle accelerators", Health Phys. 11 (1965) 353-361.

(Ba67) G. Bathow, E. Freytag, and K. Tesch, "Measurements on $6.3 \mathrm{GeV}$ electromagnetic cascades and cascade producing neutrons", Nucl. Phys. B2 (1967) 669-689.

(Ba69) M. Barbier, Induced radioactivity (North-Holland Publishing Company, Amsterdam and London, Wiley Interscience Division, John Wiley and Sons, Inc, New York, 1969).

(Ba84) S. I. Baker, C. R. Kerns, S. H. Pordes, J. B. Cumming, A. Soukas, V. Agoritsas, and G. R. Stevenson, "Absolute cross section for the production of ${ }^{24} \mathrm{Na}$ in $\mathrm{Cu}$ by $400 \mathrm{GeV}$ protons", Nucl. Instr. and Meth. A222 (1984) 467-473.

(Ba89) S. Ban, H. Hirayama, and S. Muiri, "Estimation of absorbed dose due to gas bremsstrahlung from electron storage rings", Health Phys. $\underline{57}$ (1989) 407-412.

(Ba91) S. I. Baker, R. A. Allen, P. Yurista, V. Agoritsas, and J. B. Cumming, " $\mathrm{Cu}(\mathrm{p}, \mathrm{x})^{24} \mathrm{Na}$ cross section from 30 to $800 \mathrm{GeV}$ ", Phys. Rev. $\underline{\text { C43 }}$ (1991) 2862-2865.

(Ba98) V. Batu, Aquifer hydraulics (John Wiley and Sons, Inc., New York, 1998).

(Be70) M. J. Berger and S. M. Seltzer, "Bremsstrahlung and photoneutrons from thick tunsten and tantalum targets", Phys. Rev. $\underline{\mathrm{C} 2}$ (1970) 621-631.

(Bo72) T. B. Borak, M. Awschalom, W. Fairman, F. Iwami, and J. Sedlet, "The underground migration of radionuclides produced in soil near high energy proton accelerators", Health Phys. 23 (1972) 679-687.

(Bo87) T. B. Borak and J. W. N. Tuyn, "Can uranium calorimeters become critical?", CERN Report TIS-RP/194 (European Organization for Nuclear Research, 1987).

(Br60) R. L. Bramblett, R. I. Ewing, and T. W. Bonner, "A new type of neutron dosimeter", Nucl. Instr. and Meth. $\underline{9}$ (1960) 1-12.

(Br78) L. W. Brackenbush, G. W. R. Endres, and L. G. Faust, "Measuring neutron dose and quality factors with tissue-equivalent proportional counters", in Advances in radiation monitoring, proceedings of a symposium (IAEA, Stockholm, 1978).

(Br97) J. F. Briesmeister, Editor, "MCNP: A general Monte Carlo N-particle transport code", LANL Report LA-12625-M, Version 4B (Los Alamos National Laboratory, March 1997). 


\section{REFERENCES}

(Br99) G. I. Britvich, A. A. Chumakov, R. M. Ronningen, R. A. Blue, and L. H. Heilbronn, "Measurements of thick target neutron yields and shielding studies using beams of ${ }^{4} \mathrm{He}$, ${ }^{12} \mathrm{C}$, and ${ }^{16} \mathrm{O}$ at $155 \mathrm{MeV} /$ nucleon from the $\mathrm{K} 1200$ cyclotron at the National Superconducting Cyclotron Laboratory", Rev. Sci. Instr. 70 (1999) 2314-2324.

(Bu89) S. W. Butala, S. I. Baker, and P. M. Yurista, "Measurements of radioactive gaseous releases to air from target halls at a high-energy proton accelerator", Health Phys. $\underline{57}$ (1989) 909-916.

(Ca87) D. C. Carey, The optics of charged particle beams (Harwood Academic Publishers, New York 1987).

(Ce69) H. Cember, Introduction to health physics (Pergamon Press, New York, 1969). Subsequent editions of this text have been produced.

(Ce80) R. A. Cecil, B. D. Anderson, A. R. Baldwin, R. Madey, A. Galonsky, P. Miller, L. Young, and F. M. Waterman, "Neutron angular and energy distributions from 710-MeV alphas stopping in water, carbon, steel, and lead, and 640-MeV alphas stopping in lead",

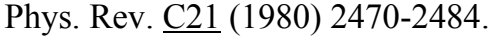

(CFR76) United States Code of Federal Regulations, Title 40, Part 141.16, "National primary drinking water standard for beta- and gamma- emitting radionuclides", 1976.

(CFR89) United States Code of Federal Regulations, Title 40, Part 61, Subpart H, "National emissions standard for hazardous air pollutants (NESHAP) for the emission of radionuclides other than radon from Department of Energy Facilities", 1989.

(CFR93) United States Code of Federal Regulations, Title 10 Part 835, "Occupational radiation protection at department of energy facilities", 1993. These Regulations were amended in November 1998 and again in June 2007 (see CFR07).

(CFR00) United States Code of Federal Regulations, Title 40, Parts 9, 141, and 142, "National primary drinking water regulations; final rule", December 2000.

(CFR07) Procedural rules for DOE nuclear activities and occupational radiation protection; final rule. Washington, DC: U. S. Government Printing Office; 10 CFR Parts 820 and 835. Federal Register 72:31904-31941; 8 June 2007. Appendix C of 10 CFR Part 835 was amended in April 2011 to revise some of the values of Derived Air Concentrations.

(Ch63) A. B. Chilton and C. M. Huddleston, "A semi-empirical formula for differential dose albedo for gamma rays on concrete", Nucl. Sci. Eng. 17 (1963) 419.

(Ch64) A. B. Chilton, "Backscatter for gamma rays from a point source near a concrete plane surface", Univ. Illinois Eng. Sta. Bull. No 471 (University of Illinois, Urbana, Illinois, 1964).

(Ch65a) A. B. Chilton, "Backscattering of gamma rays from point sources by an infinite-plane concrete surface", Nucl. Sci. Eng. 21 (1965) 194-200.

(Ch65b) A. B. Chilton, C. M. Davisson, and L. A. Beach (1965), "Parameters for C-H albedo formula for gamma rays reflected from water, concrete, iron, and lead", Trans. Am. Nucl. Soc. $\underline{8}$, No. 2, (1965) 656-657. 


\section{REFERENCES}

(Ch84) A. B. Chilton, J. K. Shultis, and R. E. Faw, Principles of radiation shielding (PrenticeHall, Englewood Cliffs, New Jersey, 1984).

(Ch99) A. W. Chao and M. Tigner, editors, Handbook of accelerator physics and engineering (World Scientific Publishing Co., Singapore, 1999).

(Ci65) A. Citron, L. Hoffmann, C. Passow, W. R. Nelson, and M. Whitehead, "A study of the nuclear cascade in steel initiated by $19.2 \mathrm{GeV} / \mathrm{c}$ protons", Nucl. Instrum. Meth. $\underline{32}$ (1965) $48-52$.

(C171) F. H. Clark, "Shielding Data" Appendix E, in Protection against neutron radiation, NCRP Report No. 38 (National Council on Radiation Protection and Measurements, Washington, DC, 1971).

(C183) F. Clapier and C. S. Zaidins, "Neutron dose equivalent rates due to heavy ion beams", Nucl. Instr. and Meth. 217 (1983) 489-494.

(Co73) M. O. Cohen, W. Guber, E. S.Troubezkoy, H. Lichtenstein, and H. A. Steinberg, "SAM$\mathrm{CE}$, a three dimensional Monte-Carlo code for the solution of the forward neutron and forward and adjoint gamma ray transport equations", Rept. DNA-2830-F, Rev. B Defense Nuclear Agency, Washington, DC (1973).

(Co78) B. L. Cohen, "Nuclear cross sections", Handbook of radiation measurement and protection, Section A: Volume I Physical science and engineering data, A. Brodsky, editor (CRC Press, Inc., West Palm Beach, Florida, 1978).

(Co82a) J. D. Cossairt, N. V. Mokhov, and C. T. Murphy, "Absorbed dose measurements external to thick shielding at a high energy proton accelerator: Comparison with Monte-Carlo calculations", Nucl. Instr. and Meth. 197 (1982) 465-472.

(Co82b) J. D. Cossairt, "A collection of CASIM calculations", Fermilab Report TM-1140 (Fermi National Accelerator Laboratory, 1982).

(Co83) J. D. Cossairt, "Recent muon fluence measurements at Fermilab", Health Phys. $\underline{45}$ (1983) 651-658.

(Co85a) J. D. Cossairt, S. W. Butala, and M. A. Gerardi, "Absorbed dose measurements at an 800 $\mathrm{GeV}$ proton accelerator, comparison with Monte-Carlo calculations", Nucl. Instr. and Meth. in Phys. Res. A238 (1985) 504-508.

(Co85b) J. D. Cossairt, J. G. Couch, A. J. Elwyn, and W. S. Freeman, "Radiation measurements in a labyrinth penetration at a high-energy proton accelerator", Health Phys. 49 (1985) 907917.

(Co85c) J. D. Cossairt and L. V. Coulson, "Neutron skyshine measurements at Fermilab", Health Phys. $\underline{48}$ (1985) 171-181.

(Co87) J. D. Cossairt and A. J. Elwyn, "Personal dosimetry in a mixed field of high-energy muons and neutrons", Health Phys. $\underline{52}$ (1987) 813-818.

(Co88) J. D. Cossairt, A. J. Elwyn, W. S. Freeman, W. C. Salsbury, and P. M. Yurista, "Measurement of neutrons in enclosures and outside of shielding at the Tevatron", Report Fermilab-Conf-88/106 (1988). Also in Proceedings of the $22^{\text {nd }}$ midyear meeting of the Health Physics Society; Topical meeting on instrumentation (San Antonio, TX, December, 1988) 190-199. 


\section{REFERENCES}

(Co89a) J. D. Cossairt, A. J. Elwyn, W. S. Freeman, and S. W. Butala, "A study of the transport of high energy muons through a soil shield at the Tevatron", Nucl. Instr. and Meth. in Phys. Res. A276 (1989) 78-85.

(Co89b) J. D. Cossairt, A. J. Elwyn, and W. S. Freeman, "A study of the production and transport of high energy muons through a soil shield at the Tevatron", Nucl. Instr. and Meth. in Phys Res. A276 (1989) 86-93.

(Co95) J. D. Cossairt, "Approximate technique for estimating labyrinth attenuation of accelerator-produced neutrons", Fermilab Radiation Physics Note No. 118 (Fermi National Accelerator Laboratory, October 1995, Revised April 2013).

(Co96) J. D. Cossairt, "On residual dose rate within particle accelerator enclosures", Health Phys. $\underline{71}$ (1996) 315-319.

(Co97) J. D. Cossairt, N. L. Grossman, and E. T. Marshall, "Assessment of dose equivalent due to neutrinos", Health Phys. 73 (1997) 894-898.

(Co98) J. D. Cossairt, "Rule of thumb for estimating groundwater activation from residual dose rates", Fermilab Environmental Protection Note No. 15 (Fermi National Accelerator Laboratory, June 1998).

(Co08) J. D. Cossairt, "Accelerator and beam physics for health physicists", in Topics in accelerator health physics (J. D. Cossairt, V. Vylet, and J. W. Edwards, editors; Medical Physics Publishing, Madison, WI, 2008) 1-45.

(Co09a) J. D. Cossairt and K. Vaziri, "Neutron dose per fluence and weighting factors for use at high energy accelerators", Health Phys. $\underline{96}$ (2009) 617-628.

(Co09b) J. D. Cossairt, D. W. Grobe, and M. A. Gerardi, "Measurements of radiation quality factors using a recombination chamber", Fermilab Report TM-1248-REV (Fermi National Accclerator Laboratory, 1984, Revised 2009).

(Co13) J. D. Cossairt, "Approximate technique for evaluating the Moyer integral in the Moyer model of hadron shielding", Fermilab Radiation Physics Note No. 117 (Fermi National Accelerator Laboratory, October 1994, revised April 2013).

(Cu55) S. C. Curran, "Proportional counter spectroscopy", in Alpha, Beta and gamma-ray spectroscopy, K. Siegbahn, editor (Elsevier-North Holland, Amsterdam, 1955).

(De65) H. DeStaebler, "Similarity of shielding problems at electron and proton accelerators", SLAC Report SLAC Pub. 179 (Stanford Linear Accelerator Center, 1965). These results are also quoted in $(\mathrm{Fa} 90)$.

(DeP66) J. DePangher and L. L. Nichols, "A precision long counter for measuring fast neutron flux density", PNL Report BNWL-260 (Pacific Northwest Laboratory, 1966).

(D'H68) M. M. D'Hombres, C. Devillers, F. Gervaise, B. De Sereville, and P. Tardy-Joubert, "Propagation des neutrons dans les tunnes d'Accès à un accélérateur de haute energie à protons", Rep. CEA-R-3491 (Centre d'études nucléaires de Saclay, 1968). Some of this work was also reported in (Se71).

(DOE90) U. S. Department of Energy Order 5400.5, "Radiation protection of the public and the environment" (1990). Subsequent revisions to this Order were made. As of 2011, this Order has been superseded by (DOE11) 


\section{REFERENCES}

(DOE11) U. S. Department of Energy Order 458.1, "Radiation protection of the public and the environment" (June, 2011, with subsequent editorial updates). The values of the Derived Concentration Standards (DCSs) for air and water are tabulated in DOE-STD-1196-2011 "Derived concentration technical standard", April, 2011.

(Ed93) D. A. Edwards and M. J. Syphers, An introduction to the physics of high energy accelerators (John Wiley and Sons, Inc., New York, 1993).

(E186) A. J. Elwyn and J. D. Cossairt, "A study of neutron leakage through an Fe shield at an accelerator", Health Phys. 51 (1986) 723-735.

(E191) A. J. Elwyn to J. D. Cossairt, private communication (1991).

(En66) H. A. Enge, Introduction to nuclear physics (Addison-Wesley, Reading, Massachusetts, 1966).

(Fa84) A. Fassò, K. Goebel, M. Höfert, G. Rau, H. Schönbacher, G. R. Stevenson, A. H. Sullivan, W. P. Swanson, and J. W. N. Tuyn, "Radiation problems in the design of the large electron-positron collider (LEP)", CERN Report 84-02 (European Organization for Nuclear Research, 1984).

(Fa90) A. Fassò, K. Goebel, M. Höfert, J, Ranft, and G. Stevenson, Landolt-Börnstein numerical data and functional relationships in science and technology new series; Group I: nuclear and particle physics Volume II: Shielding against high energy radiation (O. Madelung, Editor in Chief, H. Schopper, Editor, Springer-Verlag, Berlin, Heidelberg, 1990).

(Fa93) A. Fassò, A. Ferrari, J. Ranft, P.R. Sala, "FLUKA: present status and future developments", in Proc. IV $V^{\text {th }}$ Int. Conf. on calorimetry in high energy physics, La Biodola, Italy, 21-26 Sept. 1993, Ed. A. Menzione and A. Scribano, p. 493 (World Scientific Publishing Co, Singapore, 1993).

(Fe88) C. W. Fetter, Applied hydrogeology (Merrill Publishing Company, Columbus, OH, 1988).

(Fe96) A. Ferrari, M. Pelliccioni, and M. Pillon, "Fluence to effective dose and effective dose equivalent conversion coefficients for photons from $50 \mathrm{keV}$ to $10 \mathrm{GeV}$ ", Rad Prot. and Dos. 67 (1996) 245-251.

(Fe97a) A. Ferrari, M. Pelliccioni, and M. Pillon, "Fluence to effective dose and effective dose equivalent conversion coefficients for electrons from $5 \mathrm{MeV}$ to $10 \mathrm{GeV}$ ", Rad Prot. and Dos. 69 (1997) 97-104.

(Fe97b) A. Ferrari, M. Pelliccioni, and M. Pillon, "Fluence to effective dose conversion coefficients for protons from $5 \mathrm{MeV}$ to $10 \mathrm{TeV}$ ", Rad. Prot. and Dos. 71 (1997) 85-91.

(Fe97c) A. Ferrari, M. Pelliccioni, and M. Pillon, "Fluence to effective dose conversion coefficients for neutrons from up to 10 TeV", Rad. Prot. and Dos. 71 (1997) 165-173.

(Fr84) W. S. Freeman and F. P. Krueger, "Neutron calibration tests of Fermilab radiation detectors", Fermilab Radiation Physics Note No. 48 (Fermi National Accelerator Laboratory, 1984).

(Ga73) T. A. Gabriel and R. T. Santoro, "Photon dose rates from the interactions of $100 \mathrm{GeV}$ protons in iron and iron-lead beam stops", Part. Accel. 4 (1973) 169-186. 


\section{REFERENCES}

(Ga85) T. A. Gabriel, "The high energy transport code HETC", ORNL-TM-9727 (Oak Ridge National Laboratory, 1985).

(Ga94) T. A. Gabriel, D. E. Groom, P. K. Job, N. V. Mokhov, and G. R. Stevenson, "Energy dependence of hadronic activity", Nucl. Instrum. and Meth. in Phys. Res. A338 (1994) 336-347.

(Gi68) W. S. Gilbert, D. Keefe, J. B. McCaslin, H. W. Patterson, A. R. Smith, L. D. Stephens, K. B. Shaw, G. R. Stevenson, R. H. Thomas, R. D. Fortune, and K. Goebel, "1966 CERNLRL-RHEL shielding experiment at the CERN proton synchrotron", UCRL-17941 (University of California Radiation Laboratory, 1968). Much of this material is summarized in reference (Pa73).

(Gi69) W. S. Gilbert, "Shielding measurements at the CERN $25 \mathrm{GeV}$ proton synchrotron", in Proceedings of the second international conference on accelerator radiation dosimetry and experience, (Stanford, CA 1969), CONF-691101, National Technical Information Service, pp. 323-340 .

(Go71) P. J. Gollon and M. Awschalom, "Design of penetrations in hadron shields", CERN Report 71-16 Vol. 2 (European Organization for Nuclear Research, 1971). Also published in IEEE Trans. Nucl. Sci. NS-18 (1971) 697-713.

(Go75) K. Goebel, G. R. Stevenson, J. T. Routti, and H. G. Vogt, "Evaluating dose rates due to neutron leakage through the access tunnels of the SPS", CERN Report LABIIRA/Note/75-10 (European Organization for Nuclear Research, 1975).

(Go76) P. J. Gollon, "Production of radioactivity by particle accelerators", Fermilab Report TM609 (Fermi National Accelerator Laboratory, 1976) and also IEEE Trans. Nucl. Sci. NS $\underline{23}$ No. 4 (1976) 1395-1400.

(Go78) P. J. Gollon, "Soil activation calculations for the anti-proton target area", Fermilab Report TM-816 (Fermi National Accelerator Laboratory, 1978).

(Gr85) R. V. Griffith, and J. H. Thorngate, "Neutron spectrometers for radiation monitoring at Lawrence Livermore National Laboratory", Rad. Prot. Dos. 10 (1-4) (1985) 125-135.

(Gr87) N. A. Greenhouse, T. M. De Castro, J. B. McCaslin, A. R. Smith, R.-K. Sun, and D. E. Hankins, "An evaluation of NTA film in an accelerator environment and comparisons with CR-39", Rad. Prot. Dos. 20 (1987) 143-147.

(Gr90) R. V. Griffith and L. Tommasino, in "Dosimetry for radiological protection at high energy particle accelerators", Chapter 1 in The dosimetry of ionizing radiation, Volume IV (K. R. Kase, B. E. Bjärngard, and F. H. Attix, editors, Academic Press, 1990).

(Gu01) K. K. Gudima, S. G. Mashnik, A. J. Sierk, "User manual for the code LAQGSM", Los Alamos National Laboratory Report LA-UR-01-6804 (Los Alamos, NM, 2001).

(Ha47) A. O. Hanson and M. L. McKibben, "A neutron detector having uniform sensitivity from $10 \mathrm{keV}$ to $3 \mathrm{MeV}$ ", Phys. Rev. 72 (1947) 673-677.

(Ha62) D. E. Hankins, "Neutron monitoring instrument having a response approximately proportional to the dose rate from thermal to $7.0 \mathrm{MeV}$ ", LA-2717 (Los Alamos Scientific Laboratory, 1962). 


\section{REFERENCES}

(Ha88) W. K. Hagan, B. L. Colborn, T. W. Armstrong, and M. Allen, "Radiation shielding calculations for a 70-250 MeV proton therapy facility", Nucl. Sci. Eng. 98 (1988) 272278.

(He85) N. E. Hertel and J. W. Davidson, "The response of Bonner spheres to neutrons from thermal energies to $17.3 \mathrm{MeV} "$, Nucl. Instr. and Meth. in Phys. Res. A238 (1985) 509516.

(He99) L. Heilbronn, R. S. Cary, M. Cronqvist, F. Deák, K. Frankel, A. Galonsky, K. Holabird, Á. Horvath, Á. Kiss, J. Kruse, R. M. Ronningen, H. Schelin, and Z. Seres, "Neutron yields from $155 \mathrm{MeV} /$ nucleon carbon and helium stopping in aluminum", Nucl. Sci. and Eng. I32 (1999) 1-15.

(Hö69) M. Höfert, "Radiation hazard of induced activity in air as produced by high energy accelerators", in Proceedings of the second international conference on accelerator radiation dosimetry and experience (Stanford, CA 1969) CONF-691101, National Technical Information Service, pp. 111-120. Results from this work are also found in (Sw79a).

(Hö80) M. Höfert and Ch. Raffnsøe, "Measurement of absolute absorbed dose and dose equivalent response for instruments used around high-energy proton accelerators", Nucl. Instr. and Meth. 176 (1980) 443-448.

(Hö84a) M. Höfert and G. R. Stevenson, "The assessment of dose equivalent in stray radiation fields around high-energy accelerators", CERN Report TIS-RP/131/CF (European Organization for Nuclear Research, 1984).

(Hö84b) M. Höfert, "The NTA emulsion: an ill-reputed but misjudged neutron detector", in PNLSA 12352 CONF-8308140 (Pacific Northwest Laboratory, 1984) or CERN Report TISRP/110/CF (European Center for Nuclear Research, 1984).

(Hu60) E. L. Hubbard, R. M. Main, and R. V. Pyle, "Neutron production by heavy-ion bombardments", Phys. Rev. 118 (1960) 507-514.

(Hu98) M. Huhtinen, "Method for estimating dose rates from induced radioactivity in complicated hadron accelerator geometries-Write-up of the FIASCO Code", CERN Report CERN/TIS-RP/IR/98-28 (European Organization for Nuclear Research, 1998).

(Hu03) M. Huhtinen, "Radioactivation at supercolliders", in Proc. $42^{\text {nd }}$ Workshop on Innovative Detectors for Supercolliders, Erice, Sicily, Italy, 28 September-4 October, 2003.

(IC59) International Commission on Radiation Protection, Report of Committee II on permissible dose for international radiation, ICRP Report No. 2 (Washington, DC, 1959).

(IC71) International Commission on Radiation Units and Measurements, Radiation protection instrumentation and its application, ICRU Publication 20 (ICRU, Bethesda, MD, 1971).

(IC73) International Commission on Radiological Protection, Data for protection against ionizing radiation from external sources, supplement to ICRP Publication 15, ICRP Publication 21 (ICRP, Washington, DC, 1973).

(IC78) International Commission on Radiation Units and Measurements, Basic aspects of highenergy particle interactions and radiation dosimetry, ICRU Report No. 28 (ICRU, Washington, DC, 1978). 


\section{REFERENCES}

(IC87) International Commission on Radiological Protection, Data for use in protection against external radiation, ICRP Report No. 51 (Pergamon Press, New York, 1987).

(IC91) International Commission on Radiological Protection, 1990 Recommendations of the International Commission on Radiological Protection, ICRP Report 60 (Pergamon Press, New York, 1991).

(IC96) International Commission on Radiological Protection, Conversion coefficients for use in radiological protection against external radiation, ICRP Publication 74 (Pergamon Press, Oxford, 1996).

(IC07) International Commission on Radiological Protection, The 2007 recommendations of the International Commission on Radiological Protection. Elsevier; ICRP Publication 103 (Oxford: Elsevier, 2007).

(In84) H. Ing and H. C. Birnboim, "A bubble-damage polymer detector for neutrons", Nuclear Tracks Radiation Measurements $\underline{8}$, No 1.4 (1984) 285-288.

(Ip94) N. E. Ipe and A. Fassò, "Gas bremsstrahlung considerations in the shielding design of the Advanced Photon Source synchrotron radiation beam lines", Nucl. Instr. and Meth. A351 (1994) 534-544.

(Ja68) R. G. Jaeger, Editor-in-chief, E. P. Blizard, A. B. Chilton, M. Grotenhuis, A. Hönig, Th. A. Jaeger, H. H. Eisenlohr, Engineering compendium on radiation shielding, Volume 1 Shielding fundamentals and methods (Springer-Verlag, New York, 1968).

(Ja75) J. D. Jackson, Classical electrodynamics, $2^{\text {nd }}$ edition (John Wiley and Sons, New York, 1975). Subsequent editions of this text have been published.

(Ja87) J. D. Jackson, editor, M. G. D. Gilchriese, D. E. Groom, J. R. Sanford, G. R. Stevenson, W. S. Freeman, K. O'Brien, R. H. Thomas, "SSC environmental radiation shielding", Superconducting Super Collider Central Design Group Report SSC-SR-1026 (Berkeley, CA, 1987).

(Ka89) A.N. Kalinovskii, N.V. Mokhov, and Yu.P. Nikitin, in Passage of high-energy particles through matter (American Institute of Physics, NY, 1989).

(Ka08) Kaye and Laby. Tables of physical constants; section 4.5.4, radiation quantities and units. Institute of Physics, National Physical Laboratory, UK. Available online at http://www.kayelaby.npl.co.uk/toc/. (Accessed October 29, 2013.)

(Kn79) G. F. Knoll, Radiation detection and measurement (John Wiley and Sons, New York, 1979). Subsequent editions of this classic text have been published.

(Ko81) E. J. Konopinski, Electromagnetic fields and relativistic particles (McGraw Hill, New York, 1981).

(Ko85) T. Kosako, T. Nakamura, and S. Iwai, "Estimation of response functions of moderating type neutron detectors by the time-of-flight method combined with a large lead pile", Nucl. Instr. and Meth. in Phys. Res. A235 (1985) 103-122.

(Ko93) A. Yu. Konobeyev and Yu. A. Korovin, "Tritium production in materials from C to Bi irradiated with nucleons of intermediate and high energies", Nucl. Instrm. and Meth. in Phys. Res. B82 (1993) 103-115. 


\section{REFERENCES}

(Kr97) O.E. Krivosheev and N.V. Mokhov, "A new MARS and its applications", FermilabConf-98/043 (1998) and SARE3 KEK Proceedings 97-5 (1997).

(Kr02) F. Krueger and J. Larson, "Chipmunk IV: Development of and experience with a new generation of radiation area monitors for accelerator applications", Nucl. Instrm. and Meth. in Phys. Res A495 (2002) 20-28.

(Ku99) T. Kurosawa, N. Nakao, T. Nakamura, Y. Uwamino, T. Shibata, N. Nakanishi, A. Fukumura, and K. Murakami, "Measurements of secondary neutrons produced from thick targets bombarded by high-energy helium and carbon ions", Nucl. Sci. and Eng. 132 (1999) 30-57.

(Ku00) T. Kurosawa, N. Nakao, T. Nakamura, H. Iwase, H. Sato, Y. Uwamino, and A. Fukumura, "Neutron yields from thick $\mathrm{C}, \mathrm{Al}, \mathrm{Cu}$, and $\mathrm{Pb}$ targets bombarded by 400 $\mathrm{MeV}$ /nucleon Ar, Fe, Xe, and 800 MeV/nucleon Si ions", Phys. Rev. C62 (2000) 044615 (11 pages).

(Le68) J. W. Leake, "An improved spherical dose equivalent neutron detector", Nucl. Instrm. and Meth. $\underline{63}$ (1968) 329-332.

(Le72) G. S. Levine, D. M. Squier, G. B. Stapleton, G. R. Stevenson, K. Goebel, and J. Ranft, "The angular dependence of dose and hadron yields from targets in $8 \mathrm{GeV} / \mathrm{c}$ and 24 $\mathrm{GeV} / \mathrm{c}$ extracted beams", Part. Acc. $\underline{3}$ (1972) 91-101.

(Le04). $\quad$ S.Y. Lee, Accelerator physics (World Scientific, Singapore, 2004).

(Li61) S. J. Lindenbaum, "Shielding of high energy accelerators", Ann. Rev. Nucl. Sci. 11 (1961) 213-258.

(Li95) J. C. Liu, W. R. Nelson, and K. R. Kase, "Gas bremsstrahlung and associated photonneutron shielding calculations for electron storage rings", Health Phys. 68 (1995) 105213.

(Li00) D. R. Lide, Editor, CRC Handbook of chemistry and physics (Chemical Rubber Publishing Company, Boca Raton, FL, 2000) 14-16.

(Li01) J. C. Liu and V. Vylet, "Radiation protection at synchrotron radiation facilities", Rad. Prot. Dos. $\underline{96}$ (2001) 345-357.

(Li05) J. C. Liu, A. Fassò, H. Khater, A. Prinz, and S. H. Rokni, "Radiation safety design for SPEAR3 Ring and synchrotron radiation beamlines", SLAC Report RP-05-33(Stanford Linear Acclerator Center, 2005).

(Lo84) K. A. Lowry and T. L. Johnson, "Modifications to interactive recursion unfolding algorithms and computer codes to find more appropriate neutron spectra", U. S. Naval Research Laboratory Report NRL-5430, Washington, DC (1984) and L. W. Brackenbush and R. I. Scherpelz, "SPUNIT: a computer code for multisphere unfolding", in Proc. of the Health Physics Society topical meeting on computer applications in health physics (Columbia Chapter of the Health Physics Society, Richland, WA, 1984).

(Ma67) R. E. Maerker and V. R. Cain, "AMC, a Monte-Carlo code utilizing the albedo approach for calculating neutron and capture gamma-ray distributions in rectangular concrete ducts", Report ORNL-3964 (Oak Ridge National Laboratory, 1967). 


\section{REFERENCES}

(Ma68) J. B. Marion and F. C. Young, Nuclear reaction analysis, graphs and tables (North Holland, Amsterdam, 1968).

(Ma88) G. Margaritondo, Introduction to synchrotron radiation (Oxford University Press, New York, 1988).

(Ma93) A. J. Malensek, A. A. Wehmann, A. J. Elwyn, K. J. Moss, and P. M. Kesich, "Groundwater migration of radionuclides at Fermilab", Fermilab Report TM-1851 (Fermi National Accelerator Laboratory, 1993).

(McC60) J. B. McCaslin, "A high-energy neutron-flux detector", Health Phys. 2 (1960) 399-407.

(McC68) J. B. McCaslin, H. W. Patterson, A. R. Smith, and L. D. Stephens, "Some recent developments in technique for monitoring high-energy accelerator radiation", In Proc. of the first international congress of radiation protection, International Radiation Protection Association, Part 2 (Pergamon Press, New York 1968) 1131-1137.

(McC85) J. B. McCaslin, P. R. LaPlant, A. R. Smith, W. P. Swanson, and R. H. Thomas, "Neutron production by $\mathrm{Ne}$ and $\mathrm{Si}$ ions on a thick $\mathrm{Cu}$ target at $670 \mathrm{MeV} / \mathrm{A}$ with application to radiation protection", IEEE Trans. on Nucl. Sci. NS-32, No. 5 (1985) 3104-3106.

(McC87) J. B. McCaslin, W. P. Swanson, and R. H. Thomas, "Moyer model approximations for point and extended beam losses", Nucl. Instr. and Meth. in Phys. Res. A256 (1987) 418426.

(McC88) J. B. McCaslin, R.-K. Sun, W. P. Swanson, J. D. Cossairt, A. J. Elwyn, W. S. Freeman, H. Jöstlein, C. D. Moore, P. M. Yurista, and D. E. Groom, "Radiation environment in the tunnel of a high-energy proton accelerator at energies near 1.0 TeV", LBL-24640 (Lawrence Berkeley, Laboratory, 1987), presented at the Seventh Annual Congress of the International Radiation Protection Association, Sydney, Australia, April, 1988.

(Mo86) N. V. Mokhov and J. D. Cossairt, "A short review of Monte Carlo hadronic cascade calculations in the multi-TeV energy region", Nucl. Instr. and Meth. in Phys. Res. A244 (1986) 349-355.

(Mo89) L. E. Moritz, "Measurement of neutron leakage spectra at a $500 \mathrm{MeV}$ proton accelerator", Health Phys. $\underline{56}$ (1989) 287-296.

(Mo95) N.V. Mokhov, "The MARS code system user's guide, version 13", Fermilab Report FN628 (Fermi National Accelerator Laboratory, 1995).

(Mo97) N. Mokhov, private communication (1997).

(Mo99) N. V. Mokhov and A. Van Ginneken, "Neutrino induced radiation at muon colliders", presented at the 1999 Particle Accelerator Conference, New York, New York, March 19April 2, 1999, Fermilab Report, FERMILAB-Conf-99-067 (Fermi National Accelerator Laboratory, 1999).

(Mo04) N. V. Mokhov, K. K. Guidima, C. C. James, M. A. Kostin, S. G. Mashnik, E. Ng, J.-F. Ostiguy, I. L. Rakhno, A. J. Sierk, and S. I. Striganov, "Recent enhancements to the MARS15 Code", Fermilab Report FERMILAB-Conf-04/053-AD (Fermi National Accelerator Laboratory, 2004). 


\section{REFERENCES}

(Mo06) N. V. Mokhov, E. I. Rakhno, and I. L. Rakhno, "Residual activation of thin accelerator components", Fermilab Report FERMILAB FN-0788-AD (Fermi National Accelerator Laboratory, 2006).

(Na78) T. Nakamura, M. Yoshida, and T. Shin, "Spectra measurements of neutrons and photons from thick targets of $\mathrm{C}, \mathrm{Fe}, \mathrm{Cu}$, and $\mathrm{Pb}$ by $52 \mathrm{MeV}$ protons", Nucl. Instr. and Meth. $\underline{151}$ (1978) 493-503.

(Na81) T. Nakamura and T. Kosako, "A systematic study on neutron skyshine from nuclear facilities, Part I: Monte-Carlo analysis of neutron propagation in air-over-ground environment from a monoenergetic source", Nucl. Sci, and Eng. 77 (1981) 168-181.

(Na85) T. Nakamura, "Neutron energy spectra produced from thick targets by light-mass heavy ions", Nucl. Instr. and Meth. in Phys. Res. A240 (1985) 207-215.

(Na05) T. Nakamura and L. Heilbronn, Handbook on secondary particle production and transport by high-energy heavy ions (World Scientific Publishing Co., Singapore, 2005).

(NC59) National Council on Radiation Protection and Measurements, Maximum permissible body burdens and maximum permissible concentrations of radionuclides in air and in water for occupational exposure, NCRP Report No. 22 (1959). Also listed as U. S. National Bureau of Standards Handbook No 69 (1959).

(NC71) H. H. Rossi (Chair), R. S. Caswell, F. P. Cowan, W. W. Havens, F. C. Maienschein, L. D. Marinelli, W. S. Synder, and C. A. Tobias, Protection against neutron radiation, NCRP Report 38 (National Council on Radiation Protection and Measurements, Bethesda, MD, 1971)

(NC77) E. A. Burrill, Chair, J. R. Beyster, G. L. Brownell, A. B. Chilton, J. Haimson, C. J. Karzmark, W. E. Kreger, J, M. Wyckoff, Radiation protection design guidelines for 0.1$100 \mathrm{MeV}$ particle accelerator facilities, NCRP Report 51 (National Council on Radiation Protection and Measurements, Bethesda, MD, 1977). This reference has been superceded by $(\mathrm{NCO})$.

(NC90) V. P. Bond (Chair), S. Abrahamson, E. J. Hall, J. D. Boice, Jr., R. J. M. Fry, D. Grahn, P. Groer, G. Hutchison, G .Littlefield, C. W. Mays, H. Smith, R. Ullrich, and J. F. Thomson, The relative biological effectiveness of radiations of different quality, NCRP Report 104 (National Council on Radiation Protection and Measurements, Bethesda, MD, 1990).

(NC03) R. H. Thomas (Chair), W. R. Casey, J. D. Cossairt, K. O'Brien, N. Rohrig, L. A. Slaback, G. B. Stapleton, and W. P. Swanson, Radiation protection for particle accelerator facilities, NCRP Report 144 (National Council on Radiation Protection and Measurements, Bethesda, MD, 2003).

(Ne68a) W. R. Nelson, "The shielding of muons around high energy electron accelerators", Nucl. Instr. and Meth. $\underline{66}$ (1968) 293-303.

(Ne68b) R. B. Neal, editor, The Stanford two mile accelerator (Benjamin Publishing Co., New York, 1968).

(Ne74) W.R. Nelson and K. R. Kase, "Muon shielding around high-energy electron accelerators, part I, theory", Nucl. Instr. and Meth., 120 (1974) 401-411, and W.R. Nelson, K. R. Kase, and G. K. Svensson, "Muon shielding around high-energy electron accelerators, Part II, Experimental Investigation", Nucl. Instr. and Meth., 120 (1974) 413-429. 


\section{REFERENCES}

(Ne85) W. R. Nelson, H. Hirayama, and D. W. O. Rogers, "The EGS4 code system", Report SLAC-235 (Stanford Linear Accelerator Center, 1985).

(Ne90) W. R. Nelson, H. Hirayama, and D. W. O. Rogers, "The EGS4 code system", SLAC Report SLAC-265 (Stanford Linear Accelerator Center, 1985).

(Ne97) W. R. Nelson, private communication, 1997.

(No70) L. C. Northcliffe and R. F. Schilling, "Range and stopping-power tables for heavy ions", Nucl. Data Tables A7 (1970) 233-463.

(OB71) K. O'Brien, "Neutron spectra in the side-shielding of a large particle accelerator", USAEC Health and Safety Laboratory Report HASL-240 (Health and Safety Laboratory, 1971).

(OB80) K. O'Brien, "The physics of radiation transport" in Computer techniques in radiation transport dosimetry, W. R. Nelson and T. M. Jenkins, Editors, Ettore Majorana International Science Series (Plenum Press, New York, 1978) 17-56.

(OB81) K. O'Brien and R. Sanna, "Neutron spectral unfolding using the Monte-Carlo method", Nucl. Instr. and Meth. 185 (1981) 277-286.

(OD68) A. A. O'Dell, C. W. Sandifer, R. B. Knowlen, and W. D. George, "Measurements of absolute thick-target bremsstrahlung spectra", Nucl. Instr. Meth. 61 (1968) 340-346.

(Oh80) W. F. Ohnesorge, H. M. Butler, C. B. Fulmer, and S. W. Mosko, "Heavy ion target area fast neutron dose equivalent rates", Health Phys. 39 (1980) 633-636.

(Pa58) H. W. Patterson and R. Wallace, "A method of calibrating slow neutron detectors", UCRL-8359 (University of California Radiation Laboratory, 1958).

(Pa73) H. W. Patterson and R. H. Thomas, Accelerator health physics (Academic Press, New York, 1973).

(Pa94) H. W. Patterson and R. H. Thomas, editors, A history of accelerator radiaton protection (Nuclear Technology Publishing, Ashford, Kent, United Kingdom, 1994).

(PDG04) Particle Data Group, Review of particle properties, Phys. Lett. B592 (2004). This report is updated and republished every two years and is available online at http://pdg.lbl.gov/ (Accessed October 29, 2013.) Many other links to useful information are provided in the online version of this reference.

(Pe00) M. Pelliccioni, "Overview of fluence-to-effective dose and fluence-to-ambient dose equivalent conversion coefficients for high energy radiation calculated using the FLUKA code", Rad. Prot. Dos. $\underline{88}$ (2000) 279-297.

(Pr89) R. E. Prael and H. Lichtenstein, "User guide to LSC: The LAHET code system", Los Alamos National Laboratory Report No. LA-UR-89-3014 (1989).

(Ra67) J. Ranft, "Improved Monte-Carlo calculation of the nucleon-meson cascade in shielding material", Nucl. Instr. and Meth. $\underline{48}$ (1967) 133-140. 


\section{REFERENCES}

(Ra72) J. Ranft and J. T. Routti, "Hadronic cascade calculations of angular distributions of integrated secondary particle fluxes from external targets and new empirical formulae describing particle production in proton-nucleus collisions", Part. Accel. 4 (1972) 101110 .

(Ra91) R. Rameika, "Labyrinths and penetration methodology-Version 1.3", "Fermilab Research Division shielding assessment document" (private communication 1991).

(Ra01) I. Rakhno, N. Mokhov, A. Elwyn, N. Grossman, M. Huhtinen, L. Nicholas, "Benchmarking residual dose rates in a NuMI-like environment", Fermilab Report FERMILAB-Conf-01/304-E (Fermi National Accelerator Laboratory, 2001). Also given in Proc. Amer. Nucl. Soc. Topical Meeting AccApp/ADTTA'01, Reno, Nevada, USA, Nov. 11-15-2001 (Omnipress, Omnipro-CD, ISBN:0-89446-666-7, 2002).

(Re12) S. D. Reitzner, "Update to the generic shielding design criteria", Fermilab Report TM2550-ESH (Fermi National Accelerator Laboratory, November 2012).

(Ri75) ～A. Rindi and R. H. Thomas, "Skyshine-a paper tiger?", Part. Accel. 7 (1975) 23-39.

(Ri82) A. Rindi, "Gas bremsstrahlung from electron storage rings", Health Phys. 42 (1982) 187193.

(Ro41) B. Rossi and K. Greisen, "Cosmic-ray theory", Rev. Mod. Phys. 13 (1941) 240-309. See also B. Rossi, High energy particles (Prentice-Hall, Englewood Cliffs, New Jersey, 1952).

(Ro55) H. H. Rossi and W. Rosenzweig, "A device for the measurement of dose as a function of specific ionization", Radiology 64 (1955) 404-411.

(Ro68) H. H. Rossi, "Microscopic energy distribution in irradiated matter", Radiation dosimetry Vol 1, F. H. Attix and W. C. Roesch, Editors (Academic Press, New York, 1968).

(Ro76) J. T. Routti and R. H. Thomas, "Moyer integrals for estimating shielding of high-energy accelerators", Nucl. Instr. and Meth. 76 (1969) 157-163.

(Ro80) J. T. Routi and J. V. Sandbert, "General purpose unfolding LOUHI78 with linear and nonlinear regularization", Comp. Phys. Comm. 21 (1980) 119-144.

(Ro83) N N Rohrig, "Plotting neutron fluence spectra", Health Phys. $\underline{45}$ (1983) 817-818.

(Ro90) D. W. O. Rogers and A. F. Bielajew, "Monte Carlo techniques of electron and photon transport for radiation dosimetry", Chapter 5 in The dosimetry of ionizing radiation, Volume III (K. R. Kase, B. E. Bjärngard, and F. H. Attix, editors, Academic Press, New York, 1990).

(Ru76) P. Ruffin and C. Moore, "A study of neutron attenuation in the E-99 labyrinth", Fermilab Radiation Physics Note No. 9 (Fermi National Accelerator Laboratory, 1976).

(Se71) B. de Sereville and P. Tardy-Joubert, "Propagation des Neutrons dans les Tunnels D'Access aux Accelerateurs de Haute Energie A Protons", CERN Report 71-16 Vol. 2 (European Organization for Nuclear Research, 1971) 725-774. 


\section{REFERENCES}

(Se81) W. Seelmann-Eggebert, G. Pfenning, H. Münzek, H. Klewe-Nebenius, Institut für Radiochemie, Nuklidkarte (Chart of the nuclides) Kernforschungszentrum Karlsruhe $\mathrm{GmbH}$, Karlsruhe, Germany (1981) (5. Aufluge). Similar information is found on a similar Chart of the Nuclides available from the U. S. Department of Energy's Knolls Atomic Power Laboratory and may be ordered through the following website: http://knollslab.com/nuclides.html. (Accessed October 29, 2013.)

(Se85) S. M. Seltzer and M. J. Berger, "Bremsstrahlung spectra from electron interactions with screened atomic nuclei and orbital electrons", Nucl. Instr and Meth. in Phys. Res. B12 (1985) 95-134.

G. Shen, C. Ankenbrandt, M. Atac, S. Ecklund, P. J. Gollon, J. Lach, J. MacLachlan, A. Roberts, L. A. Fajardo, R. Majka, J. N. Marx, P. Nemethy, L. Rosselet, J. Sandweiss, A. Schiz, and A. J. Slaughter, "Measurement of multiple scattering at 50 to $200 \mathrm{GeV} / \mathrm{c}$, Phys. Rev. D20 (1979) 1584-1588.

(St84a) G. R. Stevenson and R. H. Thomas, "A simple procedure for the estimation of neutron skyshine from proton accelerators", Health Phys. $\underline{46}$ (1984) 115-122.

(St84b) G. R. Stevenson, "The estimation of dose equivalent from the activation of plastic scintillators", Health Phys. 47 (1984) 837-847.

(St85) G. R. Stevenson, P. A. Aarnio, A. Fassò, J. Ranft, J. V. Sandberg, and P. Sievers, "Comparison of measurements of angular hadron energy spectra, induced activity, and dose with FLUKA82 calculations", Nucl. Instr. and Meth. in Phys. Res. A245 (1985) 323-327.

(St86) G. R. Stevenson, "Dose equivalent per star in hadron cascade calculations", CERN Report TIS-RP/173 (European Organization for Nuclear Research,1986). Also in Fassò et al. (Fa90). 


\section{REFERENCES}

(St87) G. R. Stevenson, "Empirical parametrization of the shielding of end-stops at high-energy proton accelerators", CERN Report TIS-RP/183/CF (European Organization for Nuclear Research, 1987).

(St01) G. R. Stevenson, "Induced activity in accelerator structures, air and water", Rad. Prot. Dos. $\underline{96}$ (2001) 373-380.

(Su65) A. H. Sullivan and T. R. Overton, "Time variation of the dose-rate from radioactivity induced in high-energy particle accelerators", Health Phys. 11 (1965) 1101-1105.

(Su88) E. A. Sudicky, T. D. Wadsworth, J. B. Kool, and P. S. Huyakorn, "PATCH 3D; Three dimensional analytical solution for transport in a finite thickness aquifer with first-type rectangular patch source", Prepared for Woodward-Clyde Consultants, Inc., Waterloo, Ontario, Canada (University of Waterloo, 1988).

(Su89) A. H. Sullivan, "The intensity distribution of secondary particles produced in high energy proton interactions", Rad. Prot. Dos. 27 (1989) 189-192.

(Su92) A. H. Sullivan, A guide to radiation and radioactivity levels near high energy particle accelerators (Nuclear Technology Publishing, Ashford, Kent, United Kingdom, 1992).

(Sw79a) W. P. Swanson, Radiological safety aspects of the operation of electron linear accelerators, IAEA Technical Report No. 188 (International Atomic Energy Agency, Vienna, 1979).

(Sw79b) W. P. Swanson, "Improved calculation of photoneutron yields released by incident electrons", Health Phys. 37 (1979) 347-358.

(Sw90) W. P. Swanson and R. H. Thomas, "Dosimetry for radiological protection at high energy particle accelerators", Chapter 1 in The dosimetry of ionizing radiation, Volume III (K. R. Kase, B. E. Bjärngard, and F. H. Attix, editors, Academic Press, 1990).

(Te82) K. Tesch, "The attenuation of the neutron dose equivalent in a labyrinth through an accelerator shield", Part. Accel. 12 (1982) 169-175.

(Te83) K. Tesch, "Comments on the transverse shielding of proton accelerators", Health Phys. $\underline{44}(1983) 79-82$.

(Te85) K. Tesch, "A simple estimation of the lateral shielding for proton accelerators in the energy range from 50 to $1000 \mathrm{MeV",} \mathrm{Rad.} \mathrm{Prot.} \mathrm{Dos.} 11$ (1985) 165-172.

(Te88) K. Tesch, "Shielding against high energy neutrons from electron accelerators-a review", Rad. Prot. Dos. 22 (1988) 27-32.

(Th84) R. H. Thomas and S. V. Thomas, "Variation and regression analysis of Moyer model parameter data", Health Phys. $\underline{46}$ (1984) 954-957.

(Th85) R. H. Thomas and G. R. Stevenson, "Radiation protection around high energy accelerators", Rad. Prot. Dos. 10 (1985) 283-301.

(Th88) R. H. Thomas and G. R. Stevenson, Radiological safety aspects of the operation of proton accelerators, IAEA Technical Report No. 283, (International Atomic Energy Agency Vienna, 1988). 


\section{REFERENCES}

(To96) M. M. C. Torres, Neutron radiation fields outside shielding at the Fermilab Tevatron, Ph.D. Thesis, The University of Michigan (Ann Arbor, Michigan, 1996).

(Tr90) G. Tromba and A. Rindi, "Gas bremsstrahlung from electron storage rings: a Monte Carlo evaluation and some useful formulae", Nucl. Instr. and Meth. A292 (1990) 700705 .

(Ts74) Y-S. Tsai, "Pair production and bremsstrahlung of charged leptons", Rev. Mod. Phys. $\underline{46}$ (1974) 815-851.

(Tu84) J. W. N. Tuyn, R. Deltenre, C. Lamberet, and G. Roubaud, "Some radiation aspects of heavy ion acceleration", Proc. $6^{\text {th }}$ Int. Cong. International Radiation Protection Association, Berlin (1984) 673. Also in CERN Report TIS-RP/125/CF (European Organization for Nuclear Research, 1984).

(Tu05) J. K. Tuli, Nuclear wallet cards, $7^{\text {th }}$ edition, National Nuclear Data Center; (Brookhaven National Laboratory, Upton, NY, 2005.) Available at http://www.nndc.bnl.gov/ (Accessed October 29, 2013.)

(Uw82) Y. Uwamino, T. Nakamura, and K. Shin, "Penetration through shielding materials of secondary neutrons and photons generated by $52 \mathrm{MeV}$ protons", Nucl. Sci. Eng. $\underline{80}$ (1982) 360-369.

(Va71) A. Van Ginneken, "Na ${ }^{22}$ production cross section in soil", Fermilab Report TM-283 (Fermi National Accelerator Laboratory, 1971).

(Va75) A. Van Ginneken and M. Awschalom, High energy particle interactions in large targets: Volume I, hadronic cascades, shielding, and energy deposition (Fermi National Accelerator Laboratory, 1975).

(Va78) A. Van Ginneken, "AEGIS, a program to calculate the average behavior of electromagnetic showers", Fermilab Report FN-309 (Fermi National Accelerator Laboratory, 1978).

(Va85) D. G. Vasilik, G. H. Erkkila and D. A. Waechter, "A portable neutron spectrometer/dosemeter", Rad. Prot. Dos. 10 (1985) 121-124.

(Va87) A. Van Ginneken, P. Yurista, and C. Yamaguchi, "Shielding calculations for multi-TeV hadron colliders", Fermilab Report FN-447 (Fermi National Accelerator Laboratory, 1987).

(Va93) K. Vaziri, V. Cupps, D. Boehnlein, D. Cossairt, A. Elwyn, and T. Leveling, "AP0 stack monitor calibration", Fermilab Radiation Physics Note No. 106 (Fermi National Accelerator Laboratory, May 1993).

(Va96) K. Vaziri, V. R. Cupps, D. Boehnlein, D. Cossairt, and A. Elwyn, "A detailed calibration of a stack monitor used in the measurement of airborne radionuclides at a high energy proton accelerator", Fermilab Report FERMILAB-Pub-96/037 (Fermi National Accelerator Laboratory, 1994).

(Va04) K. Vaziri, B. Hartman, S. Hawke, F. Krueger, and J. Larson, "Muon scope: characteristics and operation", Fermilab Radiation Physics Note No. 149 (Fermi National Accelerator Laboratory, May 2004). 


\section{REFERENCES}

(Vy91) V. Vylet, "Estimated sensitivity of the 'muon gun' to neutrons", Fermilab Radiation Physics Note. No. 92 (Fermi National Accelerator Laboratory, 1991).

(Wi52) R. R. Wilson, "Monte Carlo study of shower production", Phys. Rev. $\underline{86}$ (1952) 261-269.

(Wi03) H. Wiedemann, Synchrotron radiation (Springer-Verlag, New York, 2003).

(Yu83) P. M. Yurista and J. D. Cossairt, "Concrete shielding exterior to iron," Fermilab Report TM-1204 (Fermi National Accelerator Laboratory, 1983).

(Za87) J. M. Zazula, "Derivation of accelerator shielding parameters from adjoining high energy neutron transport calculations", in Theory and practices in radiation protection and shielding, Proceedings of the Radiation Protection and Shielding Division (American Nuclear Society, Knoxville, Tennessee, April 22-24, 1987) Vol 2, 434-443.

(Ze67) M. Zel'chinskij and K. Zharnovetski, "Differential recombination chamber", Badan Jadrowych Nuclear Research institute Report INR No. 739/XIX/D (Translated at CERN, 1967).

(Zi62) M. Zielczynski, "Use of columnar recombination to determine the biological effectiveness of radiation", Nucleonika $\underline{Z}$ (1-12) 175-182, Nukleonika (English translation) $\underline{7}(1-12)$ (1965) 213-223.

(Zi96) J. F. Ziegler, J. P. Biersack, and U. Littmark, The stopping and range of ions in solids (Pargamon, Press, New York, 1996), and J. P. Biersack and L. G. Haggmark, "A Monte Carlo computer program for the transport of energetic ions in amorphous targets", Nucl. Instr. and Meth. 174 (1980) 257-269. The SRIM code is available at the following URL: http://www.srim.org. (Accessed October 29, 2013.) 
INDEX

1973 Radiation Protection System (1973 System)

1990 Radiation Protection System (1990 System)

$1 / E$ spectrum

$1 / v$ law

aberrations

absorbed dose

absorbed dose rate

absorbed dose response (ion chambers)

accelerator environments (instrumentation)

activation detectors

activation due to high energy hadrons

activation equation

activity

advective (hydraulic flow)

AEGIS (code)

air gaps

airborne radioactivity

airborne radionuclides at electron accelerators

airborne radionuclides at proton accelerators

albedo coefficients

aluminum (as component material)

ambient dose equivalent

Andersson-Braun detector

angular deflection

angular distributions

angular flux density

anisotropic thermal expansion (of uranium)

Approximation B

atmospheric abundances of nuclides

atomic and nuclear properties

attenuation beyond the exit

attenuation in curved tunnels

attenuation in first leg of penetrations

attenuation in second leg of penetrations

attenuation length

attenuation length of the cascade

attenuation length (skyshine)

average energy (thermal neutrons)

azimuthal dependence

background counting rate

Becquerel

bending magnet spectrum

beryllium (as component material)

beta emitters

binomial distribution

bismuth (as a shielding material)

Boltzmann equation

Boltzmann operator

Bonner sphere technique

borated polyethlyene

boron (in shielding, see borated polyethyline)

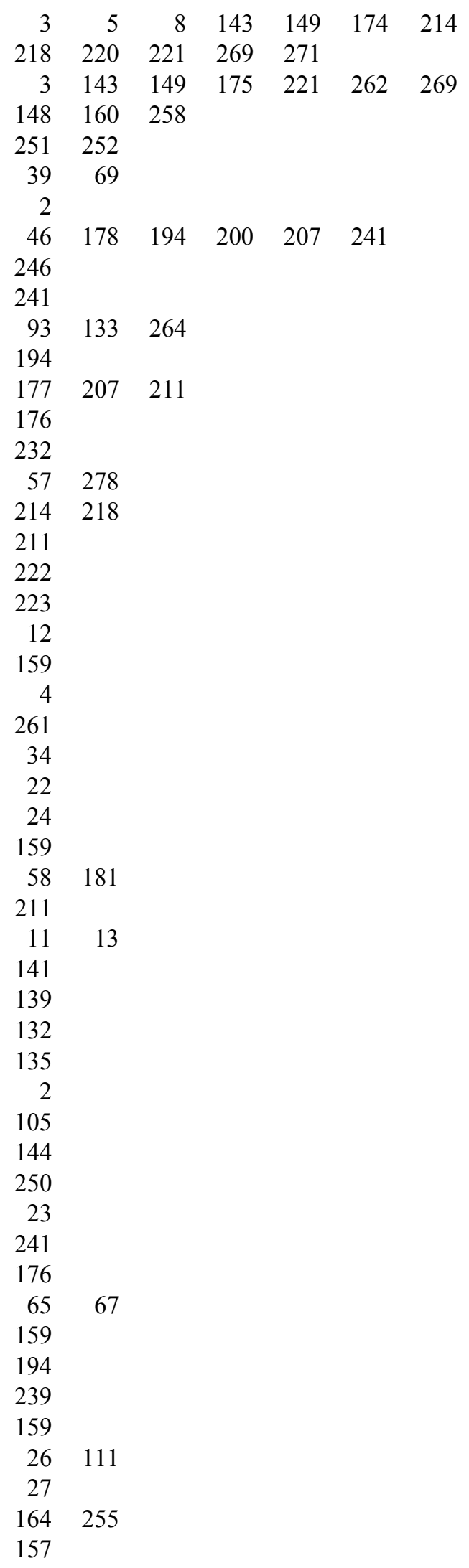


INDEX

\begin{tabular}{|c|c|c|c|c|c|c|c|}
\hline boron-10 (thremal capture reaction) & 157 & 251 & & & & & \\
\hline Bragg-Gray principle & 244 & 269 & & & & & \\
\hline bremsstrahlung & 41 & 42 & 72 & 181 & 222 & 281 & \\
\hline bremsstrahlung (spectra) & 44 & & & & & & \\
\hline bremsstrahlung path length & 222 & & & & & & \\
\hline Bremsstrahlung power & 75 & & & & & & \\
\hline bubble detectors & 249 & & & & & & \\
\hline buildup factor (hadronic cascade) & 1050 & 97 & & & & & \\
\hline Buildup factor (skyshine) & 144 & & & & & & \\
\hline BUNKI (code) & 258 & & & & & & \\
\hline cadmium (thermal capture reaction) & 254 & & & & & & \\
\hline calibration data (long counter) & 264 & & & & & & \\
\hline capacitance & 244 & & & & & & \\
\hline carbon-11 (reactions) & 266 & & & & & & \\
\hline cascade neutrons & 84 & 101 & 143 & & & & \\
\hline CASIM (code) & 113 & 199 & 204 & 278 & 282 & & \\
\hline characteristic angle (bremsstrahlung) & 47 & 74 & & & & & \\
\hline characteristic energy (synchrotron radiation) & 64 & & & & & & \\
\hline charge amplification (see gas multiplication) & 244 & & & & & & \\
\hline chemical toxicity of beryllium & 159 & & & & & & \\
\hline chemical toxicity of lead & 148 & & & & & & \\
\hline chicane (see labyrinth) & 127 & & & & & & \\
\hline chipmunk (Fermilab) & 245 & & & & & & \\
\hline Clapier and Zaidins parametrization & 94 & & & & & & \\
\hline Clark's principles & 97 & & & & & & \\
\hline collision length & 13 & 86 & & & & & \\
\hline columnar recombination & 270 & & & & & & \\
\hline Compton minimum & 53 & 56 & & & & & \\
\hline Compton scattering & 53 & 57 & 130 & & & & \\
\hline concentration model & 231 & & & & & & \\
\hline concrete (shielding) & 13 & 101 & 156 & 169 & 266 & & \\
\hline concrete density & 156 & & & & & & \\
\hline concrete shielding for hadrons & 100 & & & & & & \\
\hline concrete water content & 156 & & & & & & \\
\hline continuity equation (angular flux density) & 27 & & & & & & \\
\hline continuity equation (of water flow) & 233 & & & & & & \\
\hline cooling curves & 184 & 200 & & & & & \\
\hline cooling time & 177 & 194 & 204 & & & & \\
\hline cosmic ray muons & 258 & & & & & & \\
\hline counter telescopes & 274 & & & & & & \\
\hline counting rate & 240 & & & & & & \\
\hline counting statistics & 151 & 239 & & & & & \\
\hline CR-39 dosimeters & 249 & & & & & & \\
\hline critical energy & 42 & 53 & 65 & 101 & & & \\
\hline critical energy (synchrotron radiation) & 65 & & & & & & \\
\hline \multirow[t]{3}{*}{ cross section } & 2 & 24 & 47 & 53 & 55 & 82 & 97 \\
\hline & 102 & 133 & 177 & 200 & 225 & 251 & 225 \\
\hline & 251 & 264 & & & & & \\
\hline cumulative probability & 29 & & & & & & \\
\hline Curie & 176 & & & & & & \\
\hline current mode (instrumentation) & 244 & & & & & & \\
\hline curved tunnels (labyrinth) & 139 & & & & & & \\
\hline
\end{tabular}


INDEX

cylinders (moderators) 263

danger parameter 200

Darcy's Law

dead time

$232 \quad 234$

decay constant

$241 \quad 242$

176

decay length

decay muons

decay path

deflection parameter (see wiggler strength parem.)

14

50

defocused (beam)

density of earth

DePhanger long counter

$242 \quad 247 \quad 250$

$119 \quad 120$

$119 \quad 109 \quad 112$

depleted uranium (shielding)

Derived Air Concentrations (DACs)

Derived Concentration Guides (DCGs)

70

36

156

264

159

$218 \quad 221$

determination of the source factor (labyrinths)

219

143

deuteron (binding energy)

$220 \quad 221$

deuteron stripping and breakup reactions

92

differential neutron flux

92

258

differential probability

differential yield

differential yield (giant resonance)

28

23

$\begin{array}{lll}44 & 49 & 93 \\ 93 & \end{array}$

diffusion (in hydraulic flow)

dipole magnets

216

33

direct beam of electrons $\quad 41$

direct beams of protons $\quad 82$

direct muons 120

directional sensitivity 242

dispersion $\quad 39$

dose equivalent per star fluence 113

dose equivalent

dose equivalent per fluence

dose equivalent per star density

$\begin{array}{rrrrrrr}3 & 8 & 41 & 48 & 52 & 60 & 90 \\ 149 & 229 & 245 & 250 & & & \\ 4 & 78 & 97 & 143 & 149 & 261 & 274 \\ 113 & & & & & & \end{array}$

dose equivalent response (recombination chamber)

273

dose equivalent response (rem-meter) 260

dose rate effects (see dead time) 249

dosimeters 243

drinking water standards $\quad 230$

drop points (in LET spectrometry) 268

duty factor 242

dynamic range 241

earth (shielding) 155

effective dose 5

effective dose per fluence 5

effective porosity 231

EGS (code) 57

elastic scattering of neutrons (in a shield)

electric field 32

electrical charge 243

electromagnetic cascade

electromagnetic fields 
INDEX

electromagnetic forces

electron accelerators

electron accelerators (activation at)

electron volt

electronic equilibrium

electron-initiated shower

endothermic (nuclear reaction)

excitation function (energy dependence)

energy deposition

energy groups

energy loss per ion pair

energy loss per revolution

energy transfer

energy units

environmental effects

environmental factors

environmental media

equivalent dose

evaporation neutrons

exothermic nuclear reaction

exposure rate

factorization approximation

fading

field integral

fission counters

fluence

fluence, total

FLUKA (code)

flux density

focal length

focused (beam)

gas bremsstrahlung

gas bremsstrahlung-secondary photons

gas bremsstrahlung-neutron production rates

gas multiplication

Gauss Law (electrostatics)

Gaussian distribution

Gaussian plume model

Geiger-Müller

geometrical cross section

geometrical optics

giant photonuclear resonance neutrons

glow curve

Gollon's Rules of Thumb

gradient (atmospheric or hydraulic)

gradient (of quadrupole)

Gray

gross counting rate

groundwater resources

hadron shielding (low energy protons)

hadronic cascade

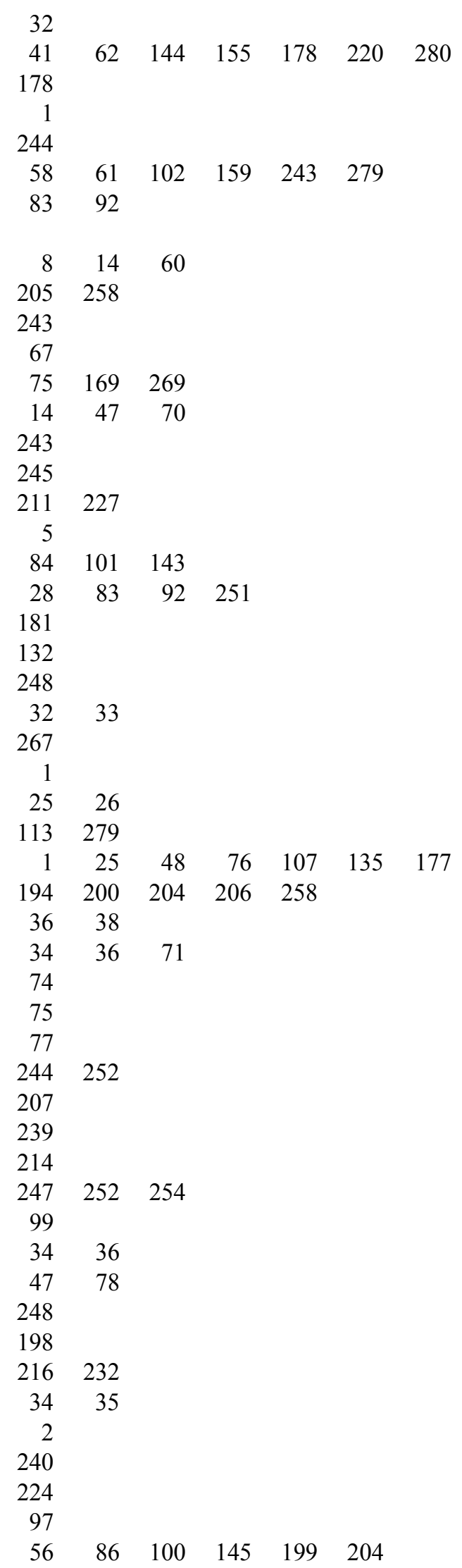


INDEX

282

hadrons produced by electromagnetic cascade 62

half-life, half-lives 176

head (hydraulic) 232

heat transfer characteristics $\quad 155$

heavy ion neutron yield formula 96

heavy ions 15

helium-3 (thermal capture reaction) 253

HETC (code)

high atomic number (shielding) materials

high energy particles

113

$\begin{array}{rrrrrr}43 & 46 & 155 & 158 & 173 & \\ 15 & 41 & 50 & 63 & 72 & 194 \\ 267 & 278 & & & & \end{array}$

histories (Monte Carlo) 28

hydraulic conductivity 233

hydraulic gradient 232

hydraulic head 230

hydrogenous materials (detectors) 255

hydrogenous materials (shielding) 157

ICRP (International Commission on Radiation Protection

ICRU (International Commission on Radiation Units and

Measurement)

ICRU Sphere

image

21

immersion

$92 \quad 111 \quad 270$

$200 \quad 279$

278

232

induced activation

induced radioactivity

induced radioactivity (environmental)

4

inelastic scattering of neutrons (in a shield)

infinite cloud

220

injection events (light sources) 68

insertion devices 285

instruments 239

integration mode

interaction length (high energy limit)

244

Interative recursion method 258

ionization chamber

ISO (geometry)

iron (density of)

iron (shielding)

iron shield "capped" (with concrete)

irradiation period

isotropic (giant resonance)

kaon decay

kinetic energy

labyrinth, see penetration

labyrinth (neutron spectrum in) 164

labyrinth scaling rule 134

labyrinth source term rule of thumb 143

LAHET (code) 279

large energy domain of neutron radiation fields 242

lateral shower development $\quad 60$

$\begin{array}{ll}\text { leachability } & 227\end{array}$

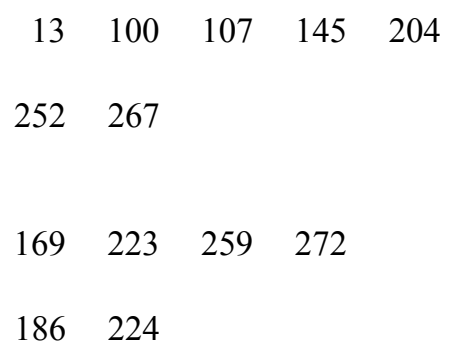


INDEX

leachable activity 227

lead (shielding)

leakage of low energy neutrons through iron

169

least squares fitting

$258 \quad 272$

legs (penetrations)

$\begin{array}{llll}130 & 132 & 135 & 164\end{array}$

LET spectrometry

269

lethargy plot

160

light source

limiting attenuation at high energy

64

Lindenbaum model

linear absorption coefficient

$98 \quad 102$

102

linear energy transfer (LET)

line source

4

76

Liouville's theorem

39

lithium-6 (thermal capture reaction)

252

long counter

263

longitudinal shower development

57

Lorentz force

32

LOUHI (code)

low atomic number (shielding) materials

258

43

macroscopic cross sections

macroscopic removal cross section

magnetic deflection

magnetic field

magnetic focusing

maintenance activities (residual activity)

MARS (code)

mass attenuation length

mass stopping power

maximum angle (muons)

Maximum Permissible Concentrations (MPCs)

maximum permissible dose equivalent

Maxwell-Boltzmann distribution

Maxwellian distribution

Maxwell's equations

maze (see penetrations, labyrinth)

$\begin{array}{llll}158 & 178 & 194 & 218\end{array}$

155

113

$\begin{array}{lll}278 & 280 & 282\end{array}$

MCNP (code)

mean energy per photon (synchrotron radiation)

mean free paths (for tunnel transmission)

mean-life

133

14

median energy (synchrotron radiation)

meteorological conditions

metric of radiation detriment

$\begin{array}{ll}65 & 282\end{array}$

$215 \quad 217$

microscopic removal cross section

minimum (muon) momentum

minimum ionizing (particle)

mixed radiation fields

moderated neutron detectors 
INDEX

\begin{tabular}{|c|c|c|c|c|c|c|c|}
\hline Molière radius & 55 & 76 & & & & & \\
\hline momentum & 11 & 15 & 33 & 39 & 155 & 204 & 252 \\
\hline momentum spectrum (muons) & 119 & & & & & & \\
\hline \multirow[t]{3}{*}{ Monte Carlo } & 15 & 25 & 28 & 41 & 57 & 74 & 82 \\
\hline & 86 & 109 & 111 & 113 & 118 & 132 & 143 \\
\hline & 200 & 204 & 228 & 255 & 258 & 278 & 282 \\
\hline Monte Carlo inverse transform & 28 & & & & & & \\
\hline Monte Carlo method & 28 & & & & & & \\
\hline most probable energy (thermal neutrons) & 250 & & & & & & \\
\hline mouth (penetrations) & 132 & & & & & & \\
\hline Moyer integral & 111 & & & & & & \\
\hline Moyer model & 105 & & & & & & \\
\hline Moyer model for a line source & 110 & & & & & & \\
\hline Moyer model for a point source & 105 & & & & & & \\
\hline Moyer model (labyrinth) & 143 & & & & & & \\
\hline Moyer model parameters & 108 & & & & & & \\
\hline Moyer's simplifying assumptions & 108 & & & & & & \\
\hline multi-nucleon transfer reactions & 185 & 193 & & & & & \\
\hline multiple Coulomb scattering & 15 & 20 & 39 & 42 & 105 & & \\
\hline multiplicity & 104 & 108 & 143 & 214 & 242 & & \\
\hline muon (radiation) fields & 91 & 242 & 247 & 274 & & & \\
\hline muon finder (Fermilab) & 275 & & & & & & \\
\hline muons & 3 & 8 & 15 & 28 & 42 & 91 & 101 \\
\hline muons (at electron accelerators) & 50 & & & & & & \\
\hline muons (at proton accelerators) & 90 & 105 & 118 & 120 & & & \\
\hline \multicolumn{8}{|c|}{ NCRP (National Council on Radiation Protection and } \\
\hline Measurements) & 3 & 6 & 21 & 218 & & & \\
\hline net counting rate & 240 & & & & & & \\
\hline neutron albedo coefficients & 128 & & & & & & \\
\hline neutron attenuation in labyrinths & 131 & & & & & & \\
\hline neutron emission (giant resonance) & 47 & & & & & & \\
\hline neutron energy spectra & 133 & 146 & 160 & 172 & & & \\
\hline neutron fluence and dosimetry & 174 & & & & & & \\
\hline neutron importance & 146 & & & & & & \\
\hline neutron spectra (due to protons) & 86 & & & & & & \\
\hline neutron spectra (due to electrons) & 47 & 49 & & & & & \\
\hline neutron spectra due to ions & 92 & & & & & & \\
\hline neutron spectrum measurements & 155 & 255 & & & & & \\
\hline neutron yields & 48 & 78 & 84 & 92 & 96 & 143 & 160 \\
\hline neutrons (electromagnetic cascade) & 62 & & & & & & \\
\hline normal (distribution) & 239 & & & & & & \\
\hline normalized transverse emittance & 39 & & & & & & \\
\hline nuclear interaction length & 2 & 13 & 93 & 100 & 113 & 118 & 199 \\
\hline nuclear material & 159 & & & & & & \\
\hline nuclear temperature & 48 & & & & & & \\
\hline nuclear track emulsions (NTA) & 248 & & & & & & \\
\hline number density & 62 & 177 & 226 & & & & \\
\hline off-axis source point (in labyrinths) & 134 & & & & & & \\
\hline omega factor ( $\omega$-factor) & 204 & & & & & & \\
\hline operating modes (light sources) & 72 & & & & & & \\
\hline operational quantity & 5 & & & & & & \\
\hline optic axis & 34 & & & & & & \\
\hline
\end{tabular}


INDEX

pair production

pair production (muons)

50

52

paraffin (shielding)

partial density

penetrations (see also labyrinths)

period (light source insertion devices)

personal dose equivalent

phoswich detector

photon albedo coefficients

photoelectric effect

photographic film

photon mean free path

photon-initiated shower

physical constants

physical quantity

pion decay

point source

Poisson distribution

polarization

polyethylene (detectors)

polyethylene (shielding)

porosity (see effective porosity)

primary particles (or beam)

primary radiation fields

production cross sections

proportional counters

protection quantity

proton recoils

proton recoils (detectors)

pseudospheres (moderators)

pulsed radiation fields

pyrophoric (property of uranium)

quadrupoles

quality factor

quasi-deuteron neutrons

Q-value (nuclear reaction)

$\mathrm{rad}$

radiated power (synchrotron radiation)

radiation length

radiation weighting factors

radiative processes

radiofrequency radiation

radiological standards

random number

Ranft model

range

range (muons)

range straggling

range straggling (muons)

ratemeter mode

ray tracing (light sources)
158

$97 \quad 207$

$\begin{array}{lll}79 & 112 & 127\end{array}$

69

4

259

127

57

247

$\begin{array}{ll}53 & 75\end{array}$

$58 \quad 61$

11

2

28

25

142

240

21

255

157

$231 \quad 236$

$6 \quad 23$

$\begin{array}{llll}23 & 41 & 82 & 92\end{array}$

$\begin{array}{llll}55 & 62 & 177 & 194\end{array}$

$\begin{array}{llll}252 & 254 & 261 & 269\end{array}$

5

248

268

263

247

159

34

3

49

82

2

67

21

5

41

34

21

29

$86 \quad 278$

$\begin{array}{lll}15 & 41 & 91\end{array}$

$\begin{array}{llll}15 & 50 & 80 & 101\end{array}$

19

19

244

79 
INDEX

reaction threshold

real image 36

recombination chamber technique

$139 \quad 270$

reflection coefficients (see albedo coefficients)

regulatory standards

relative error

relativistic kinematics

relativistic mass

rem

rem-meter

removable induced activity (contamination)
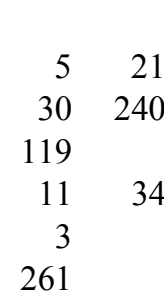

removal cross section theory

$\begin{array}{llll}5 & 21 & 218 & 228\end{array}$

$30 \quad 240$

119

11

34

3

261

247

97

resolving time

241

response functions

255

rest energy

RF cavities

right-hand rule

ROT (geometry)

Rossi counter

saturation (activity)

saturation cross sections

scarecrow (Fermilab)

scintillation telescopes

scintillators

secondary particles (or beam)

self-reading pocket ion chambers

shielding against muons (at proton accelerators)

shielding materials

shower maximum

SI (Système Internationale) units

Sievert

silver (thermal capture reaction )

single resident model

11

258

$\begin{array}{lllll}15 & 70 & 86 & 101 & 130\end{array}$

35

5

269

177

212

246

274

253

6

186

247

118

80

58

121
77

$181 \quad 186$

single-nucleon transfer reactions

skyshine

sodium (in concrete)

soil (activation)

soil shielding

soil water content

solid angle

source strength (skyshine)

source terms (electromagnetic cascade)

1

3

254

231

185

127

207

211

20

155

5

144

59

260

spatially nonuniform neutron fields

11

special theory of relativity

176

specific activity

specific energy

specific gamma-ray constant

92

181

spherical moderators

255

stability classes (meteorological)

214

stable isotopes (in the atmosphere)

211

stacks (ventilation)

$215 \quad 217$ 
INDEX

standard deviation $\quad 238$

standard deviation (net counting rate) 239

star density

star fluence

steering (quadrupole)

stopping power

store (light source)

straight penetration

streaming

Sullivan's approximation for muons

Sullivan's formula

Sullivan-Overton approximation

superheated droplets

superlinearity

Sutton's equation

Swanson's rules of thumb

SWIFT (code)

synchrotron radiation

temperature gradient (meteorology)

tertiary particles (or beam)

Tesch approximation

the 1973 System (The 1973 Radiation Protection System)

the 1990 System (The 1990 Radiation Protection System)

thermal neutron cross section

thermal neutron detectors

thermal neutron flux density

thermal neutrons

thermoluminescent dosimeters (TLDs)

thick targets

thin lens approximation

thin lens equation

thin targets

threshold energy (nuclear reaction)

thresholds (for activation)

tissue equivalent (materials)

tissue-equivalent proportional chamber (TEPC)

top-off mode (light sources)

total energy

total yield

track etch dosimeters

trajectories (Monte Carlo)

transition curve

transverse emittance

tungsten (shielding)

undulator

undulator frequency

unfolding (neutron energy spectra)

uniform irradiation (of walls)

uniform magnetic field

unit length (in penetrations)

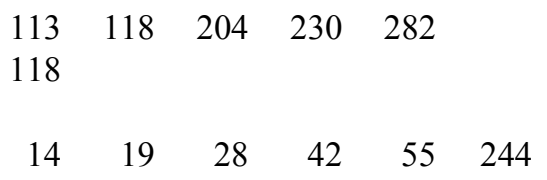


INDEX

\author{
universal dose attenuation curves (in labyrinths) \\ unshielded radiation field \\ upper energy (in skyshine) \\ uranium (depleted) \\ uranium (shielding) \\ ventilation \\ very high thresholds (reactions) \\ virtual image \\ water (shielding) \\ water and geological media activation \\ wavelength shifter \\ weights (Monte Carlo) \\ wiggler \\ wiggler strength parameter \\ wood (shielding) \\ $\omega$-factor (omega-factor) \\ yield (of particles) \\ zirconium (as component material)
}

134

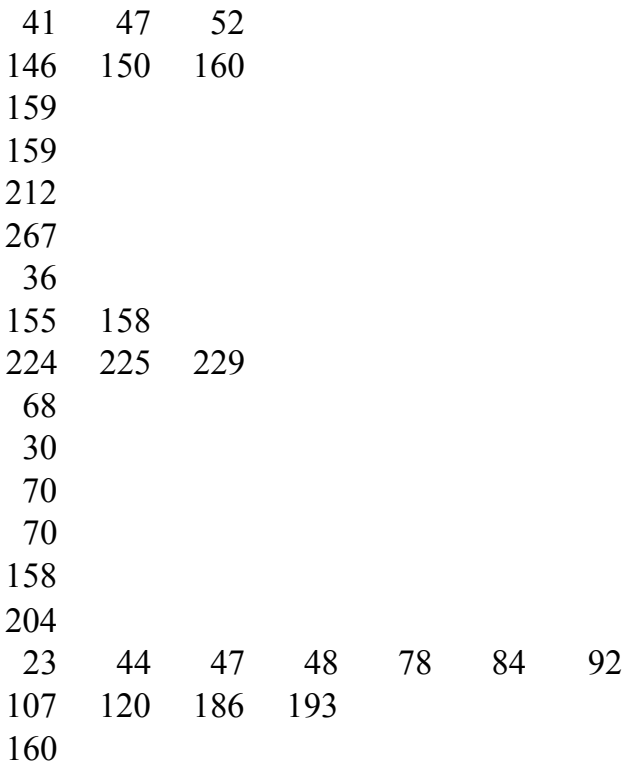

